\title{
FORMULATION OF A SEARCH STRATEGY FOR SPACE DEBRIS AT GEO
}

\author{
A Thesis \\ Presented to the \\ Faculty of California Polytechnic State University, \\ San Luis Obispo \\ In Partial Fulfillment \\ Of the Requirements for the Degree \\ Master of Science in Aerospace Engineering
}

By

James Patrick Biehl

July 2010 
(C) 2010

James Patrick Biehl

ALL RIGHTS RESERVED 


\section{Committee Membership}

TITLE:

AUTHOR: James Patrick Biehl

DATE SUBMITTED: July 6, 2010
FORMULATION OF A SEARCH STRATEGY FOR SPACE DEBRIS AT GEO

Signature

Committee Chair

Dr. Ed Barker

Committee Member

Dr. Eric A. Mehiel

Committee Member

Dr. Jordi Puig-Suari

Committee Member
Signature

Signature

Signature 


\title{
ABSTRACT
}

\section{FORMULATION OF A SEARCH STRATEGY FOR SPACE DEBRIS AT GEO}

\author{
By \\ James Patrick Biehl
}

The main purpose of this thesis is to develop a search strategy for space debris that are in the geosynchronous orbit (GEO) region. The search strategy is not an effort to find the object initially but rather if found one time to aid in finding it again within a small time frame. This was a request from NASA Johnson Space Center Orbital Debris Program Office through the MODEST, Michigan Orbital Debris Survey Telescope, program. A single definitive search pattern was not found, however depending on the COEs of the orbit specific search strategy can be employed. These search strategies are far from perfect and can be improved upon with more rigorous testing as well as a larger data sample.

Another goal is to look for correlation between the orbital parameters and the errors in the predicted right ascension (RA) and the declination (DEC). This was accomplished by varying the different orbital parameters by $\pm 10 \%$ individually while holding the other parameters constant. This showed some correlation existed between some parameters and their errors, in particular there was correlation between a variation in right ascension of ascending node (RAAN) and the value of RAAN itself. The correlation found was that with the higher the value of RAAN the larger the RMS error. 


\section{TABLE OF CONTENTS}

LIST OF FIGURES …......................................................................................... vii

LIST OF ACRONYMS _............................................................................................ ix

1 INTRODUCTION...............................................................................................

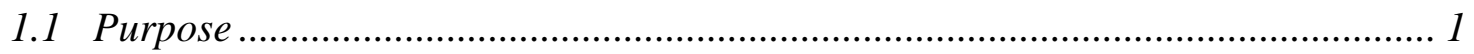

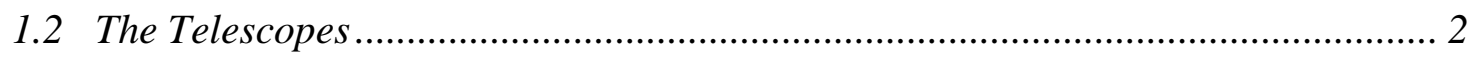

2 LITERATURE REVIEW ......................................................................................2

2.1 Asteroid Search Strategy............................................................................. 2

2.2 Faint GEO Objects ...................................................................................... 3

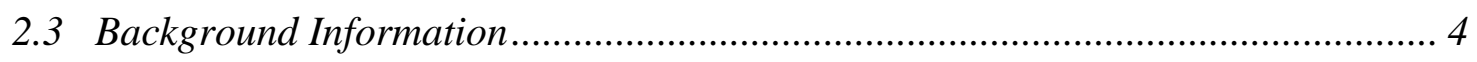

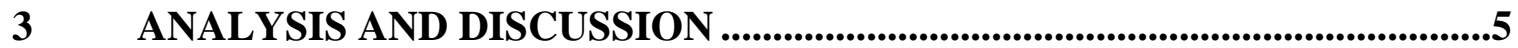

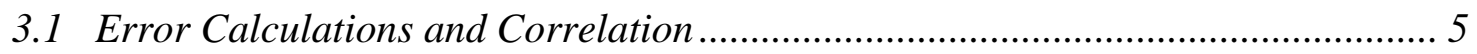

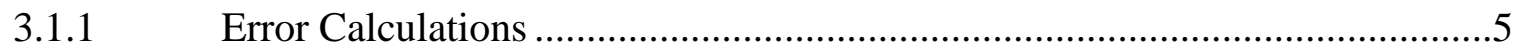

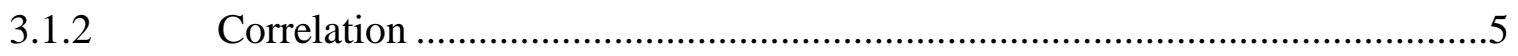

3.2 The Data

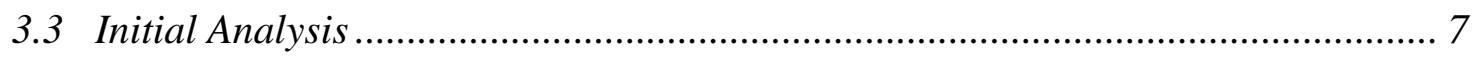

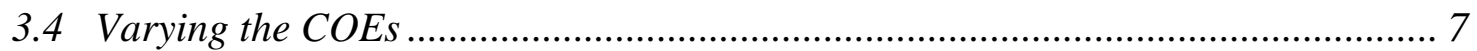

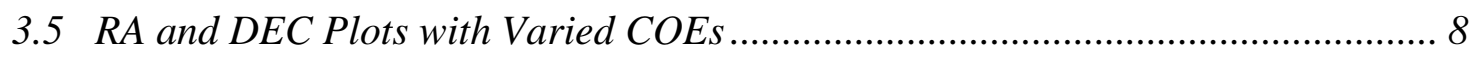

3.5.1 Trends in Errors with Different COEs ........................................................

3.5.2 The Relationship between INC and Error in DEC......................................13

3.5.3 COEs That Most Impact the Error ............................................................15

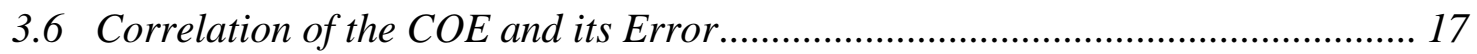

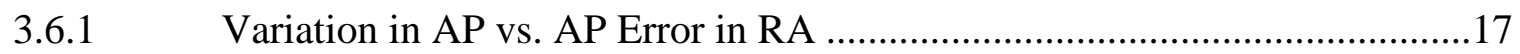

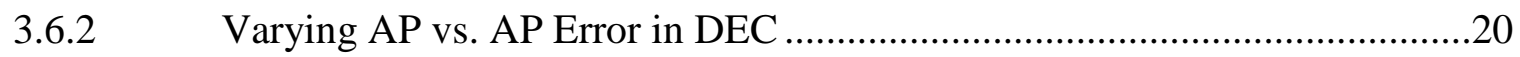

3.6.3 Varying MANOM vs. MANOM Error in RA and DEC ..............................21

3.6.4 Varying RAAN vs. RAAN Error in RA and DEC ......................................23

3.6.5 Varying AP, MANOM and RAAN vs. Themselves Error in RMS ................25

3.6.6 What Errors Fall within the Field of View .................................................28

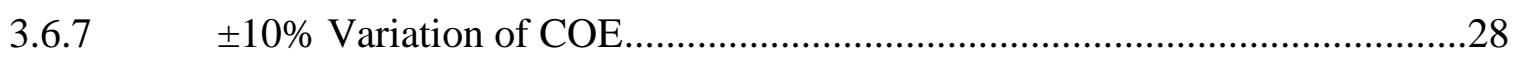




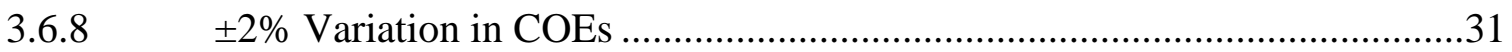

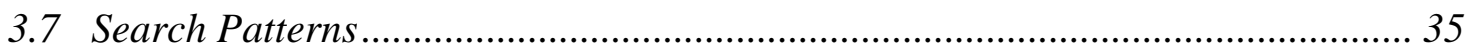

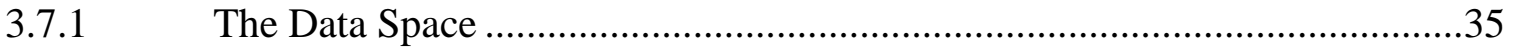

3.7.2 Type 1: Search Strategy for High Inclination Greater than 12 Degrees ........38

3.7.3 Type 2: Search Strategy for Low Inclination Less Than or Equal to 12

Degrees $\quad 41$

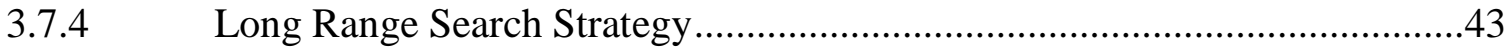

3.7.5 Summary of Search Patterns .............................................................44

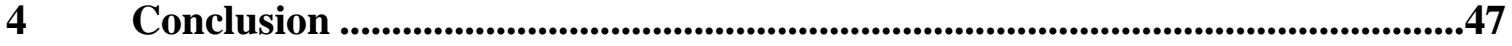

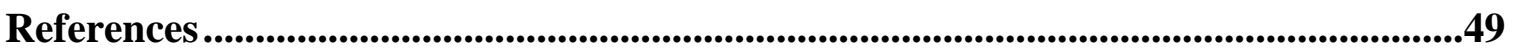

APPENDIX A: FULL ORBIT PLOTS 10\% VARIATION...........................................50

APPENDIX B: FIRST PREDICTED POINT 10\% VARIATION.............................115

APPENDIX C: FULL ORBIT SELECT PLOTS 2\% VARIATION.......................245

APPENDIX D: FIRST PREDICTED POINT 2\% VARIATION ................................248

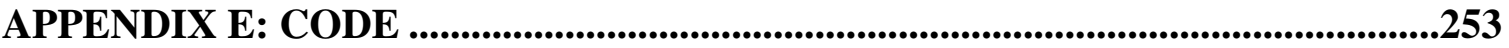




\section{LIST OF FIGURES}

Figure 1. Search Area from Milani ${ }^{1}$ .3

Figure 2. Showing the error in inclination appears only in DEC and error in right ascension of the ascending node appears only in RA

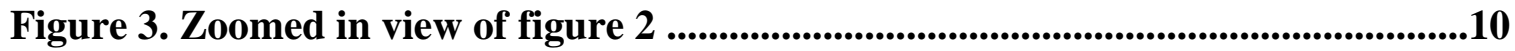

Figure 4. Shows the variation in the RAAN affecting the RA .................................11

Figure 5. All COEs of Figure 2 ............................................................................ 12

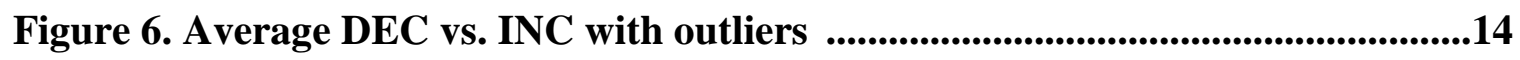

Figure 7. Average DEC vs. INC with largest DEC error removed ............................15

Figure 8. Example of high error for AP and MANOM ................................................16

Figure 9. RA Varying AP vs. AP from Original Prediction ......................................18

Figure 10. RA Varying AP vs. AP from Original Prediction ....................................19

Figure 11. RA Varying AP vs. AP from Actual Position...........................................20

Figure 12. DEC Varying AP vs. AP from Original Prediction ......................................21

Figure 13. RA Varying MANOM vs. MANOM from Original Prediction ...............22

Figure 14. DEC MANOM vs. MANOM from Original Prediction ............................23

Figure 15. RA Varying RAAN vs. RAAN from Original Prediction .........................24

Figure 16. DEC Varying RAAN vs. RAAN from Original Prediction ........................25

Figure 17. RMS Varying RAAN vs. RAAN from Original Prediction ......................26

Figure 18. RMS Varying MANOM vs. MANOM from Original Prediction ............27

Figure 19. RMS Varying AP vs. AP from Original Prediction .................................27

Figure 20. 0.2 Degree View (C9 Telescope) for an object .......................................29

Figure 21. 2 Degree View (MODEST Telescope) for the same object ..........................30

Figure 22. Full view of the same object with $10 \%$ variation ......................................31 


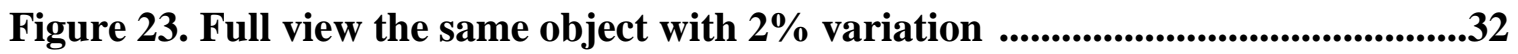

Figure 24. 0.2 degree (C9 Telescope) of same object with $2 \%$ variation ...................33

Figure 25. 2 degree (MODEST Telescope) of same object with 2\% variation ..........34

Figure 26. Full View of the 1st prediction for all observation sets ............................36

Figure 27. Full View of the 1st prediction for observation sets with a base

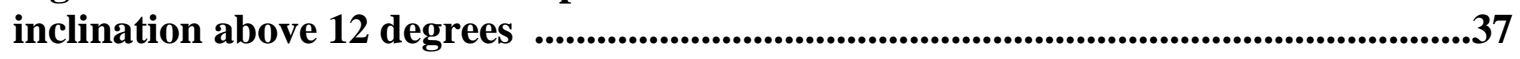

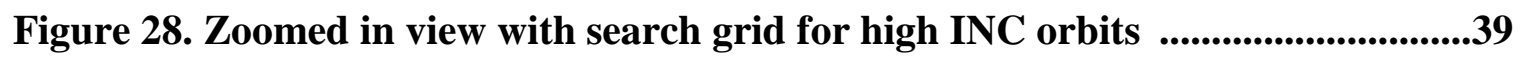

Figure 29. Zoomed in view with search grid for high INC orbits MM low priority .39

Figure 30. Zoomed in view with search grid for high INC orbits INC low priority..40

Figure 31. Zoomed in view with search grid for high INC orbits telescope ...............40

Figure 32. Zoomed in view with search grid for low INC orbits MM low priority ...42

Figure 33. Zoomed in view with search grid for low INC orbits telescope................. 43

Figure 34. Multiple COE Variation Full Orbit .................................................................45

Figure 35. Multiple COE Variation First Prediction, Equal Axis .................................46 


\section{LIST OF ACRONYMS}

$\begin{array}{ll}\text { AP } & \text { Argument of Perigee } \\ \text { COE } & \text { Smarts } 0.9 \text { m Telescope } \\ \text { CTIO } & \text { Classical Orbital Elements } \\ \text { DEC } & \text { Declination } \\ \text { ECC } & \text { Eccentricity } \\ \text { GEO } & \text { Geosynchronous Orbit } \\ \text { INC } & \text { Inclination } \\ \text { MANOM } & \text { Mean Anomaly } \\ \text { MM } & \text { Mean Motion } \\ \text { MODEST } & \text { Michigan Orbital Debris Survey Telescope } \\ \text { NASA } & \text { National Aeronautics and Space Administration } \\ \text { RA } & \text { Right Ascension } \\ \text { RAAN } & \text { Right Ascension of the Ascending Node } \\ \text { RMS } & \text { Root Mean Square } \\ \text { Rwo Line Element }\end{array}$




\section{INTRODUCTION}

\subsection{Purpose}

The purpose of this thesis is to create a search strategy to reacquire orbital debris when the when the object fails to appear at the predicted location for NASA Johnson Space Center Orbital Debris Program Office through the MODEST, Michigan Orbital

Debris Survey Telescope, program. Objects are located using the MODEST telescope, which tracks a specific RA and DEC all night. These objects orbit is then predicted following the assumption that the objects path is circular. This information is then transferred to another telescope, referred to as $\mathrm{C} 9$, which performs the tracking of the object. The data collected from this telescope is then used to further refine the orbit. The C9 telescope will track the object several times over the course of the night, the question arises, what happens when the object is not in C9's field of view?

At this point it would be advantages to have a search strategy in place that would increase the likelihood of finding an object that is not found where it was predicted. In order to accomplish this goal two sub goals were created, one to look for a correlation between error in one of the classical orbital elements (COEs) vs. the actual COE. The COEs analyzed in this report are Argument of Perigee, Eccentricity, Inclination, Mean Anomaly, Mean Motion, and Right Ascension of the Ascending Node. The other sub goal is to look for to look for trends in the error, such as all of the error being in declination or right ascension. 


\subsection{The Telescopes}

The MODEST telescope is located at the Cerro Tololo Inter-American Observatory (CTIO) near La Serena, Chile. This telescope has a field of view of 1.3 degrees by 1.3 degrees and is used to initially find objects for later tracking. The C9 telescope has a field of view of 0.2 degrees by 0.2 degrees and is used to track objects; this telescope will move to the next predicted point and look for the object.

\section{LITERATURE REVIEW}

There are very few resources on search strategies for space debris objects when a known object is not found where it is expected. While this field is fairly unstudied there are a few pieces of literature that were found to be relevant.

\subsection{Asteroid Search Strategy}

In the paper "The Asteroid Identification Problem: Recovery of Lost Asteroids"1 a similar analysis to the one used in this report was applied to asteroids, of particular interest were those that cross Earth orbit. The paper describes creating most probable regions where an asteroid could be when it has been lost. The results from this paper showed that the asteroids had an elongated possible region, where the asteroid could be in a large area along its trajectory but a small area perpendicular to its trajectory. Figure 1 shows one of the search regions for reference. This smaller search area enabled them to allow smaller field of view telescopes to search for the asteroids in this region and keep the larger field of view telescopes on the task of searching for new asteroids. The largest difference between the methods discussed in this paper and the one used in Milani ${ }^{1}$ is 
time. The asteroids take much longer to move through the sky than the debris at GEO enabling the asteroid searchers to look through the probability area for an extended period of time; whereas the debris search must happen in a substantially shorter period of time moves across the sky at a much faster rate.

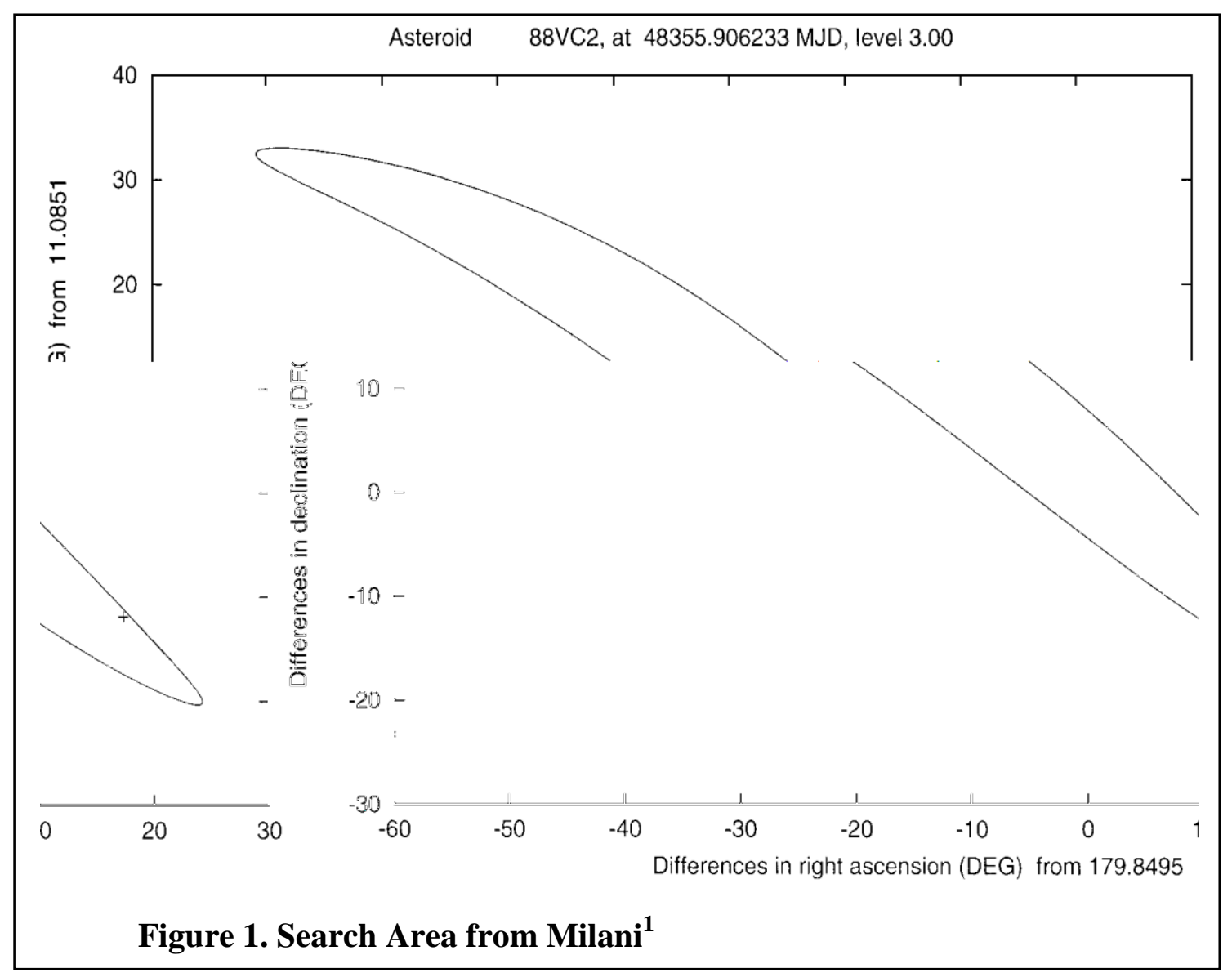

\subsection{Faint GEO Objects}

Another paper actually discusses lost objects at GEO, "Faint GEO Objects Search and Orbital Analysis"2 deals with the differences between faint objects and bright objects. It was mentioned in Agapov $^{2}$ that it is preferable to look in the direction of motion when 
searching for an object; this concept can be seen in later chapters of this report. It was also stated in Agapov that solar pressure appears to affect brighter objects different than dimmer objects; this was not something that was taken into account in this report. According to Agapov solar pressure causes a change in the phase angle, and may contribute to error in the eccentricity. This change in eccentricity if not taken into account may cause an object to be lost when observed over the course of several days.

\subsection{Background Information}

It is assumed that the reader will have basic understanding of the COEs and orbital analysis. A good educational resource for this would be the textbook "Orbital Mechanics for Engineering Students" by Curtis. ${ }^{3}$ For more information on space debris in general see “Orbital Debris: A Technical Assessment” by the National Research Council ${ }^{4}$ and "Third European Conference on Space Debris"5 a compilation of many papers about Space Debris.

A useful report on the data collected by the telescopes as well as information on the objects in the orbital region in question is "Michigan Orbital Debris Survey Telescope (MODEST) Observations of the Geosynchronous Orbital Debris Environment." ${ }^{\circ}$ The years discussed in this report are 2002-2003; and not the same as discussed below, but provide a good reference to how the data was collected, processed and analyzed, as well as the distribution of some COEs. Of major interest is the distribution of inclination, which falls between 0 degrees and 15 degrees. 


\section{ANALYSIS AND DISCUSSION}

\subsection{Error Calculations and Correlation}

\subsubsection{Error Calculations}

All of the errors in this report have been normalized for ease of comparison. The equation used for calculating the error in RA is

$$
R A_{\text {error }}=\frac{R A_{\text {Original Predicted }}-R A_{\text {Predicted }}}{R A_{\text {Original Predicted }}}
$$

The equation used to calculate the error in DEC is very similar, with RA being replaced by DEC.

$$
D E C_{\text {error }}=\frac{D E C_{\text {Original Predicted }}-D E C_{\text {Predicted }}}{D E C_{\text {Original Predicted }}}
$$

And lastly the RMS error was calculated by taking the square of the normalized RA and DEC errors adding them and the square rooting them.

$$
R M S_{\text {error }}=\sqrt{D E C_{\text {error }}^{2}+R A_{\text {error }}^{2}}
$$

\subsubsection{Correlation}

The correlation and P-value was calculated using MATLAB's built-in correlation function. Correlation is a defined as the degree to which two or more attributes or measurements on the same group of elements show a tendency to vary together. MATLAB calculates the Pearson's Product-moment Coefficient; an article by Rodgers ${ }^{7}$ 
is a good source for this type of correlation. In the type of correlation the closer the value is to one the more likely the two measurements vary together.

The P-value discussed in this report is a measurement of the percent chance that the correlation that was calculated could have occurred if the data set was completely random. The closer the value is to zero the less likely the correlation is due to random chance. So in order to see if two measurements have meaningful correlation one would need a high correlation value and a low P-value.

\subsection{The Data}

For each object the data provided by the MODEST telescope is a series of RA and DEC values as well as the time that the observation set was made. In addition to this data there are predictions made based on these observations. The prediction data includes the RA and DEC of the orbit over a large time period as well as the COEs and in some cases includes a two-line element (TLE) file. Each observation set contained a series of observations that took place over the course of approximately six minutes. This data was then used to predict a location at some later time, usually about a half hour later. Most of the objects had many subsequent observation sets and predictions. The initial observations are combined with the subsequent data to create a longer orbital arc upon which a more accurate orbit is established.

The data used for this report was gathered in March 2007 for seven nights and in August 2008 for three nights. There were 33 objects from March 2007 each with an average of three observation sets each. From August 2008 there were 14 objects each with an average of four observation sets each. Even though none of these objects were 
lost over the course of the observation set they are being used to find a pattern in the orbital parameters to find the most likely place for an object to be given an error in one of the COEs.

\subsection{Initial Analysis}

Early on in the analysis it was decided to graph the actual observation sets and the predicted observation sets on the same RA vs. DEC plot. This was done to have a visual reference of the behavior of the orbits compared to the predicted orbit. It did not take long to discover that as the time between observations increased the less likely one was to find the object within the field of view. This showed a need to create a search strategy so that when it was not possible to track an object quickly after the initial observation one could still make an attempt to find the object should it not appear in the field of view.

\subsection{Varying the COEs}

At this point it was clear that a larger data set was needed to see what would happen with many different orbit types and how the COEs would affect the error in the location between the actual position and the predicted position. In order to accomplish this, new TLEs had to be created. The method used to create these TLEs involved varying each COE individually by $\pm 10 \%$. For example, if the original RAAN was 120 degrees then the variation would be from $98 \mathrm{deg}$ to $132 \mathrm{deg}$. The $\pm 10 \%$ span was the divided up into 10 distinct COE numbers creating 10 TLE files that varied from the original. This process was repeated for each of the 6 COEs used; varying Argument of Perigee, AP; Eccentricity; ECC, Inclination, INC; Mean Anomaly, MANOM; Mean 
Motion, MM; and Right Ascension of the Ascending Node, RAAN; creating a total of 60 new TLEs for each initial TLE. The TLEs were then run through a TLE propagator program provided by NASA to create a set of predicted RA and DEC positions for the

object. These files were, in general, over a much larger time span than the actual position data, more than 24 hours vs. about 7 hours. These points were also not at exactly the same time as the actual position data, making comparison difficult. To alleviate this problem the set of predicted files were cropped to match the time span of the actual position data and then linear interpolation was used to get the RA and DEC of the predicted file at the exact time of the actual observed position. At this point the RA and DEC values were compared; all 62 sets (60 varied TLEs, the original predicted TLE, and the actual position data) of data were graphed on one plot.

\subsection{RA and DEC Plots with Varied COEs}

\subsubsection{Trends in Errors with Different COEs}

Data set of 49 objects with 133 different observation sets making 1330 different TLEs were plotted as described above. These plots made it easy to see the major contributors to error in the RA and DEC. INC had almost no affect on where the object would be in RA and only contributed to an error in DEC. This is only of minimal importance as the INC is among the easiest COE to calculate from the observation data. Figure 2 shows the full orbit of one of the objects from the 2008 data set. The purple circles represent a variation of the INC for the observation set, as one can see the INC only varies in the DEC. This is an expected outcome because the INC is directly related 
to the DEC, since inclination is a measure of the angle that the orbit makes from the equatorial plane and the DEC is a measure of the angle of the object 'up' from a reference point in the north-south direction.

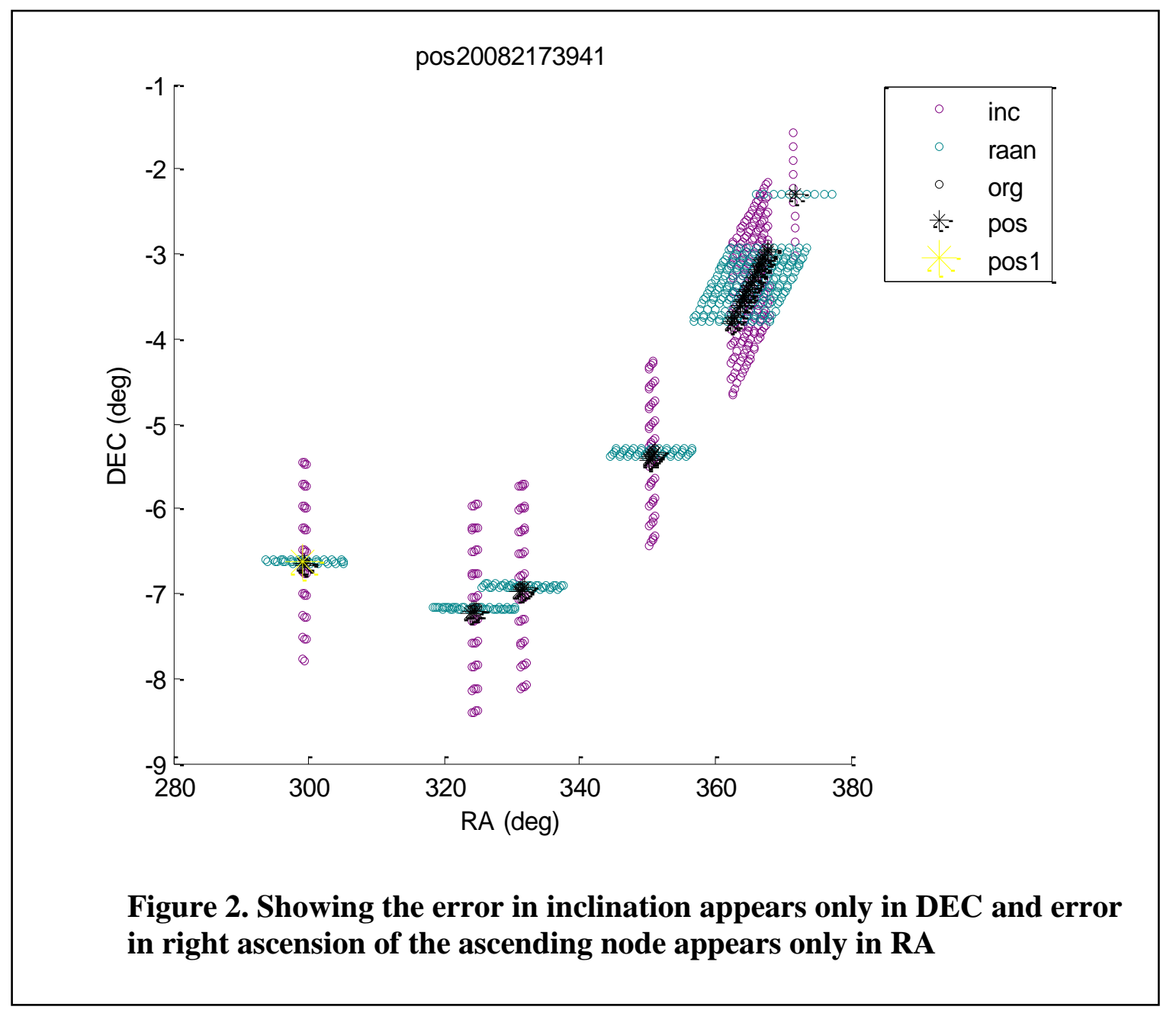

Figure 3, shows a zoomed in view of the second set of observations, for a better view of the variation on INC. From this view it is quite obvious that a variation in the INC only causes an error in the DEC. This is the case for every object analyzed regardless of the initial inclination. Any movement in RA that occurs is due to the natural motion of the object from the point of view of the observation. 


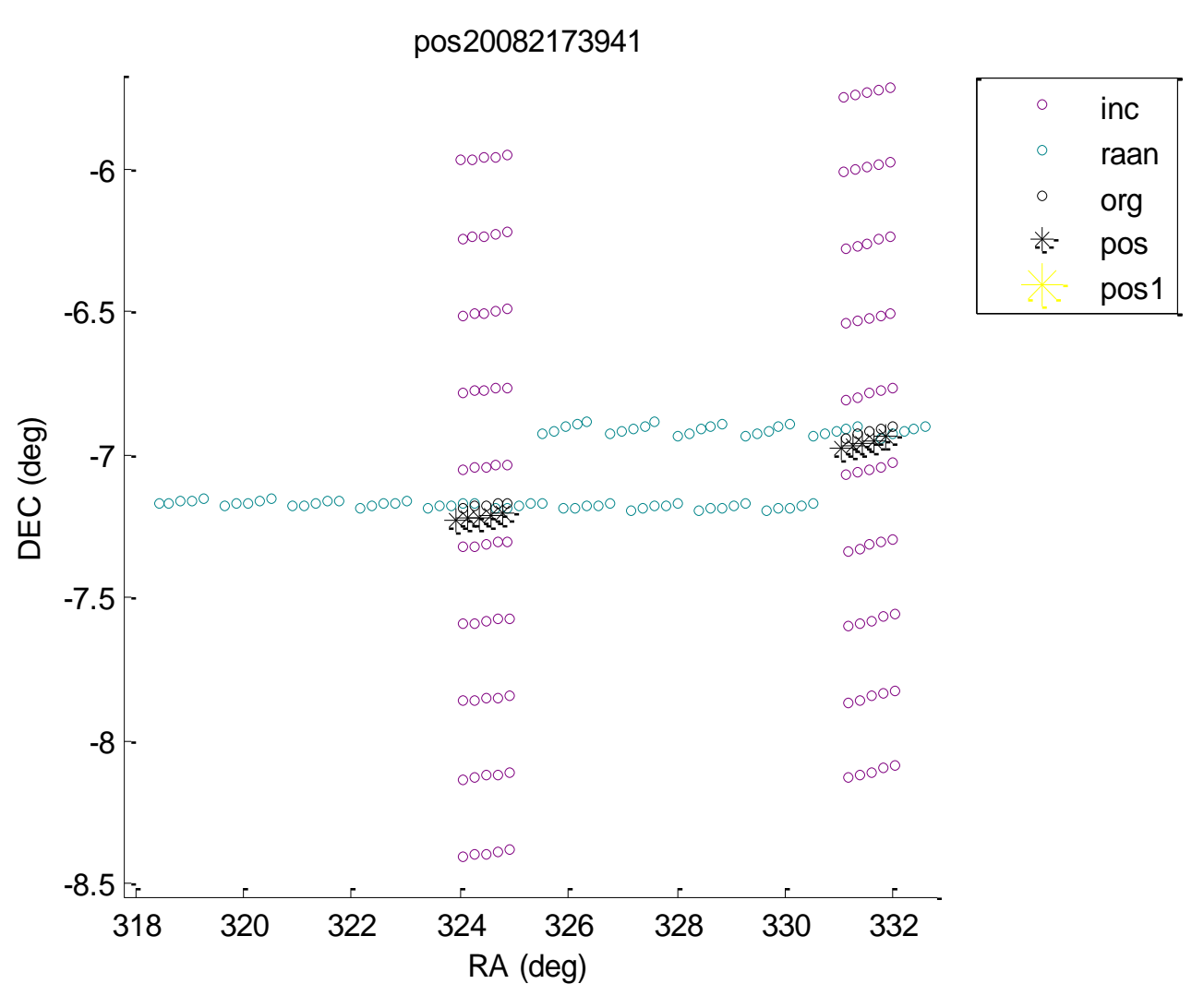

Figure 3. Zoomed in view of figure 2

Another expected trend is the relationship between RA and RAAN. Comparing the preceding figures with Figure 4 one can see a variation in RAAN only affects the RA. Although this is a slightly more useful trend than the trend in INC, RAAN is the second quickest $\mathrm{COE}$ value to converge, usually only changing a few degrees over two observation sets before settling out in the rest of the sets. For example, the RAAN for the initial orbit may be 210 degrees, the next observation set may refine this value to 212 , and from then on the RAAN stays close to 212 , varying only in the decimal places (i.e. 212.3 or 211.7 ). There is a large variation on the maximum error generated in the RA by a $10 \%$ error in RAAN from object to object however; this trend will be discussed in a bit more detail later in the report. 


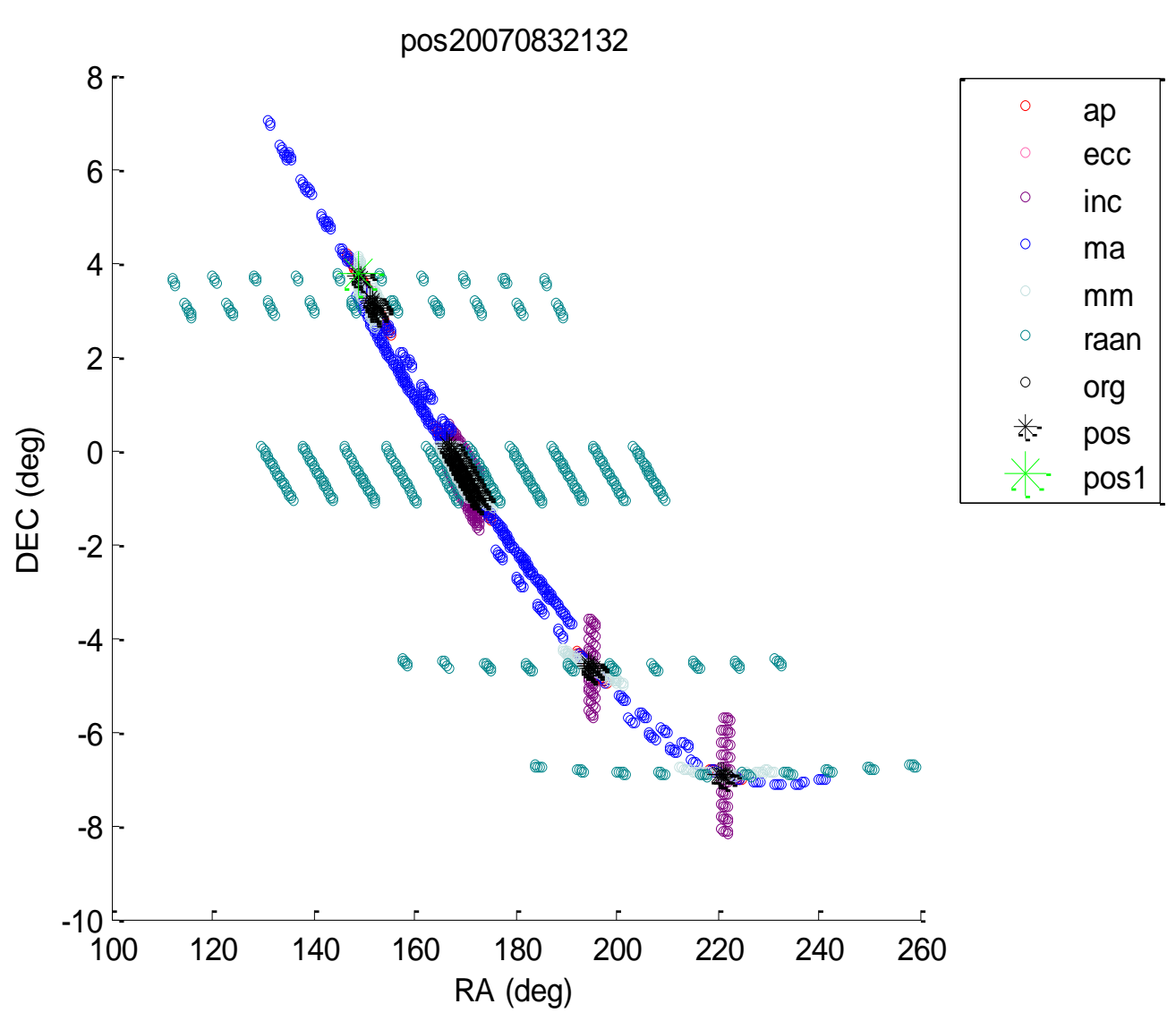

Figure 4. Shows the variation in the RAAN affecting the RA

The other COEs depend on the direction of motion as to whether their error is in the RA or the DEC. Figure 5 shows Figure 2 with all of the COEs included; from this figure and Figure 4 one can see the trend that when the object nears the lowest point, in DEC, of its orbit the predominant error is RA while when in a part of the orbit where more of the motion is in the DEC the COEs create a larger error in the DEC. This leads one to believe the inclination of the orbit has a profound impact on how large error in the DEC could be. 


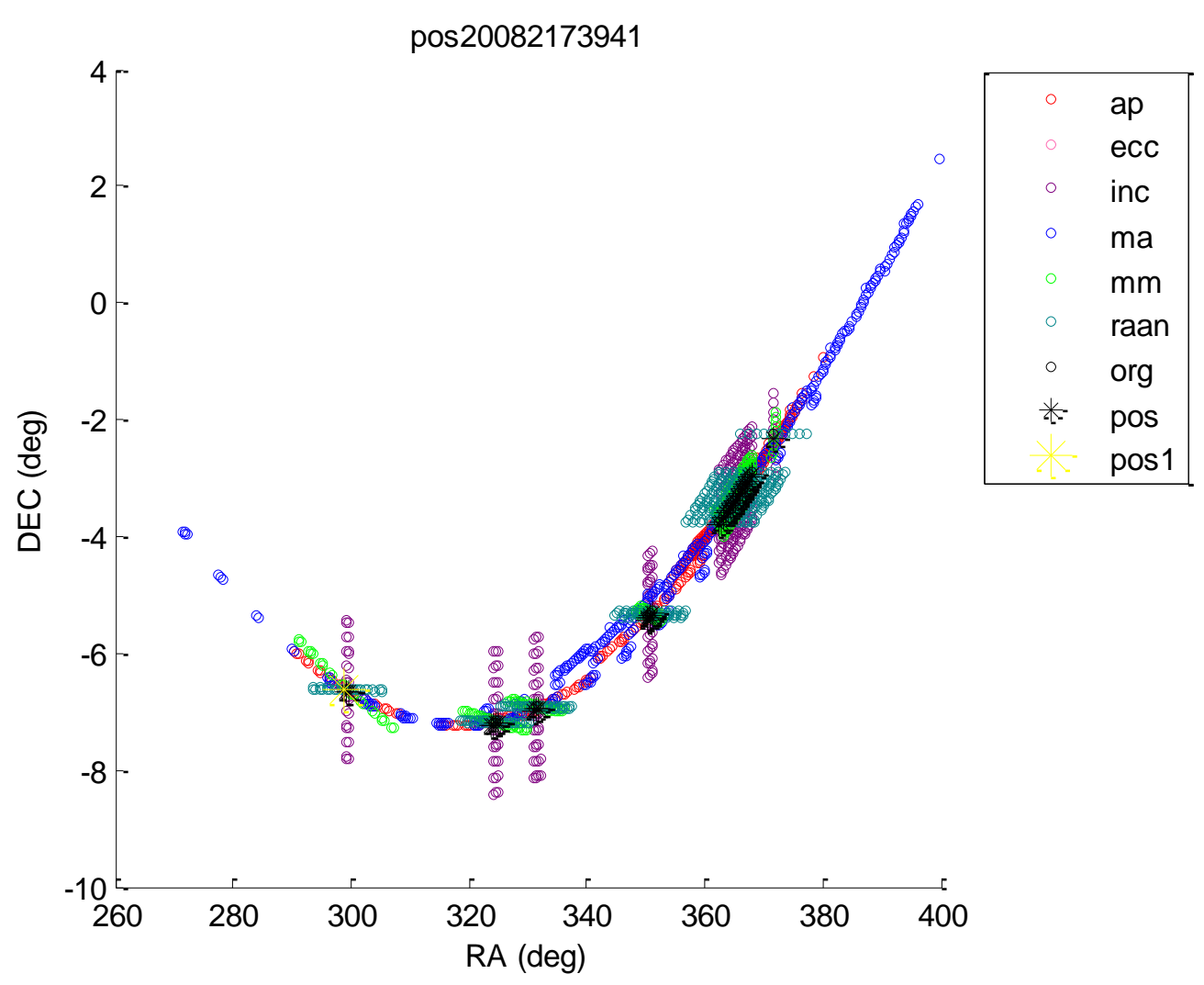

Figure 5. All COEs of Figure 2

The fact that the AP, ECC, MANOM, and MM variations appear to cause errors in the direction of motion is not a surprise, since those parameters only shift the object or orbit around in the orbital plane created by the RAAN and INC. A variation in any of these four parameters should affect where it would appear in the sky at a particular time however it would still appear in the sky at that point either at a later or earlier time than expected. That is not to say that they are less important, if the object does not appear in the field of view at the time the telescope is looking for it then it still needs to be searched 
for. To further illustrate this point see appendix A where all of the objects have been plotted.

One COE that was expected to make a big impact on the error was the eccentricity however the error generated by this COE was on average the smallest, less than 0.1 in RMS. This was a surprise since it is one of the more difficult COEs to nail down however this may be a skewed statistic since most of the objects that are in this data set are low eccentric orbits, and many of the orbits that may be higher eccentricity are lost because the initial MODEST predictions assume that the orbit is circular.

\subsubsection{The Relationship between INC and Error in DEC}

In all of these cases the error in the RA is much greater than the error in the DEC. This is due to the inclination being less than 15 degrees. Figure 6 shows a plot of the average DEC error across all variations, variations in each of the 6 COEs, for every object plotted against the INC. One can see the beginnings of a trend here however; the correlation is rather low meaning that the error does not strongly depend on the INC. 


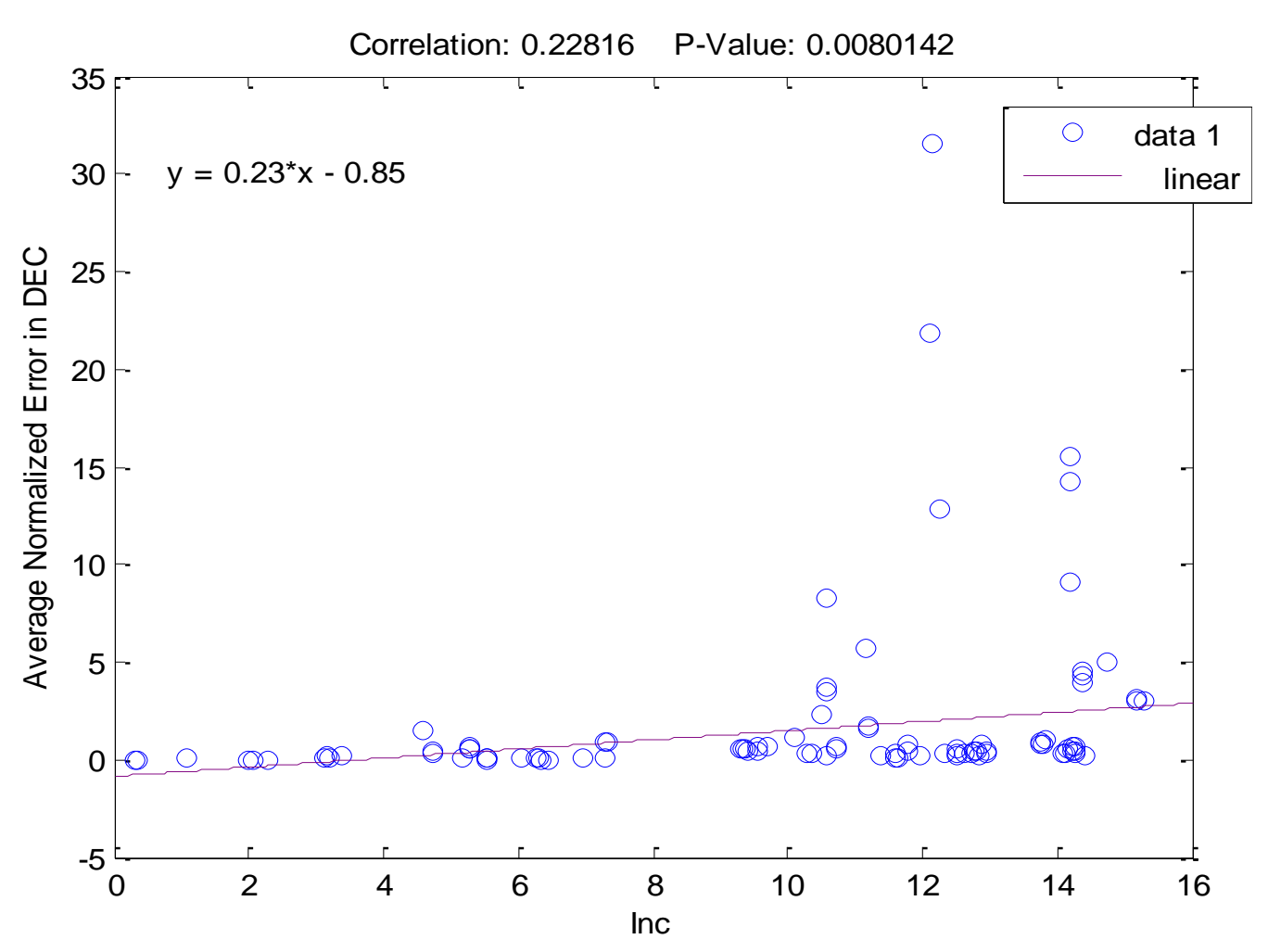

Figure 6. Average DEC vs. INC with outliers

Removing the object with the largest DEC error, shown in Figure 7, improves both the correlation coefficient and the P-value but lowers the slope of the linear line this could mean that the DEC error does not increase rapidly with INC or that the DEC error has a non linear relationship with INC. Judging from the plot it appears that the relationship is non linear but more data points are needed to prove this with certainty. 


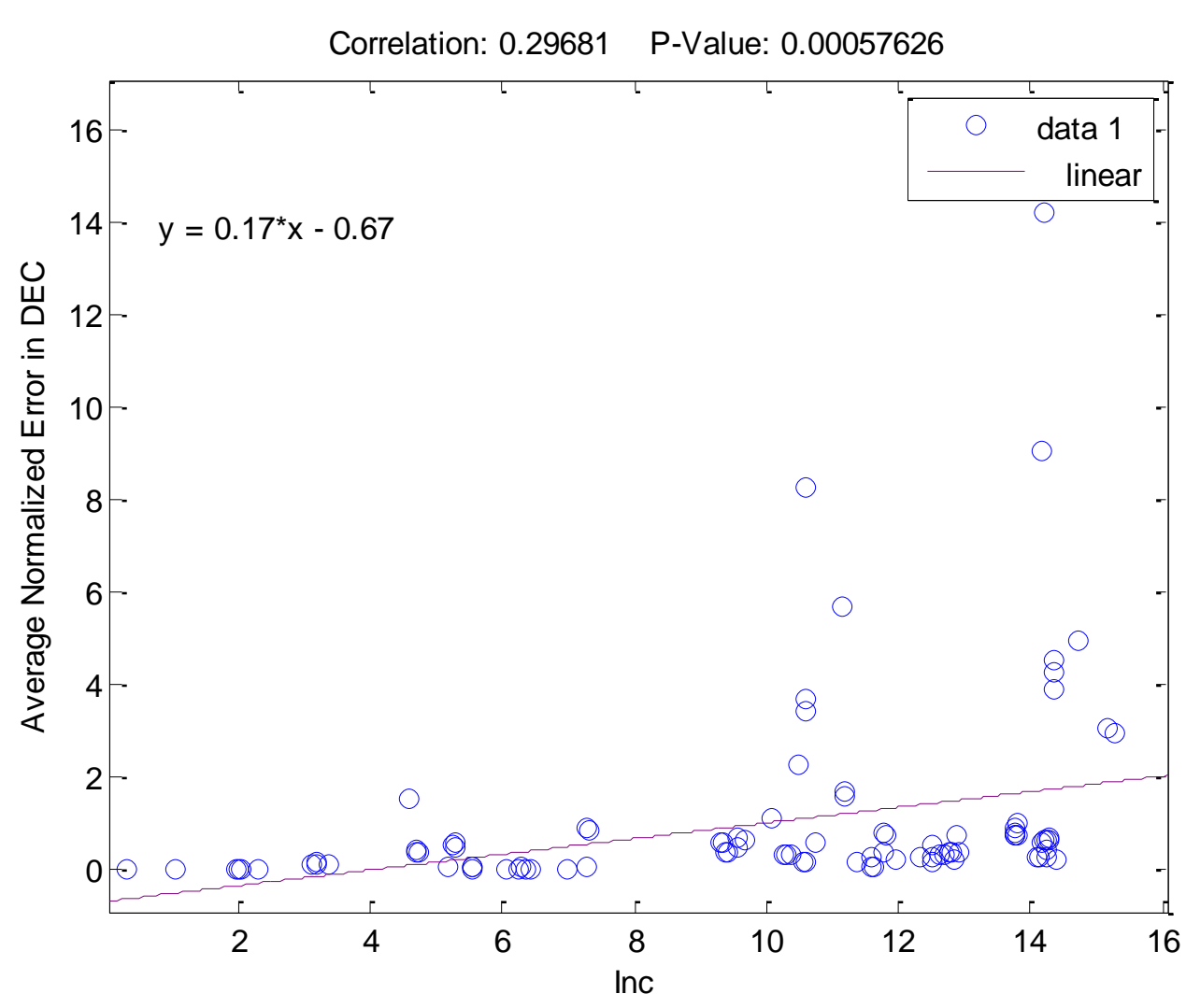

Figure 7. Average DEC vs. INC with largest DEC error removed

\subsubsection{COEs That Most Impact the Error}

It became apparent early on that the COEs that produced the most error, be it in RA or DEC, were AP and MANOM. With an average RMS error of about 4 for both COEs they were well above the rest of the COEs who were below 0.7 RMS error. Figure 8 clearly shows this phenomenon, AP and MANOM, ma on the legend, represented by the red and blue circles respectively. 


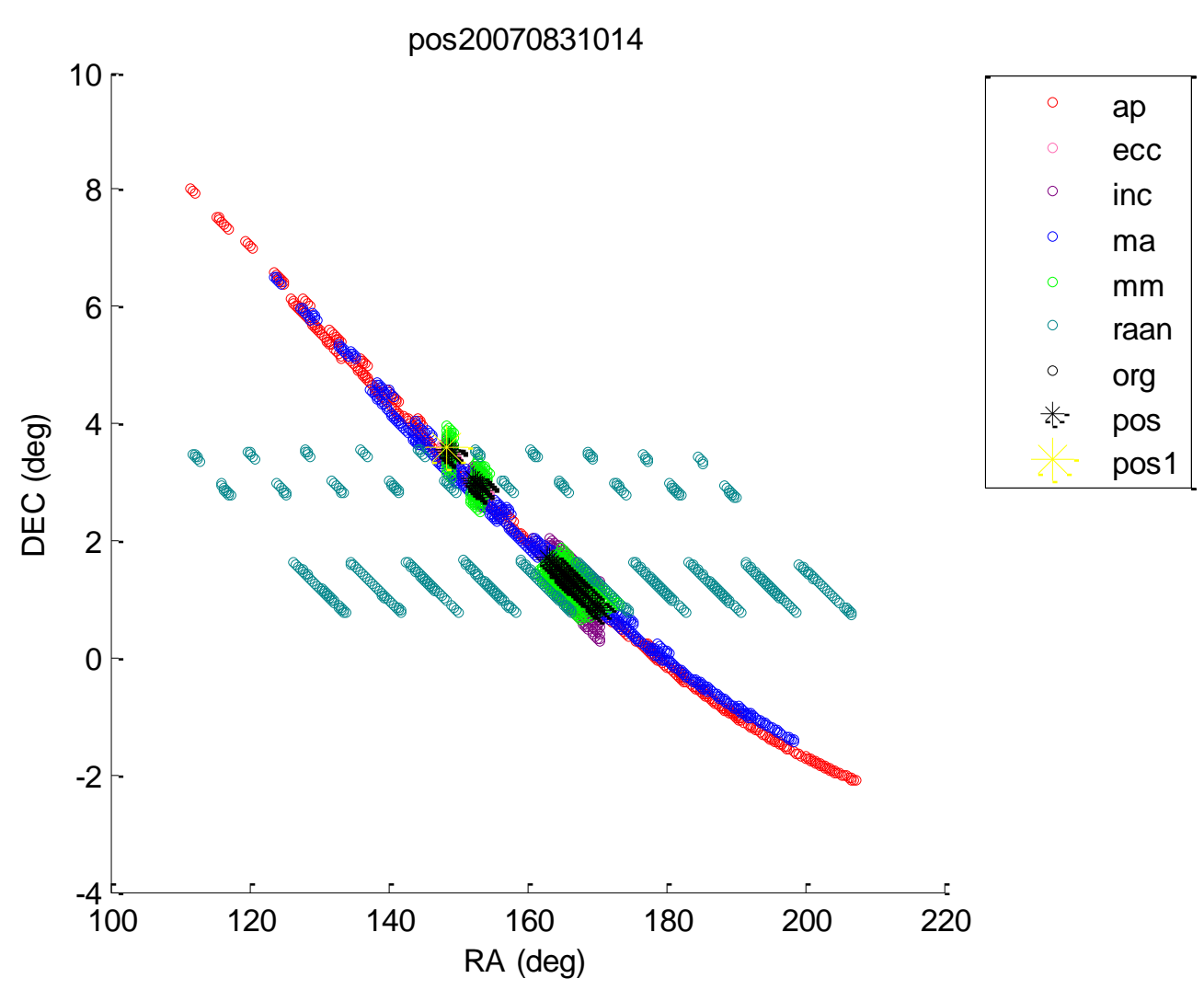

Figure 8. Example of high error for AP and MANOM

No definitive reason could be established as to why these errors were so large, but they appear to be the worst of the contributors to RMS error, the next section of this report will discuss trends in error based on a variation of the COE vs. the COE itself.

One may have noticed that the RAAN, raan (dark green) on the graph, also has a large error, RAAN had the second largest average RMS error, of about 0.7. This phenomenon will be discussed in a later portion of the paper. 


\subsection{Correlation of the COE and its Error}

This section studies the idea that there may be a correlation between a COE and the error caused by a variation and any other COE. For example a variation in AP may cause a consistently higher error when the orbit has a high INC. Statistically speaking the number of data points used in this section is minimal, with approximately 260 data points per plot the correlation has less meaning. That said however, most of the plots discussed in this section display an extraordinarily strong relationship that could point to a meaningful relationship when more data points are included.

\subsubsection{Variation in AP vs. AP Error in RA}

Interestingly it appears that there is a strong correlation between the error in RA vs. the AP when the AP is varied. This is made even more interesting when one realizes the error is normalized to prevent any interference from the fact that the variation is based on $10 \%$ of the original COE value. Figure 9 shows a variation in AP vs. error in RA for a variation in AP; The most obvious thing one can gather from this plot is that if one over predicts, predict an AP larger than the actual AP, then the object will be behind where one would expect to see it, conversely if one under predicts, predicts an AP smaller than the actual AP, then the object will be ahead of where one would expect to see it in RA. This is true whenever the variable the error is being compared to is also the one being varied. 


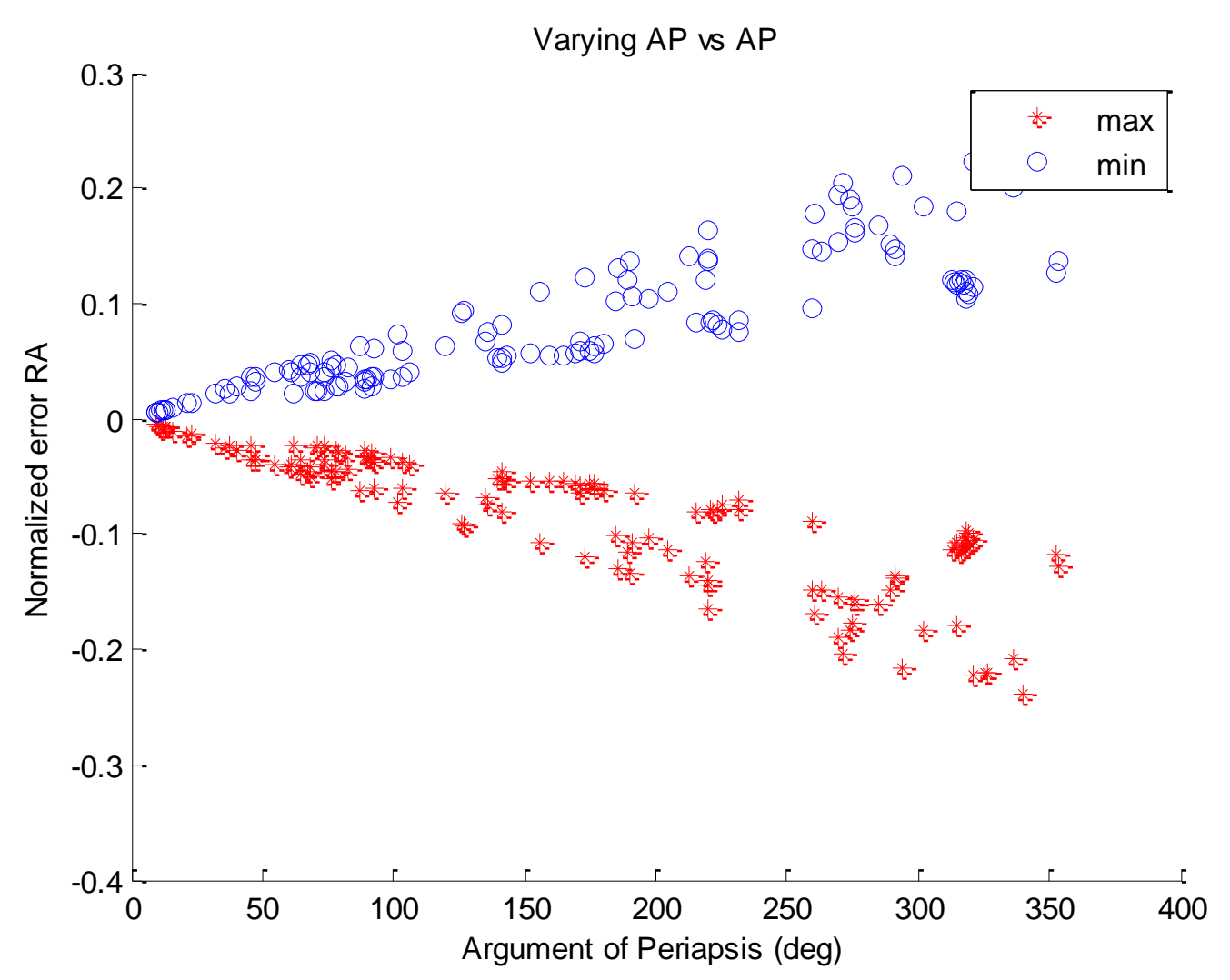

Figure 9. RA Varying AP vs. AP from Original Prediction

This is a very interesting feature, but in order to find correlation between error and COEs the magnitude of the error needs to be analyzed, this will be done for the remainder of the correlation plots. Figure 10 shows the magnitude of AP vs. Error in RA with a variation in $\mathrm{AP}$, and has a high correlation as well as an extremely low $\mathrm{P}$-value, this value is the percent chance that this formation could occur if there was no correlation. This implies a strong relationship between the error in the RA and AP when AP is increasing. 


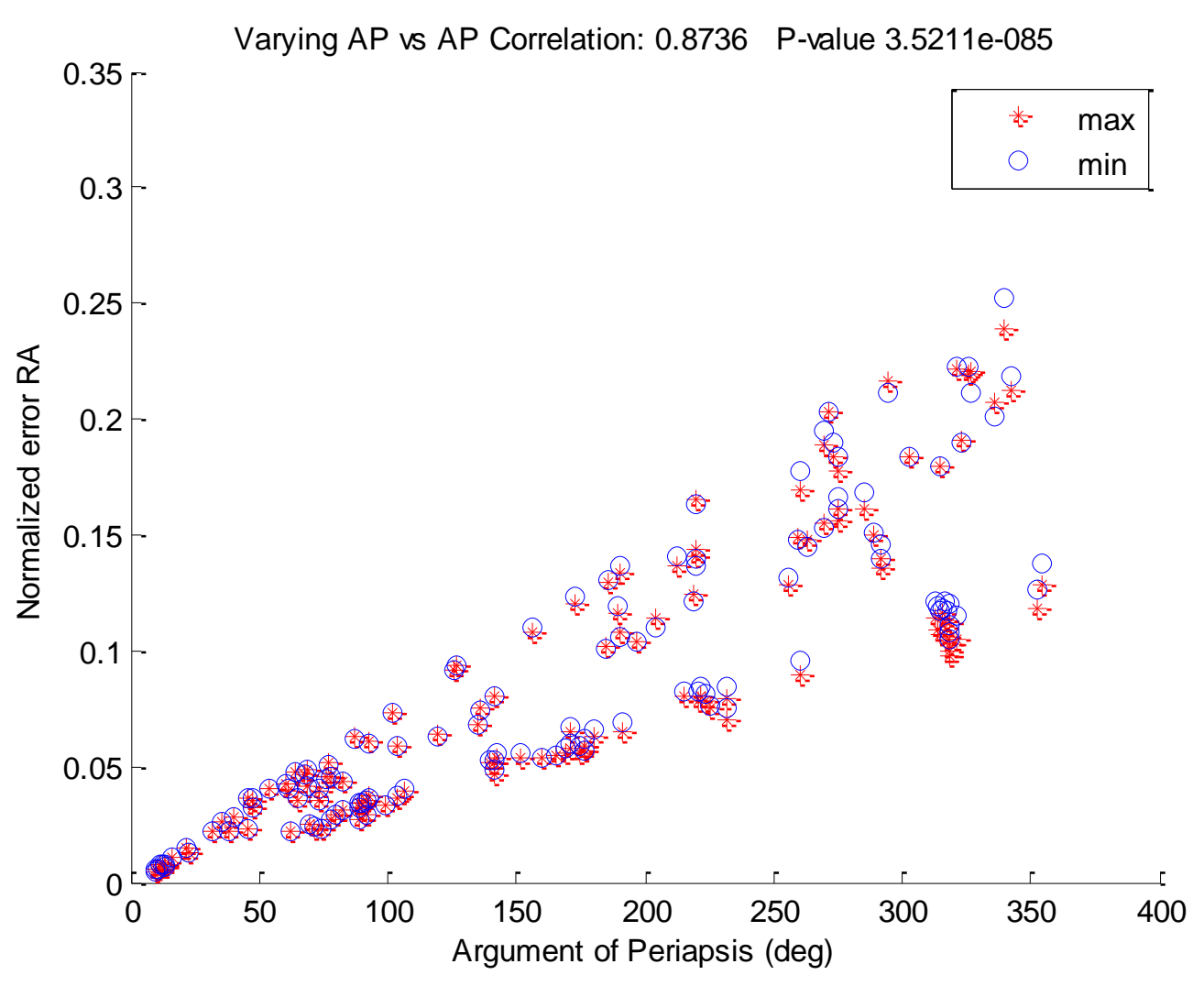

Figure 10. RA Varying AP vs. AP from Original Prediction

Figure 10 shows the error due to the $10 \%$ variation from the original prediction TLE, which was assumed to be true for these calculations. This assumption eliminated the possible inherent error in the original prediction TLE as a possible source of the trend. Figure 11 shows the error due to the $10 \%$ variation from the actual position, the graphs look slightly different, some errors farther from the center line, but the overall trend is still there. This seems to imply that the value of the predicted AP has a rather large effect on the error in the RA. 


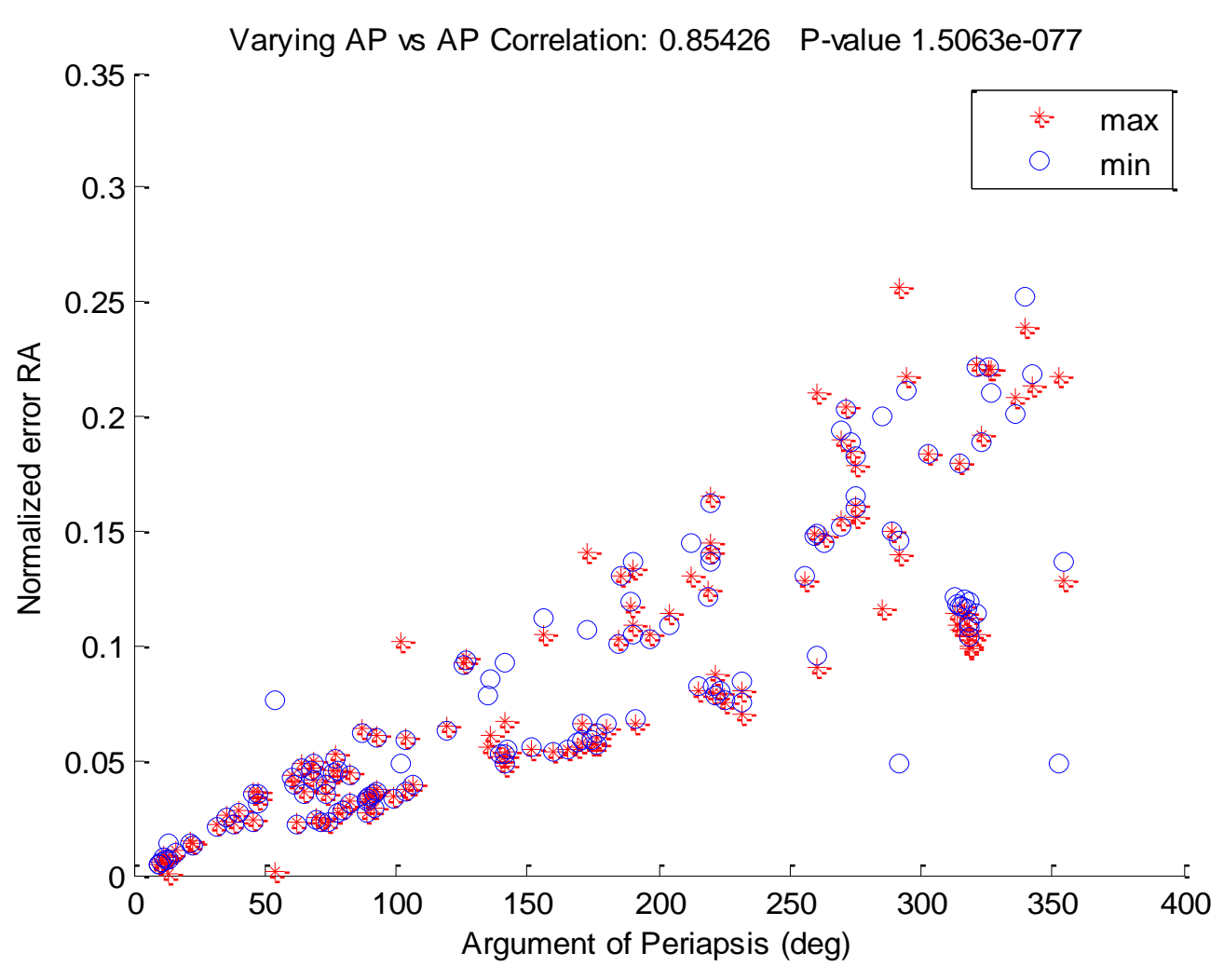

Figure 11. RA Varying AP vs. AP from Actual Position

\subsubsection{Varying AP vs. AP Error in DEC}

Figure 12 shows that while some correlation exists between the variation in AP and the error in DEC however it is much smaller than the correlation with the error in RA. One will notice that the normalized error in DEC gets extremely high for some observations; this is due to how close the observation is to 0 degrees DEC. 


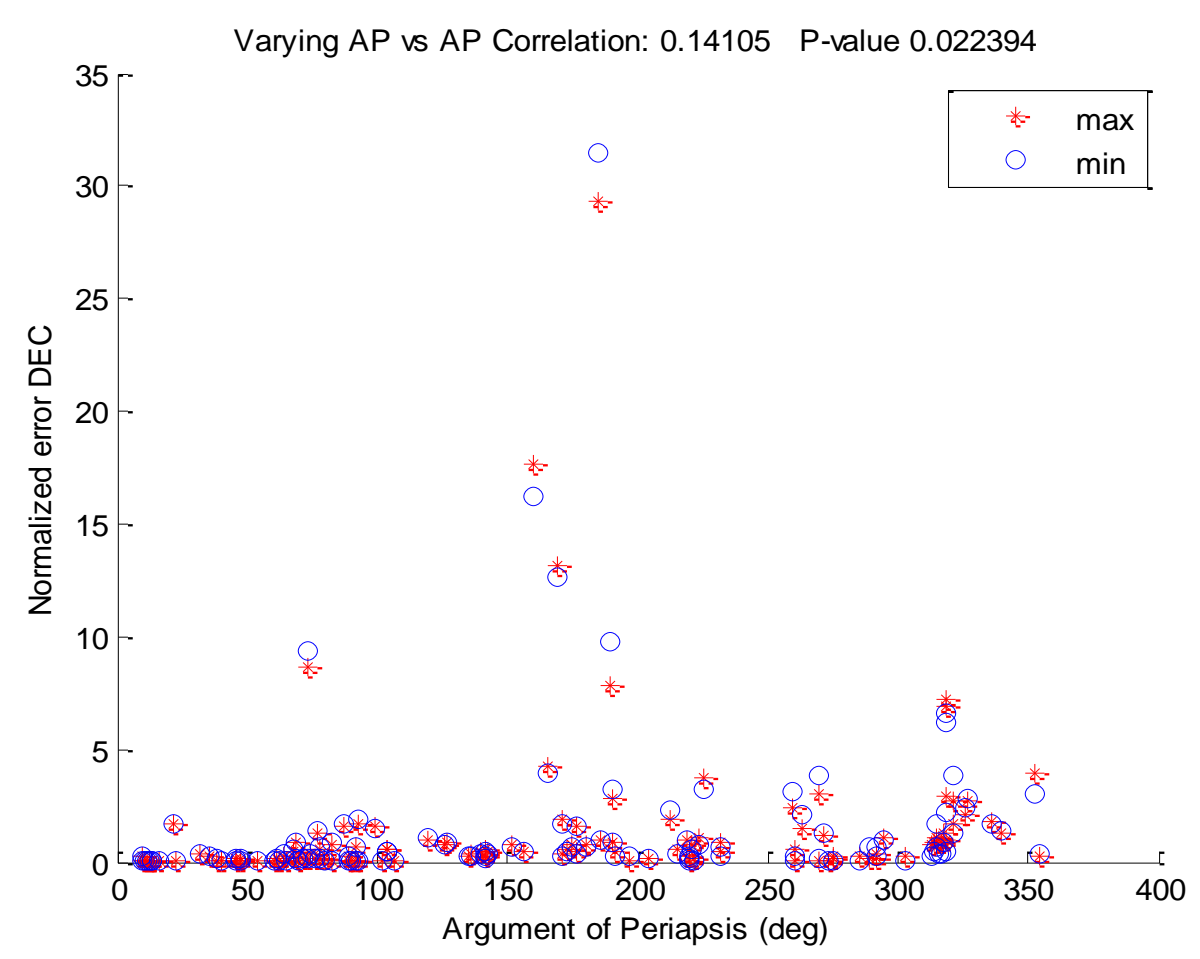

Figure 12. DEC Varying AP vs. AP from Original Prediction

From this point on, simply for ease of view, data points that cause an error in DEC above 10 degrees will be ignored. Removing these points produces a graph that more accurately represents the data set but does little to improve correlation or P-value. This holds true both for the original prediction case and the original position case. Based on this data set it is safe to assume that value of AP does not have a profound effect on the error in DEC. However that does not mean an error in AP will not cause a large error in DEC; it just means that the error is independent of the actual AP.

\subsubsection{Varying MANOM vs. MANOM Error in RA and DEC}

Much like AP, a variation in the MANOM has a very strong correlation between the MANOM and the error in RA. Figure 13 shows this relationship in detail, and as one 
can see follows a very similar pattern to the AP plot shown in Figure 10. Again this trend still exists when compared to the error in RA for the actual position.

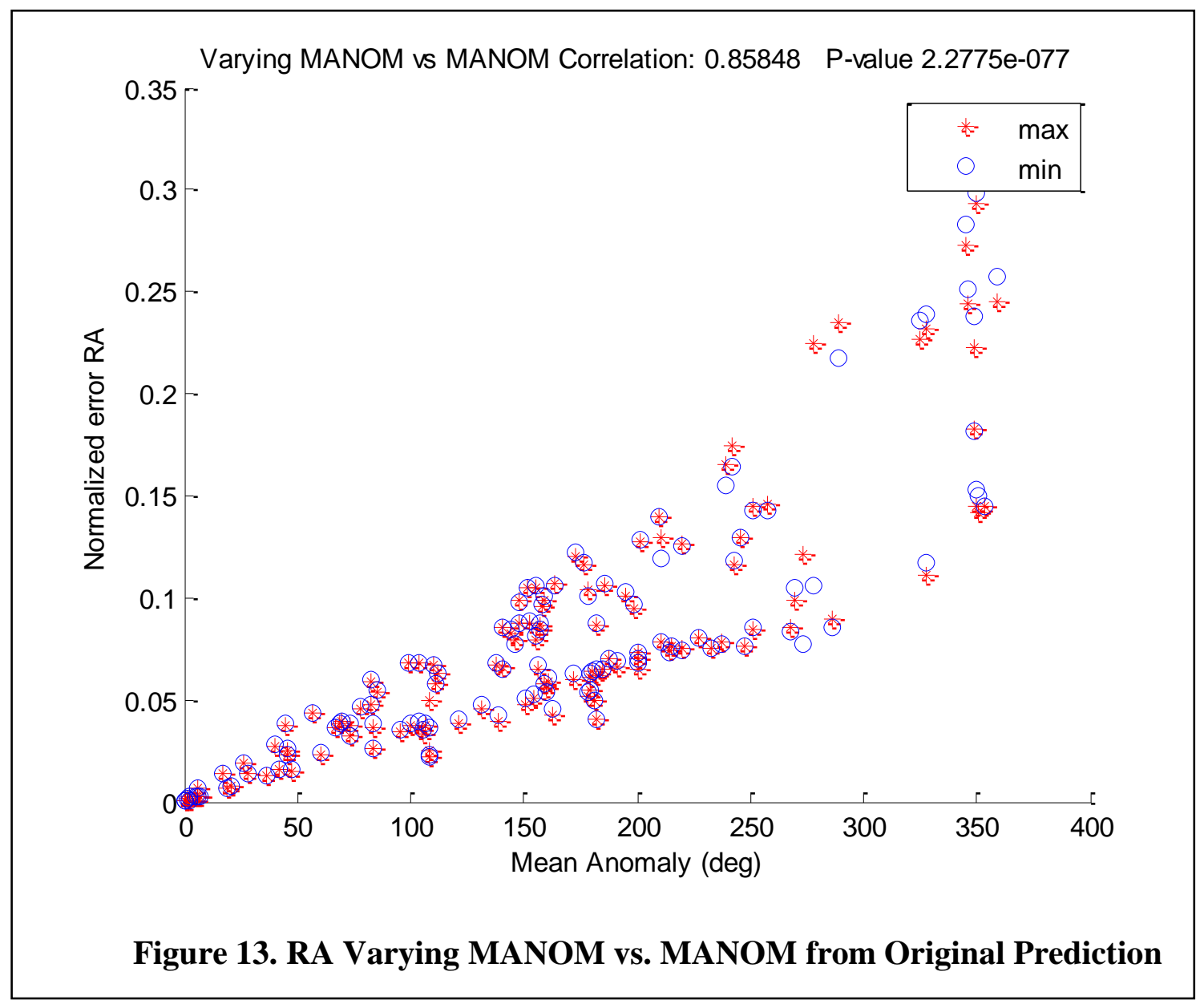

Figure 13 clearly shows a strong relationship between the magnitude of the error and the value of the predicted MANOM. Just to reiterate it is possible that this trend is an artifact of the limited data pool however based on the strength of the trend it seems unlikely.

The error in the DEC for the MANOM is also similar to the error in the DEC for $\mathrm{AP}$, that is, a very weak correlation from which little conclusion can be drawn. Figure 14 shows this relationship, the relationship is weak enough to say there is no relationship 
between these two parameters, however it is possible that a relationship would reveal itself with more data, particularly data with higher inclinations.

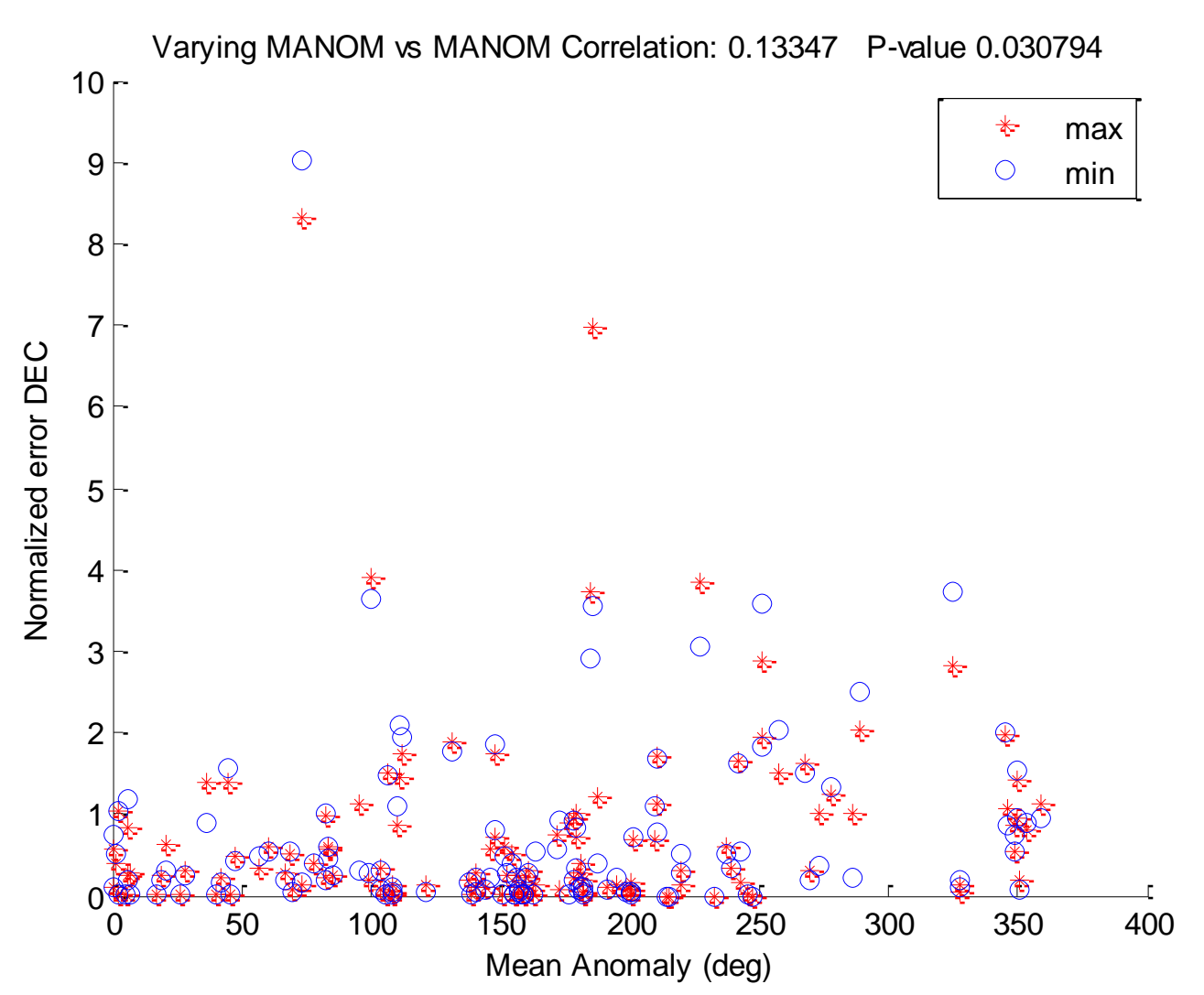

Figure 14. DEC MANOM vs. MANOM from Original Prediction

\subsubsection{Varying RAAN vs. RAAN Error in RA and DEC}

Unlike the previous two COEs RAAN has a strong correlation in RA and a stronger correlation in DEC. However the DEC errors generated by a variation in RAAN are quite small, so the trend generated in this plot is of less importance than the trend in 
RA. Figure 15 shows the relationship between variations in RAAN and RA and Figure 16 shows the relationship between variations in RAAN and DEC.

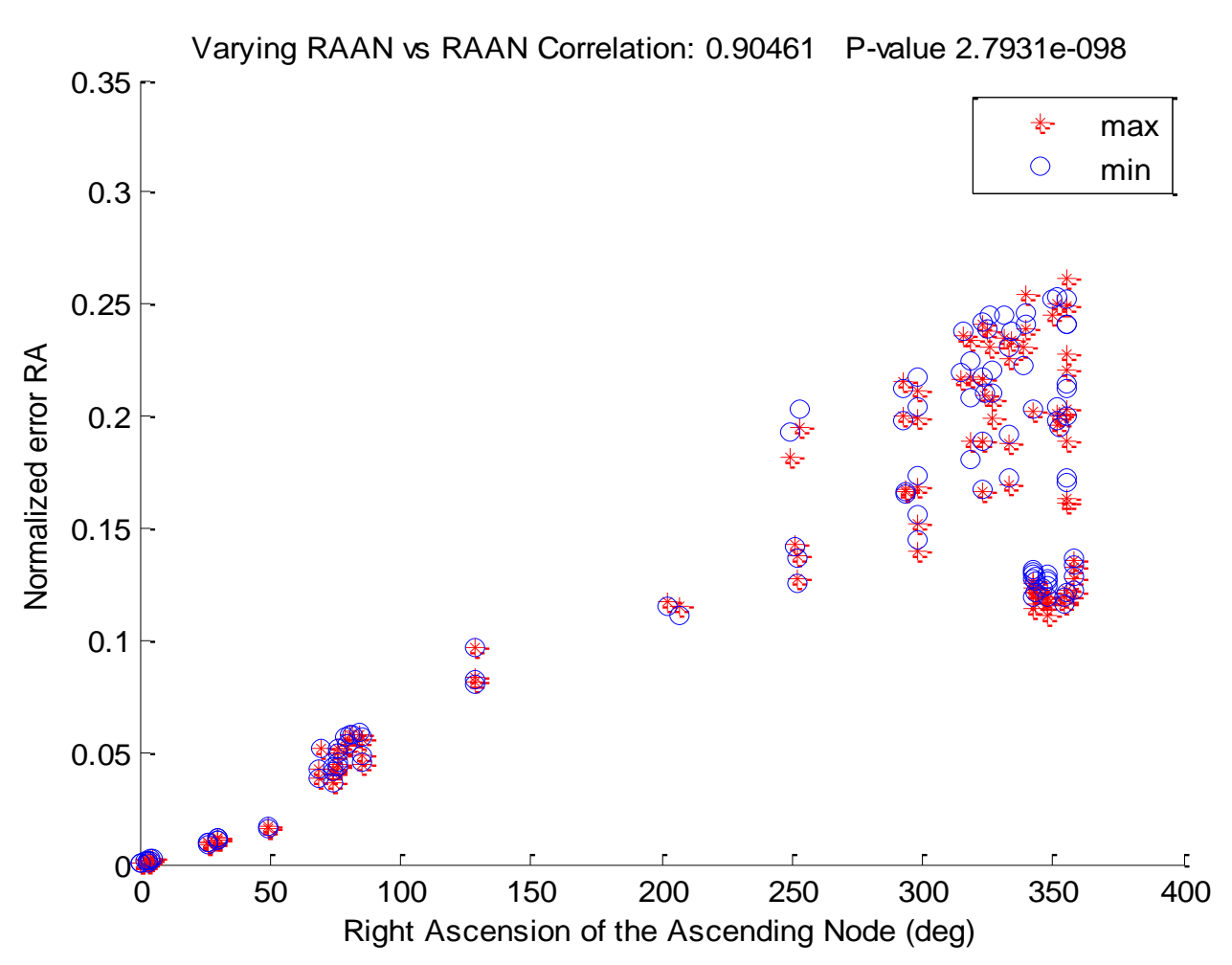

Figure 15. RA Varying RAAN vs. RAAN from Original Prediction

Figure 15 shows a strong relationship between the RAAN and the error in RA, the error clearly is larger when the RAAN is larger. The variation in the error also increases as RAAN increases. There is no obvious reason as to why this phenomenon exists; it seems counter intuitive that moving the RAAN would cause the error to change since the RAAN has little effect on the motion of the object just over what part of the sky the object travels. Because AP and MANOM depend on the value of RAAN it is likely that the trend found in them both is due to the trend found in the RAAN.

Figure 16 shows there is a relationship between RAAN and error in DEC but in comparing the magnitude of the error to some other COEs errors in DEC it is obvious 
that it has little effect; one can see in Figure 14 that the errors are several orders of magnitude different. So while there may be a trend here the impact on finding the object is minimal compared to other COEs. Again because AP and MANOM depends on RAAN it is likely that due to the low value of the errors in DEC from RAAN that the correlation does not show up in AP and MANOM. Further study into the correlation between error in RA and RAAN would be useful in discovering the reason behind this.

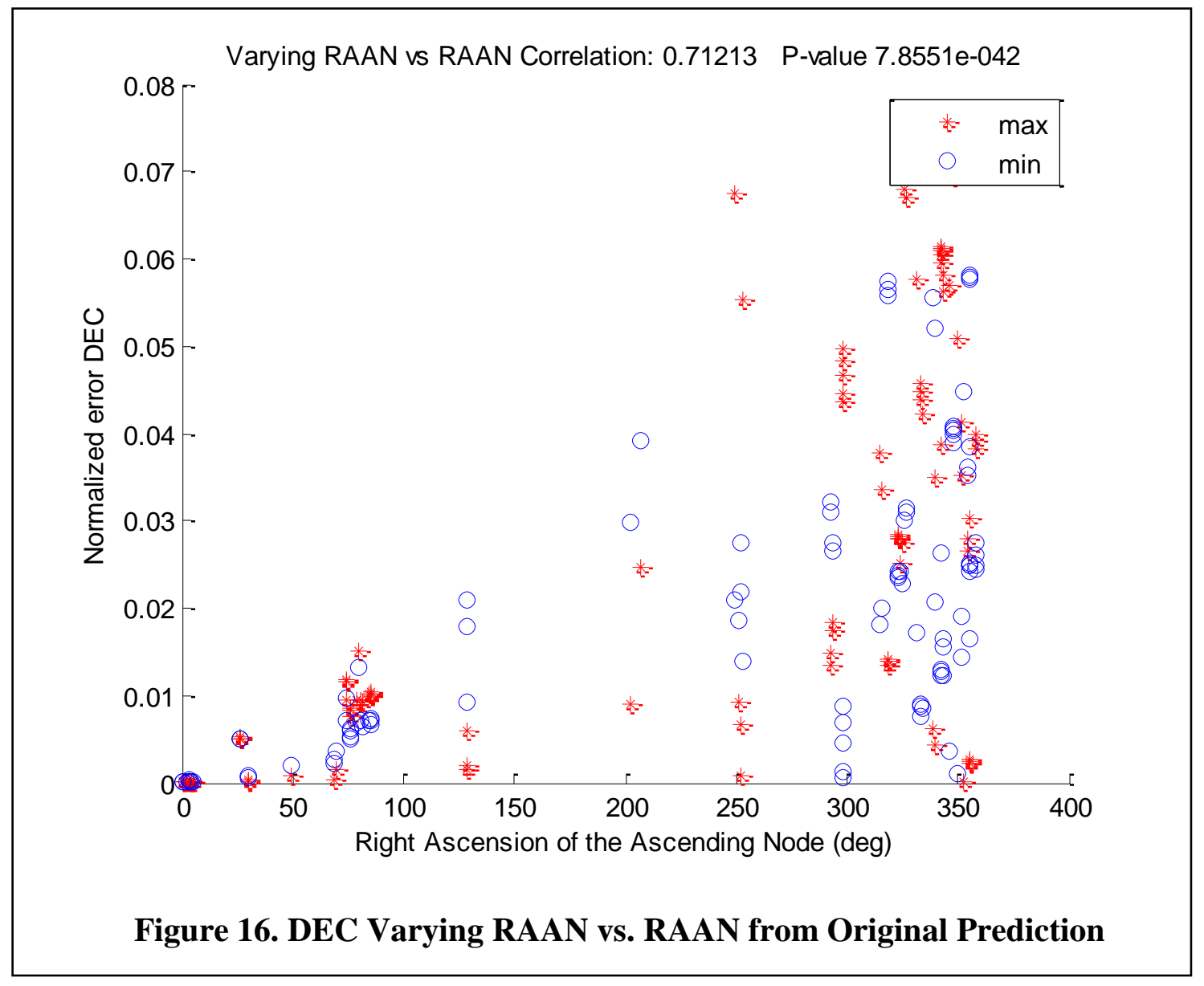

\subsubsection{Varying AP, MANOM and RAAN vs. Themselves Error in RMS}

Checking the RMS error for these three COEs shows that only RAAN keeps its strong trend in error, this is likely due to the fact that RAAN affects RA far more than 
DEC where as the other two COEs affect both RA and DEC to some degree. Figure 17, Figure 18, and Figure 19 show the trends with RMS and it is clear that the only one with a defined trend is RAAN.

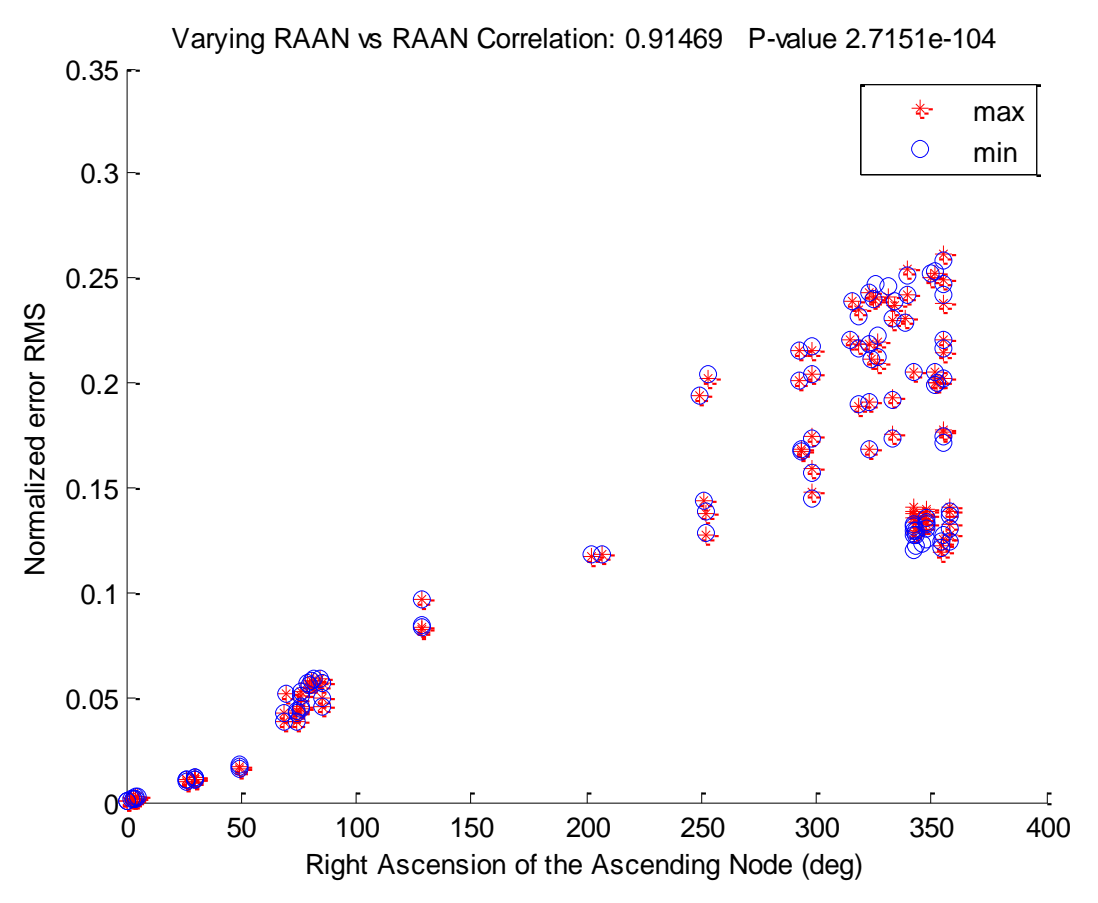

Figure 17. RMS Varying RAAN vs. RAAN from Original Prediction 


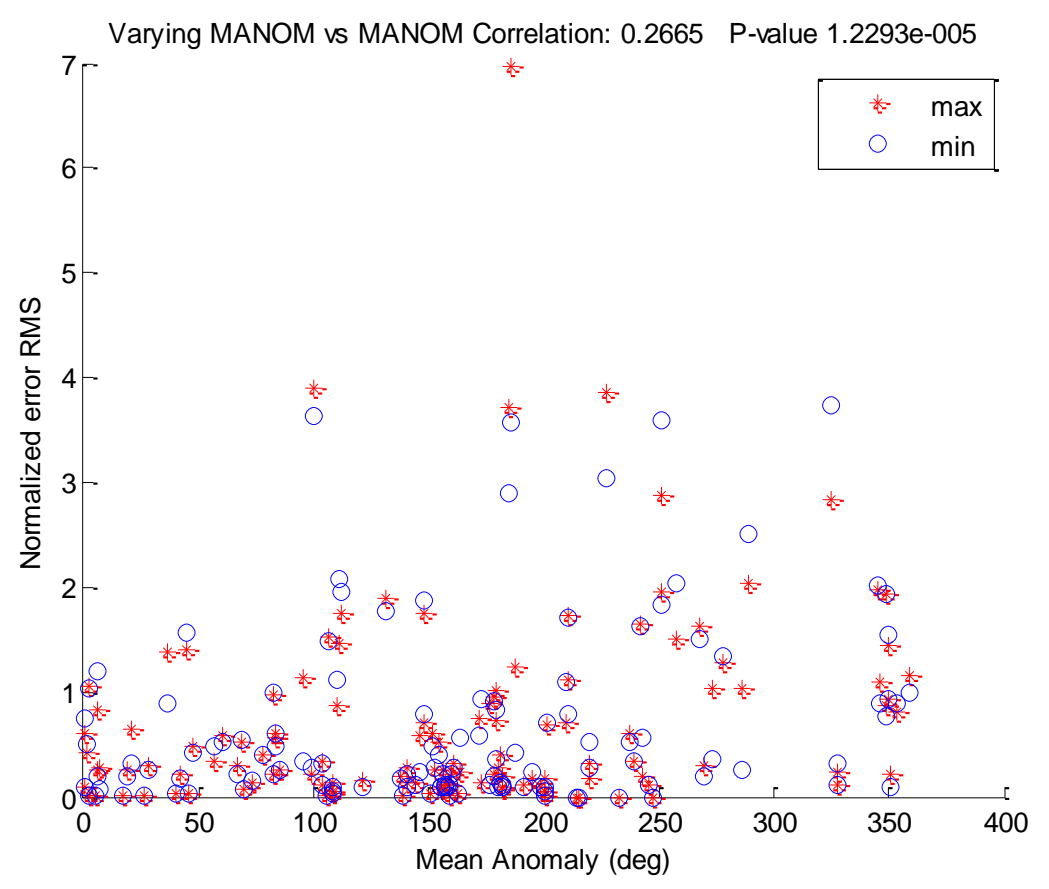

Figure 18. RMS Varying MANOM vs. MANOM from Original

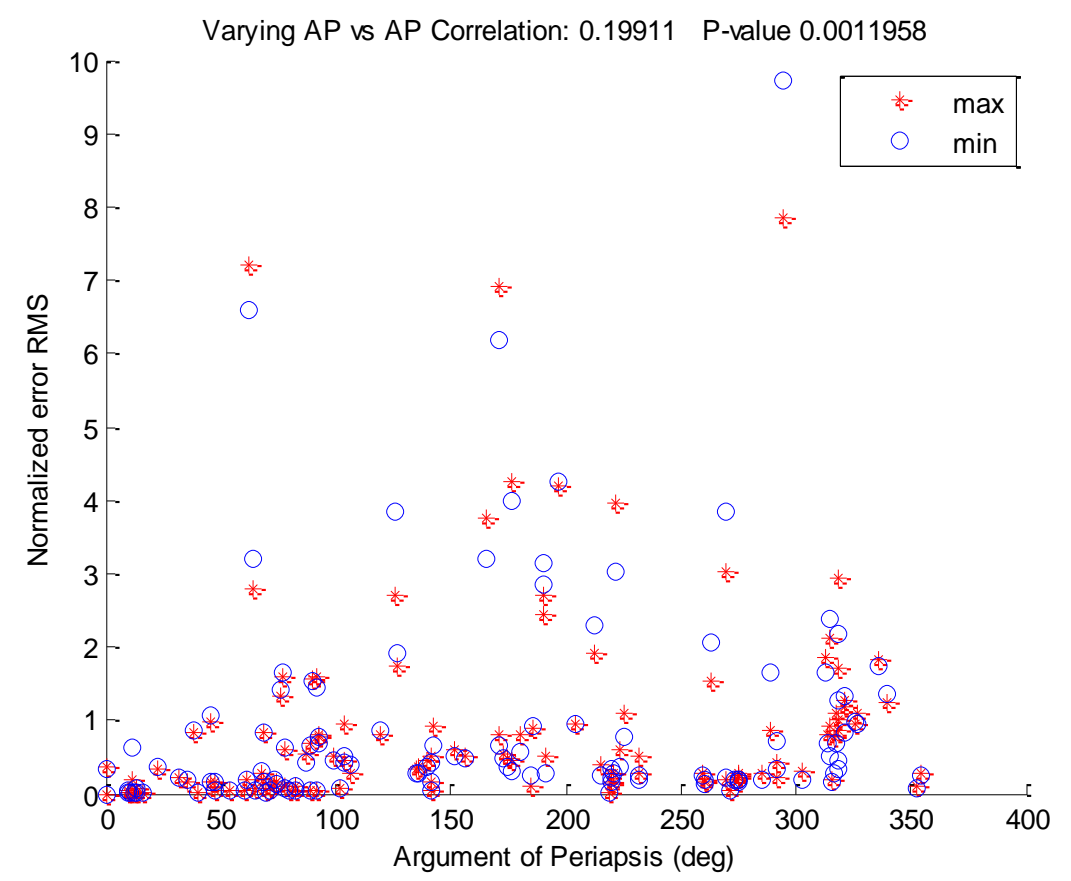

Figure 19. RMS Varying AP vs. AP from Original Prediction 
Again because AP and MANOM depend on RAAN it is probable that the trend found in those COEs is directly related to the trend found in RAAN. This idea is supported by the fact that there is no trend in the DEC for AP and MANOM where RAAN has little effect. The reasons behind the correlation between error in RA and RAAN are unclear and merit further research, even if just to prove there really is no trend.

\subsubsection{What Errors Fall within the Field of View}

Up until now very little has been discussed about whether the object can be seen by the telescope or not. This section will show in more detail how the errors affect the ability of the telescope to capture and track the object in question. It will show the maximum percent error that a $\mathrm{COE}$ can have in order to reliably find the object at the next location, as well as begin to develop search patterns for objects that do not fall inside the view.

\section{$3.6 .7 \pm 10 \%$ Variation of COE}

Most of the data was analyzed using $\pm 10 \%$ variation of the COEs. With this variation three of the COEs, INC, ECC, and MM had a few points within the field of view, and had small enough variations to allow for analysis. Figure 20 shows a view of the first predicted point using the C9 telescopes field of view. The field of view is centered on the original predicted point; this is where the telescope would be centered if it were looking for the object in question. Each point is $2 \%$ from the previous point of that color. For example the left most pink circle is a $2 \%$ change in eccentricity from the center black point. From this information it can be shown that the three COEs visible in 
the view window have two points inside the window. This means that a variation of $\pm 2 \%$ in any of these three COEs from the originally predicted COE would still be visible by the C9 telescope.

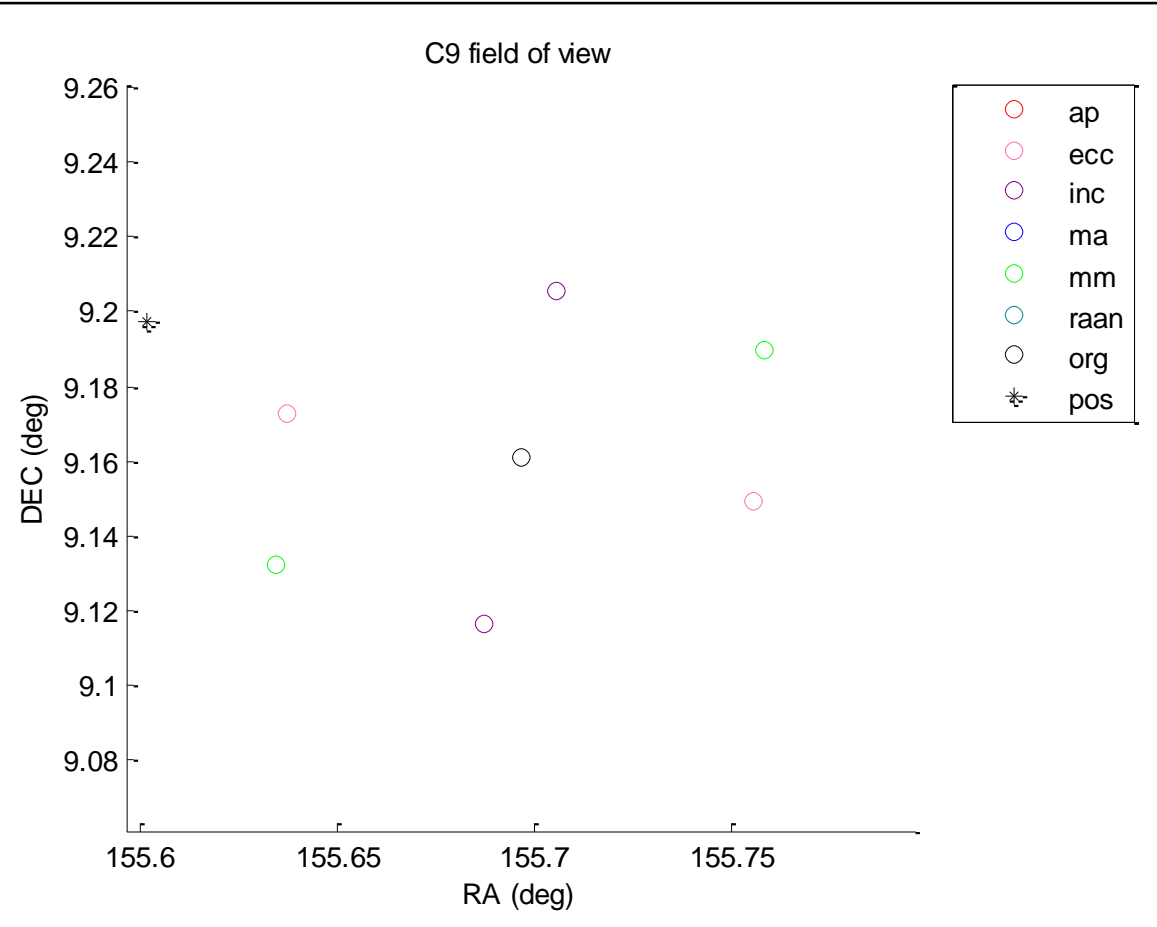

Figure 20. 0.2 Degree View (C9 Telescope) for an object

Zooming out to the field of view of the MODEST telescope, shown in Figure 21 shows more of the variation, the three COEs mentioned above are now completely visible and but none of the other COEs are visible yet. This means that even in the MODEST telescopes view an object with an error of $\pm 2 \%$ in AP and MANOM would not be found. This object had a high RAAN and therefore the error in RAAN was high, and still does not show up in the field of view. Most of MM is now visible as well; MM is the COE with the third lowest error corresponding to its variation. For completeness sake Figure 22 shows the full orbit of the object. One can see from this figure that the RAAN has a very high error in RA. 
This is essentially the same for every object with some variation on whether AP or MA are farther outside the field of view, with the exception of objects with a low RAAN where the variation in the error for RAAN shrinks.

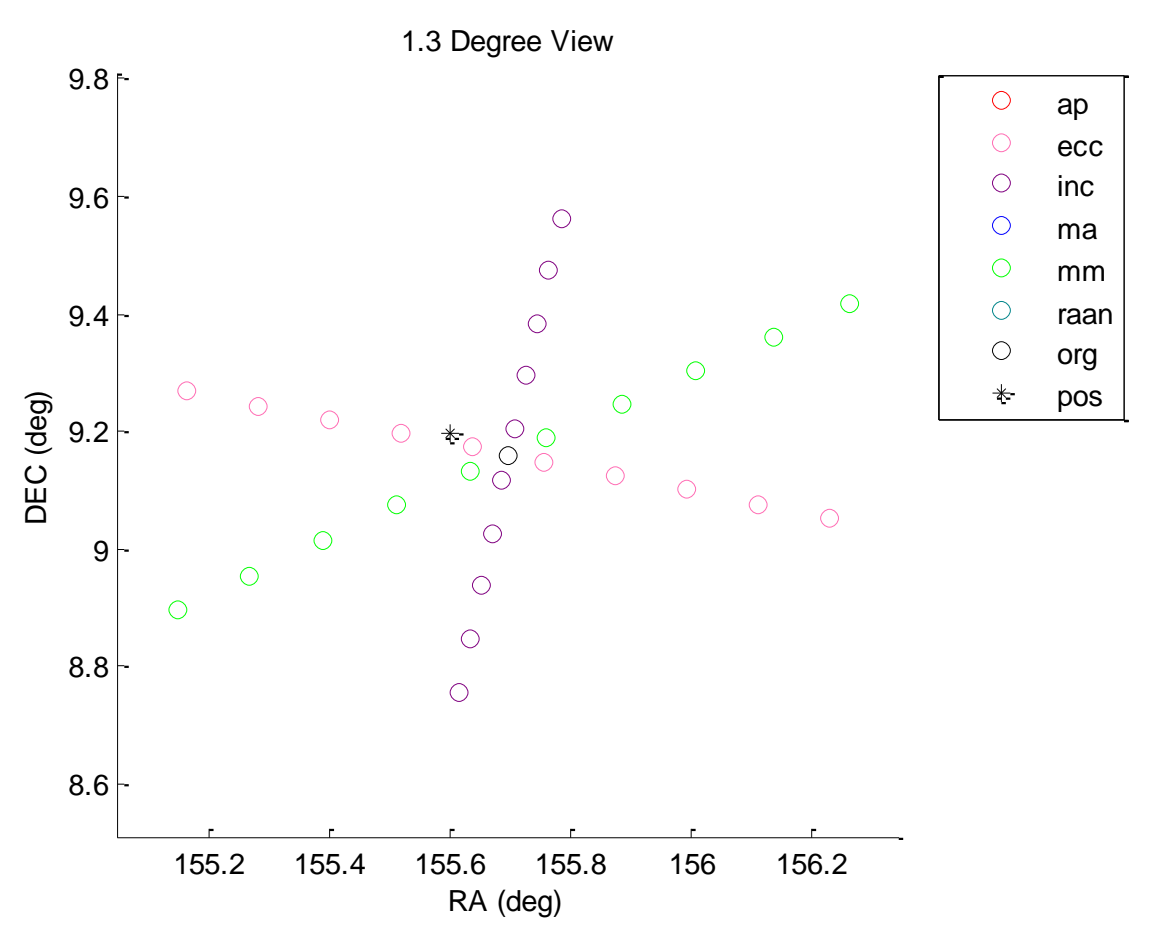

Figure 21. 2 Degree View (MODEST Telescope) for the same object 


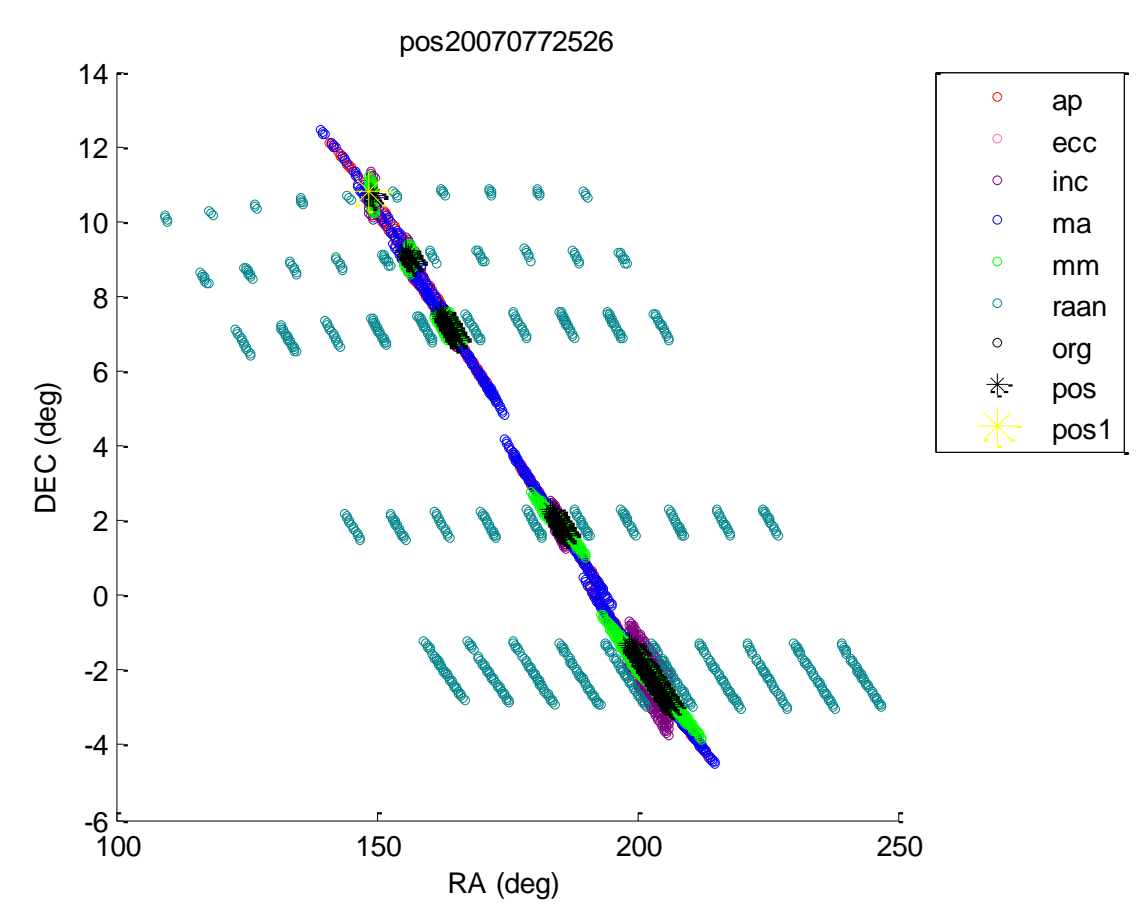

Figure 22. Full view of the same object with $10 \%$ variation

\subsection{8 $\pm 2 \%$ Variation in COEs}

For the same object the variation in the COEs was reduced to $\pm 2 \%$, Figure 23 below shows the full orbit of this variation.

This time AP and MANOM are still outside the field of view of the C9 telescope. All of INC and ECC are fully within the view as one can see from Figure 24. With a $\pm 2 \%$ error each point represents a variation of $0.4 \%$ meaning even with the variation at $0.4 \%$ the object would still not appear in the telescopes field of view. At least some variation of all of the COEs, with the exception of RAAN, appears in the MODEST telescope field of view; this is shown in Figure 25. This figure shows that AP and MANOM need to vary less than $\pm 0.8 \%$ to appear in the MODEST field of view, which translates to $\pm 0.06 \%$ variation for the $\mathrm{C} 9$ telescope. This is a very small error compared 
to the other COEs and would lead one to believe that these COEs are an important part of finding the object.

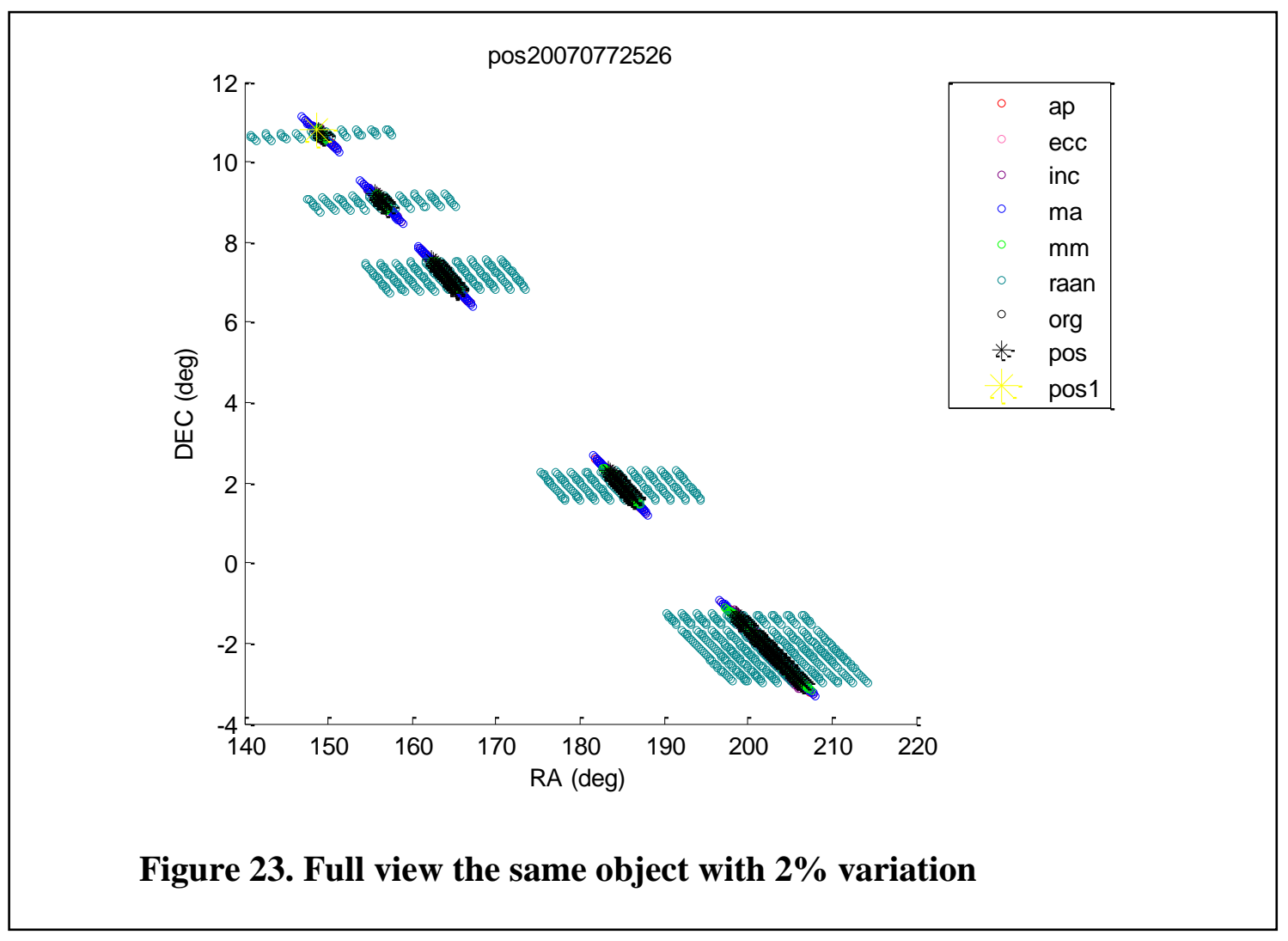




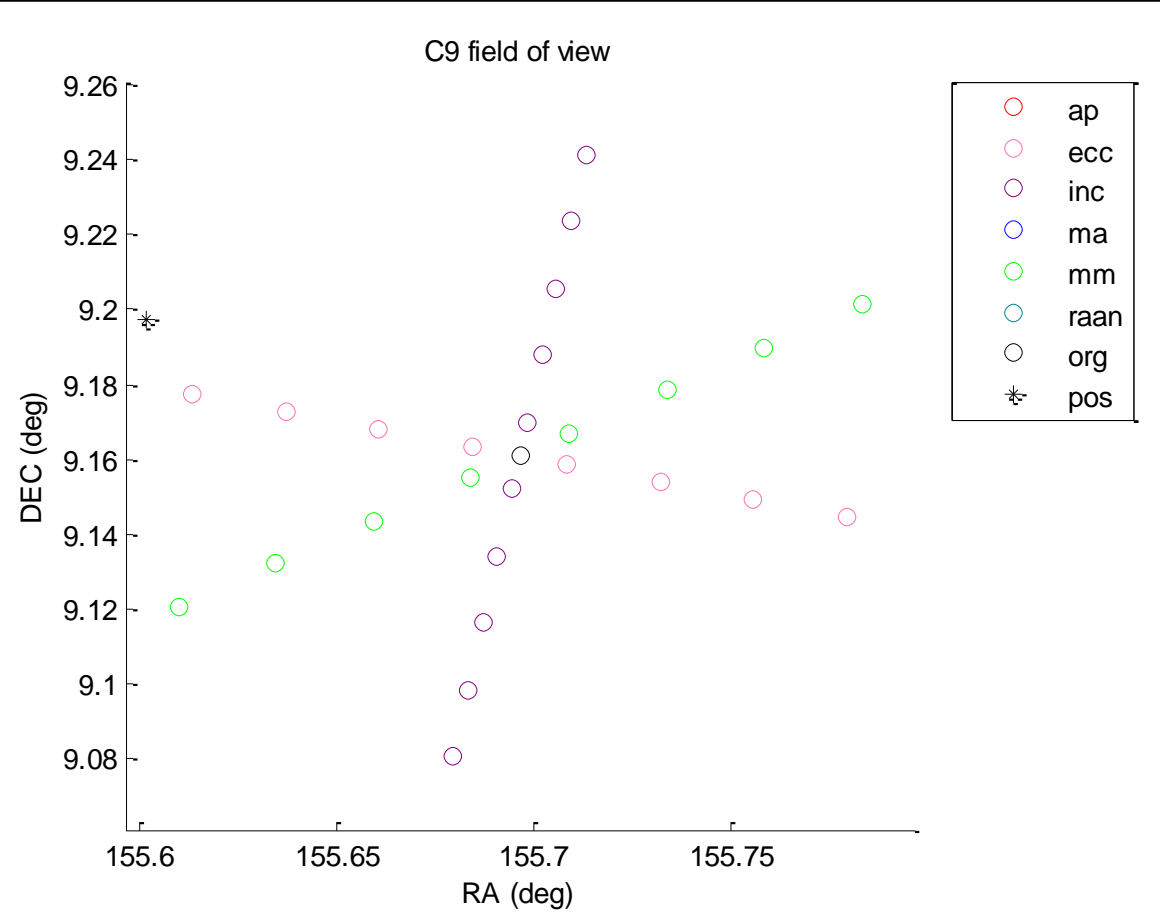

Figure 24. 0.2 degree (C9 Telescope) of same object with $2 \%$ variation 


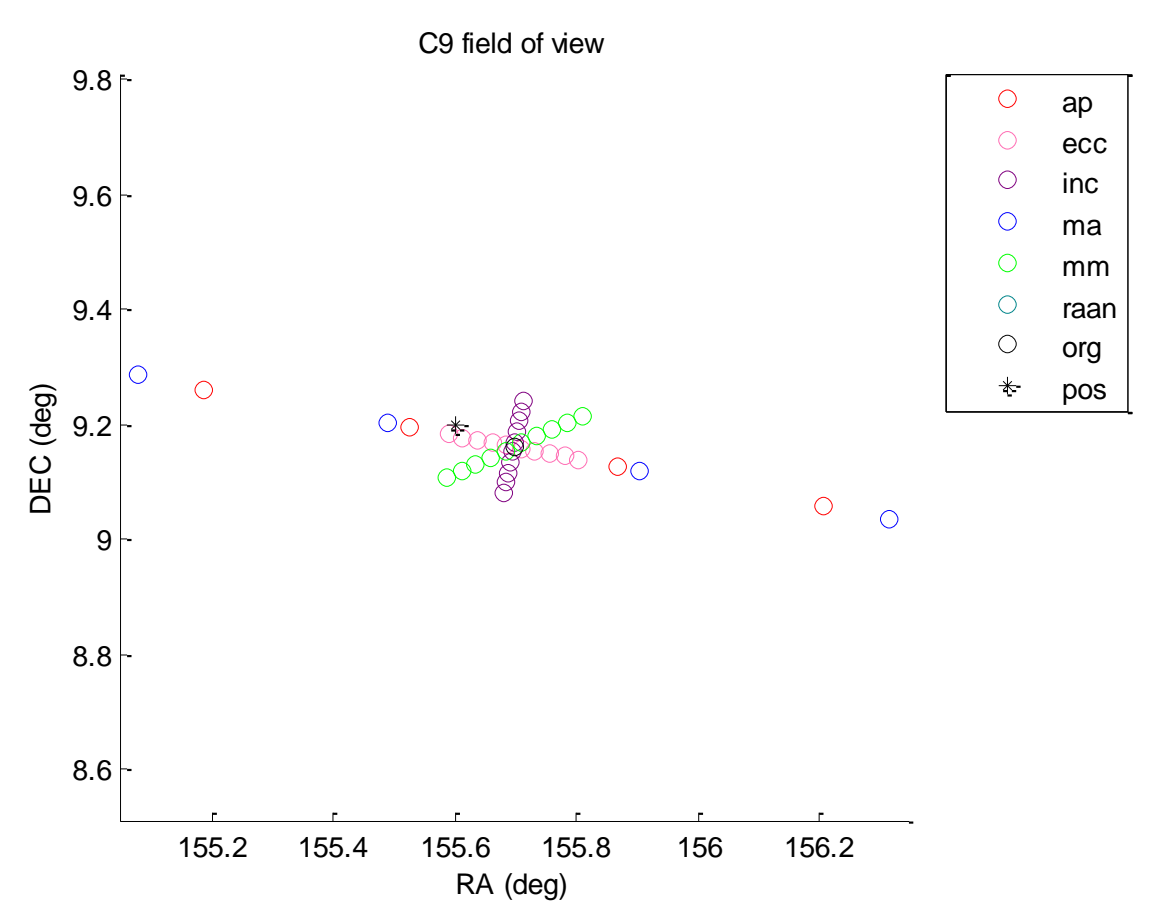

Figure 25. 2 degree (MODEST Telescope) of same object with $2 \%$ variation

Most of the set of objects analyzed followed along this pattern, AP and MA were on average the hardest to keep within the field of view, RAAN came next because in some cases it was easier to bring into view than others, as discussed earlier this was very dependent on the initial value of the RAAN prediction. MM trailed behind them as the next most difficult usually requiring a tolerance of about $\pm 1 \%$ to keep within view, and lastly ECC and INC were quite easy to keep within the field of view compared to the others, usually being well within the field of view with a $2 \%$ variation. All of these plots for $10 \%$ variation can be found in appendix B and some select objects for $2 \%$ variation can be found in appendix D. 


\subsection{Search Patterns}

The main goal of this report is to create a search pattern for when an object does not appear in the field of view of the $\mathrm{C} 9$ telescope. While most of the preceding information is important in learning how the objects move through space and the trends that exist in varying the COEs it does not offer a clear search pattern to use. This section will discuss the search patterns developed as well as reasons behind the choice of search patterns.

\subsubsection{The Data Space}

First it would be beneficial to see the entire set of data around a single point; with this regions where it is unlikely for an object to appear can be seen. To do this the difference between the first predicted point of an observation set and the first predicted point of the first observation was calculated, and then used to shift all of the other data so the first observation set was the center of all variations. This can be seen on Figure 26. It is quite clear from this figure that there are two large regions where none of the objects ever enter. These regions quite likely only exist because the inclination does not pass 15 degrees; a 90 degree inclination would cause the AP and MA errors to be completely in DEC instead of RA and would close this region. However due to the nature of the objects being sampled the inclination cannot exceed 15 degrees and therefore these regions are very real.

From this data it is clear that while an all encompassing search strategy may be desirable it would not be practical because the possible locations are wide spread. So in order to create more successful search strategies the best option would be to base them 
off of one or more COEs, preferably ones that are easy to find with reasonable accuracy. Two COEs stand out in this, INC and RAAN, both vary very little per object; that is the initial guess for these COEs change very little over the course of subsequent observations, usually on the order of less than $1 / 10^{\text {th }}$ of a degree. Inclination would be a good place to start separating search patterns since it has the most control on how much error in DEC will be generated by AP and MANOM. Figure 27 shows this concept, as one limits the inclination to only higher values in the plot the center of the cones projected by AP and MANOM are cut out. This means that the higher the inclination the more likely the object is off in DEC.

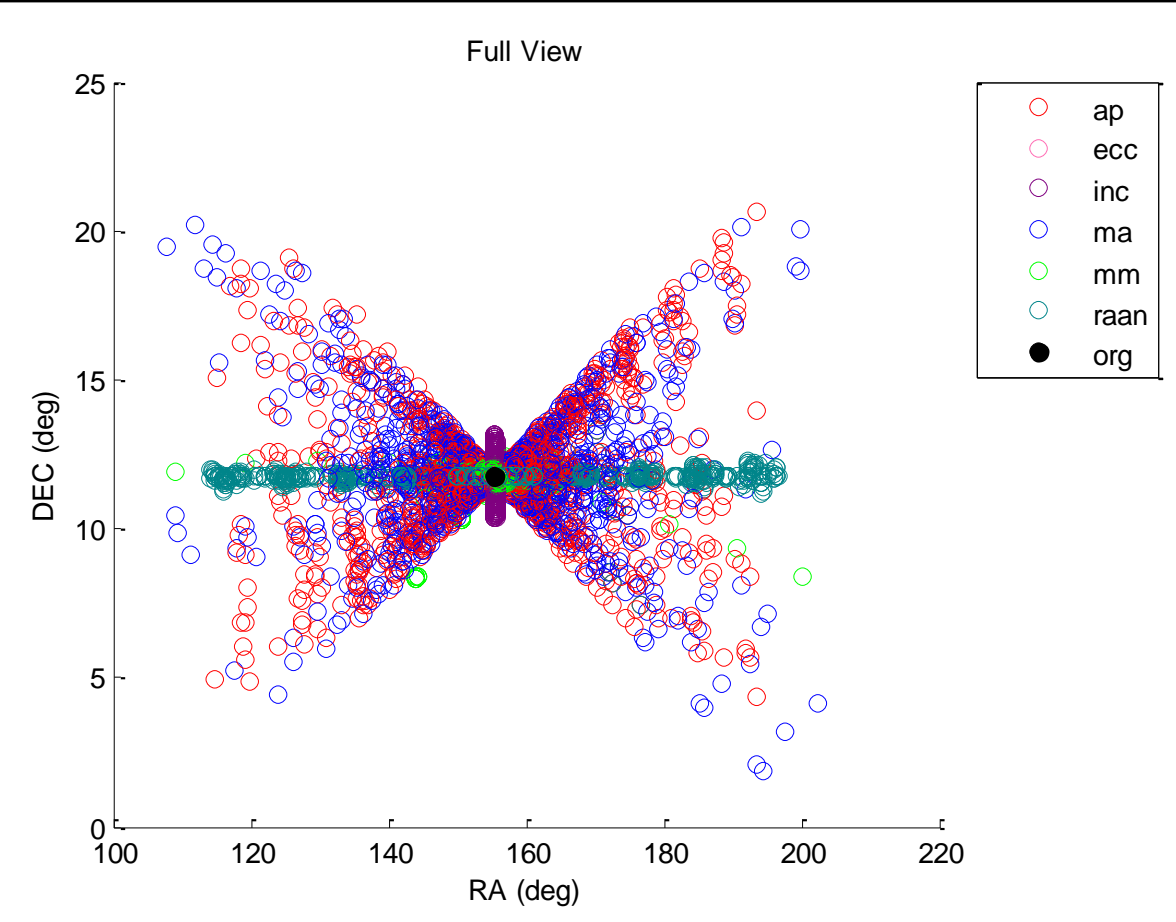

Figure 26. Full View of the 1st prediction for all observation sets 


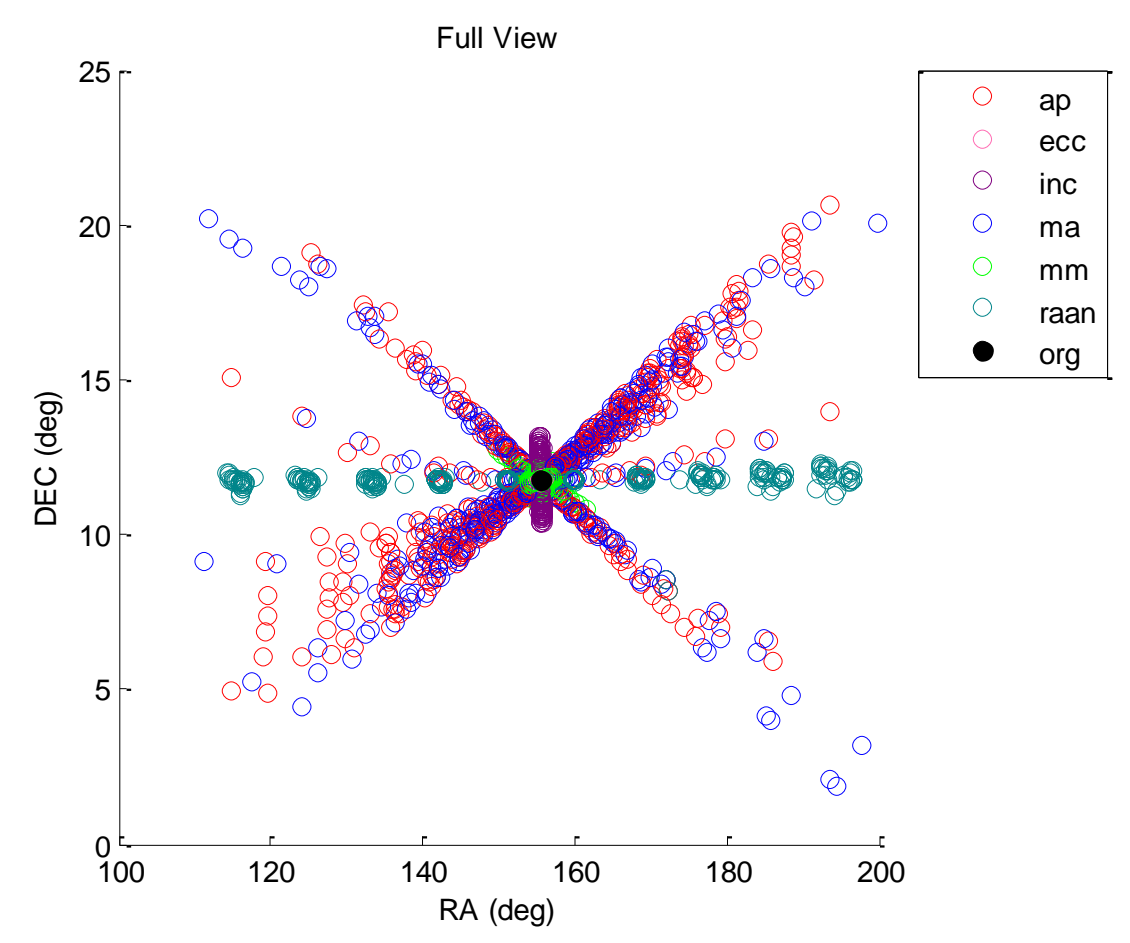

Figure 27. Full View of the 1st prediction for observation sets with a base inclination above 12 degrees

There is still a few data points for AP and MANOM in the center of the plot, this is due to the RAAN, when the observation is close to 90 degrees off of the RAAN the object will be moving mostly in the RA, it has reached a peak (or valley depending on the side of the orbit) in its orbit and from an observers standpoint will appear to be changing direction. This generates a couple criteria for when picking the search strategy that will work best:

- Is the inclination high or low?

- Will the predicted location be close to 90 degrees off of the right ascension of the ascending node?

This will potentially create four main types of search strategies to use when searching for missing objects. However when zoomed in to close to the C9 telescope field of view, 0.2 
degrees by 0.2 degrees, it is clear that the RAAN does not have enough of an impact to warrant changing the search strategy so that leaves two main strategies that will break into several patterns. All of these patterns assume that the COE is being underestimated, if it is believed the COE is being overestimated mirror the grid across the $\mathrm{x}$ and $\mathrm{y}$ axis.

\subsubsection{Type 1: Search Strategy for High Inclination Greater than 12 Degrees}

The first step in creating a search strategy for this will be to zoom in on the predicted location. Figure 28 shows this zoomed in view on the point so the initial search area can be seen. Each square in the grid is 0.2 degrees by 0.2 degrees, the size of the field of view for the C9 telescope. From here it can be seen that if mean motion, MM, is the most likely source of error then the object could be in any possible search region.

But since this is only one possible source of error it will be a low priority for the first search pattern. Figure 29, Figure 30 and Figure 31 shows three possible search patterns for this strategy. 


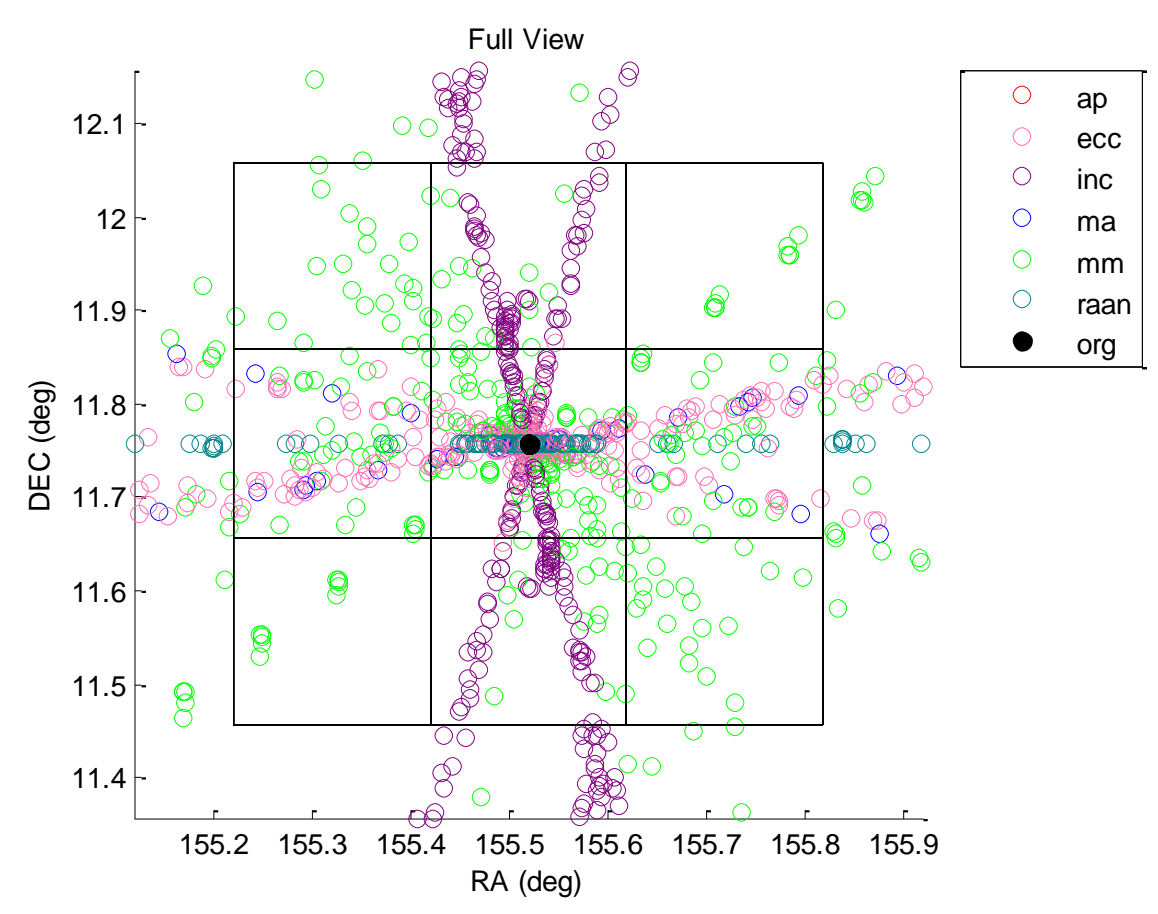

Figure 28. Zoomed in view with search grid for high INC orbits

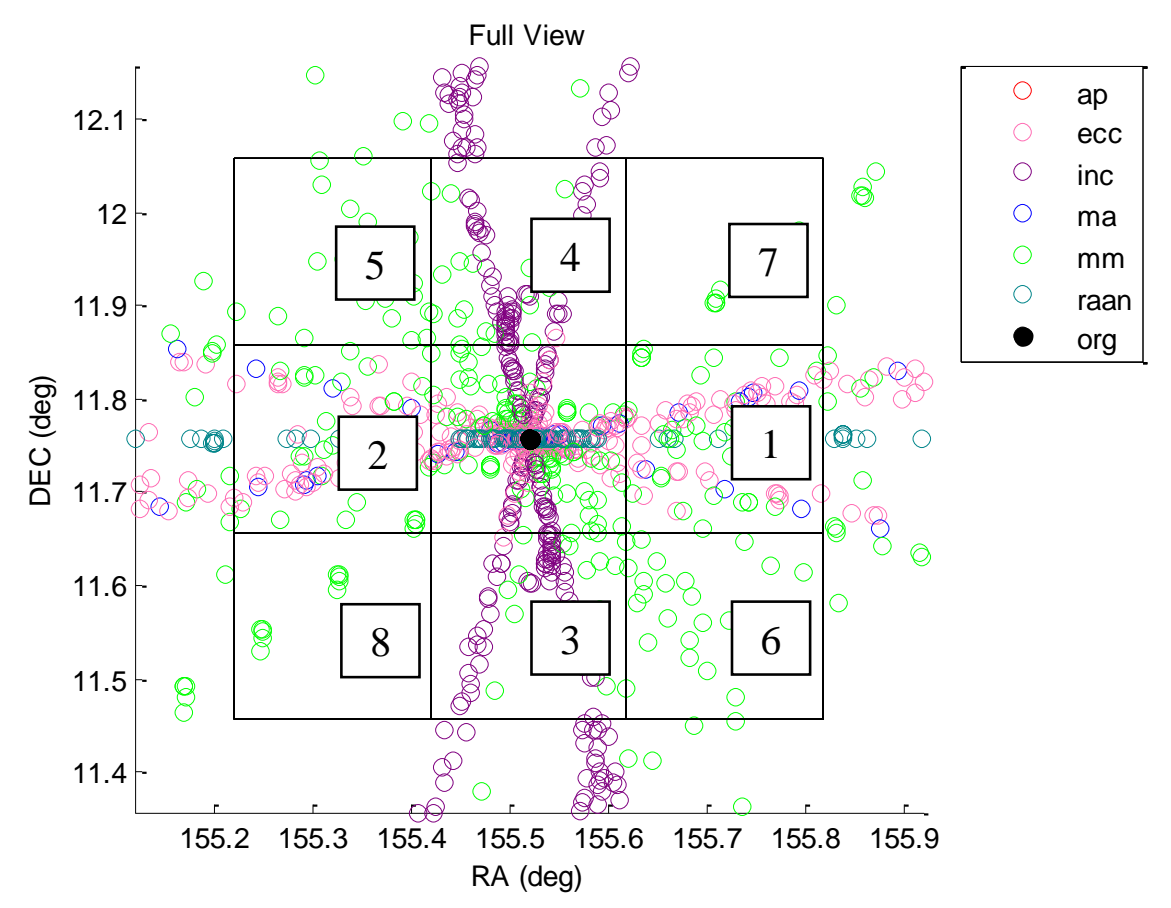

Figure 29. Zoomed in view with search grid for high INC orbits MM low priority 


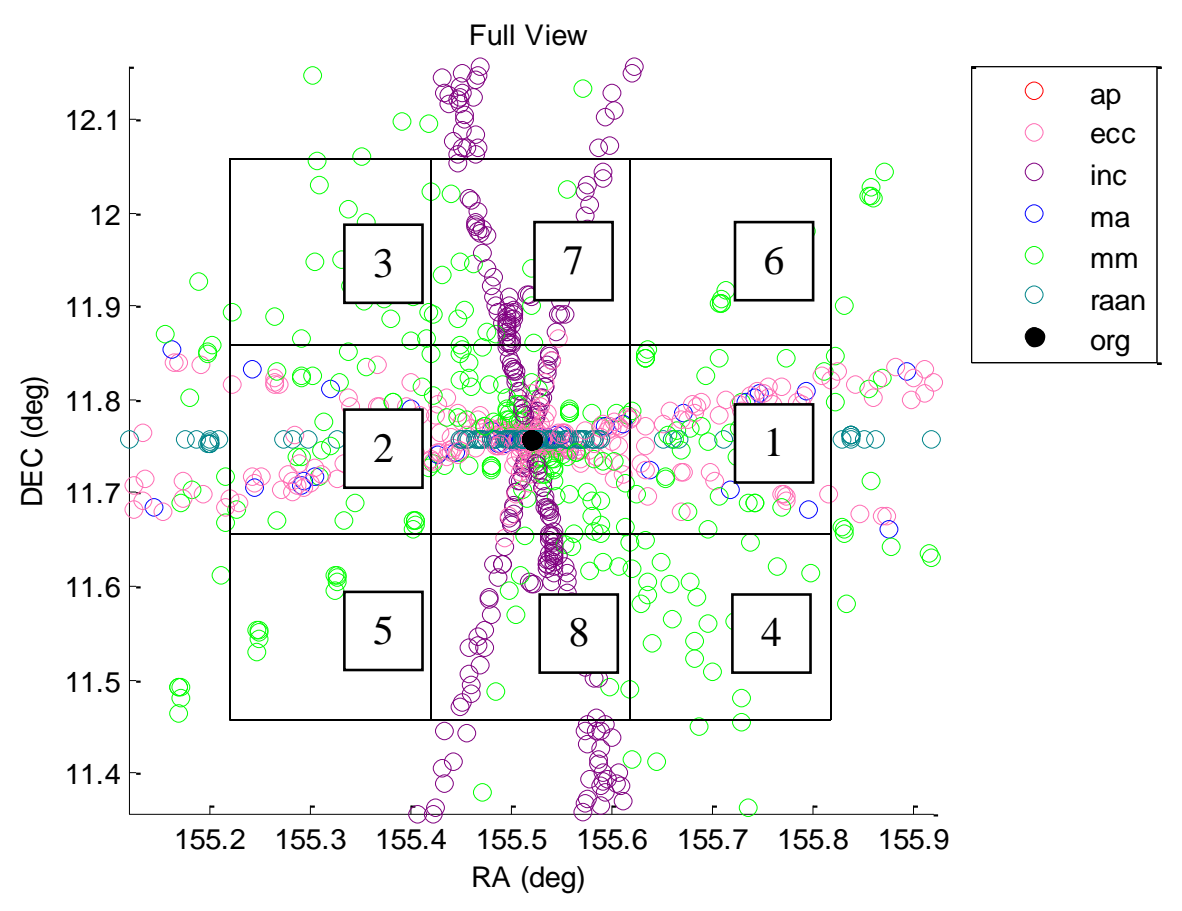

Figure 30. Zoomed in view with search grid for high INC orbits INC low priority

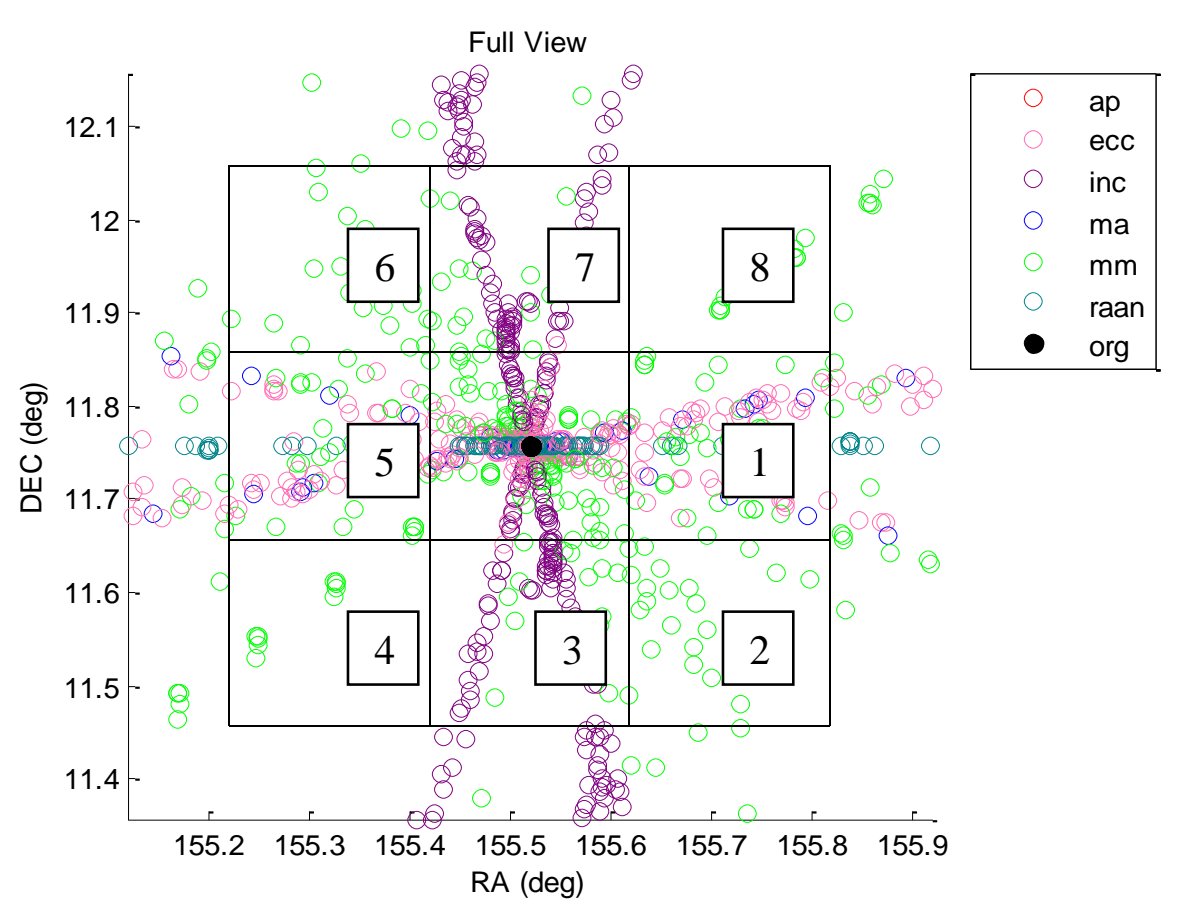

Figure 31. Zoomed in view with search grid for high INC orbits telescope limitations 
The search pattern in Figure 29 puts MM at the lowest possible priority looking everywhere there is error before looking in places where only MM error contributes. If $\mathrm{MM}$ is a higher priority Figure 30 can be used; where INC is the low priority COE. In both cases the search in RA was done first because more COEs vary into those regions. Lastly Figure 31 shows a search strategy more useful to the telescope operators because it is difficult to maneuver the telescope into a pattern that is not steady. A good strategy if the variation in MM is relatively small would be to skip grid 4 if possible and move diagonally from 3 to 5 skipping over the relatively empty grid.

\subsubsection{Type 2: Search Strategy for Low Inclination Less Than or Equal to 12 Degrees}

The second type of search strategy becomes more defined as inclination decreases, that is following this strategy will be more likely to find the object the lower the inclination is unlike the previous where a higher inclination will change the strategy here the strategy just improves. The search strategy for both this section and the preceding section will be different for searching beyond the initial grid; this will be discussed in the next section. 


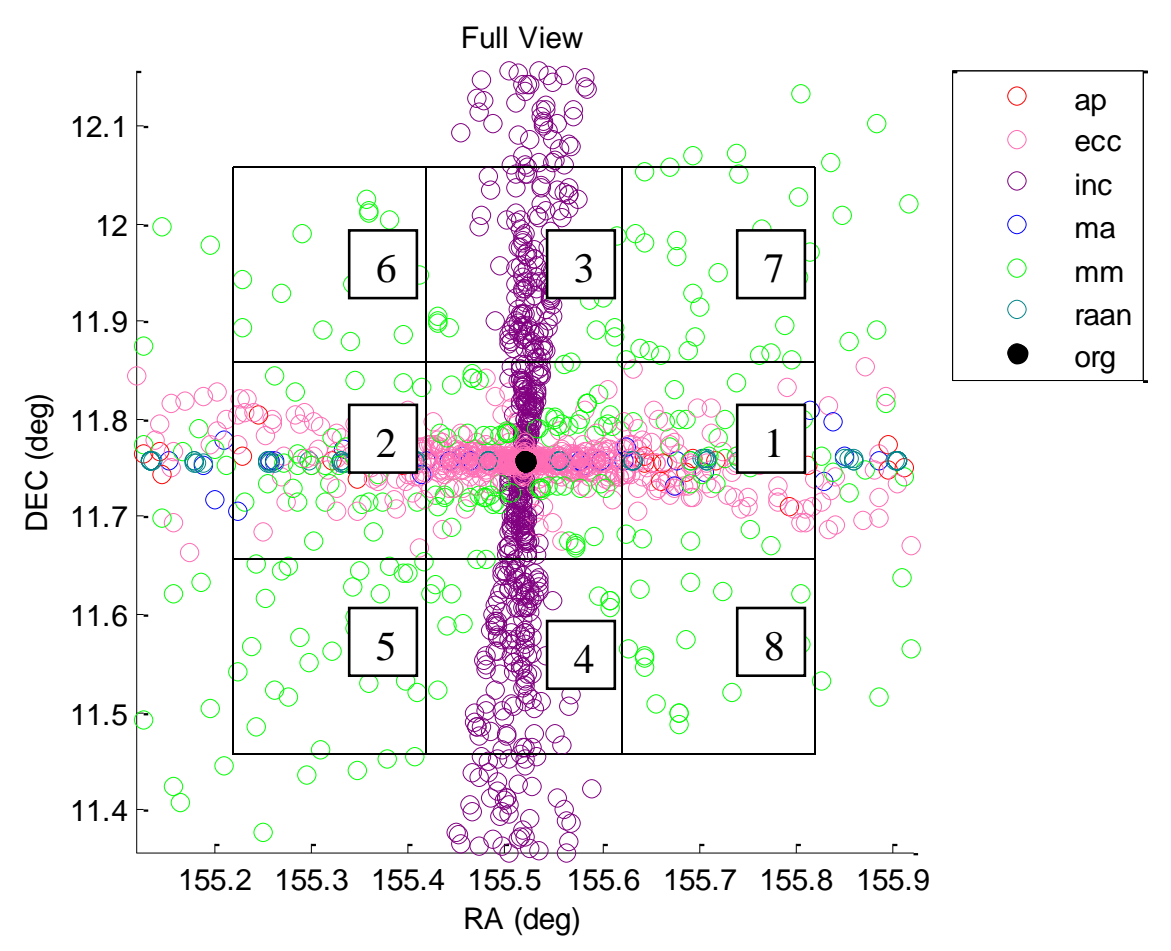

Figure 32. Zoomed in view with search grid for low INC orbits MM low priority

In the pattern in Figure 32, MM is a low priority and is essentially skipped over in favor of the other COEs. Looking in RA is a preferable to looking in DEC, switching the positions of grid 1 and 2 if it is believed that the COEs are being overestimated.

Figure 33 shows the search pattern that is easiest to use for the telescope operator. And like the previous pattern skipping the corners would be preferable if MM is not seen as a major factor in the error. 


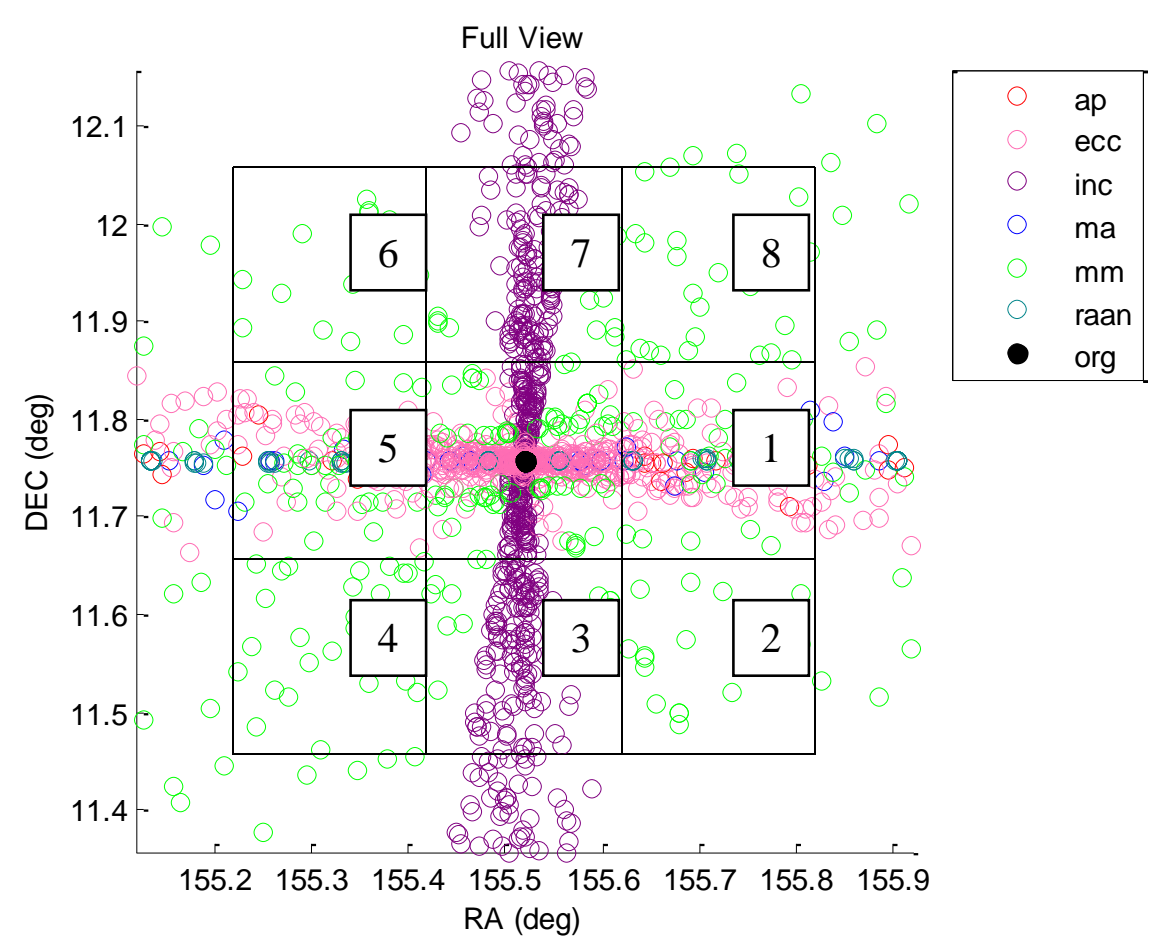

Figure 33. Zoomed in view with search grid for low INC orbits telescope limitations

\subsubsection{Long Range Search Strategy}

After the initial grid searching in the spiral formation will have increasingly depreciating results. After this point the search strategy should be broken up depending on which $\mathrm{COE}$ is believed to be the one that is most likely incorrect. If the inclination is wrong searching in the DEC would work best, if the RAAN is wrong searching in the RA would work best. When one believes the objects COEs were underestimated look forward in RA or up in DEC from the initial prediction, and when the objects COEs were overestimated look back in RA or up in DEC the initial prediction. 
For AP and MANOM one should search along the predicted orbital path, backward when one believes the COE is overestimated and forward when one believes it has been underestimated. ECC works in a similar fashion however it does not perfectly follow the orbital path and therefore may be missed; this is still the best way to search for an object in this case. And lastly MM is a fairly random error, that is when there is an error in MM the object could be in any direction from the center so it would be difficult to find a good search strategy for this COE except to spiral out from the center and continue to use the strategy discussed for when close to the original prediction.

\subsubsection{Summary of Search Patterns}

The search patterns presented in the previous section are only truly useful close to the predicted location, past a couple of field of view lengths from the original view the AP and MA vary into the DEC and it becomes more difficult to track down. In these cases moving in the direction of motion is preferred, either towards where the object is coming from if the COEs were underestimated or away from your predicted location if the COEs overestimated.

For low inclination orbits where the inclination is well known, like most of the objects in this data set, it would be acceptable to track just in the RA for a few frames the direction again being forward if the COE was overestimated and backward if it was underestimated.

These search patterns do not take into account the effect of varying multiple COEs at once; this could cause a compounding error that would create a larger, but more defined search area. This idea needs more research but to provide a look at how this could affect the above plots one object was subjected to varying every COE by $2 \%$ 
creating 64 TLE files, one for each combination of the variation; for example, INC $+2 \%$, RAAN -2\%, ECC-2\%, AP +2\%, MANOM-2\%, MM-2\%. Figure 34 and Figure 35 show this concept, one in full orbital view and one in equal axis view of the first predicted point. With this set up none of the varied predicted points fell inside the telescopes field of view.

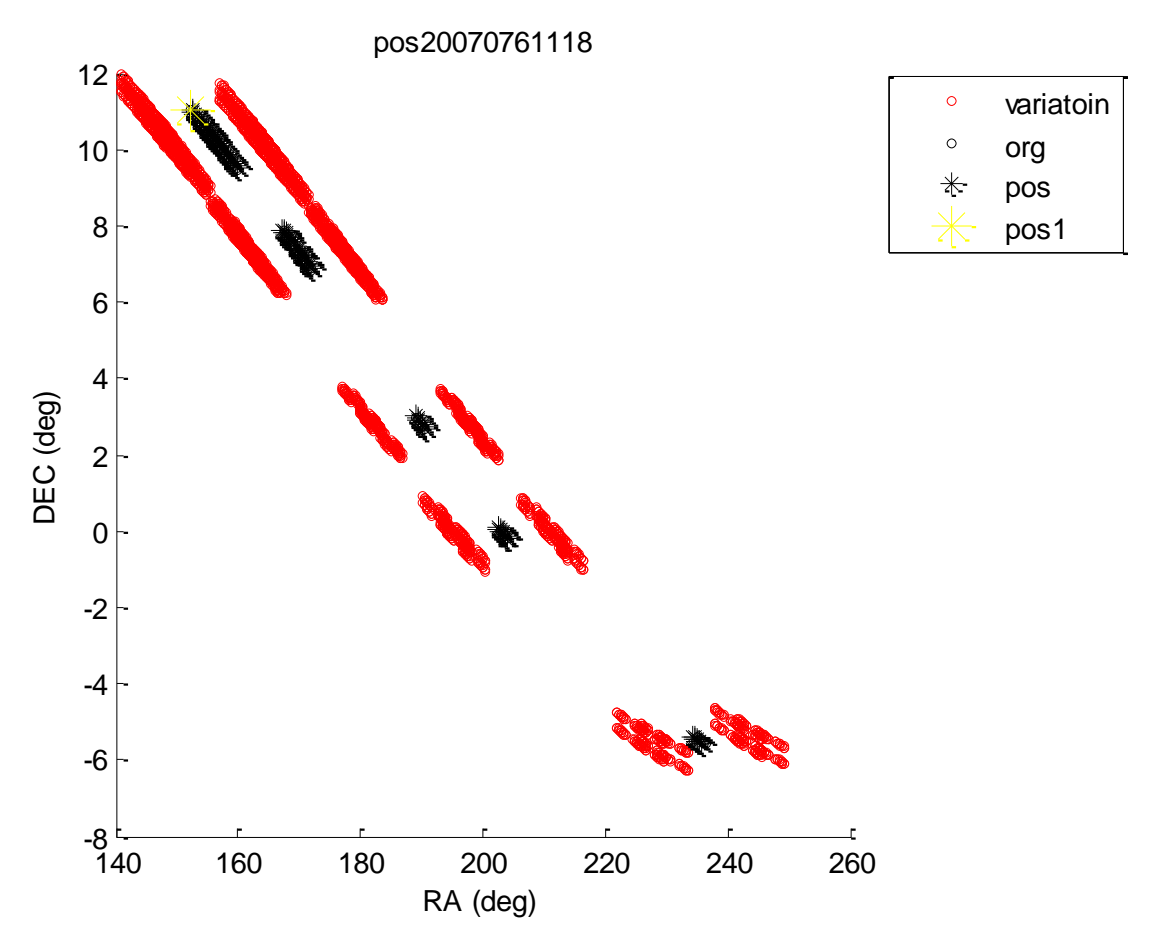

Figure 34. Multiple COE Variation Full Orbit 


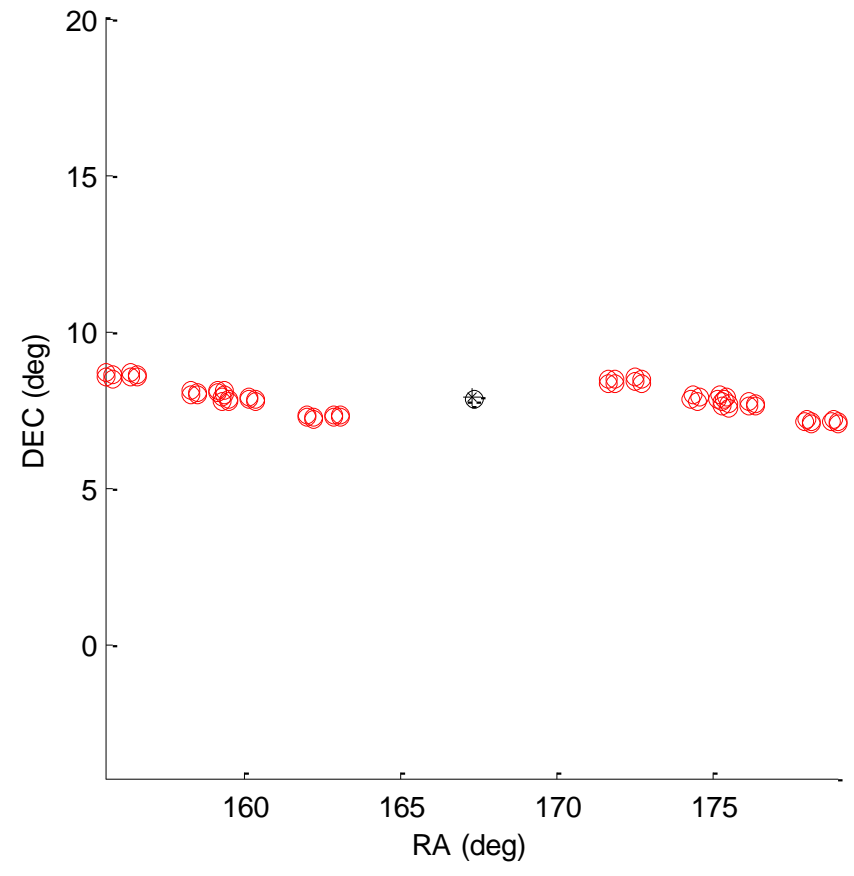

Figure 35. Multiple COE Variation First Prediction, Equal Axis 


\section{Conclusion}

In summary the search pattern used for the initial search when an object is missing is a spiral-like motion around the object. This pattern takes into account the best places to observe based on the data as well as the minimizing telescope movement time. As time progresses from the originally predicted position the search pattern is dependent upon the COE that is believed to be the largest source of error. Since there were no objects in the data set analyzed in this report that had much variation in the INC or the RAAN throughout the observations the decision is really limited to AP, MANOM, ECC and MM. MM is difficult to plan around because a variation in MM could put the object in front, behind, above or below the originally predicted location. ECC somewhat follows the orbital path and this can be used as a search direction; whereas AP and MANOM follow the orbital path exactly and makes a good search direction when they are the COE most likely incorrect.

Most of the data analyzed in this report used a variation of $\pm 10 \%$ this was sufficient to calculate the average maximum variation of INC, ECC and MM that could be allowed, and still have the object in the field of view. For the other COEs some objects were selected for the $2 \%$ variation, this showed that for these COEs the allowable variation was much smaller than for INC, ECC and MM. RAAN, AP, and MANOM have the lowest tolerance to variation, that is the error in RA and DEC is much greater for the same variation than INC, ECC and MM.

This leads to a logical next step, varying more than one COE at the same time. This would be a good starting point for future research, finding the full region that an object could be in would help limit the search areas and possibly allow a faster and more 
efficient search strategy; this could also point to one overarching search strategy for all objects. The search strategies presented in this report are based on the fact that each COE varies independently, that is it is the only thing that is varied.

A final note, investigating the reason why the error in RA vs. RAAN increases when RAAN is varied could lead to beneficial results. This was the most correlated error vs. a COE found in the data set and could be a contributor to the large errors in the AP and MANOM. More correlations may be found as the size of the data set is increased. To do a true statistical analysis of the GEO belt would require many more data points than used in this report however the data used was based on real observations and therefore the scope of the data was limited by the number of observations produced. 


\section{References}

[1] Milani, Andrea. "The Asteroid Identification Problem: Recovery of Lost Asteroids." October 13, 1998. <http://www.idealibrary.com>.

[2] Agapov, V., Biryukov, V., Kiladze, R., Molotov, I., Rumyantsev, V., Sochilina, A., Titenko V. "FAINT GEO OBJECTS SEARCH AND ORBITAL ANALYSIS" Proceedings of the 4th European Conference on Space Debris (ESA SP-587). 1820 April 2005, ESA/ESOC, Darmstadt, Germany. Editor: D. Danesy., p.153. The SAO/NASA Astrophysics Data System. 24 June 2010 $<$ http://articles.adsabs.harvard.edu//full/2005ESASP.587..153A/0000153.000.htm $1>$.

[3] Curtis, Howard D. Orbital Mechanics for Engineering Students. New York: Elsevier Ltd. 2007.

[4] National Research Council. Orbital Debris: A Technical Assessment. Washington, D.C.: National Academy Press. 1995.

[5] Proceedings of the Third European Conference on Space Debris. Darmstadt, Germany: European Space Operations Centre (ESOC). October 2001.

[6] Abercromby, K. J., Barker, E, S., Cowardin, H. M., Matney, M. J., Parr-Thumm, T. L. "Michigan Orbital Debris Survey Telescope (MODEST) Observations of the Geosynchronous Orbital Debris Environment.” NASA/TP-2010-under review. June 2010.

[7] Rogers, Joseph Lee, Nicewander, W. Alan. "Thirteen Ways to Look at the Correlation Coefficient.” The American Statistical Association. Vol. 42 no. 1 February 1988. 26 June 2010. < http://www.jstor.org/pss/2685263>. 


\section{APPENDIX A: FULL ORBIT PLOTS 10\% VARIATION}

Some plots look similar this is because they are the same object but predictions based on different observation sets.

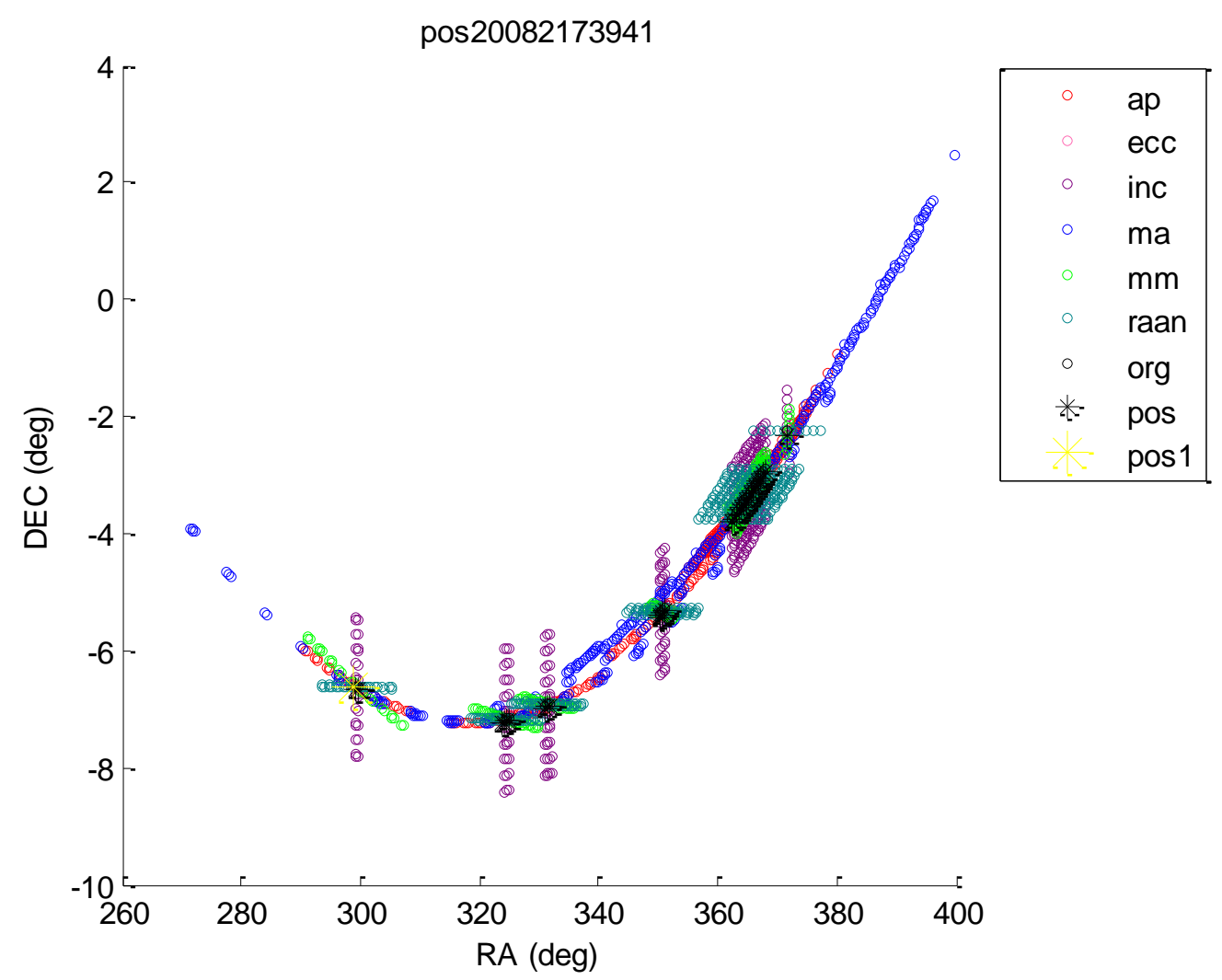



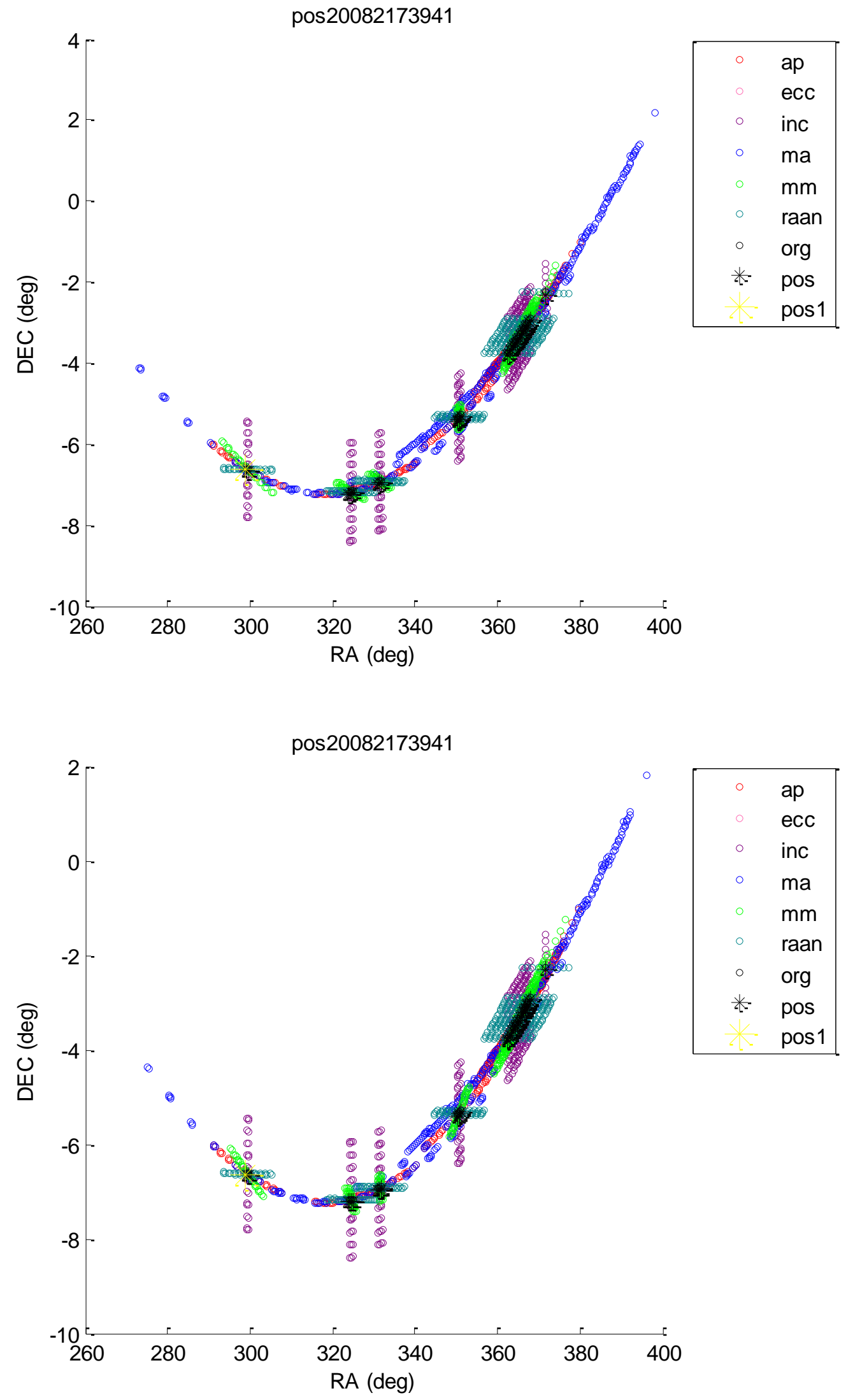

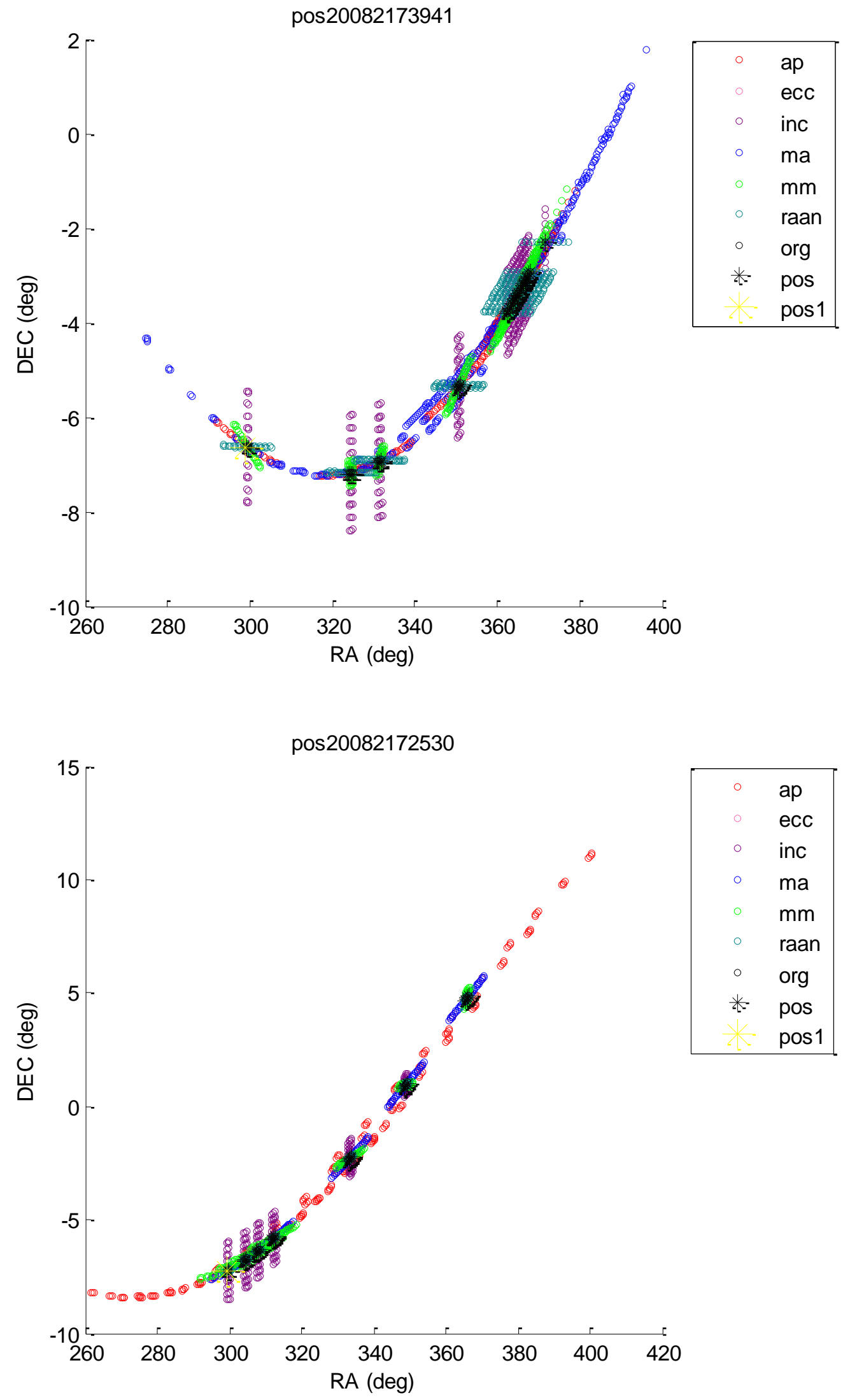

\begin{tabular}{|ll|}
\hline & ap \\
ecc \\
inc \\
ma \\
mm \\
raan \\
org \\
*- pos \\
\\
\end{tabular}



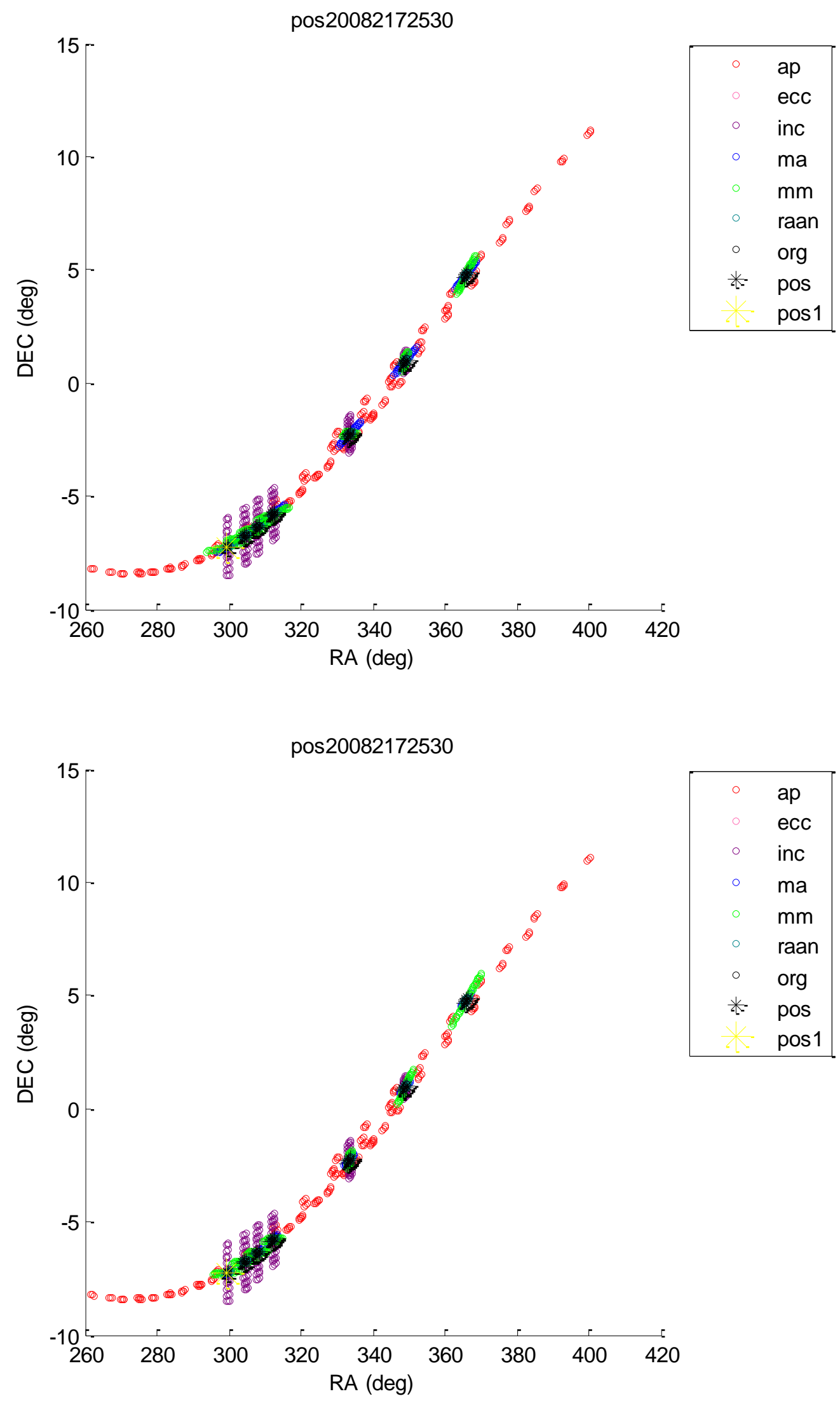

\begin{tabular}{|ll|}
\hline & ap \\
ecc \\
inc \\
ma \\
mm \\
raan \\
org \\
*- pos \\
\\
\end{tabular}



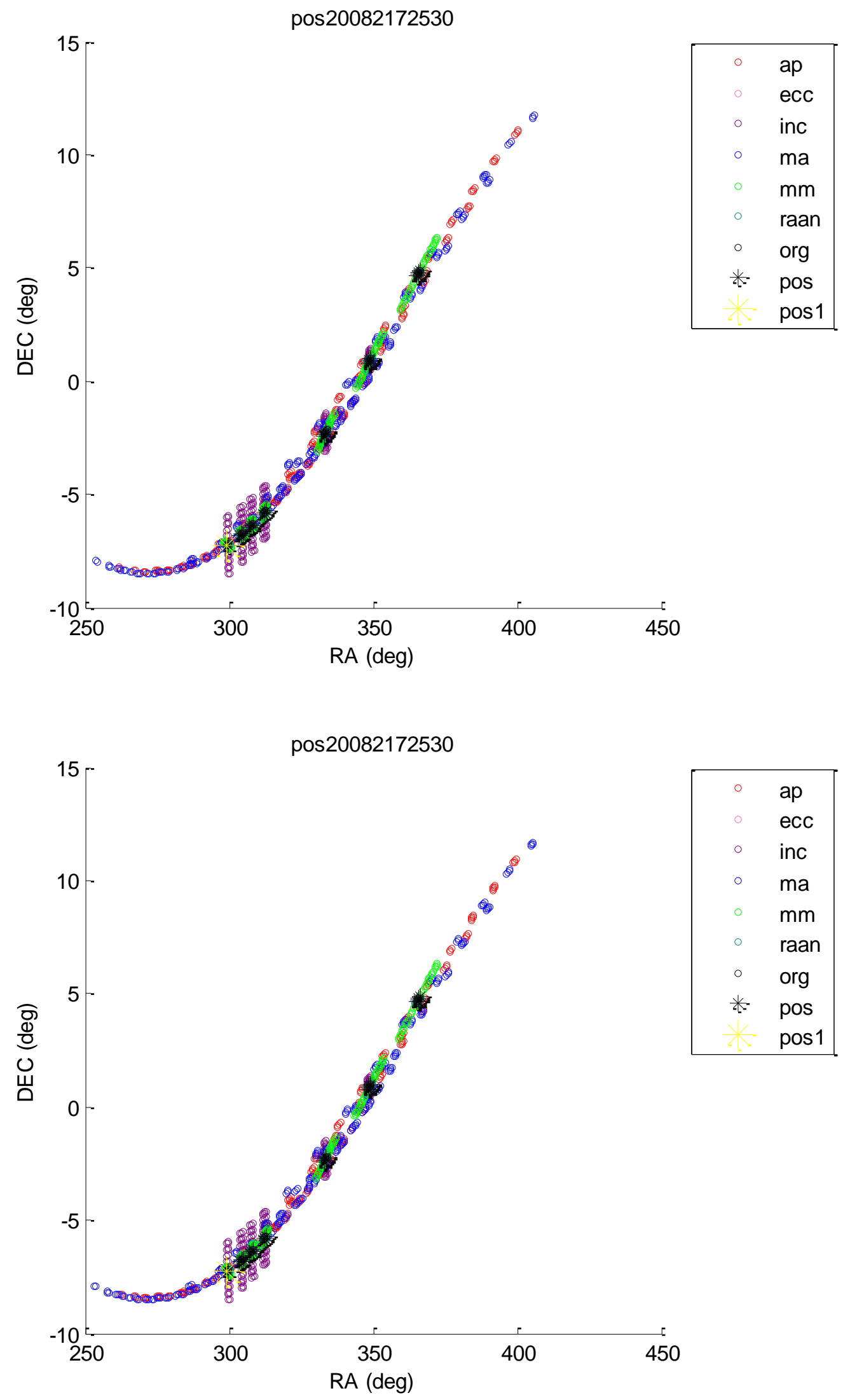

\begin{tabular}{|ll|}
\hline & ap \\
& ecc \\
& inc \\
& ma \\
& $\mathrm{mm}$ \\
& raan \\
$\circ$ & org \\
$*$ & pos \\
& pos1 \\
\hline
\end{tabular}



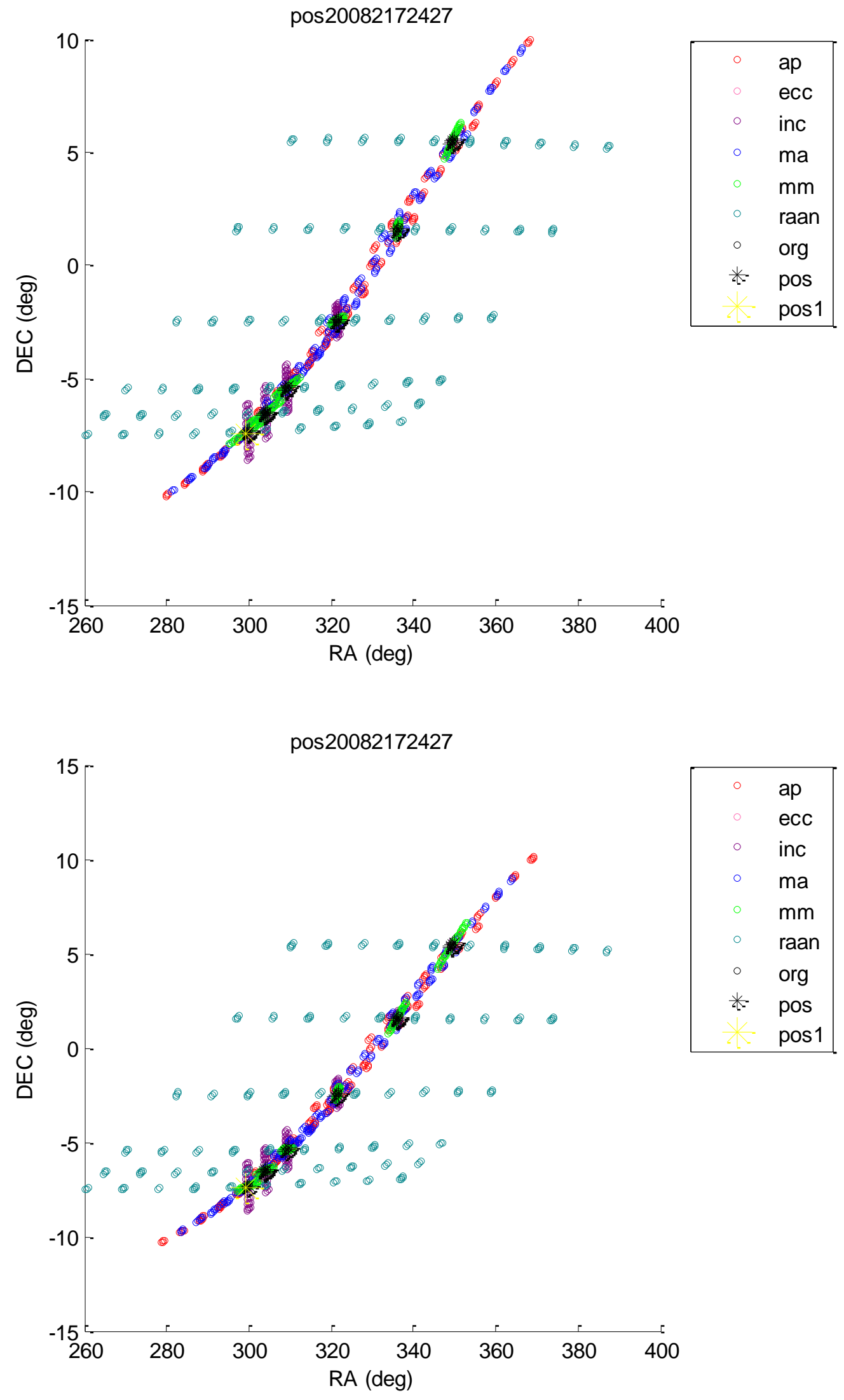

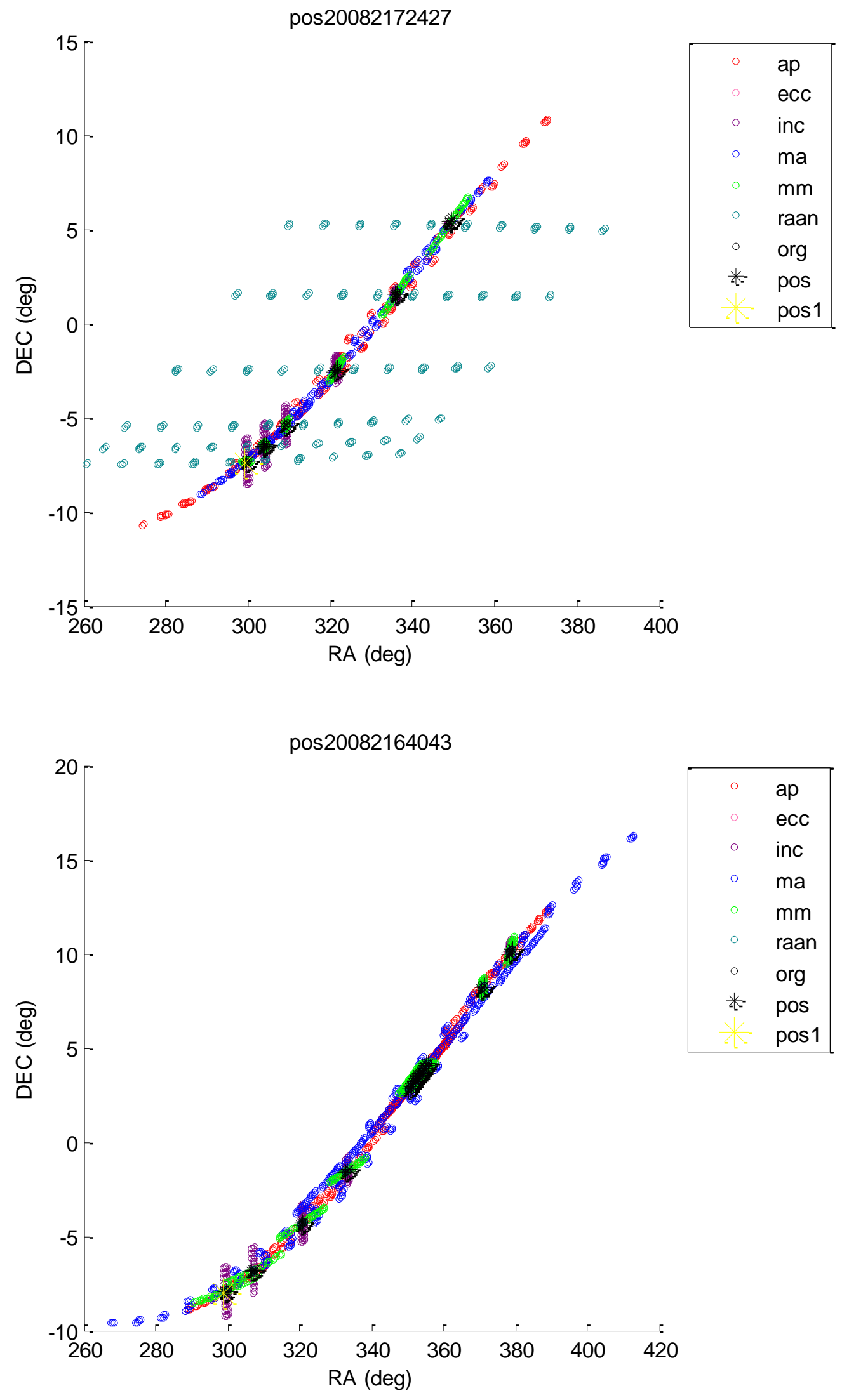

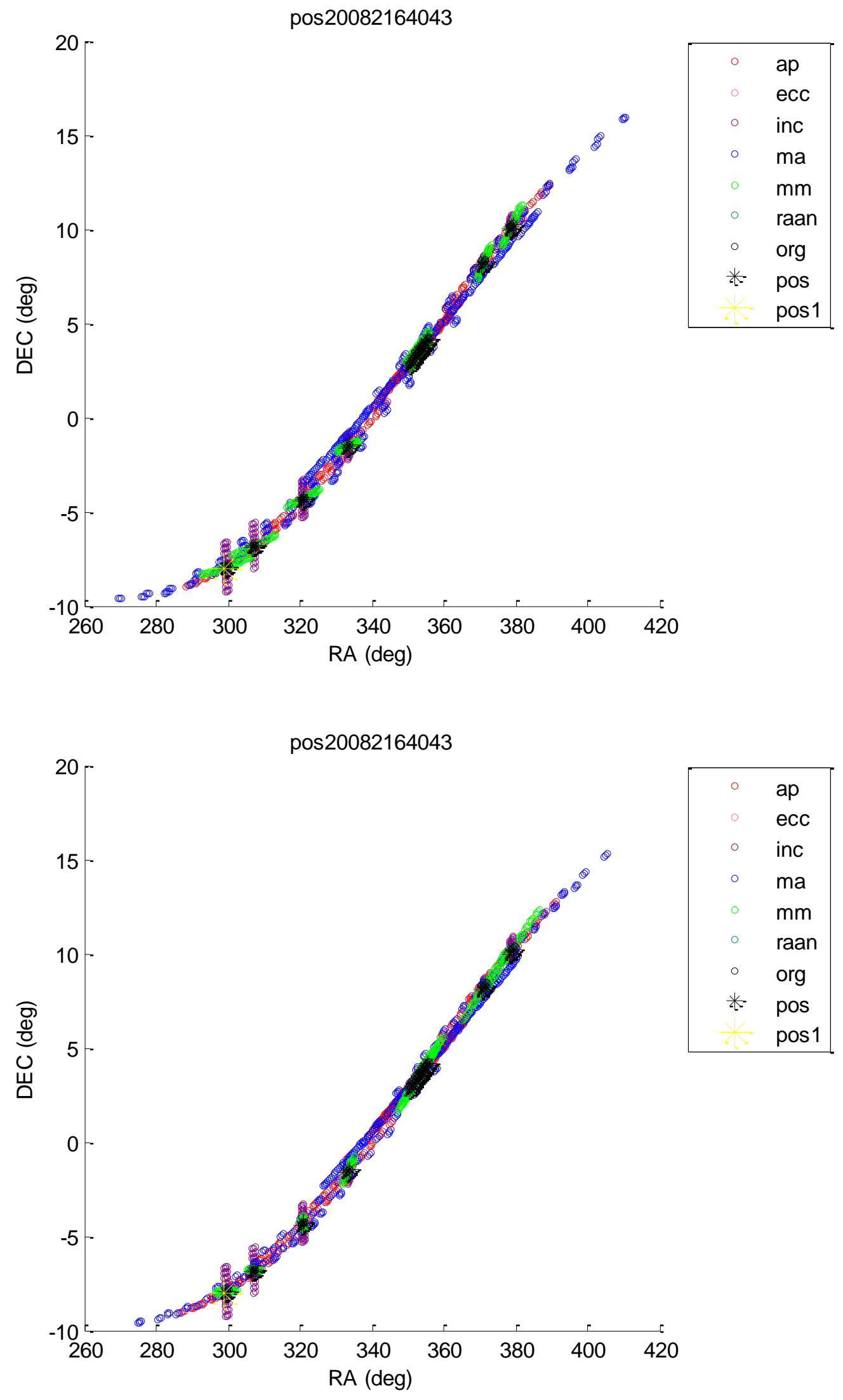

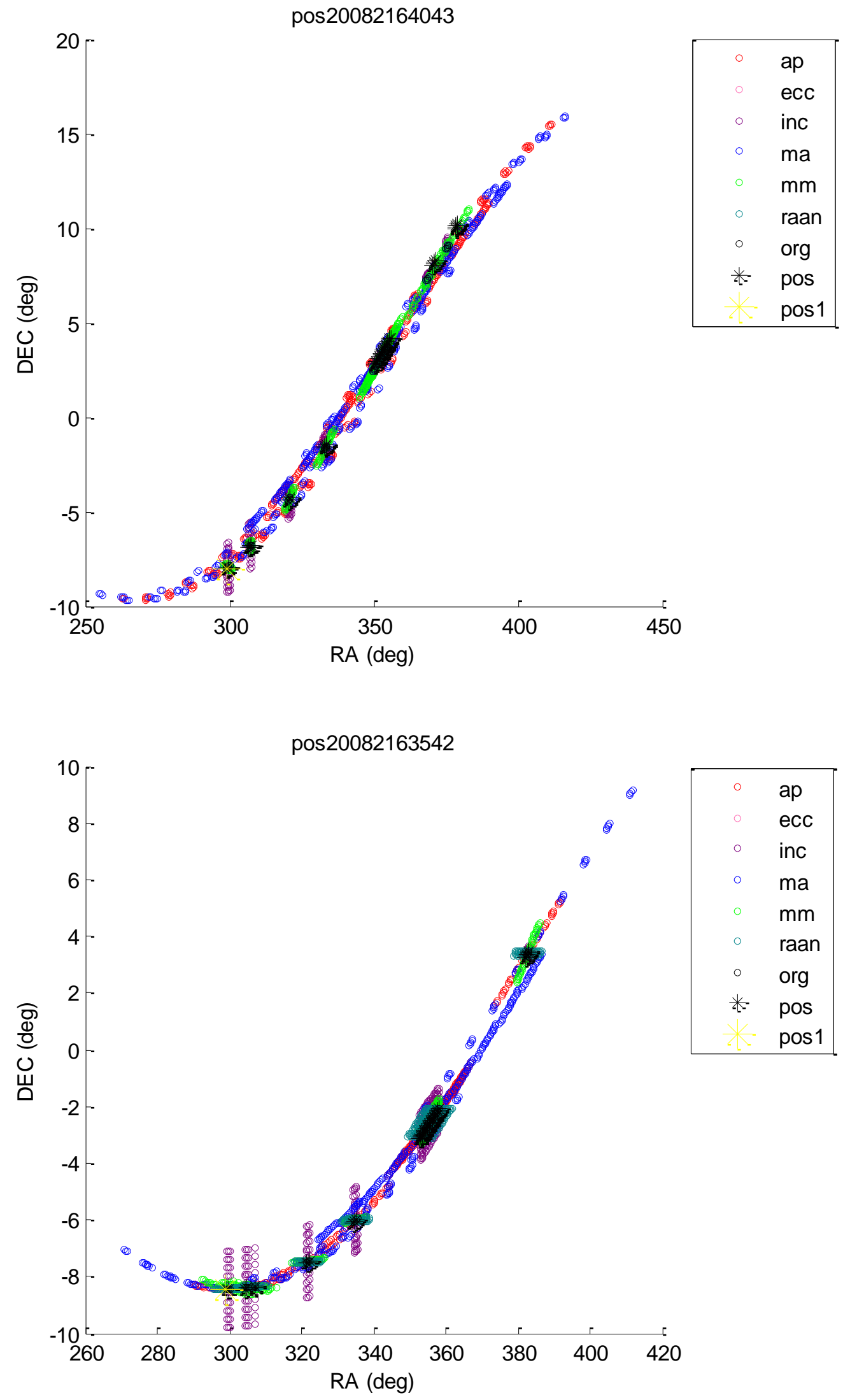

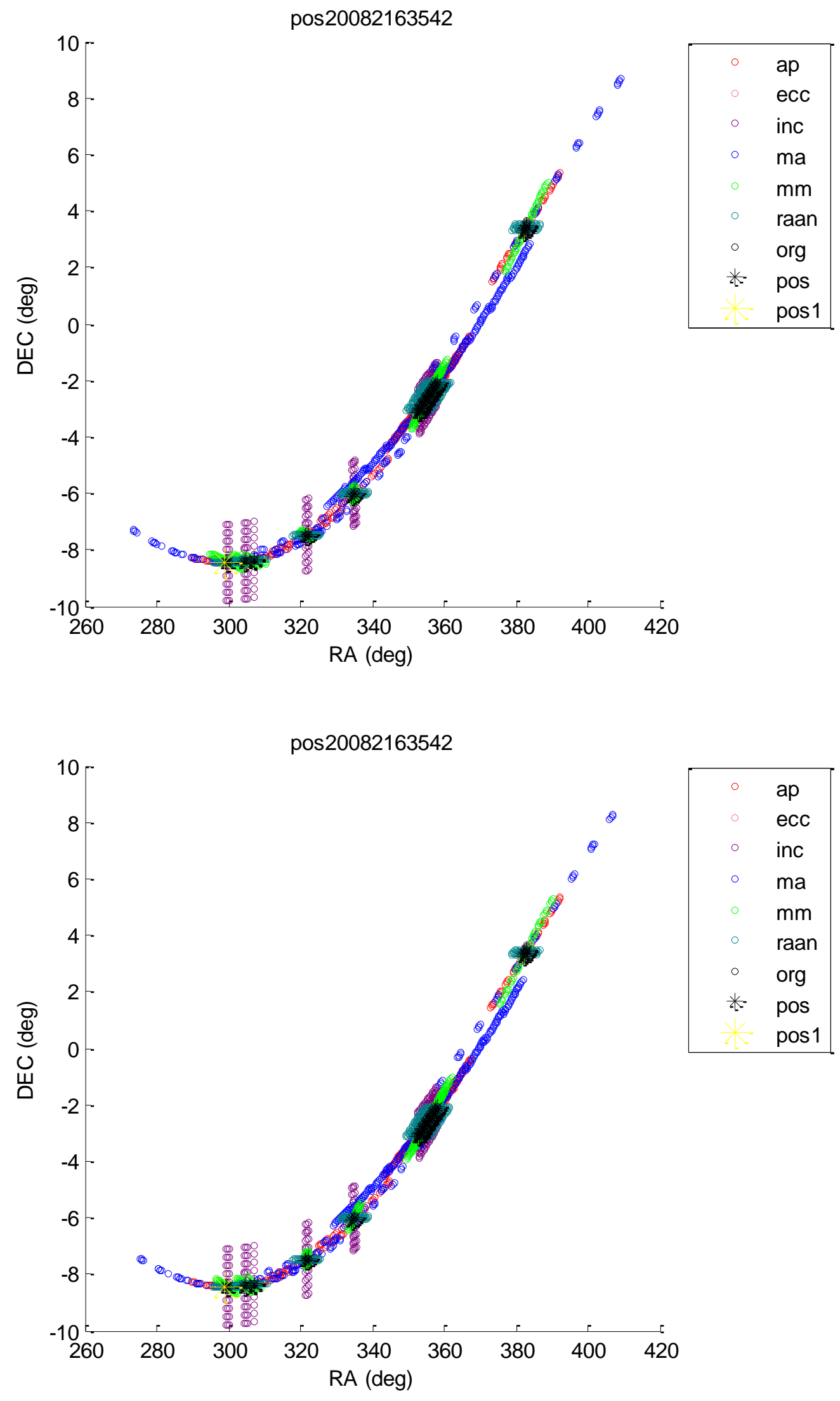

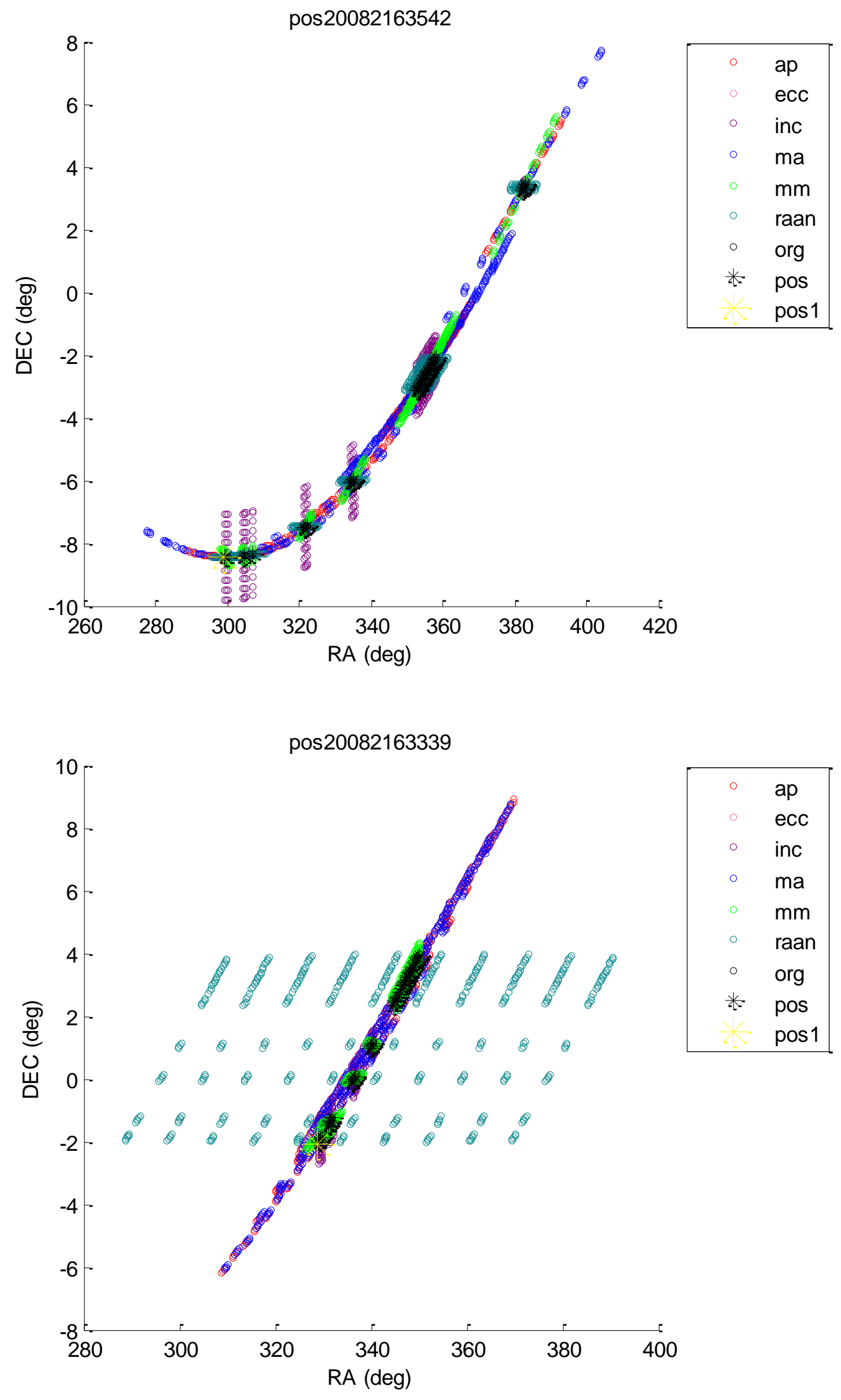

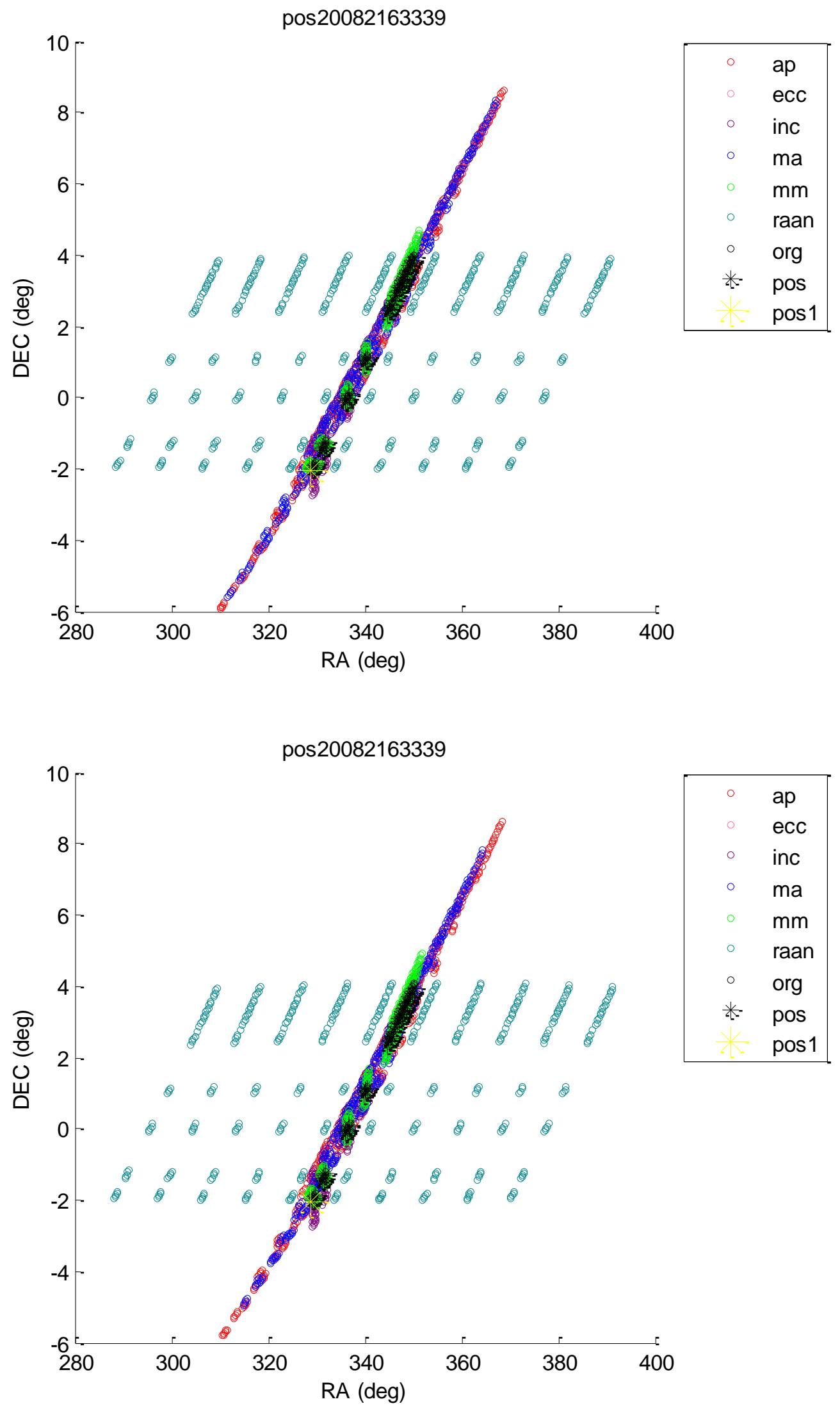

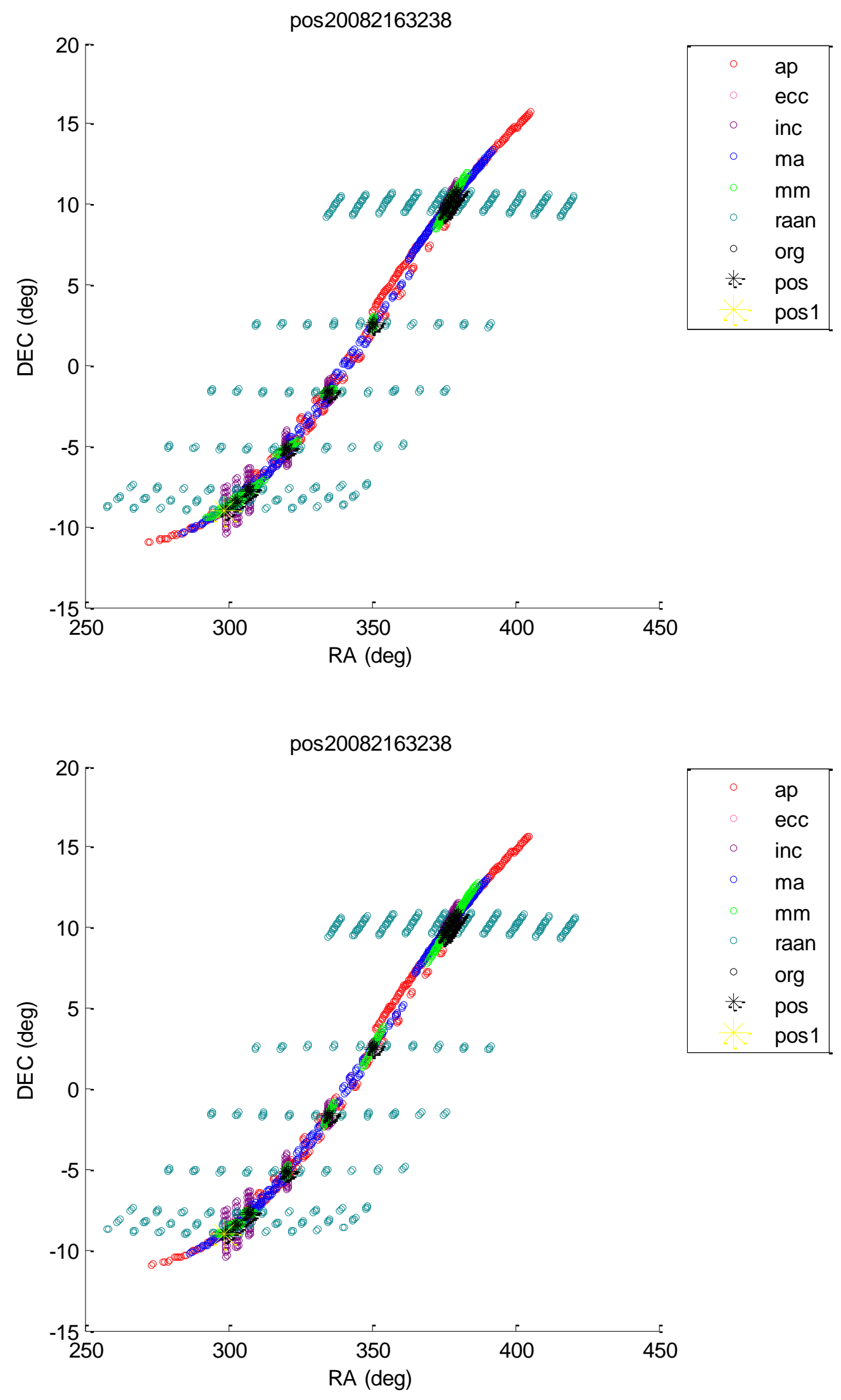

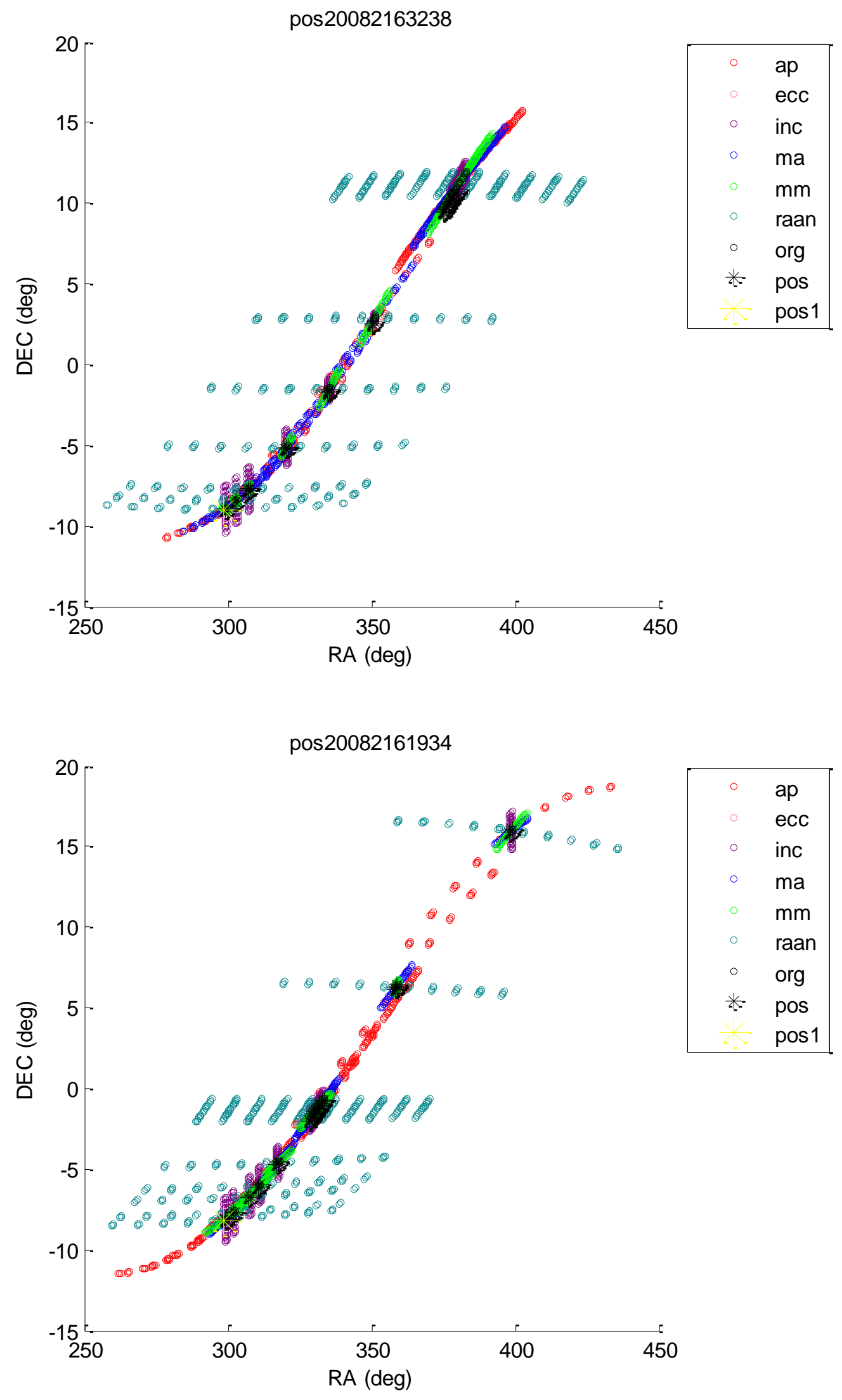

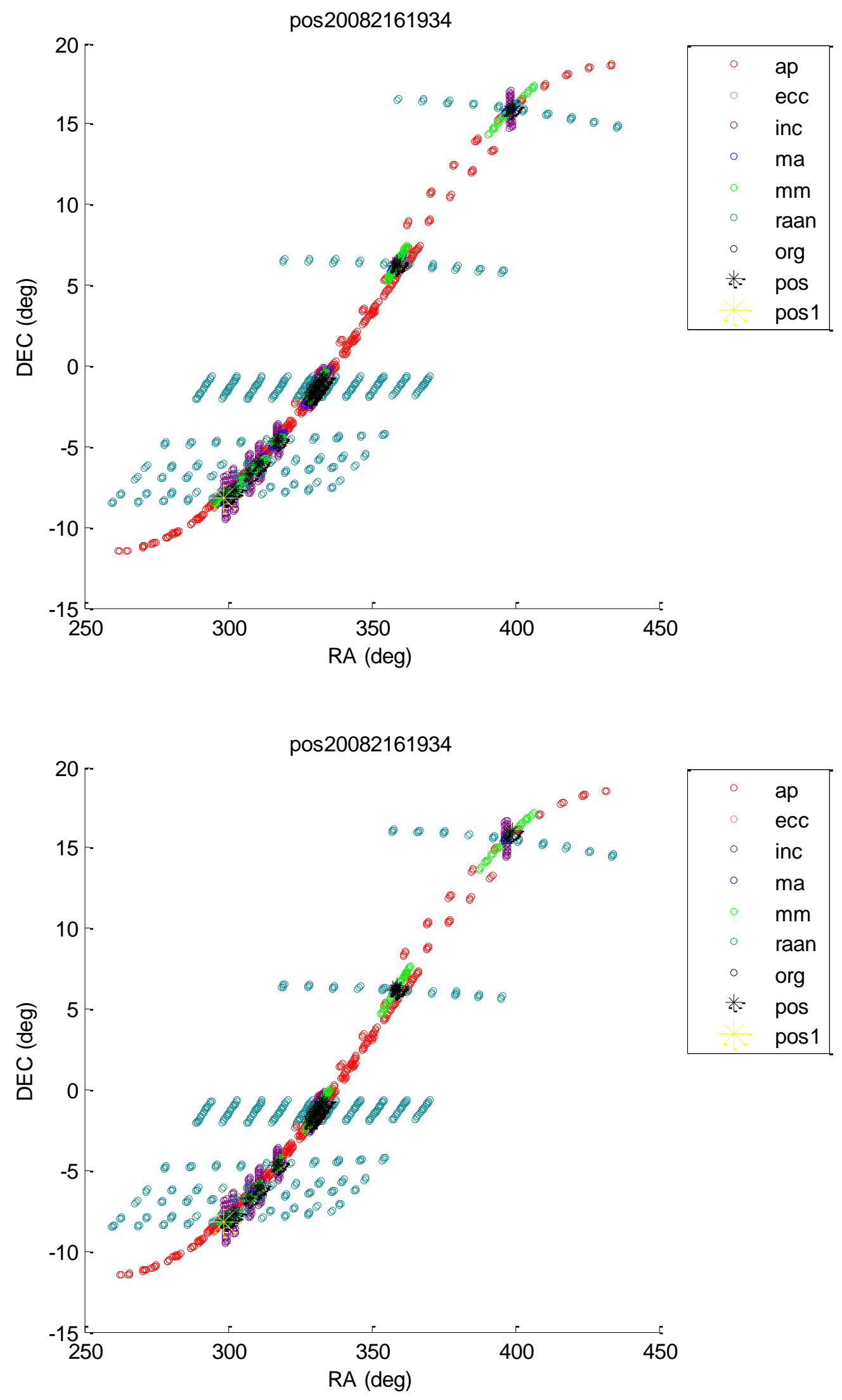

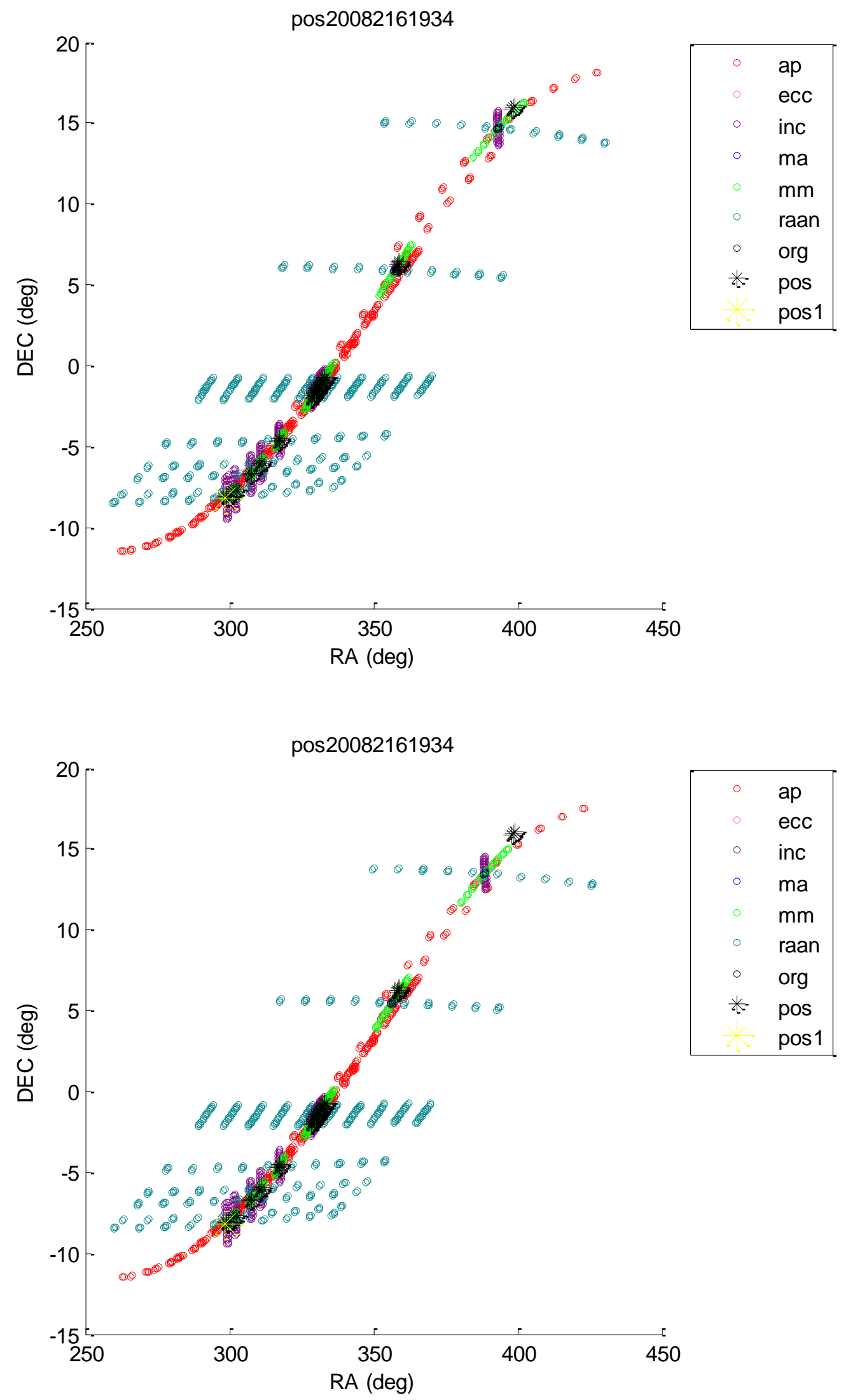

\begin{tabular}{|ll|}
\hline & ap \\
& ecc \\
& inc \\
& ma \\
& mm \\
& raan \\
$\circ$ & org \\
$*$ & pos \\
& pos1 \\
\hline
\end{tabular}



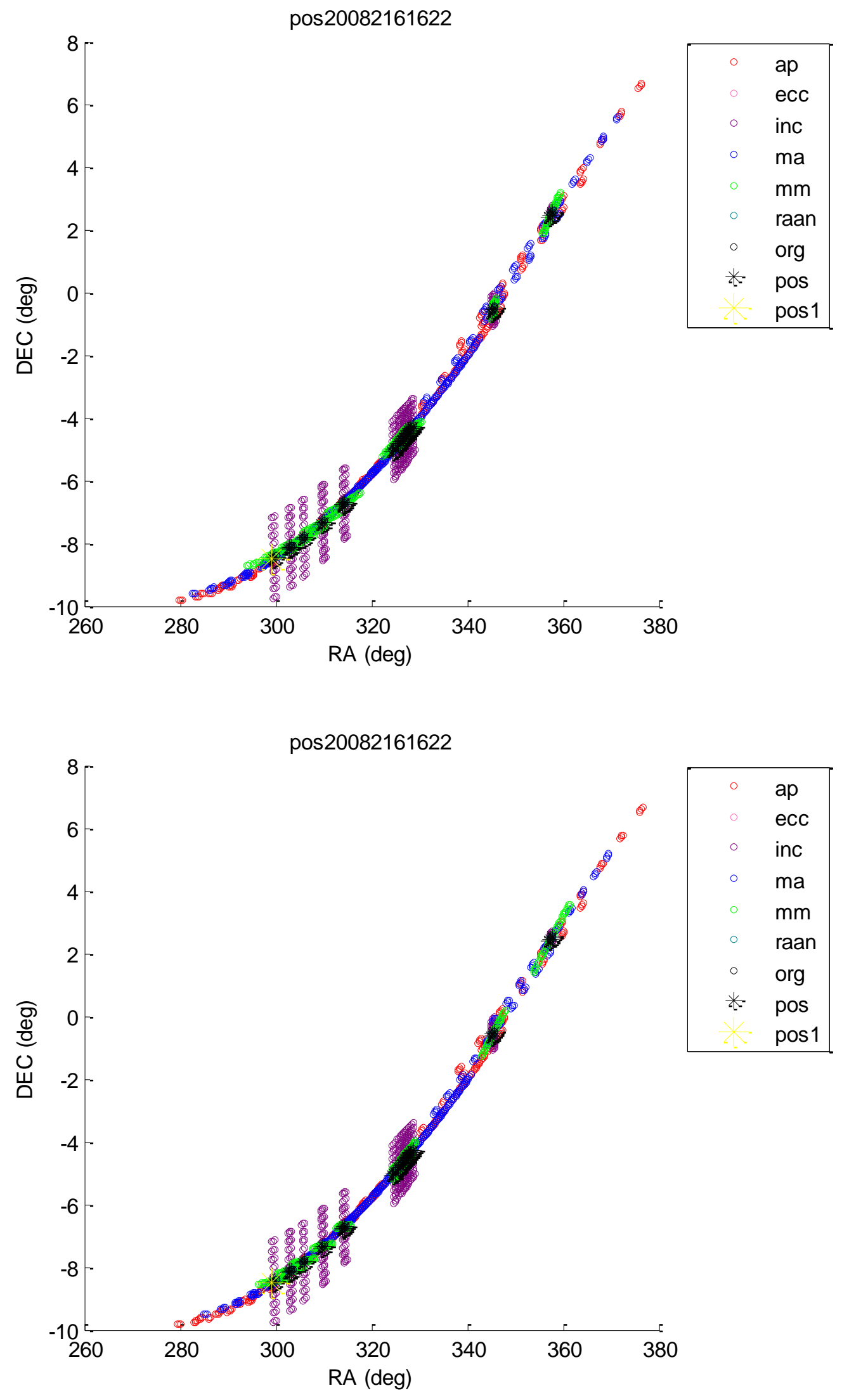

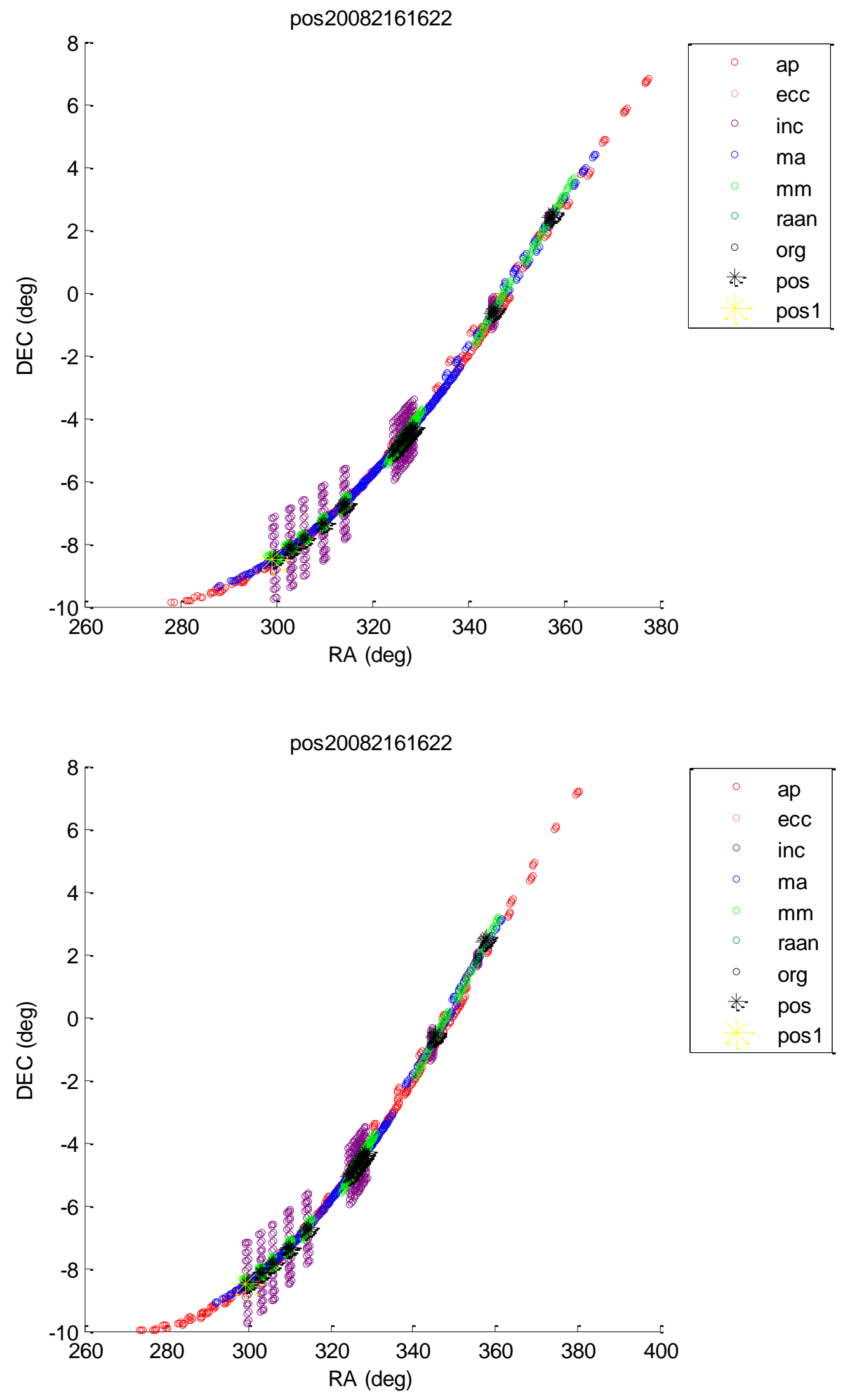

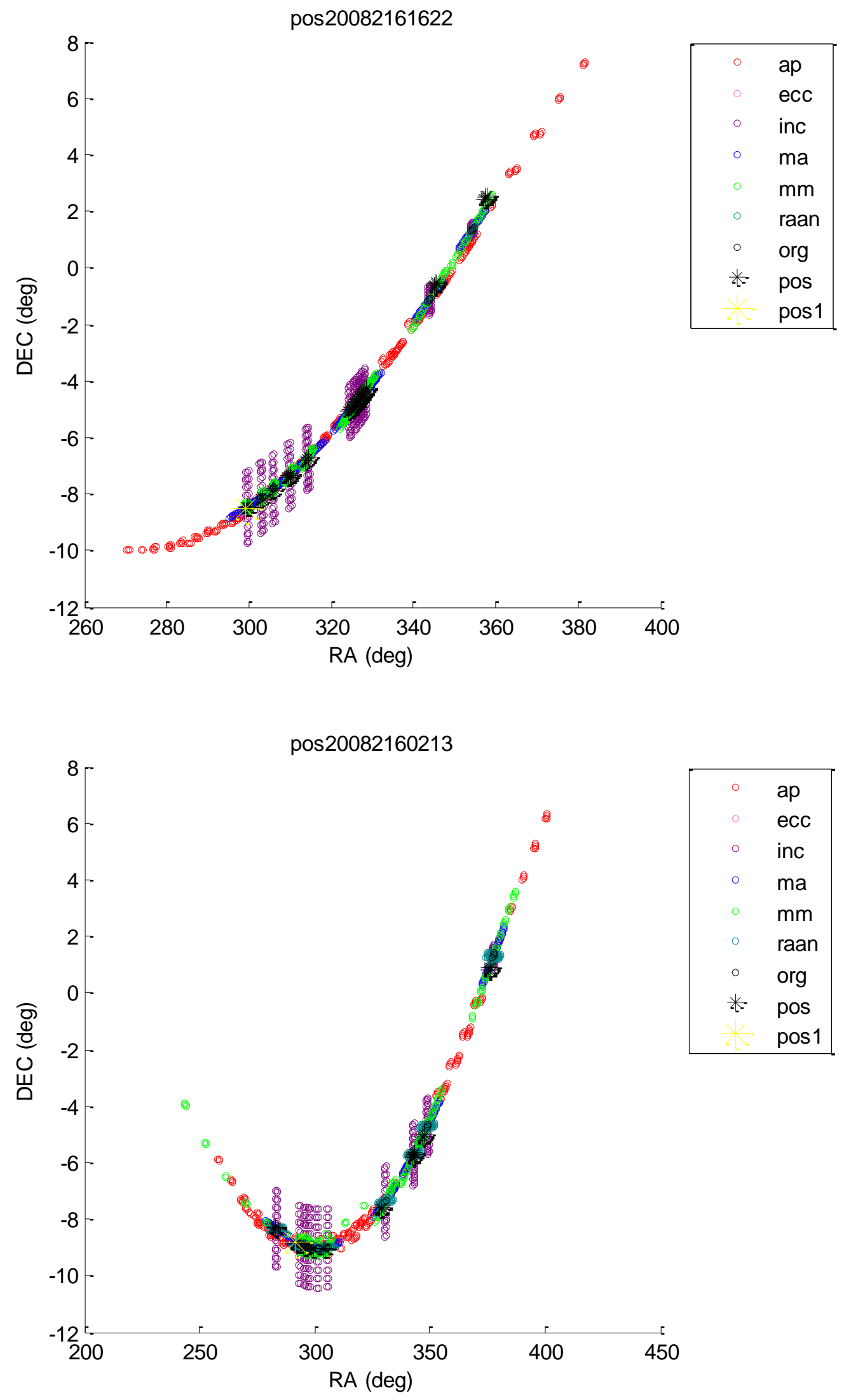

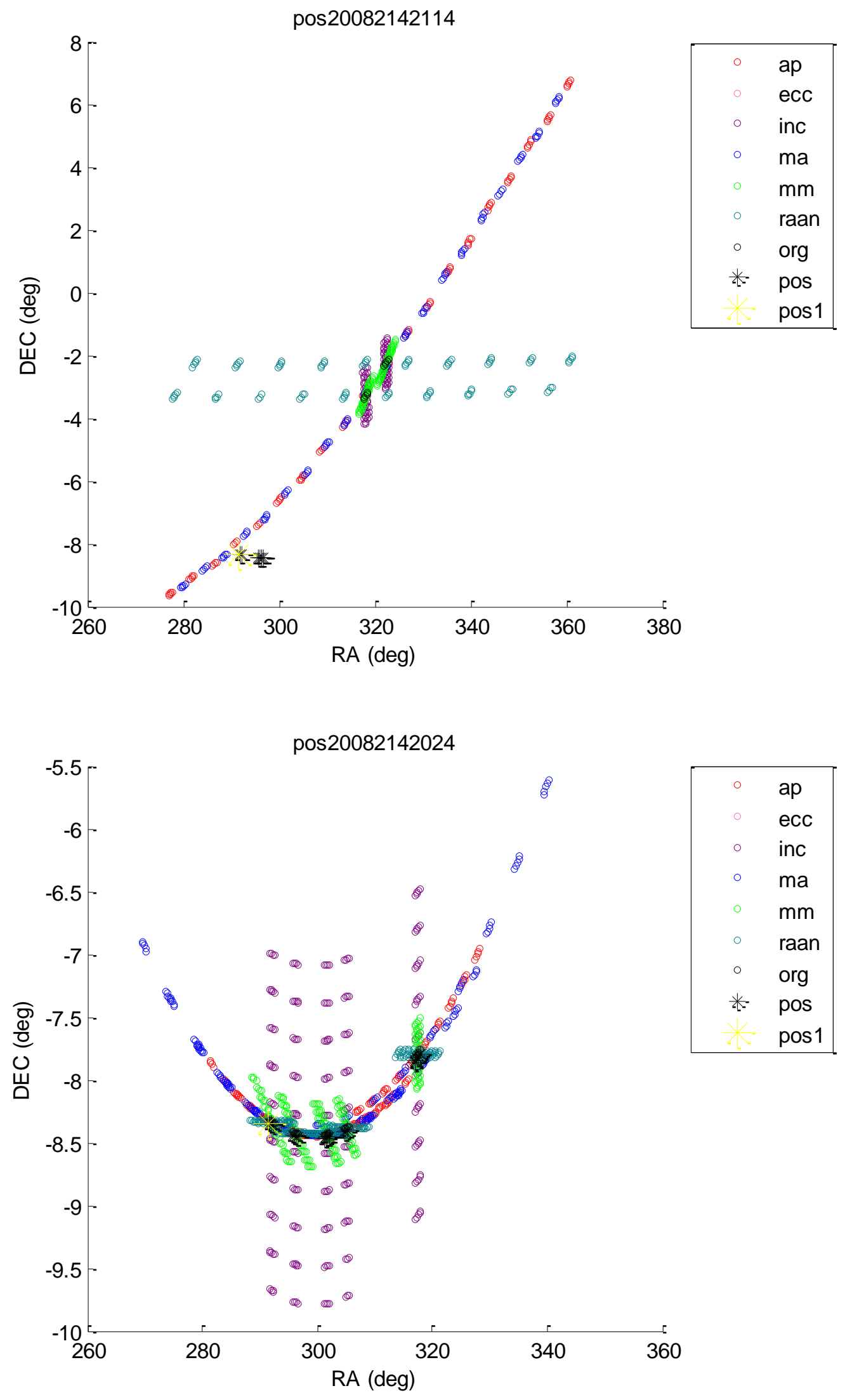

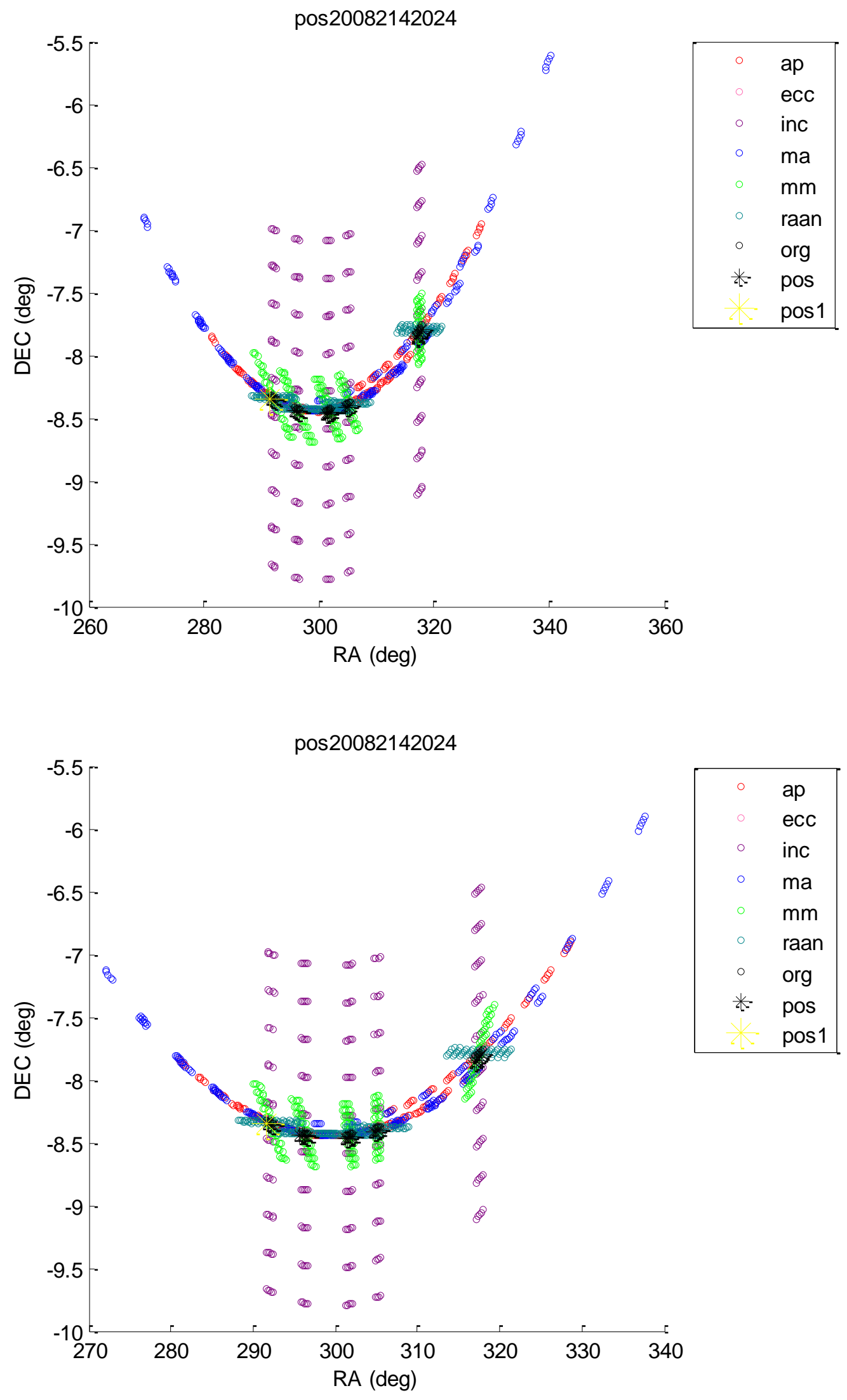

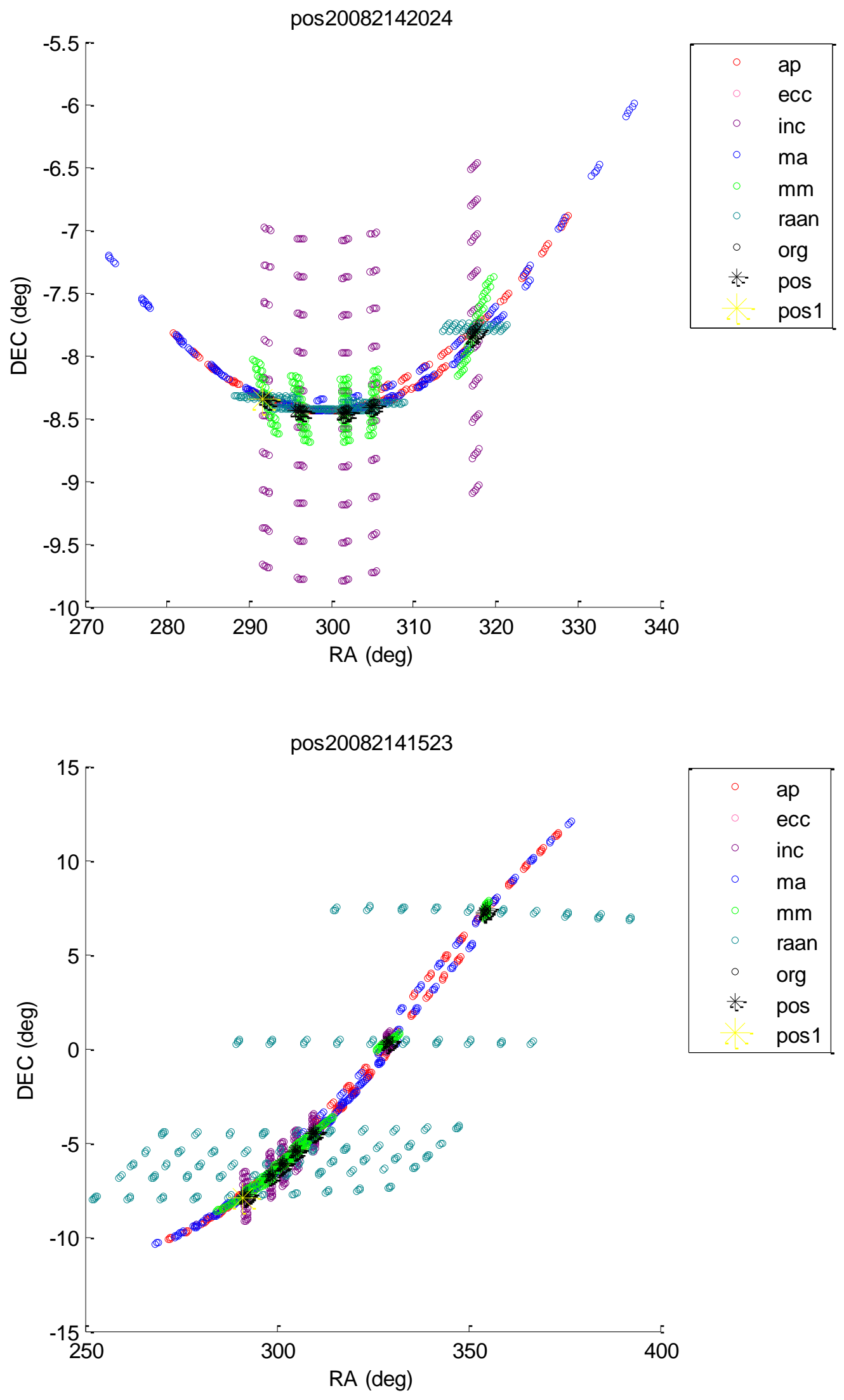

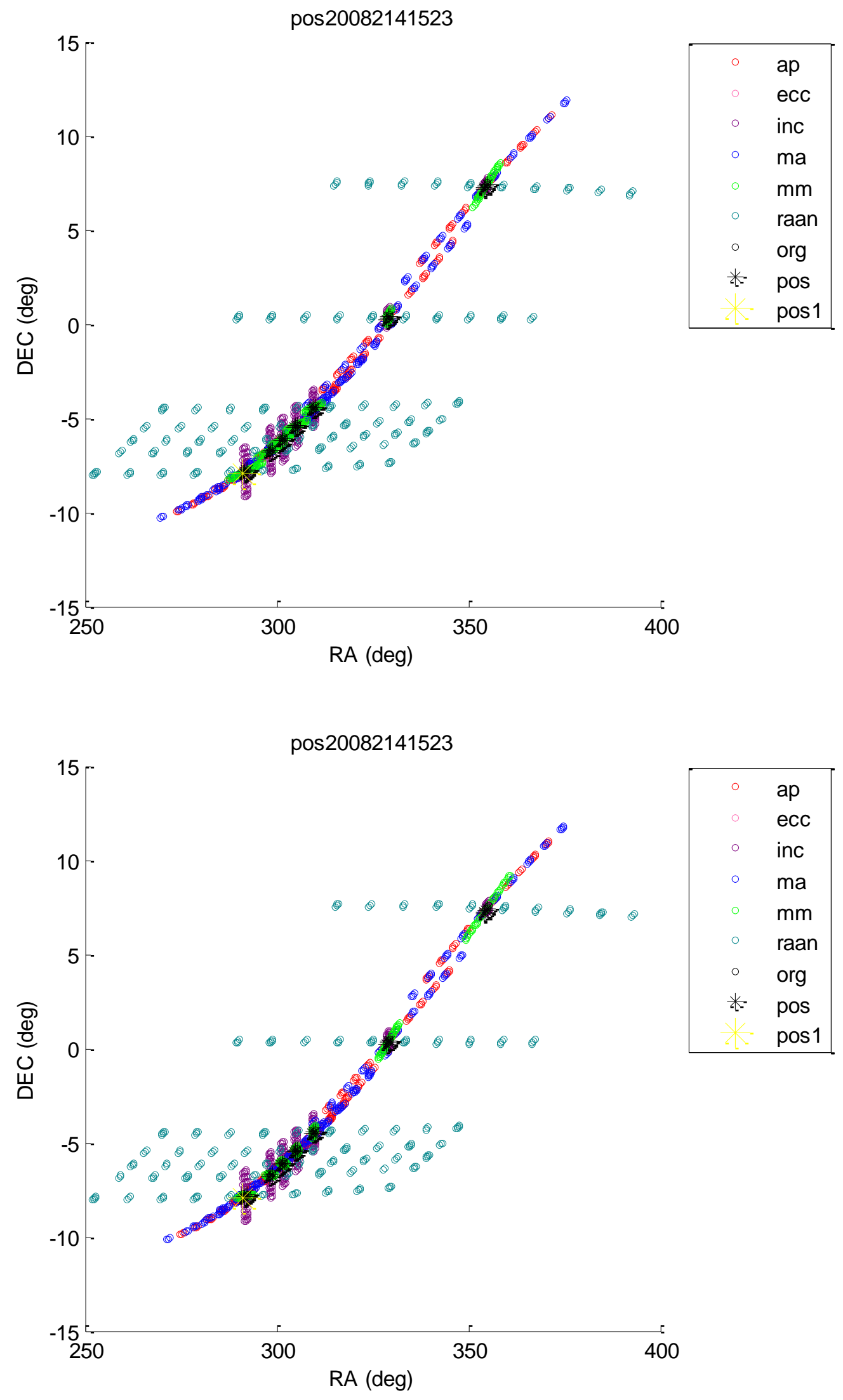

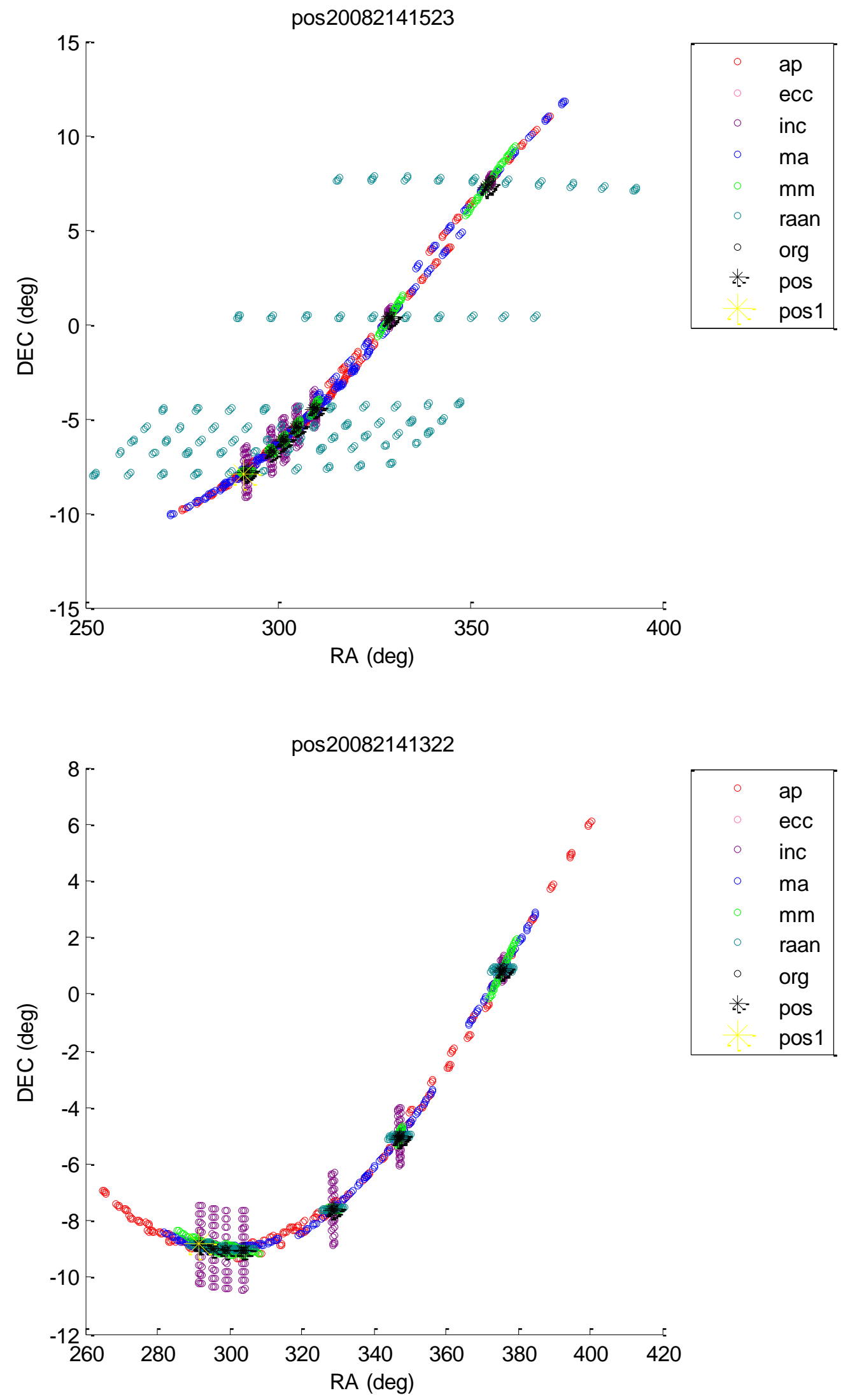

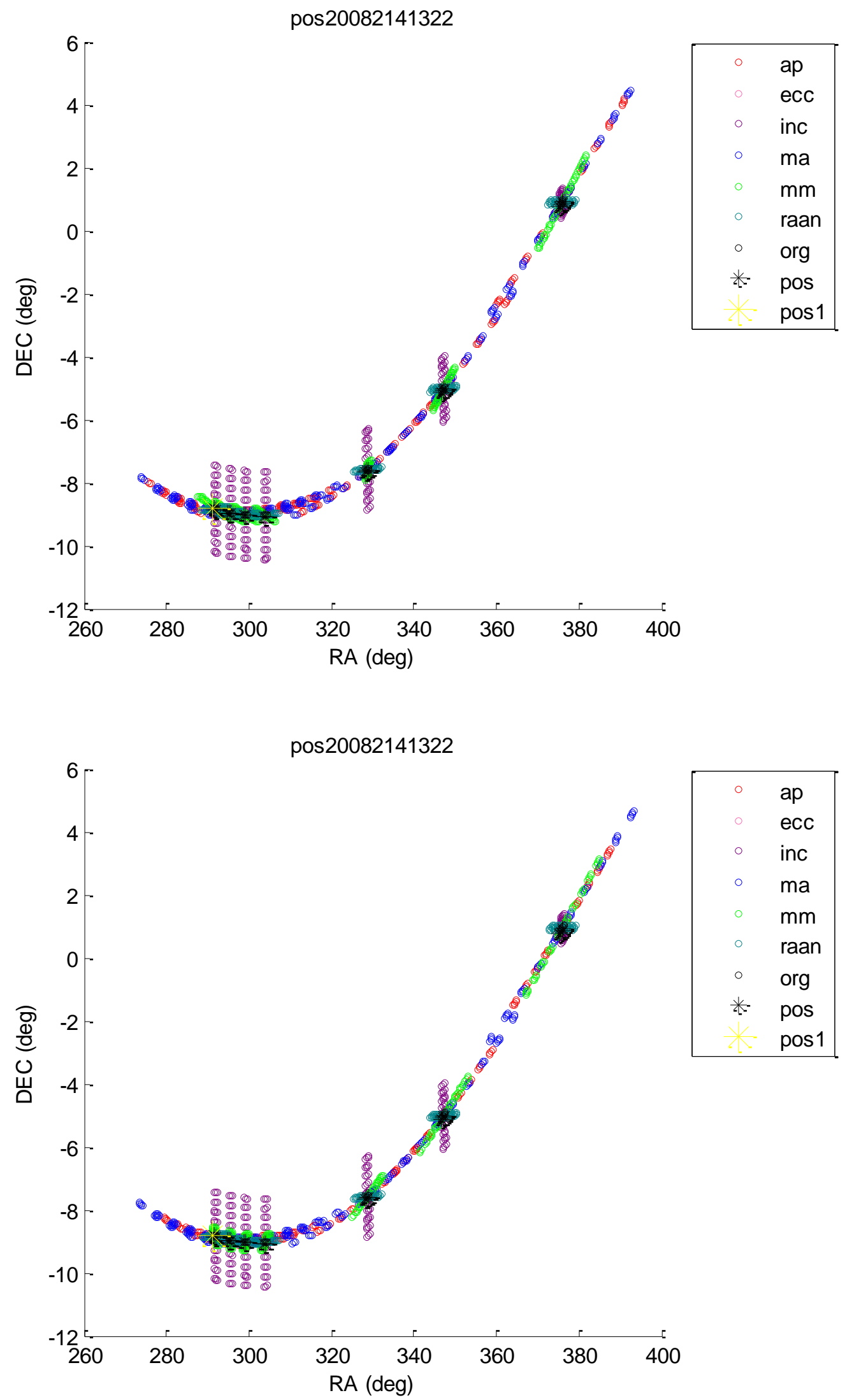

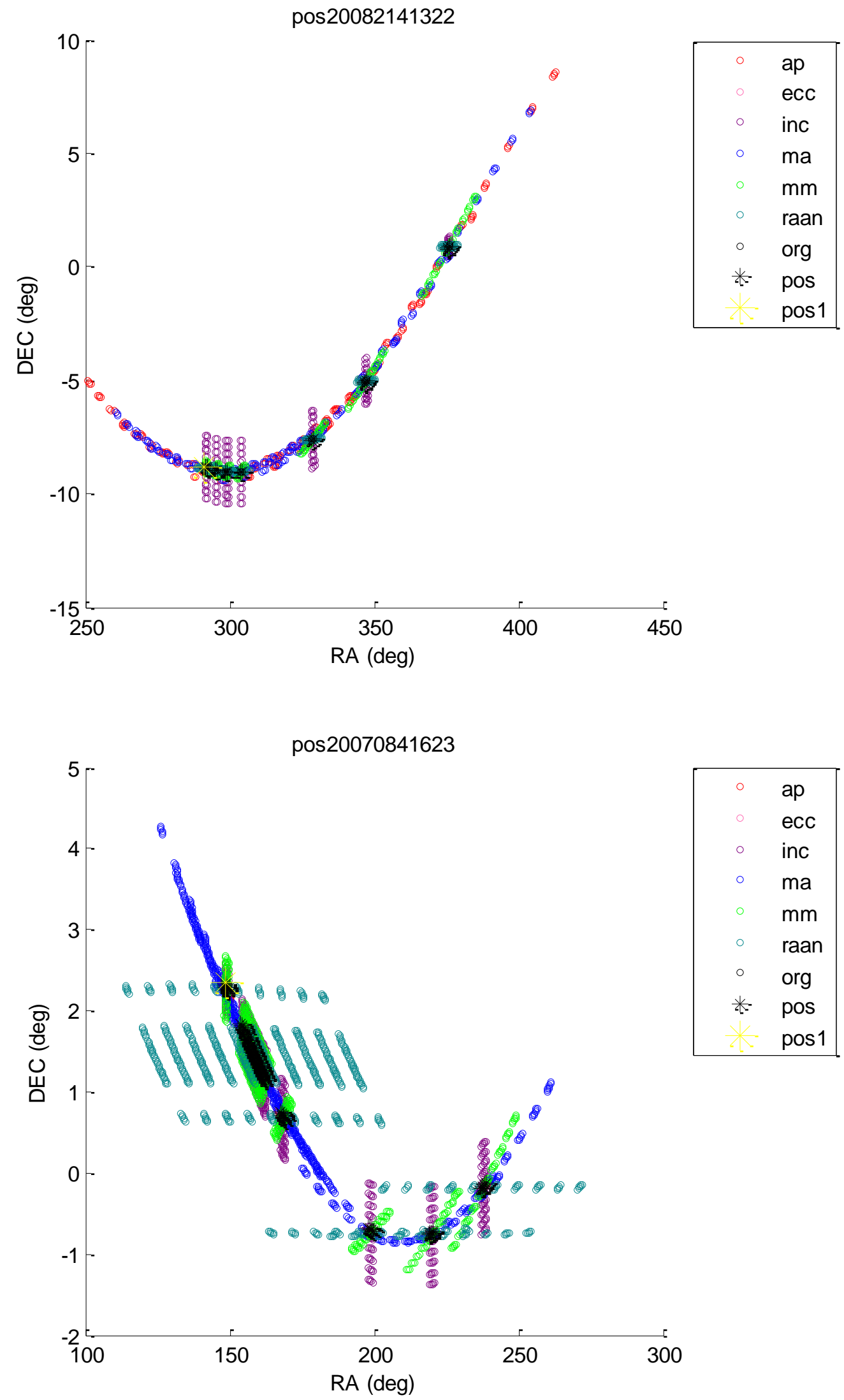

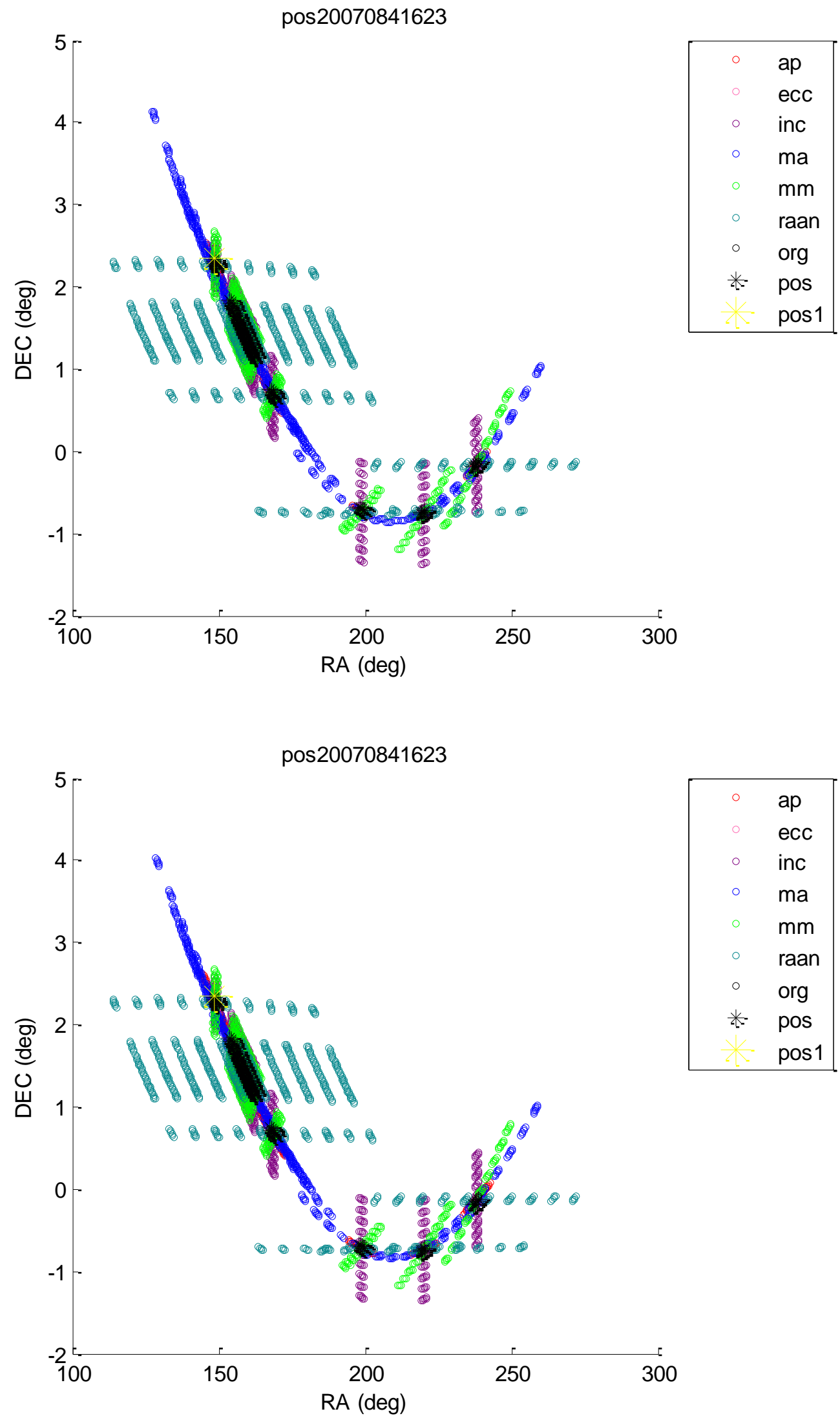

\begin{tabular}{|ll|}
\hline & ap \\
& ecc \\
$\circ$ & inc \\
$\circ$ & ma \\
& $\mathrm{mm}$ \\
$\circ$ & raan \\
$\circ$ & org \\
$*$ & pos \\
& pos1 \\
\hline
\end{tabular}



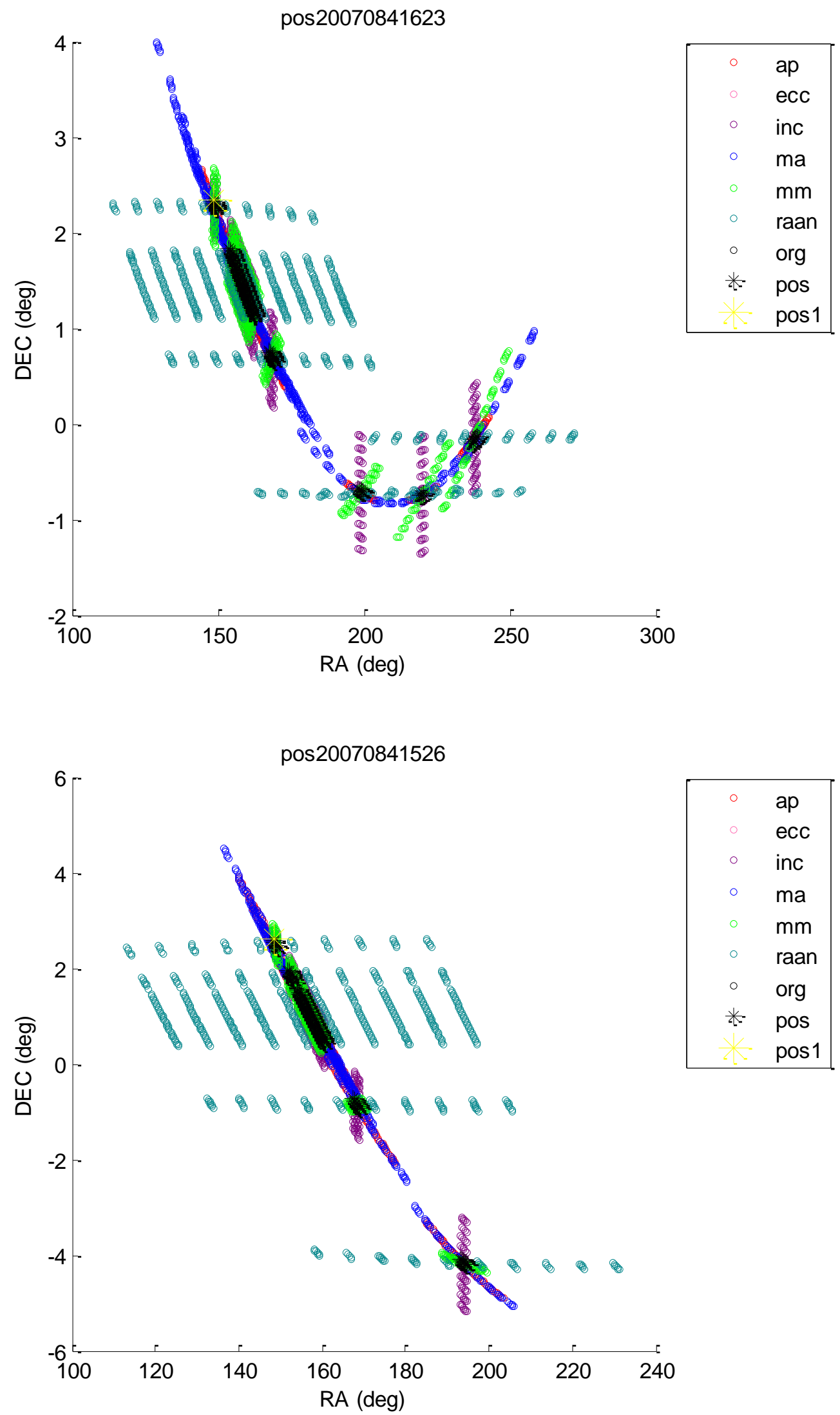

\begin{tabular}{|ll|}
\hline & ap \\
& ecc \\
& inc \\
& ma \\
& $\mathrm{mm}$ \\
& raan \\
$\circ$ & org \\
$*$ & pos \\
& pos1 \\
\hline
\end{tabular}



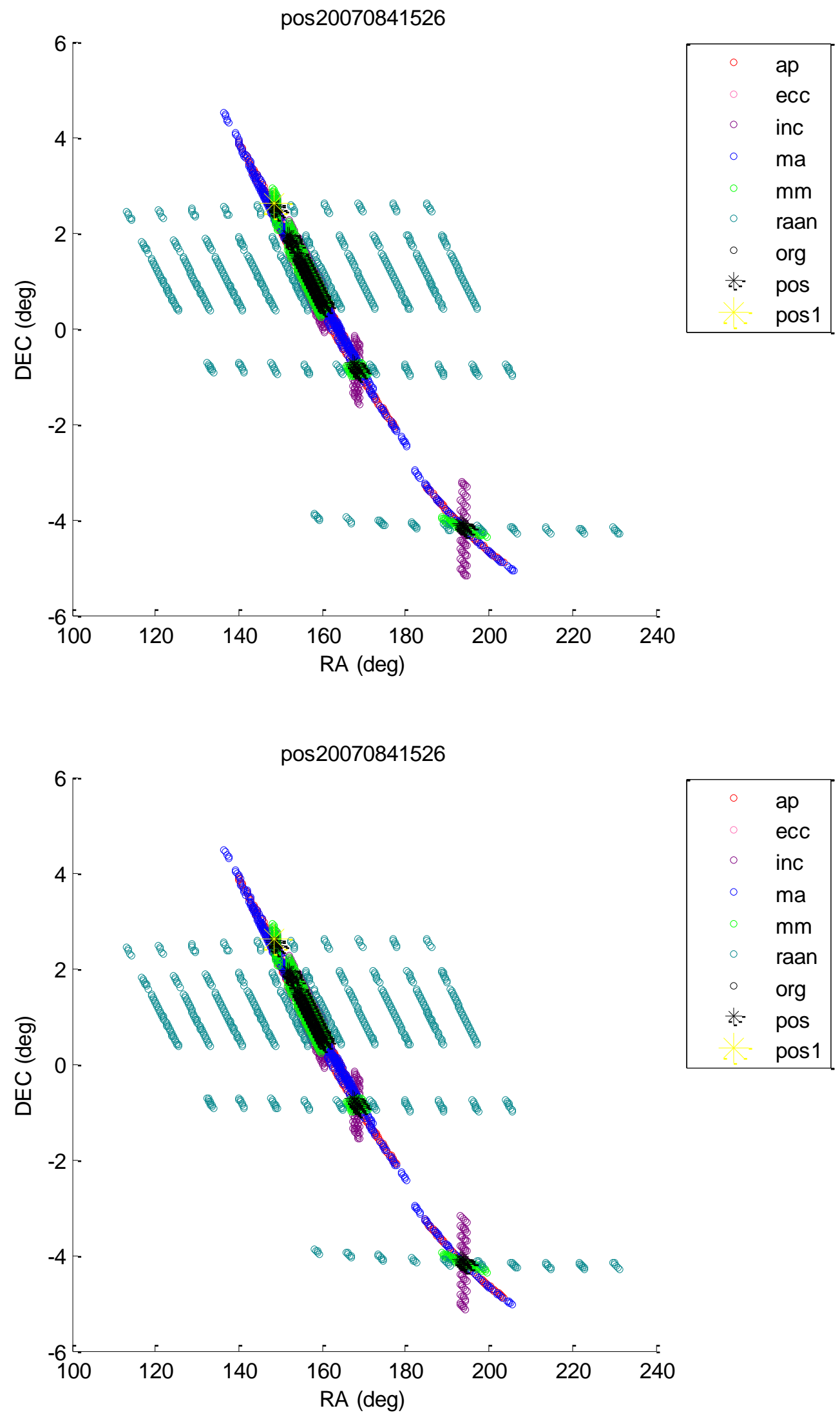

\begin{tabular}{|ll|}
\hline & ap \\
& ecc \\
& inc \\
& ma \\
& $\mathrm{mm}$ \\
& raan \\
$\circ$ & org \\
$*$ & pos \\
& pos1 \\
\hline
\end{tabular}



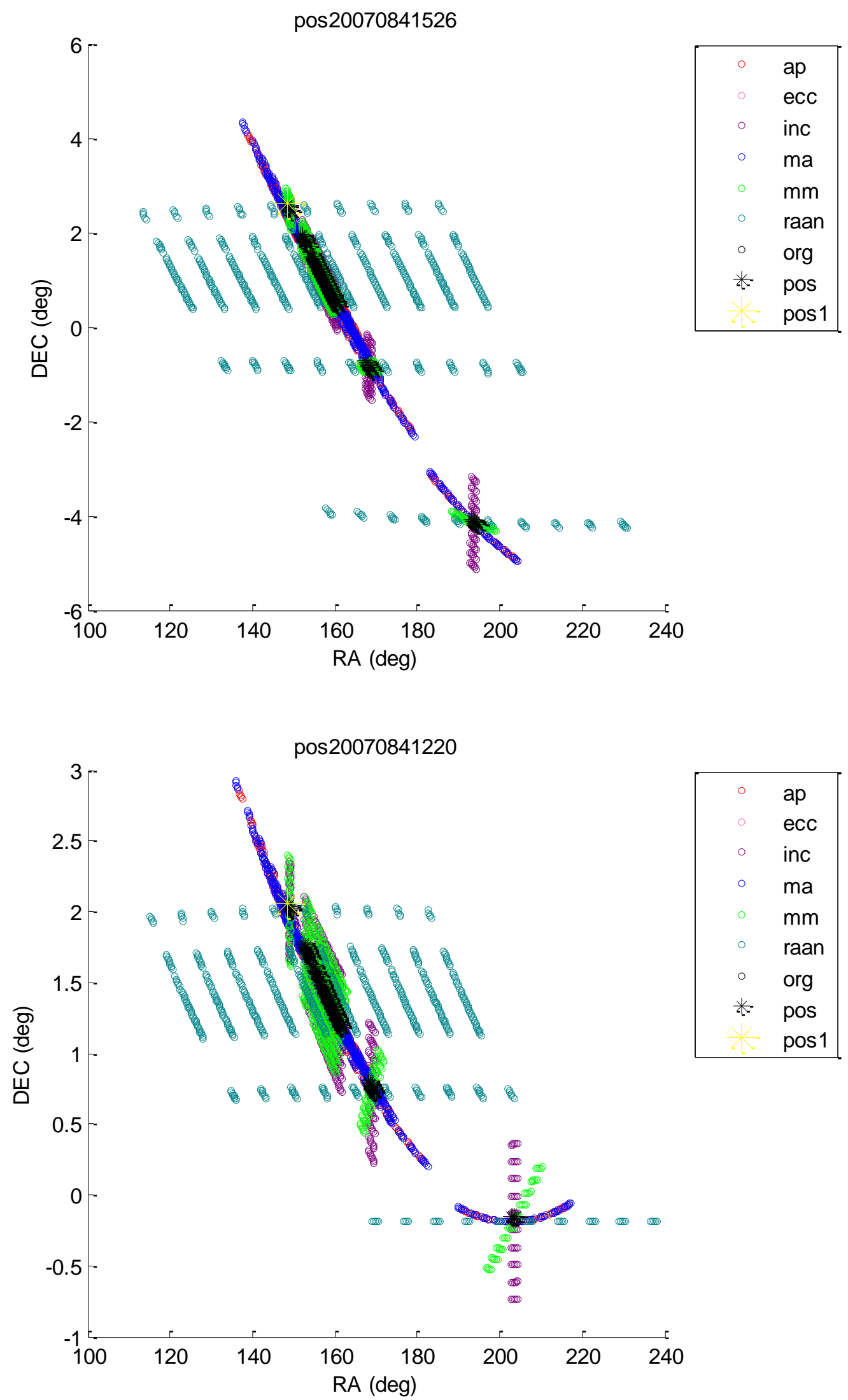

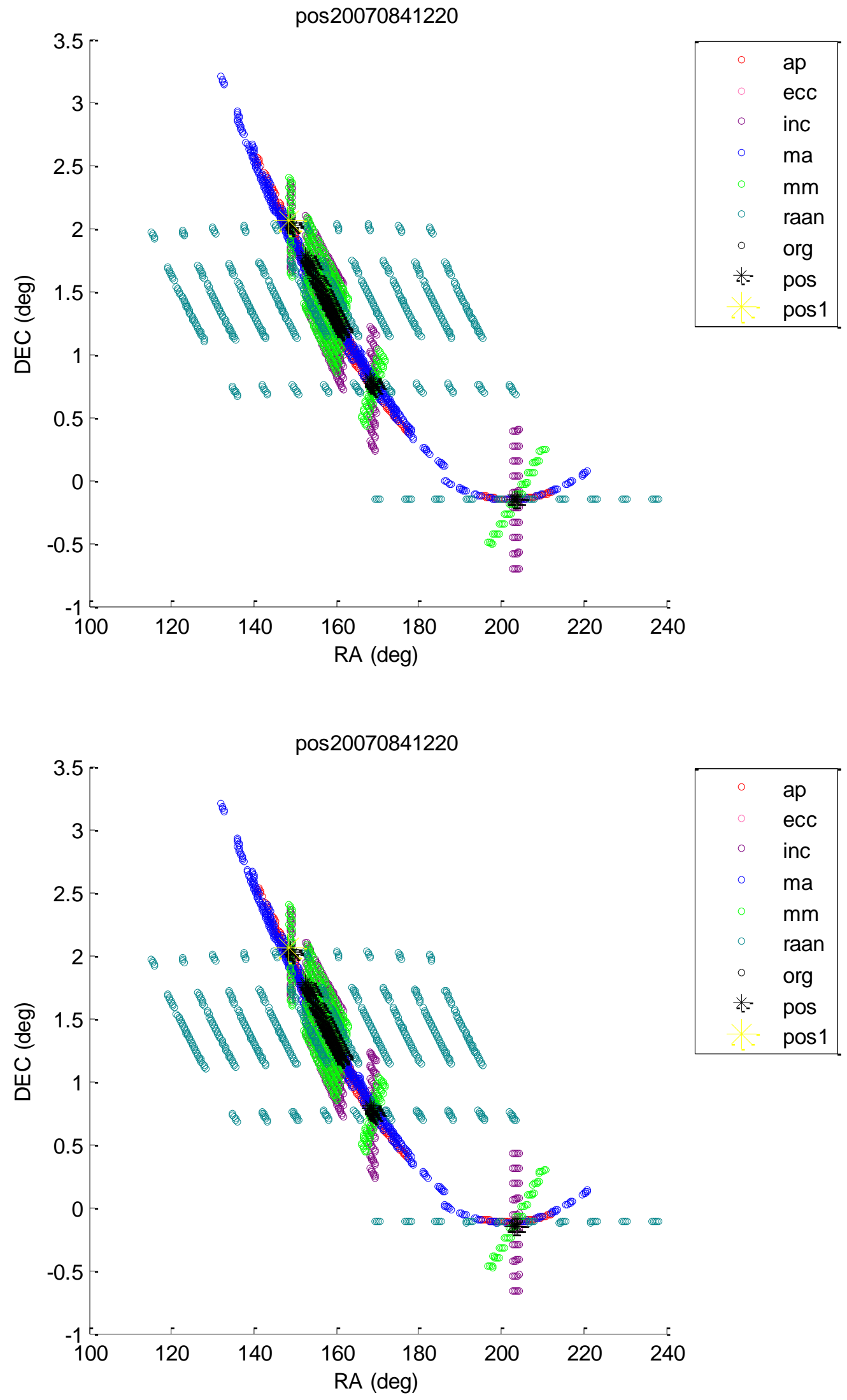

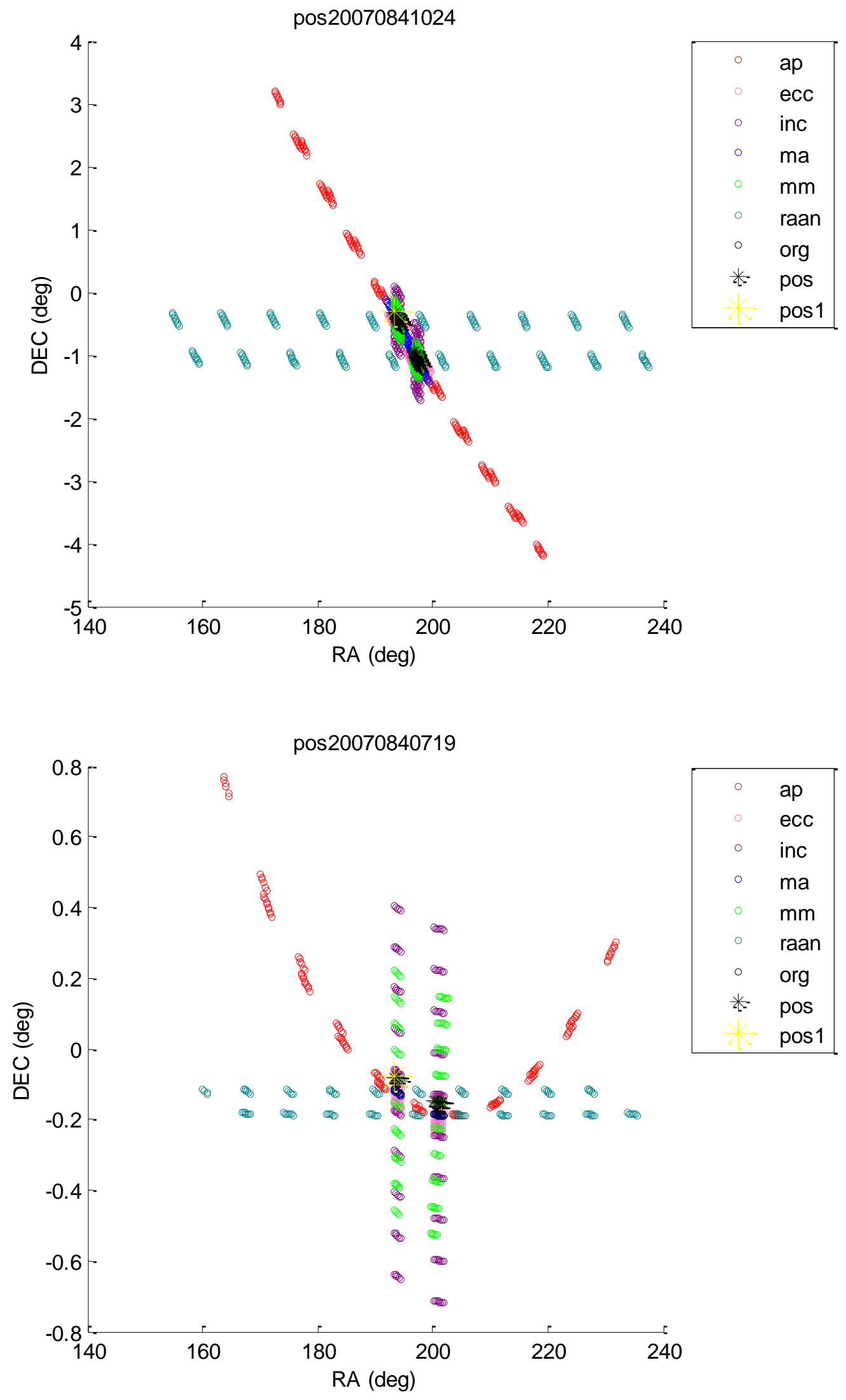

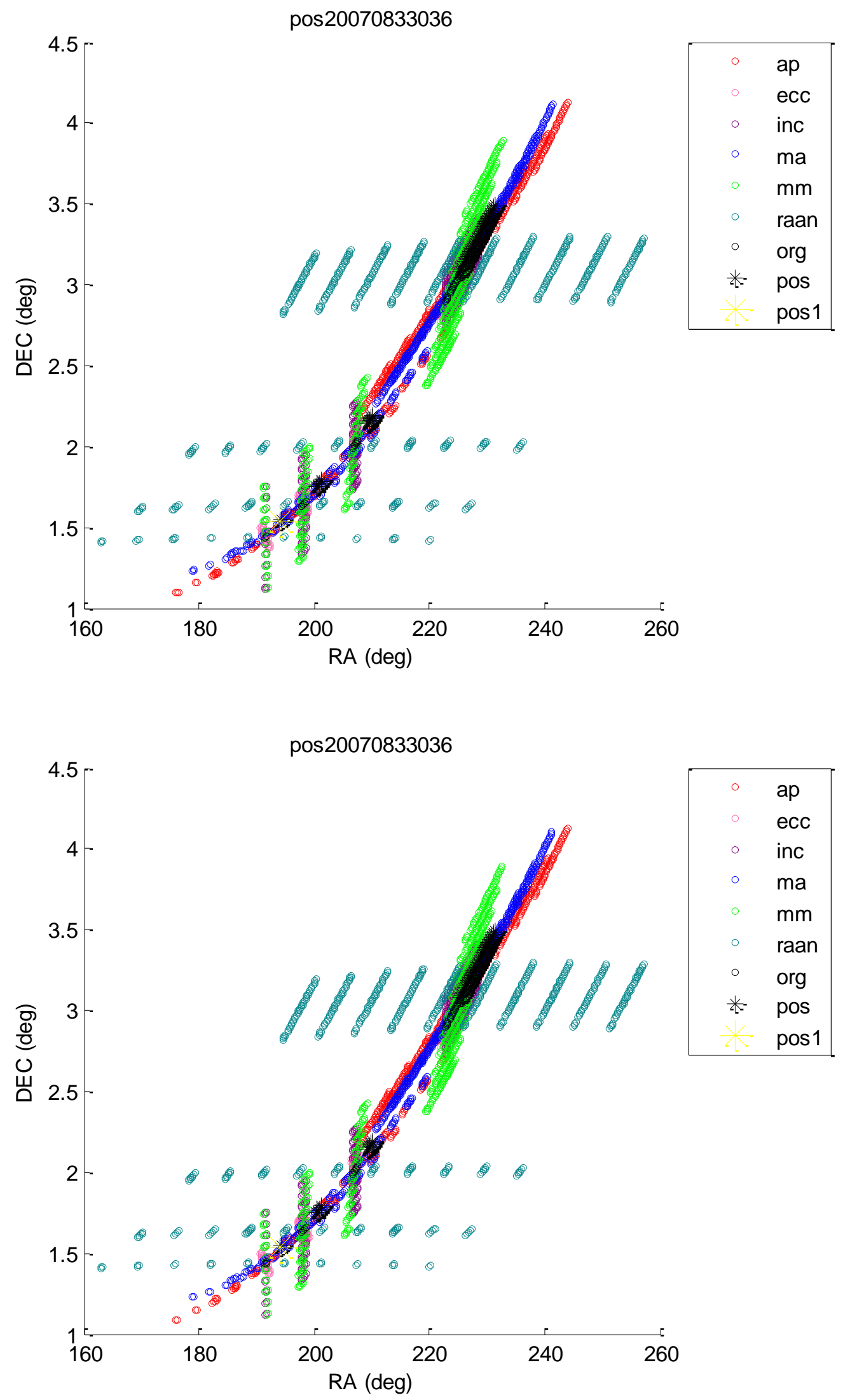

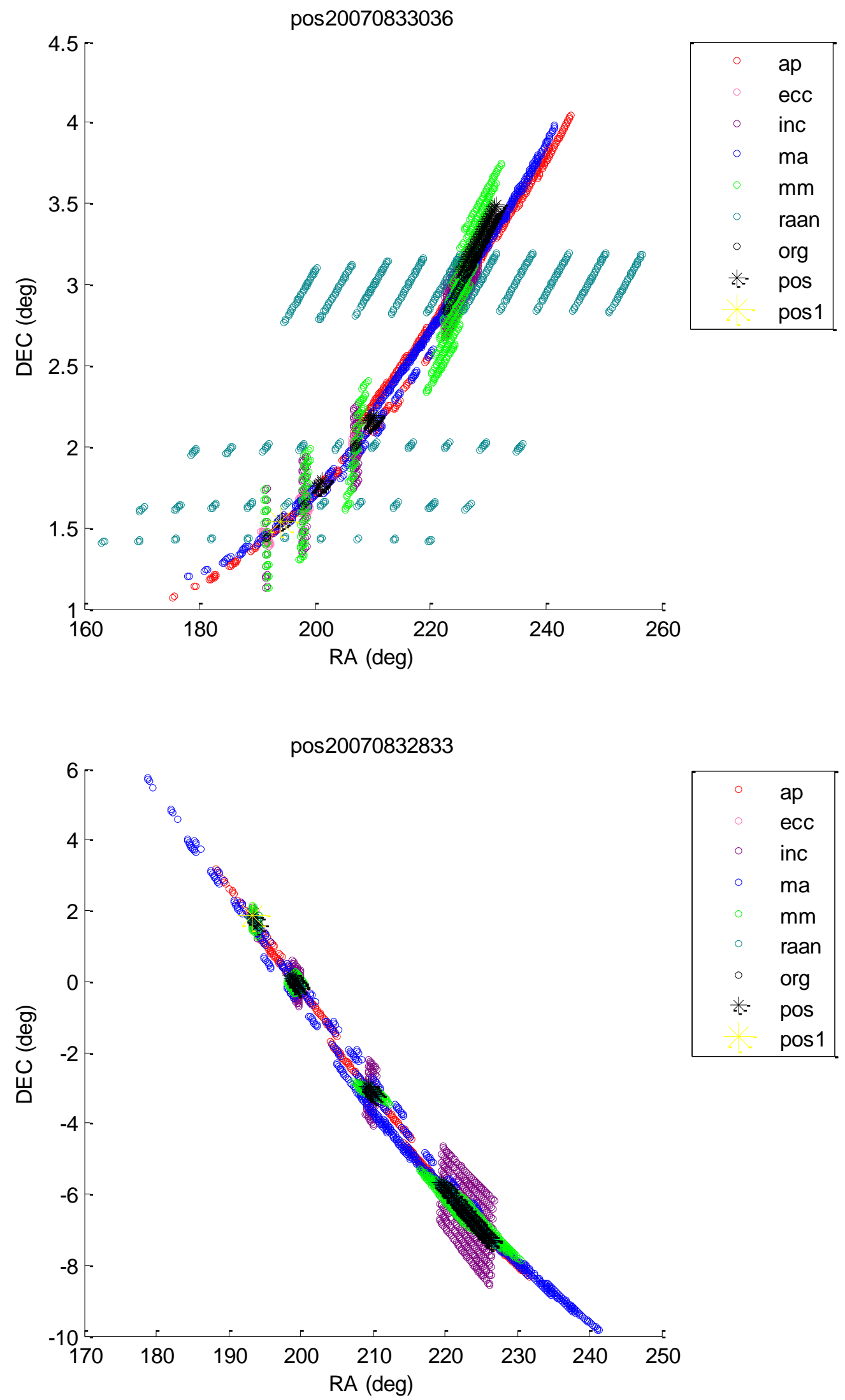

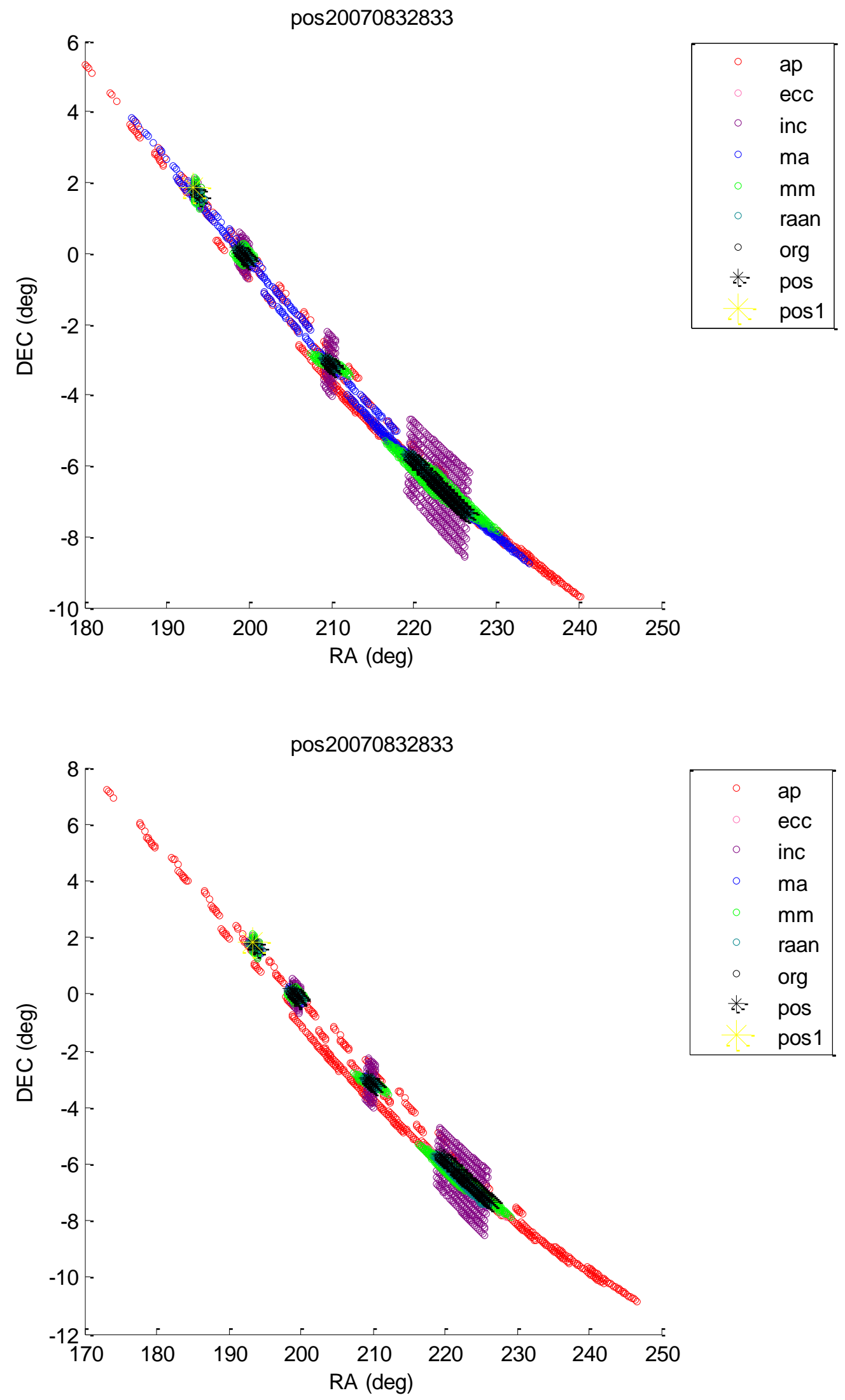

\begin{tabular}{|ll|}
\hline & ap \\
& ecc \\
inc & \\
& ma \\
& mm \\
& raan \\
$\circ$ & org \\
$*$ & pos \\
& pos1 \\
\hline
\end{tabular}



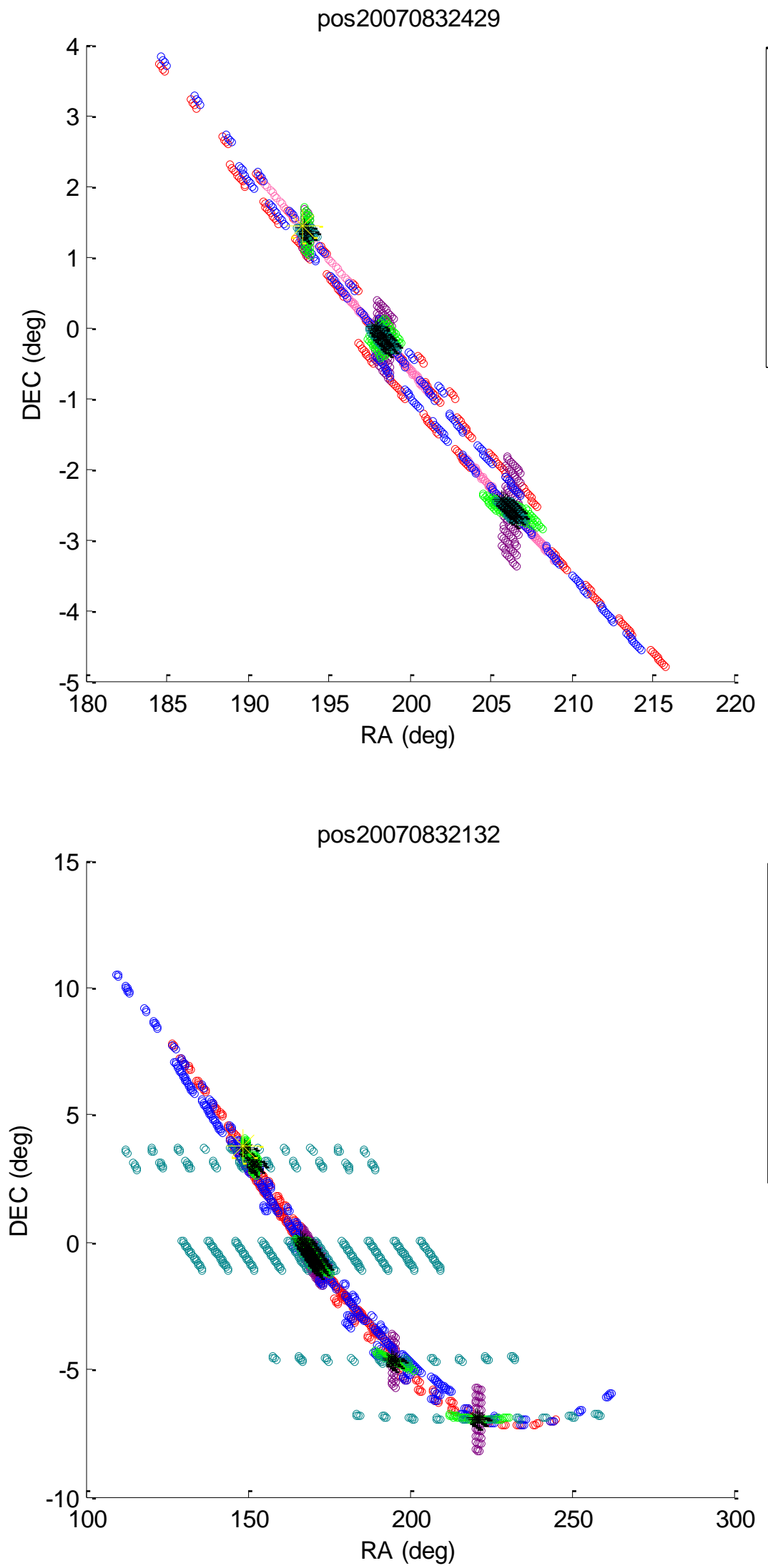

\begin{tabular}{|ll|}
\hline & ap \\
& ecc \\
& inc \\
& ma \\
& $\mathrm{mm}$ \\
& raan \\
$\circ$ & org \\
$*$ & pos \\
& pos1 \\
\hline
\end{tabular}



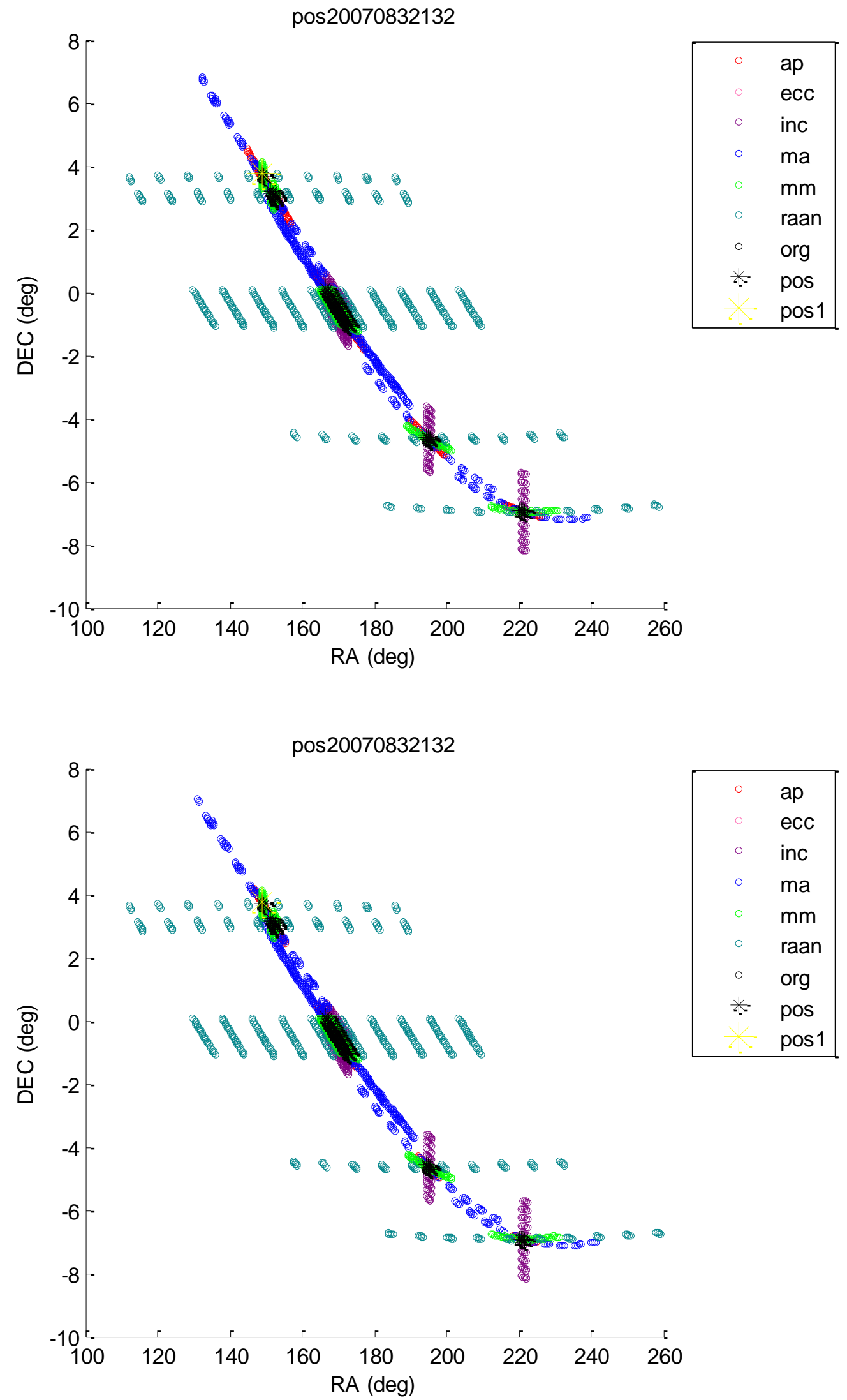

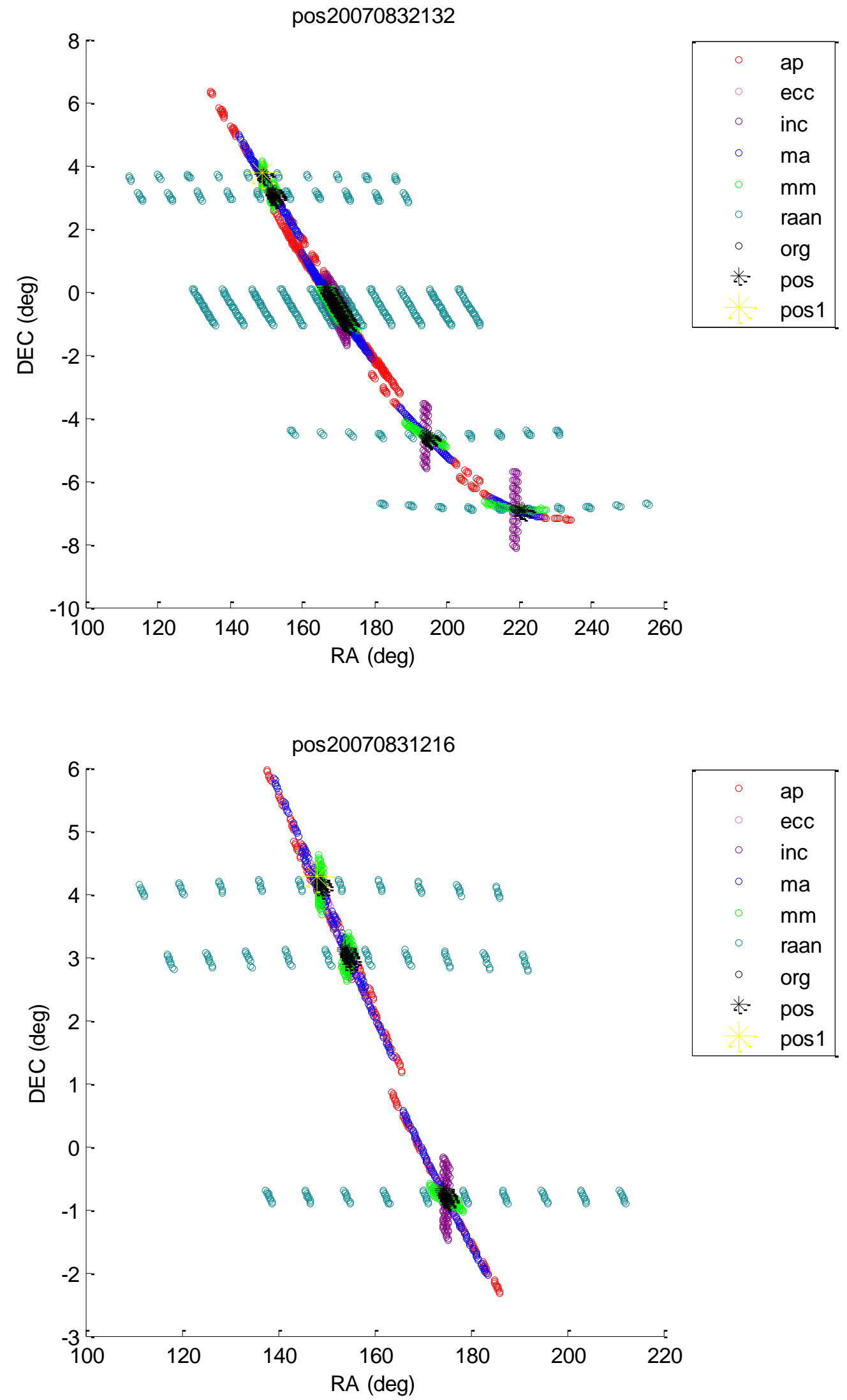

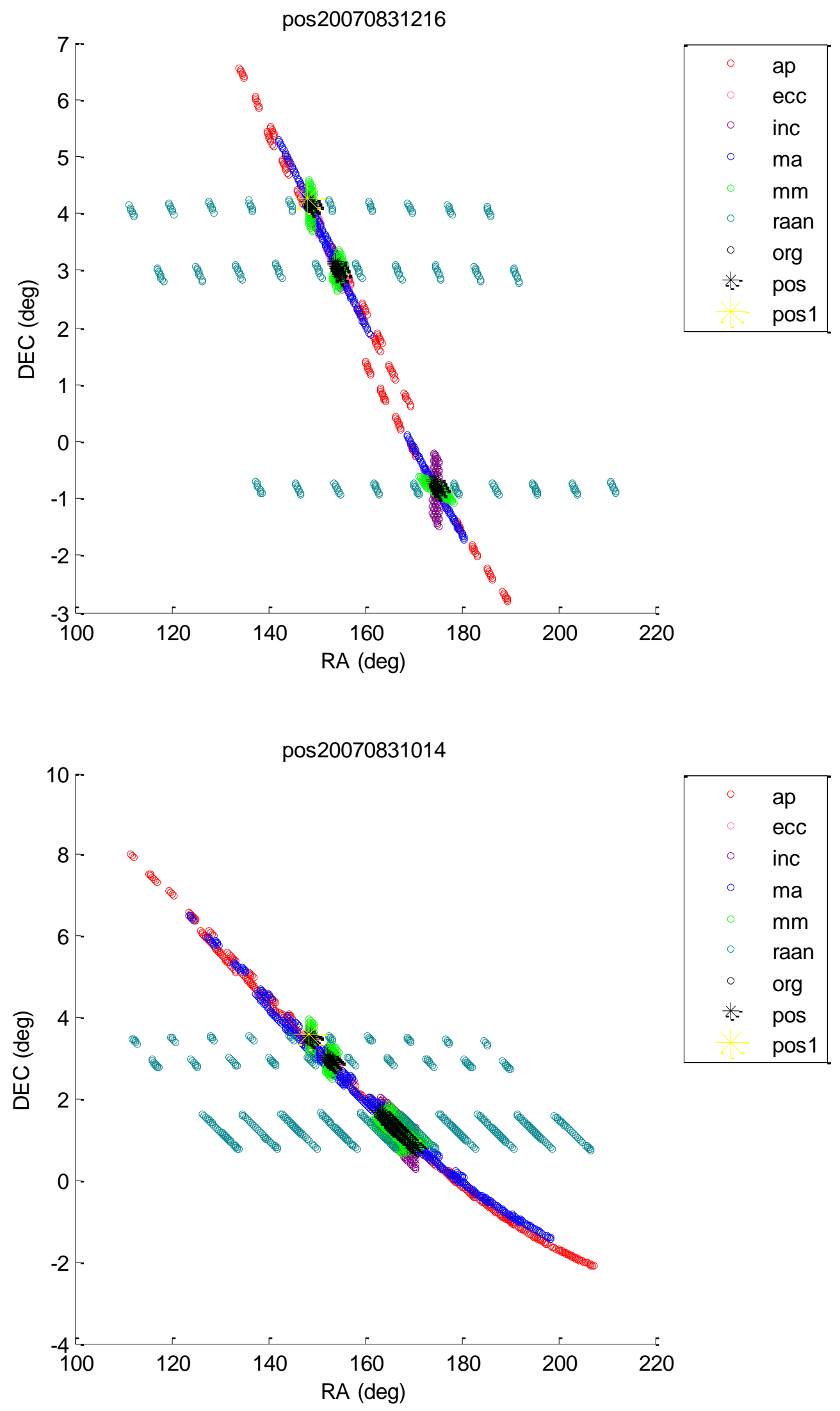

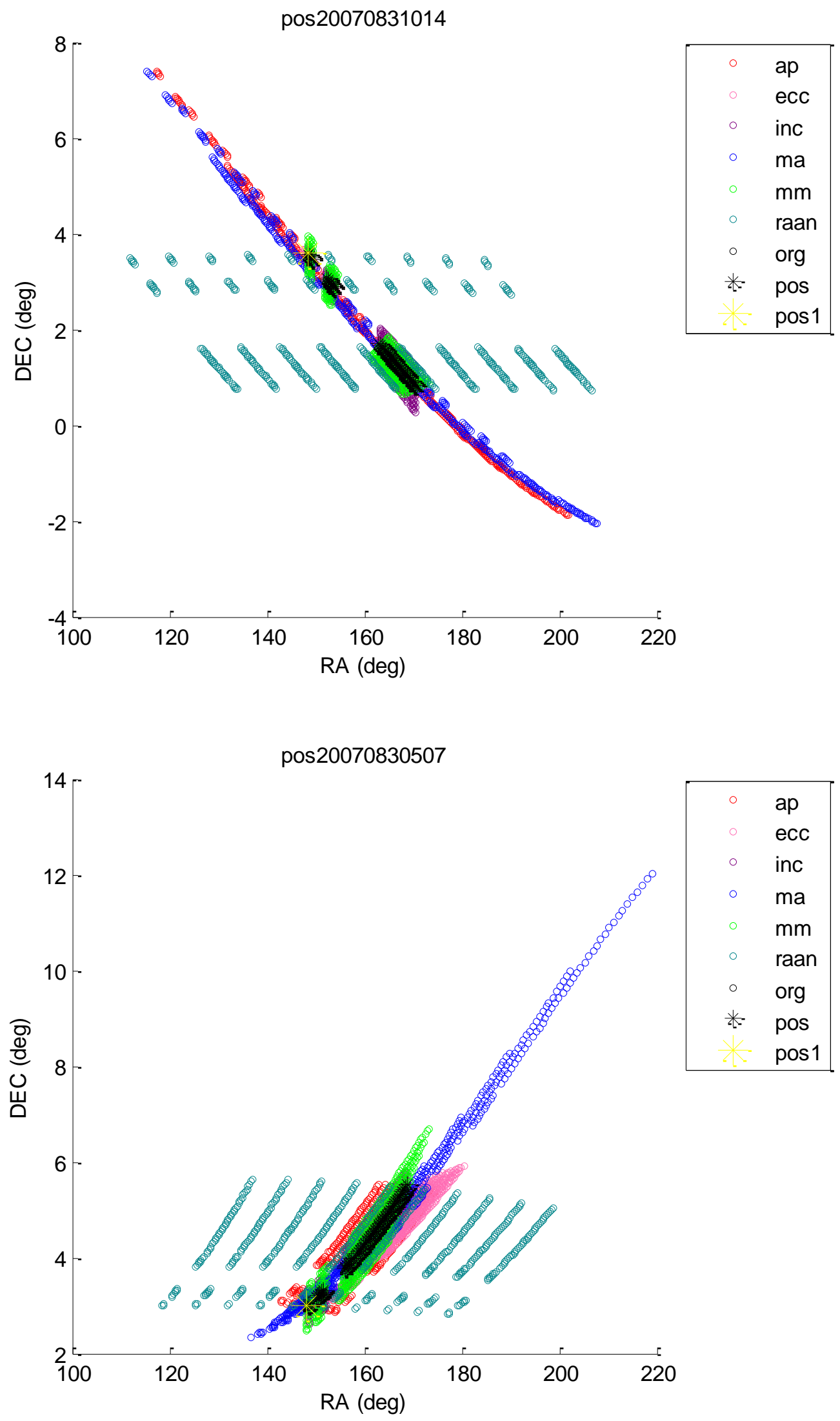

\begin{tabular}{|ll|}
\hline & ap \\
& ecc \\
& inc \\
& ma \\
& $\mathrm{mm}$ \\
& raan \\
$\circ$ & org \\
$*$ & pos \\
& pos1 \\
\hline
\end{tabular}



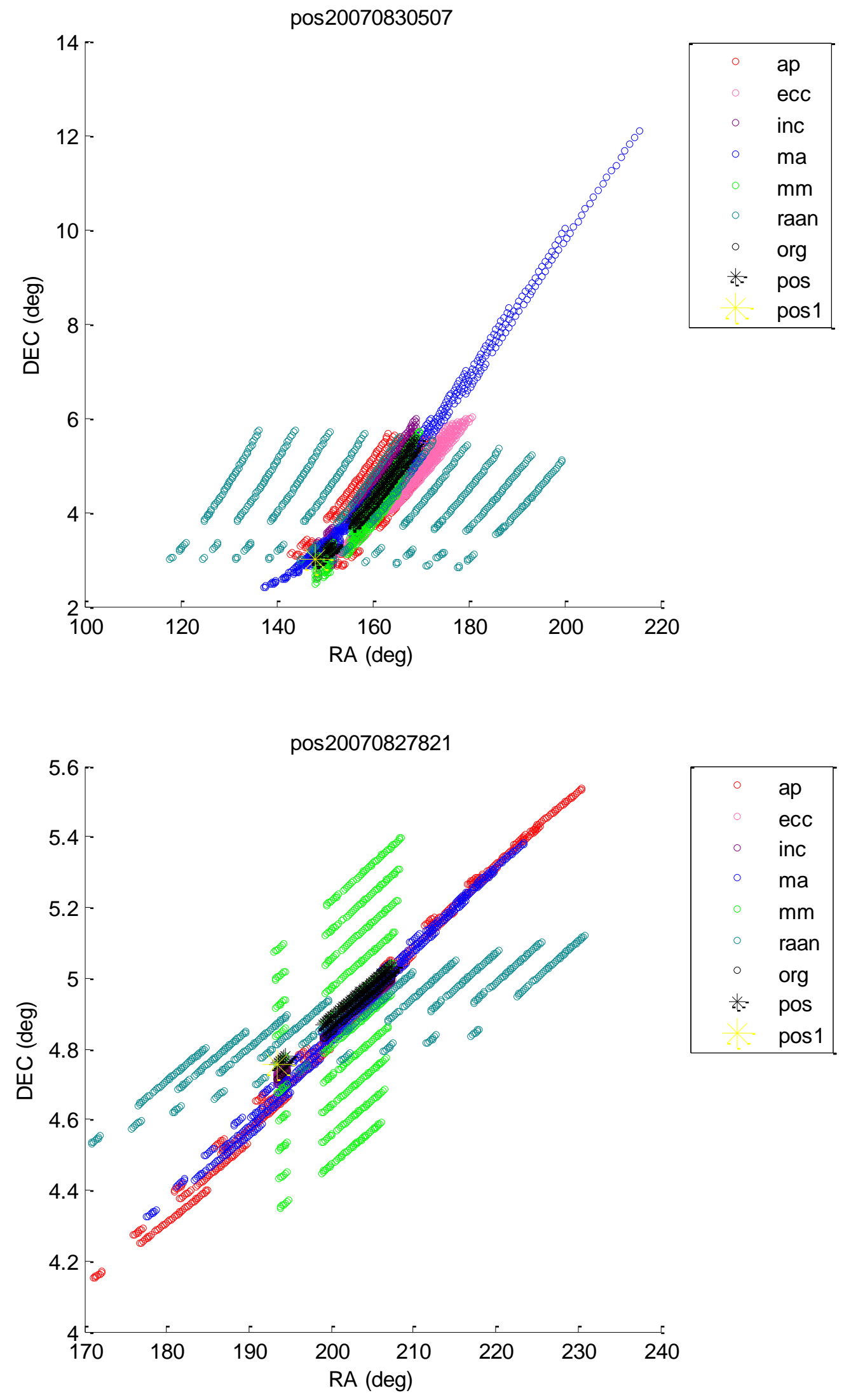

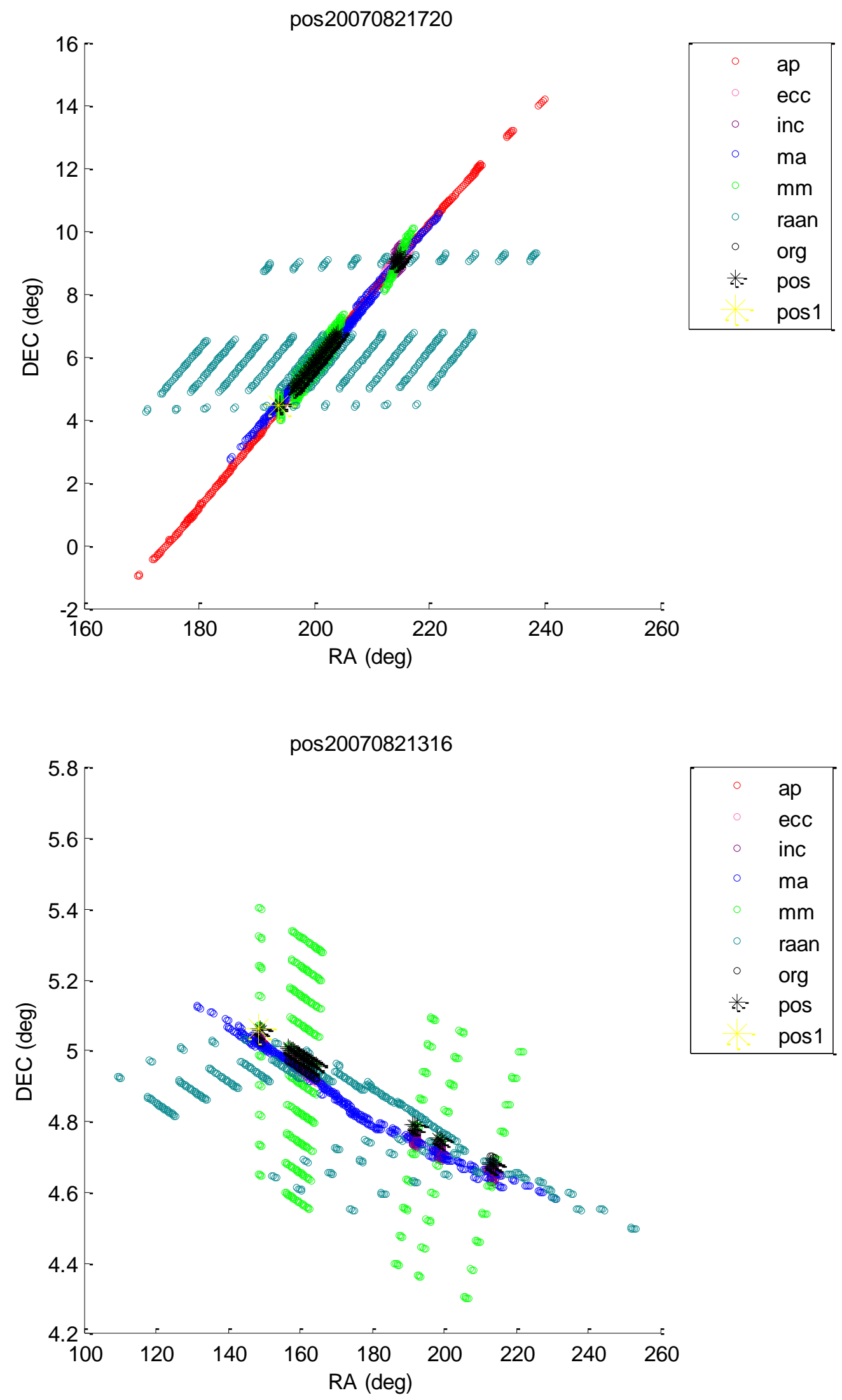

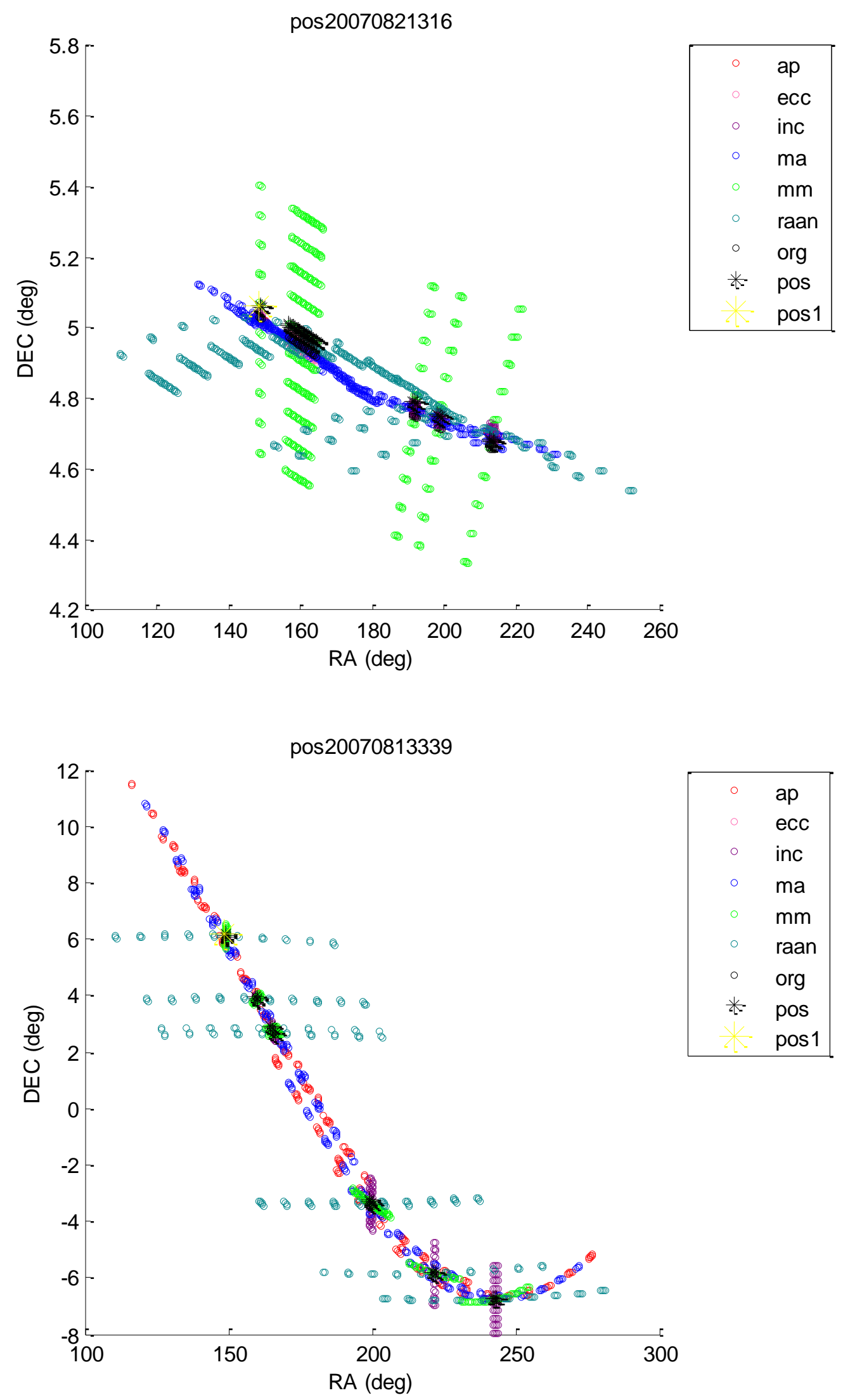

\begin{tabular}{|ll|}
\hline & ap \\
& ecc \\
inc \\
ma \\
mm \\
raan \\
$\circ$ & org \\
$*$ & pos \\
& pos1 \\
\hline
\end{tabular}




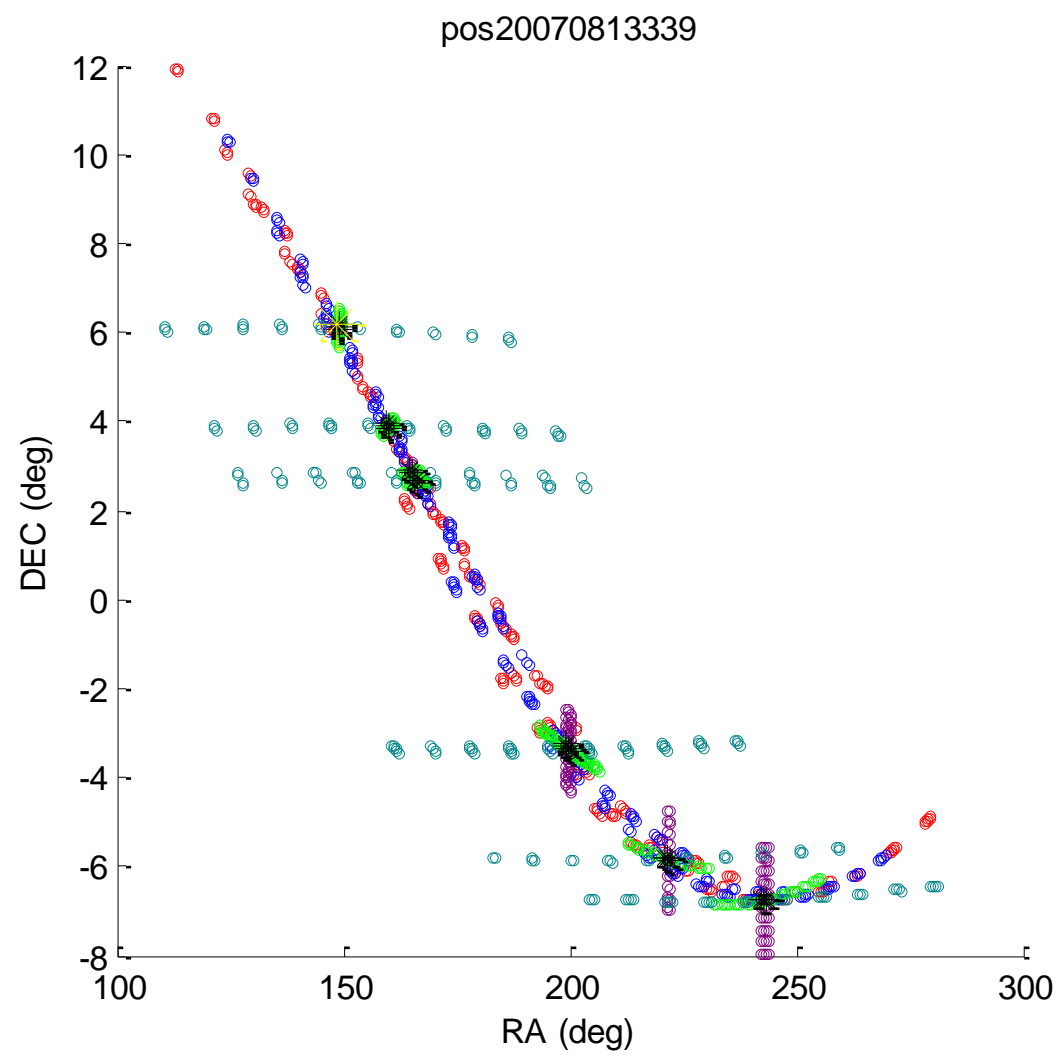

\begin{tabular}{|ll|}
\hline & ap \\
& ecc \\
& inc \\
& ma \\
& mm \\
& raan \\
$\circ$ & org \\
$*$ & pos \\
& pos1 \\
\hline
\end{tabular}

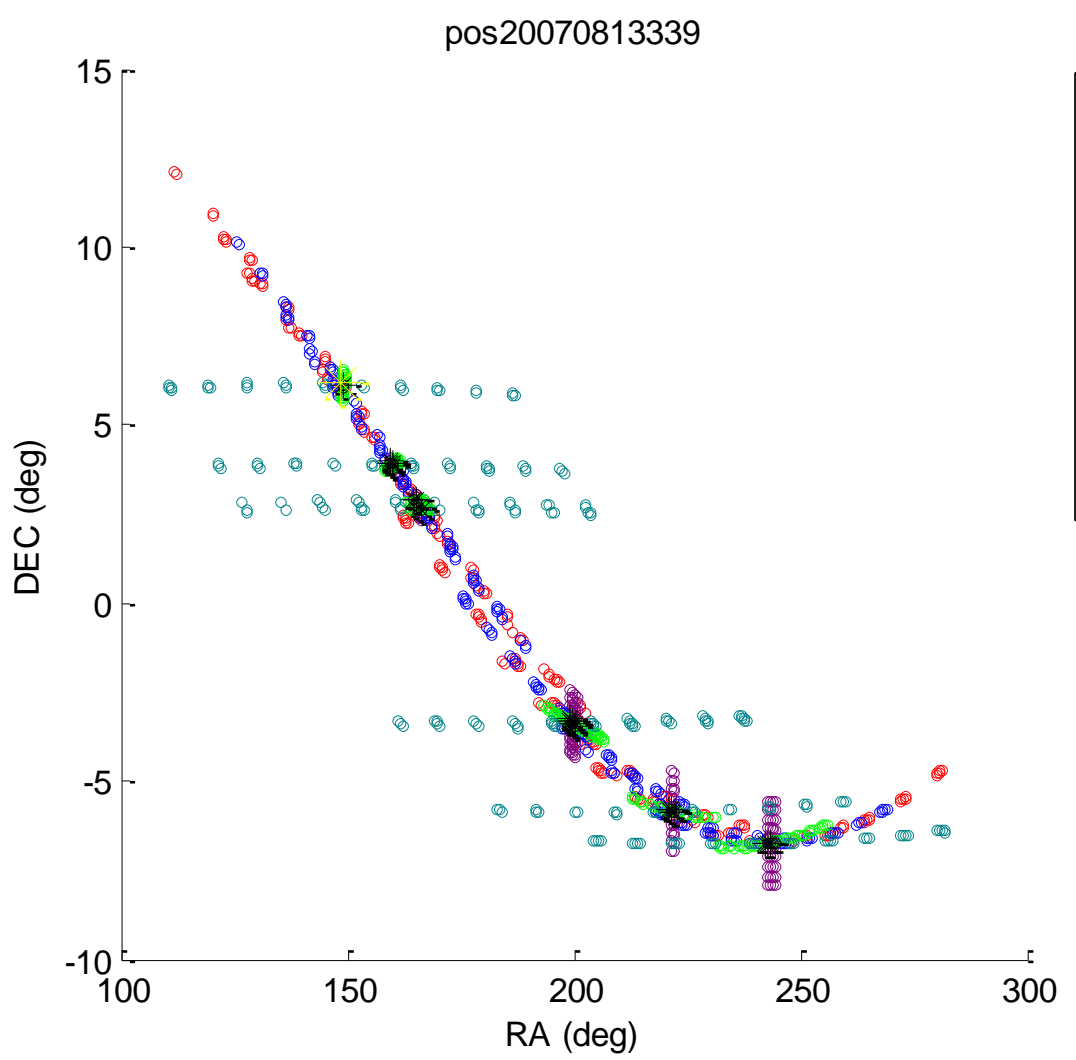

\begin{tabular}{|ll|}
\hline & ap \\
& ecc \\
& inc \\
& ma \\
& $\mathrm{mm}$ \\
& raan \\
$\circ$ & org \\
$*$ & pos \\
& pos1 \\
\hline
\end{tabular}



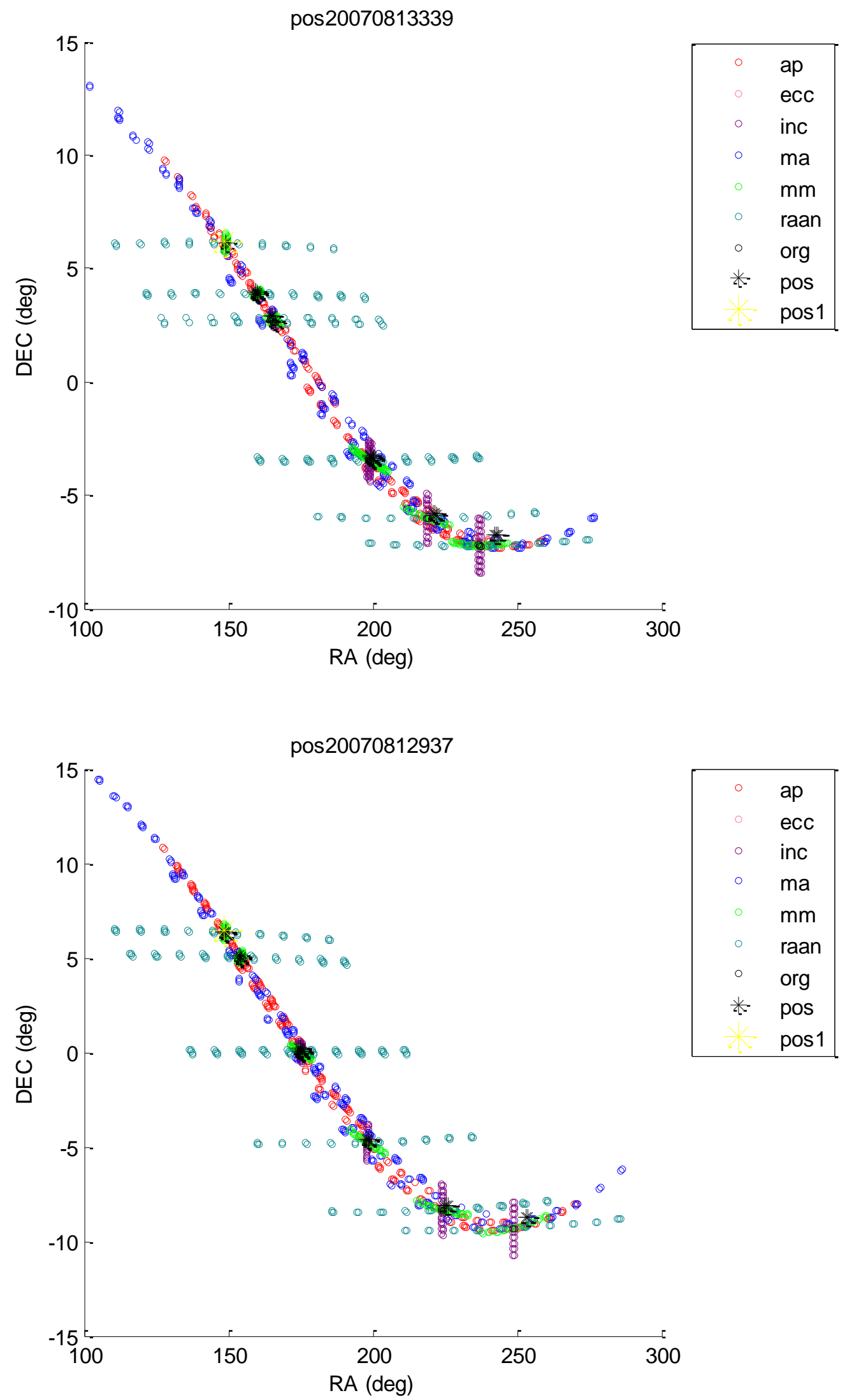

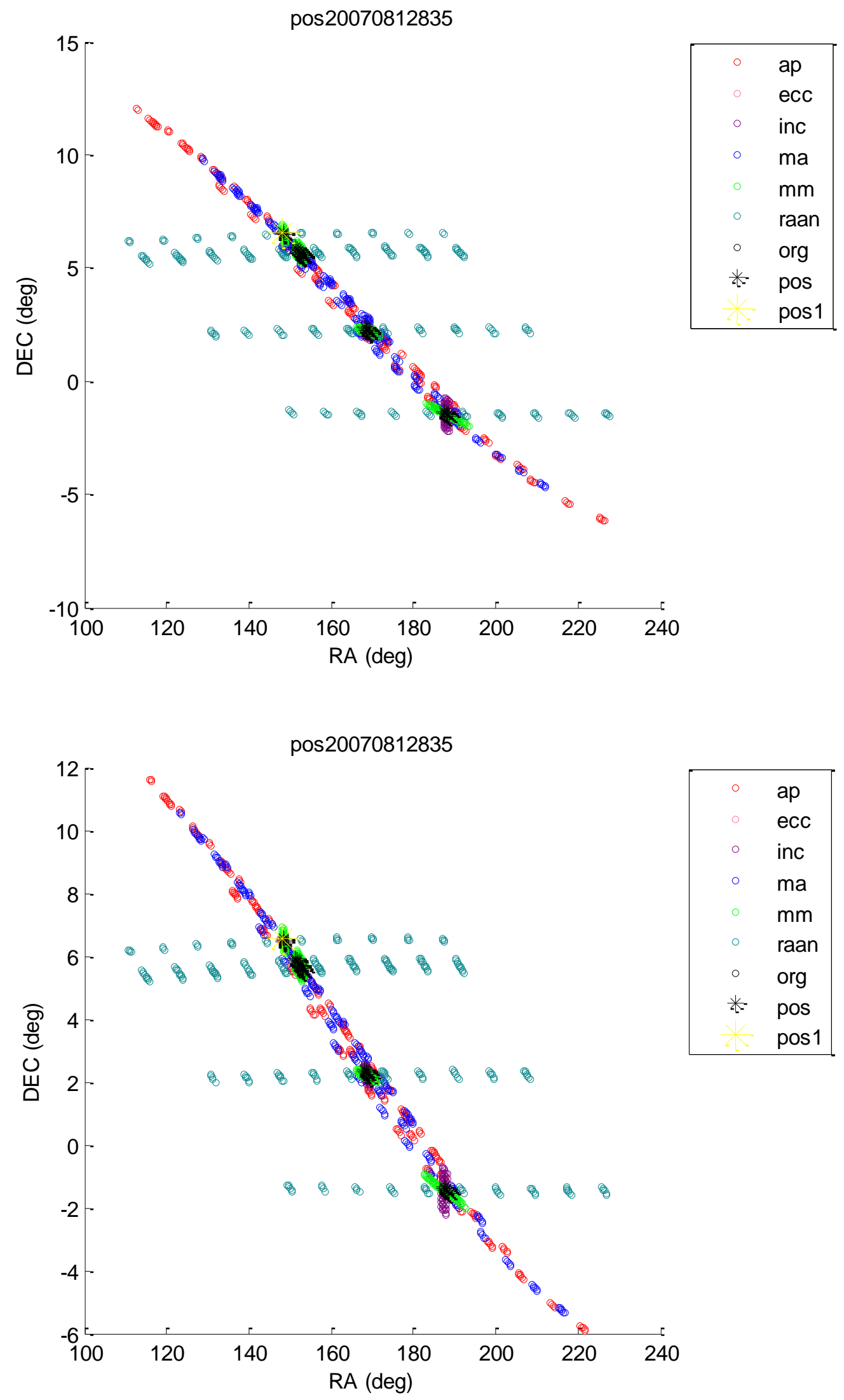

\begin{tabular}{|ll|}
\hline & ap \\
ecc \\
inc \\
ma \\
mm \\
raan \\
org \\
*- pos \\
\\
pos1
\end{tabular}



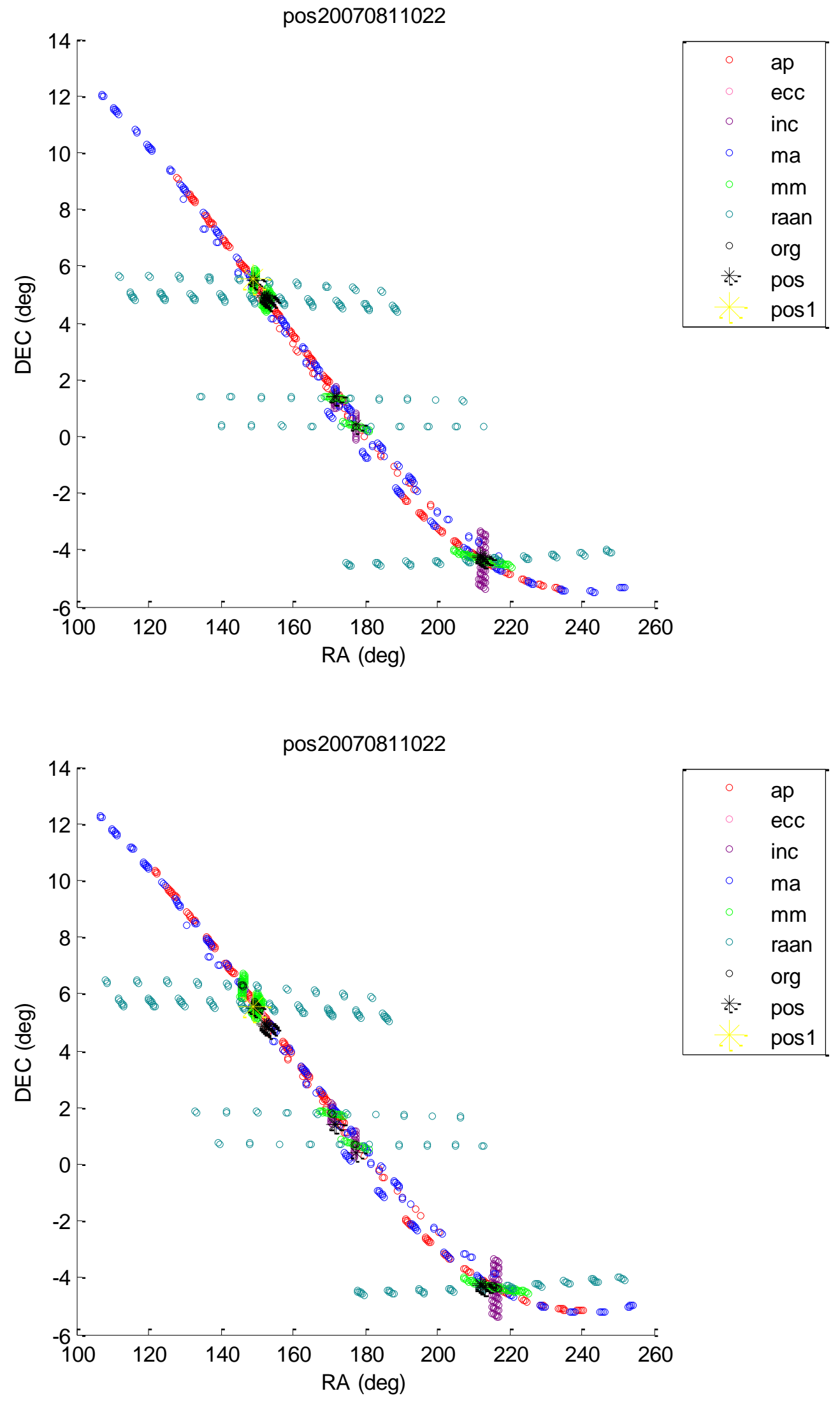

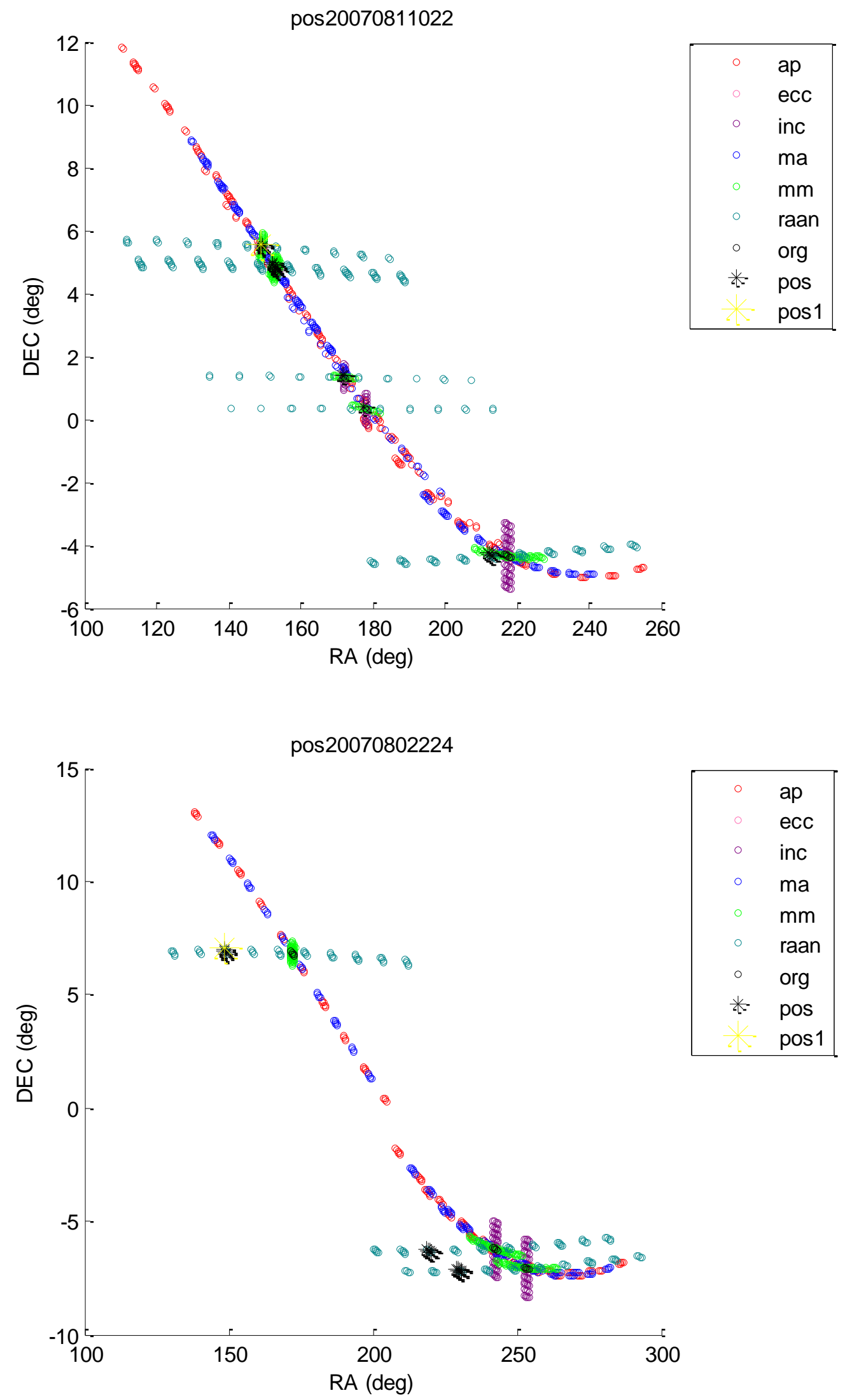

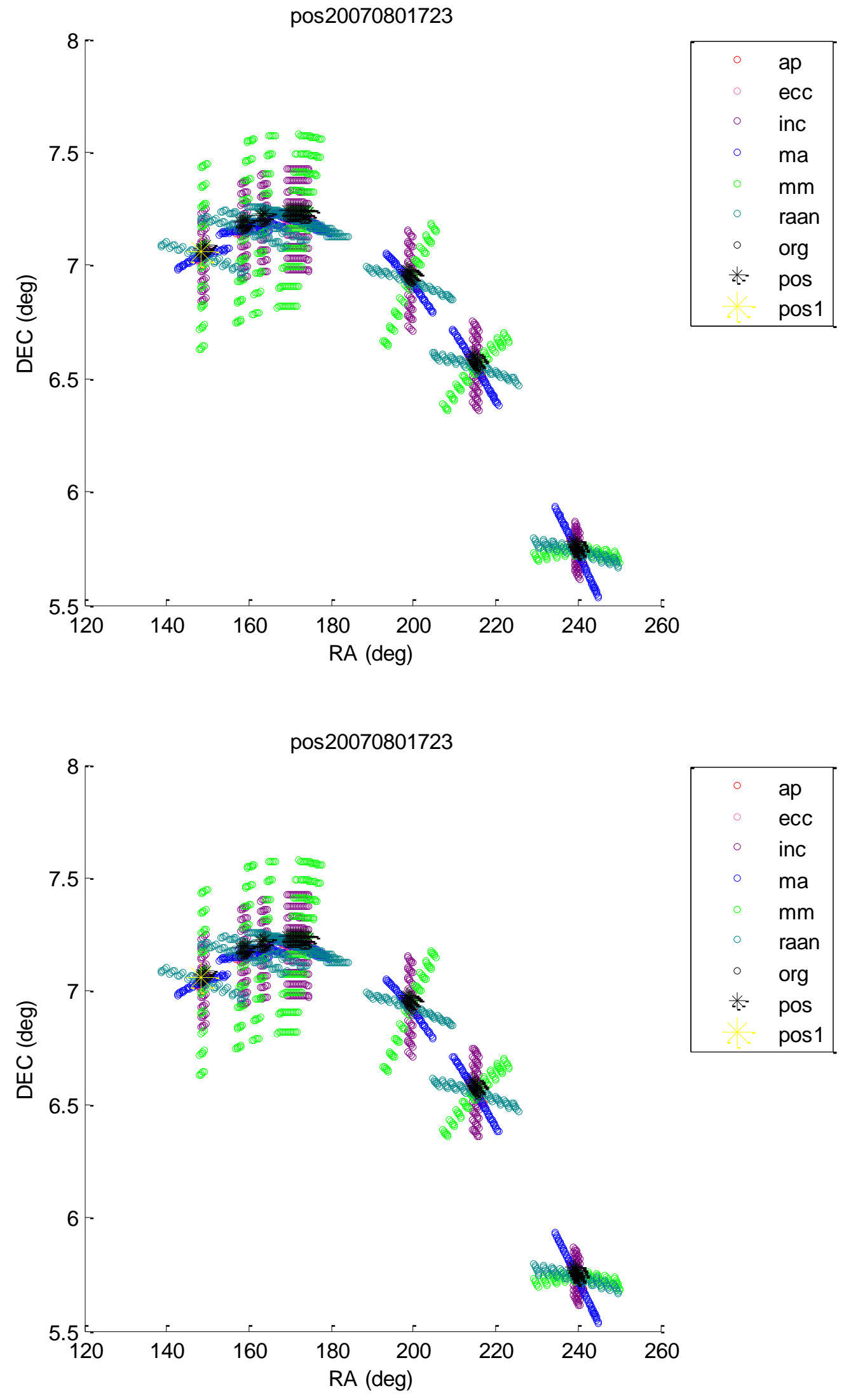

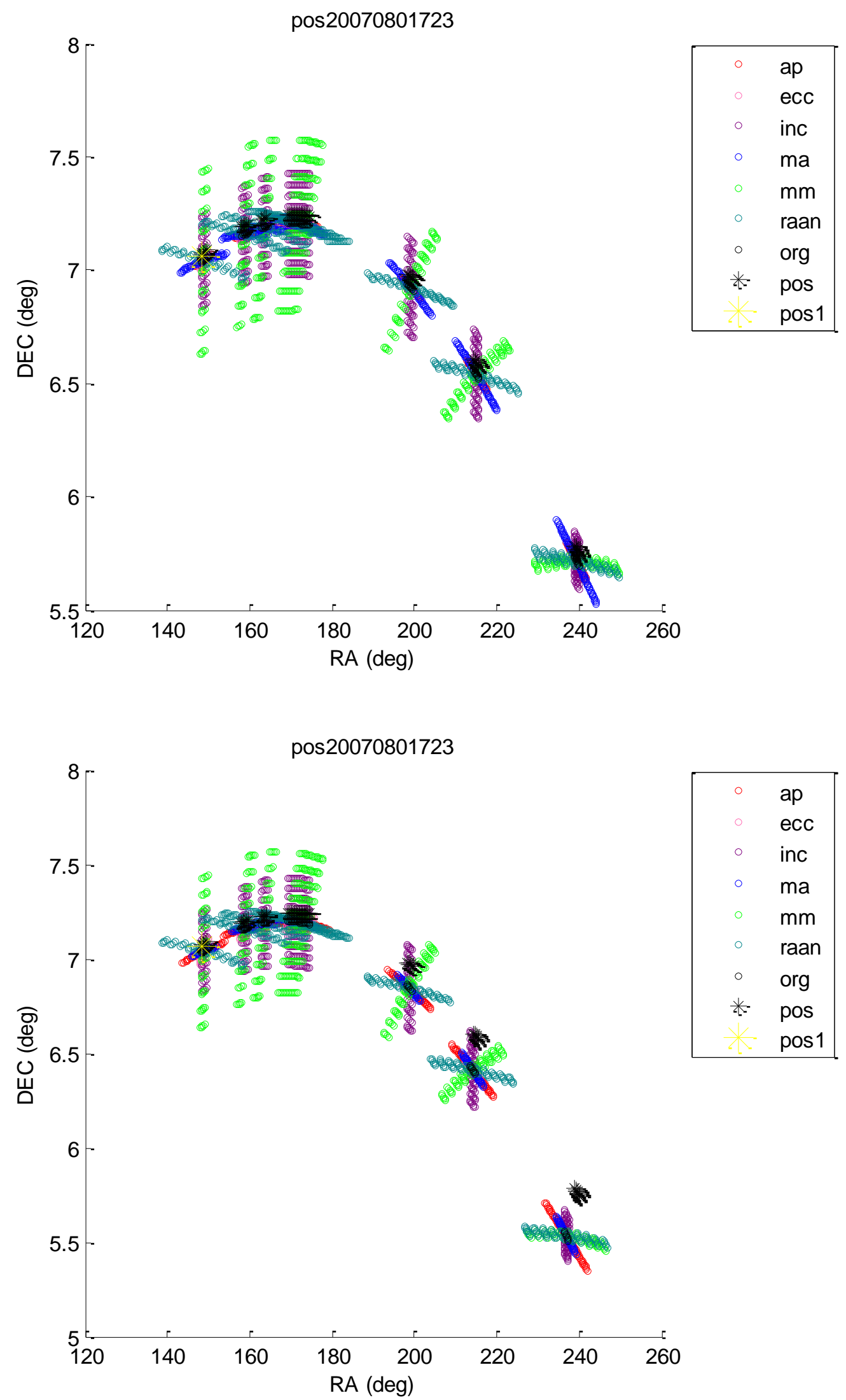

\begin{tabular}{|ll|}
\hline & ap \\
& ecc \\
inc \\
ma \\
mm \\
raan \\
$\circ$ & org \\
$*$ & pos \\
& pos1 \\
\hline
\end{tabular}



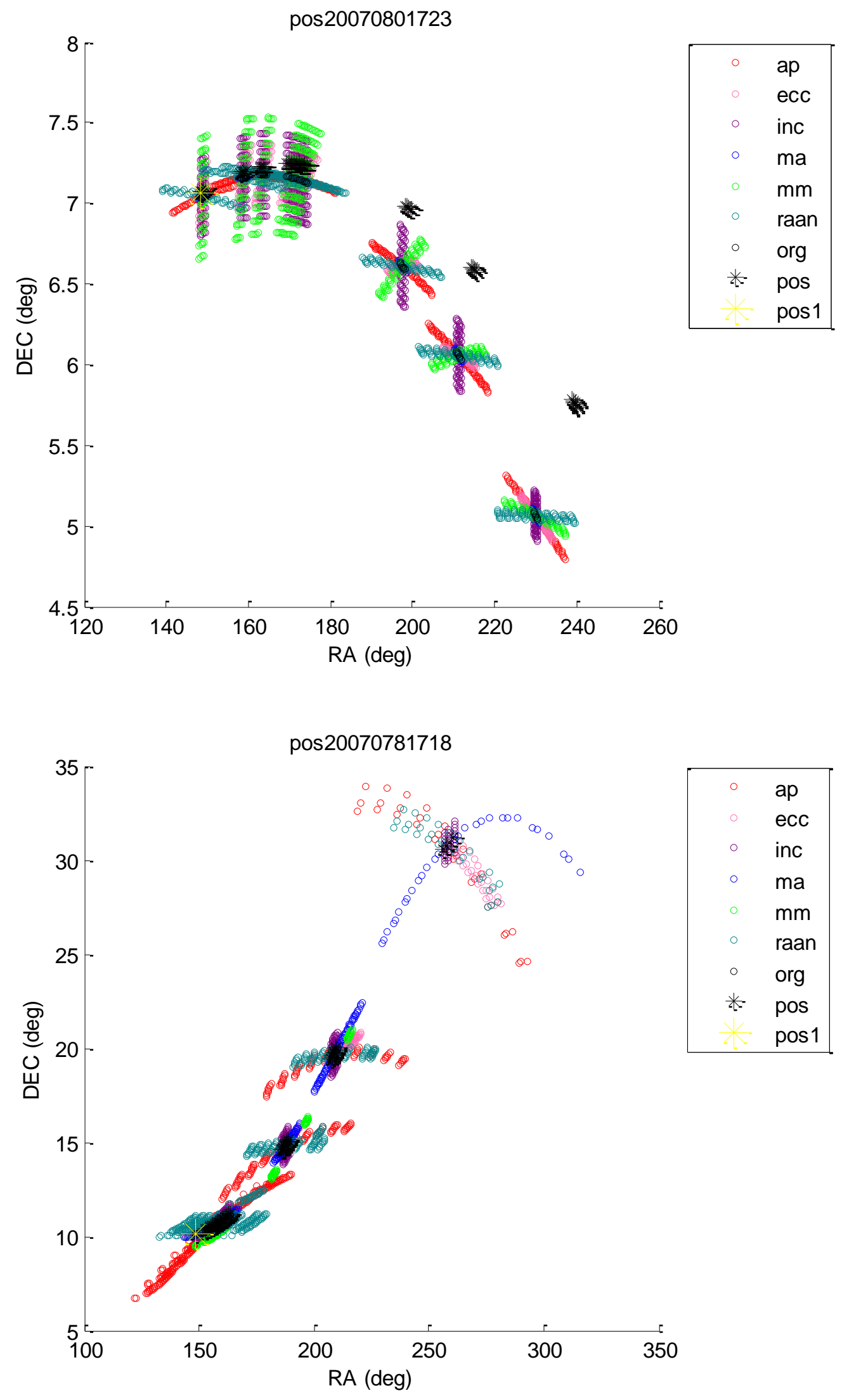

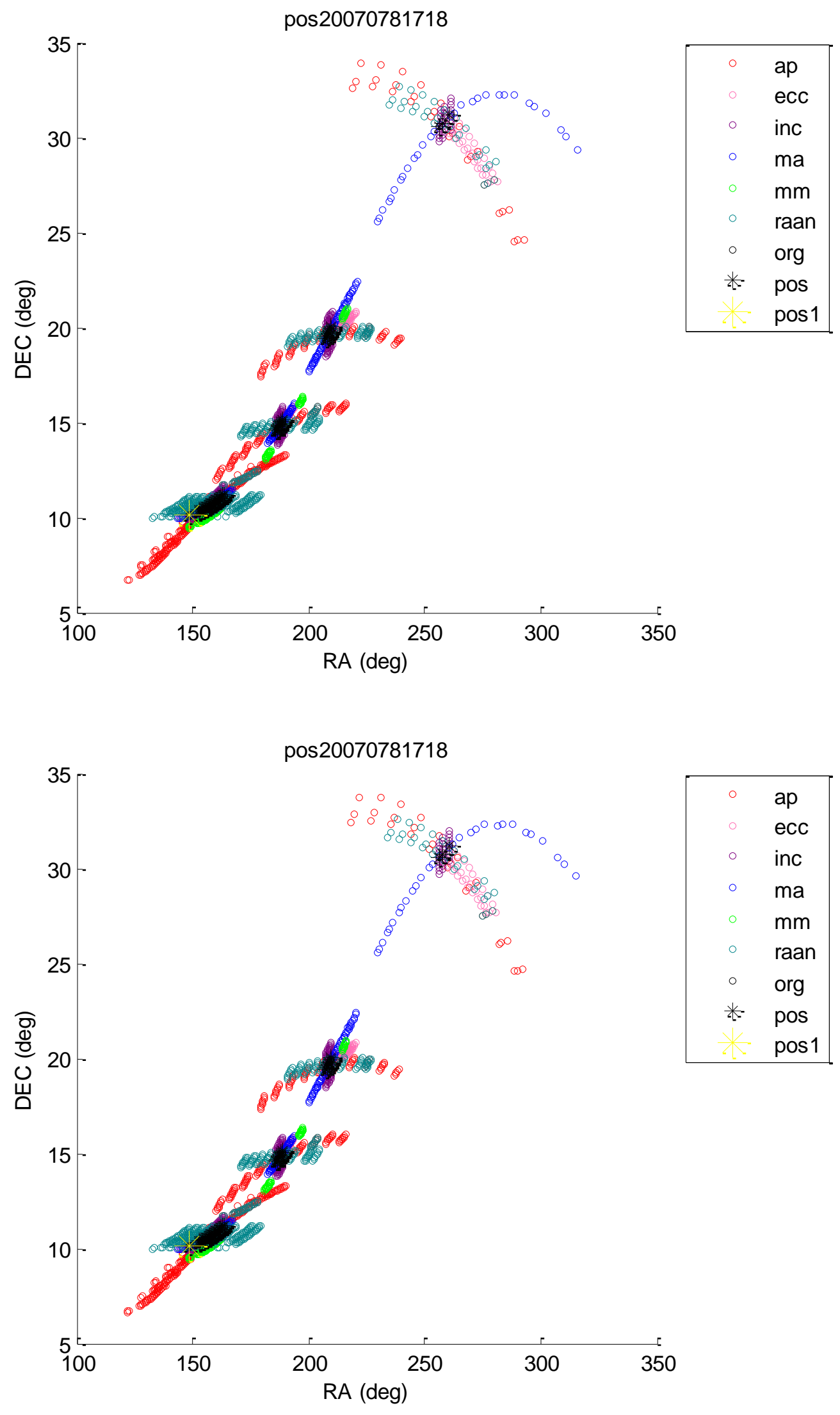

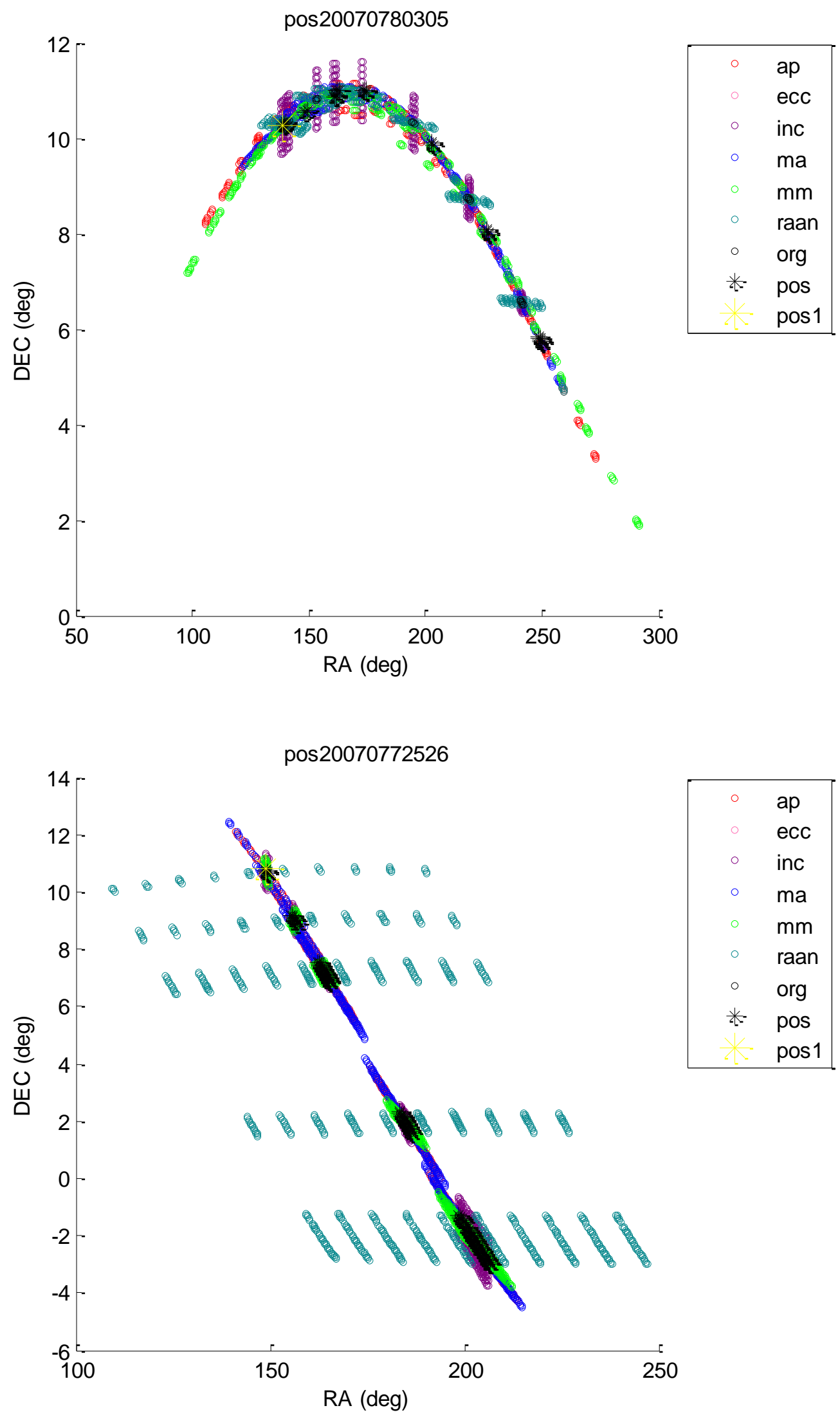

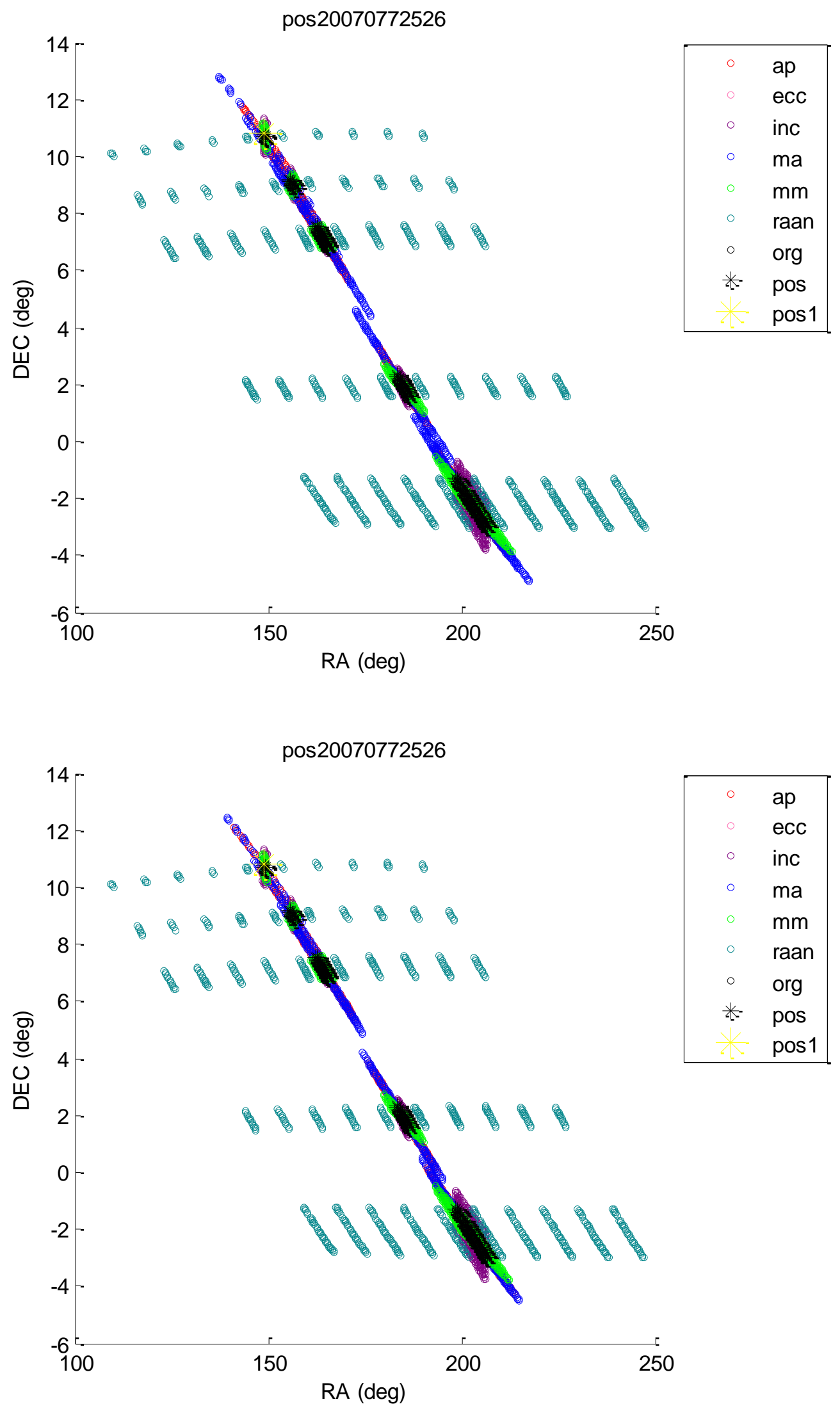

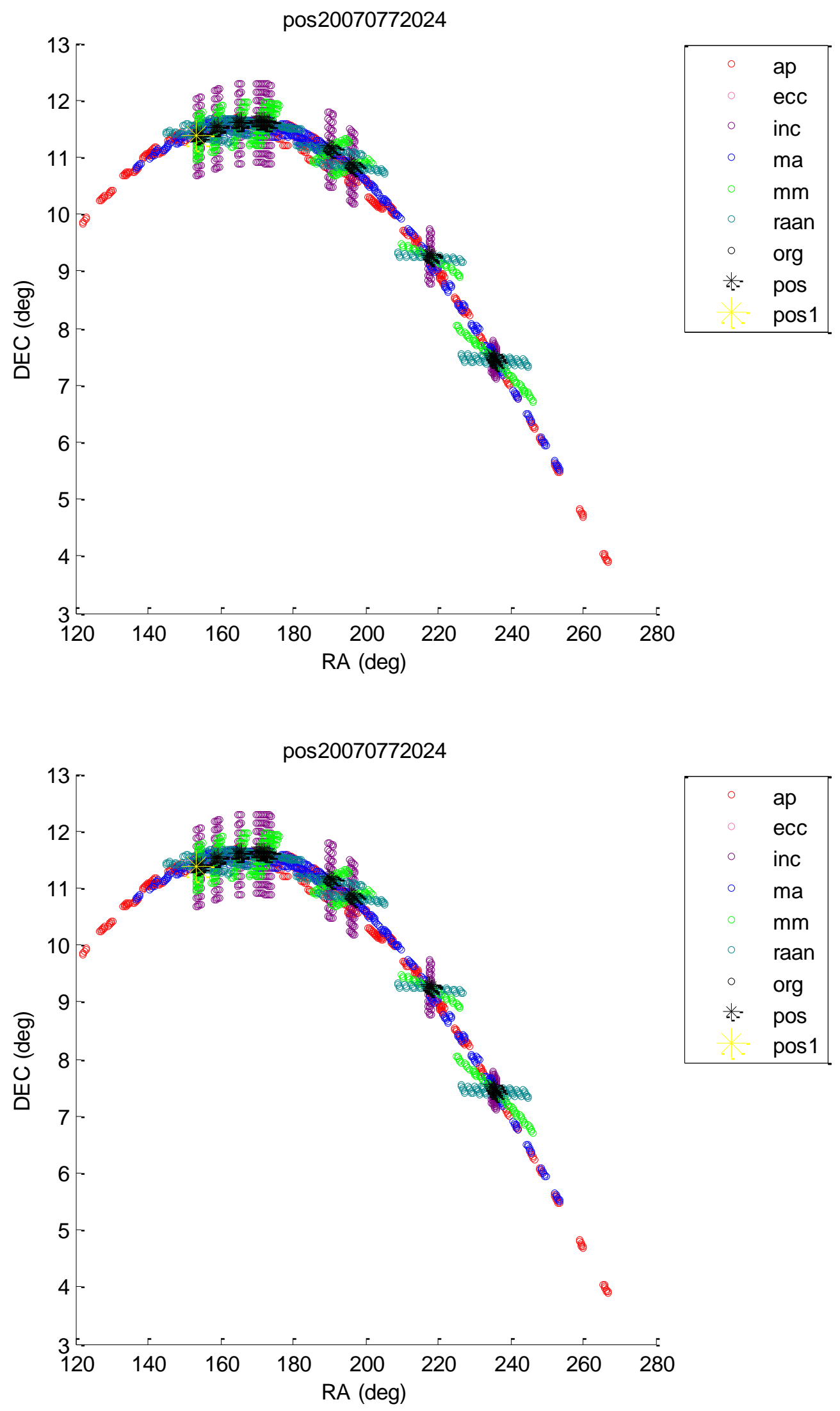

\begin{tabular}{|ll|}
\hline & ap \\
& ecc \\
& inc \\
& ma \\
& mm \\
& raan \\
$\circ$ & org \\
$*$ & pos \\
& pos1 \\
\hline
\end{tabular}



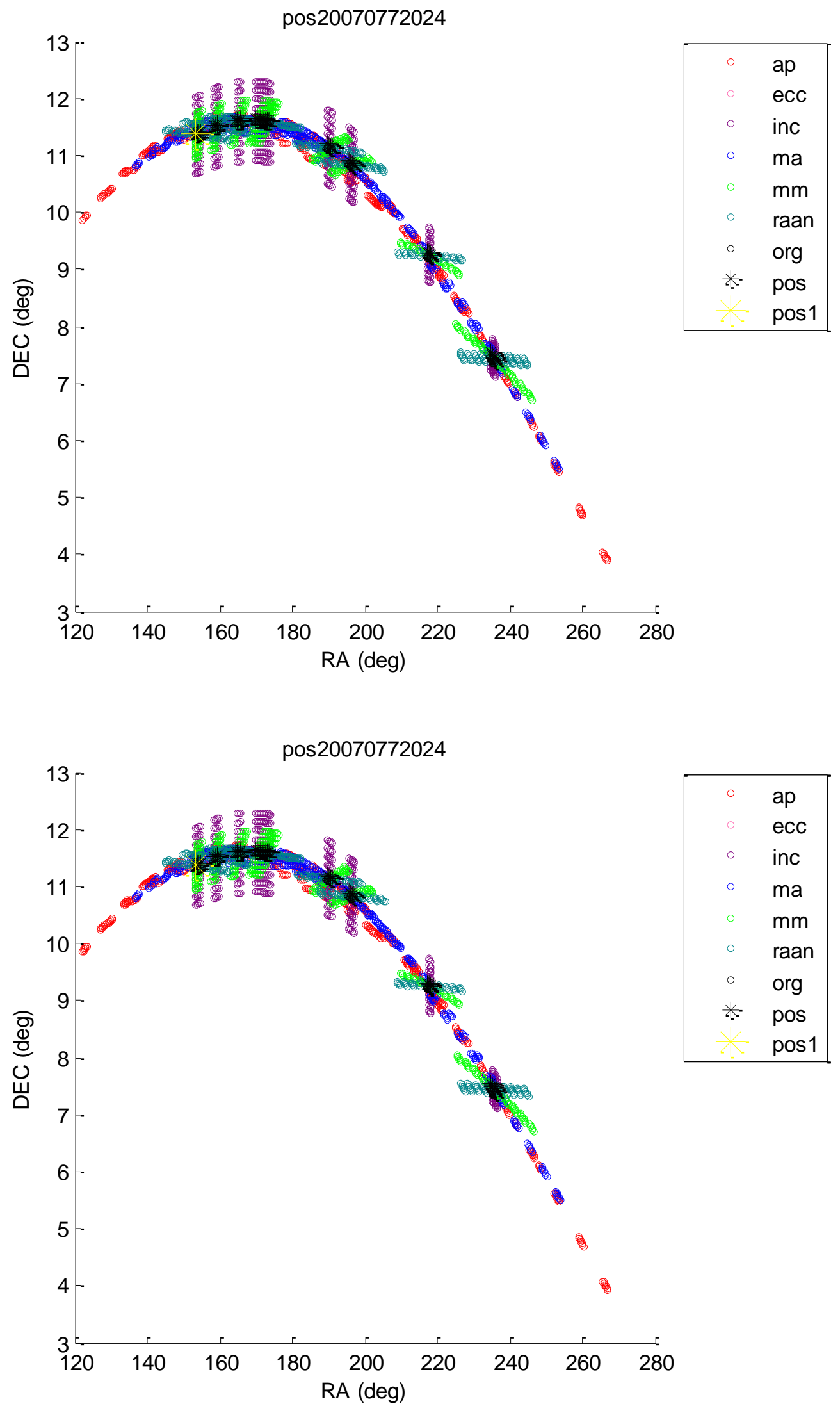

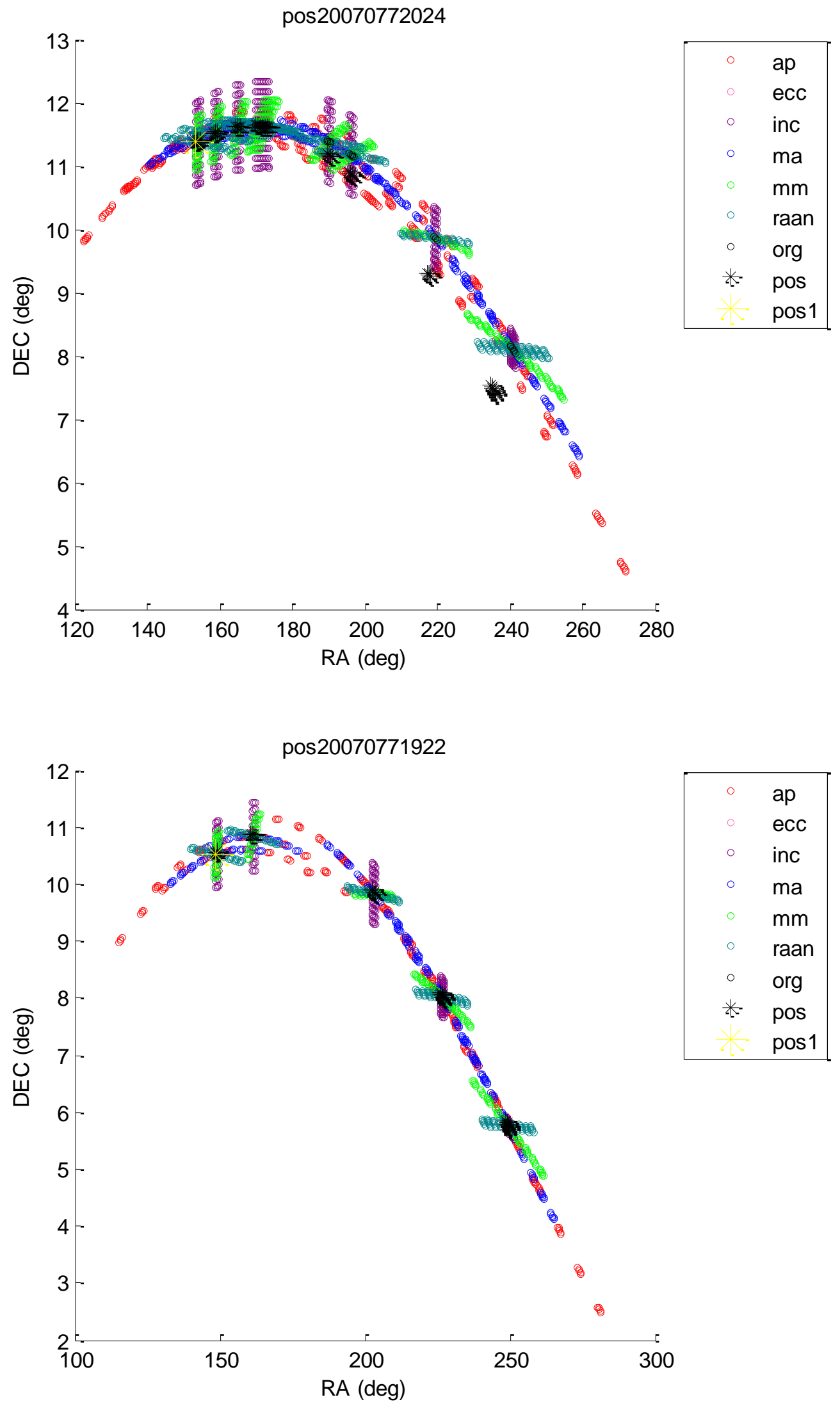

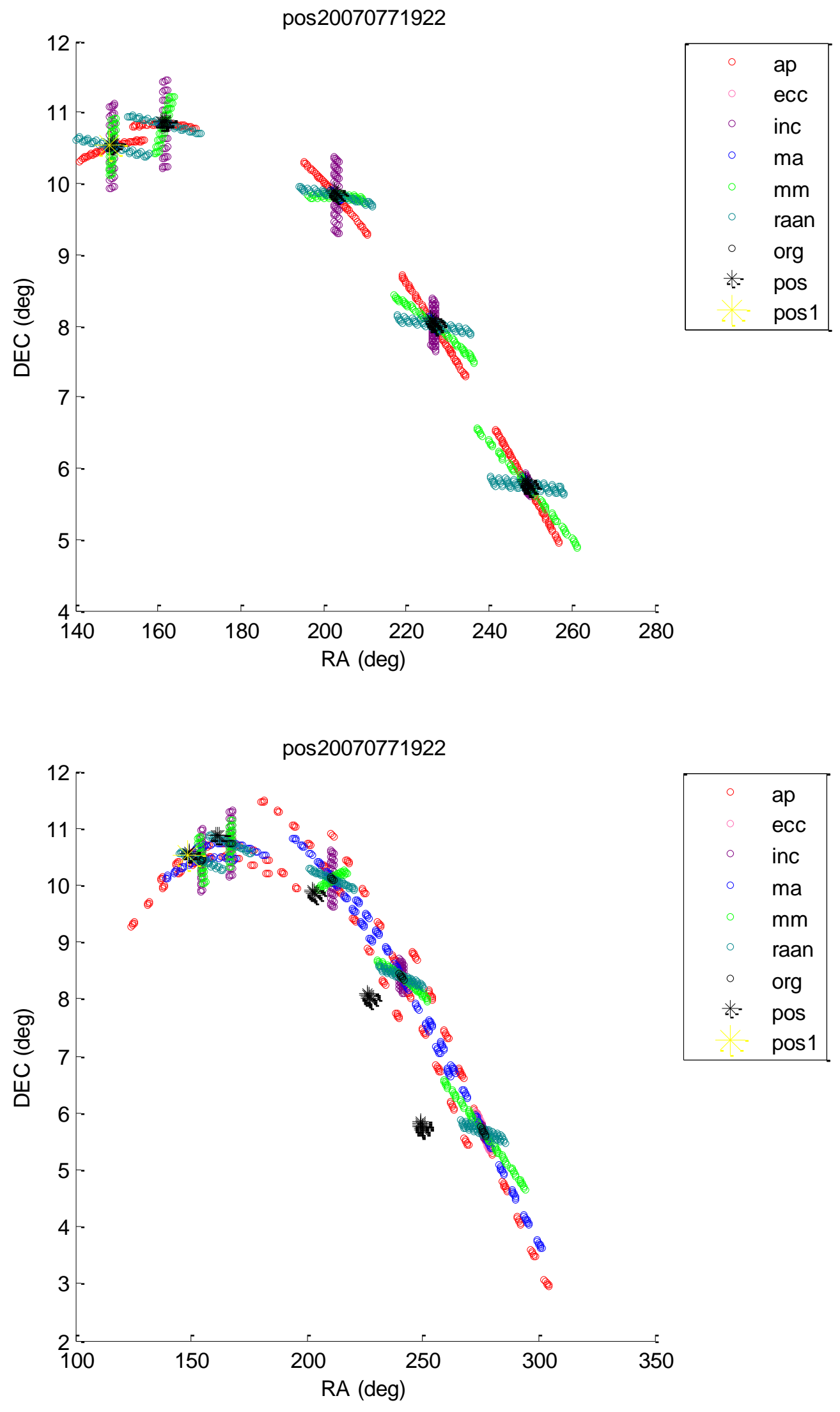


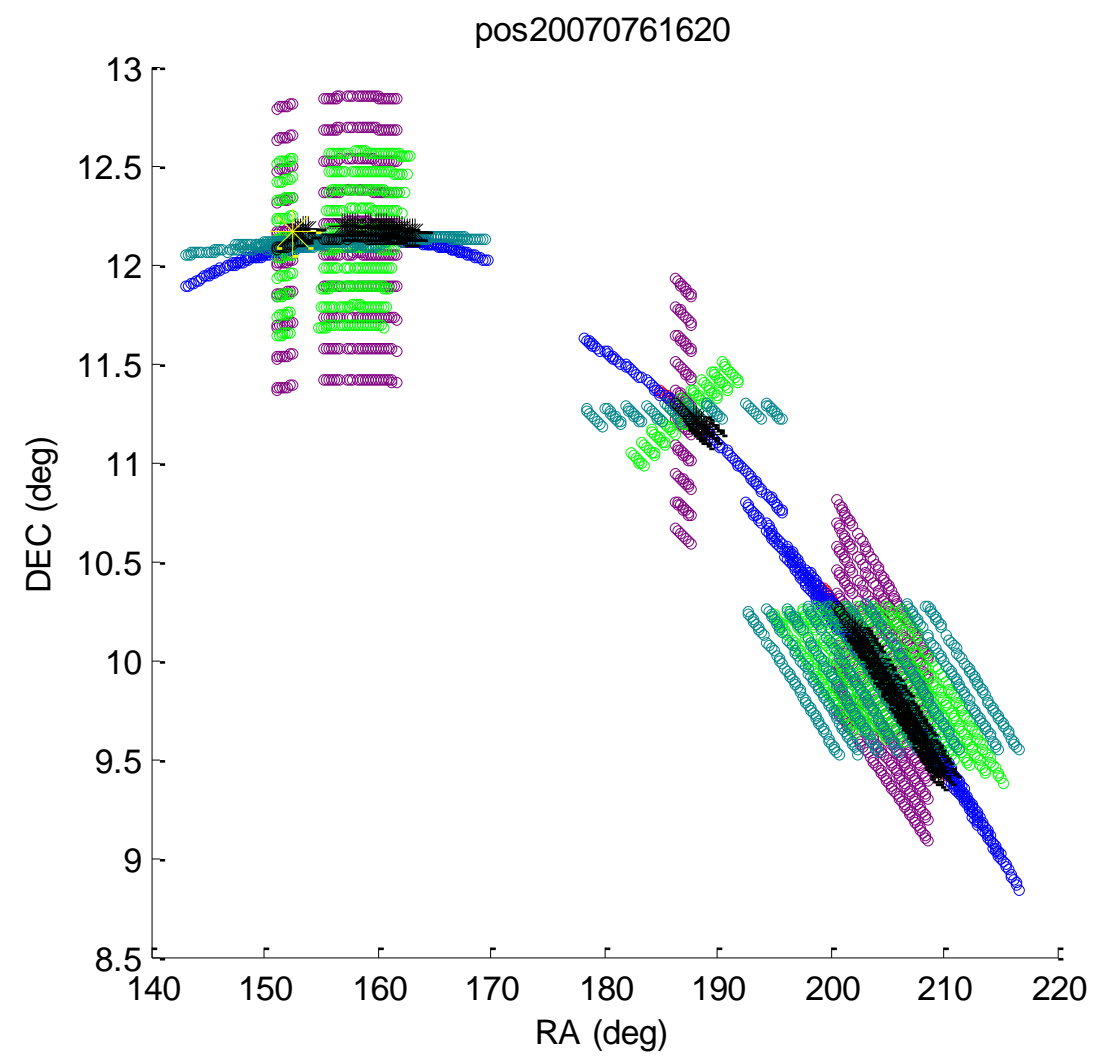

\begin{tabular}{|ll|}
\hline$\circ$ & ap \\
& ecc \\
& inc \\
$\circ$ & ma \\
& mm \\
& raan \\
$\circ$ & org \\
$*$ & pos \\
& pos1 \\
\hline
\end{tabular}

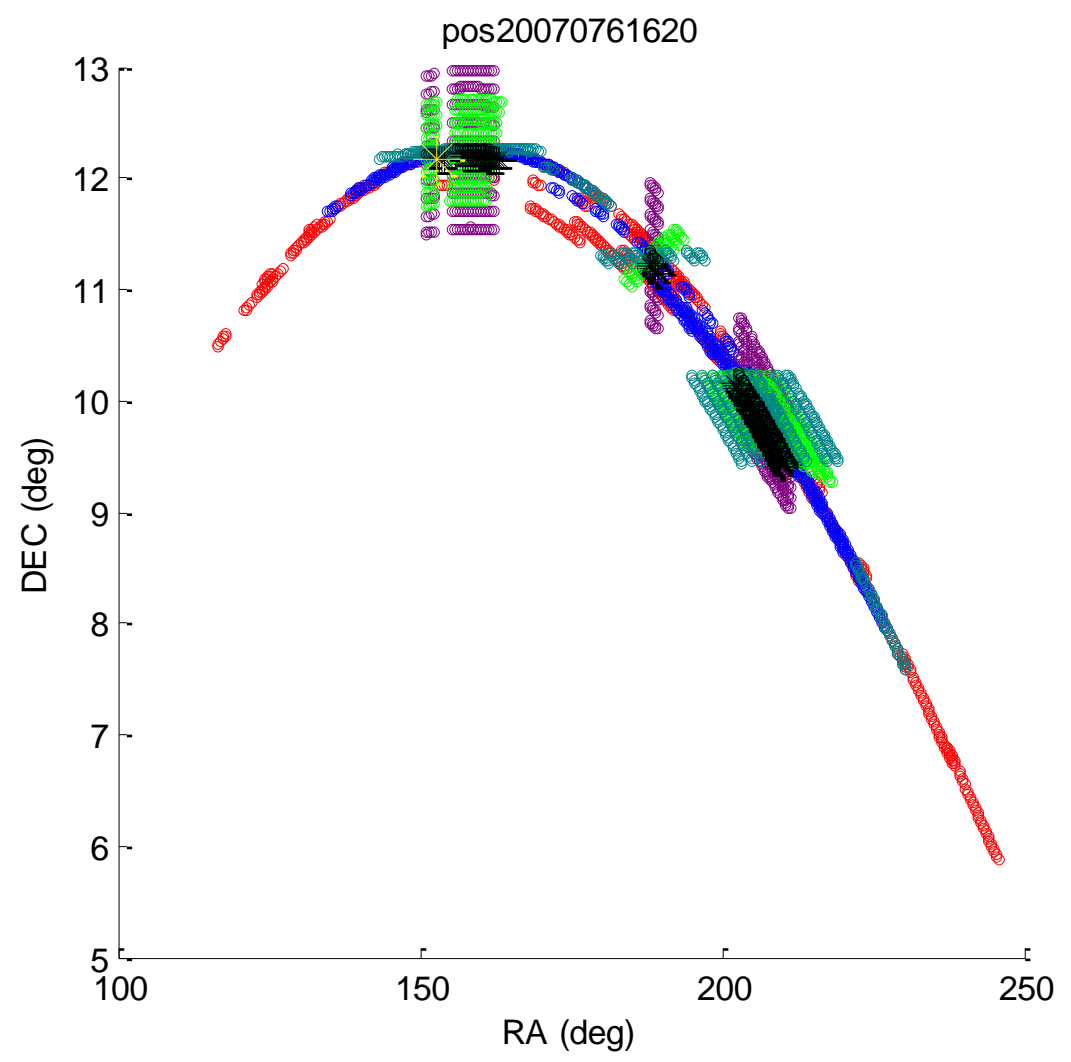

\begin{tabular}{|ll|}
\hline & ap \\
& ecc \\
& inc \\
& ma \\
& mm \\
& raan \\
$\circ$ & org \\
$*$ & pos \\
& pos1 \\
\hline
\end{tabular}



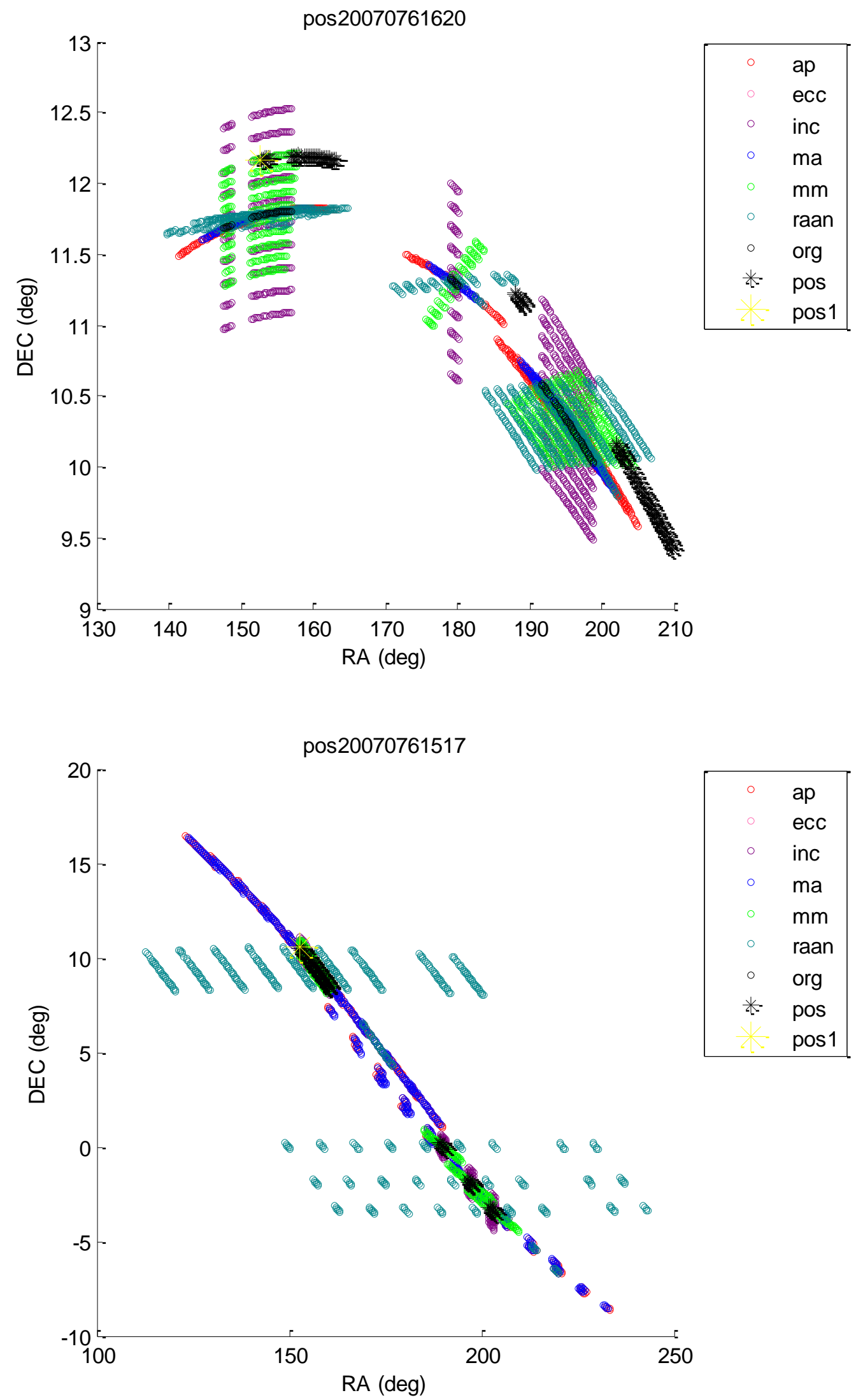

\begin{tabular}{|ll|}
\hline & ap \\
& ecc \\
& inc \\
& ma \\
& $\mathrm{mm}$ \\
& raan \\
$\circ$ & org \\
$*$ & pos \\
& pos1 \\
\hline
\end{tabular}



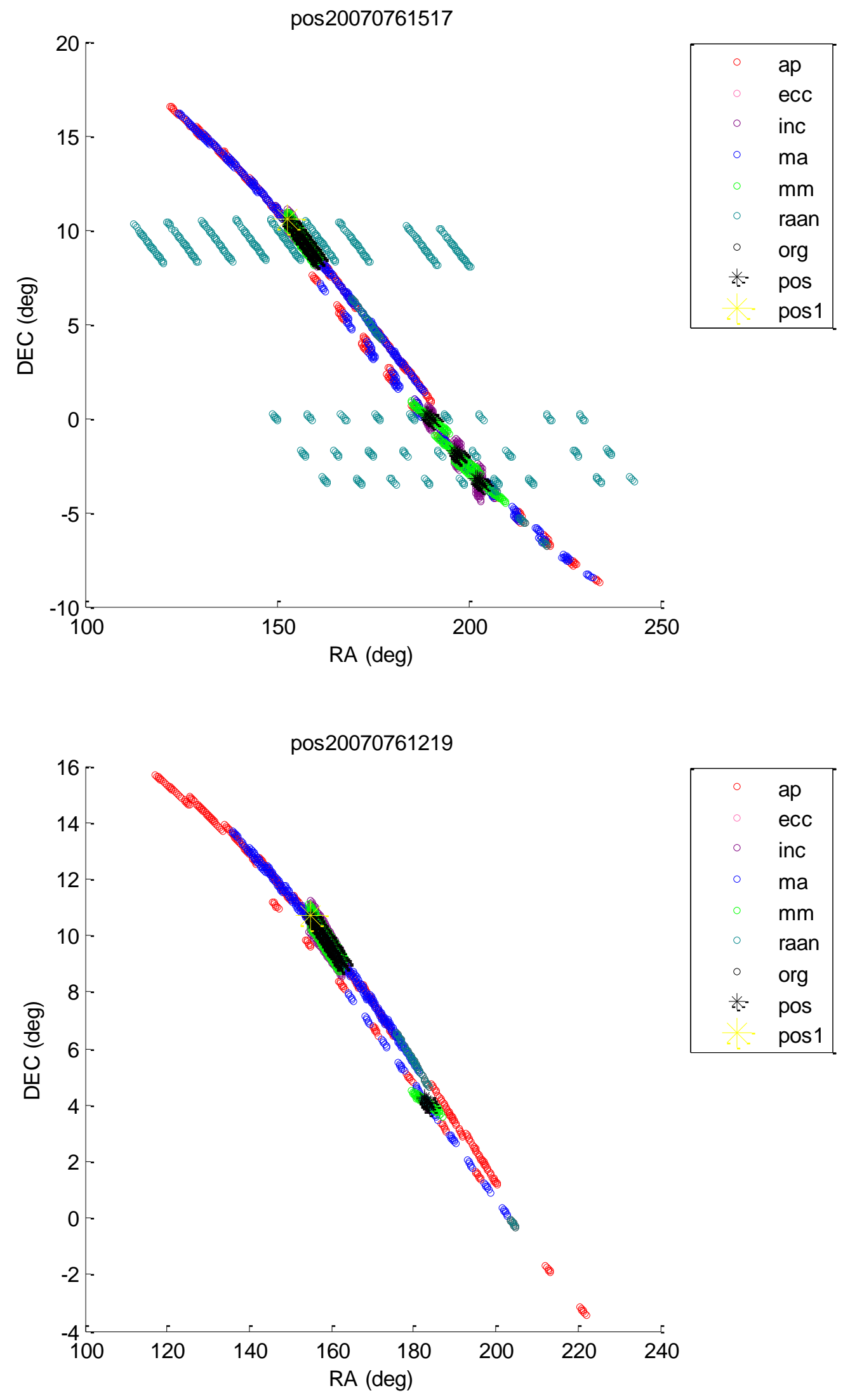

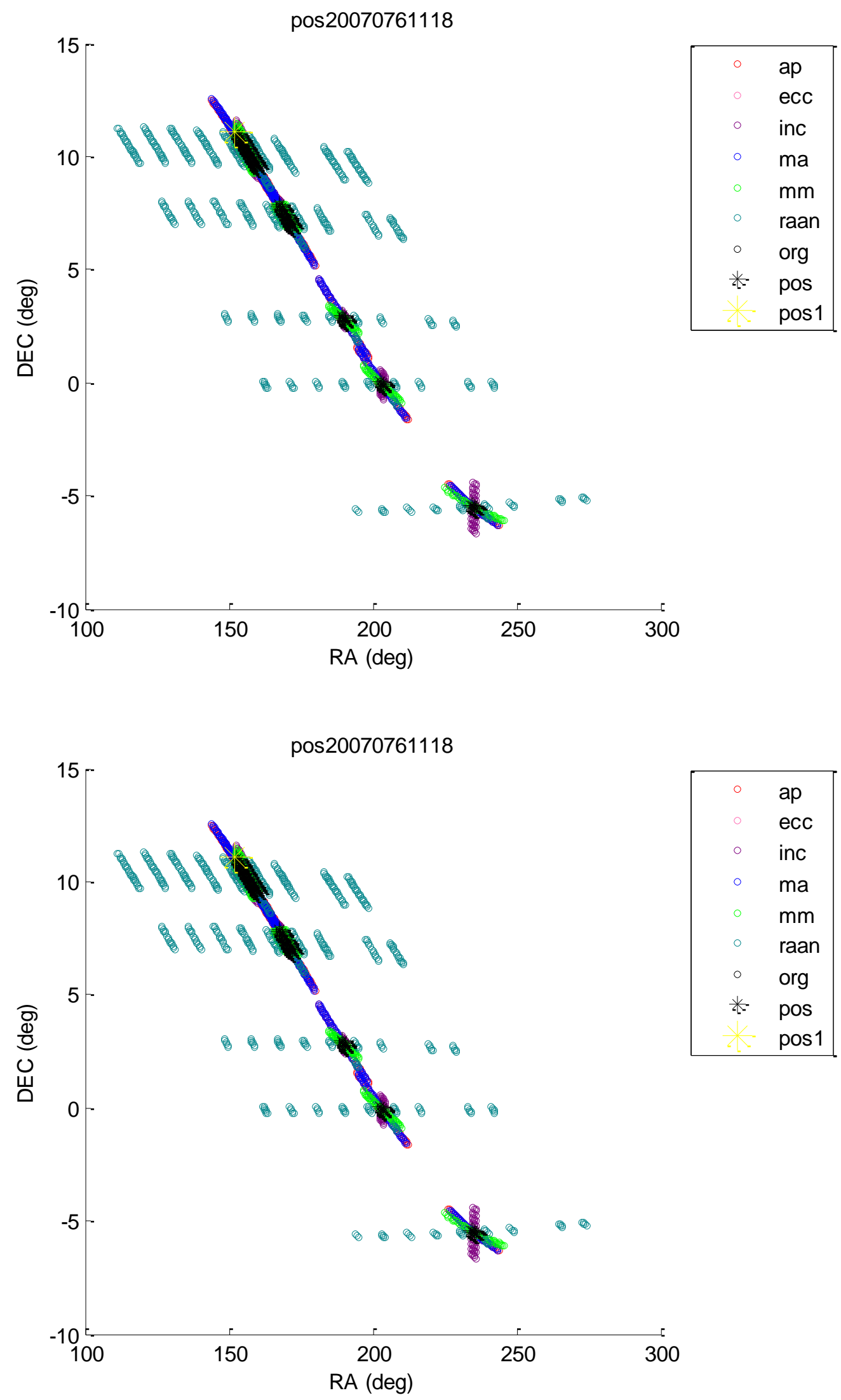

\begin{tabular}{|ll|}
\hline & ap \\
& ecc \\
& inc \\
& ma \\
& $\mathrm{mm}$ \\
& raan \\
$\circ$ & org \\
$*$ & pos \\
& pos1 \\
\hline
\end{tabular}



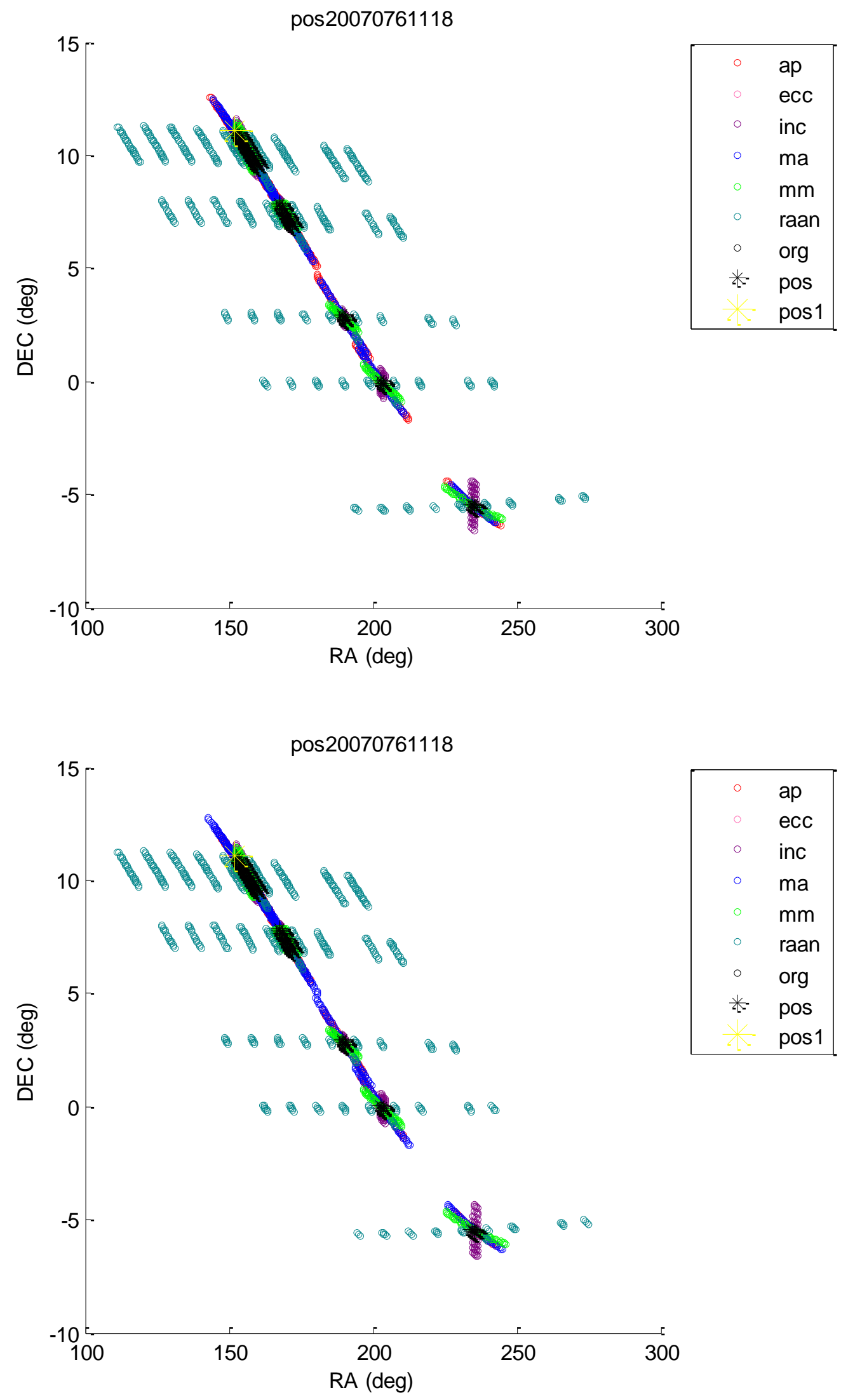

\begin{tabular}{|ll|}
\hline & ap \\
& ecc \\
inc \\
ma \\
mm \\
raan \\
$\circ$ & org \\
$*$ & pos \\
& pos1 \\
\hline
\end{tabular}



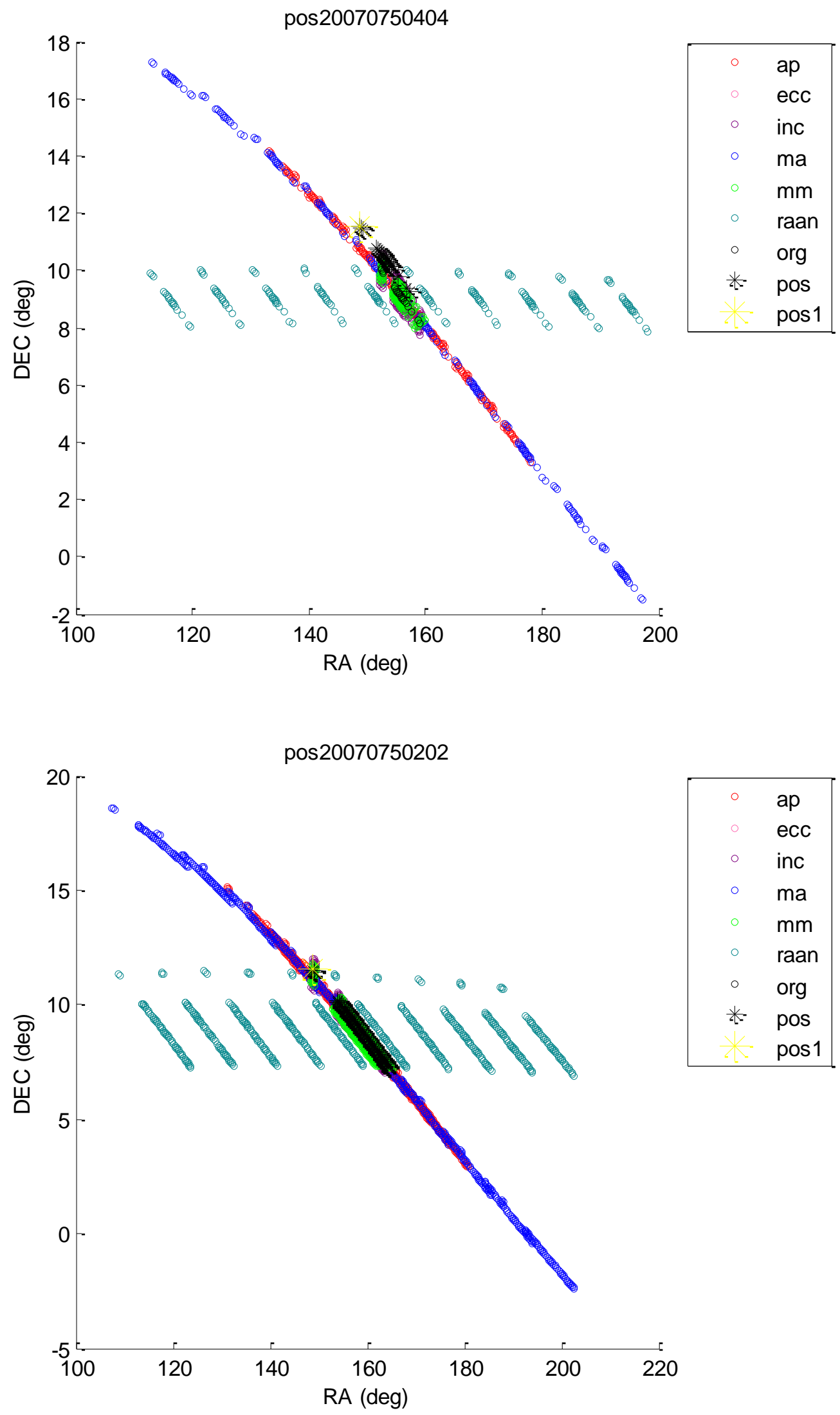

\begin{tabular}{|ll|}
\hline & ap \\
& ecc \\
& inc \\
& ma \\
& mm \\
& raan \\
$\circ$ & org \\
$*$ & pos \\
& pos1 \\
\hline
\end{tabular}




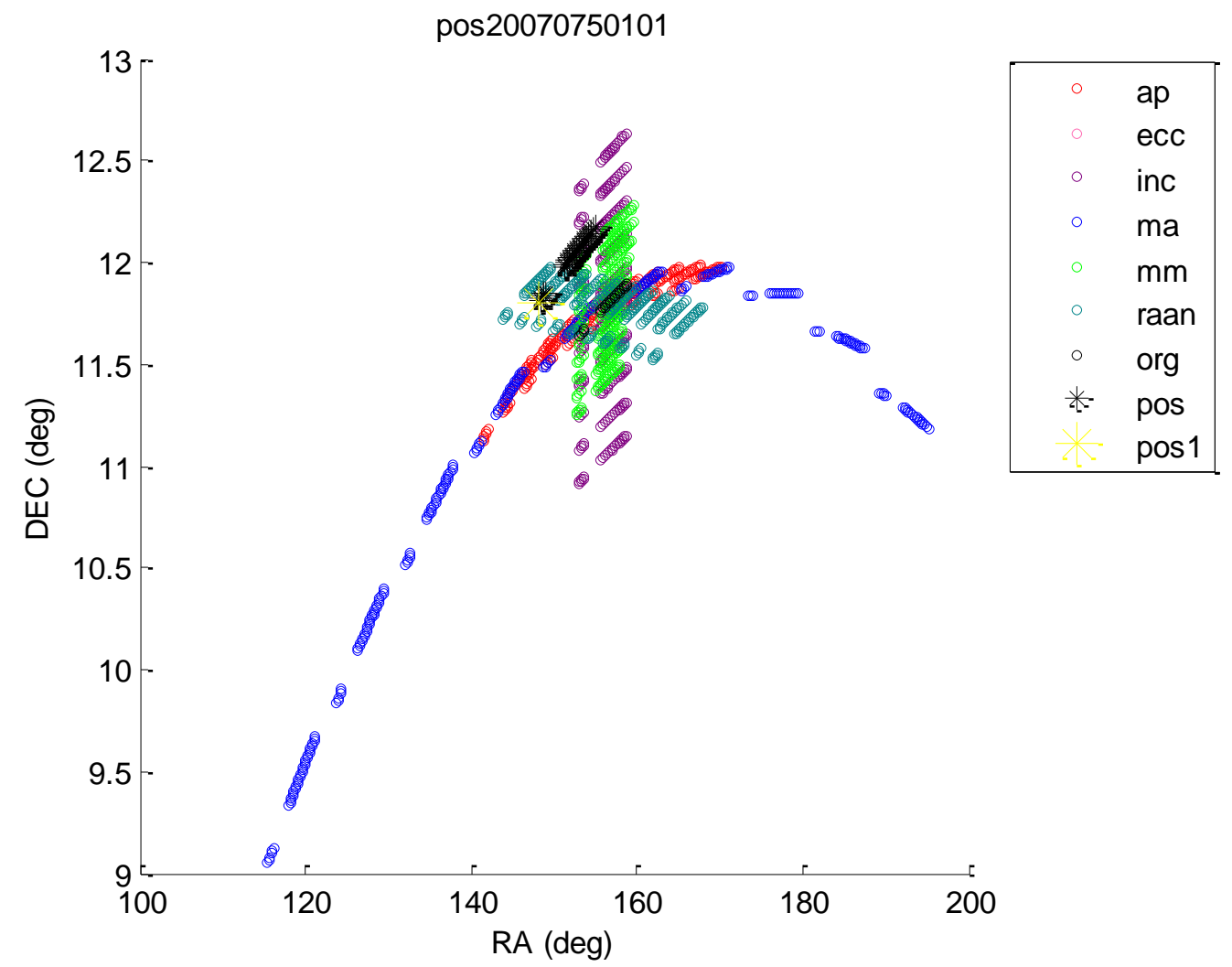




\section{APPENDIX B: FIRST PREDICTED POINT 10\% VARIATION}

The box in these images is the C9 telescope view; the images were combined to shorten the appendix.

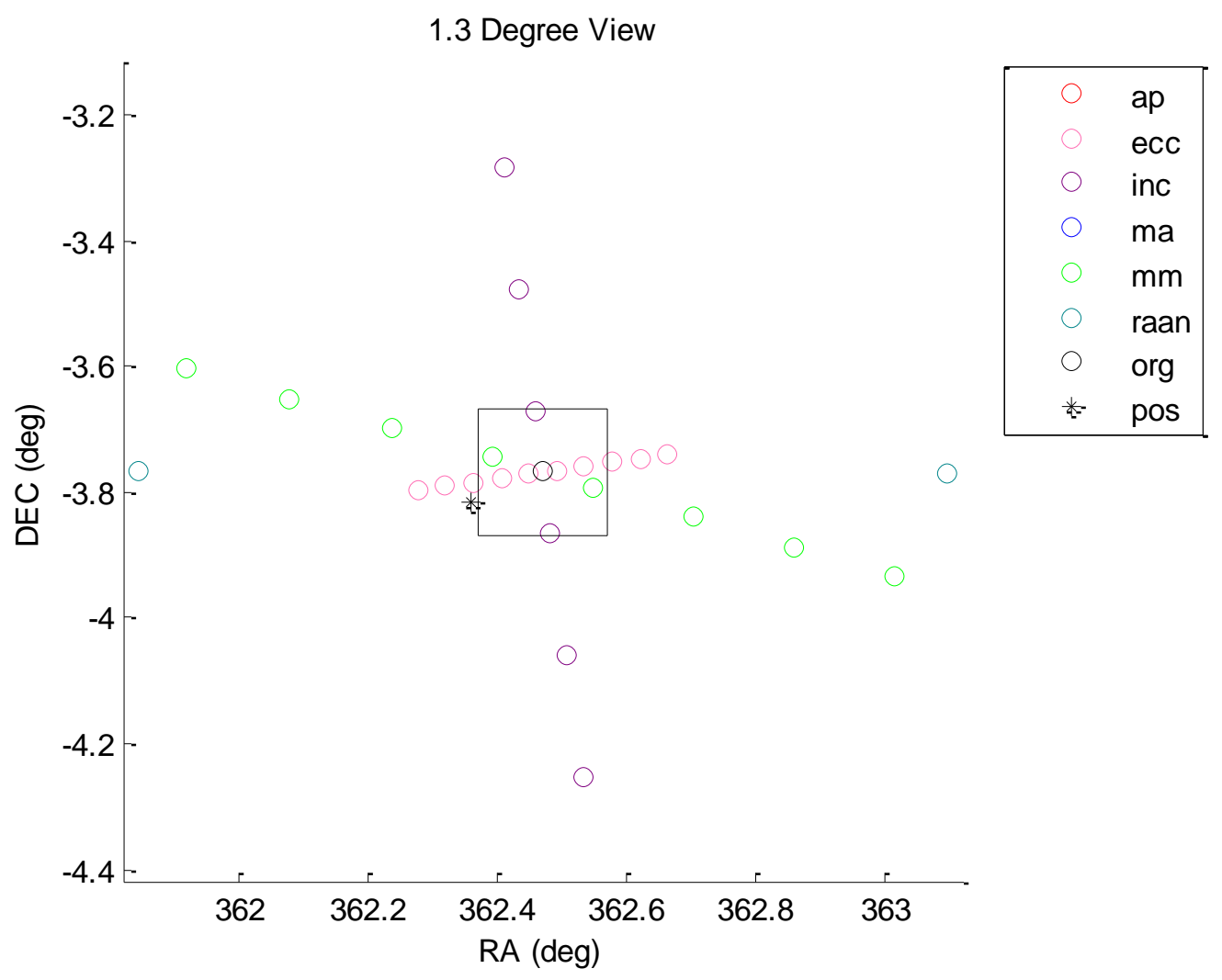


1.3 Degree View

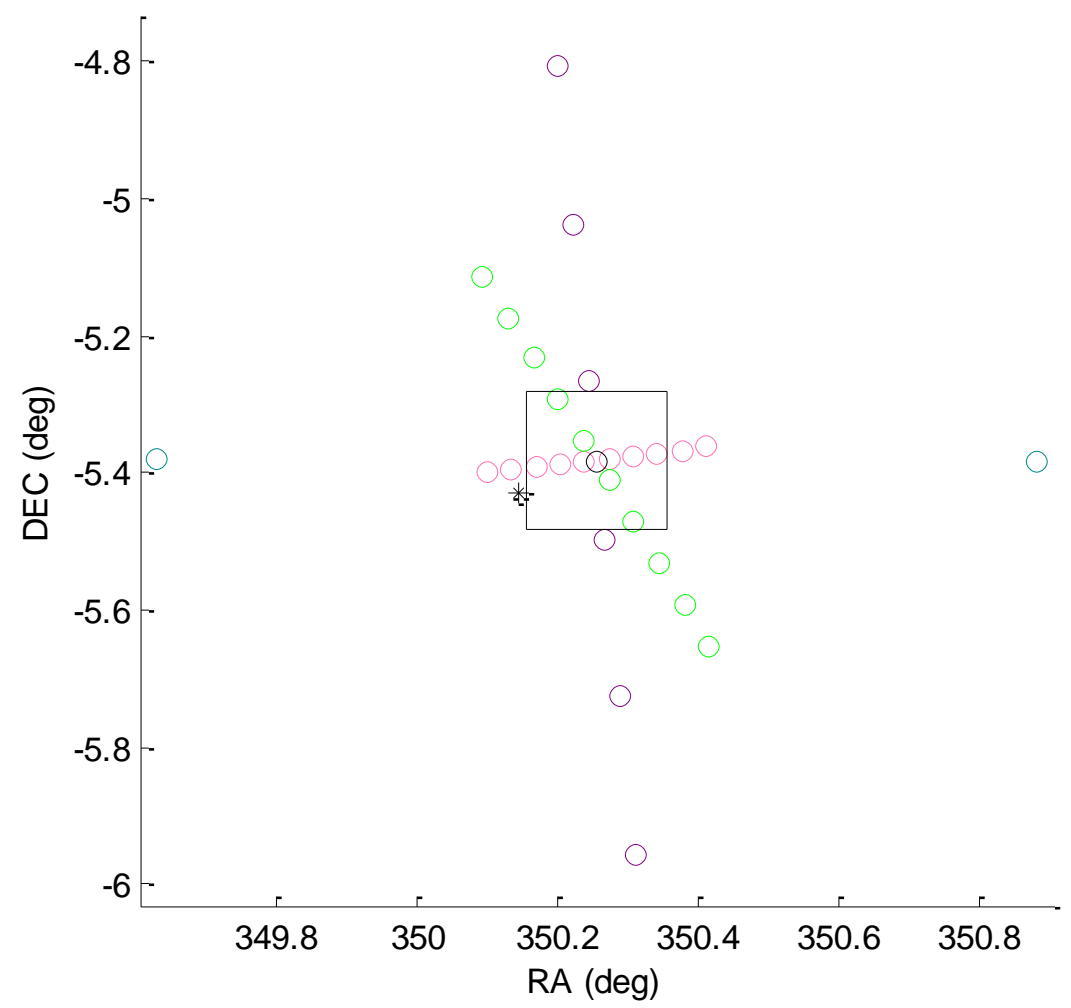

\begin{tabular}{|ll|}
\hline ap \\
ecc \\
inc \\
ma \\
mm \\
raan \\
org \\
* pos \\
\hline
\end{tabular}




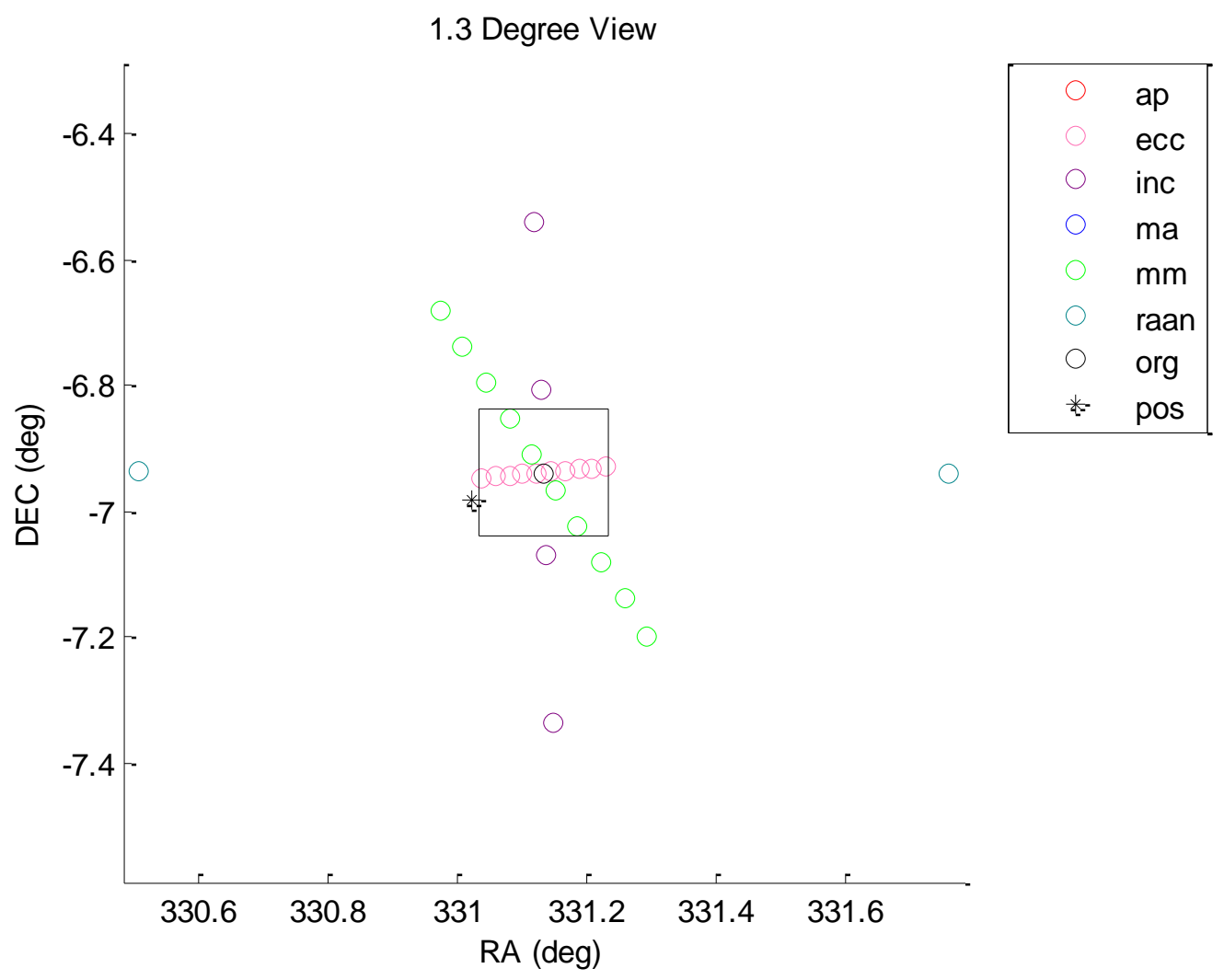


1.3 Degree View

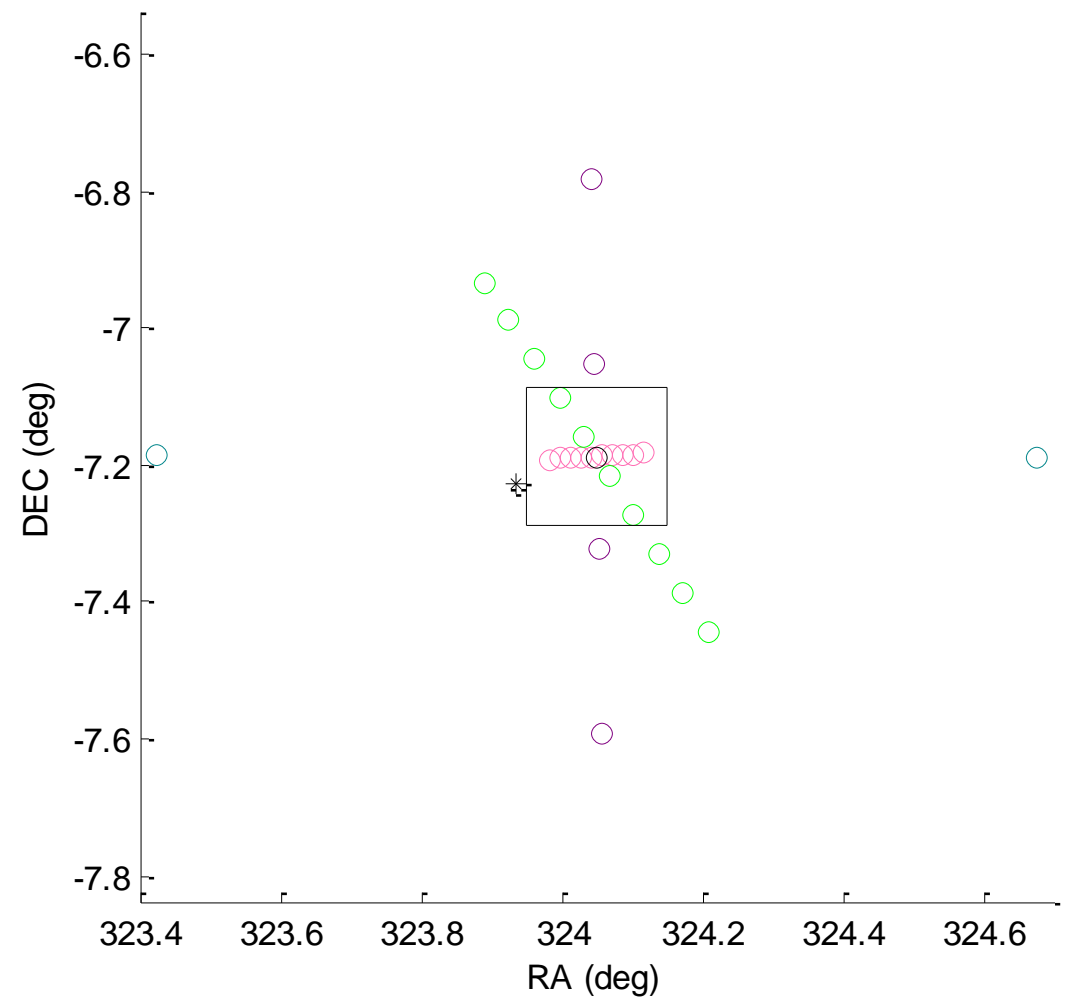

\begin{tabular}{|ll|}
\hline ap \\
ecc \\
inc \\
ma \\
mm \\
raan \\
org \\
* pos \\
\hline
\end{tabular}


1.3 Degree View

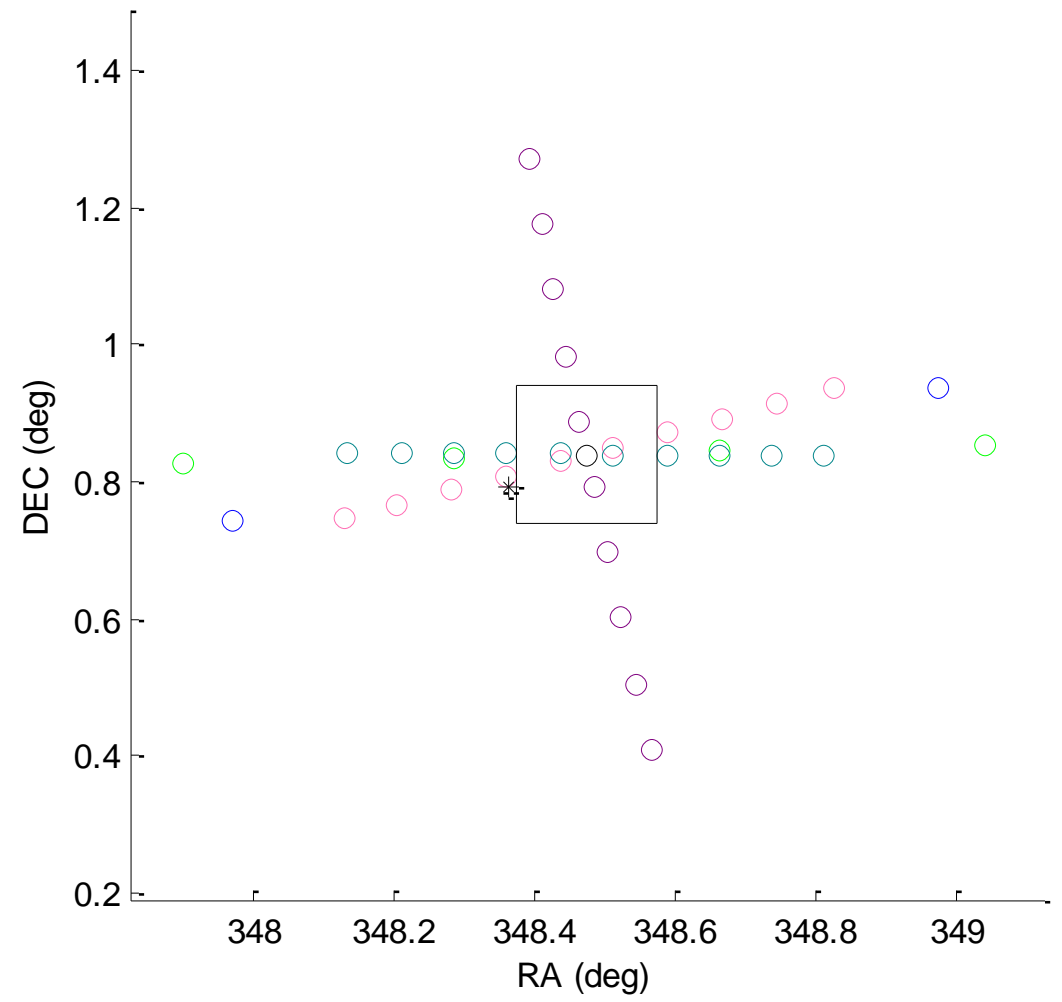

\begin{tabular}{|ll|}
\hline ap \\
ecc \\
inc \\
ma \\
mm \\
raan \\
org \\
* & pos \\
\hline
\end{tabular}




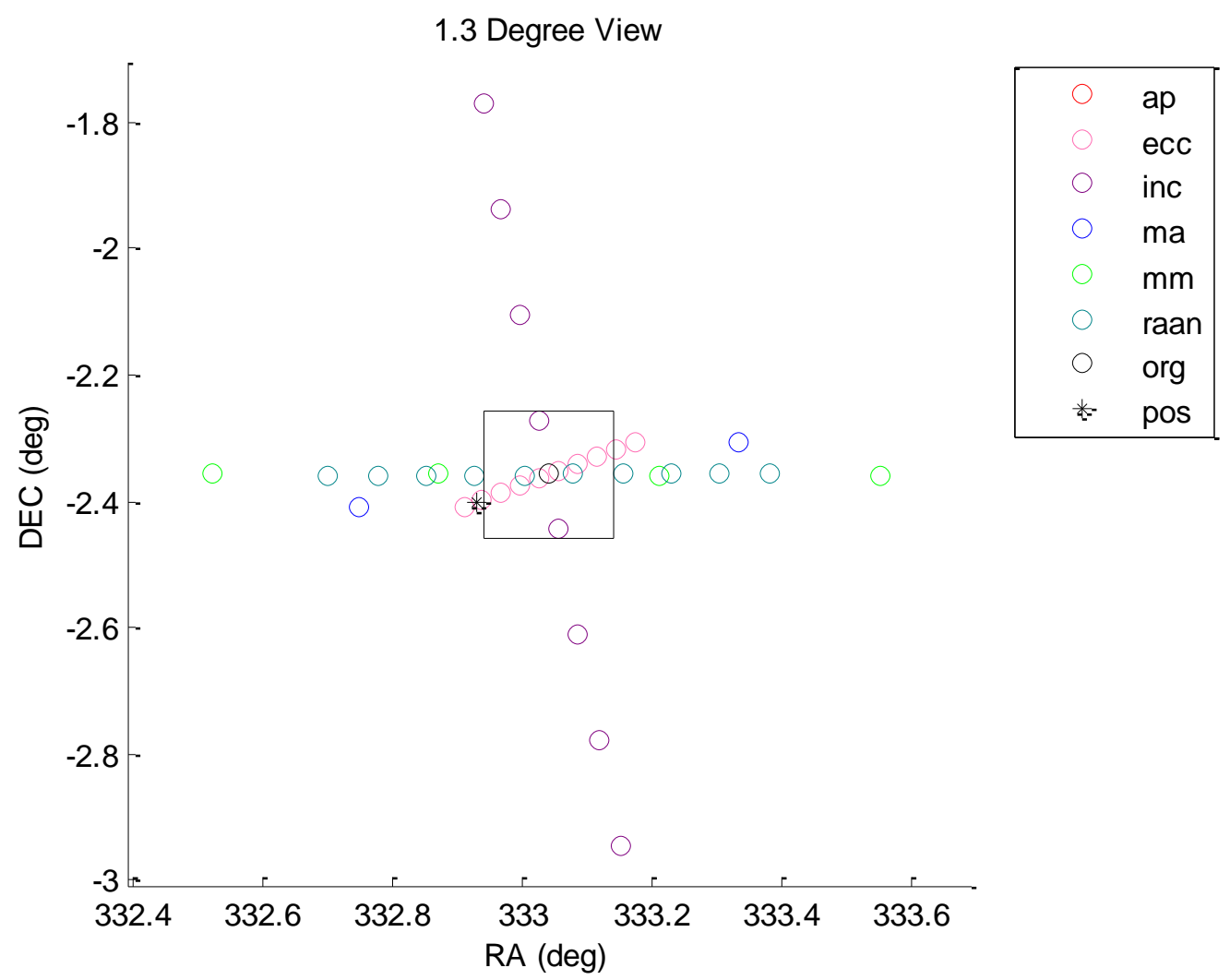




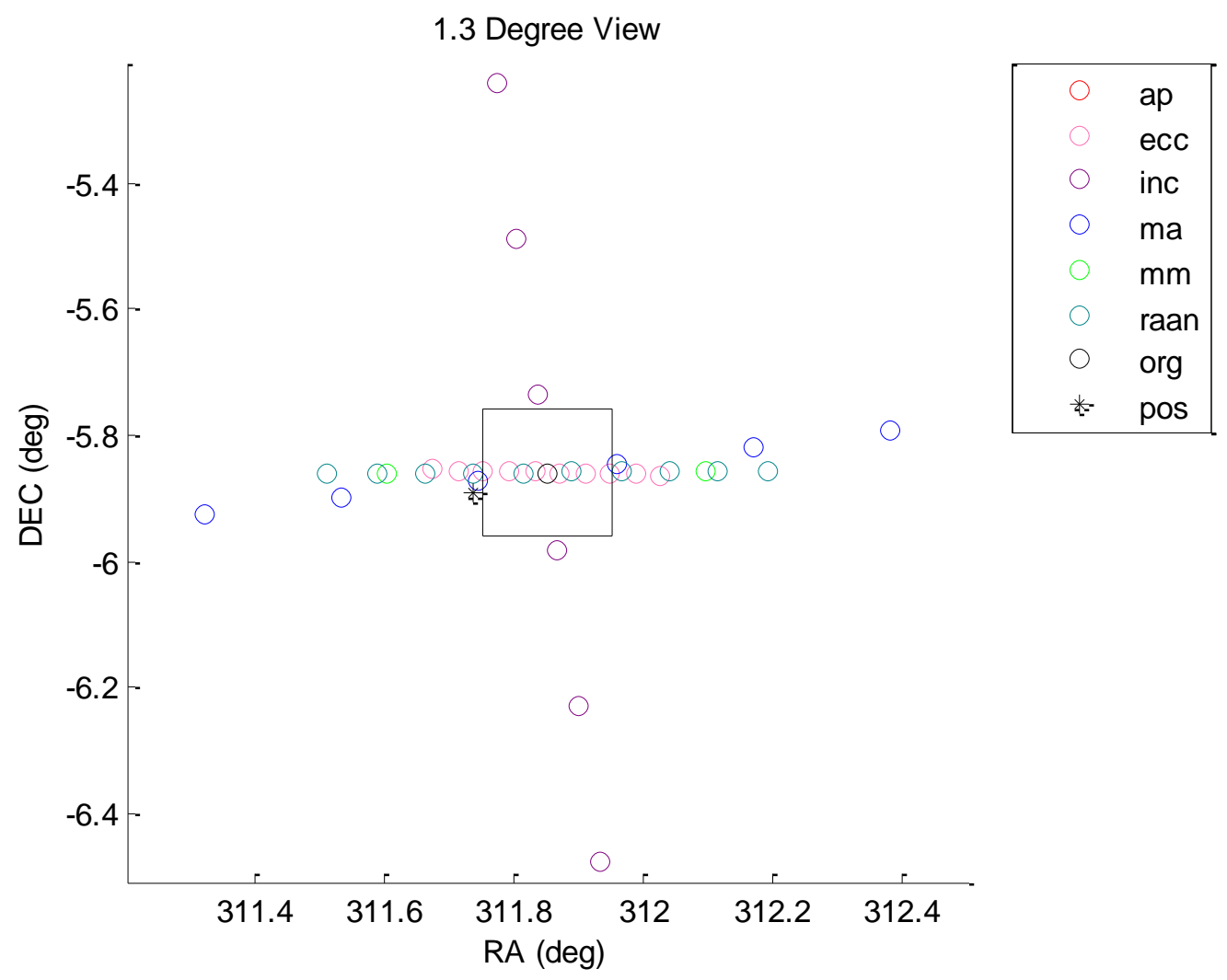




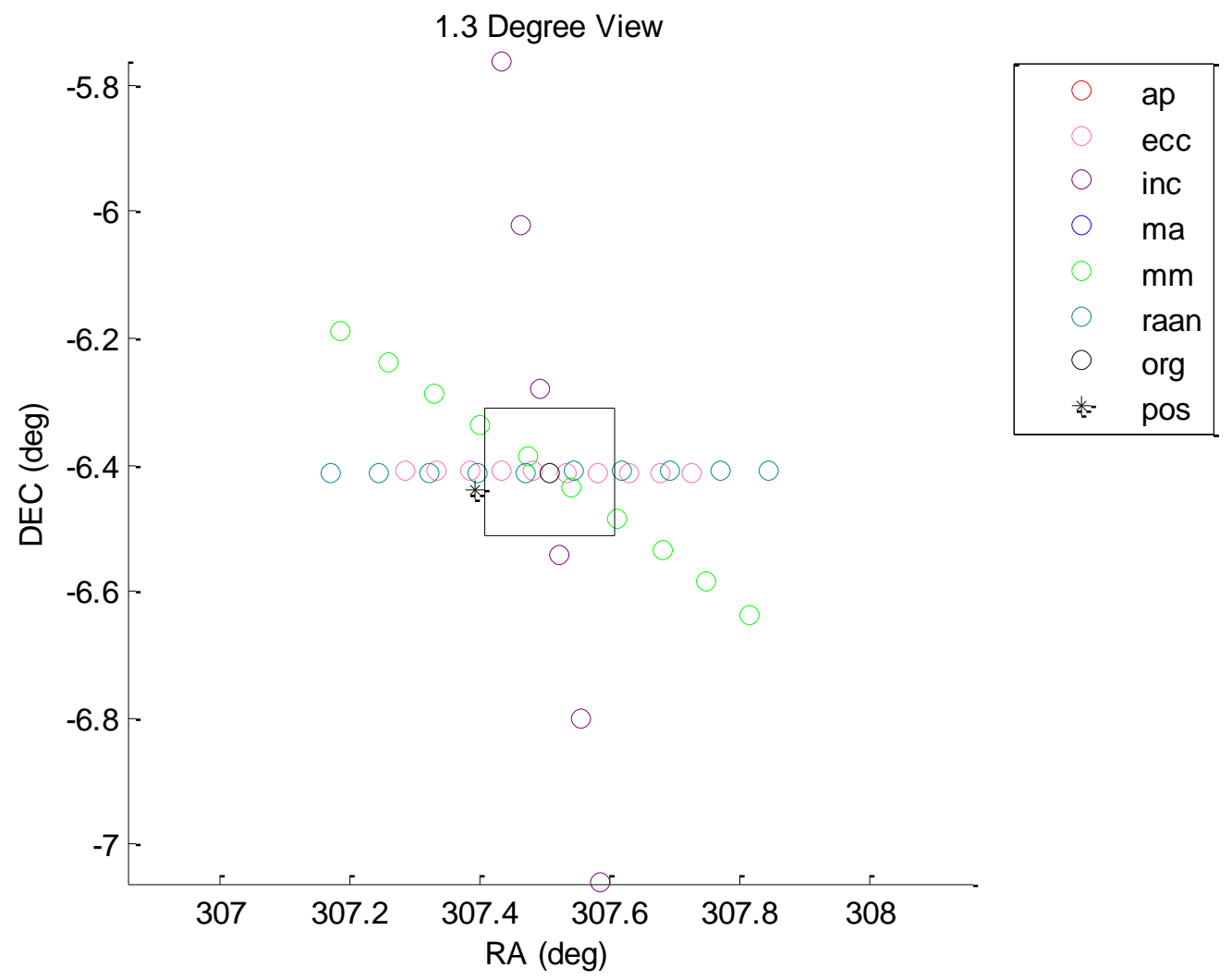




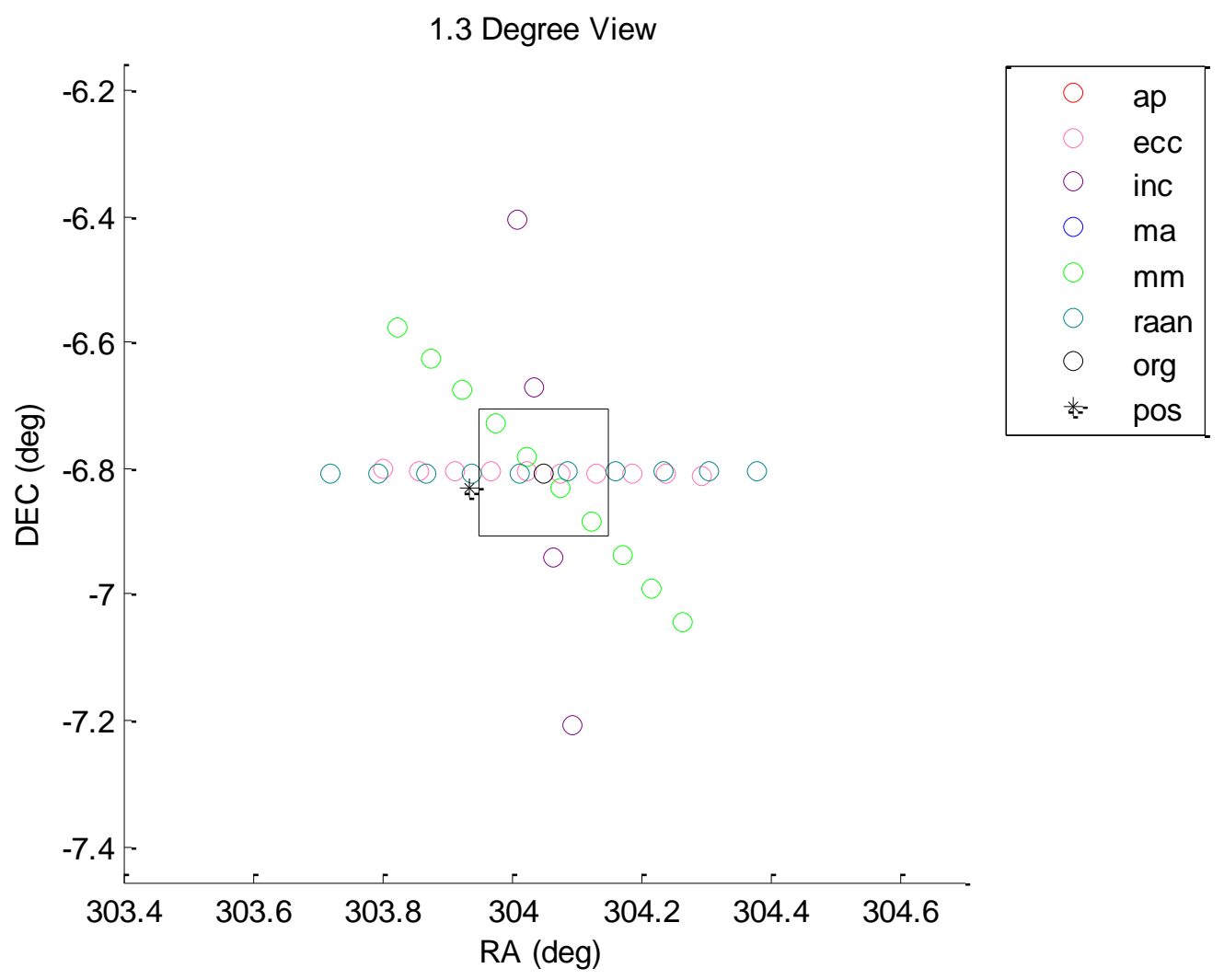




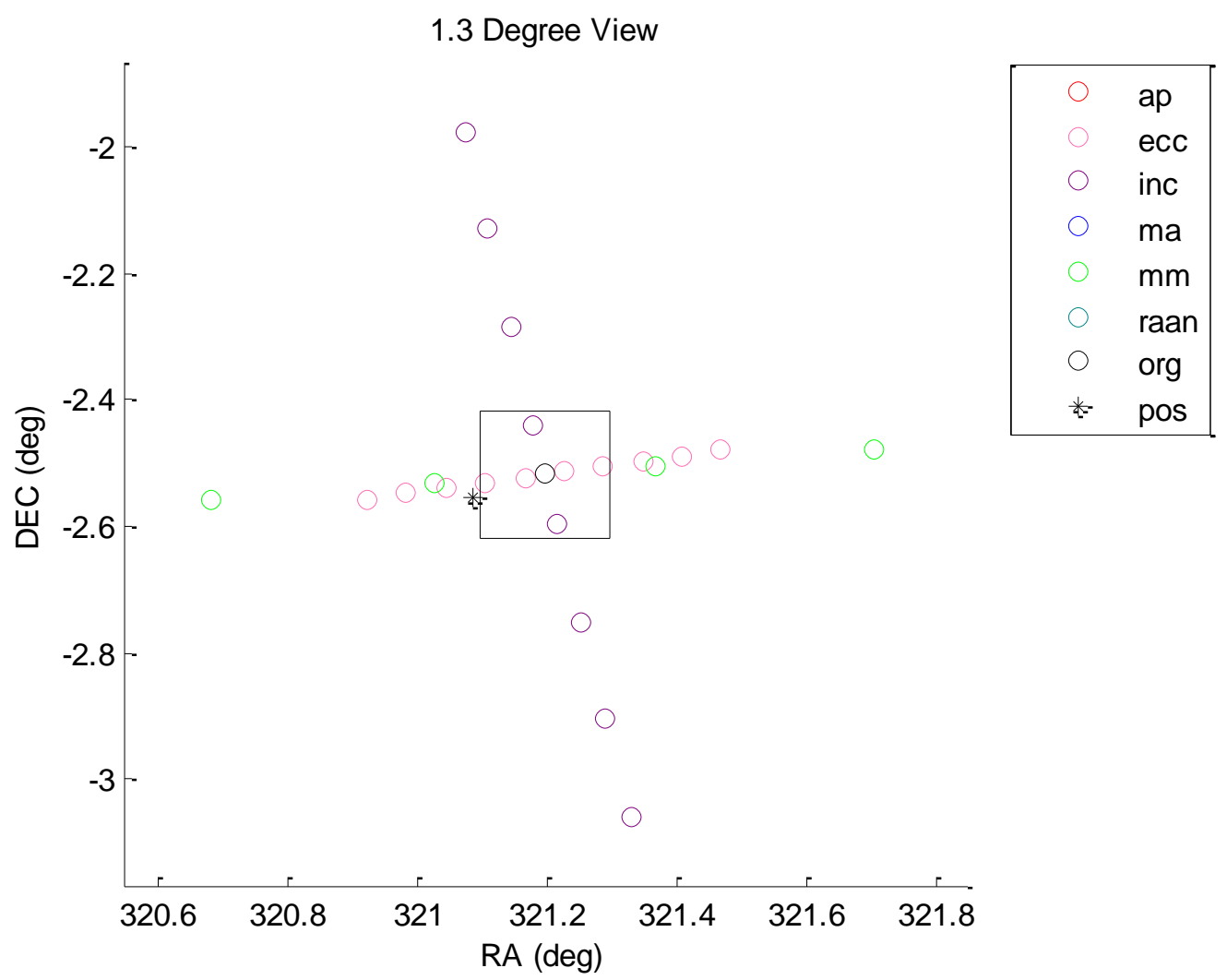


1.3 Degree View

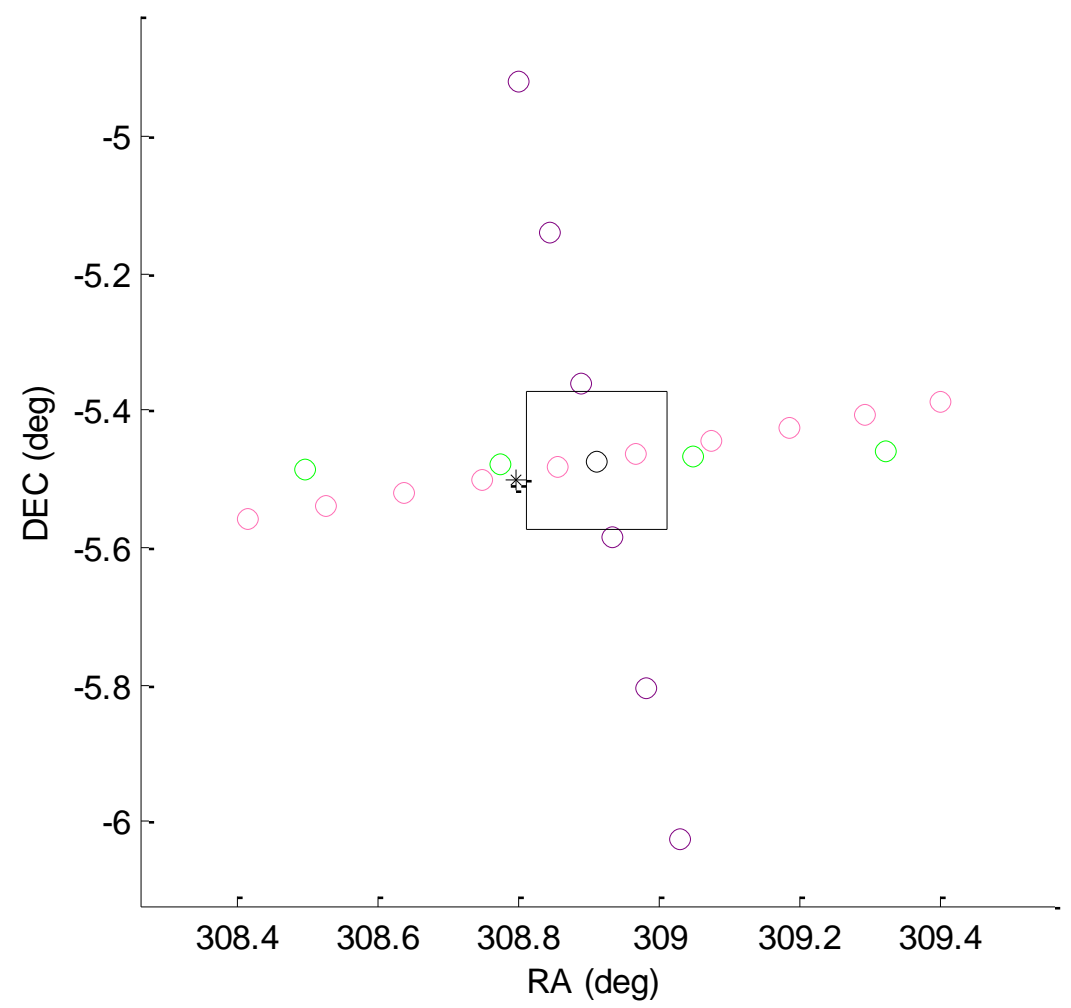

\begin{tabular}{|cl|}
\hline & ap \\
ecc \\
0 & inc \\
ma \\
mm \\
0 \\
raan \\
org \\
*- pos
\end{tabular}




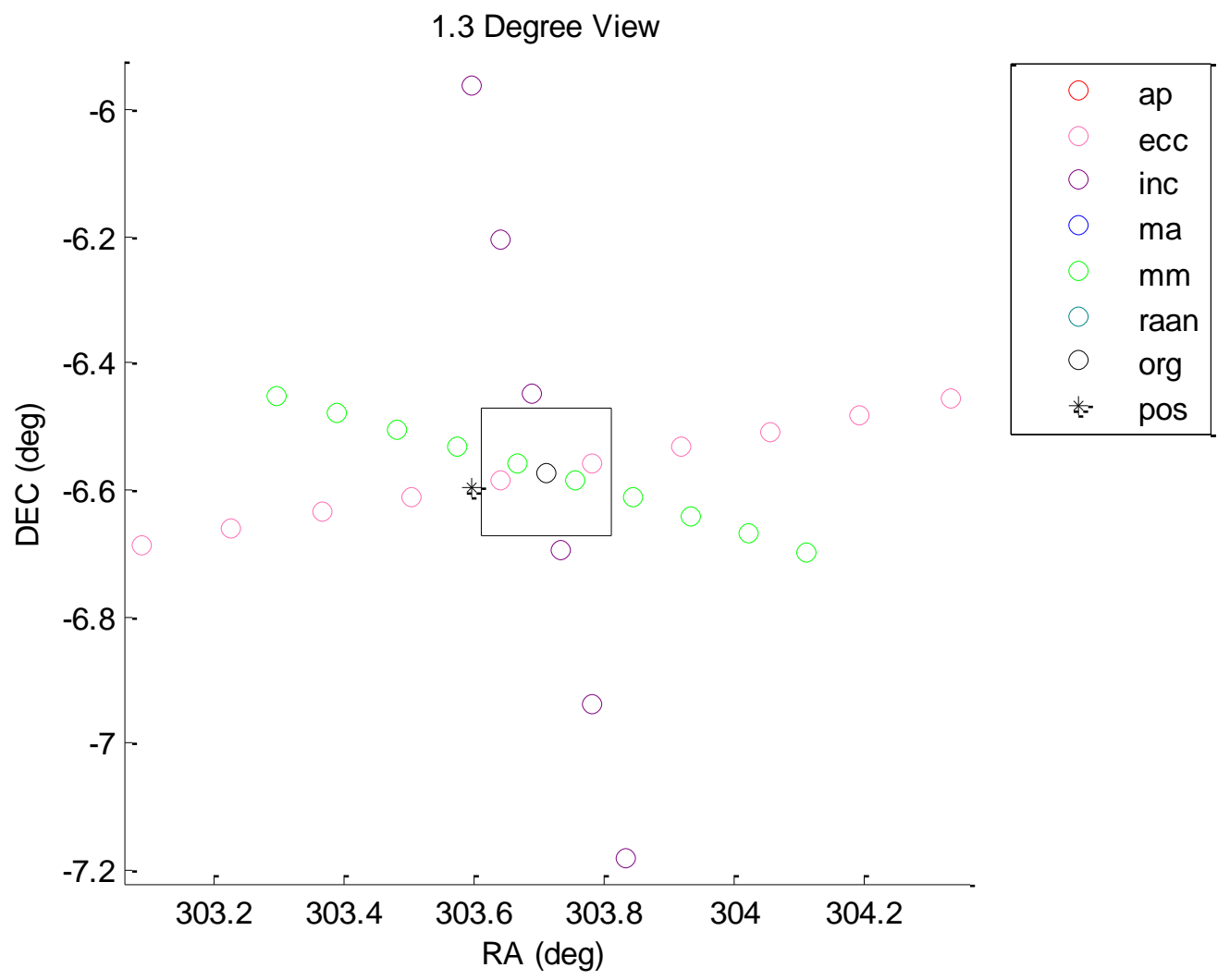




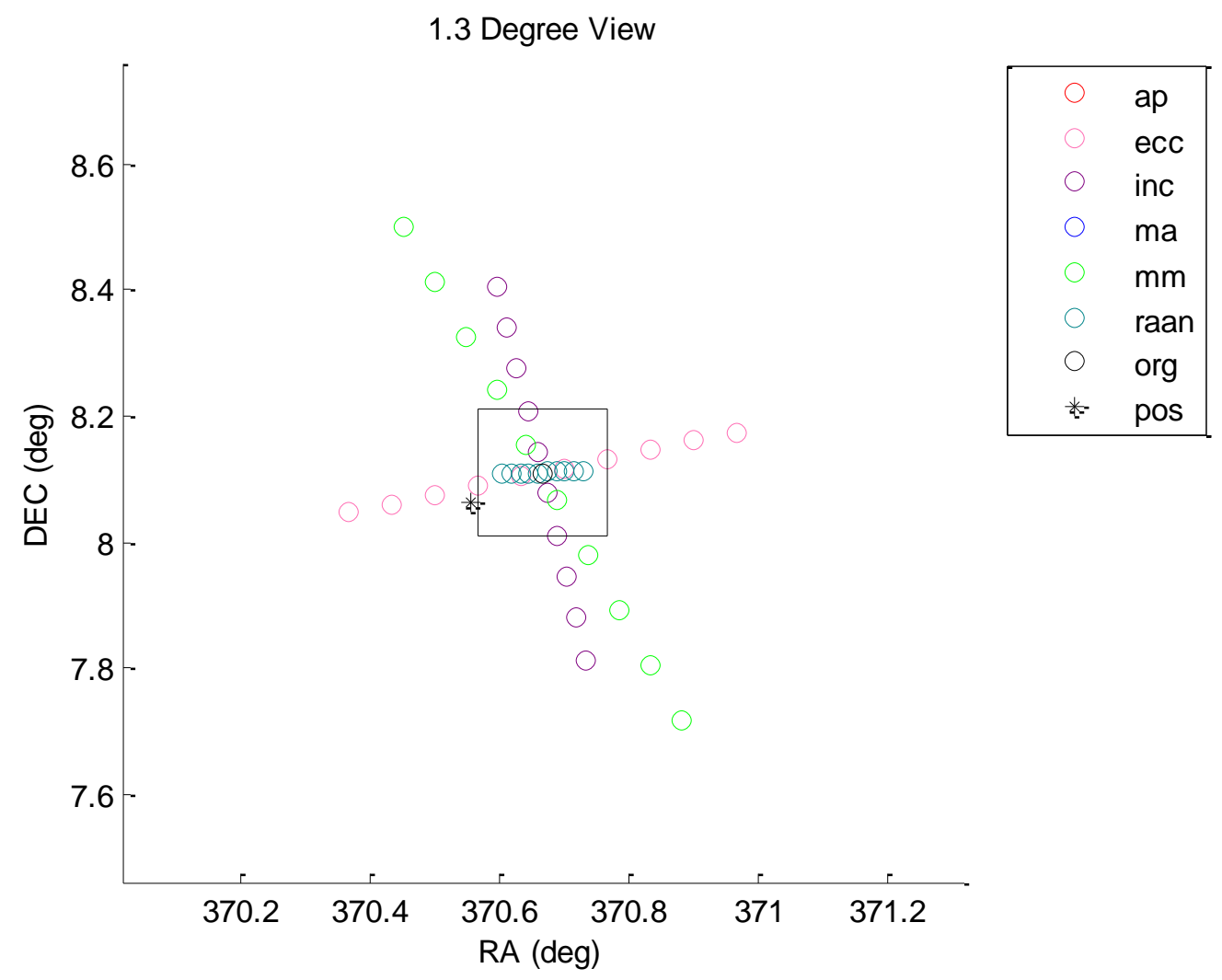


1.3 Degree View

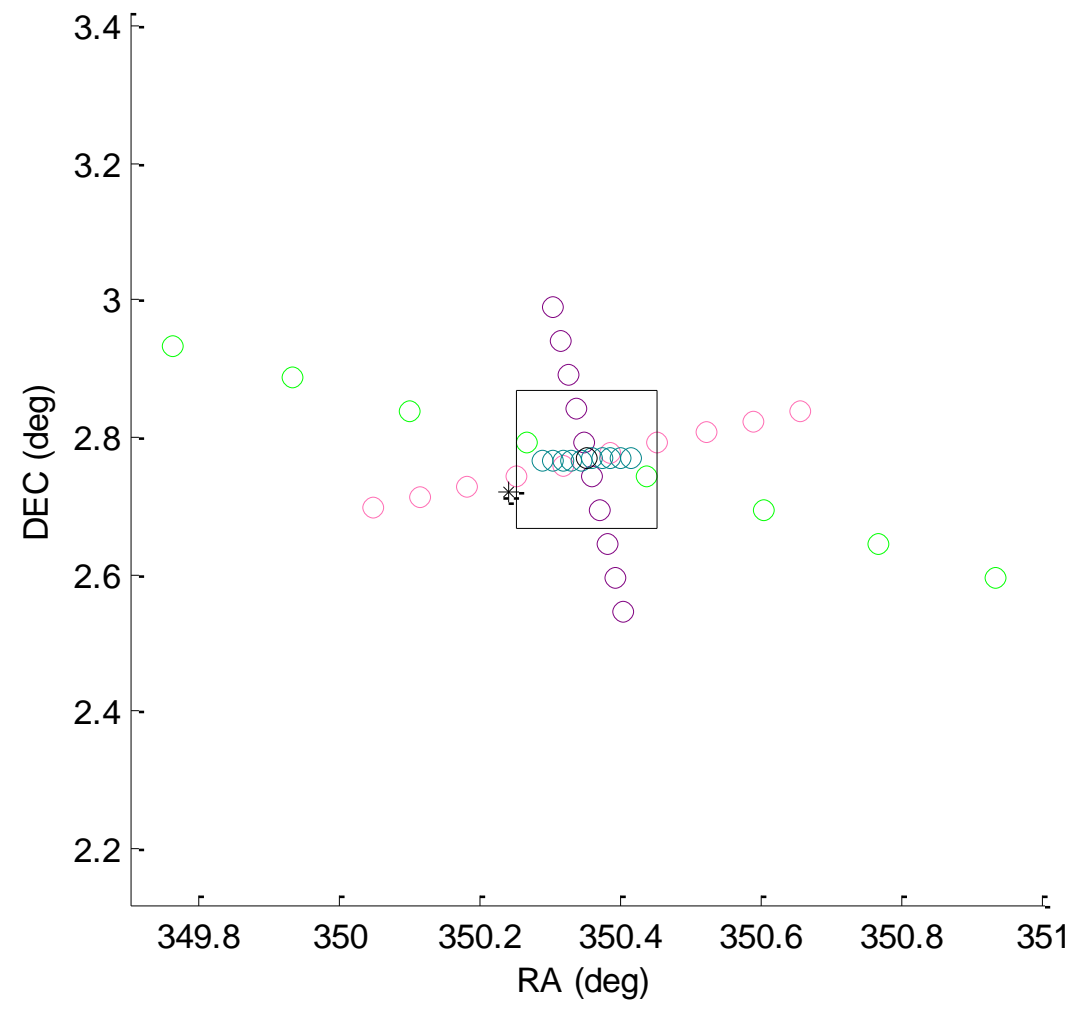

\begin{tabular}{|ll|}
\hline ap \\
ecc \\
inc \\
ma \\
mm \\
raan \\
org \\
* pos \\
\hline
\end{tabular}




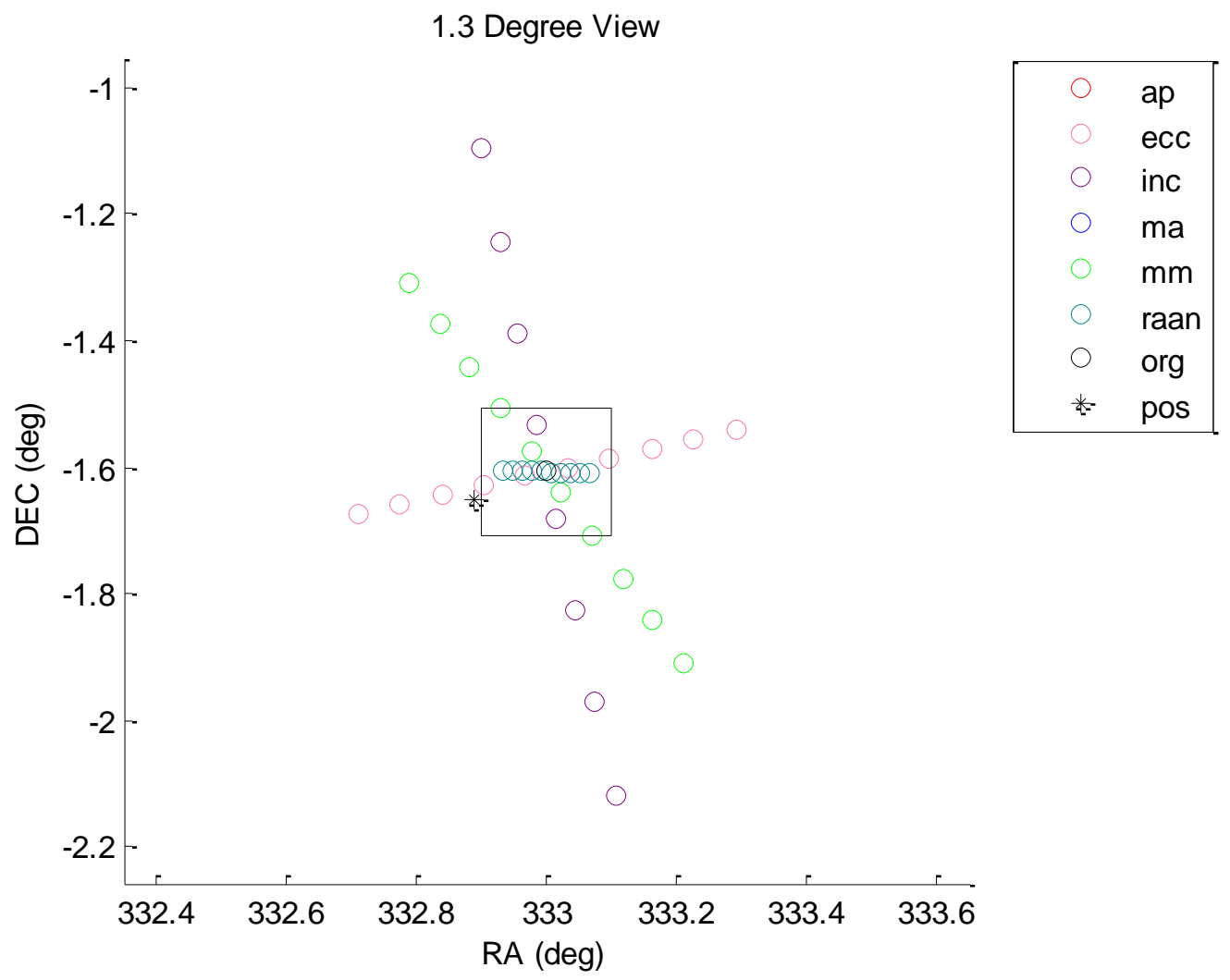


1.3 Degree View

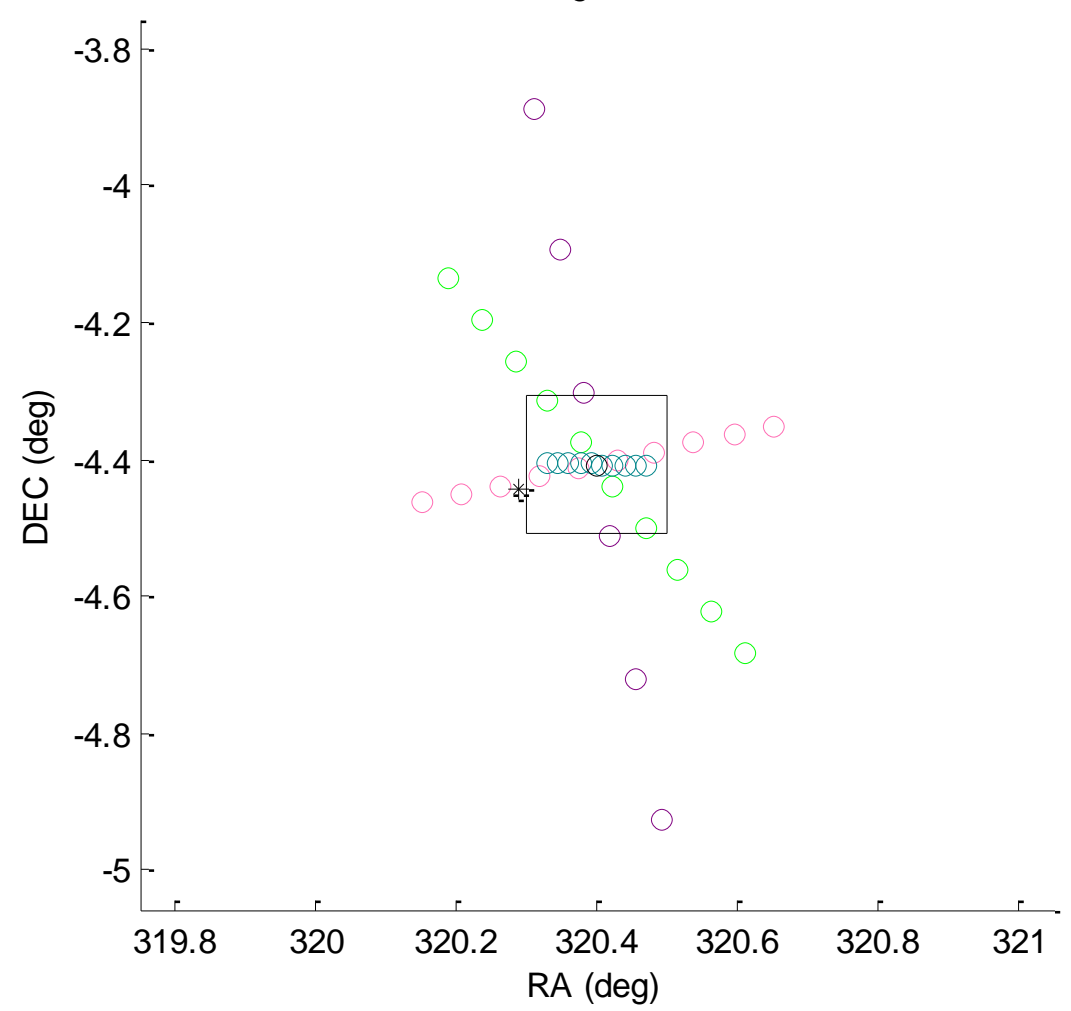

\begin{tabular}{|ll|}
\hline & ap \\
ecc \\
inc \\
ma \\
mm \\
raan \\
org \\
* pos \\
\hline
\end{tabular}


1.3 Degree View

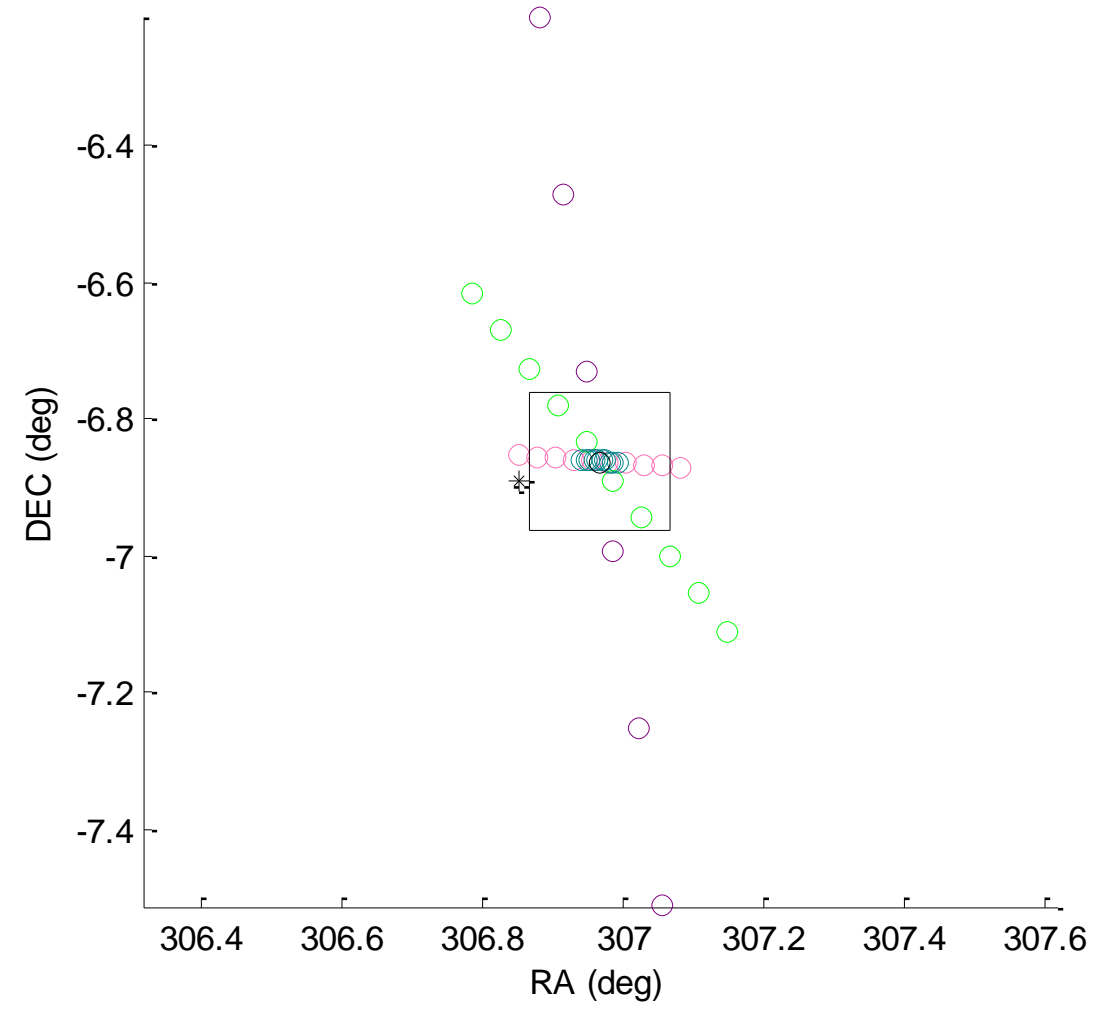

\begin{tabular}{|ll|}
\hline ap \\
ecc \\
inc \\
ma \\
mm \\
raan \\
org \\
* pos \\
\hline
\end{tabular}




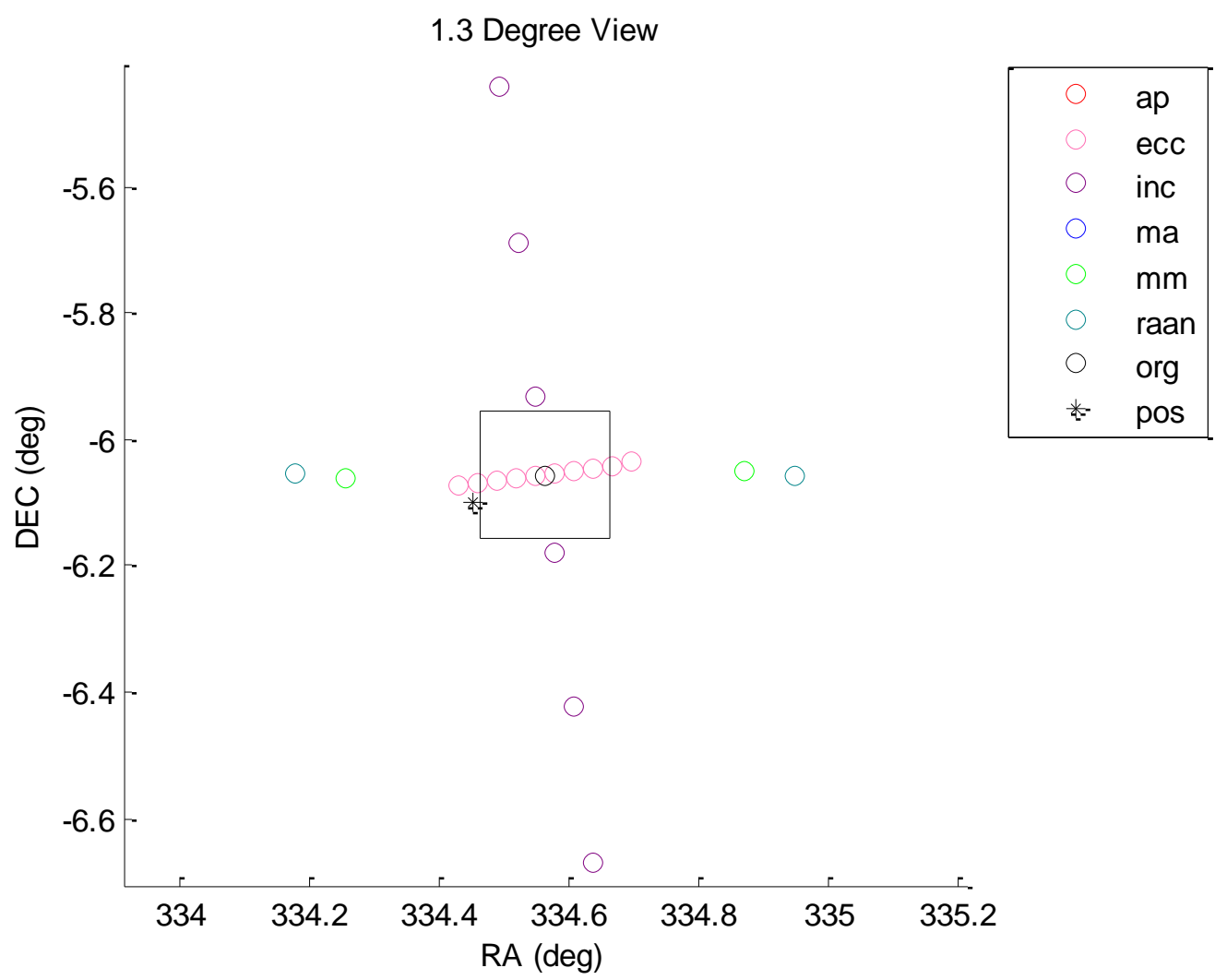




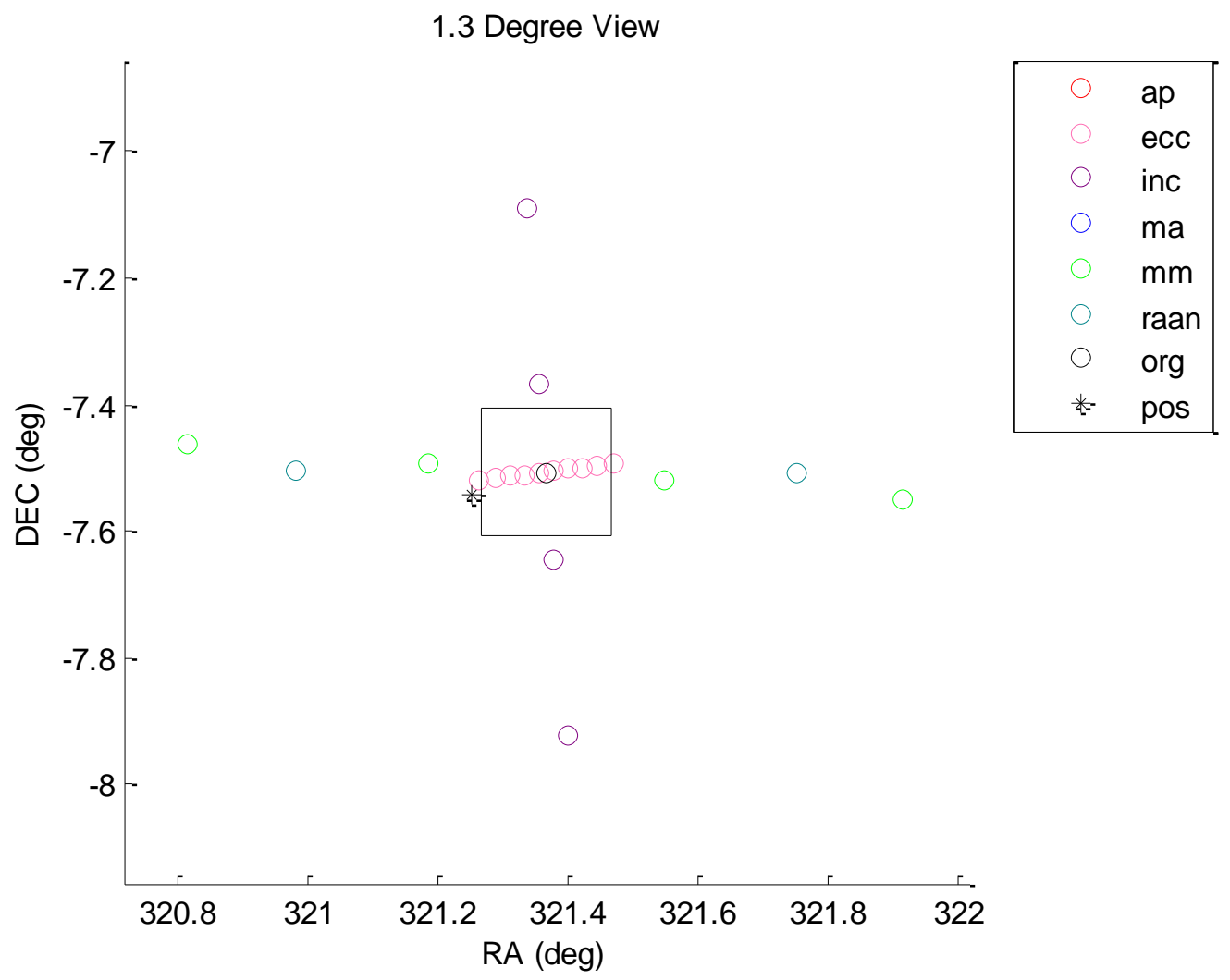




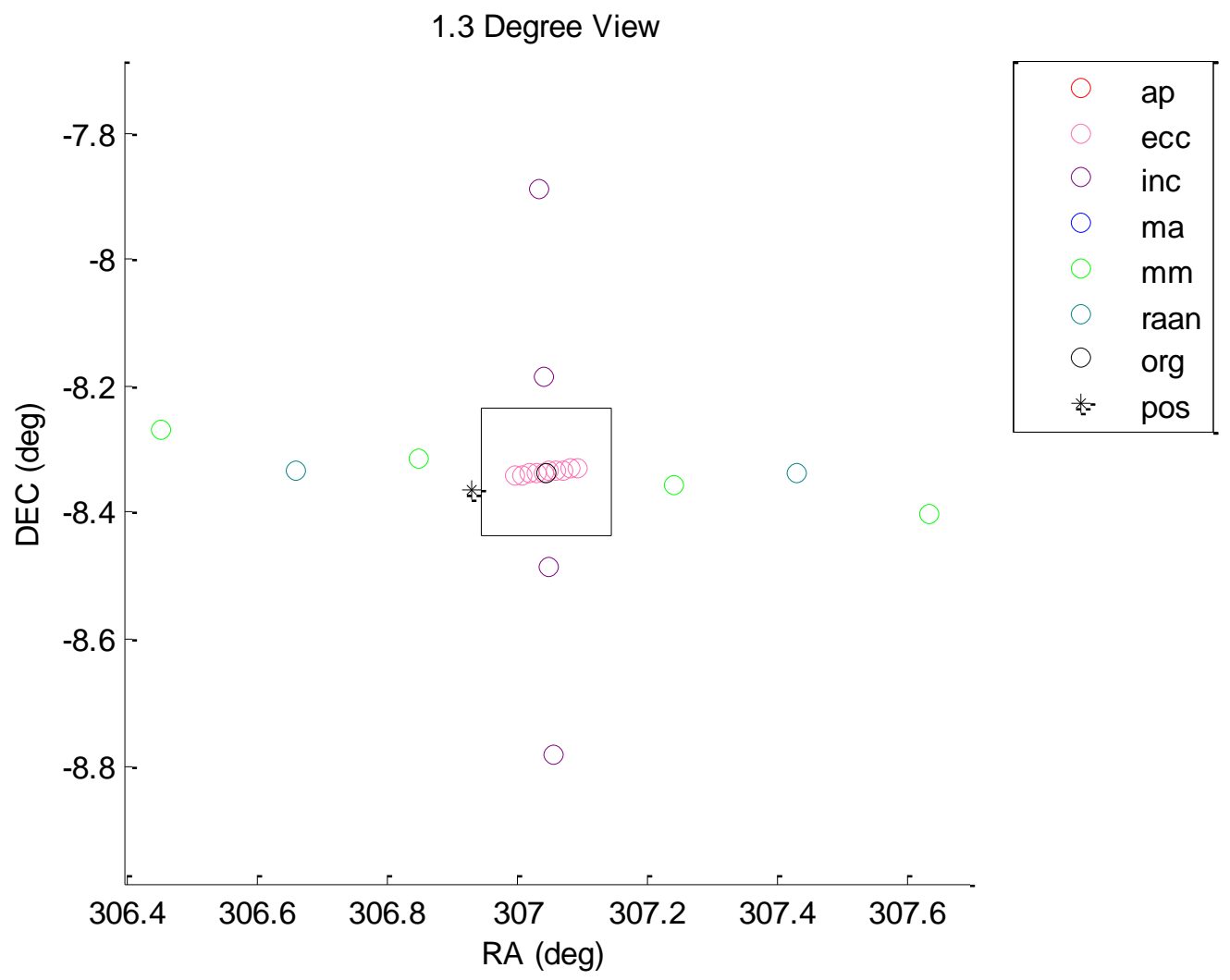


1.3 Degree View

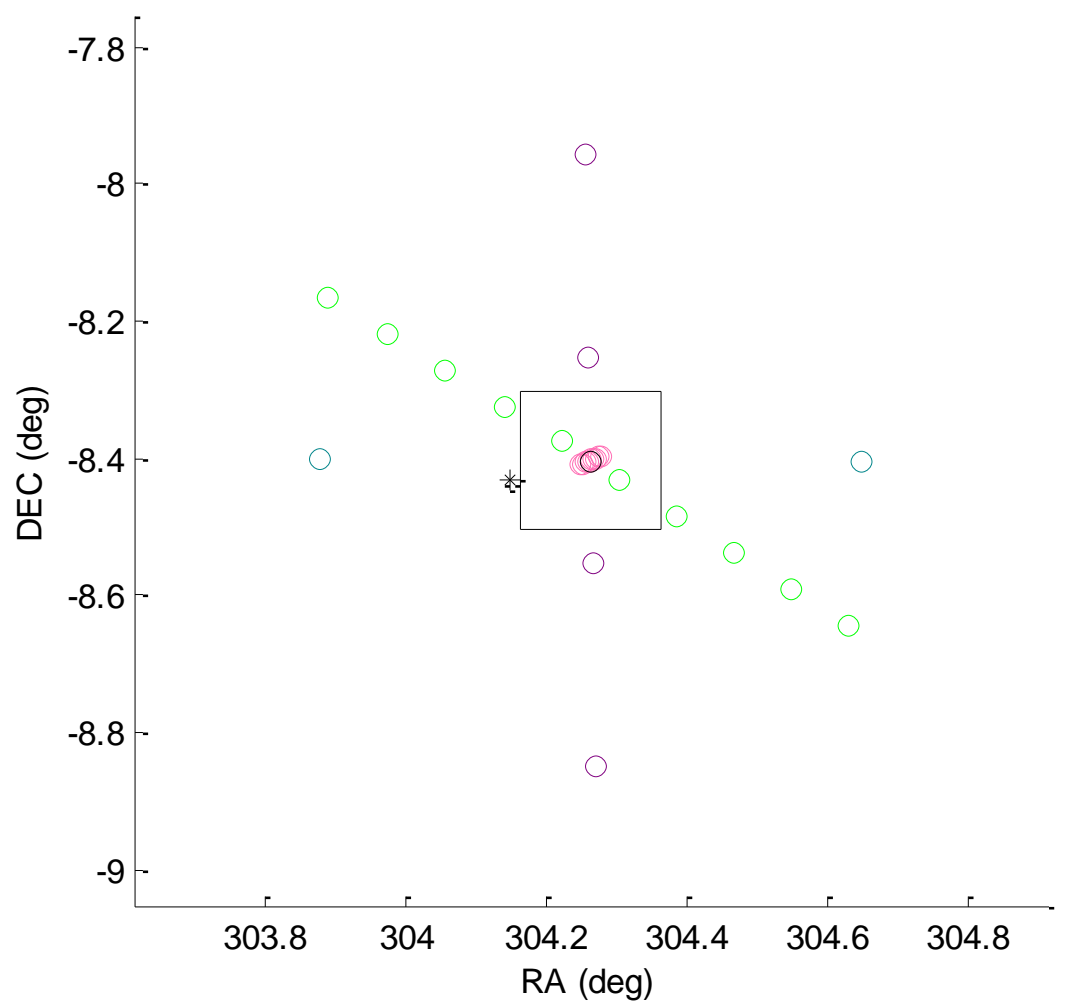

\begin{tabular}{|ll|}
\hline ap \\
ecc \\
inc \\
ma \\
mm \\
raan \\
org \\
* pos \\
\hline
\end{tabular}




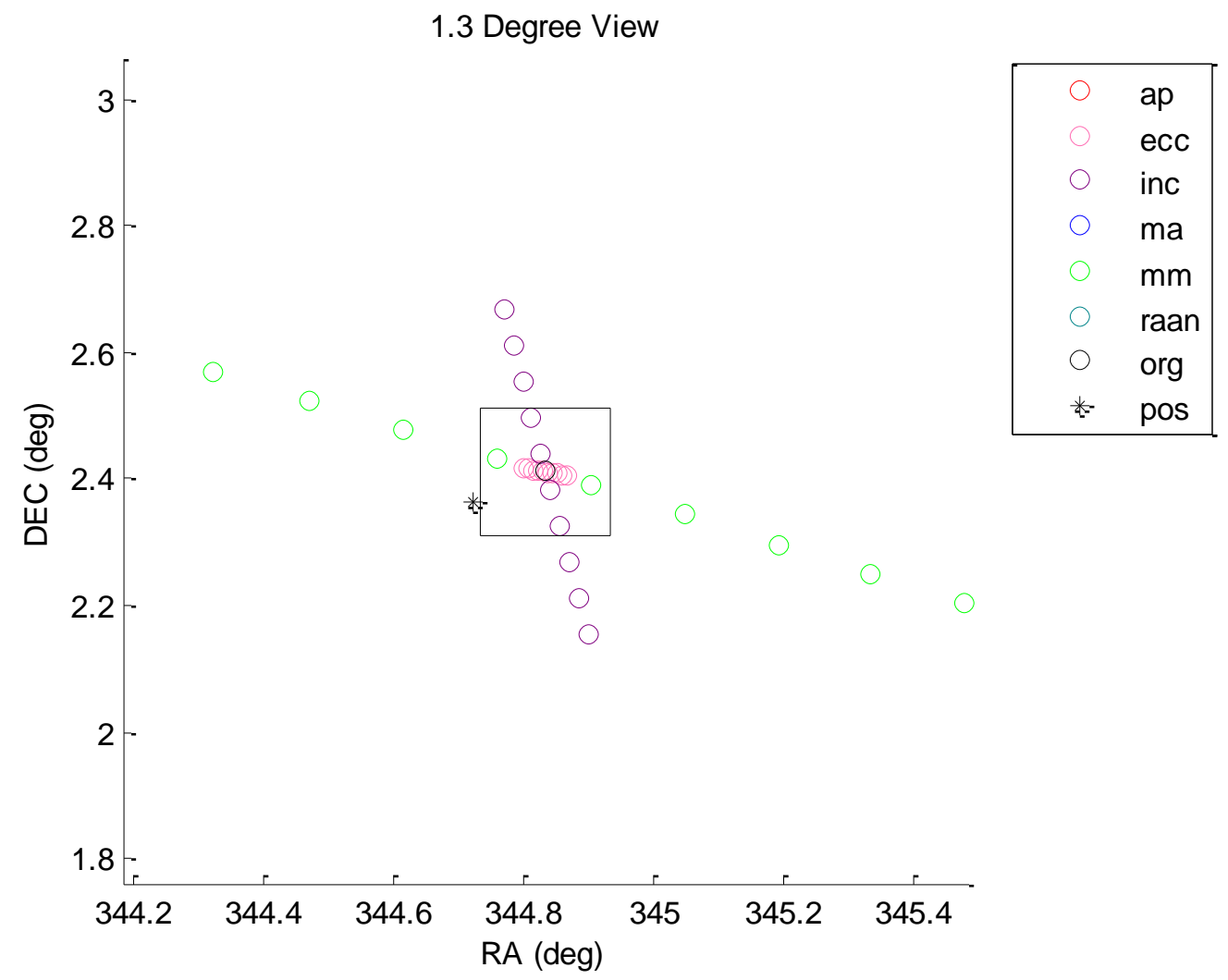


1.3 Degree View

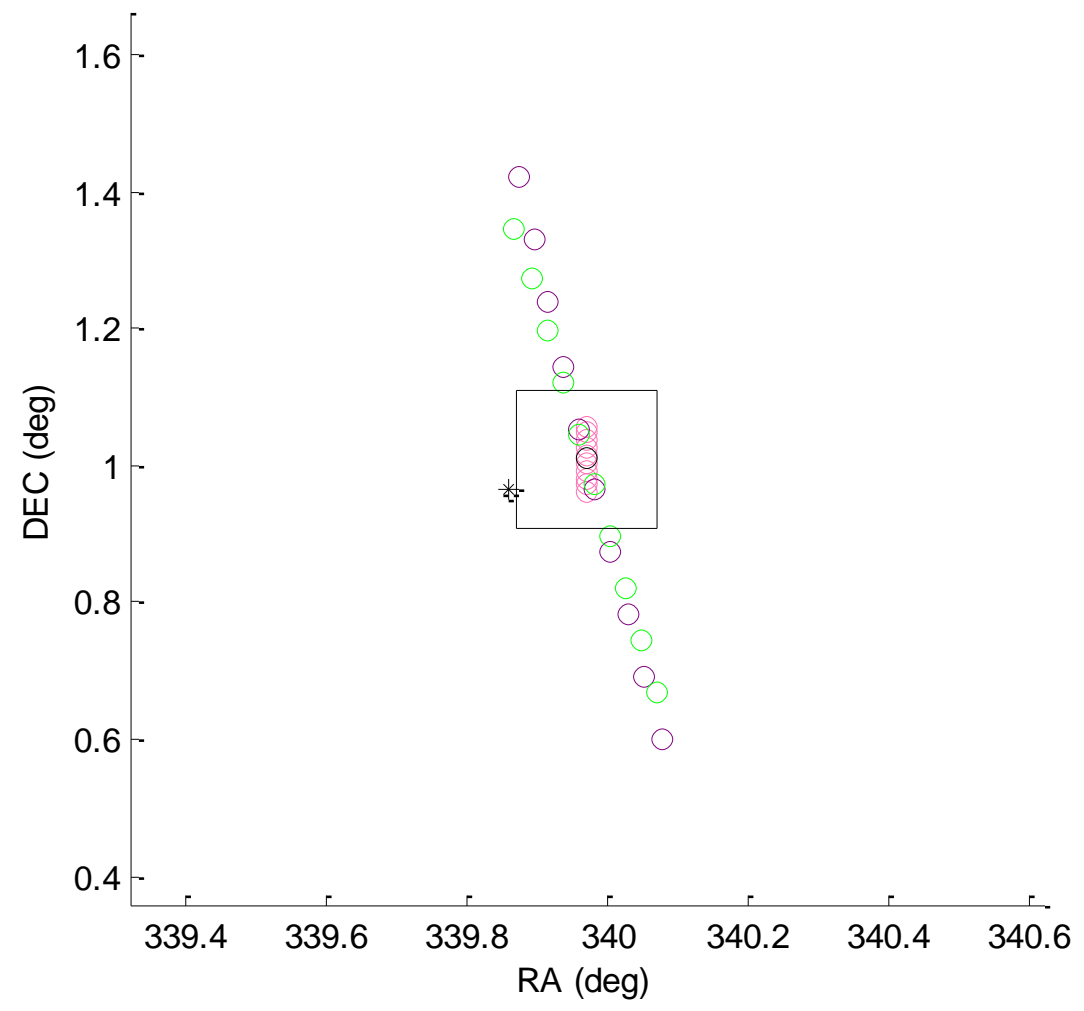

\begin{tabular}{|ll|}
\hline ap \\
ecc \\
inc \\
ma \\
mm \\
raan \\
org \\
* pos \\
\hline
\end{tabular}




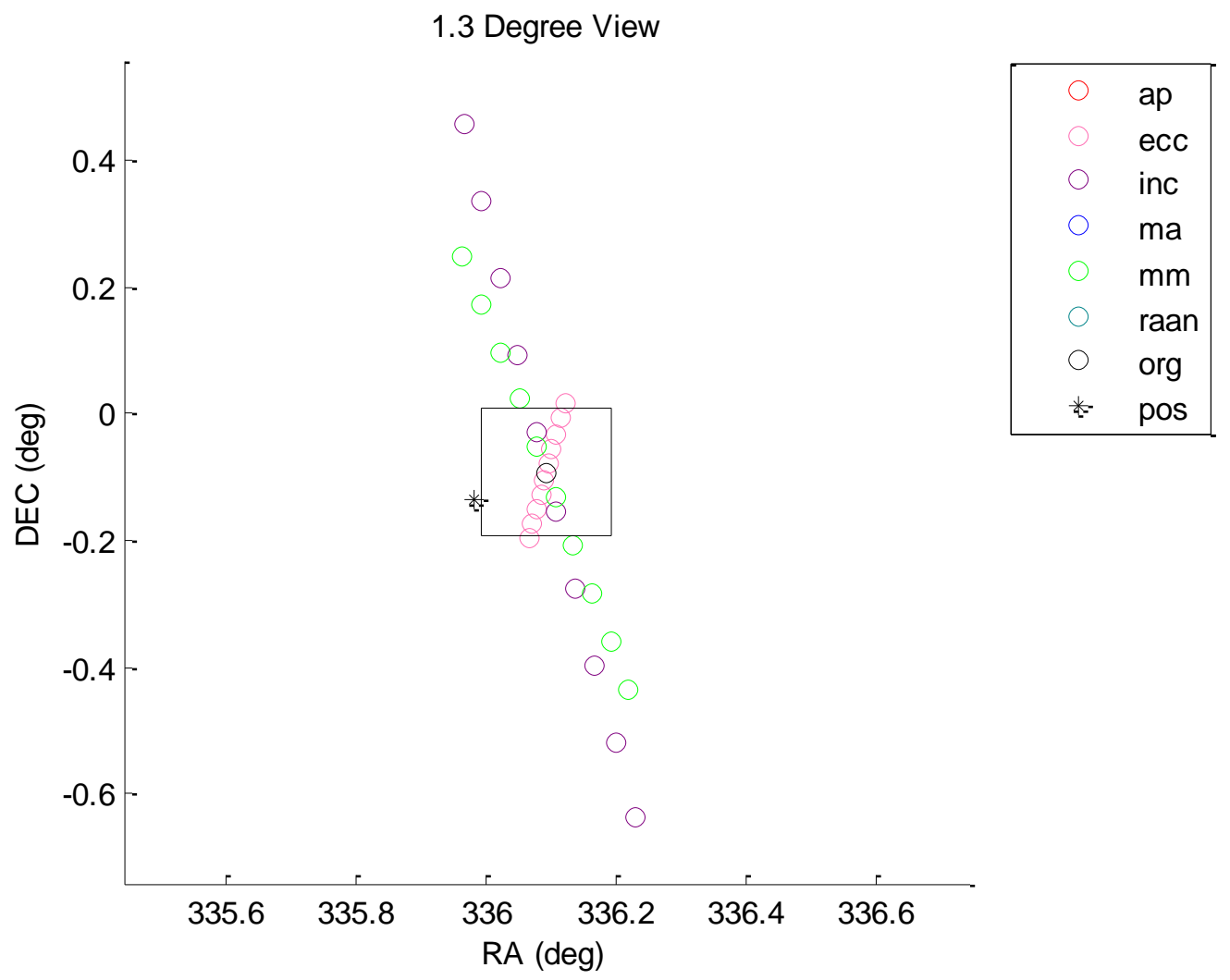




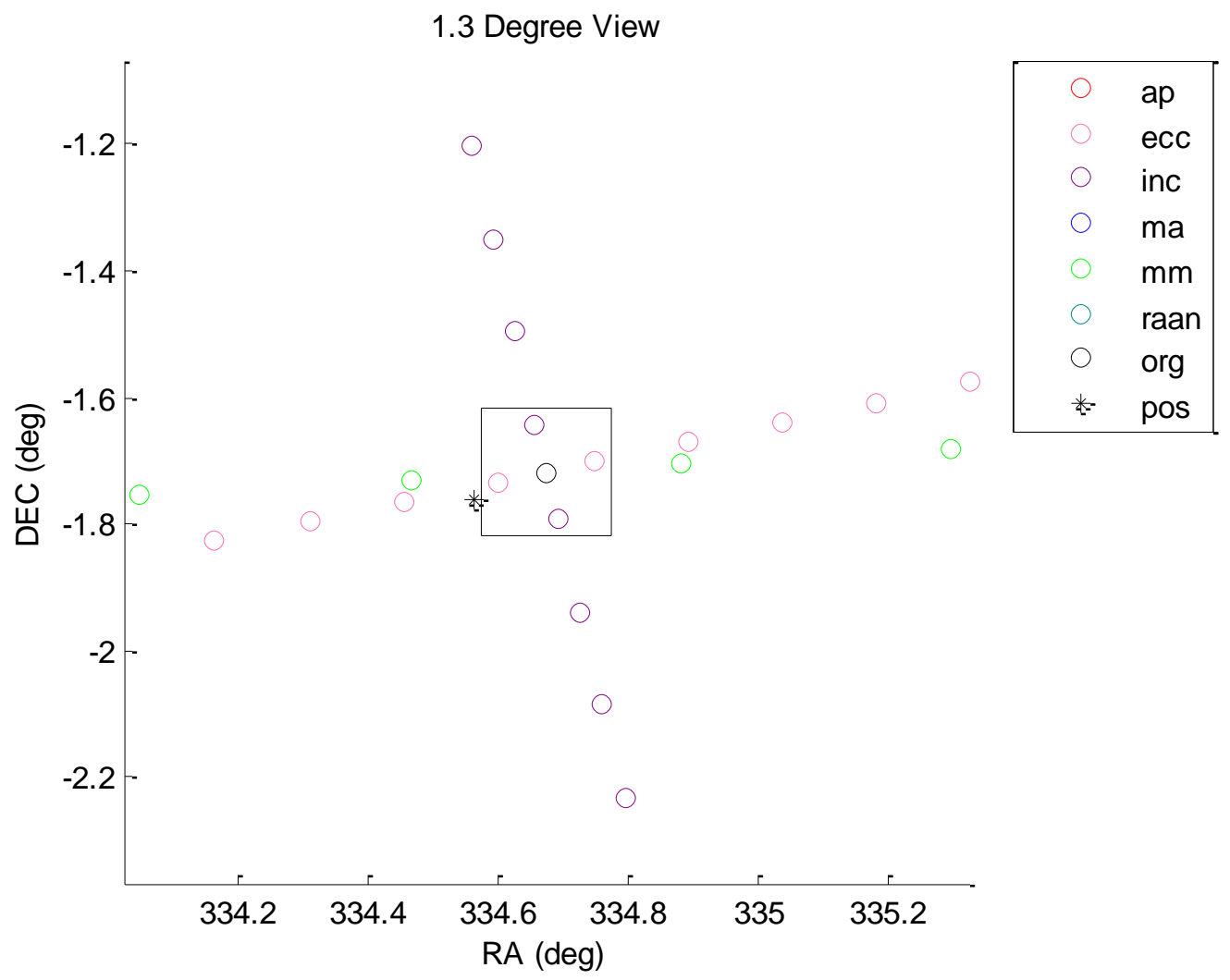




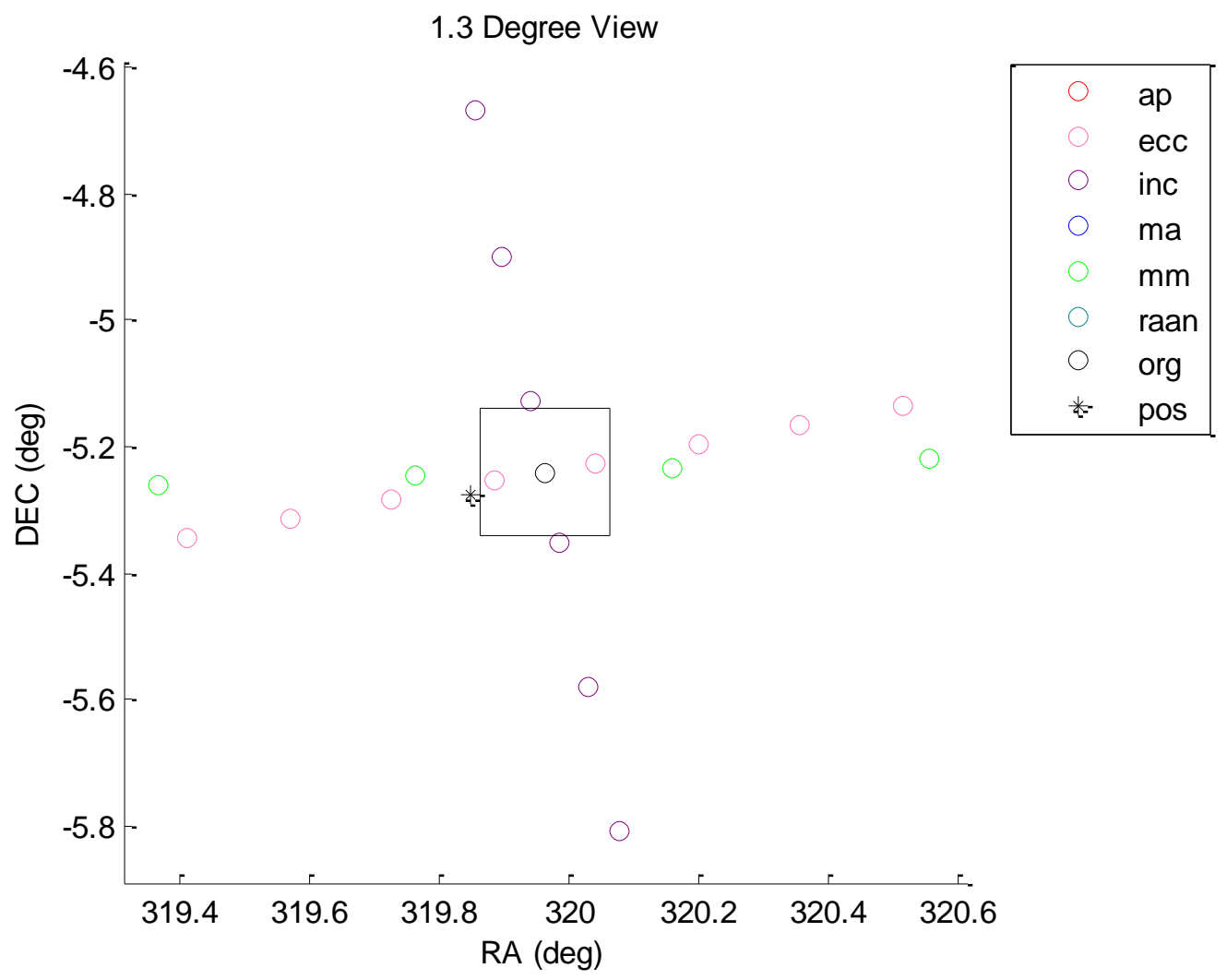




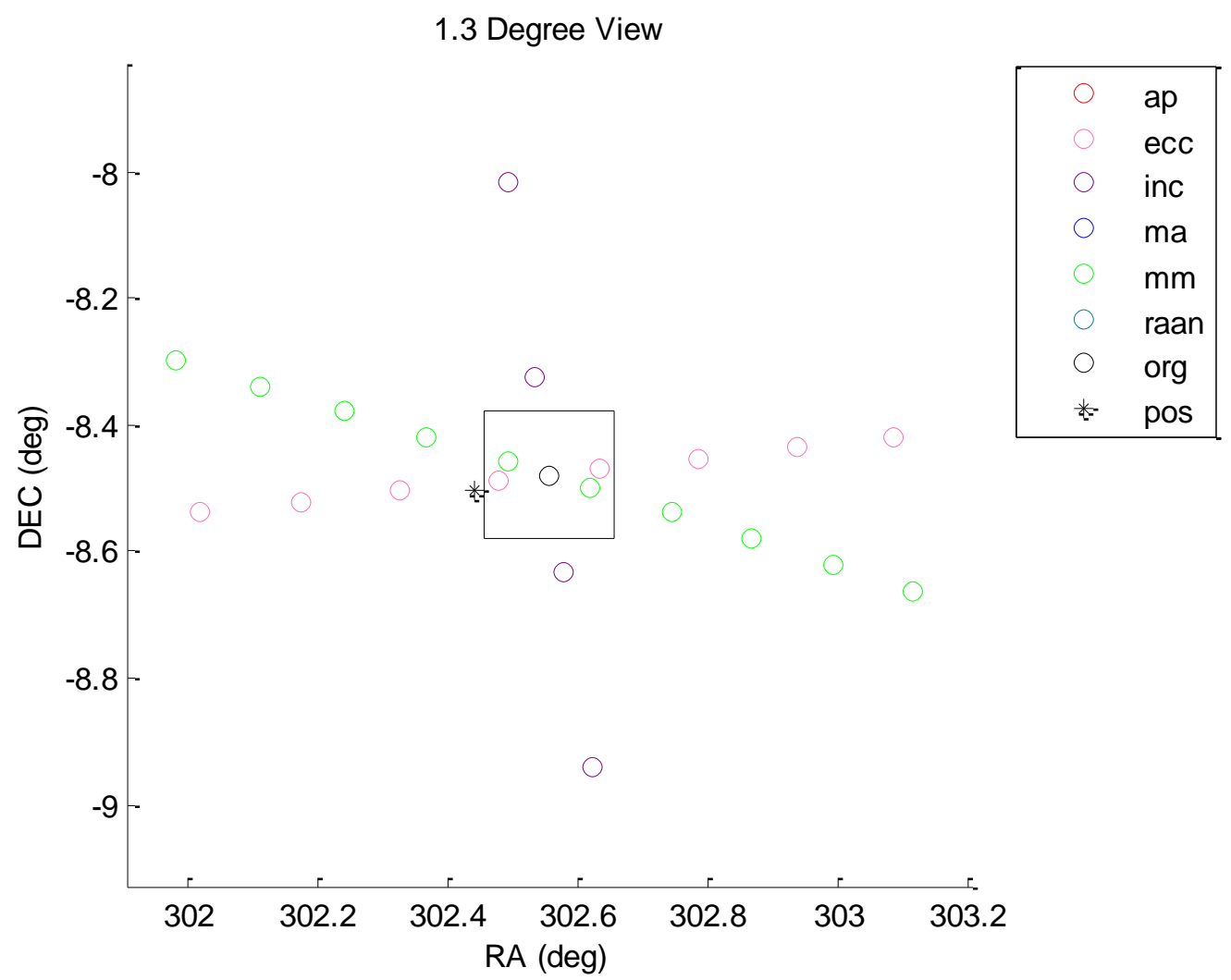




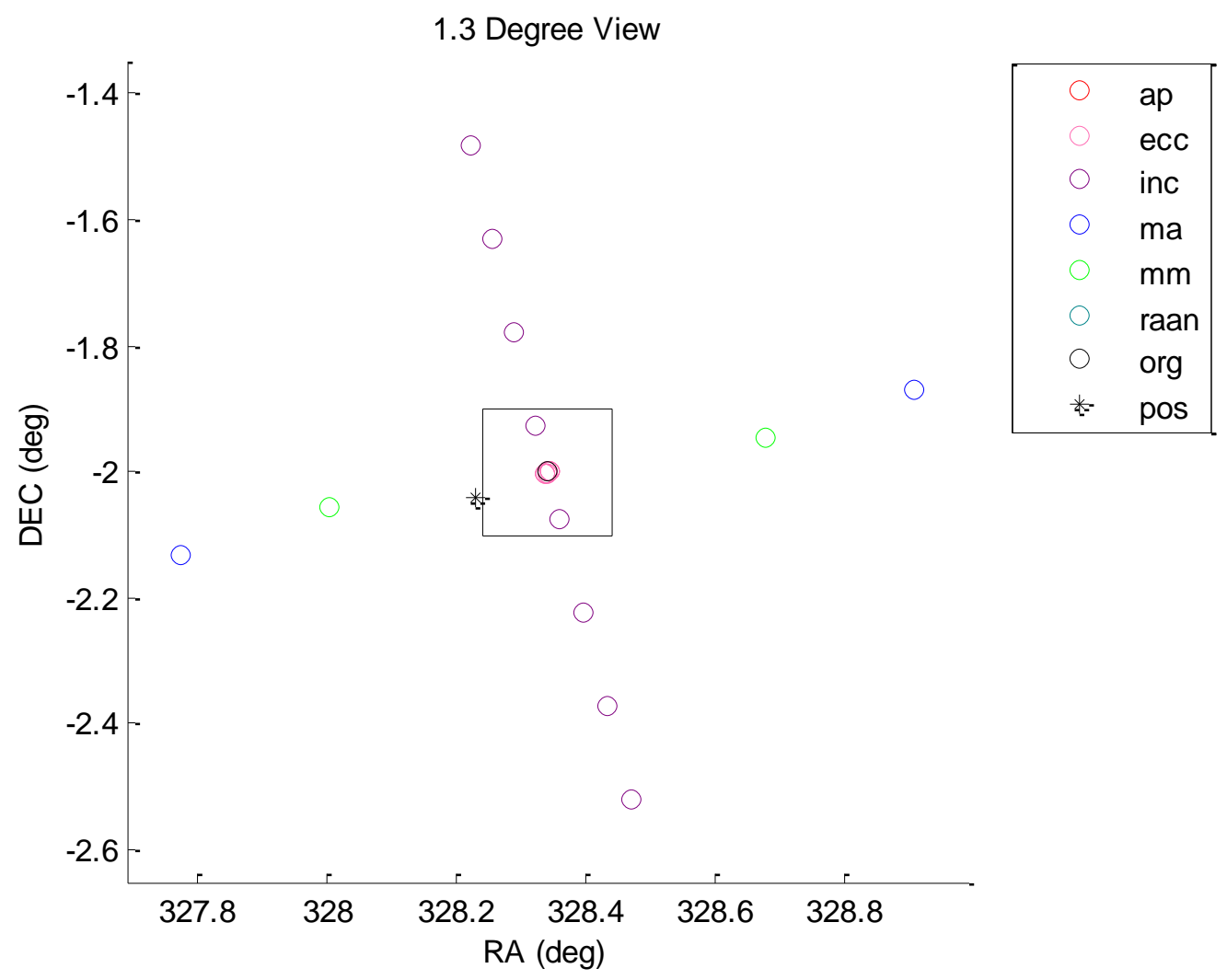




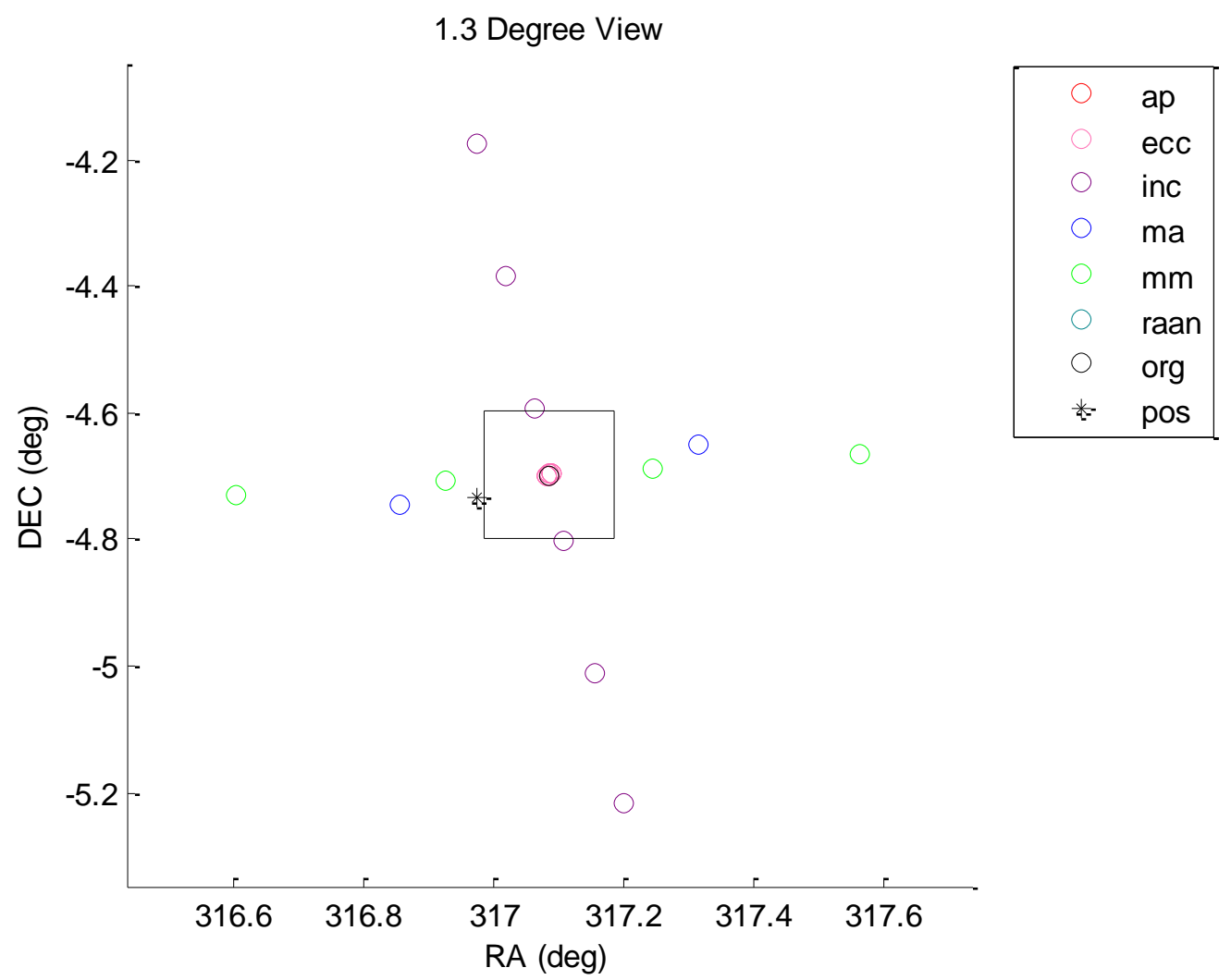




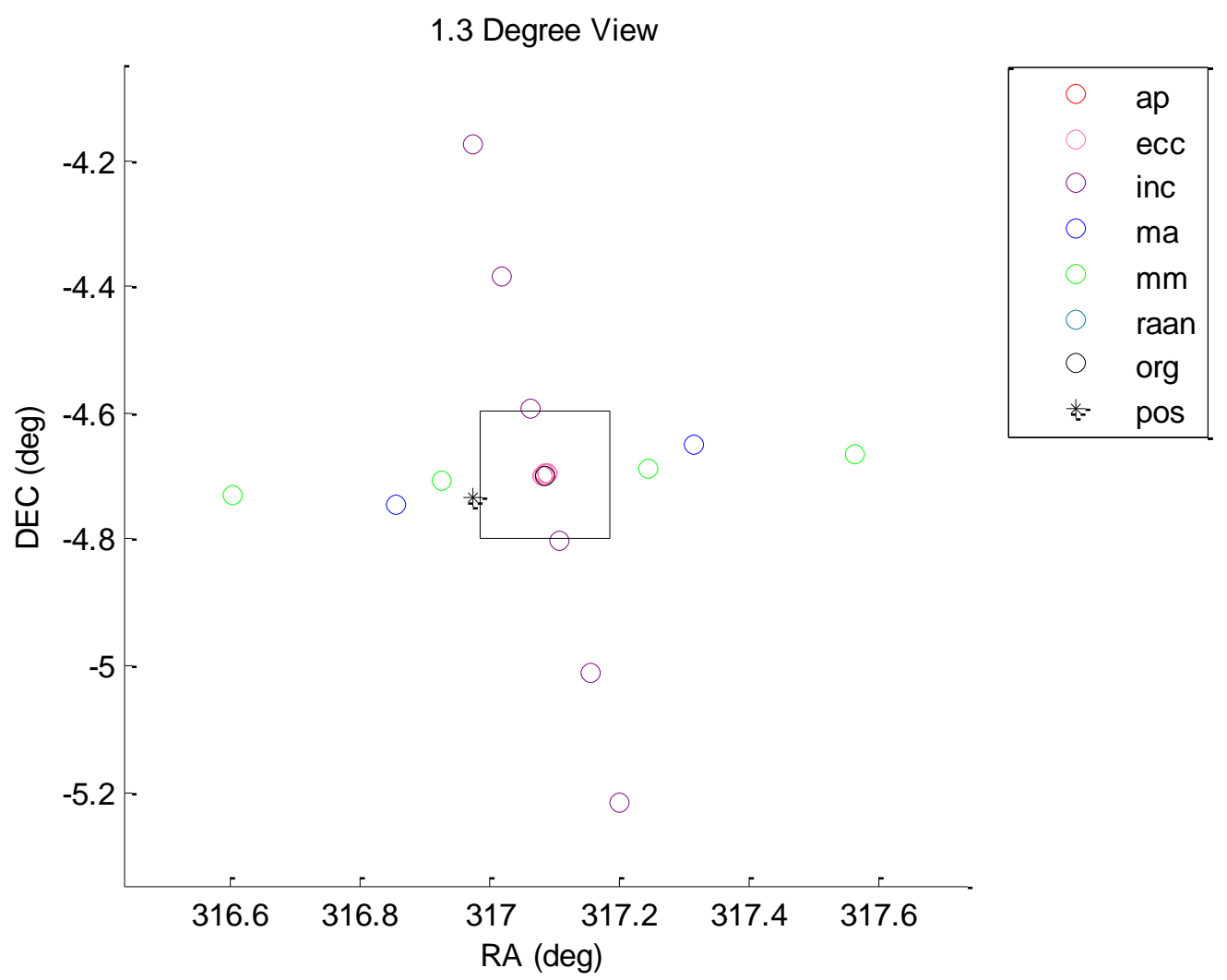




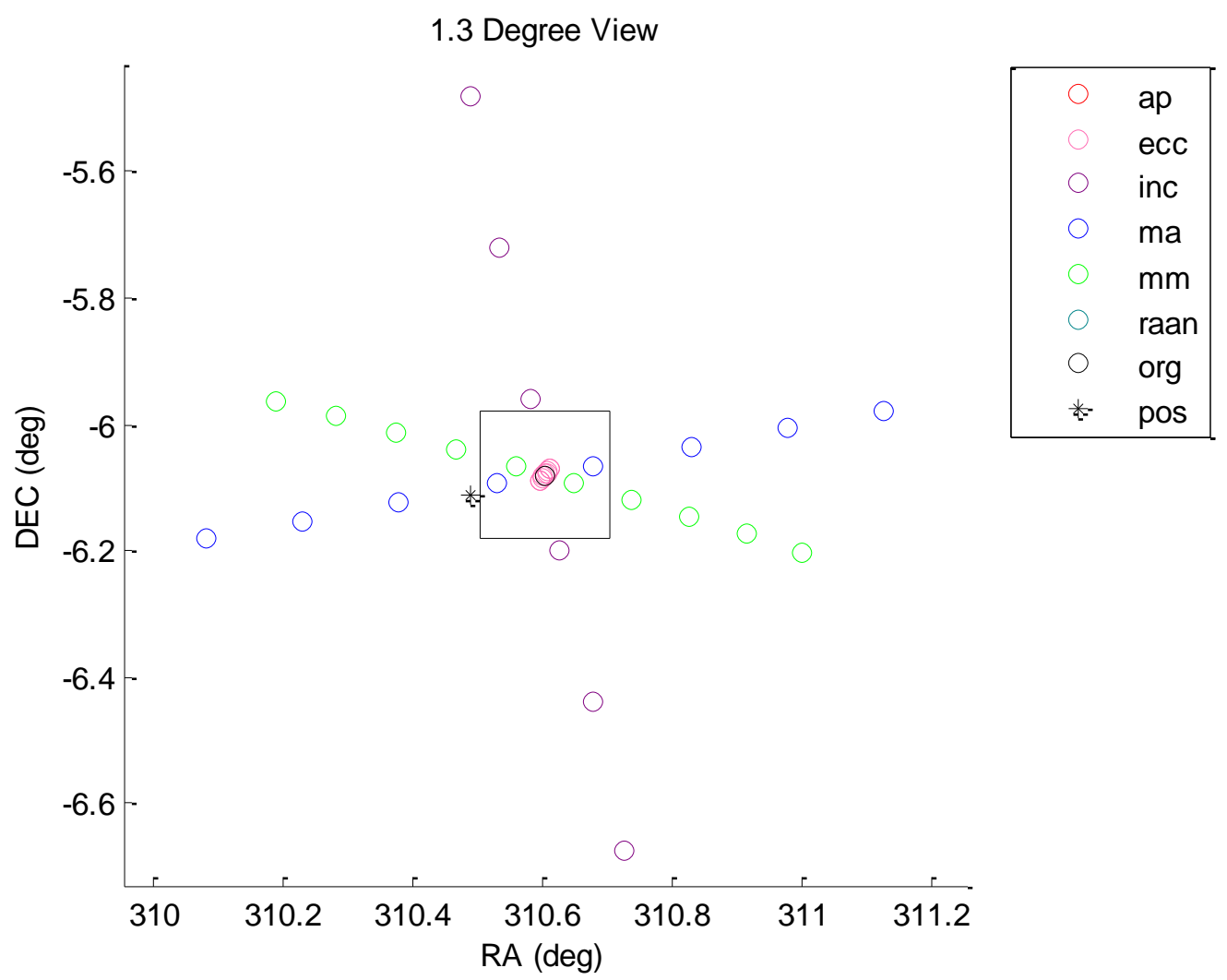




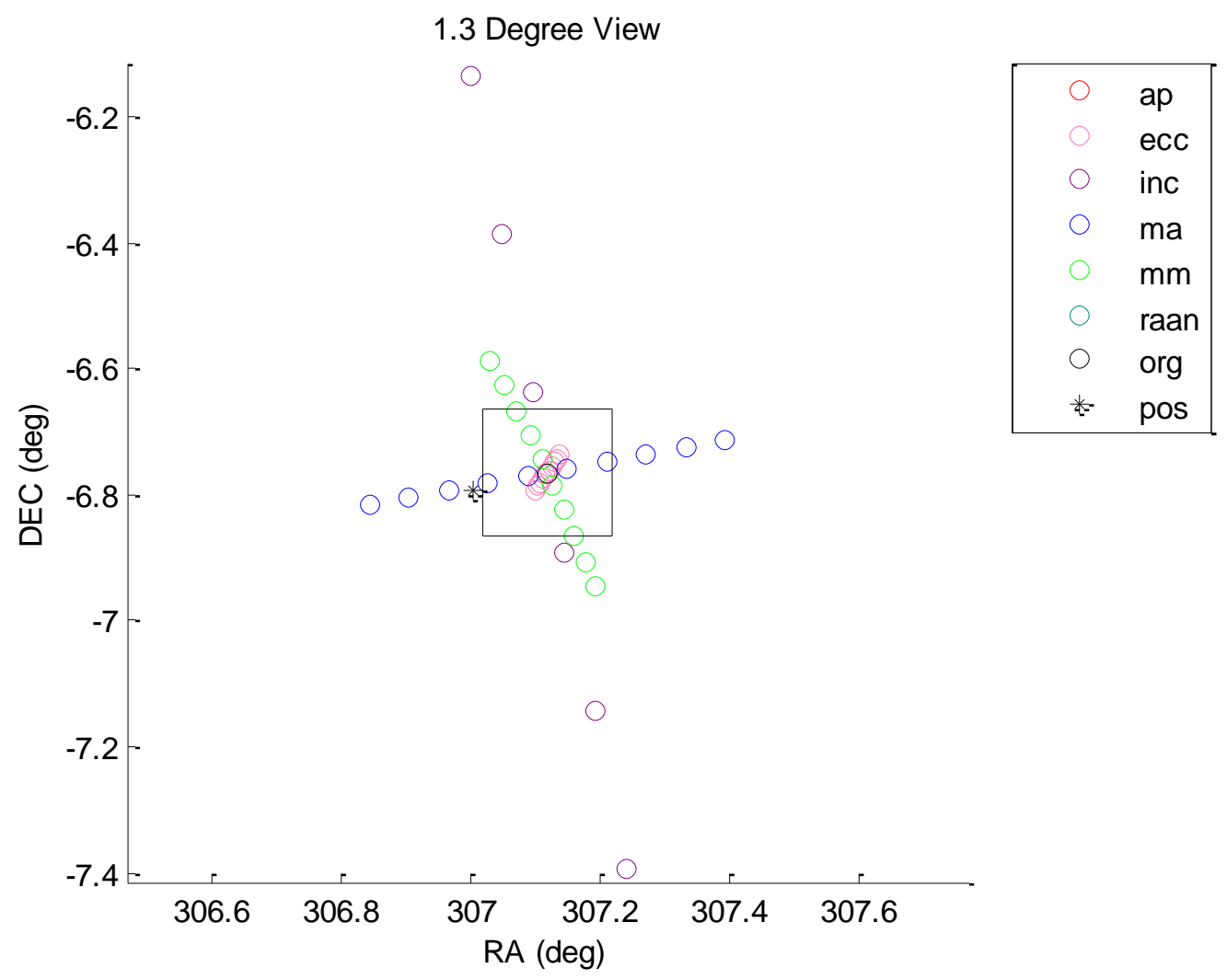




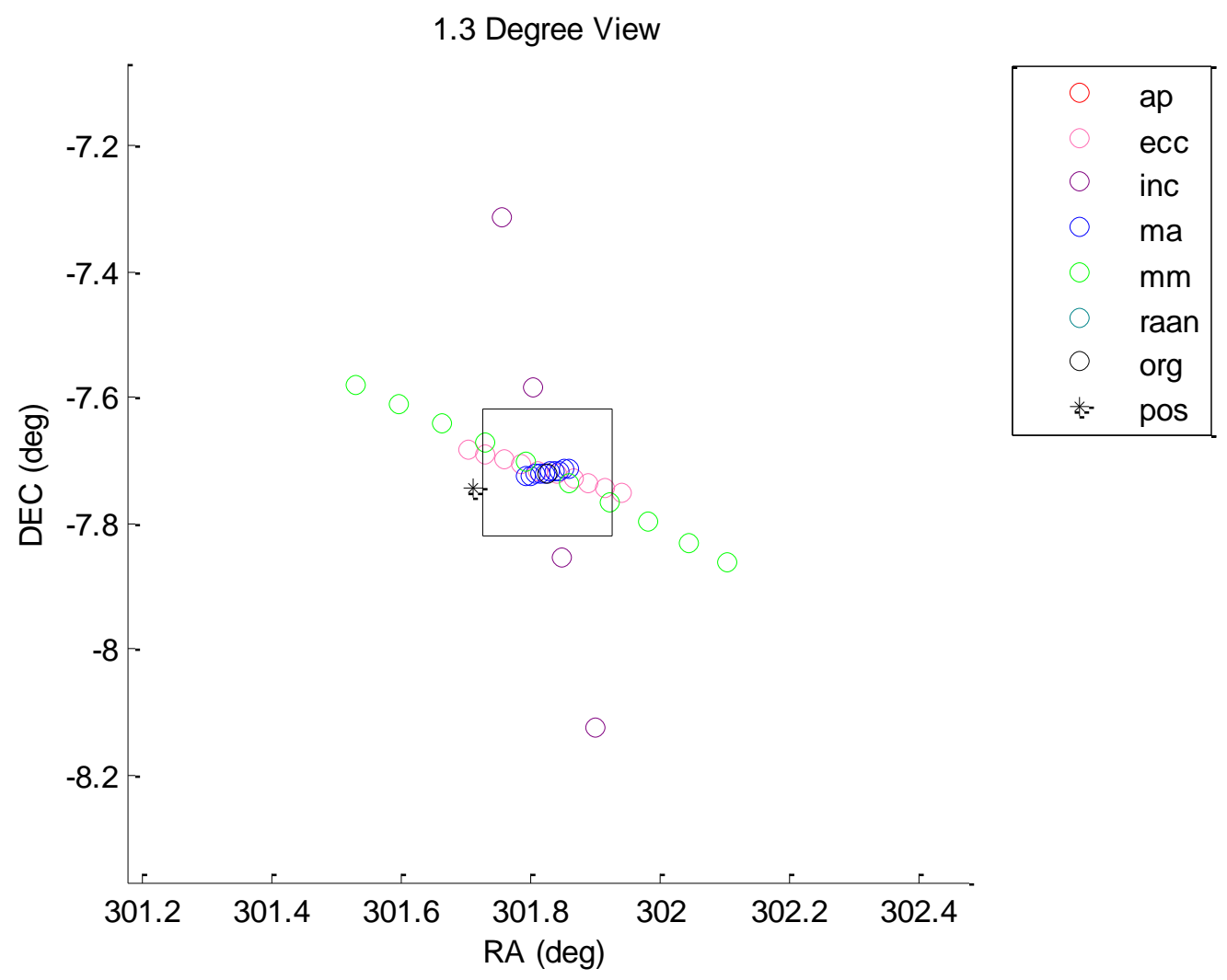




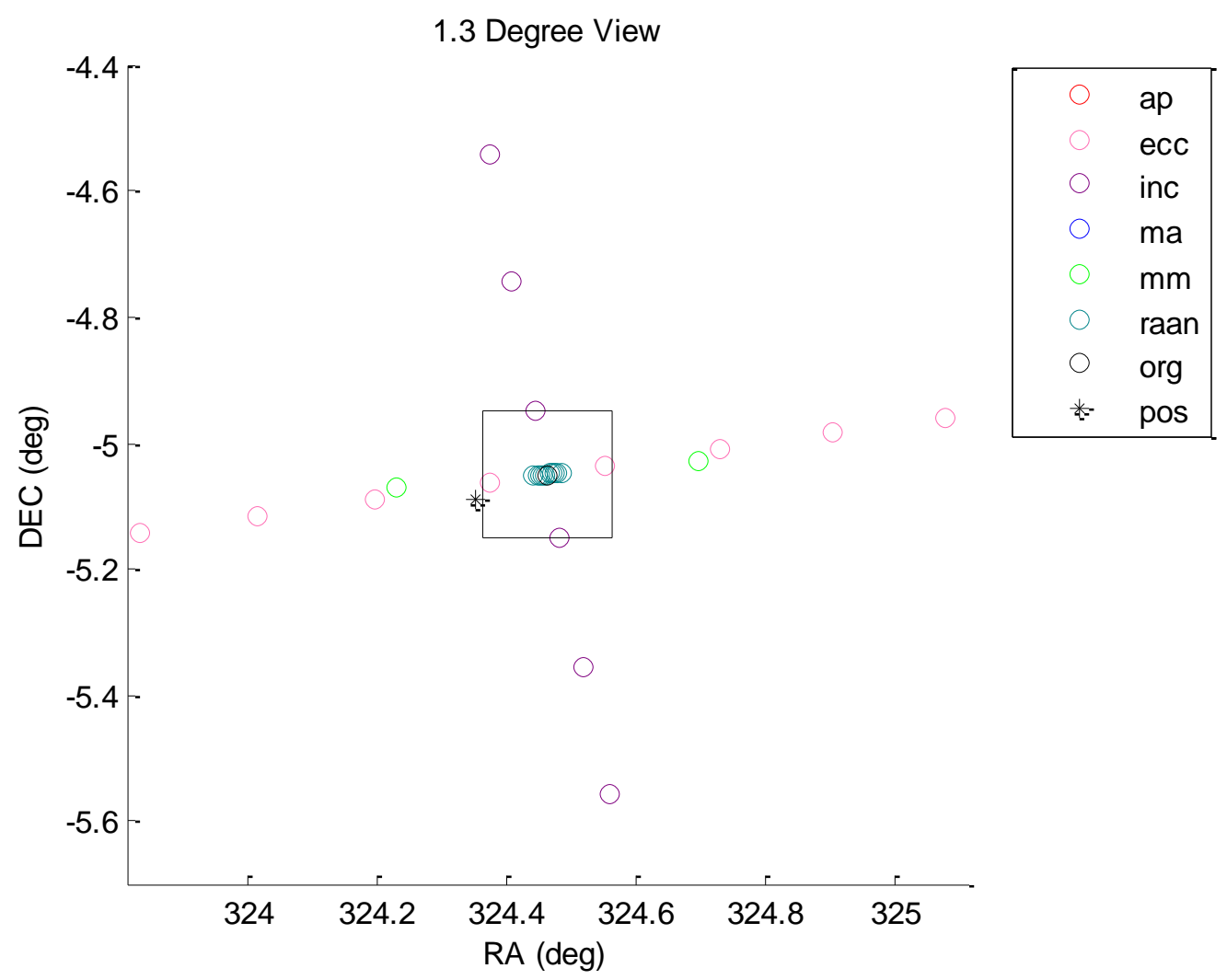




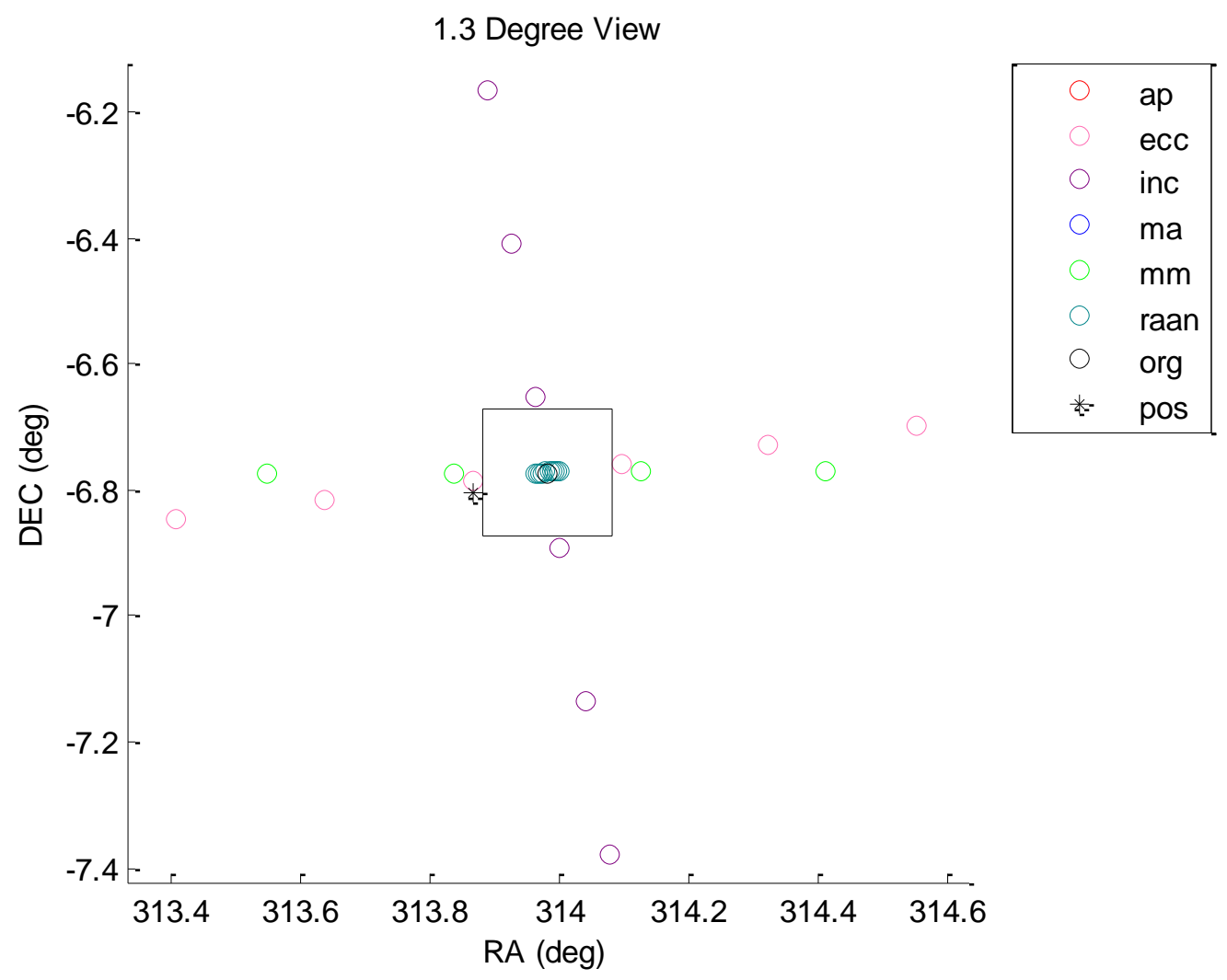




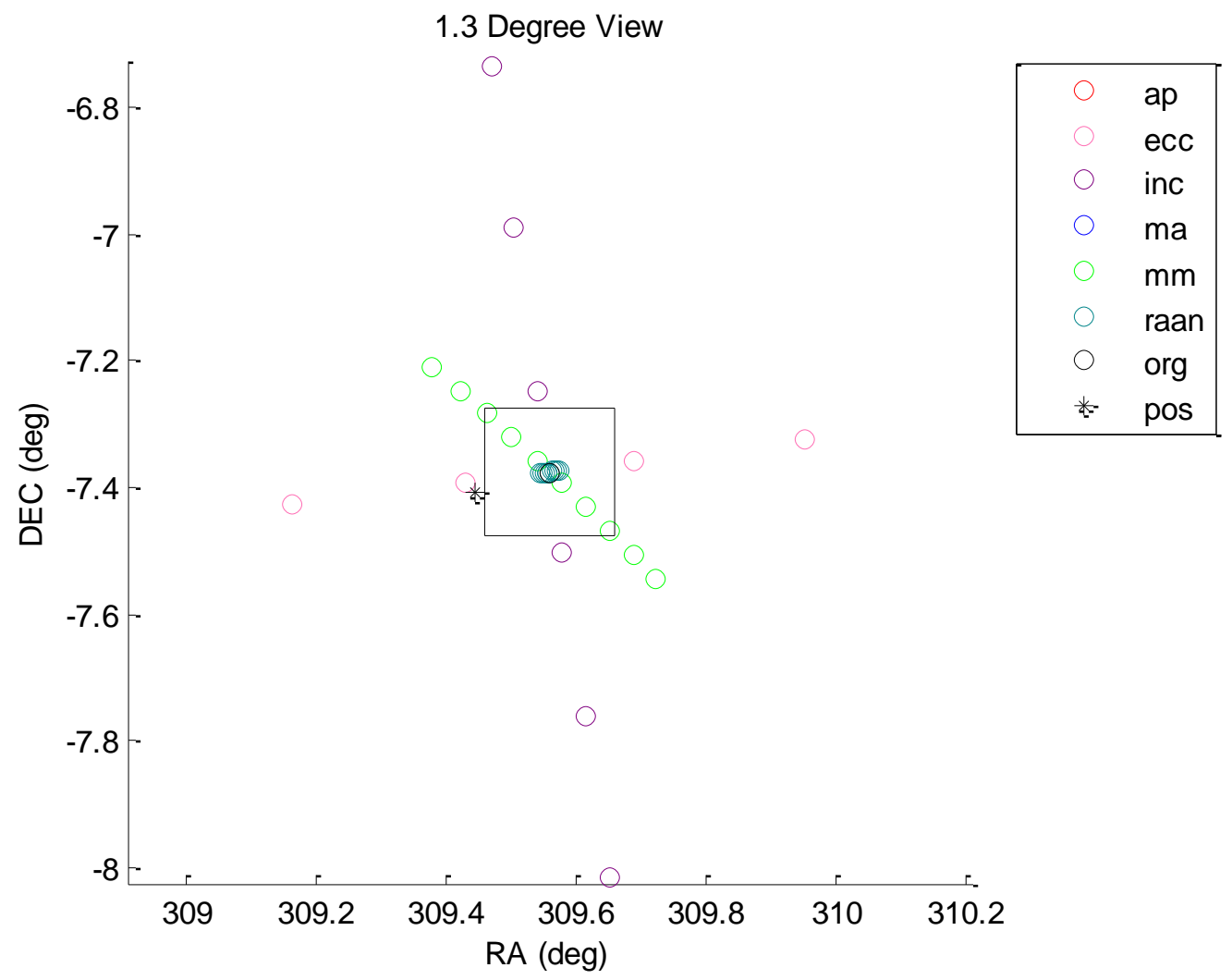




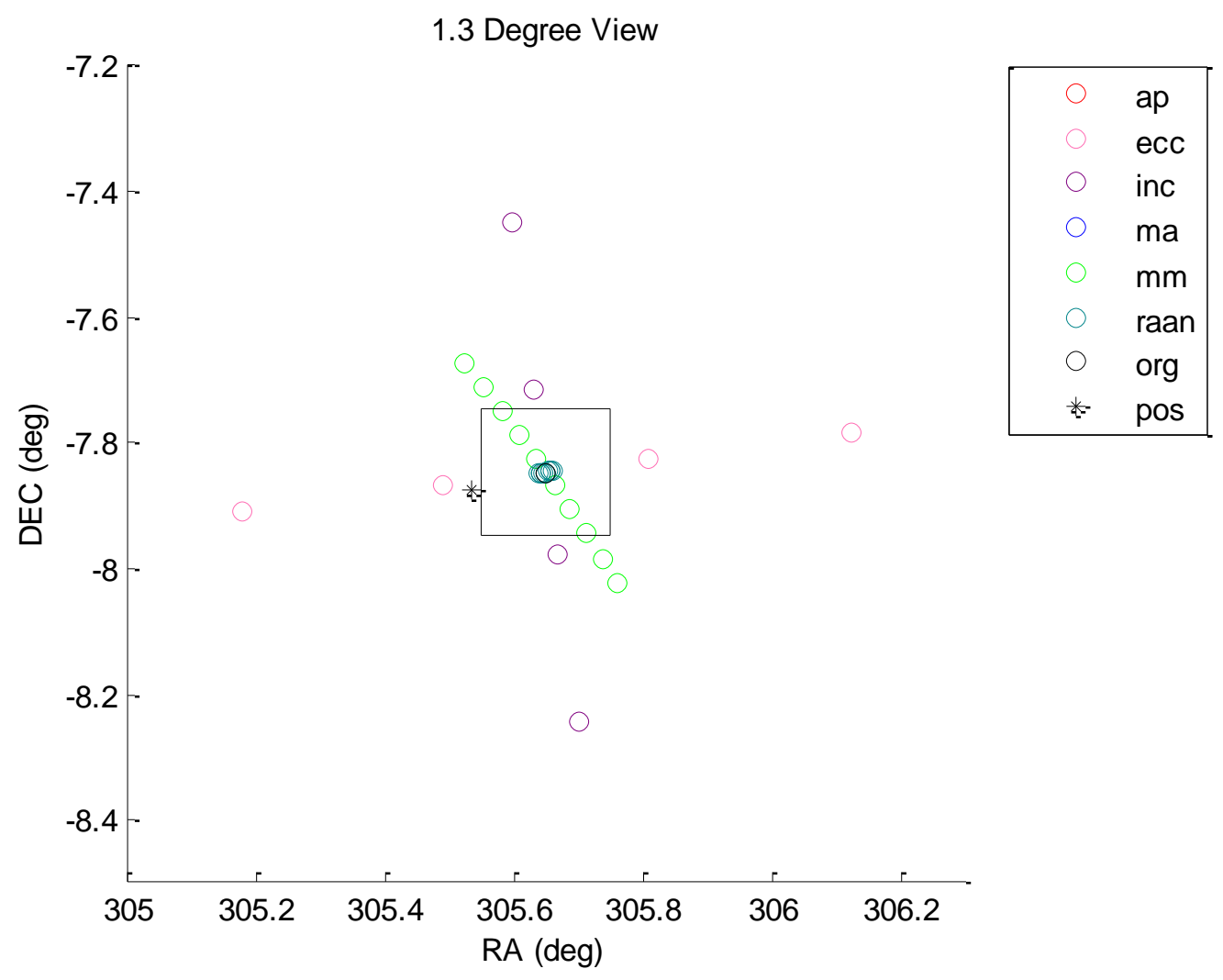




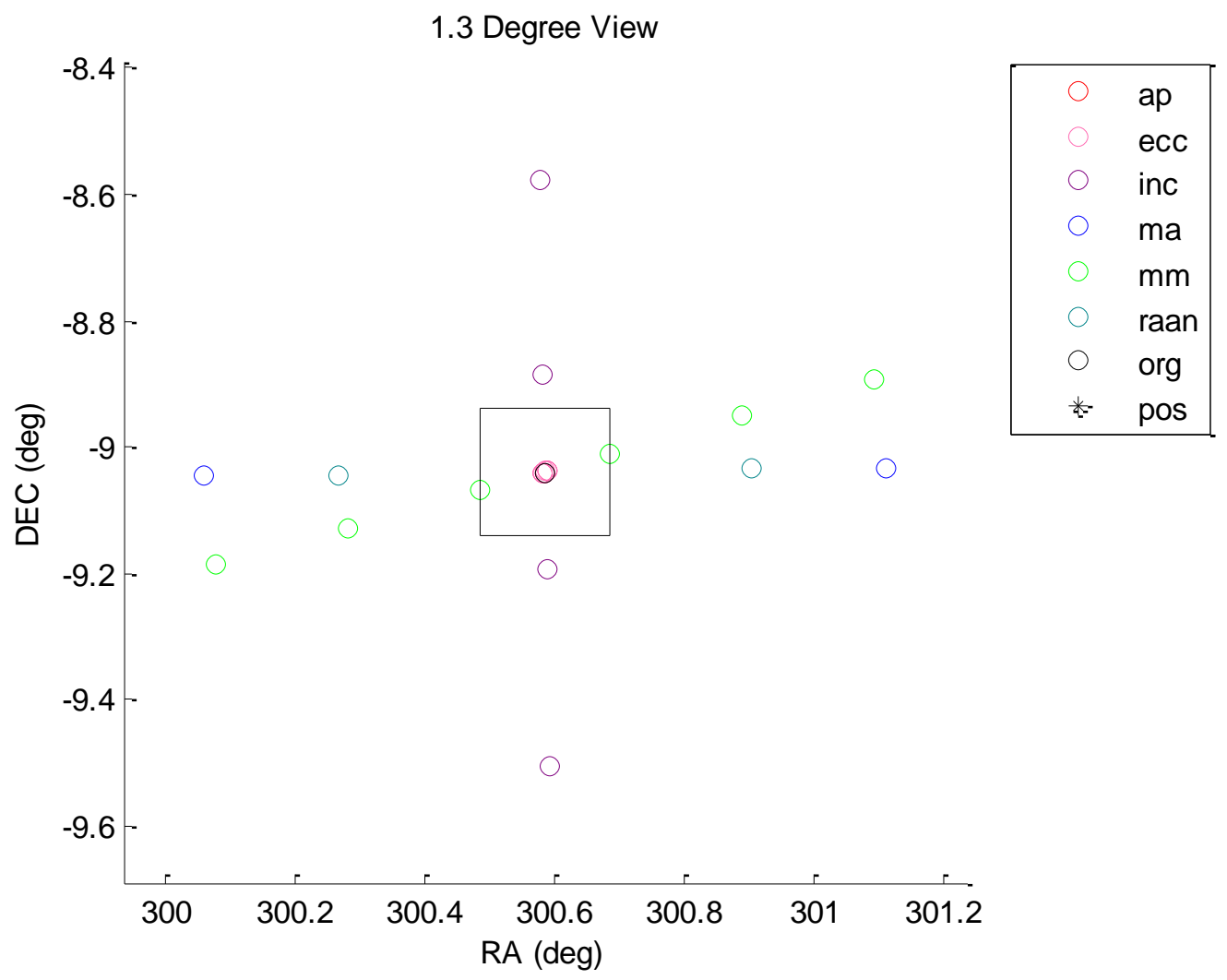




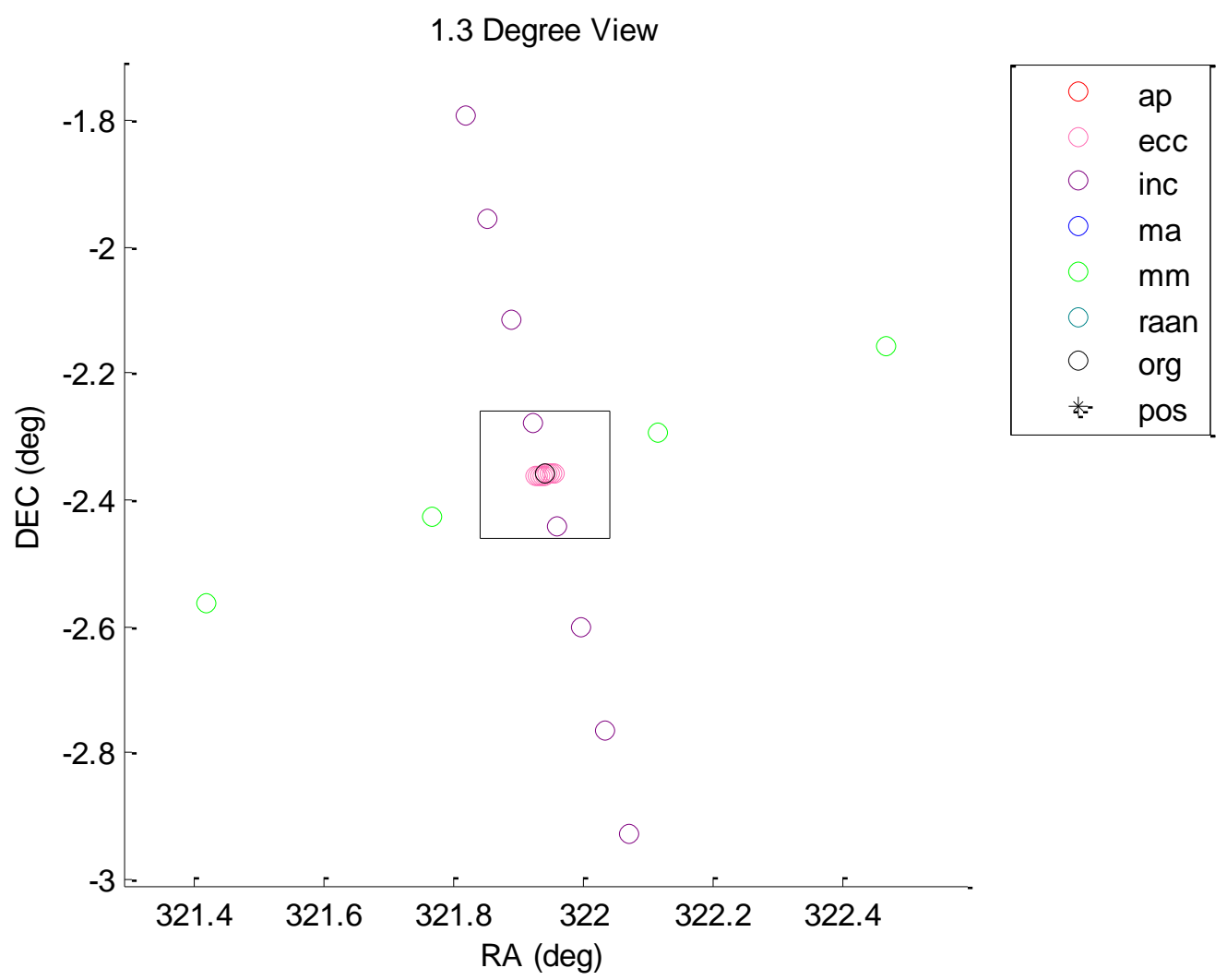




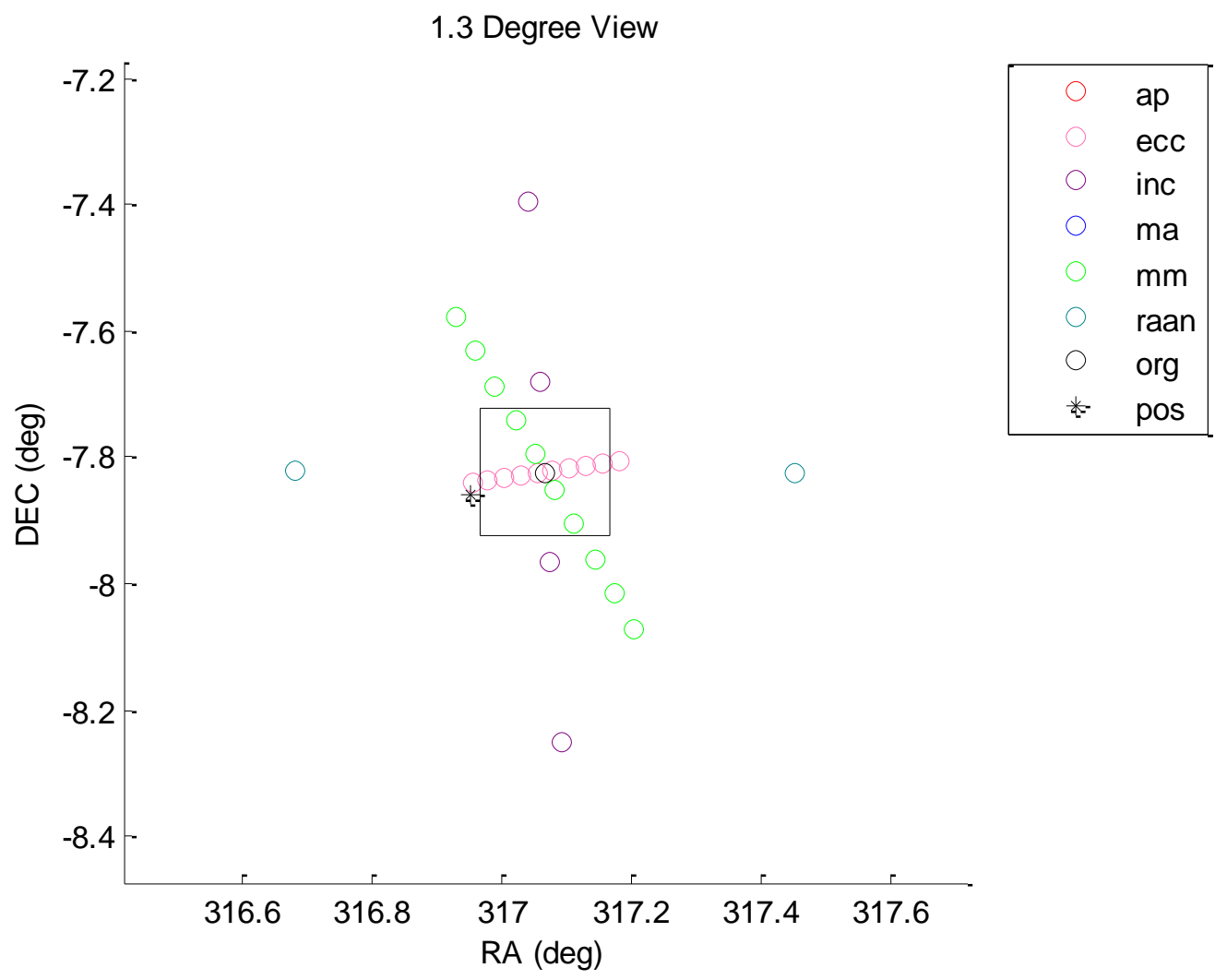




\subsection{Degree View}

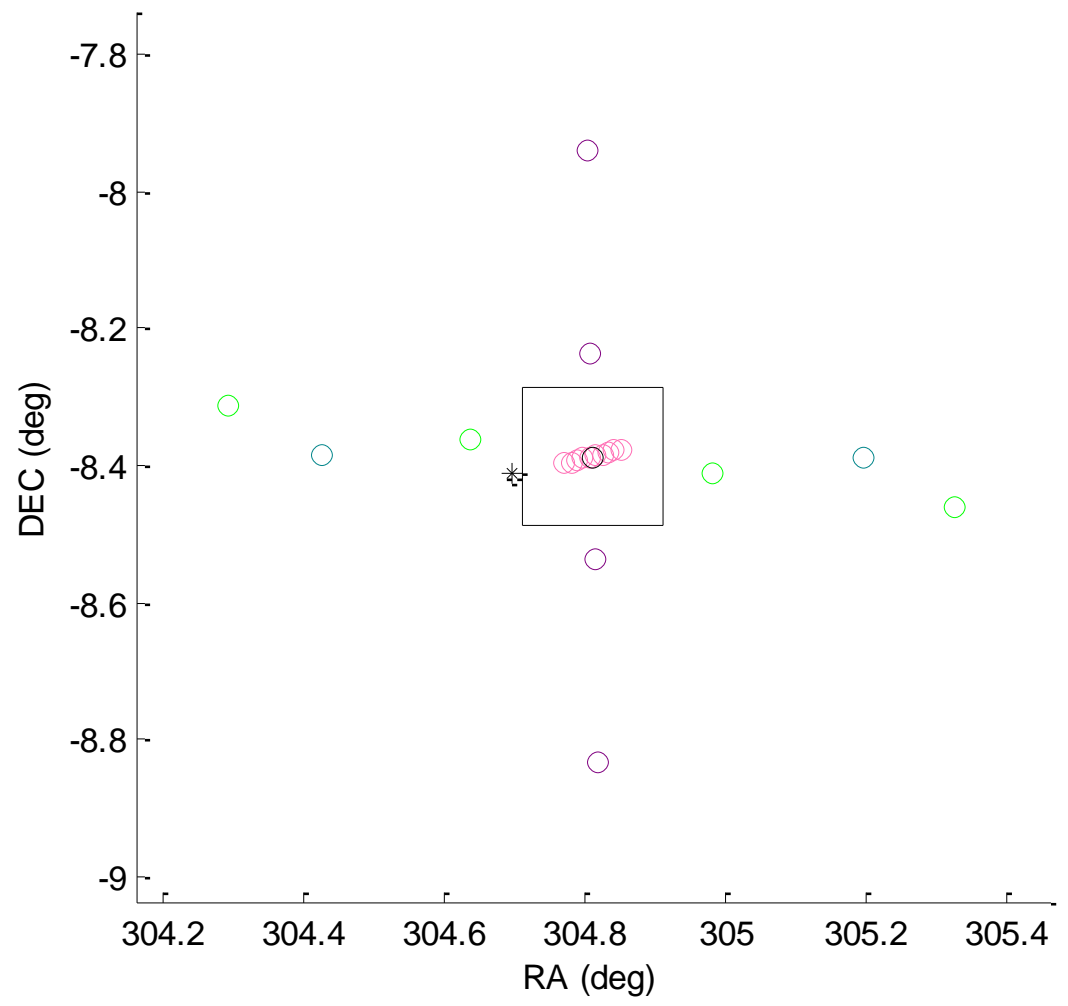




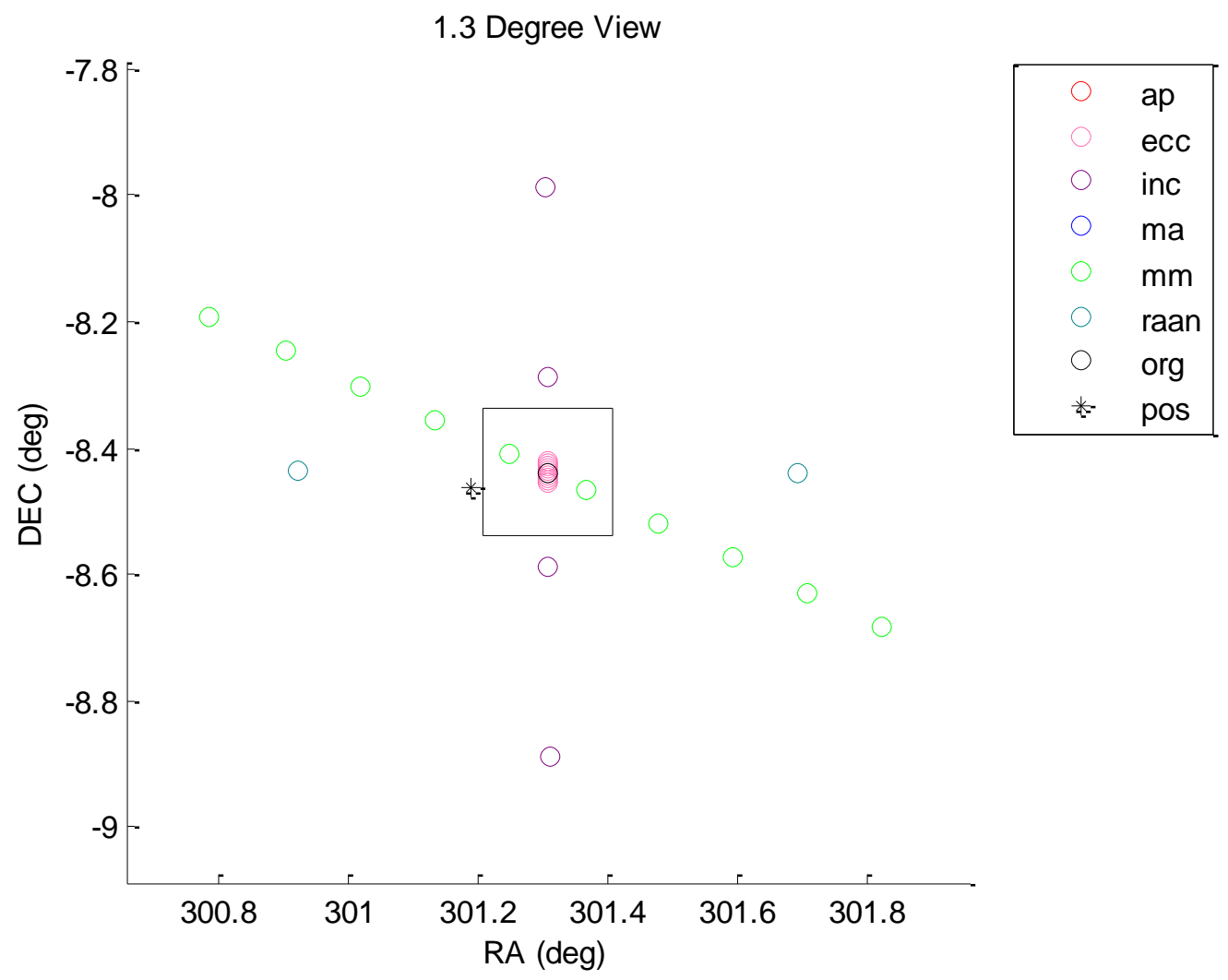




\subsection{Degree View}

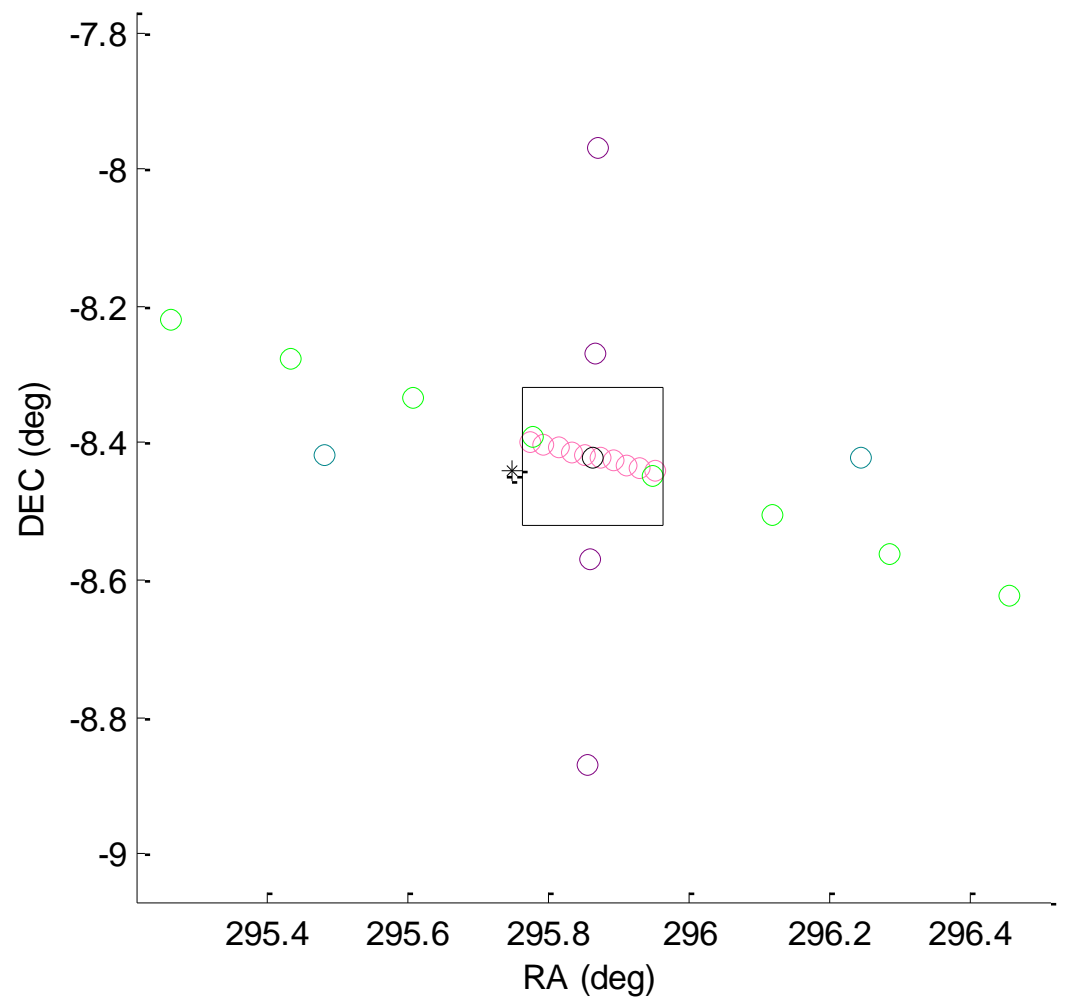

\begin{tabular}{|ll|}
\hline ap \\
ecc \\
inc \\
ma \\
mm \\
raan \\
org \\
* pos \\
\hline
\end{tabular}




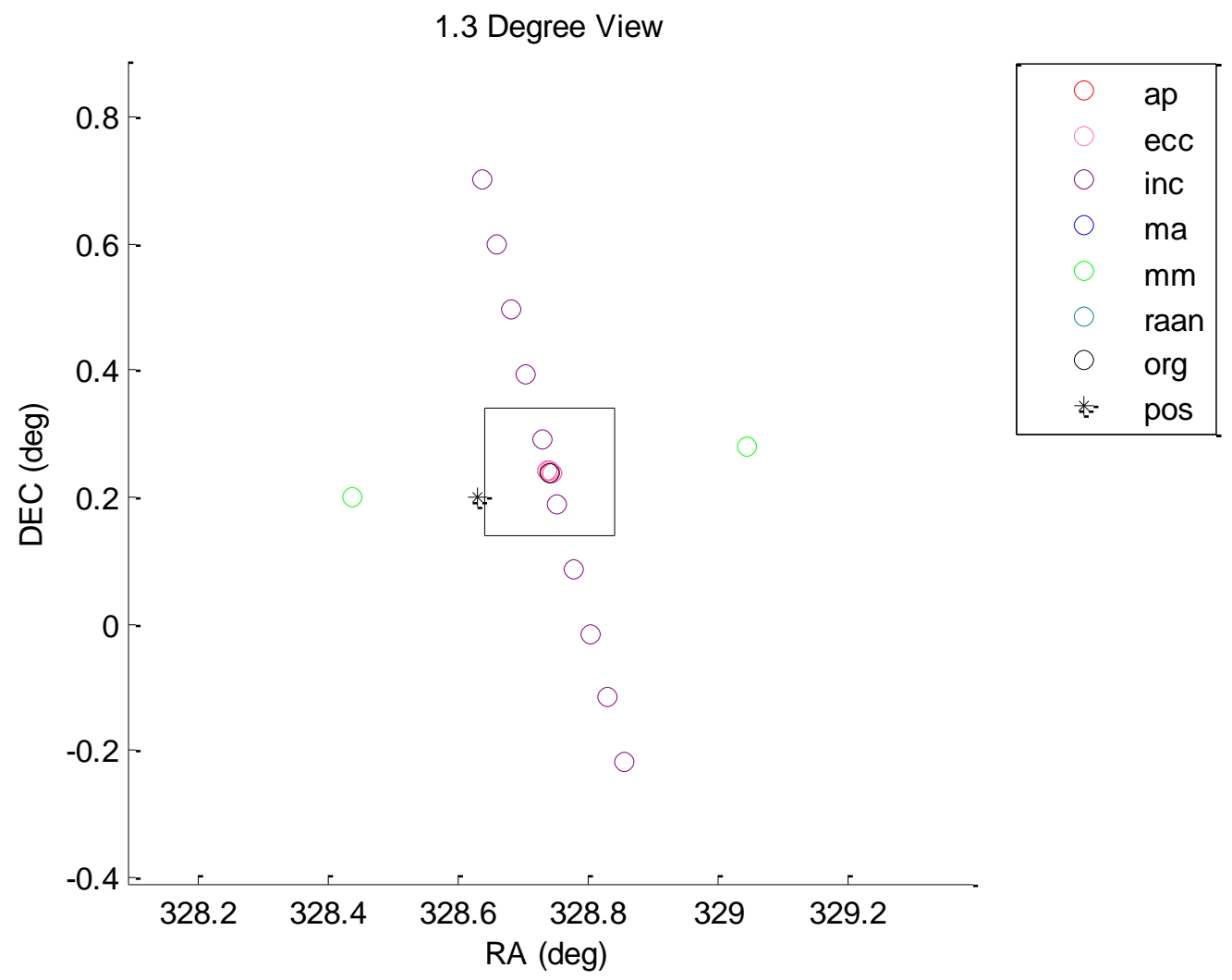




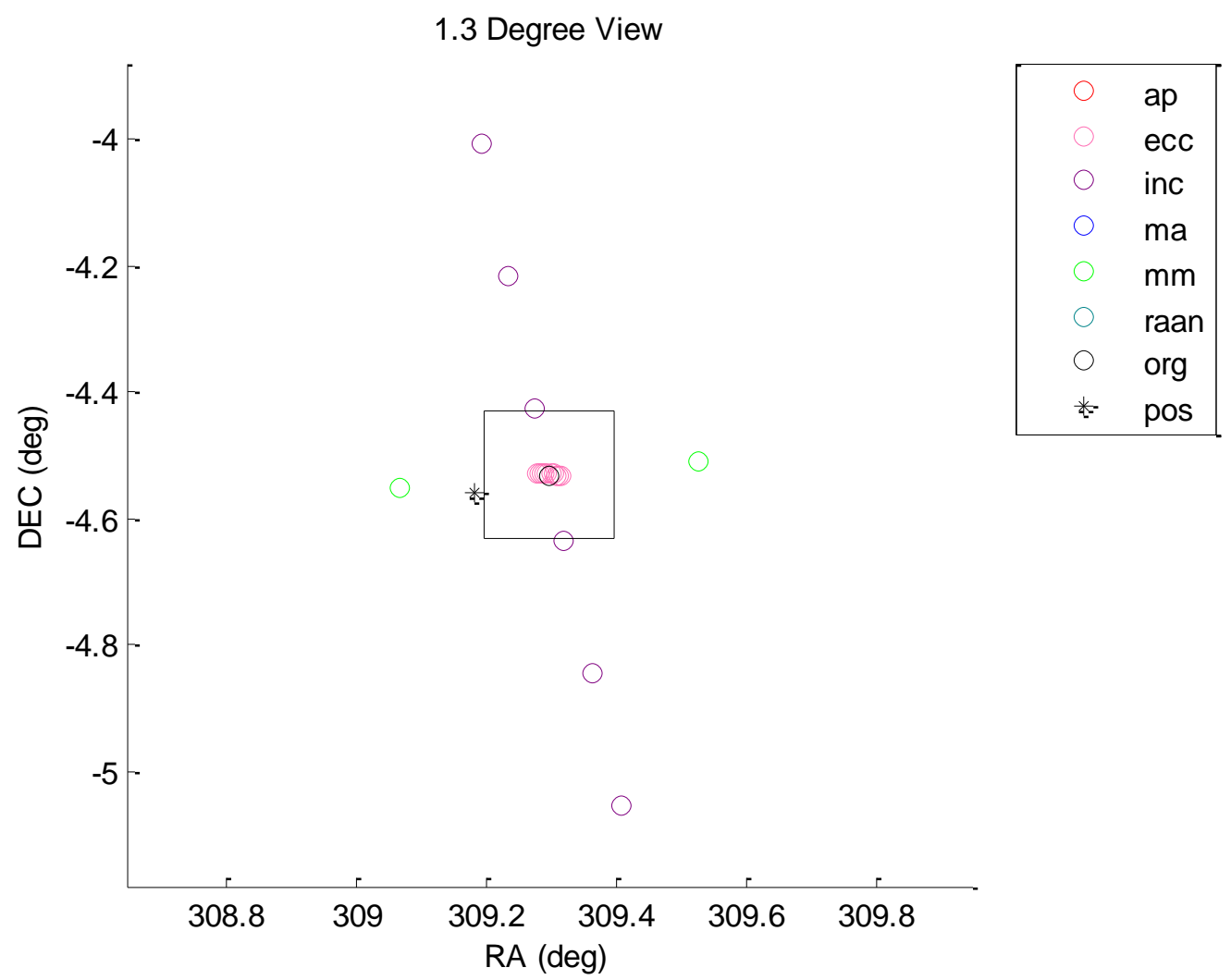


1.3 Degree View

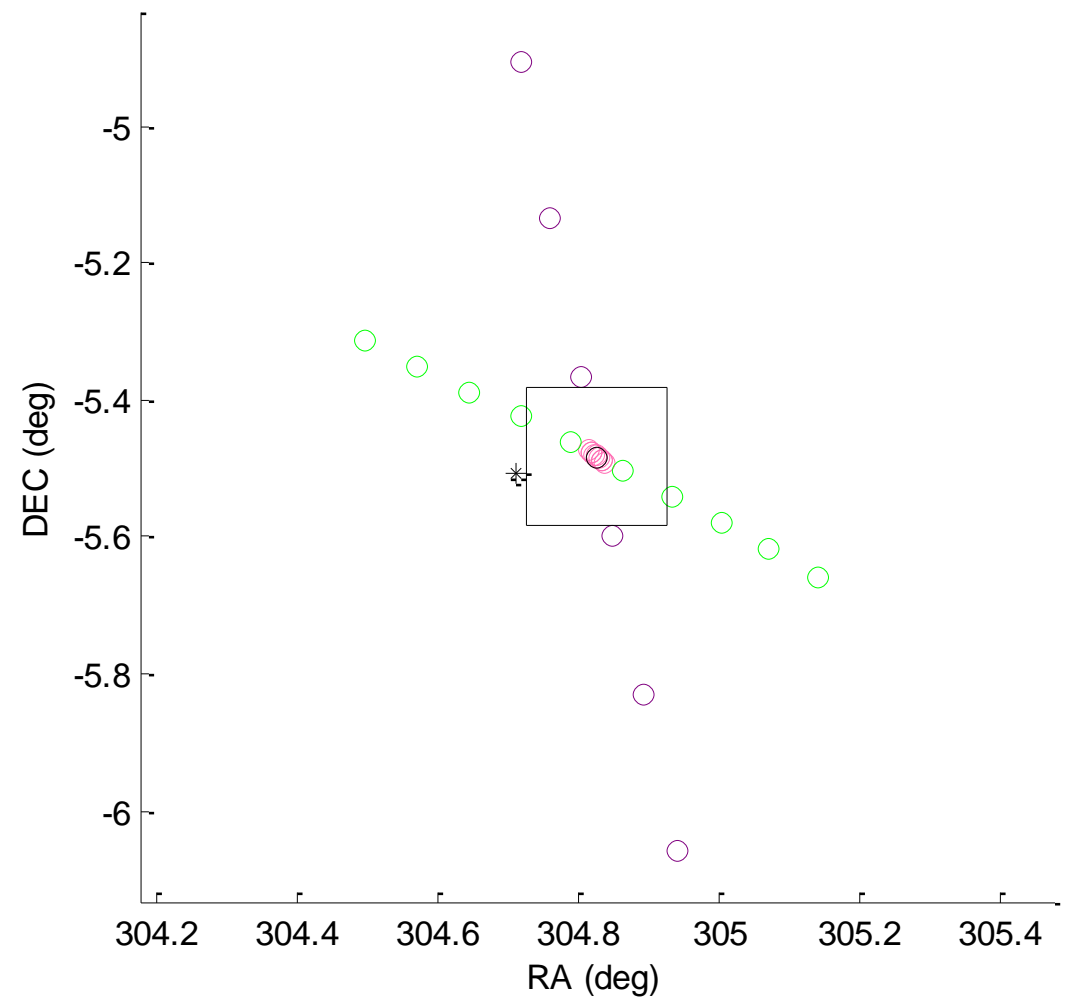

\begin{tabular}{|ll|}
\hline ap \\
ecc \\
inc \\
ma \\
mm \\
raan \\
org \\
* pos \\
\hline
\end{tabular}


1.3 Degree View

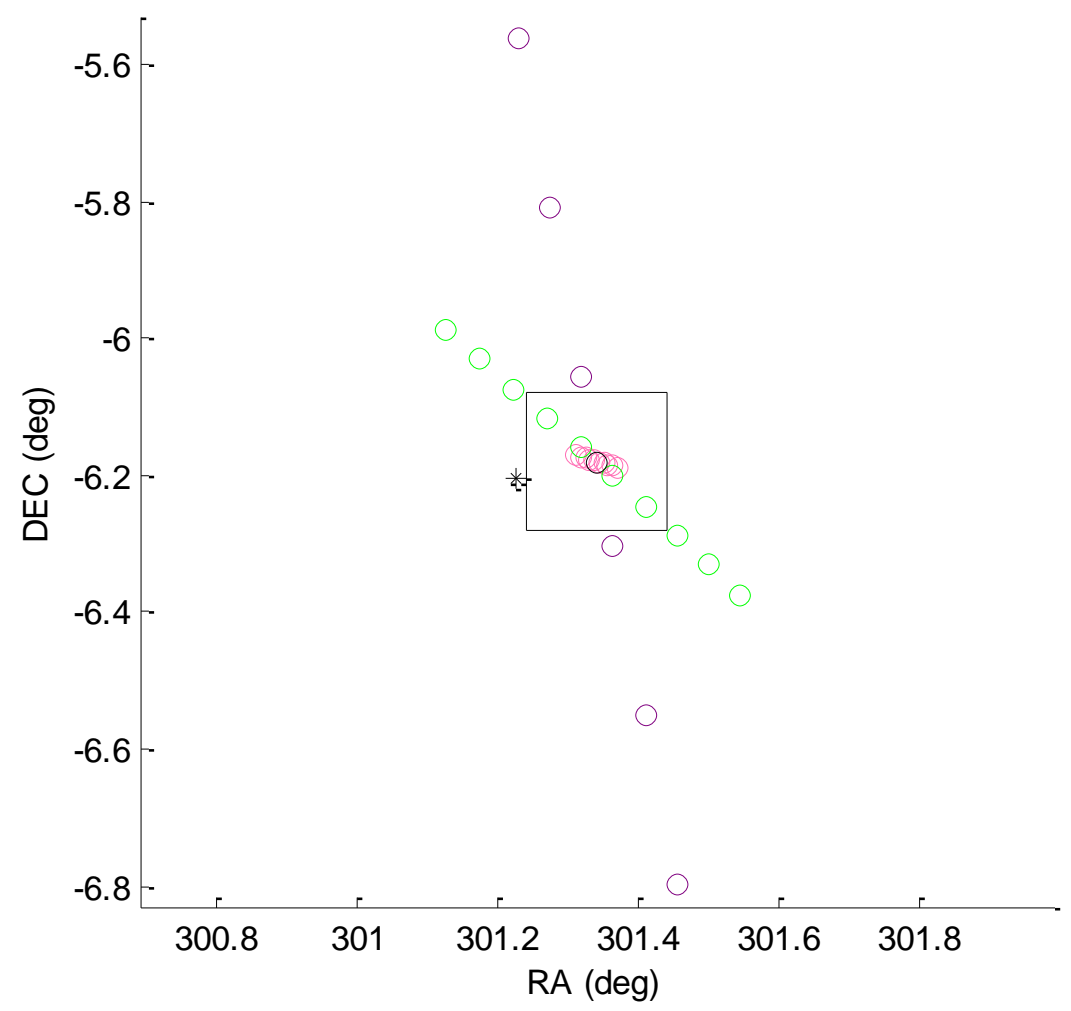

\begin{tabular}{|ll|}
\hline ap \\
ecc \\
inc \\
ma \\
mm \\
raan \\
org \\
* pos \\
\hline
\end{tabular}




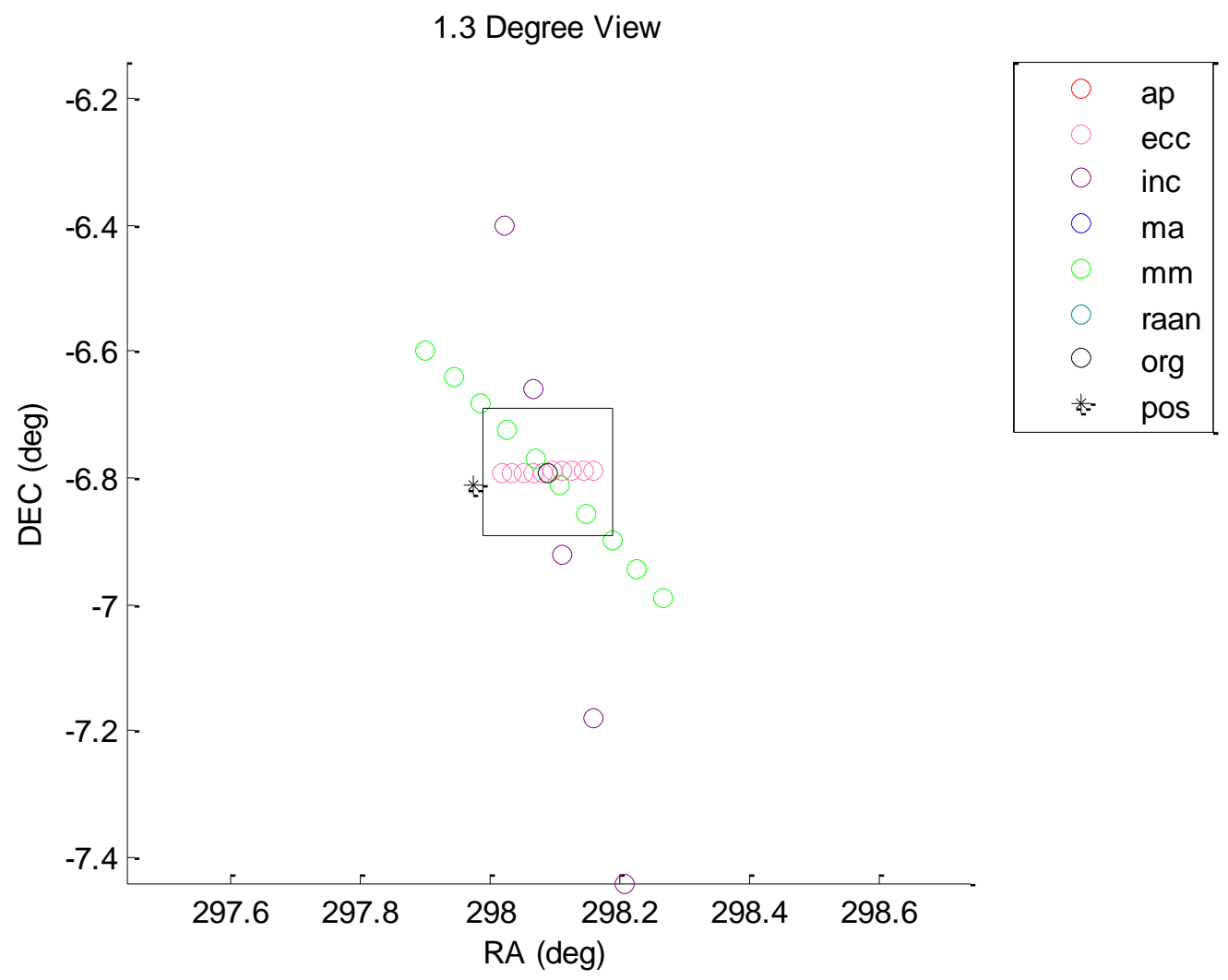




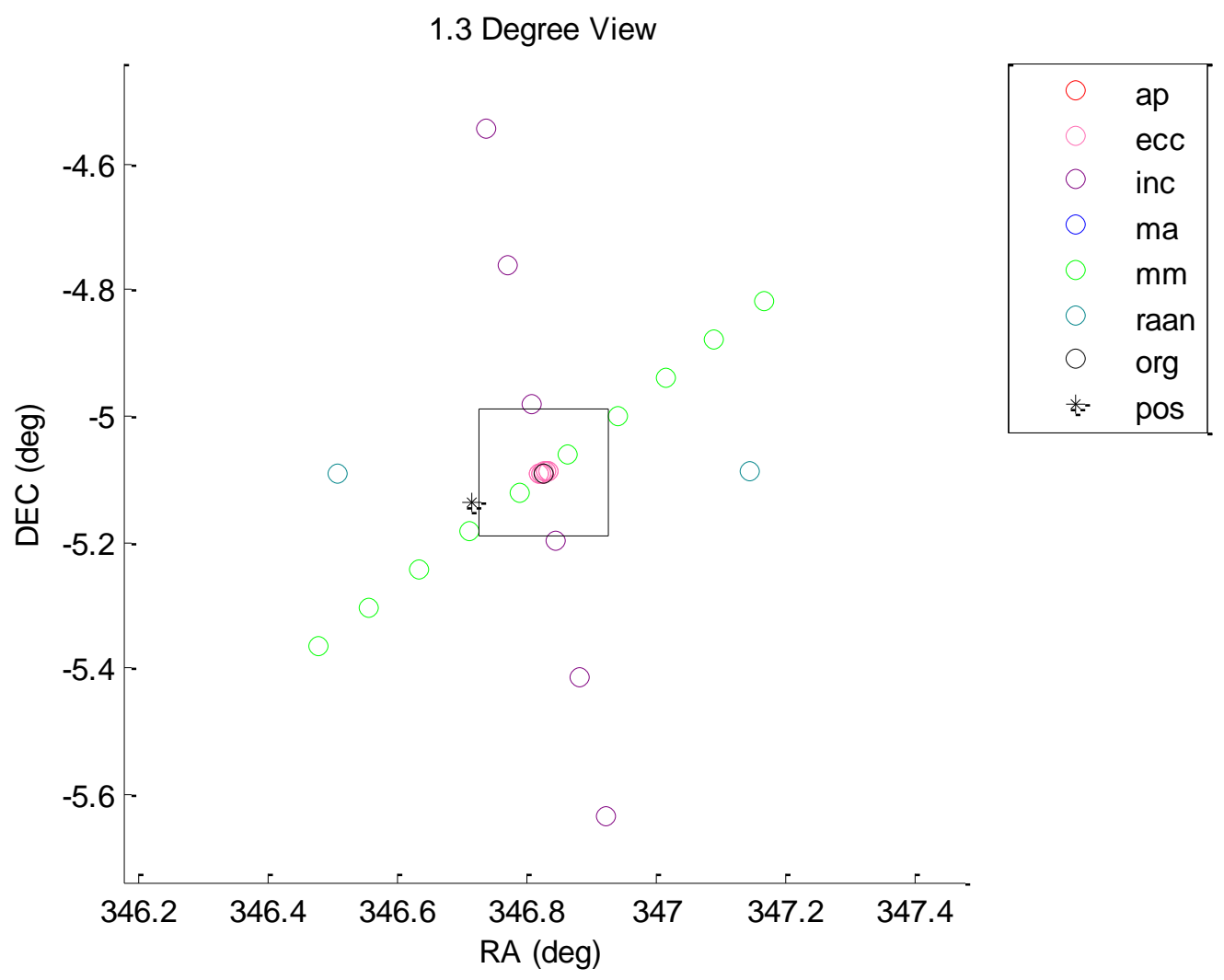




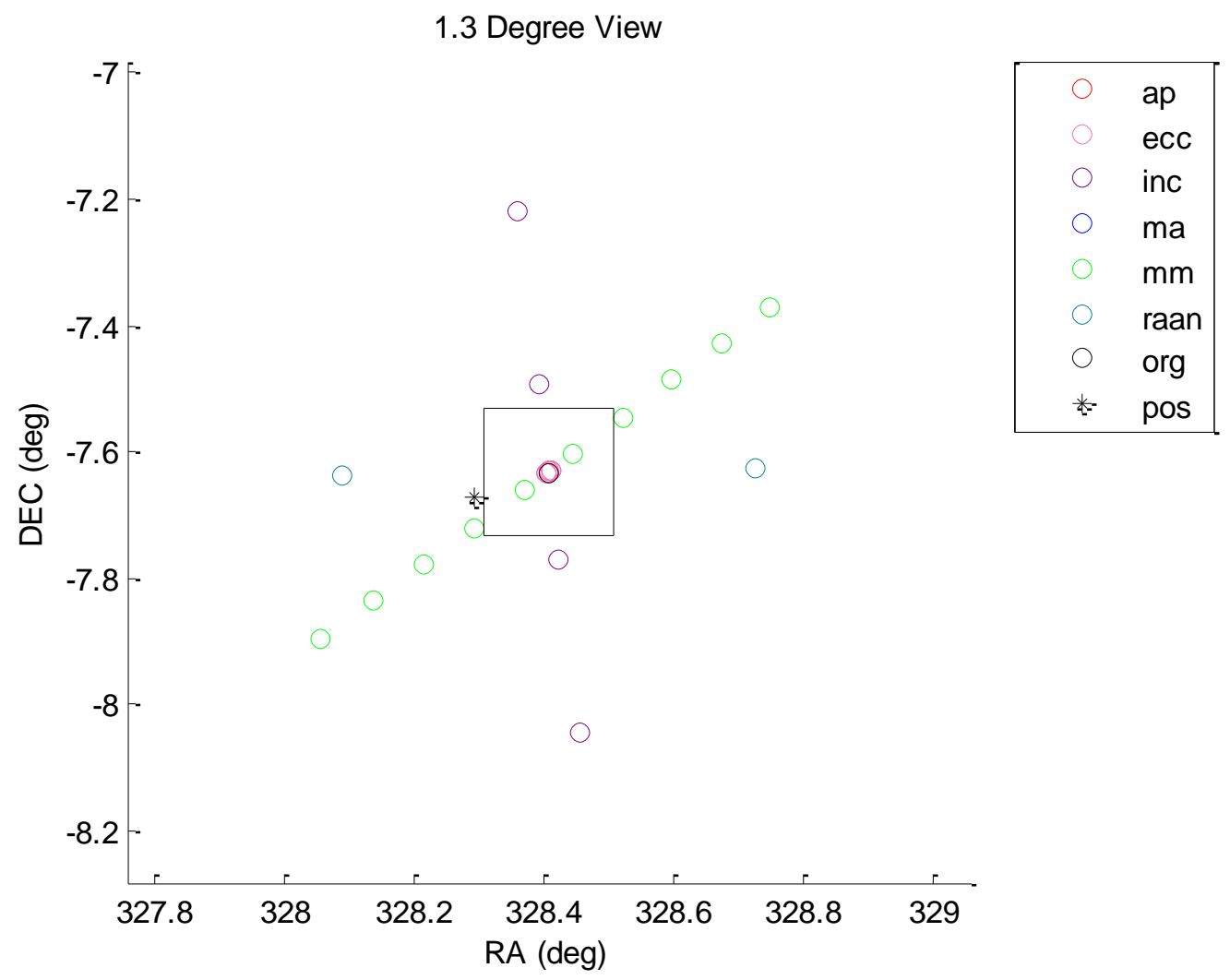




\subsection{Degree View}

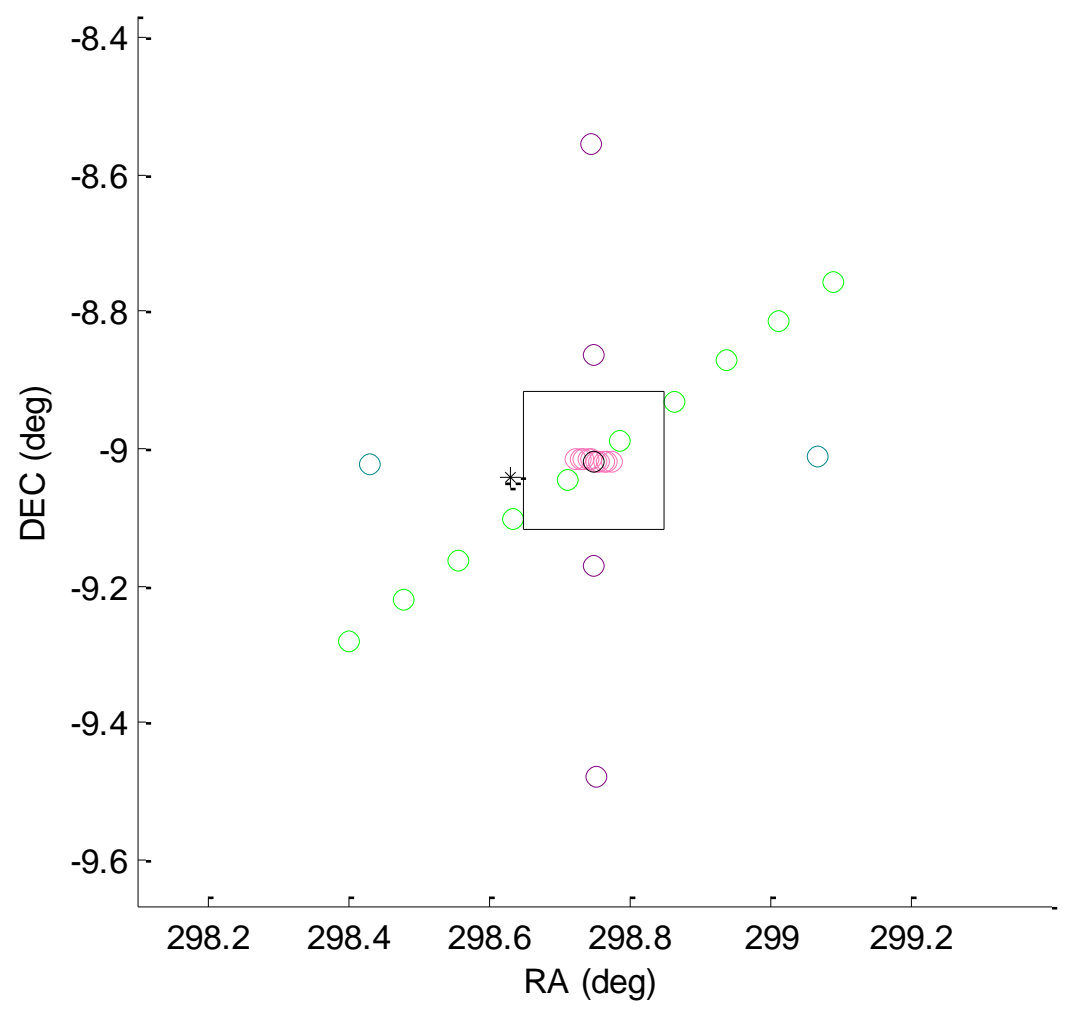

\begin{tabular}{|ll|}
\hline ap \\
ecc \\
inc \\
ma \\
mm \\
raan \\
org \\
* pos \\
\hline
\end{tabular}




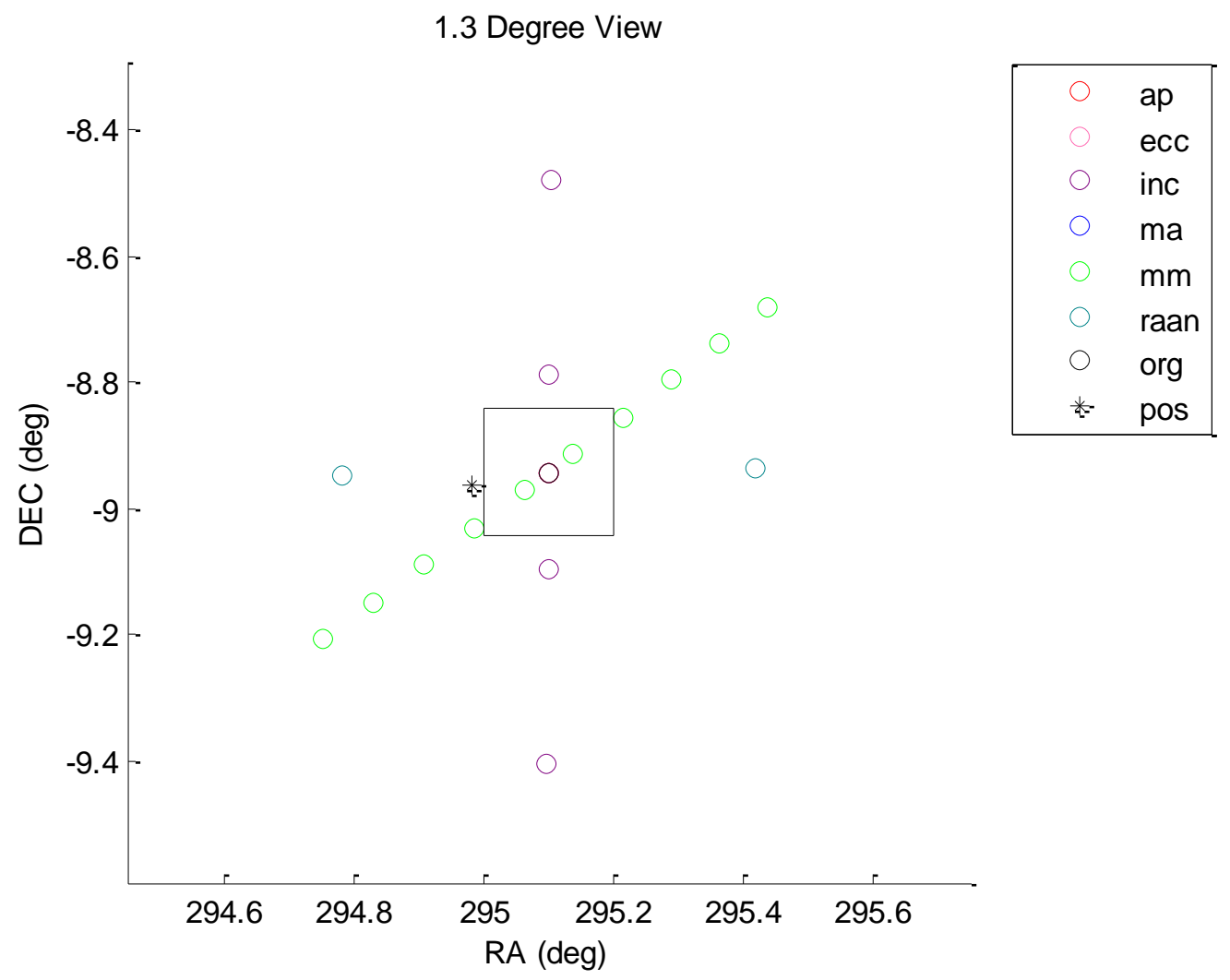




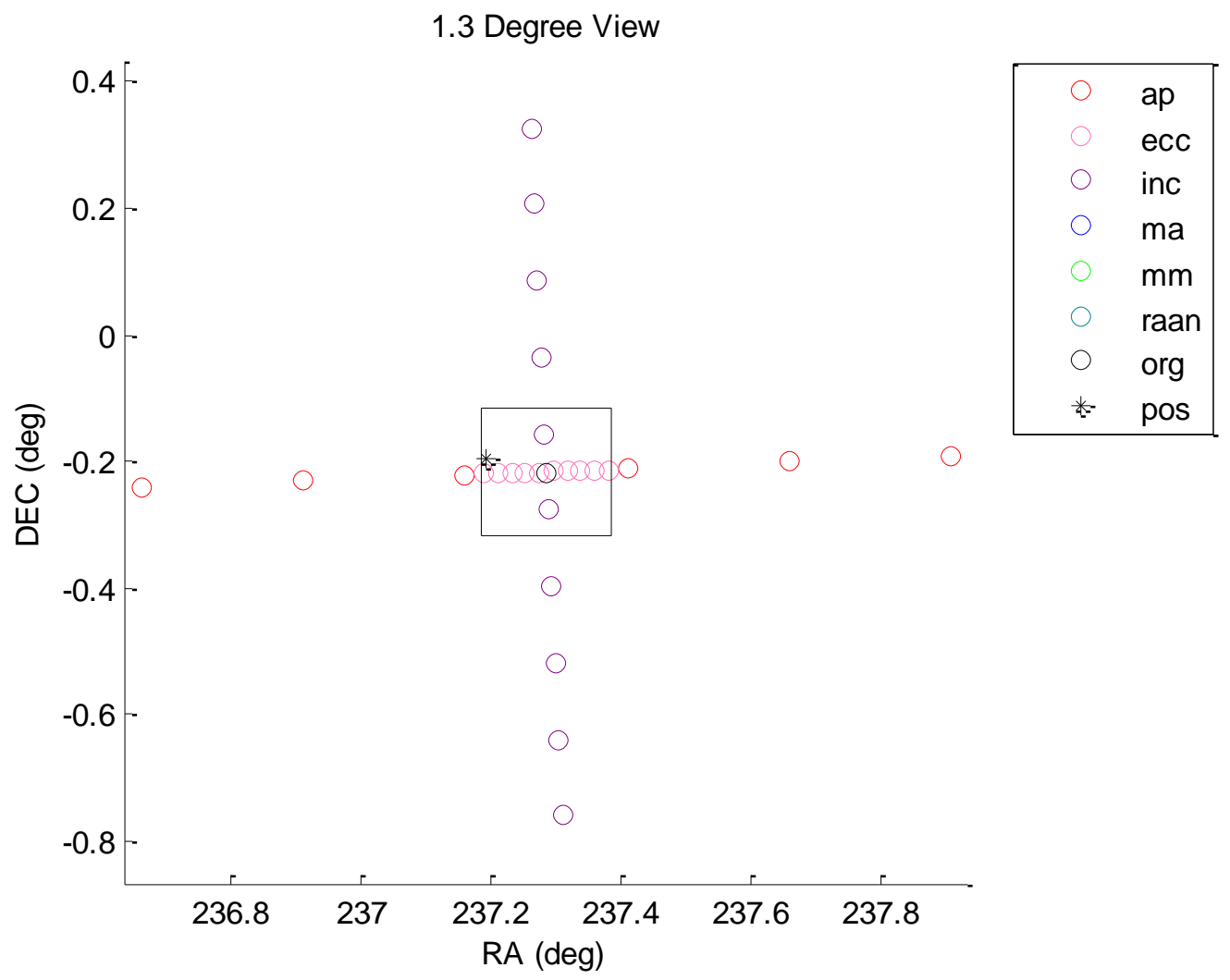


1.3 Degree View

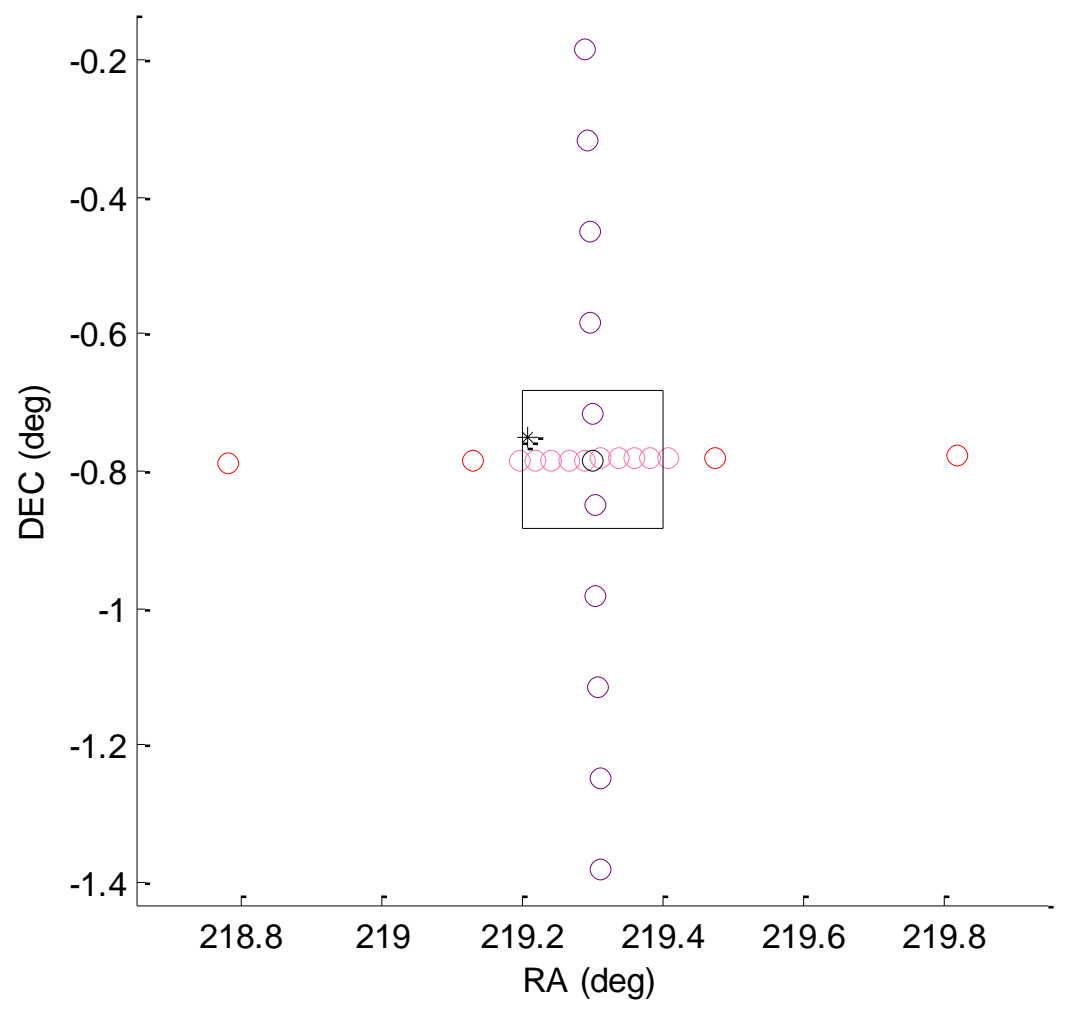

\begin{tabular}{|ll|}
\hline ap \\
ecc \\
inc \\
ma \\
mm \\
raan \\
org \\
* pos \\
\hline
\end{tabular}




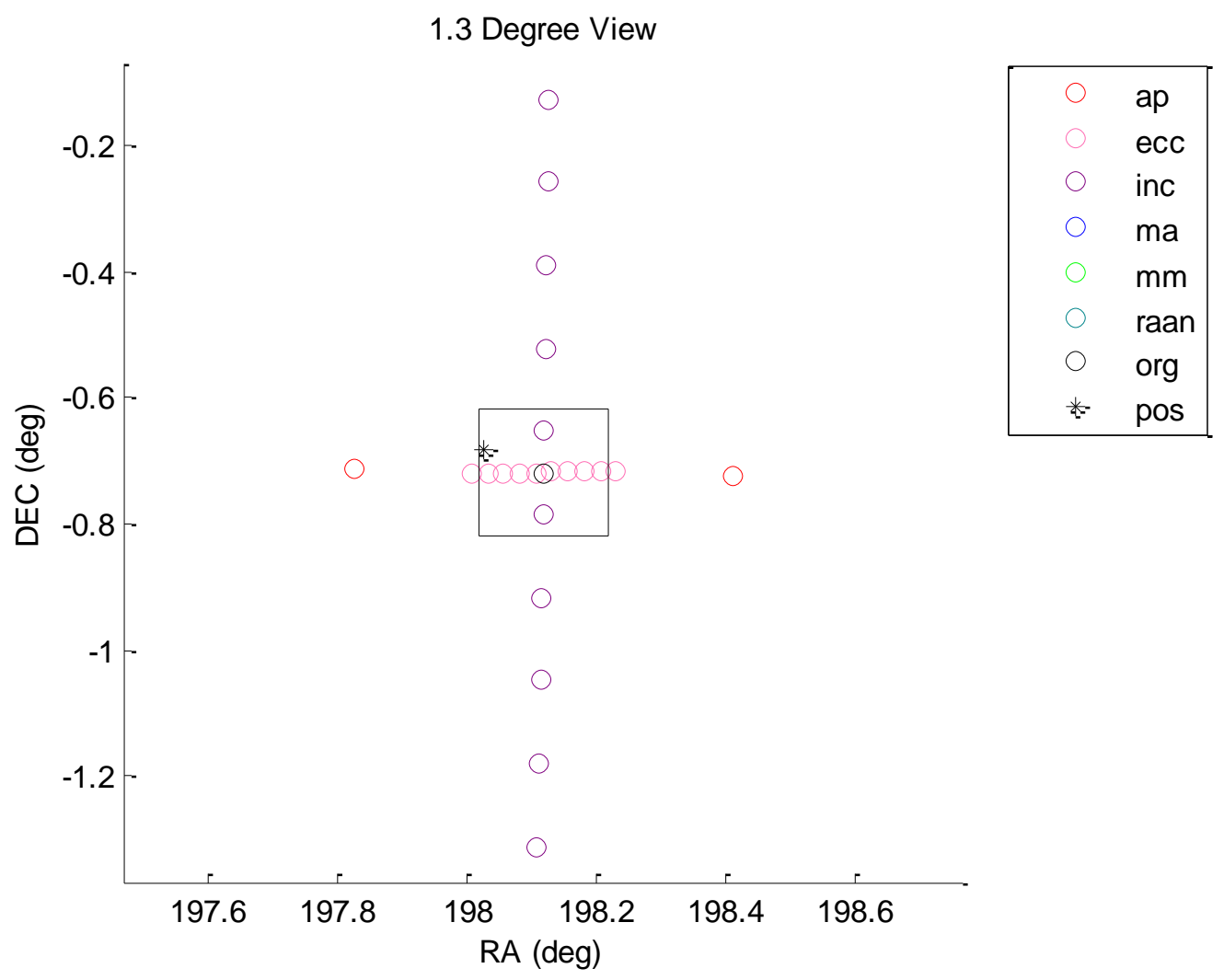




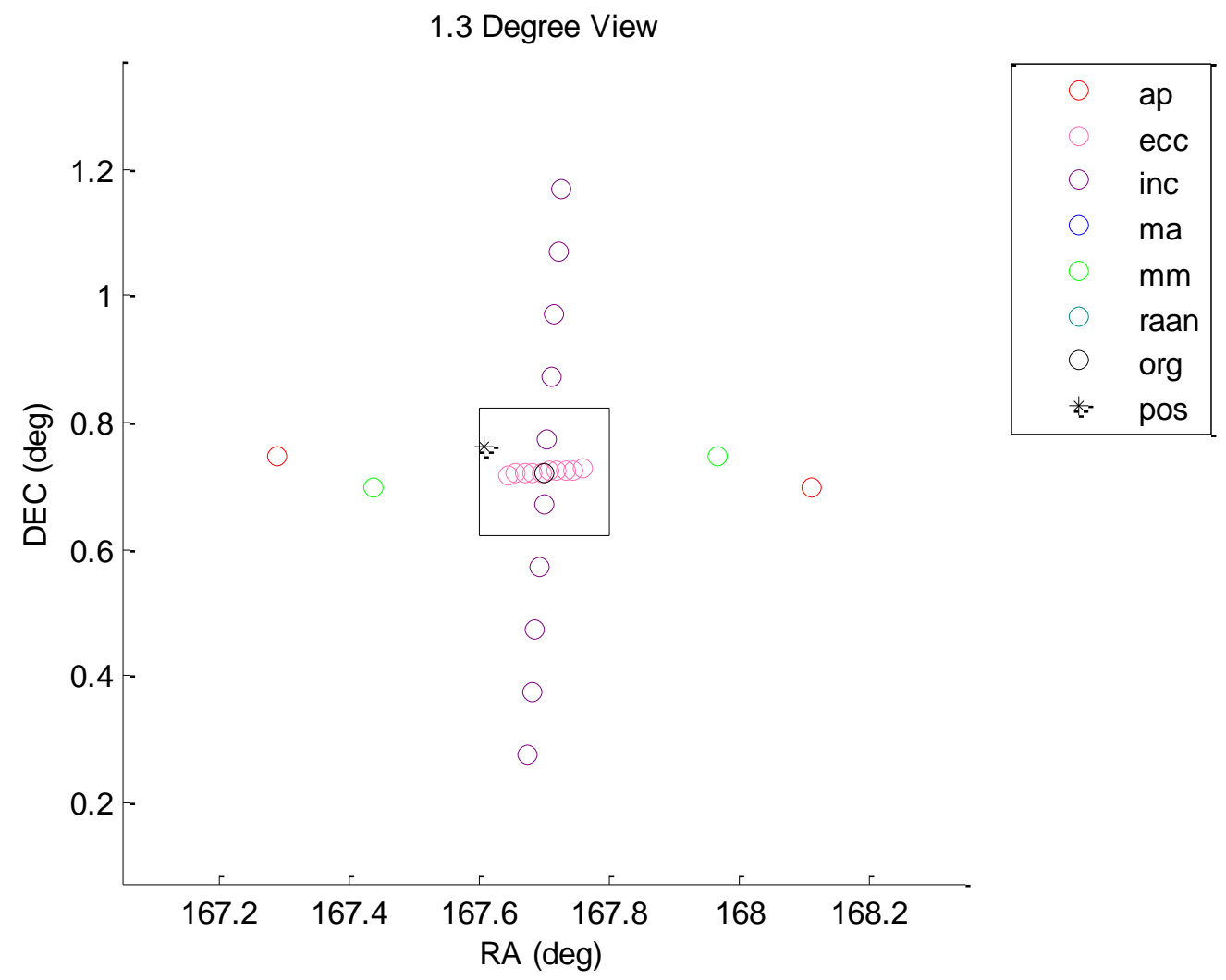


1.3 Degree View

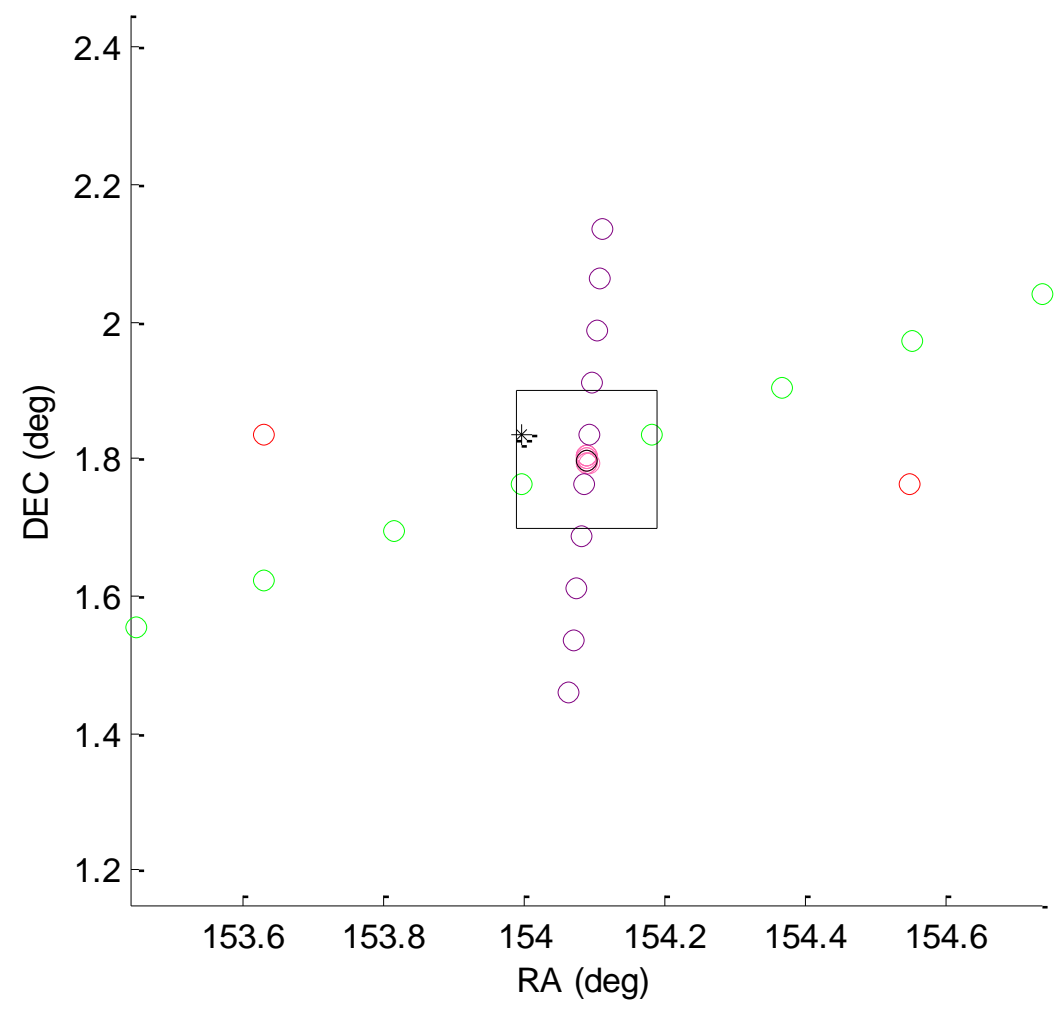

\begin{tabular}{|ll|}
\hline ap \\
ecc \\
inc \\
ma \\
mm \\
raan \\
org \\
* pos \\
\hline
\end{tabular}




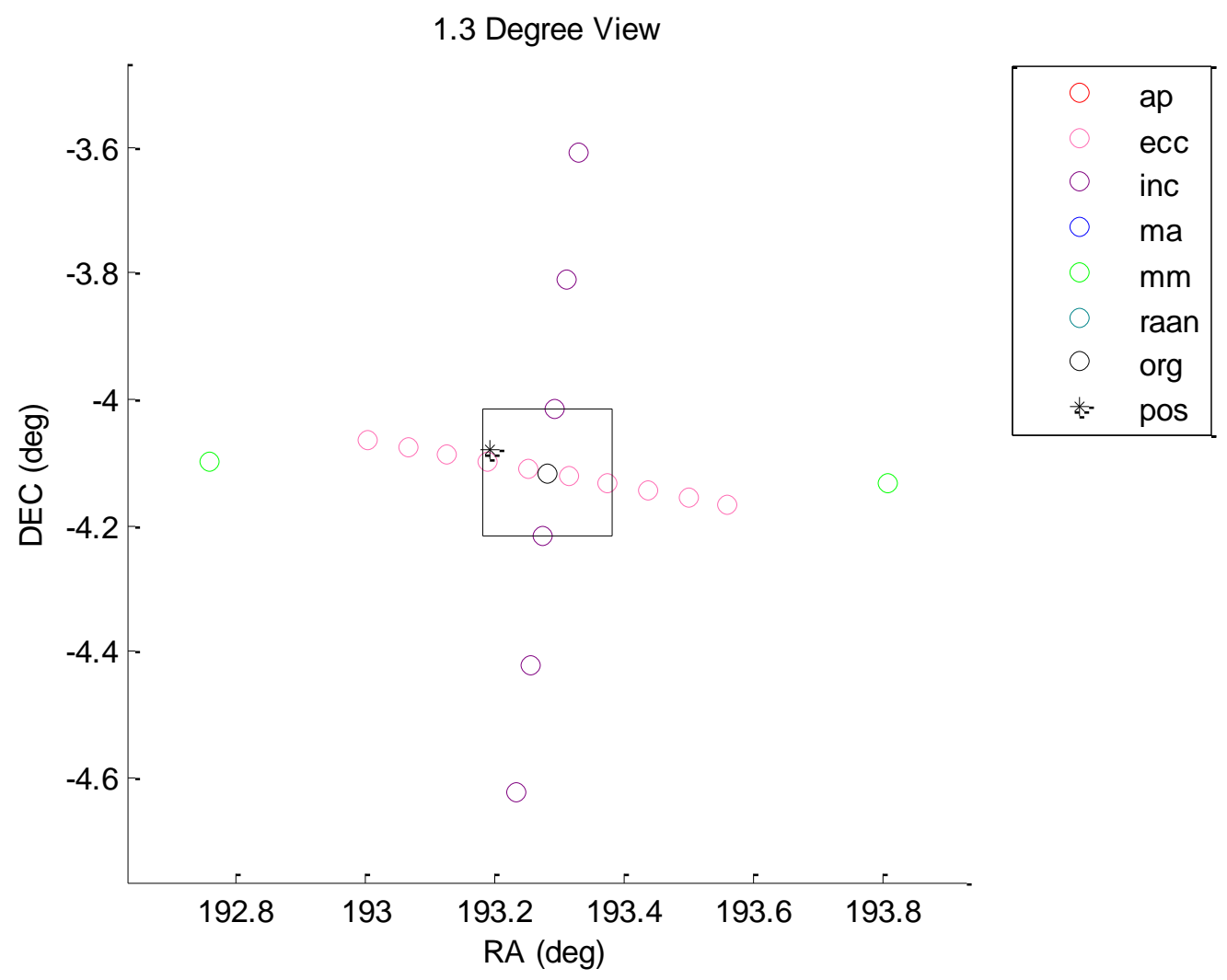




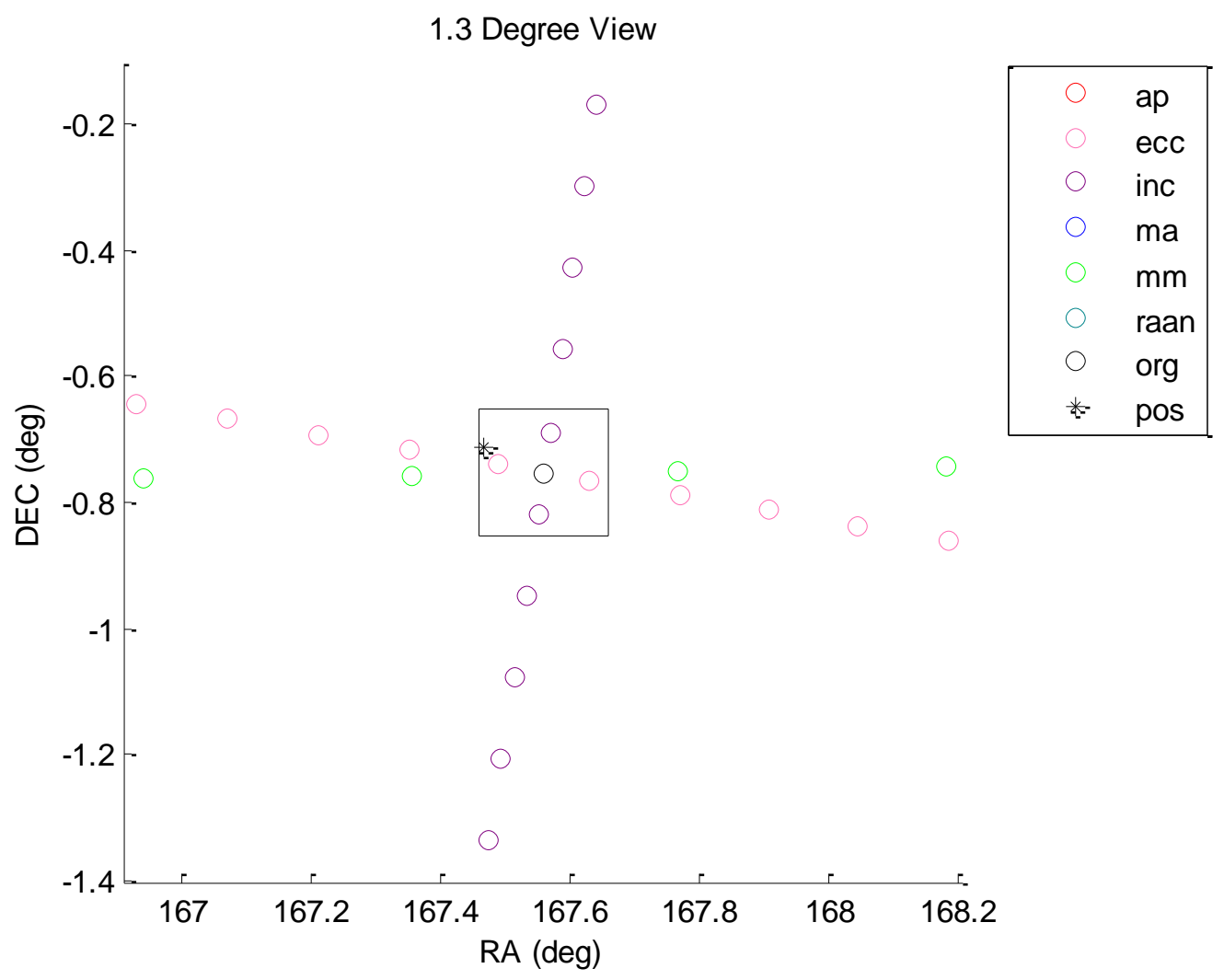




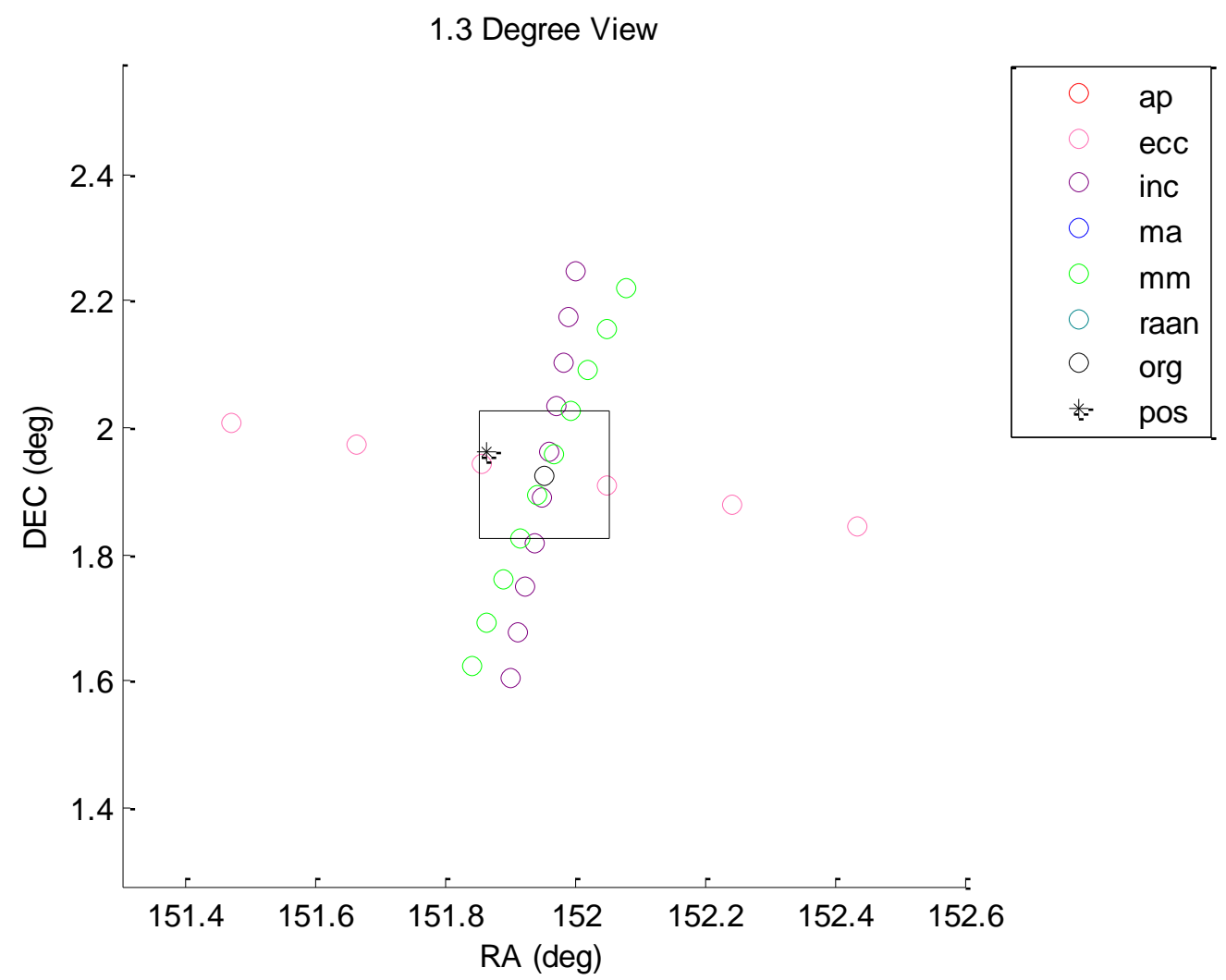


1.3 Degree View

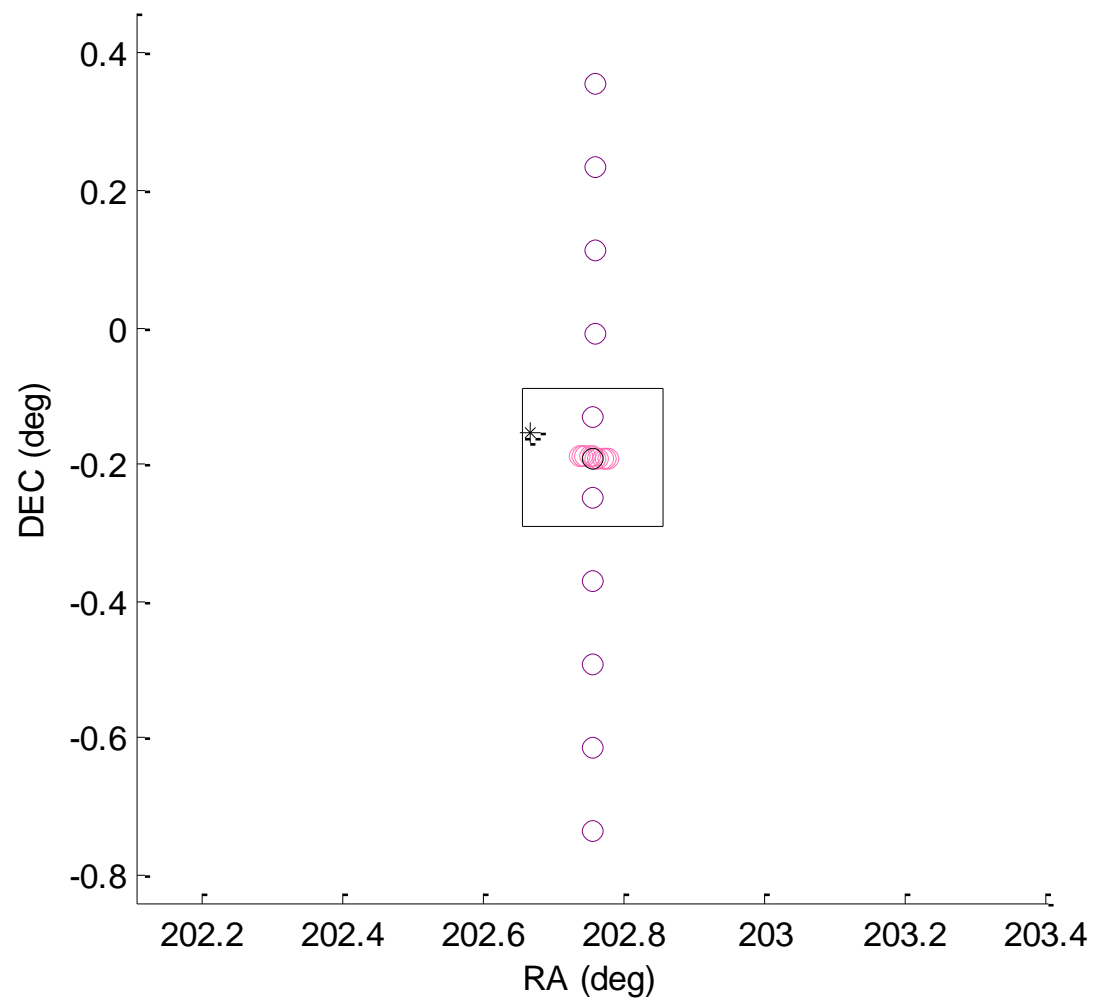

\begin{tabular}{|ll|}
\hline ap \\
ecc \\
inc \\
ma \\
mm \\
raan \\
org \\
* pos \\
\hline
\end{tabular}




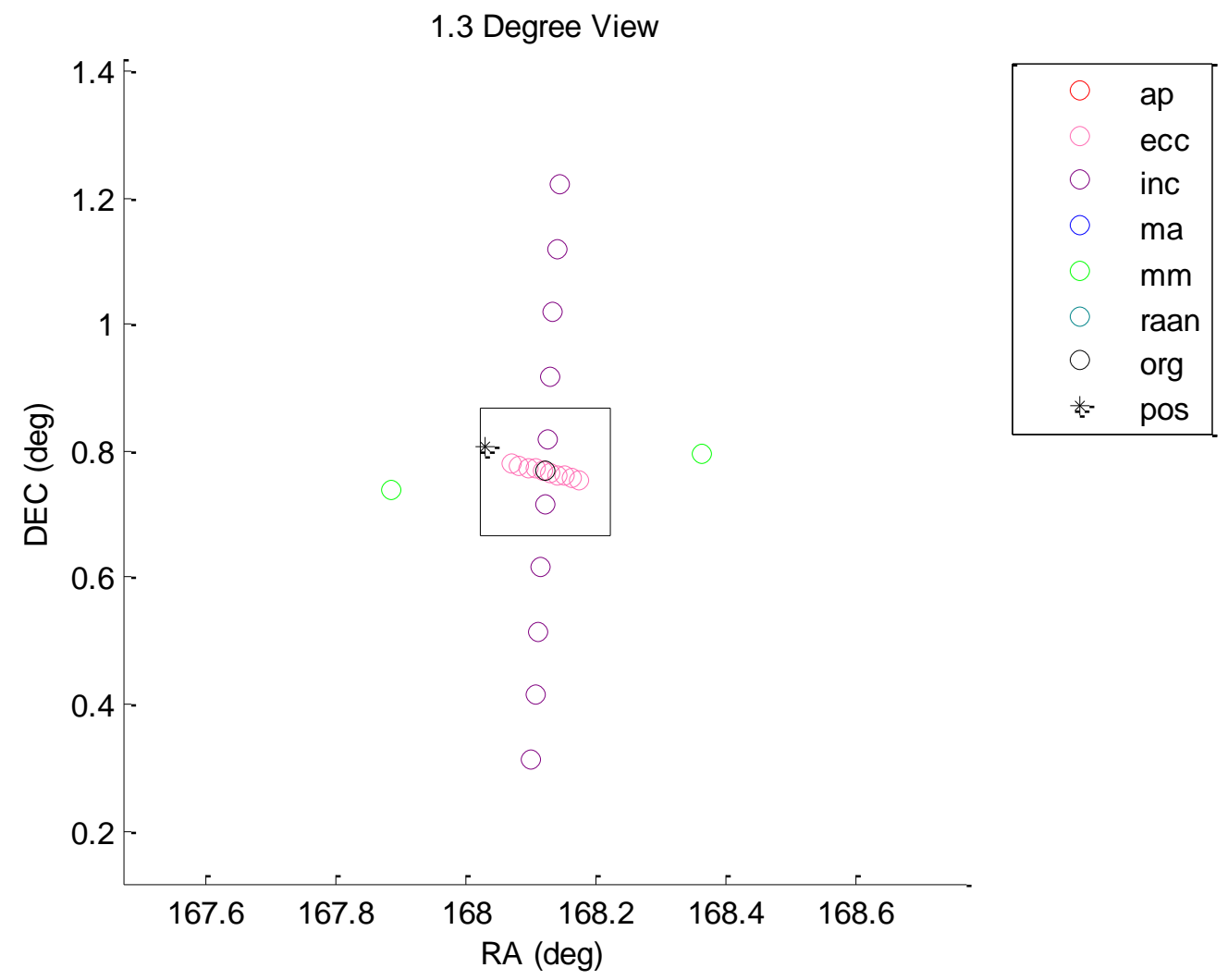




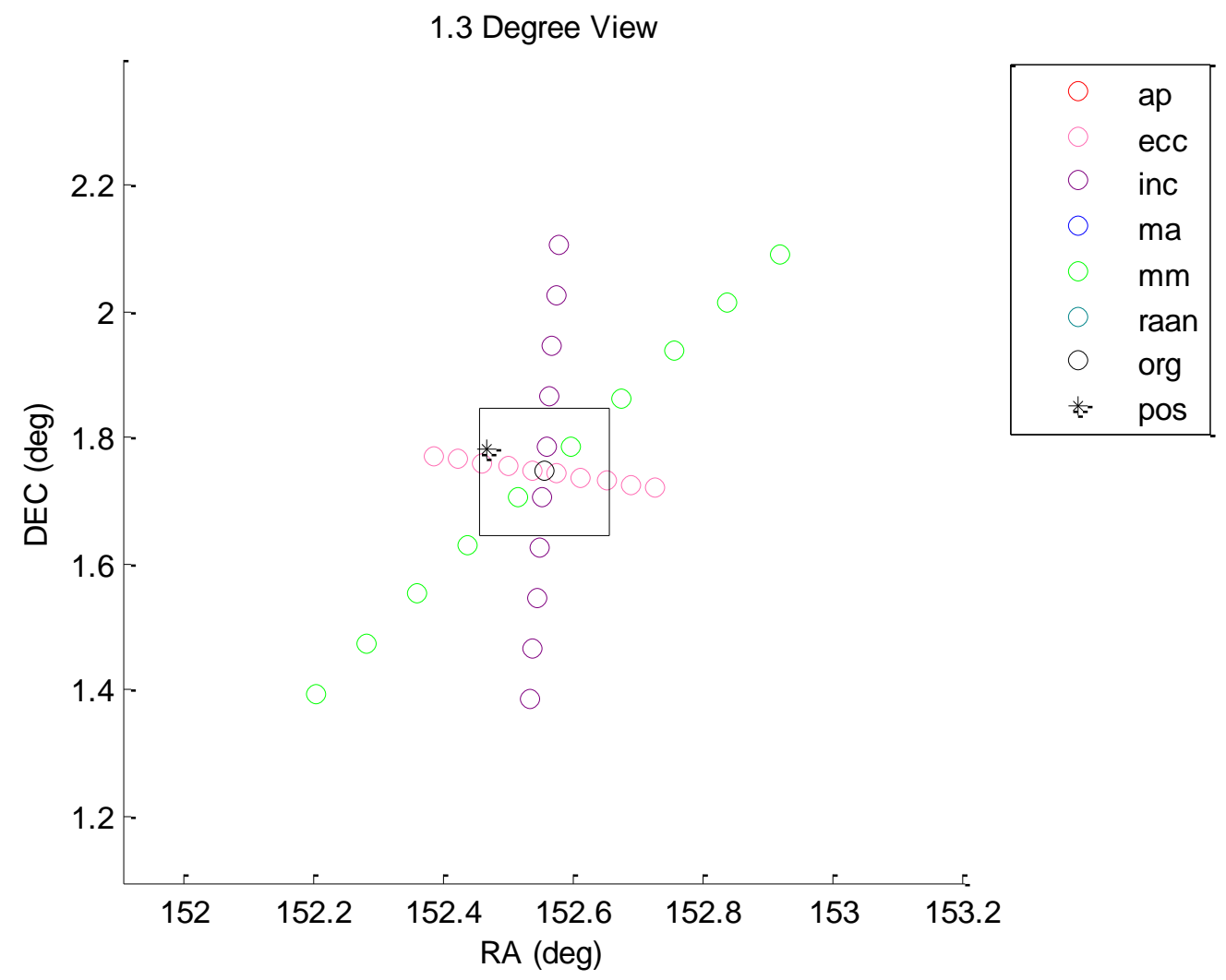




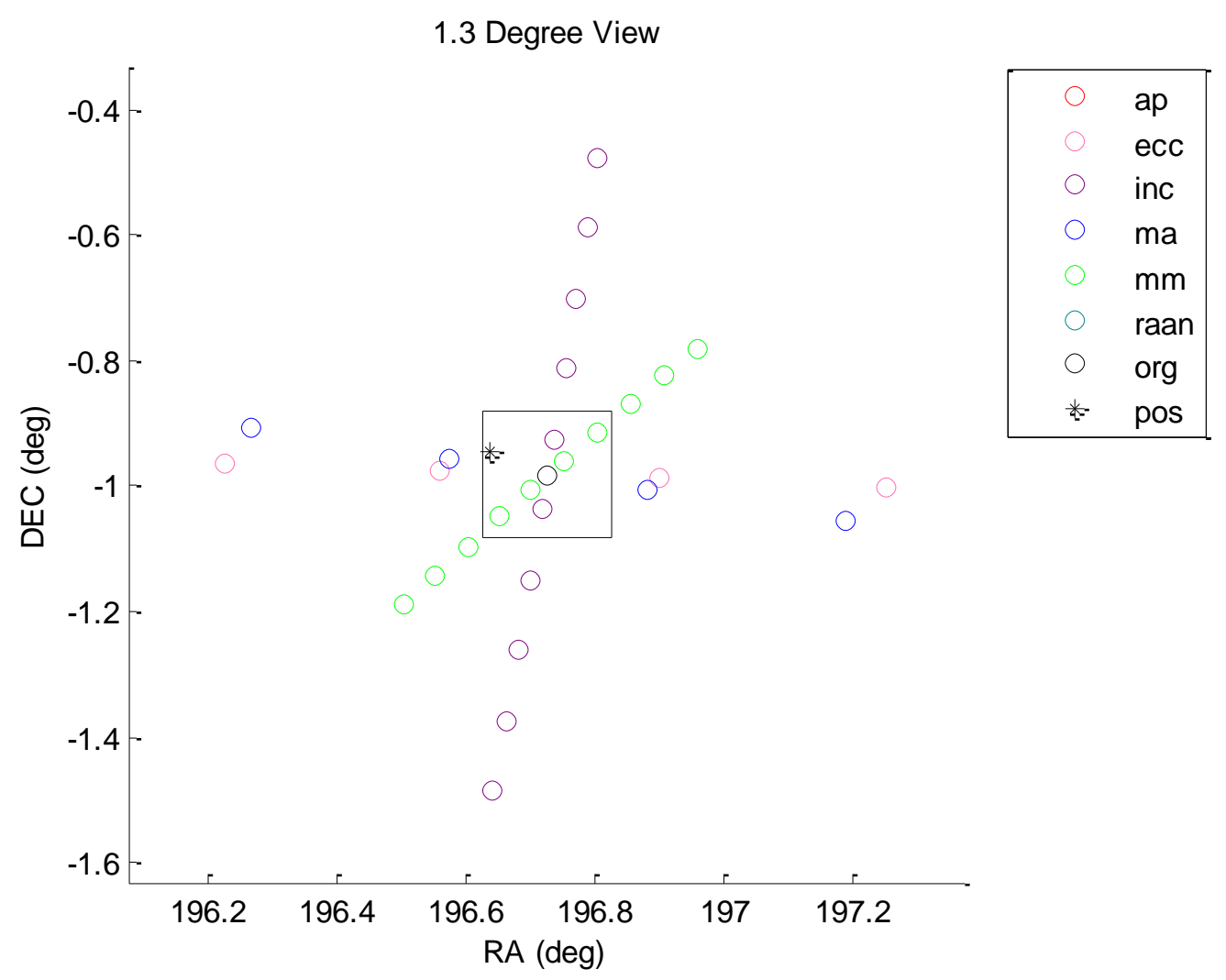




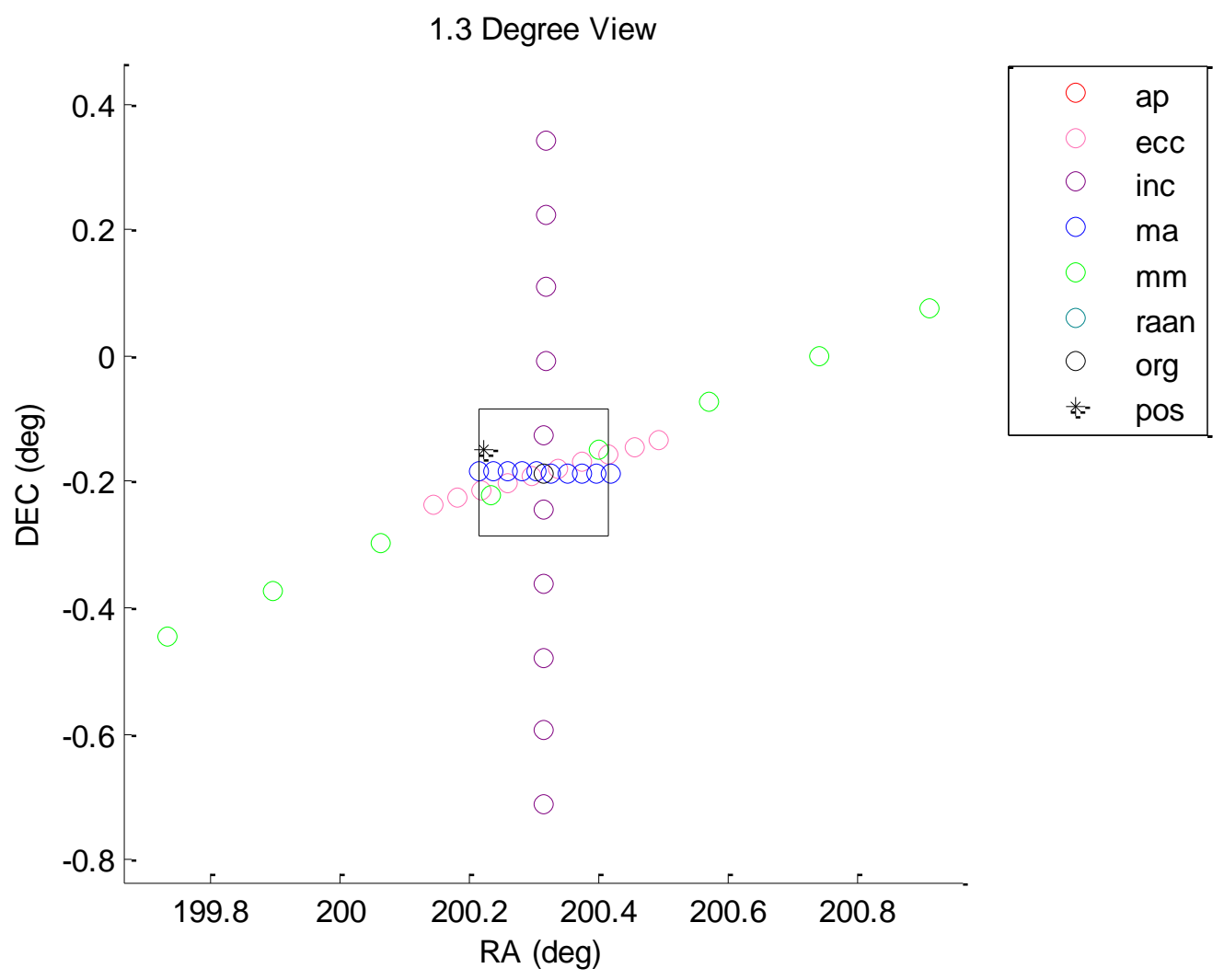




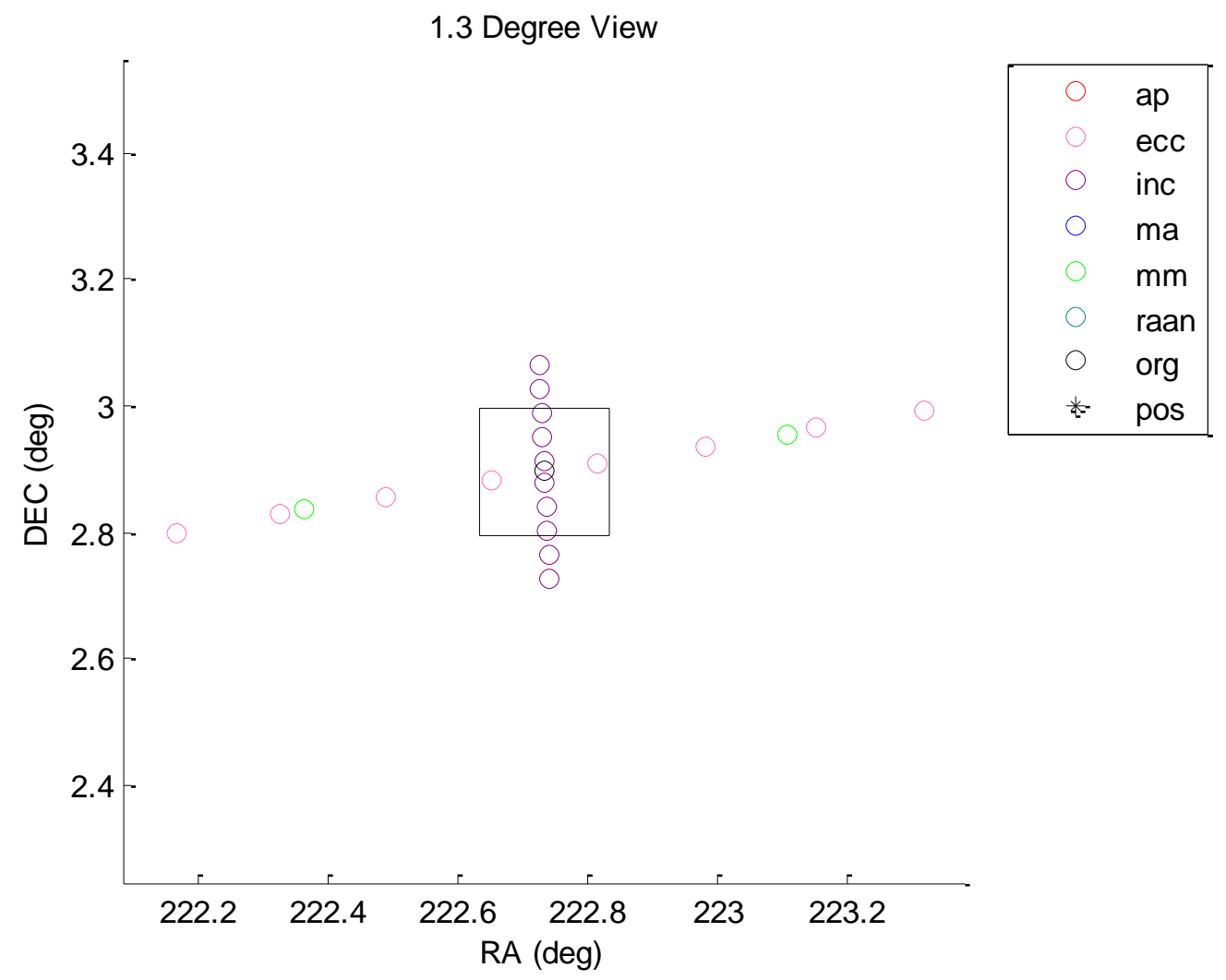


1.3 Degree View

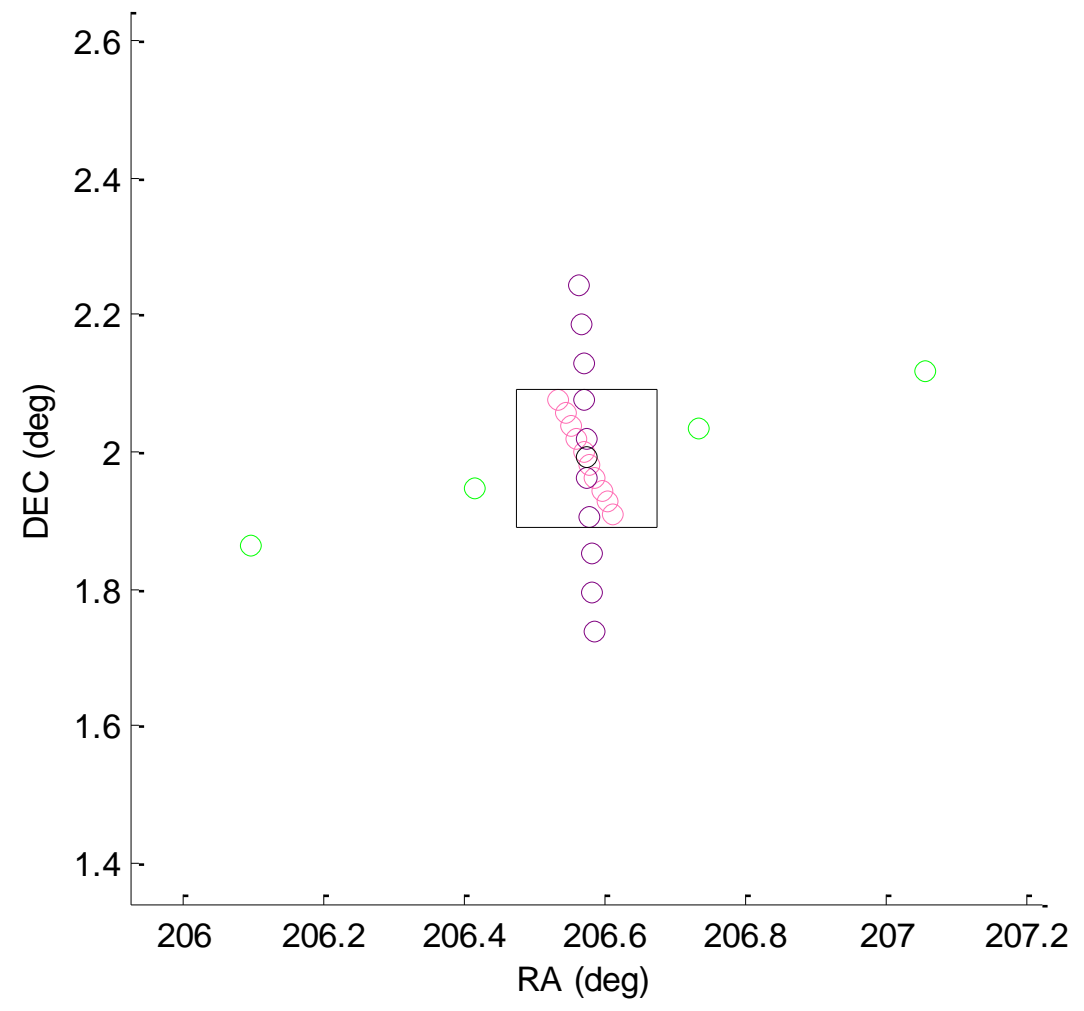

\begin{tabular}{|ll|}
\hline ap \\
ecc \\
inc \\
ma \\
mm \\
raan \\
org \\
* pos \\
\hline
\end{tabular}




\subsection{Degree View}

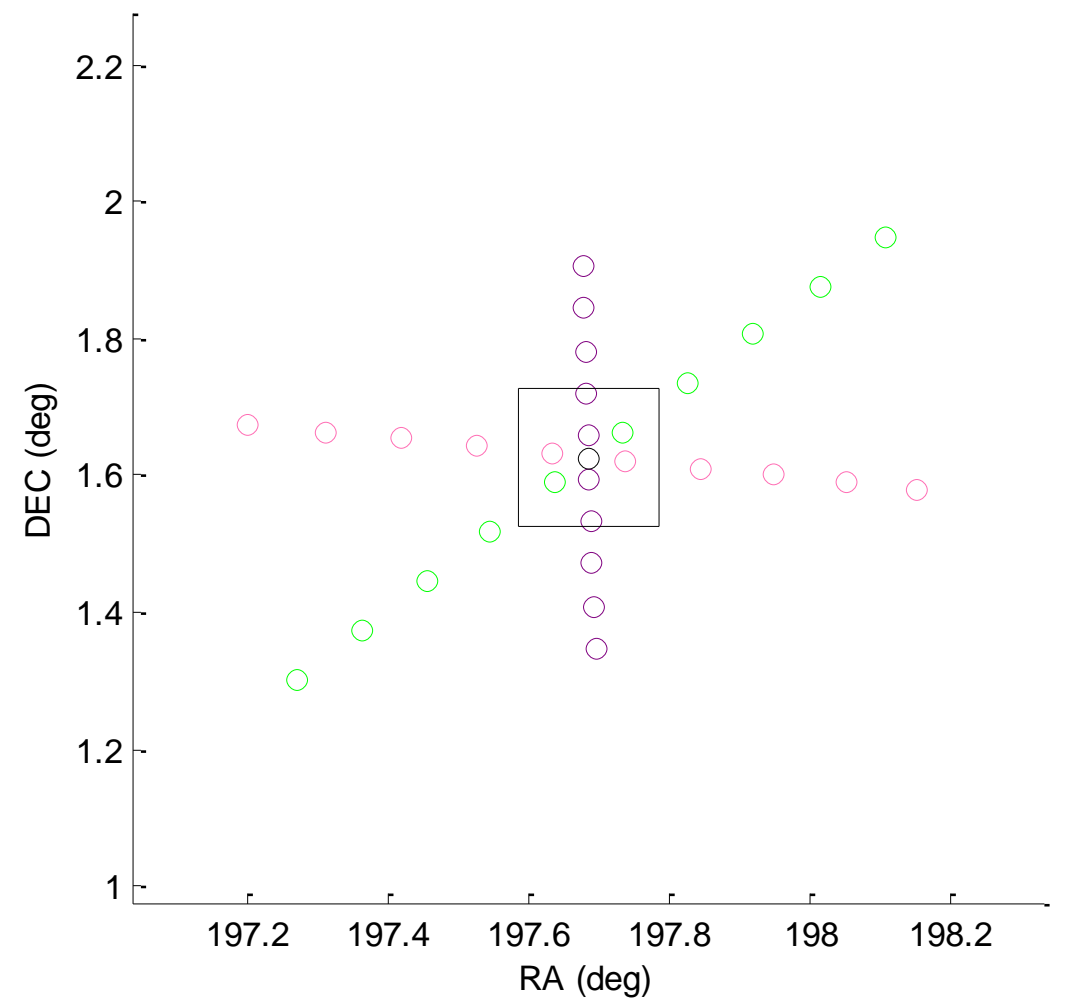

\begin{tabular}{|ll|}
\hline & ap \\
ecc \\
inc \\
ma \\
mm \\
raan \\
org \\
* pos \\
\hline
\end{tabular}

RA (deg) 


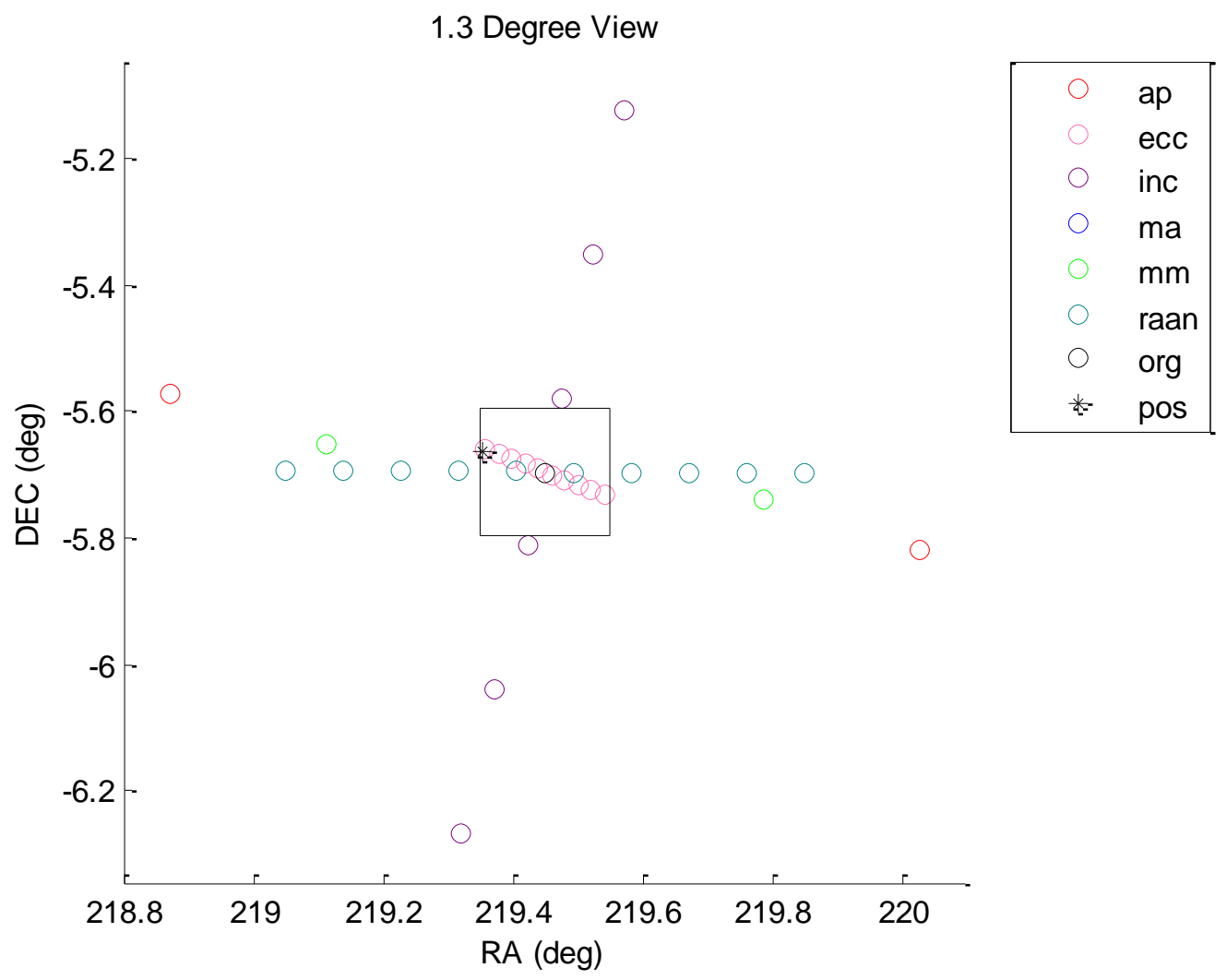




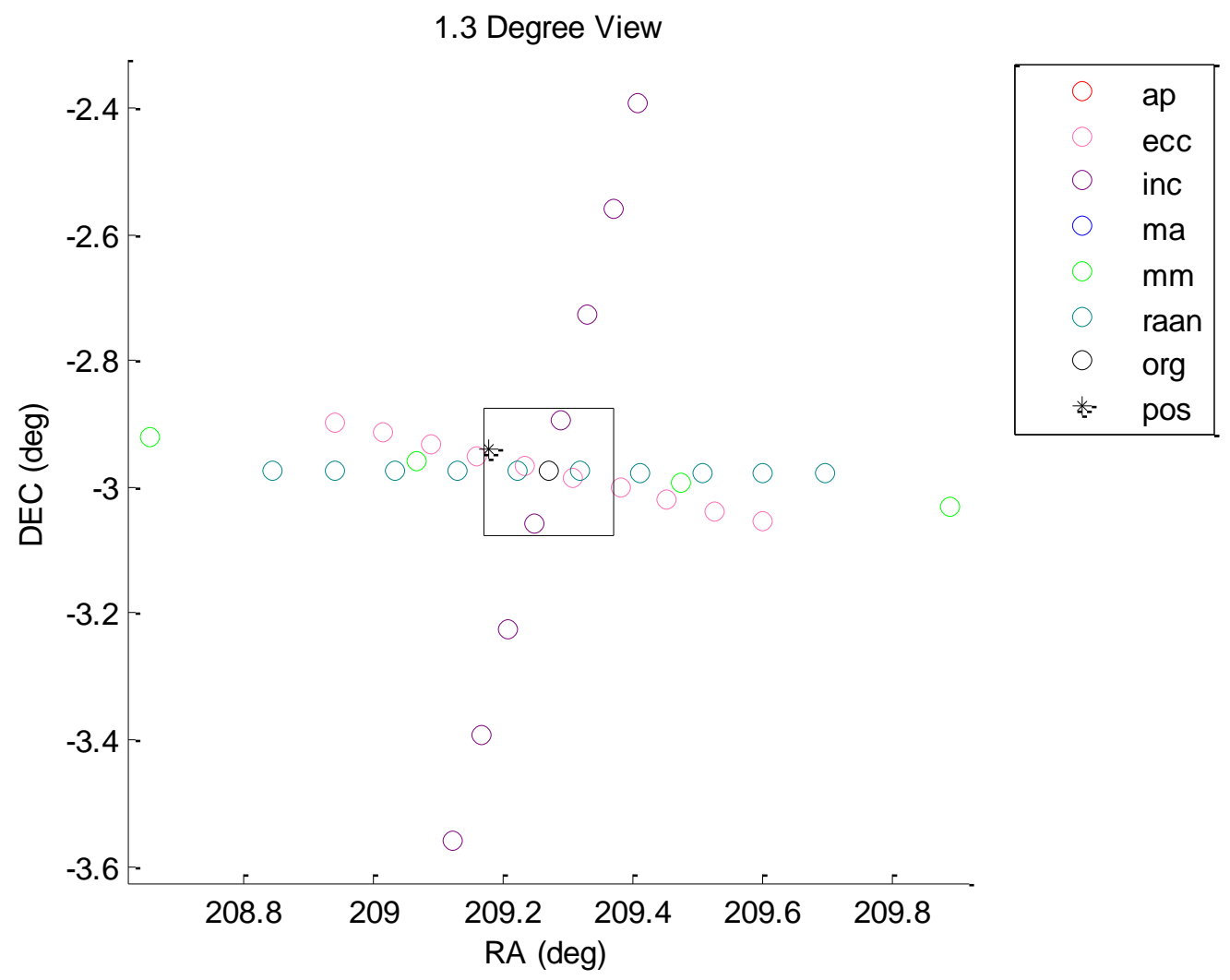




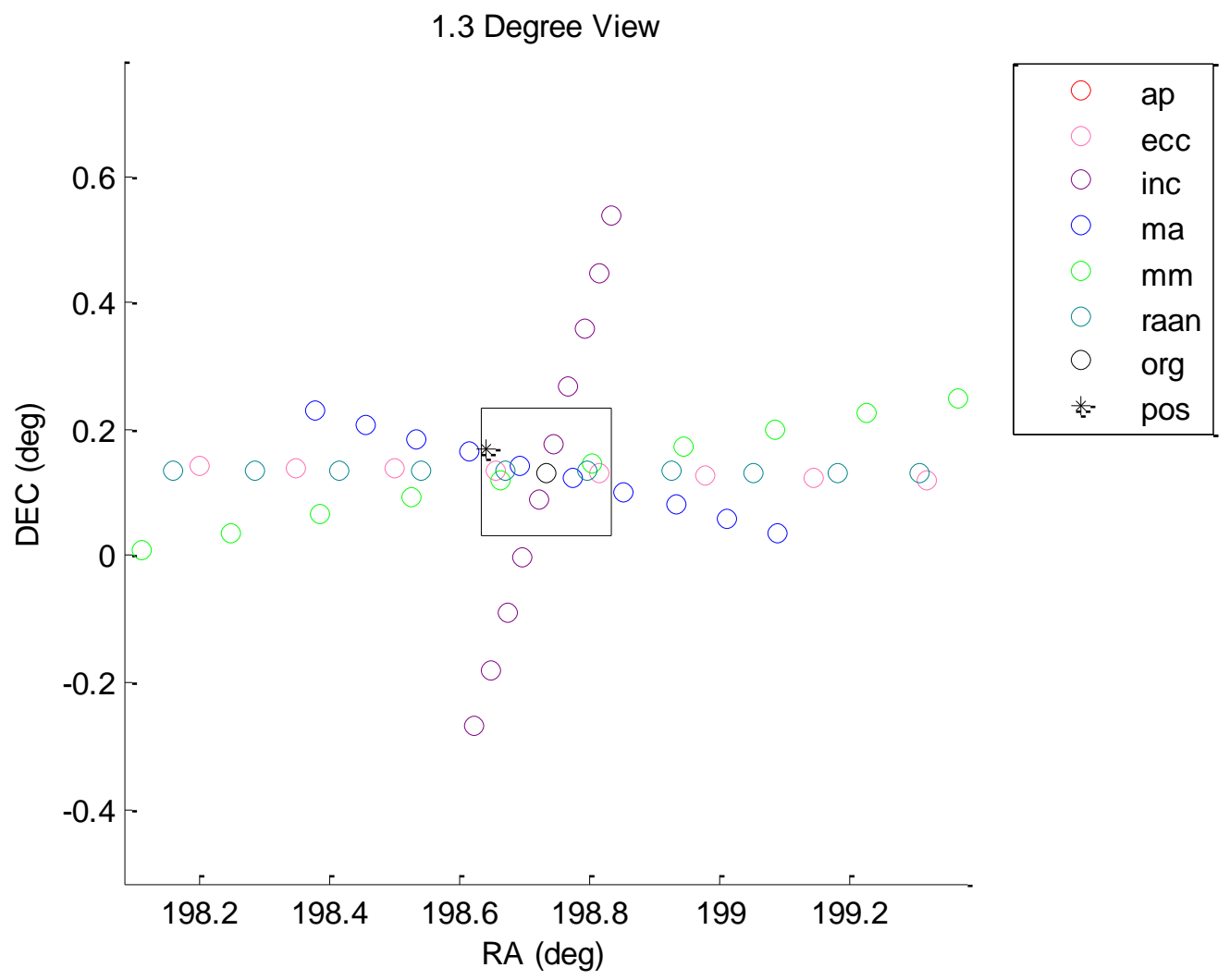




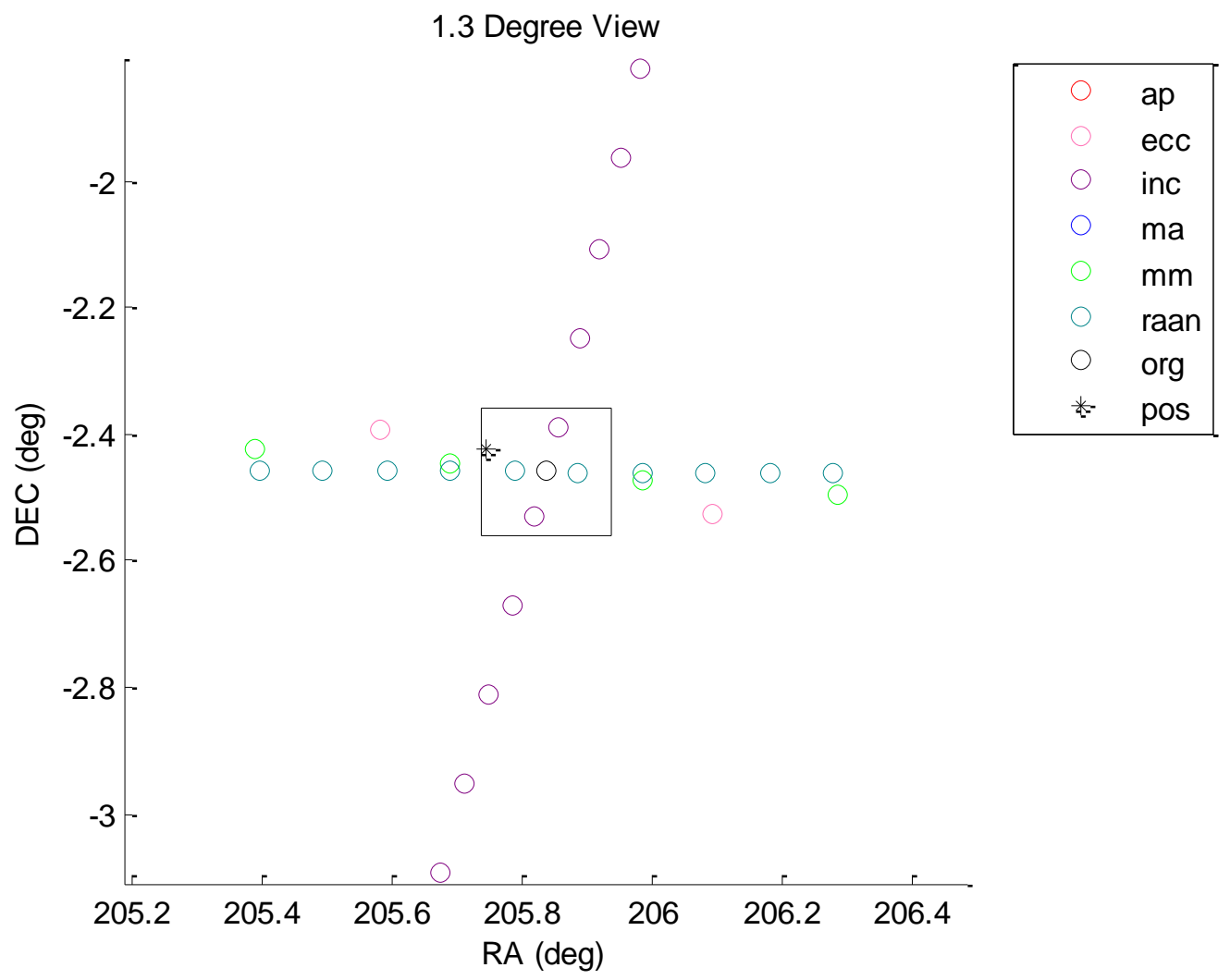




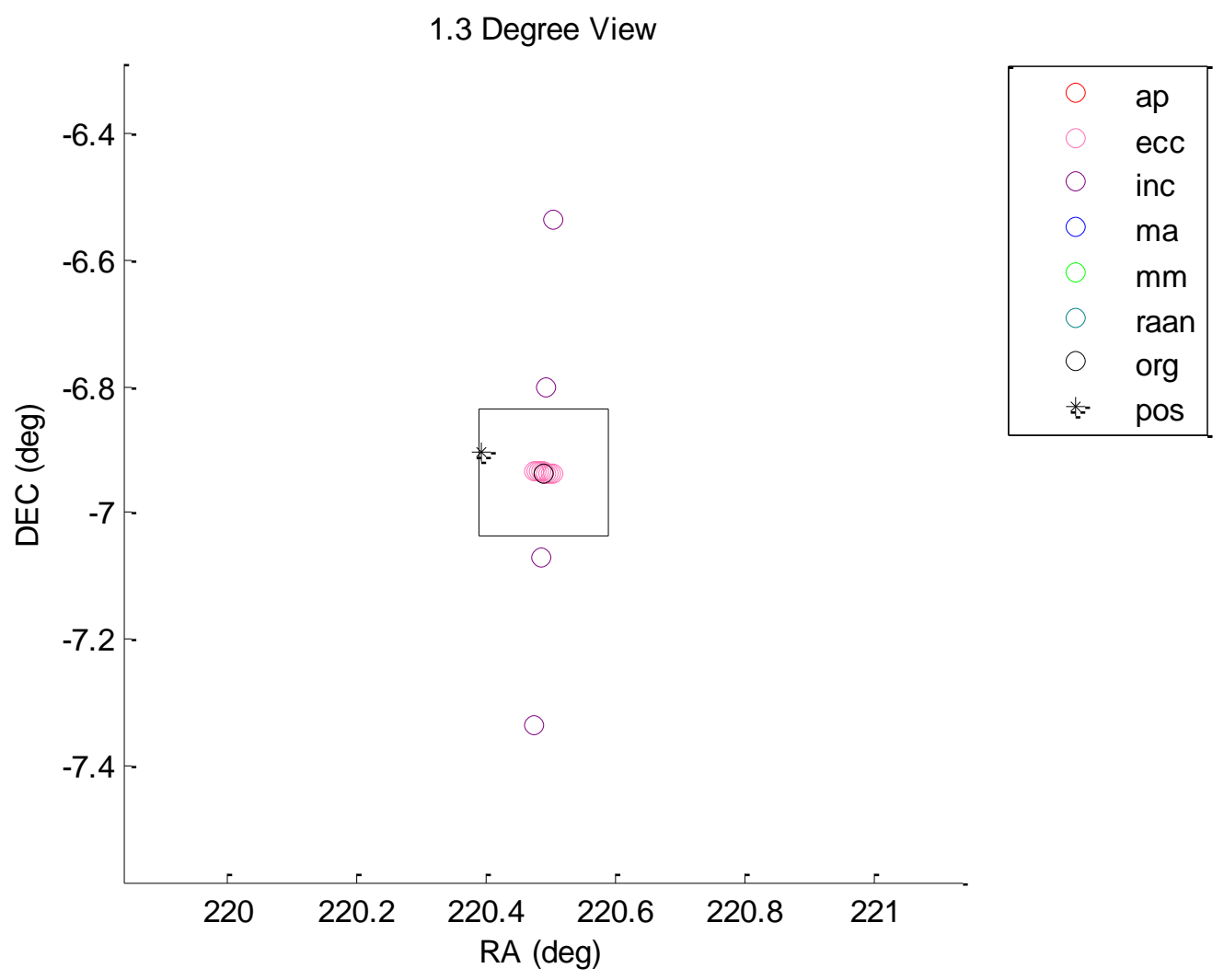




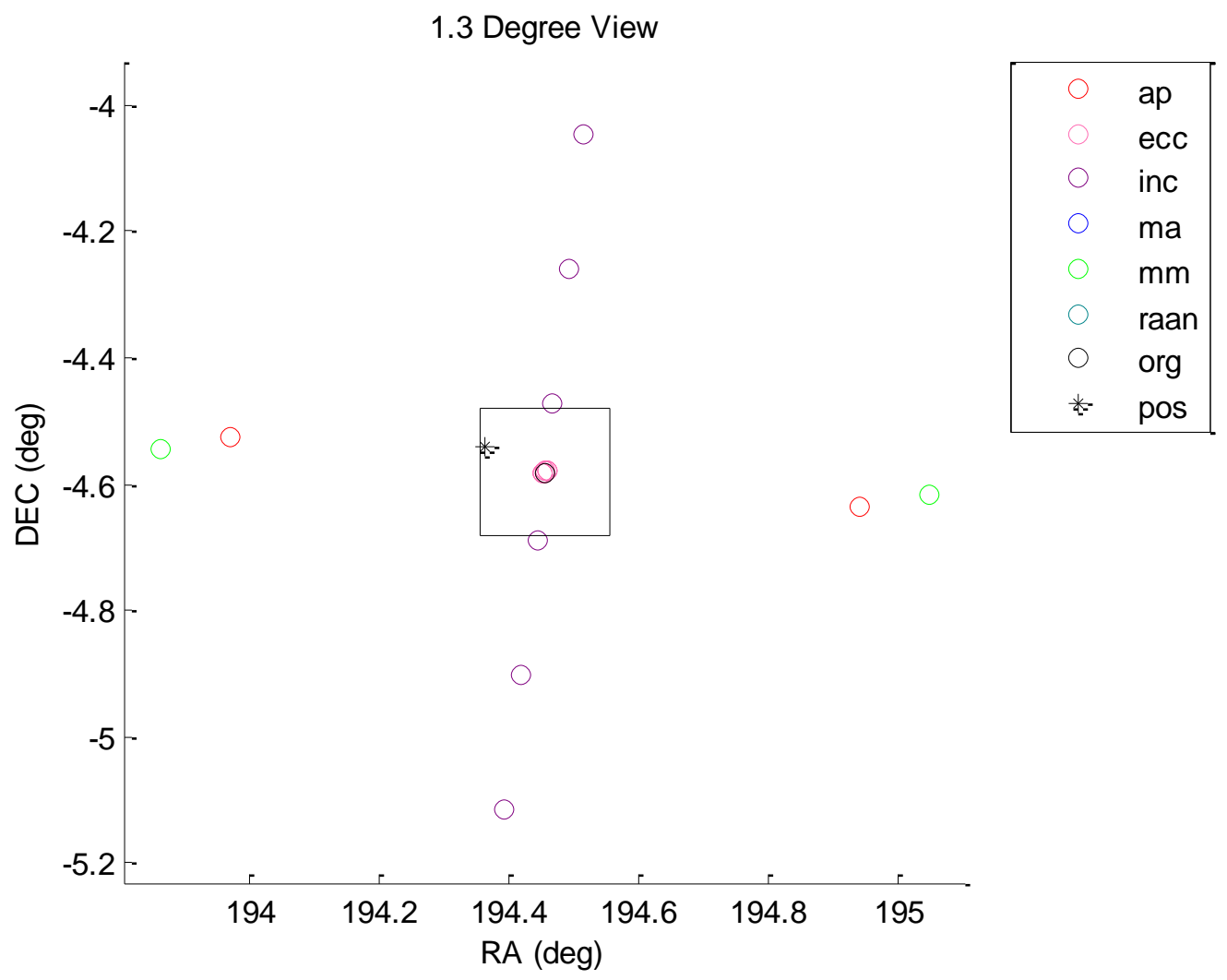




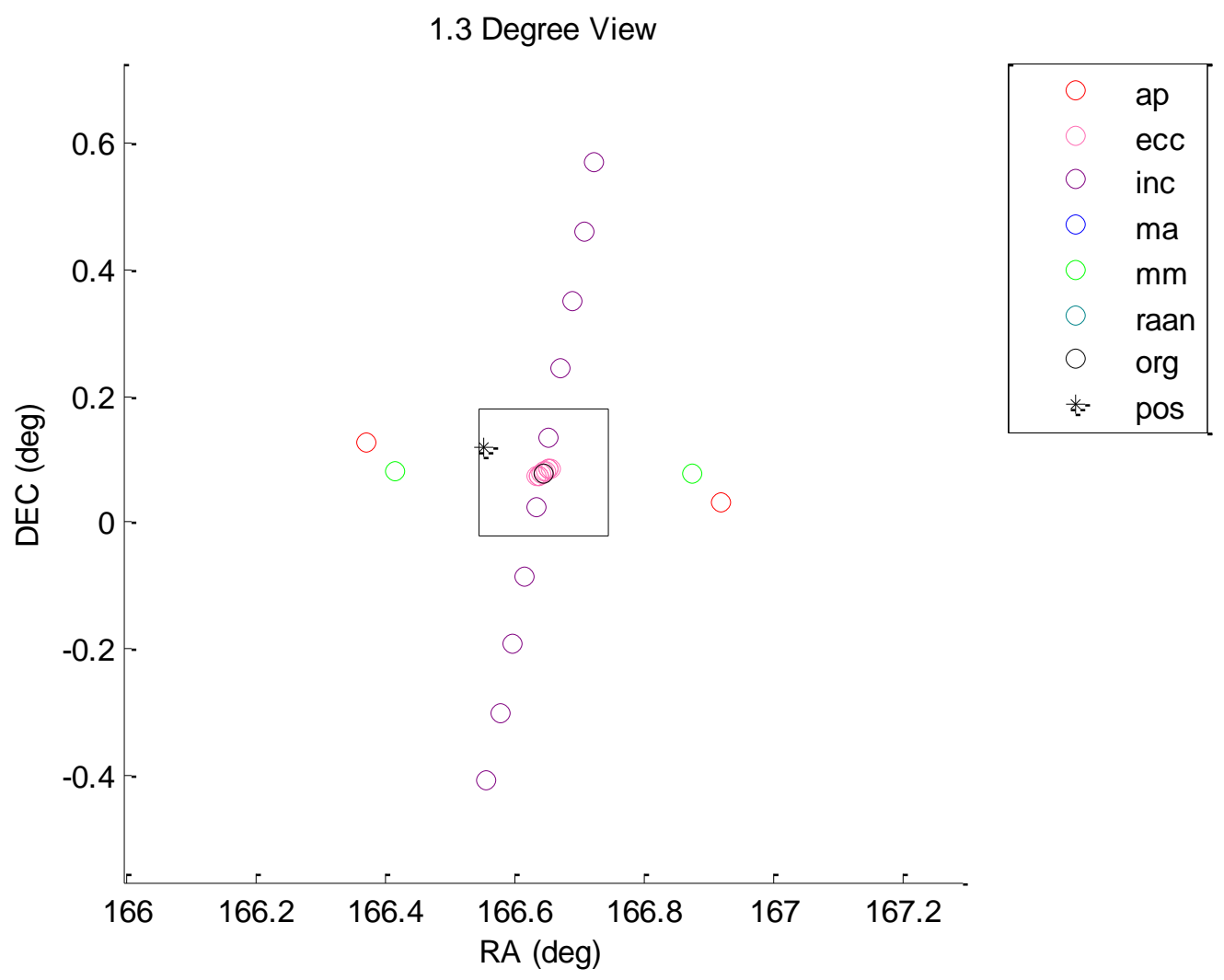




\subsection{Degree View}

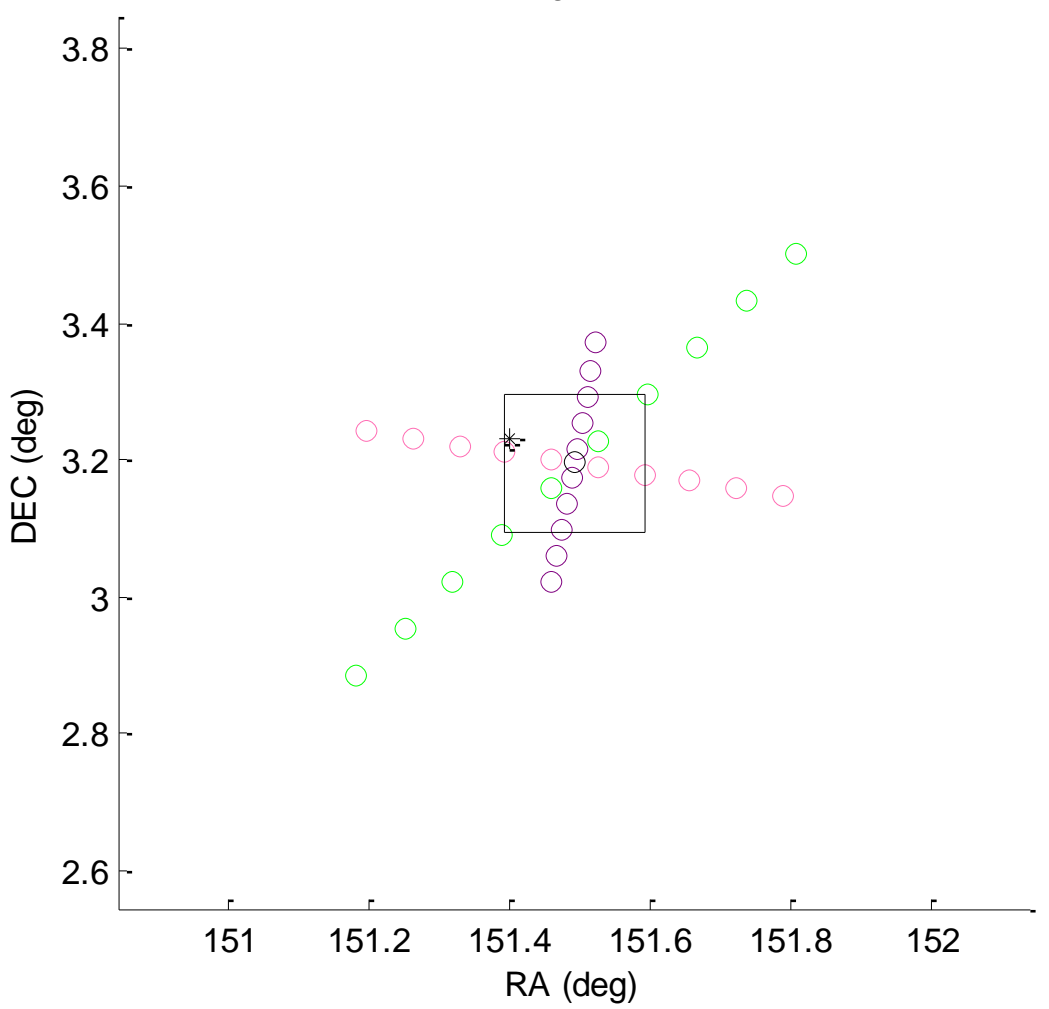

\begin{tabular}{|ll|}
\hline & ap \\
ecc \\
inc \\
ma \\
mm \\
raan \\
org \\
* pos \\
\hline
\end{tabular}




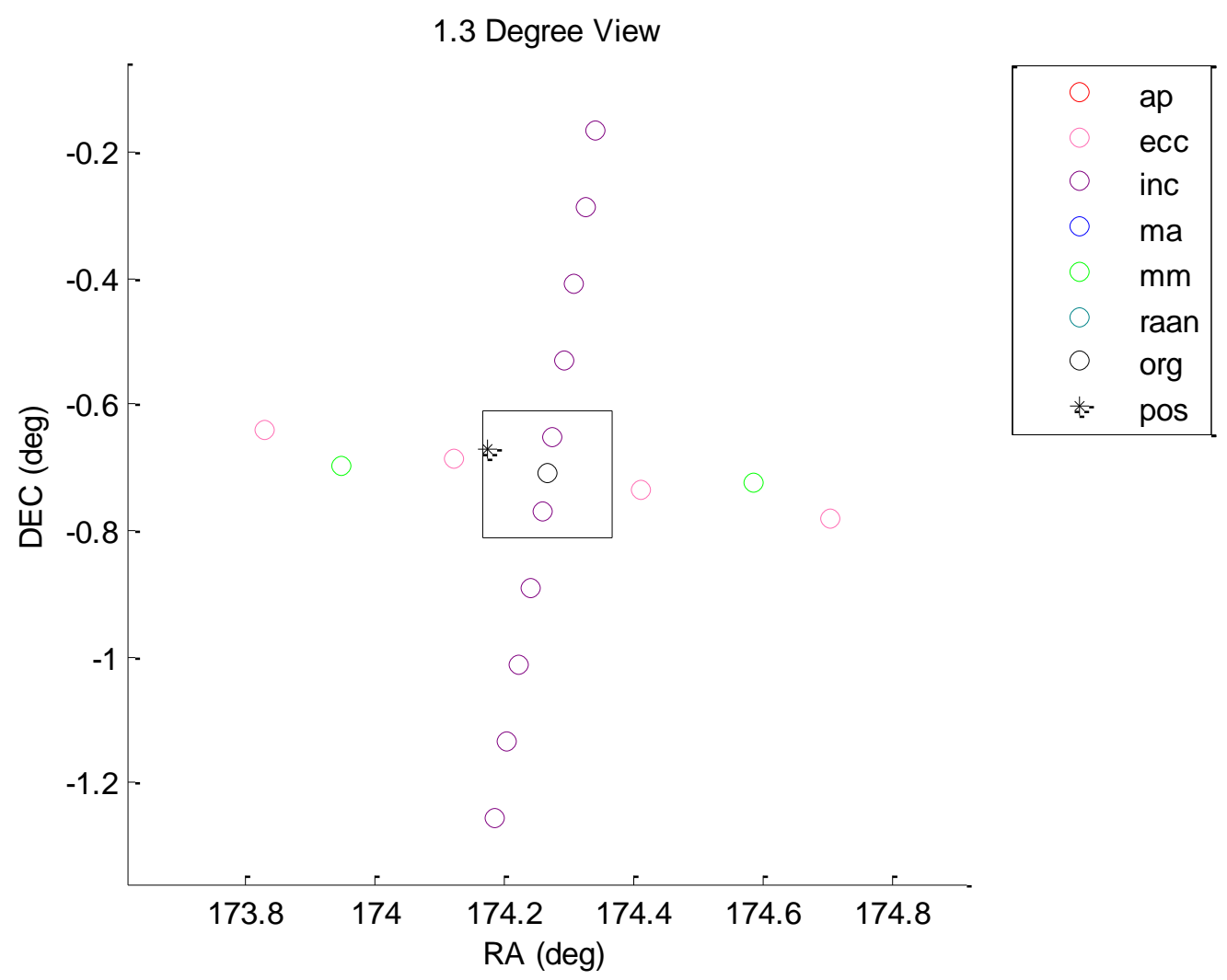




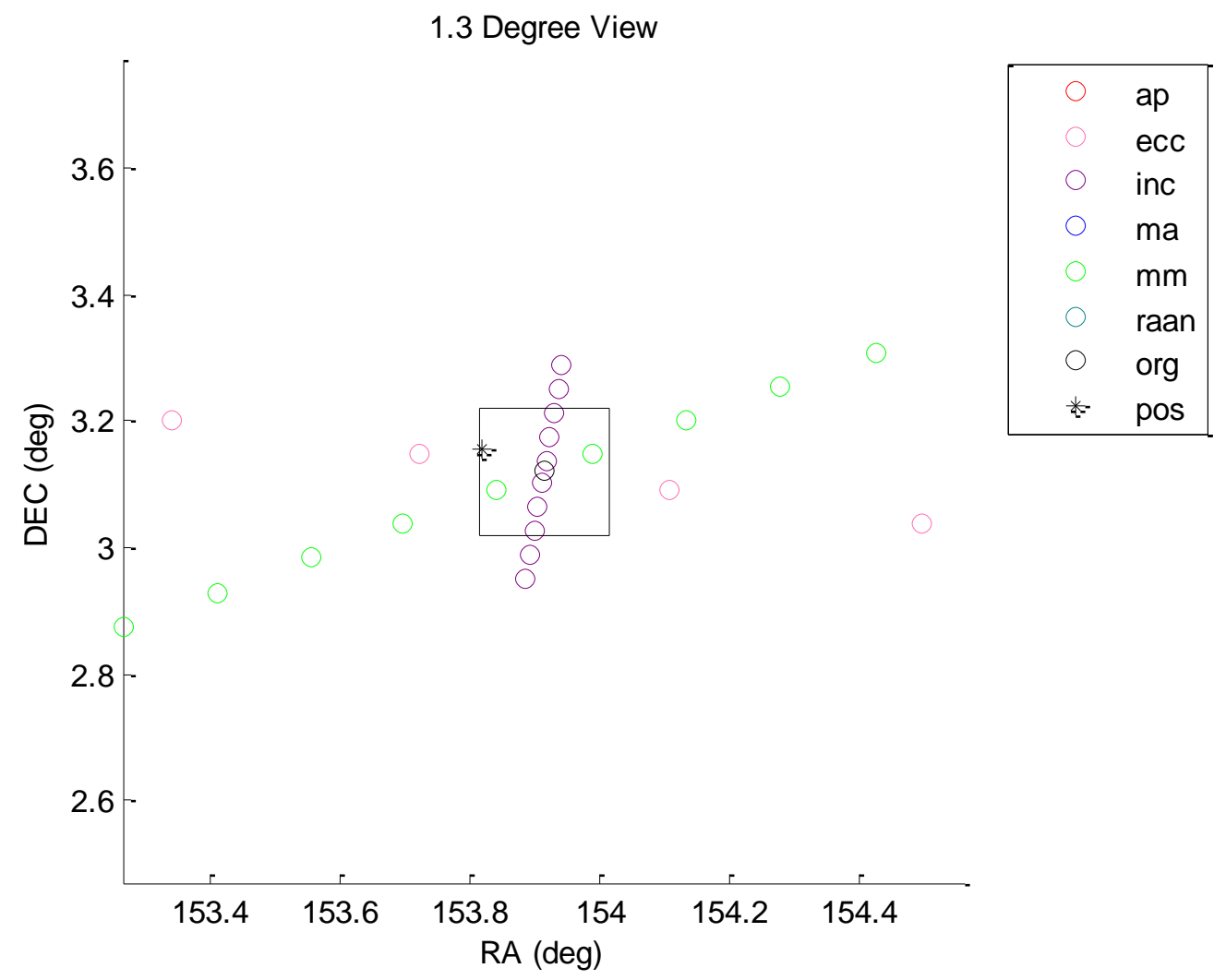




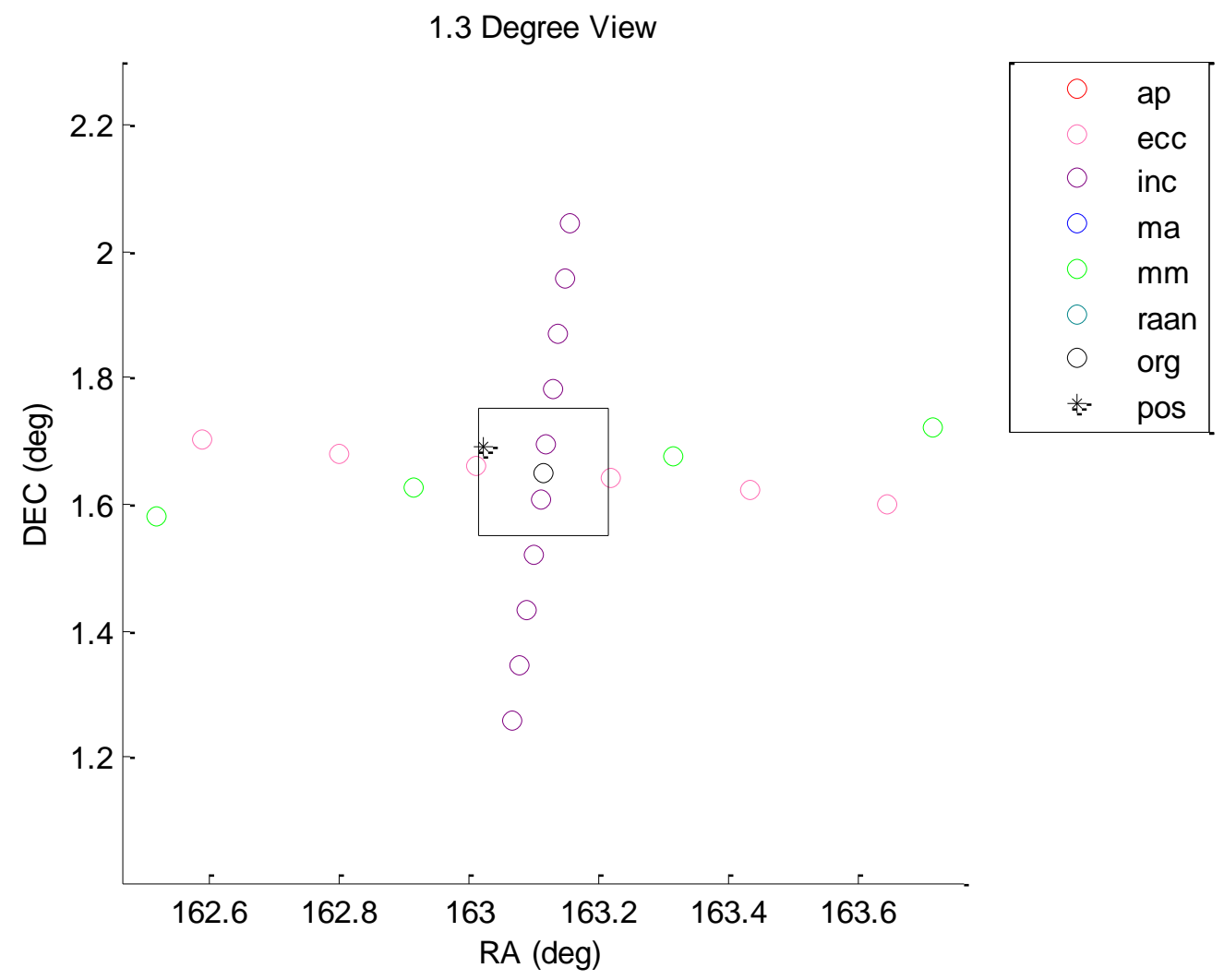




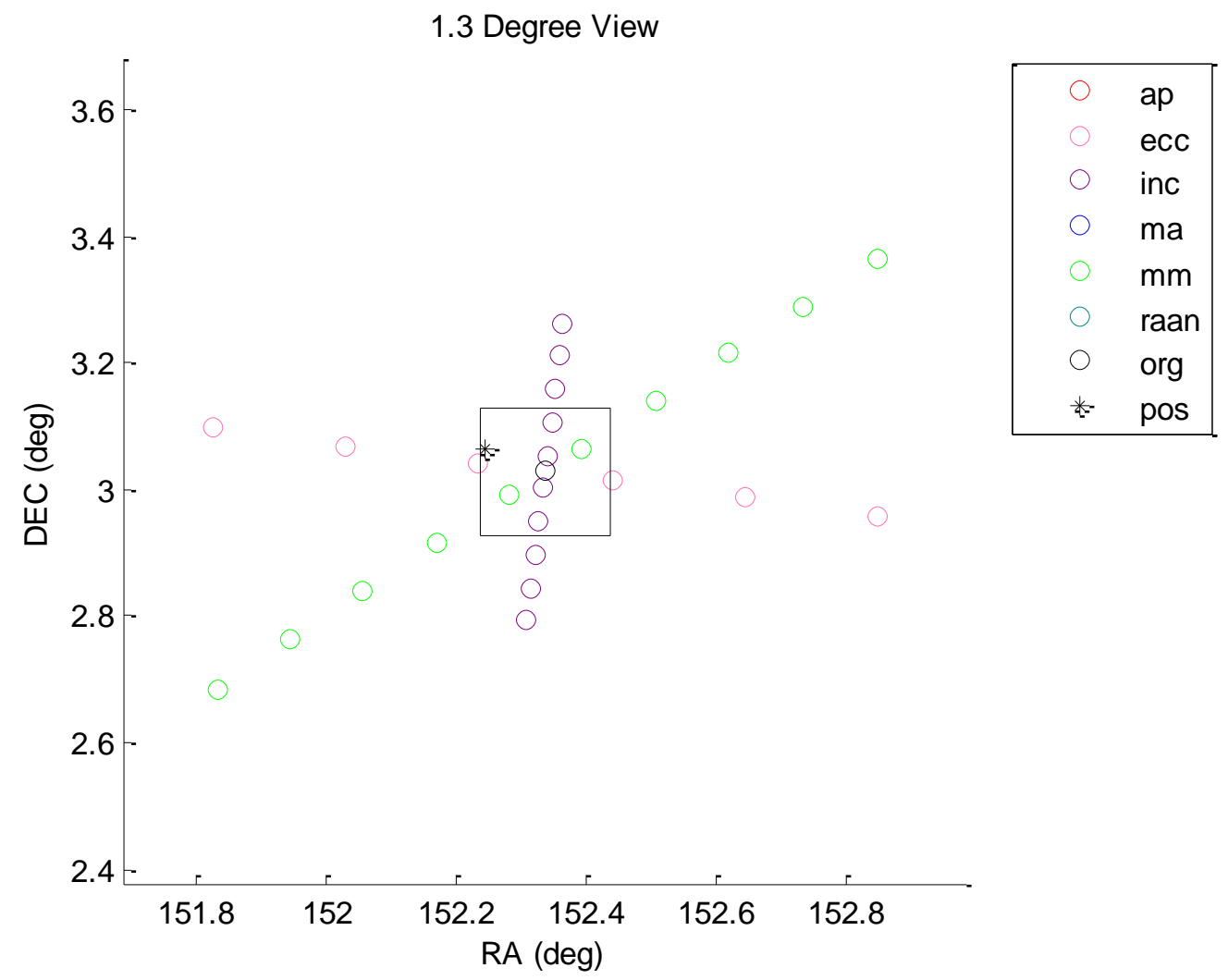




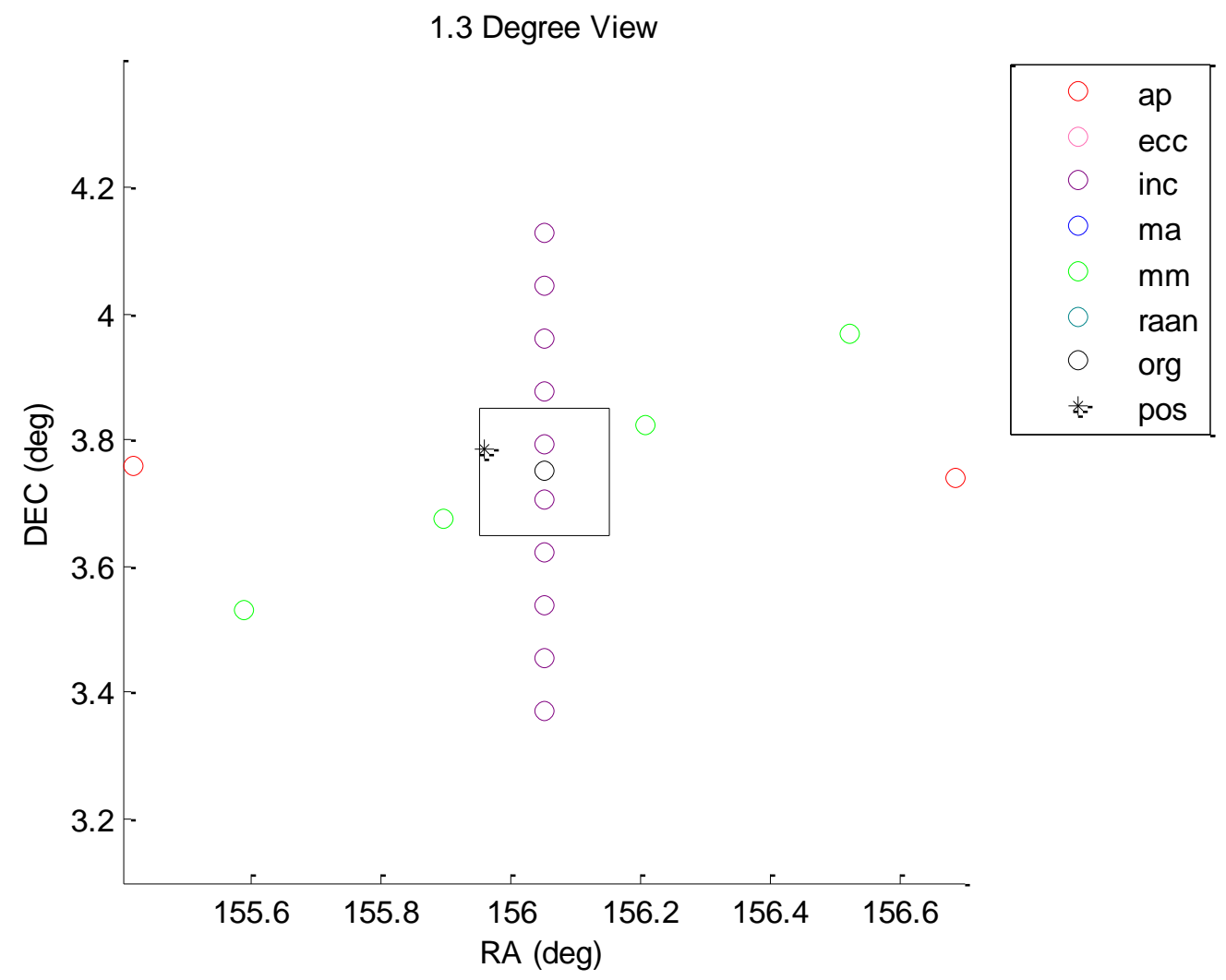




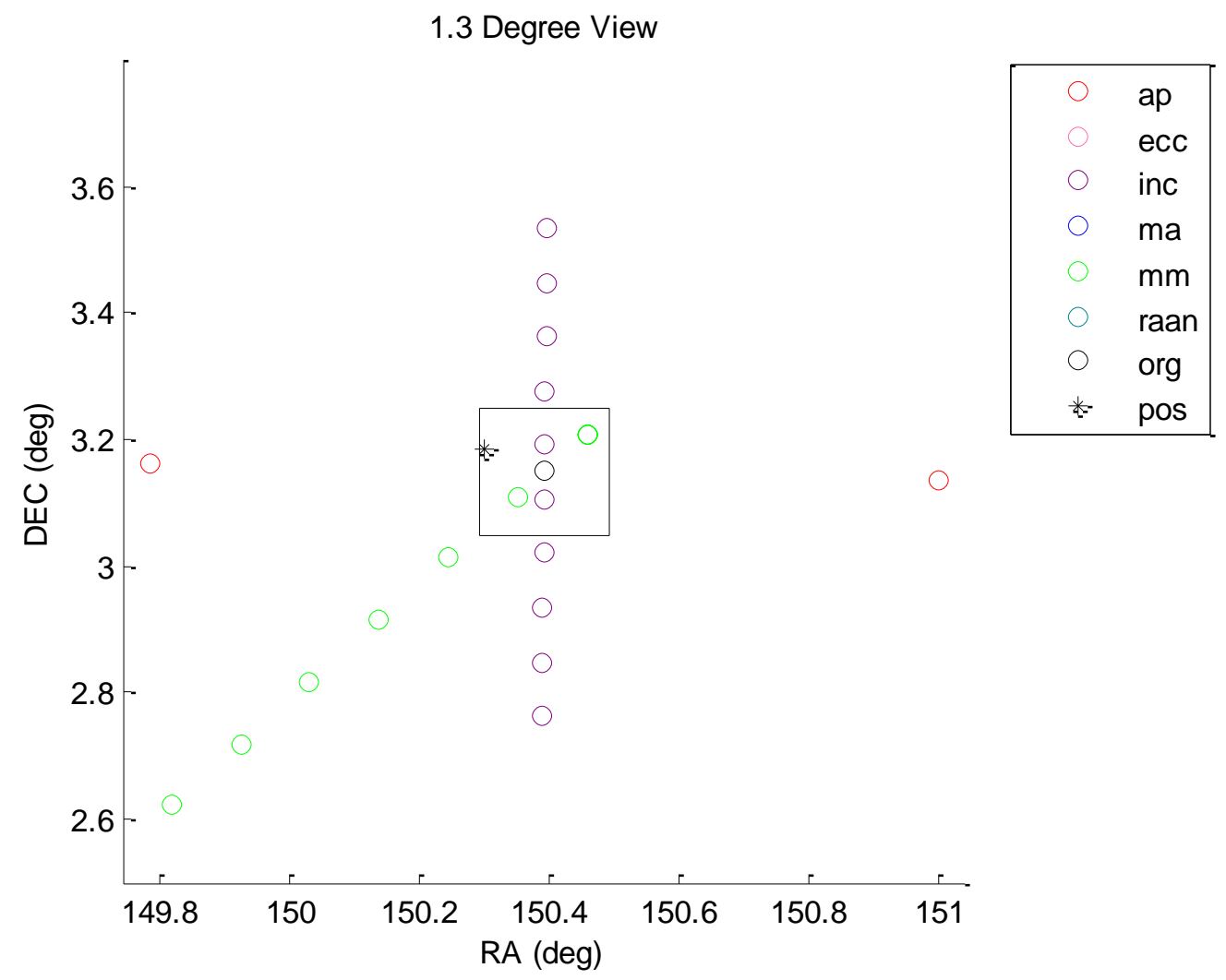




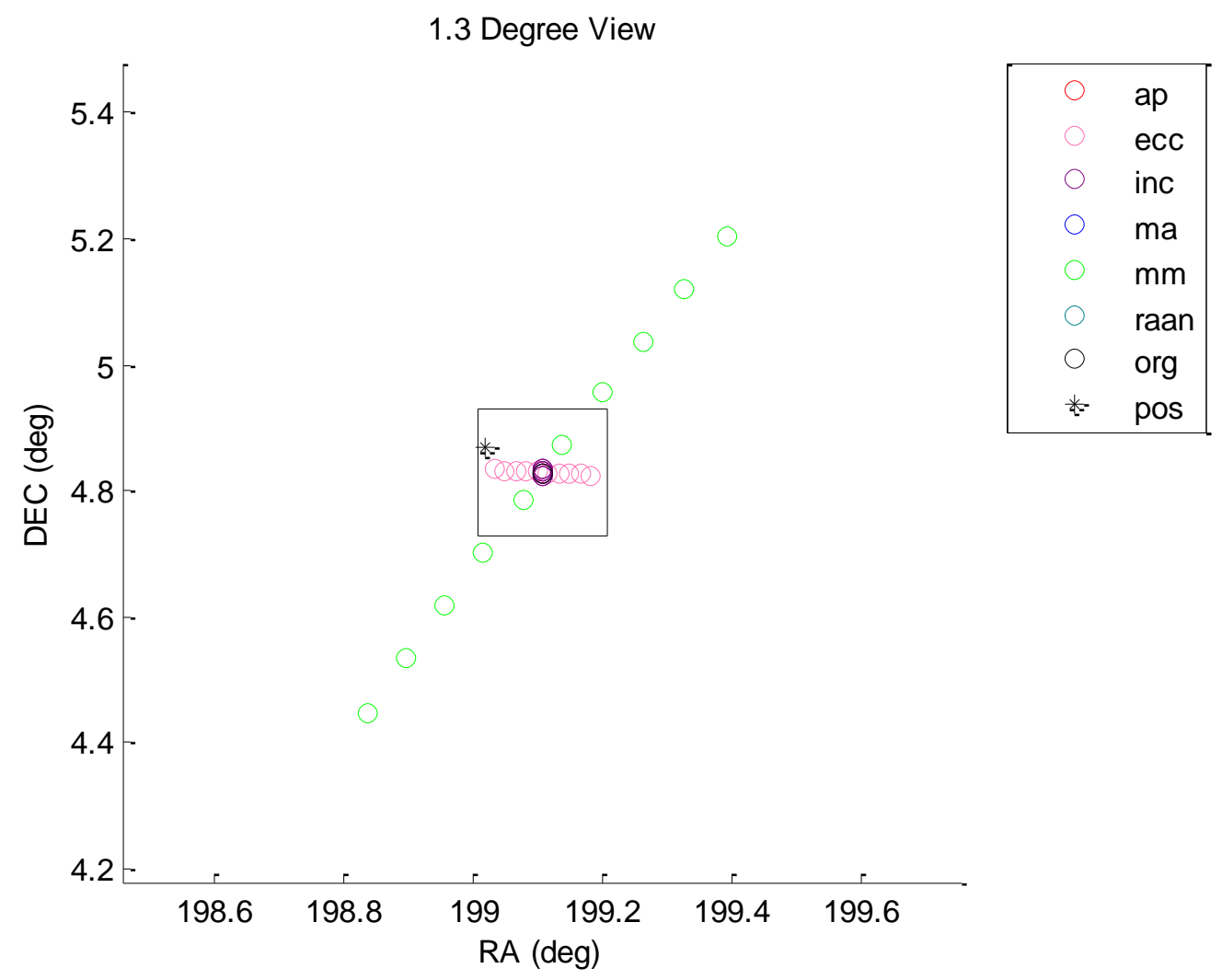




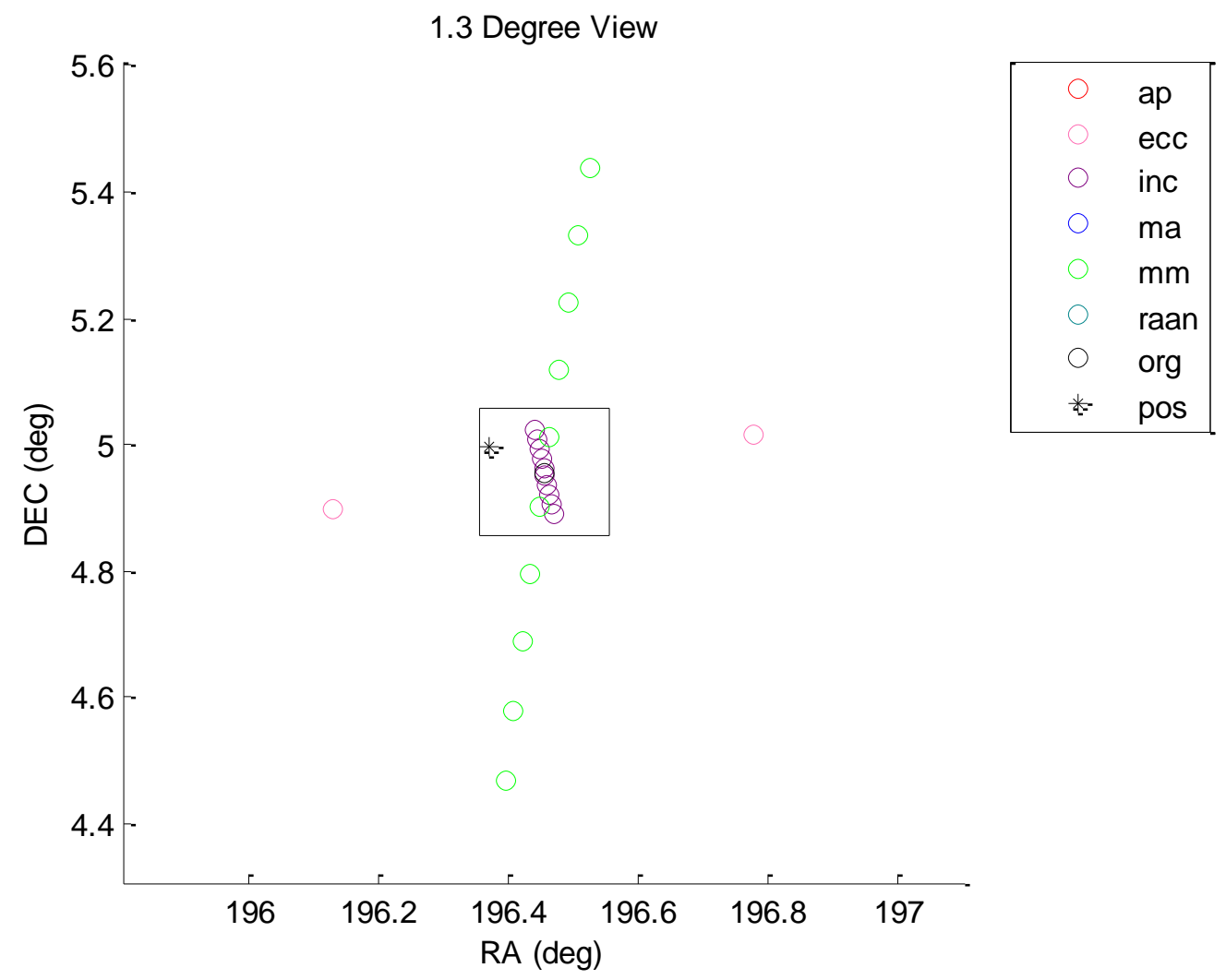




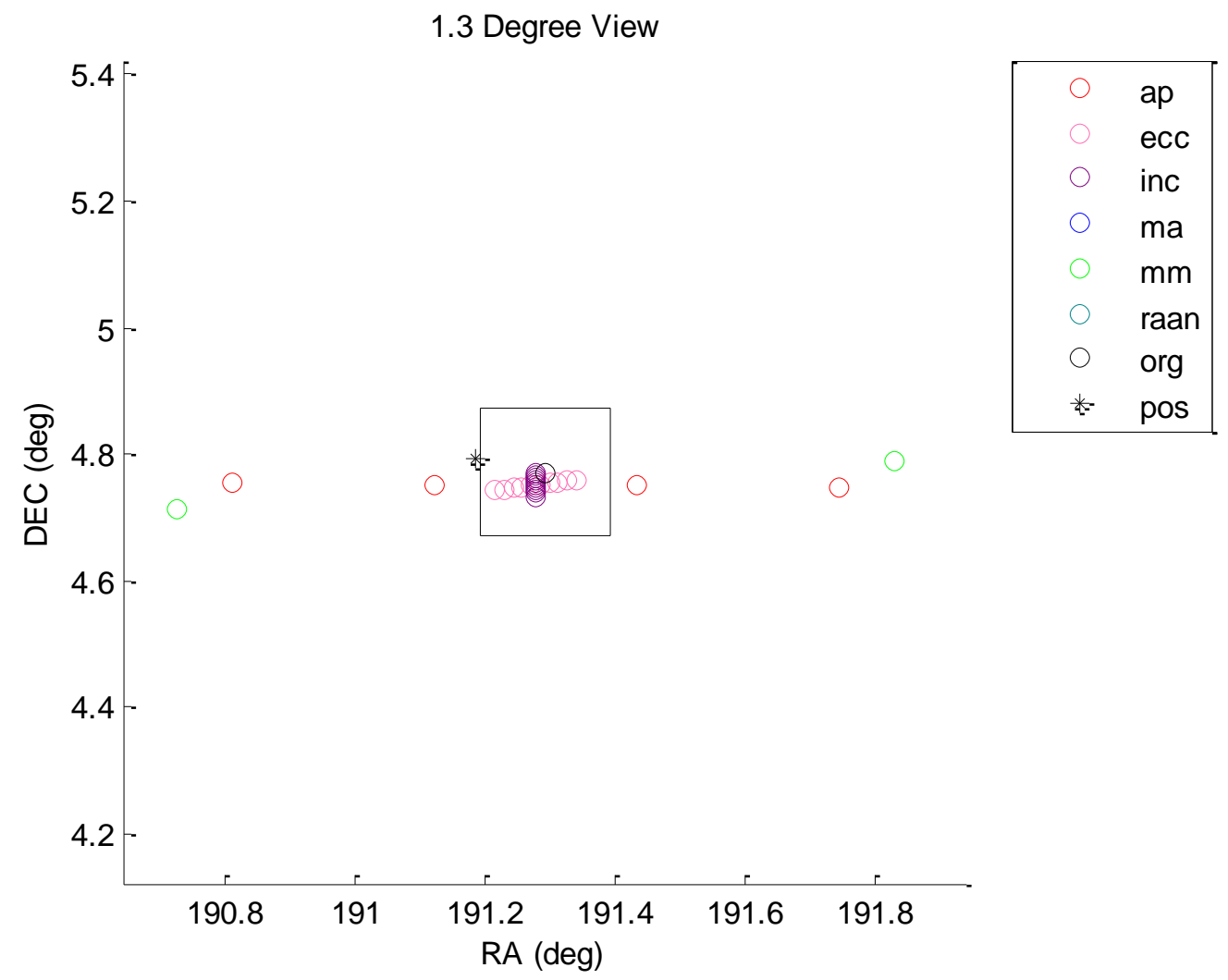


1.3 Degree View

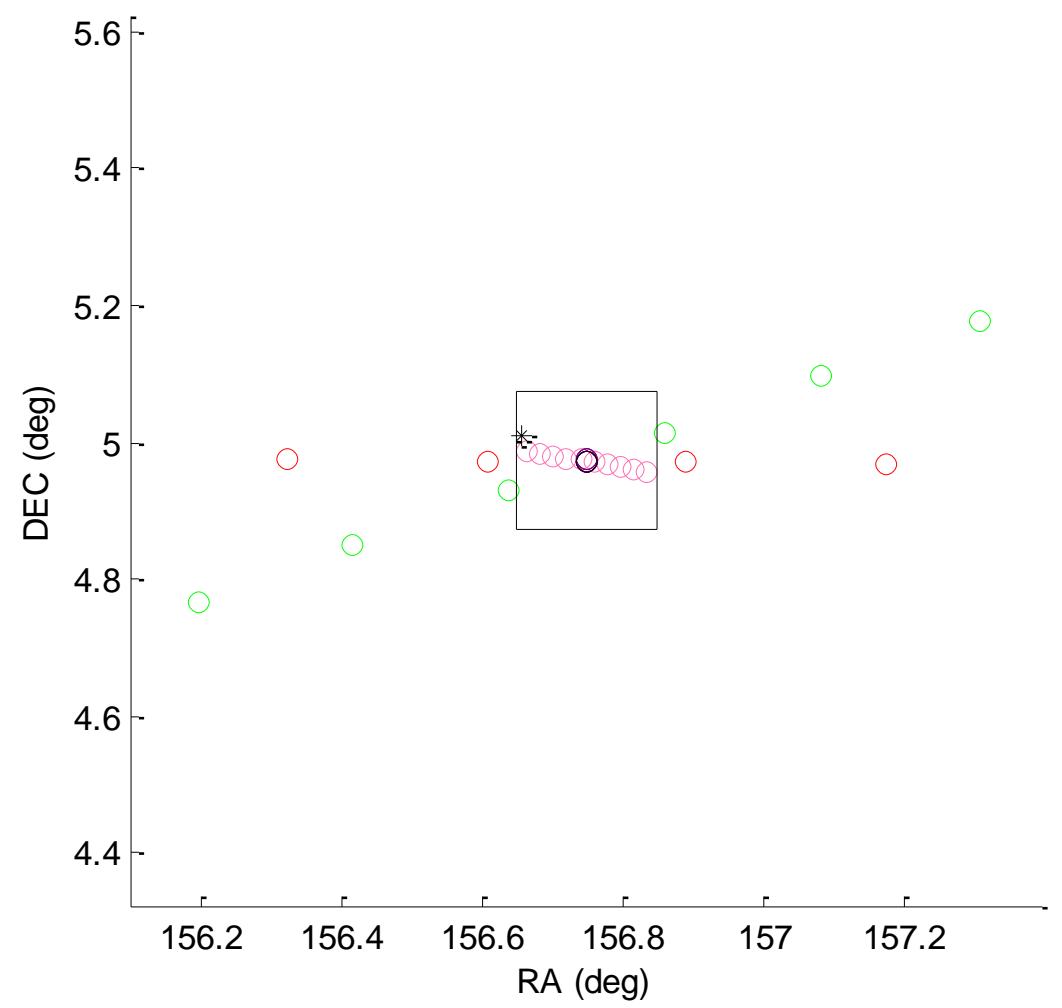

\begin{tabular}{|cl|}
\hline & ap \\
ecc \\
inc \\
ma \\
mm \\
0 \\
raan \\
org \\
- pos
\end{tabular}


1.3 Degree View

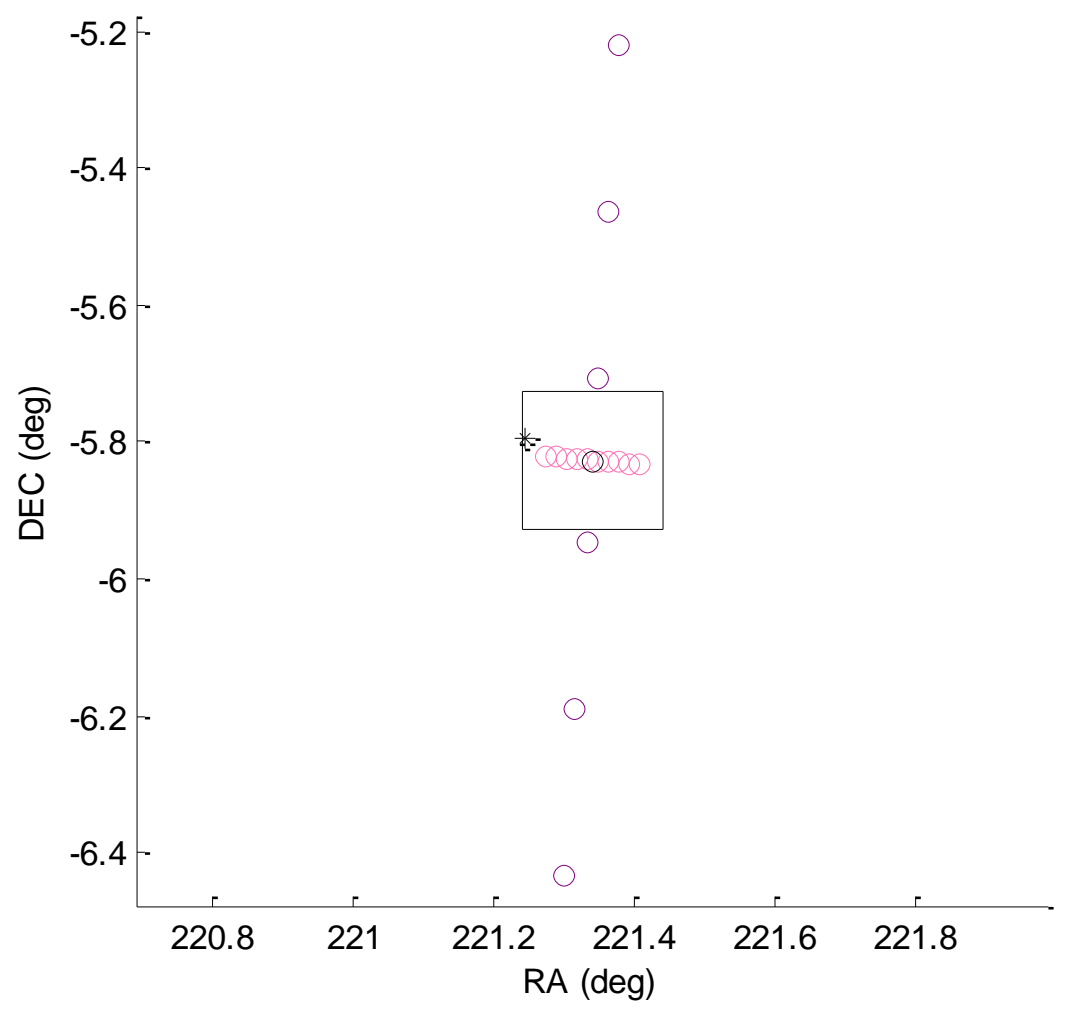

\begin{tabular}{|ll|}
\hline ap \\
ecc \\
inc \\
ma \\
mm \\
raan \\
org \\
$*$ pos \\
\hline
\end{tabular}




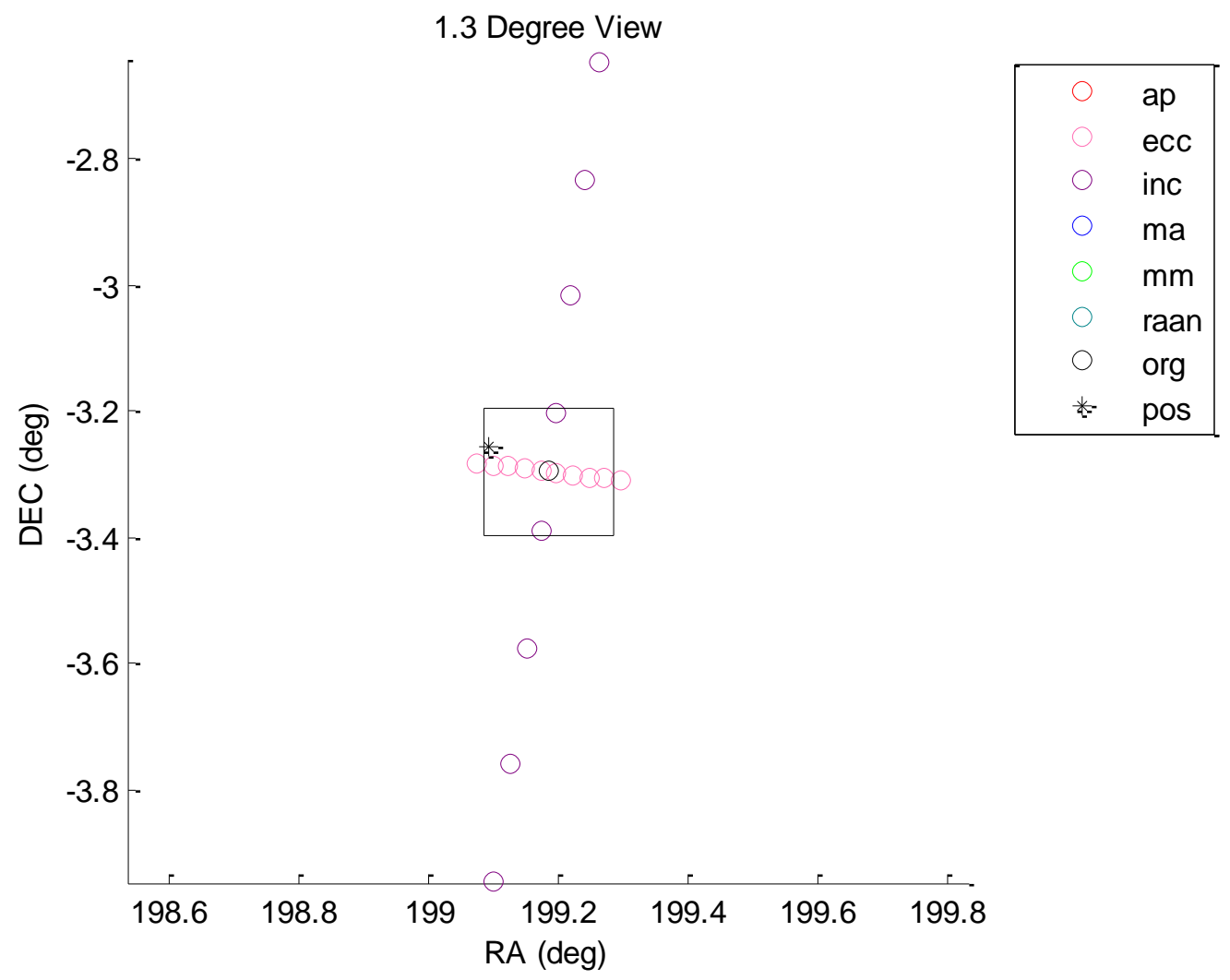




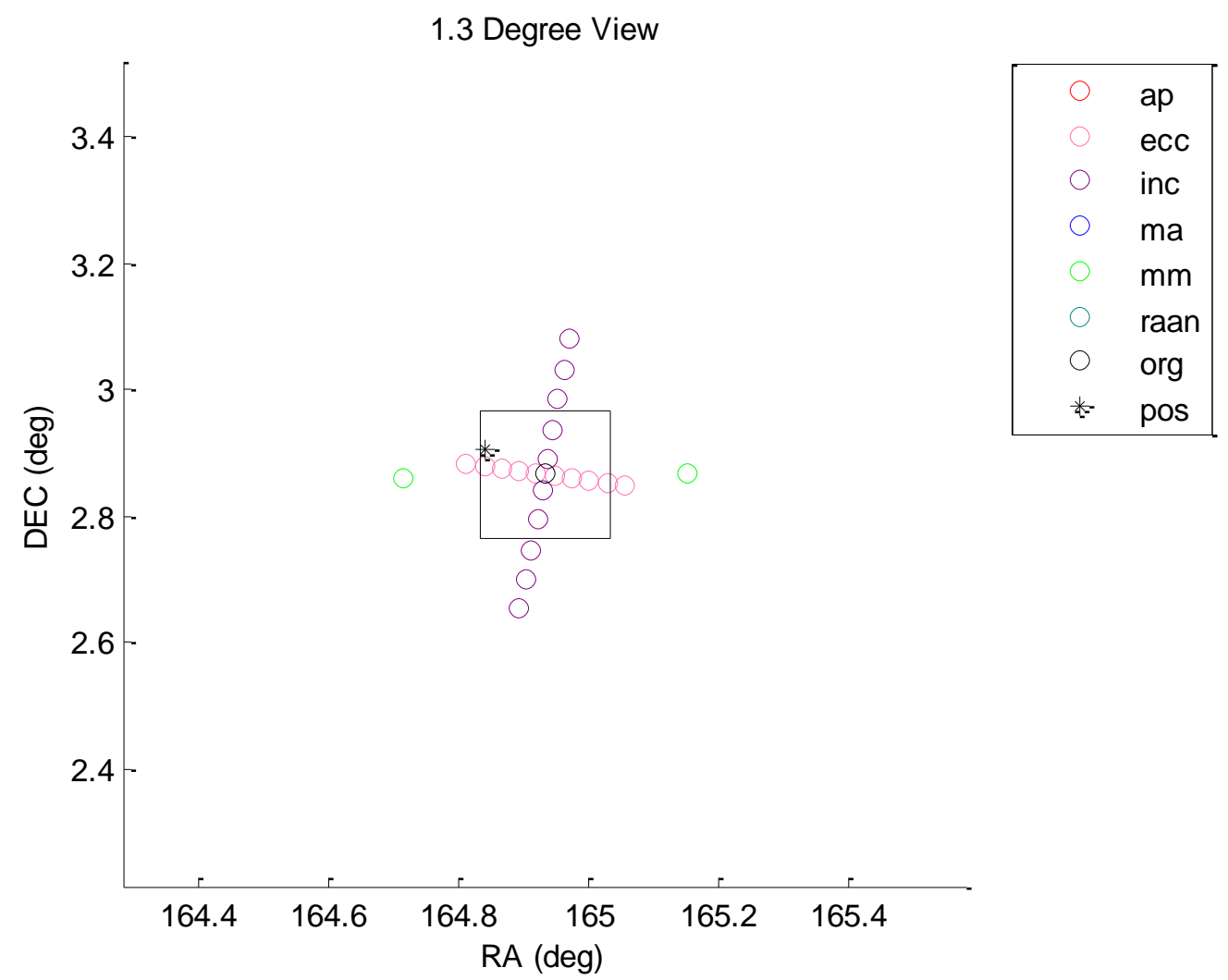




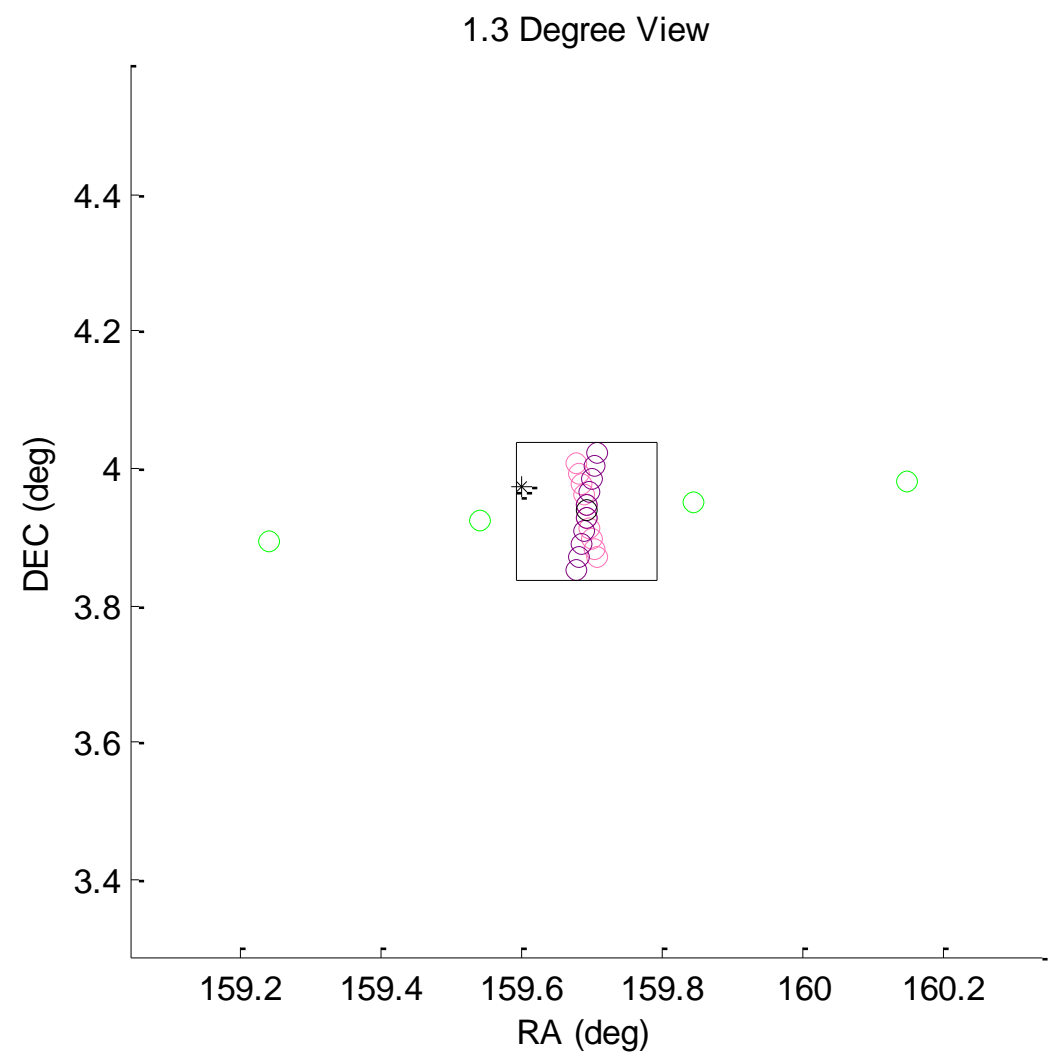

\begin{tabular}{|ll|}
\hline & ap \\
ecc \\
inc \\
ma \\
mm \\
raan \\
org \\
* pos \\
\hline
\end{tabular}




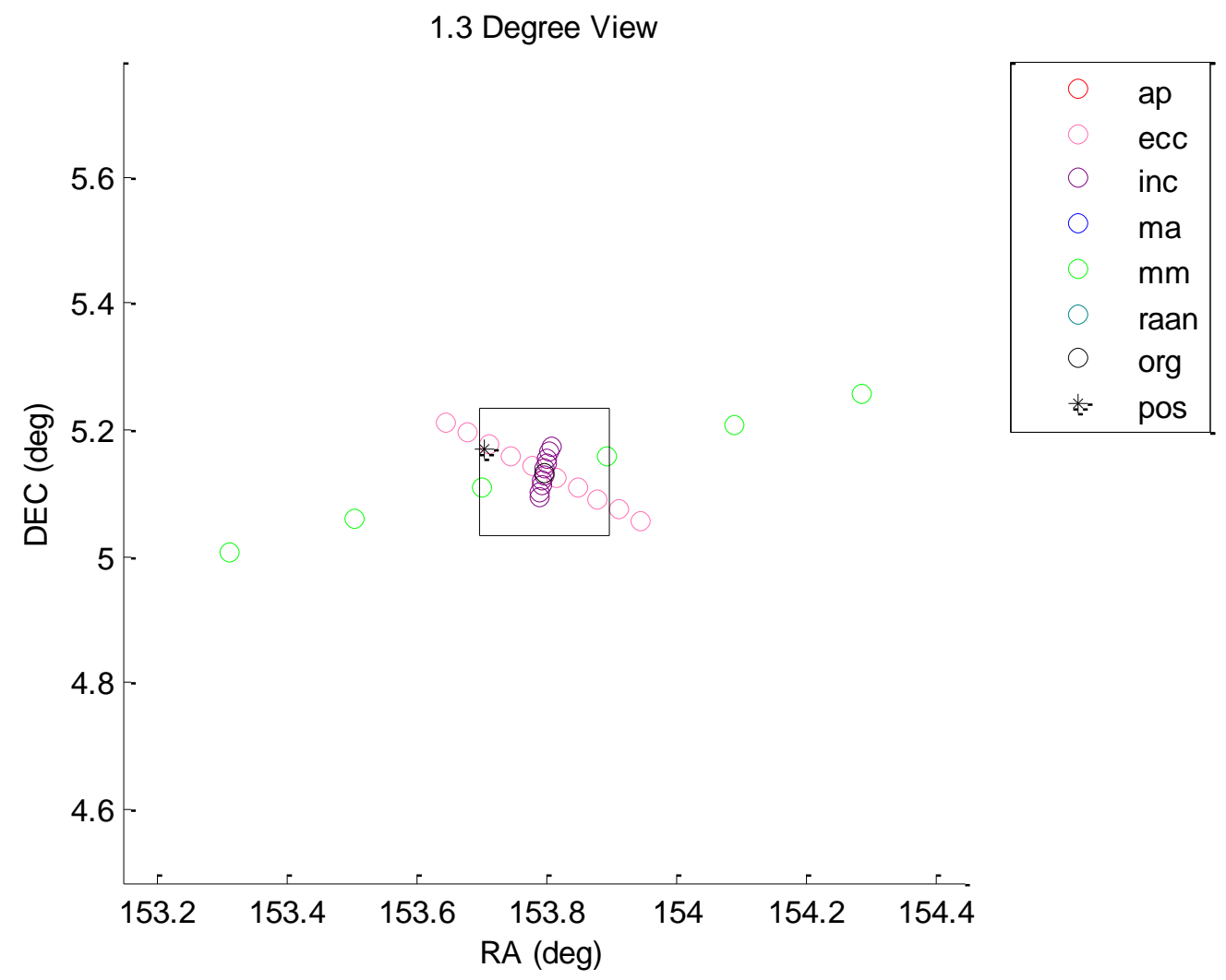




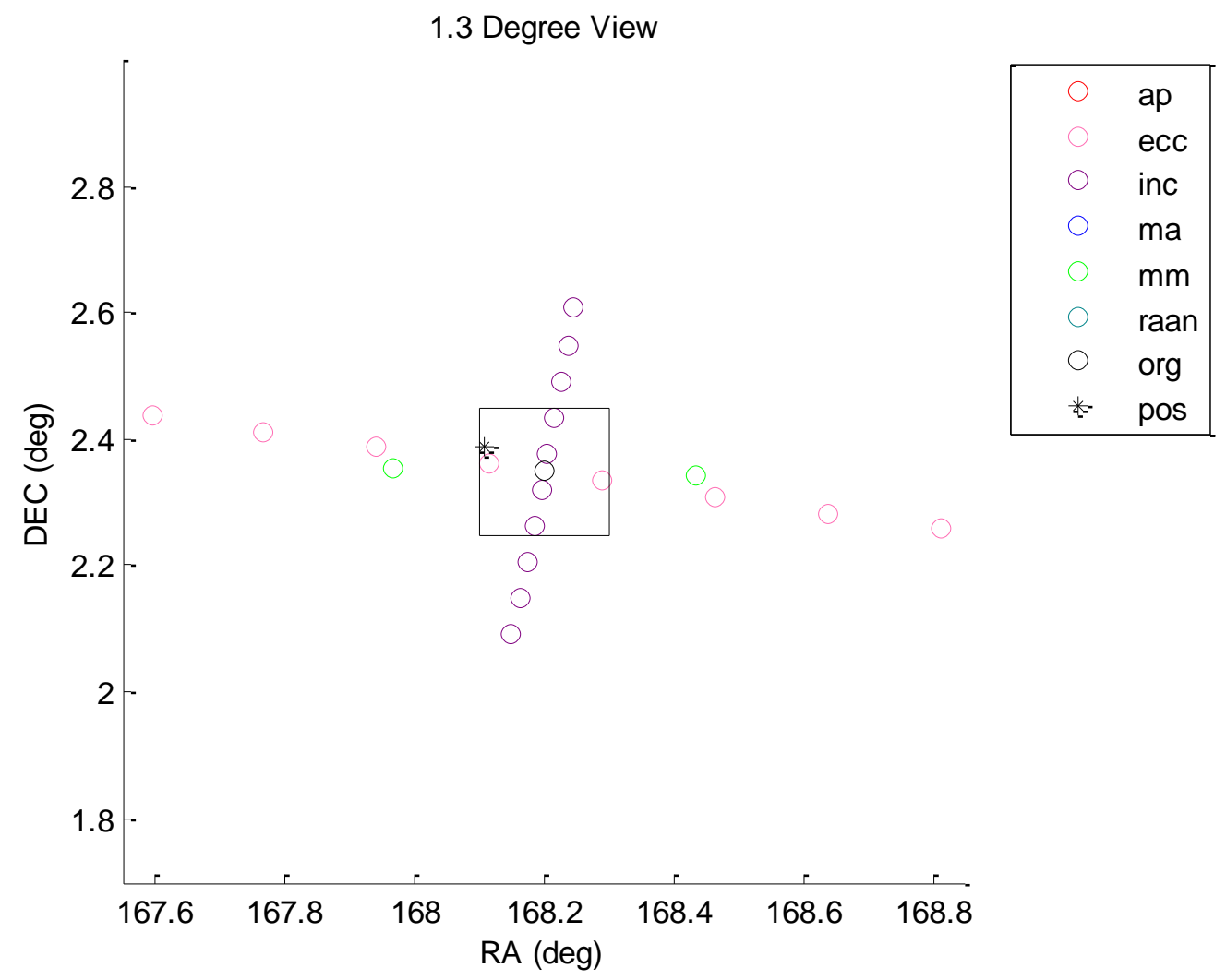




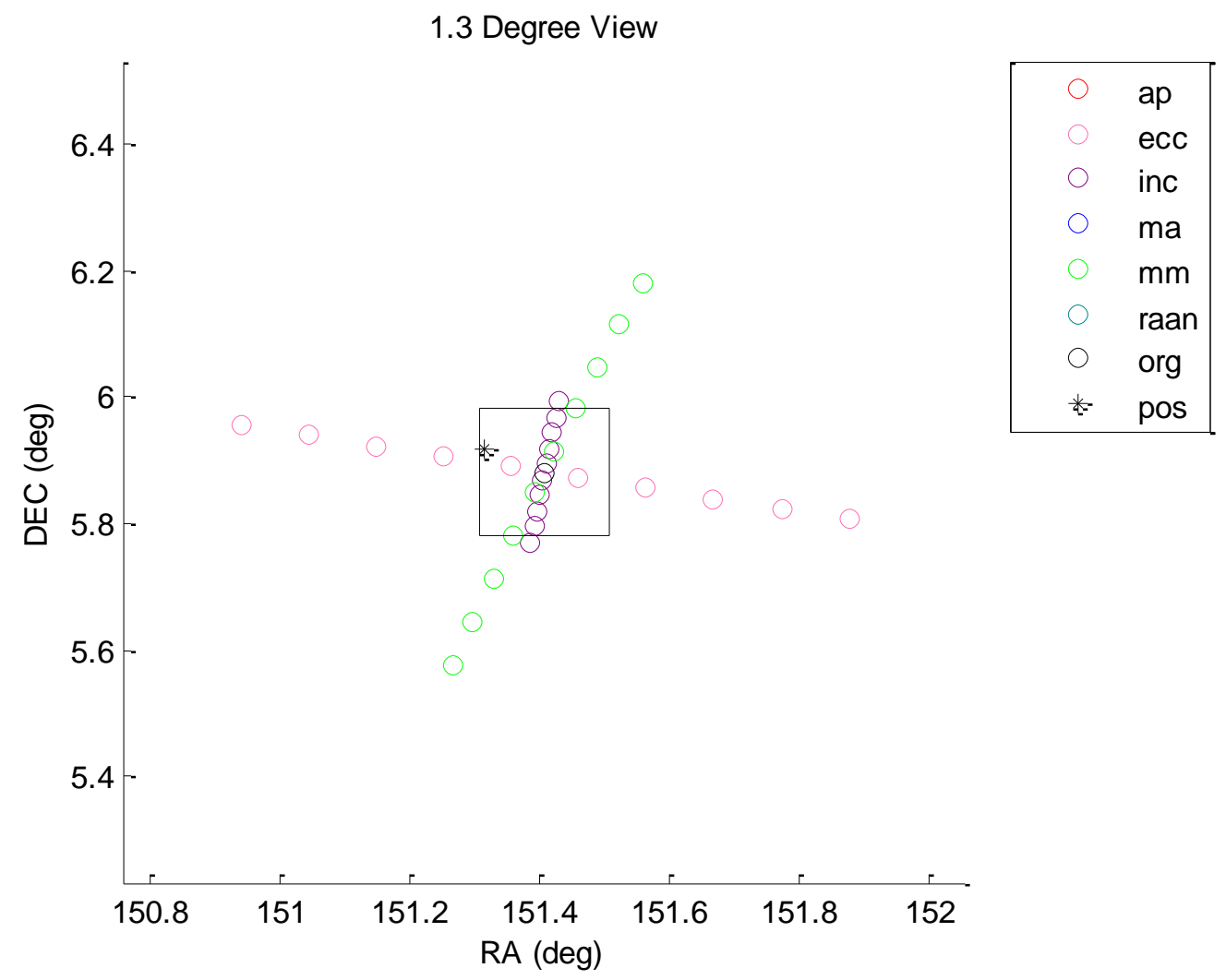




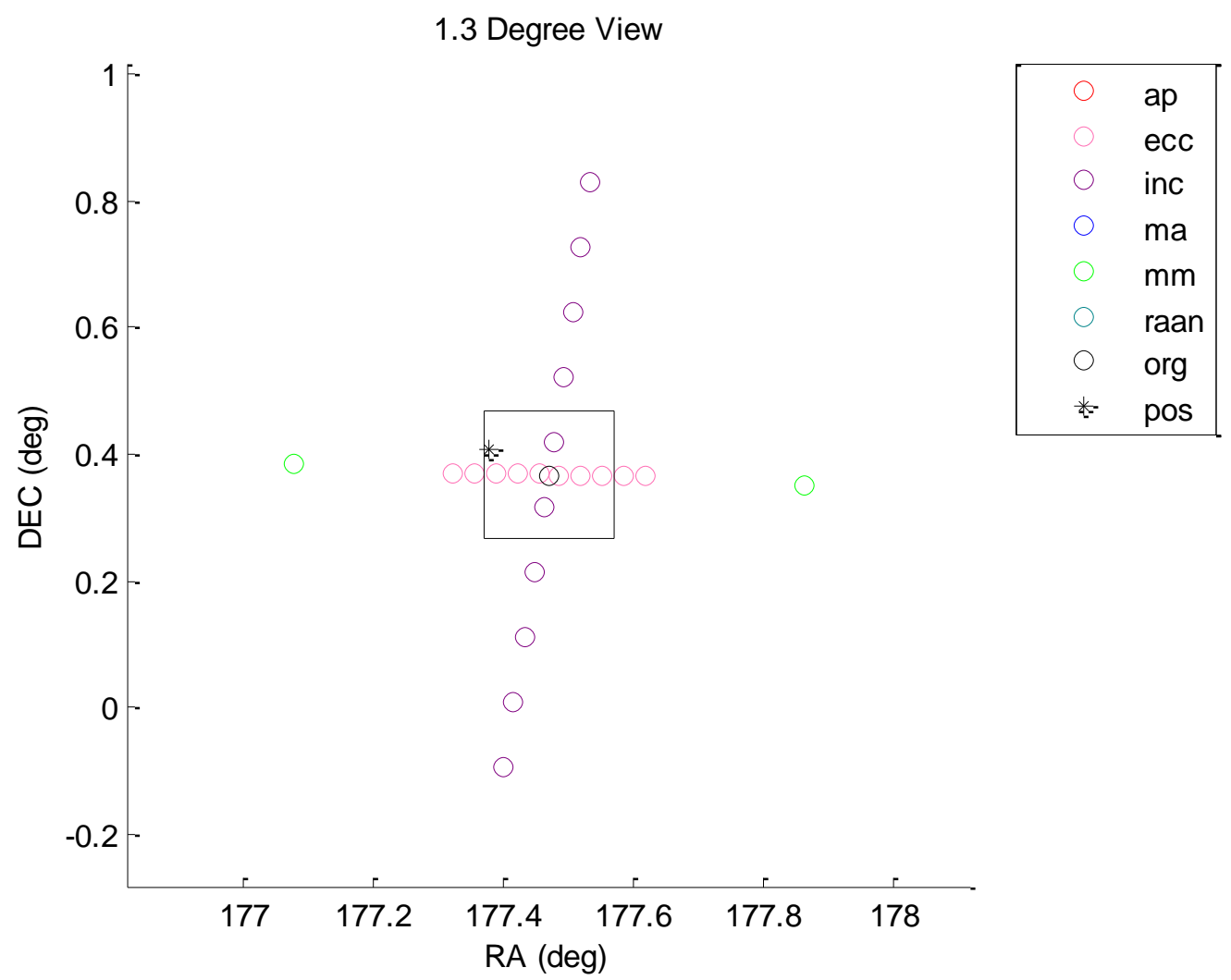


1.3 Degree View

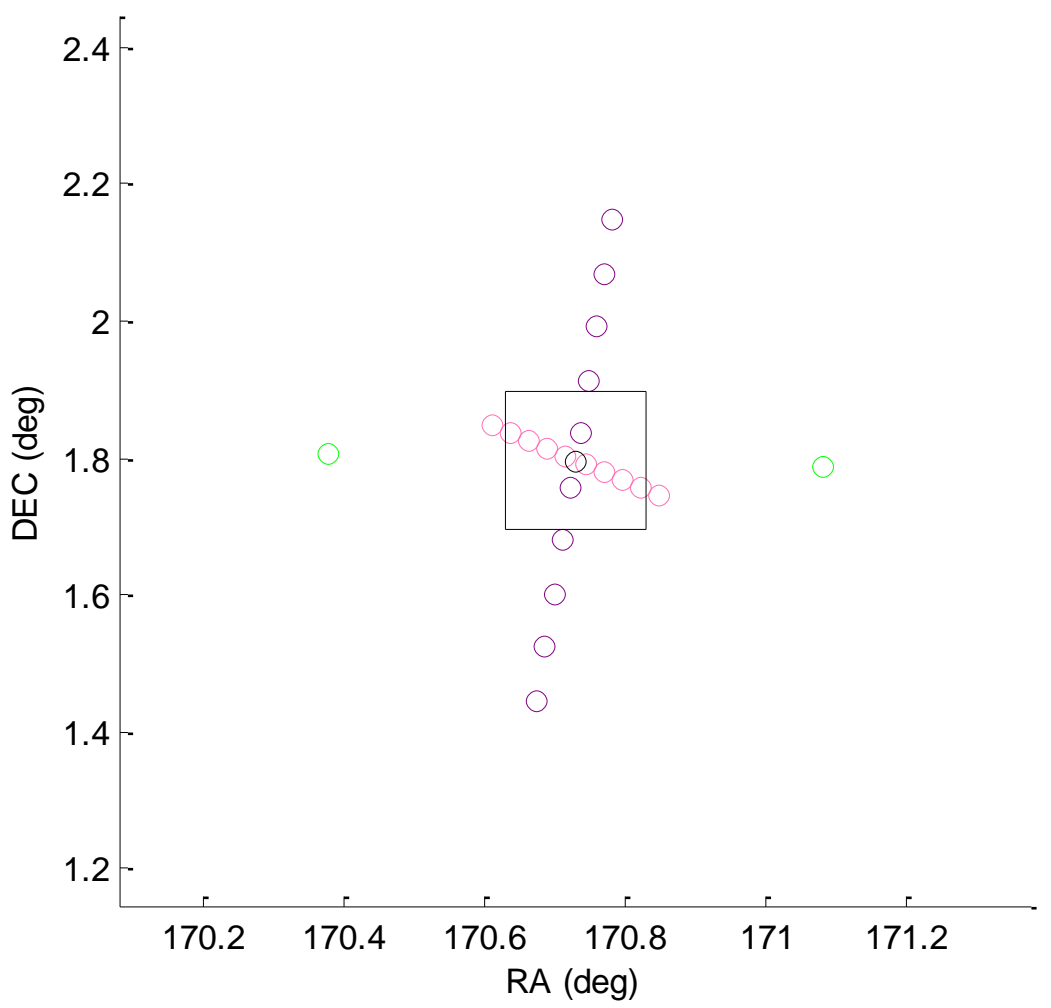

\begin{tabular}{|ll|}
\hline ap \\
ecc \\
inc \\
ma \\
mm \\
raan \\
org \\
* pos \\
\hline
\end{tabular}




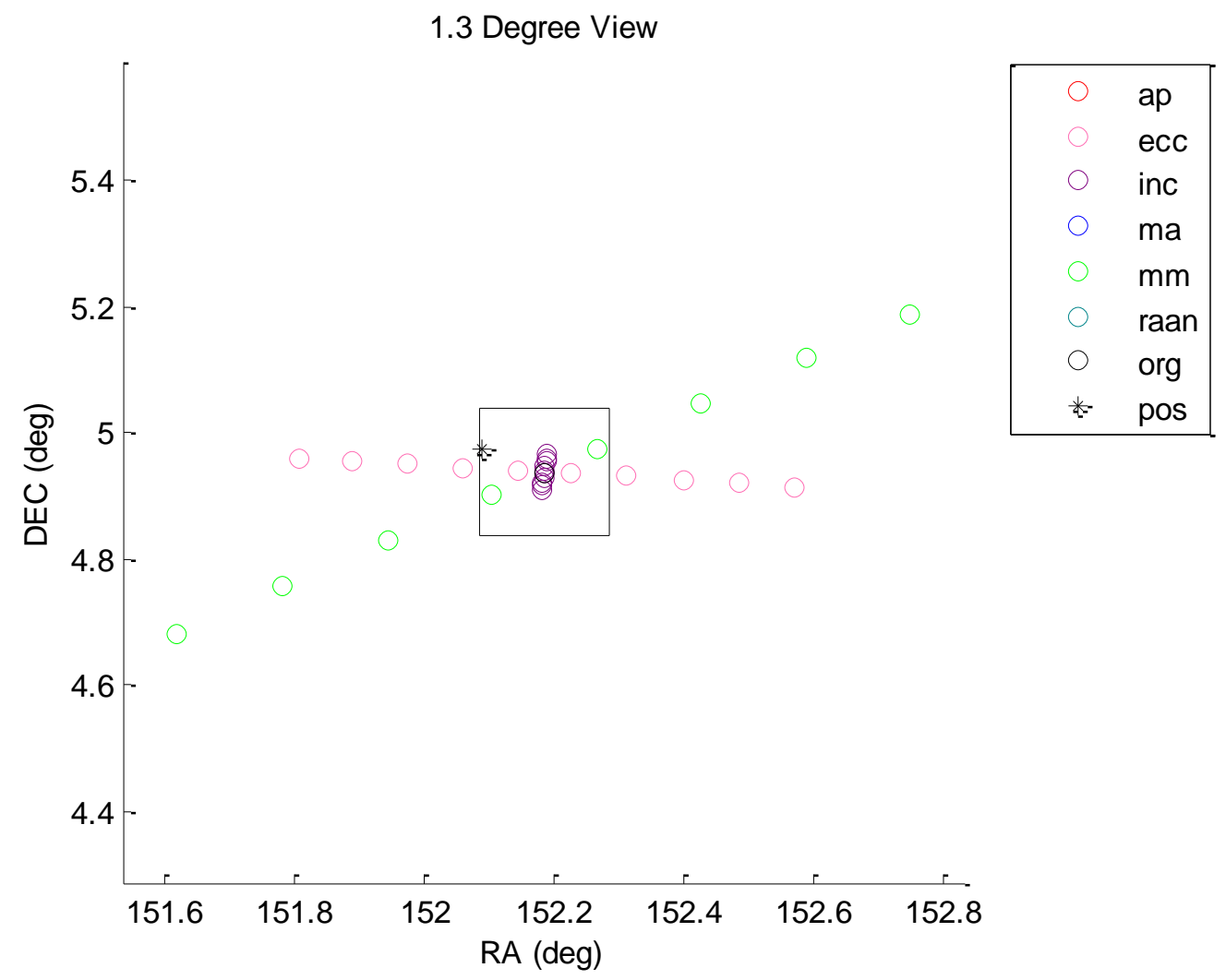




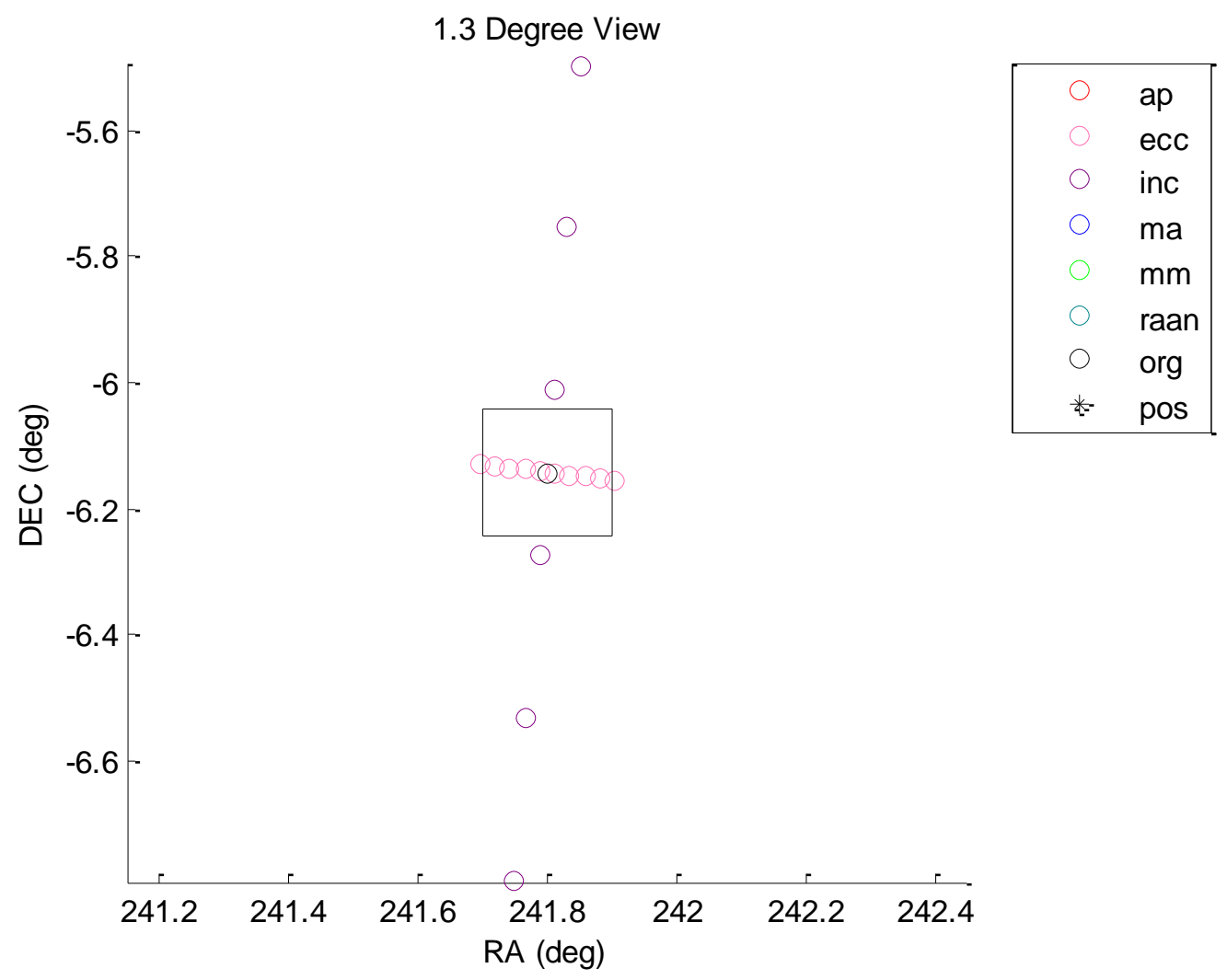


1.3 Degree View

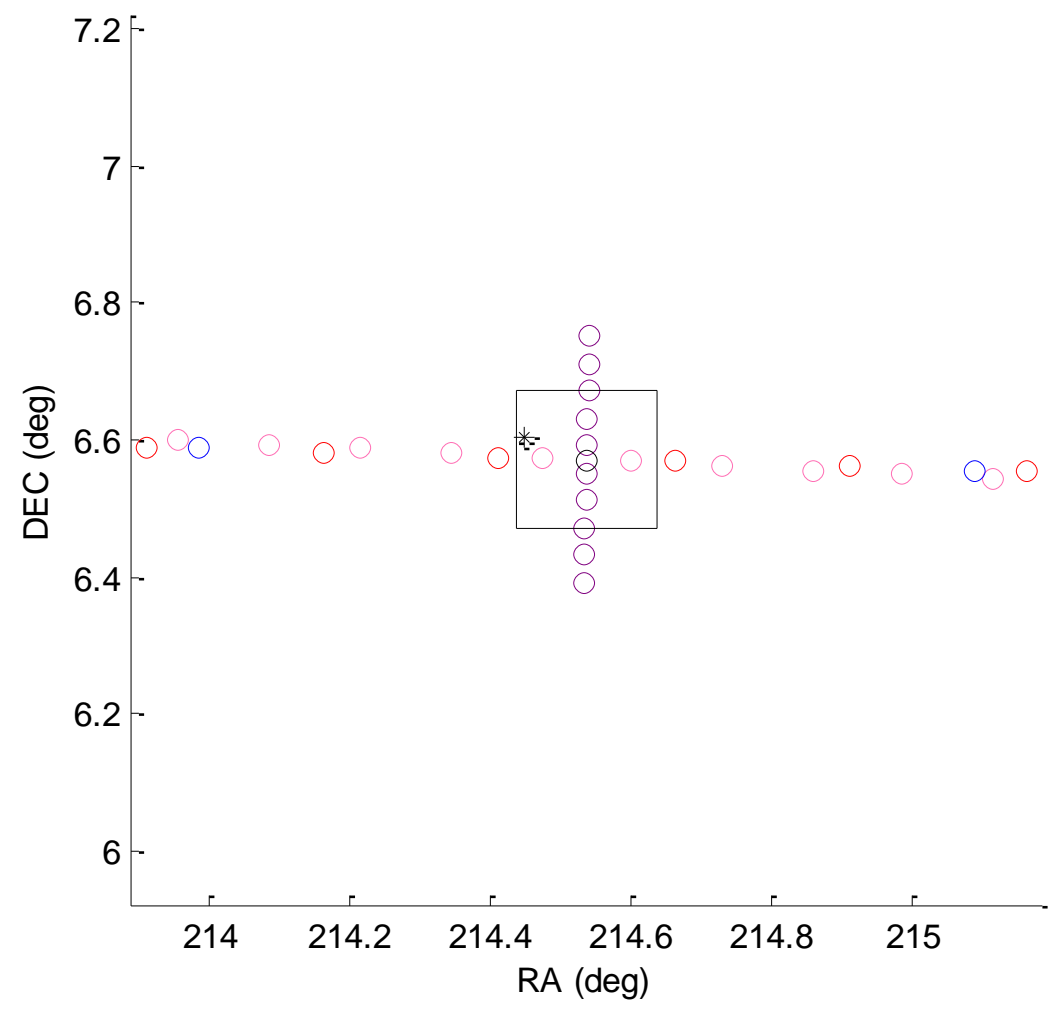

\begin{tabular}{|ll|}
\hline ap \\
ecc \\
inc \\
ma \\
mm \\
raan \\
org \\
$*$ pos \\
\hline
\end{tabular}




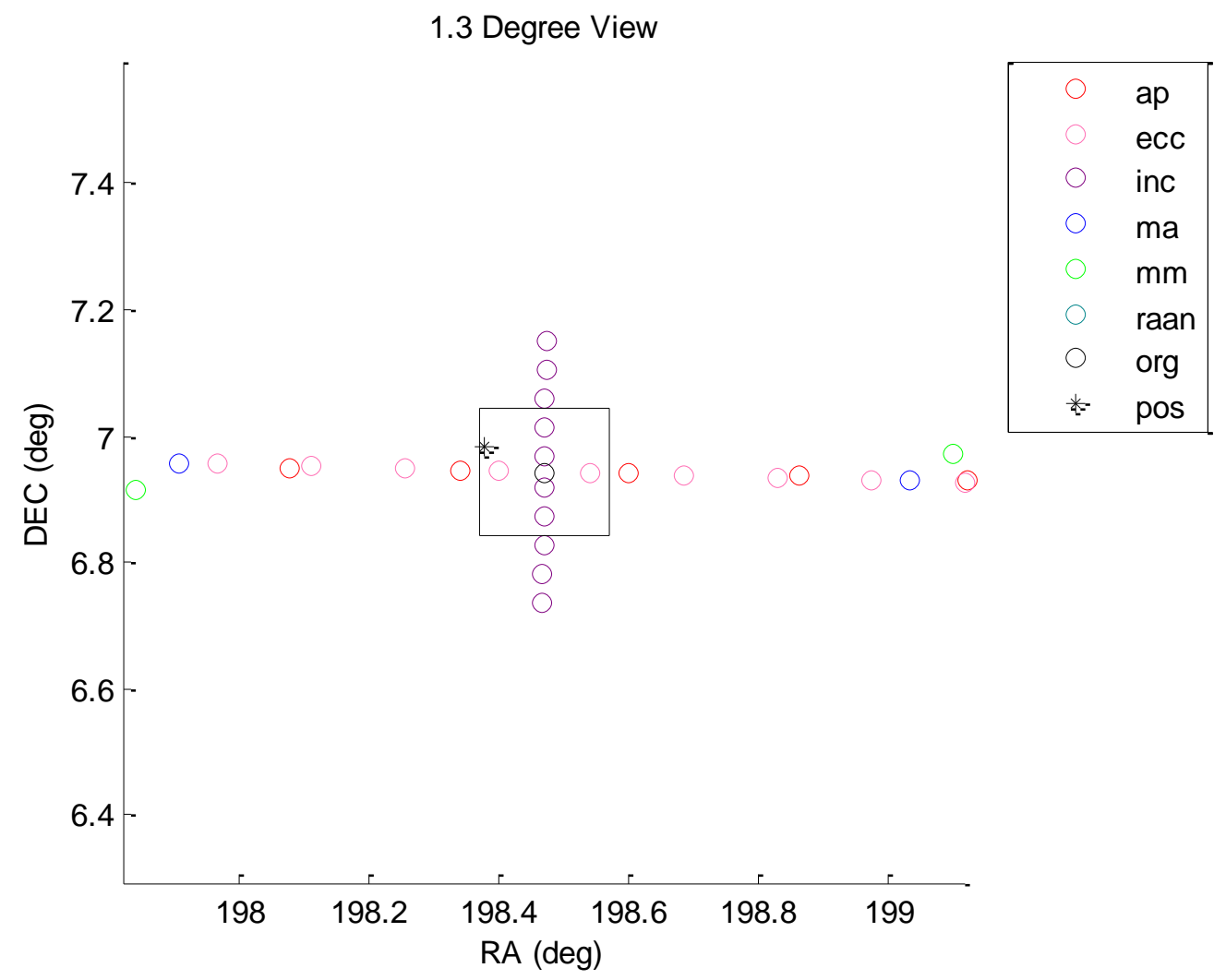




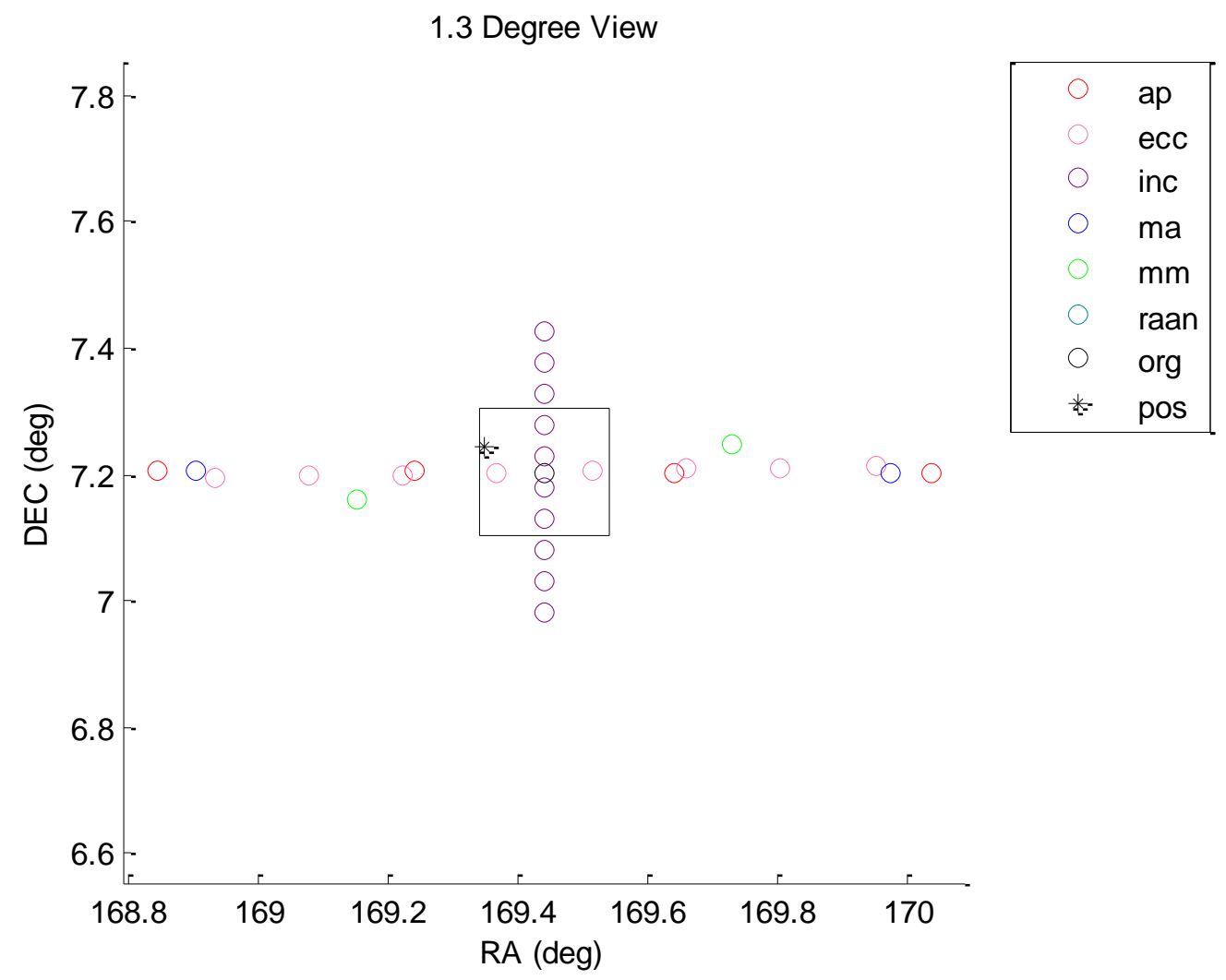


1.3 Degree View

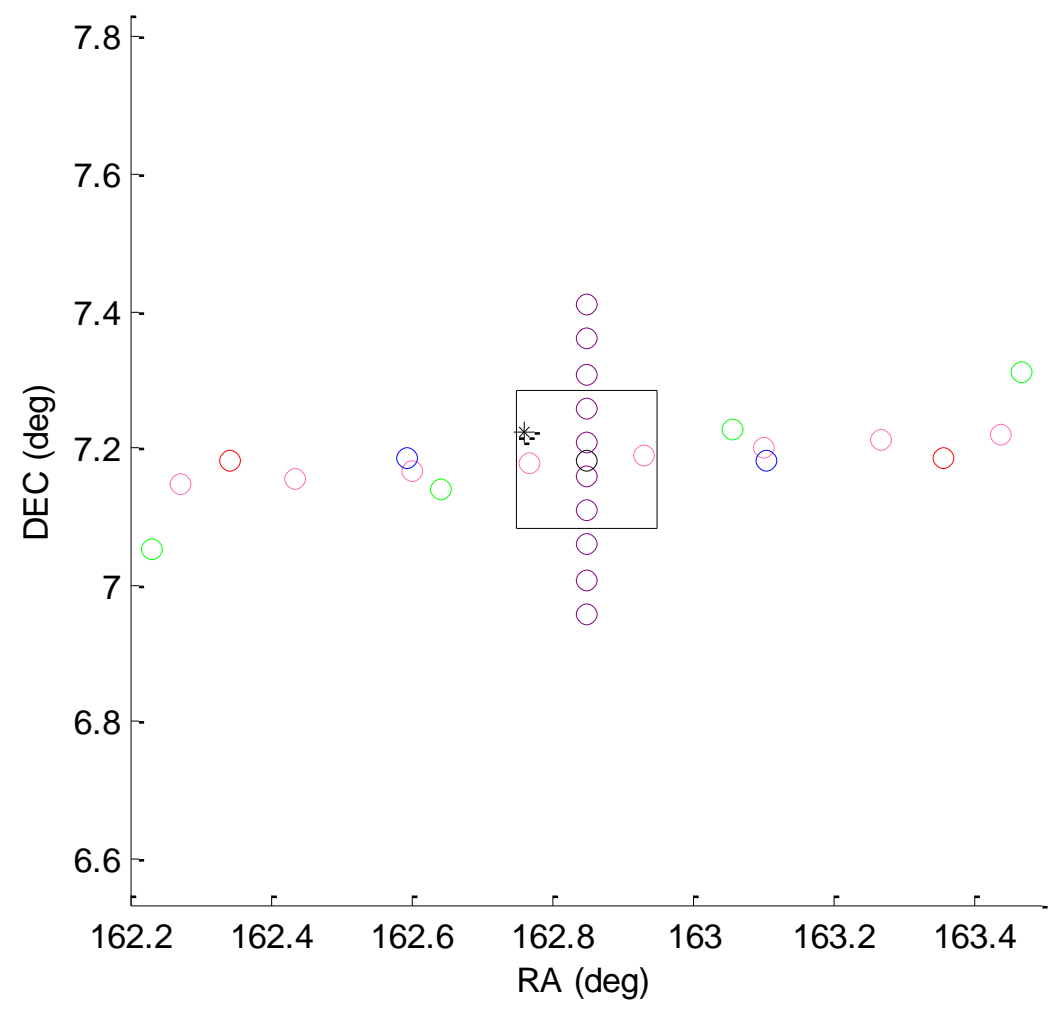

\begin{tabular}{|cl|}
\hline & ap \\
ecc \\
0 & inc \\
ma \\
mm \\
0 \\
raan \\
org \\
- pos
\end{tabular}




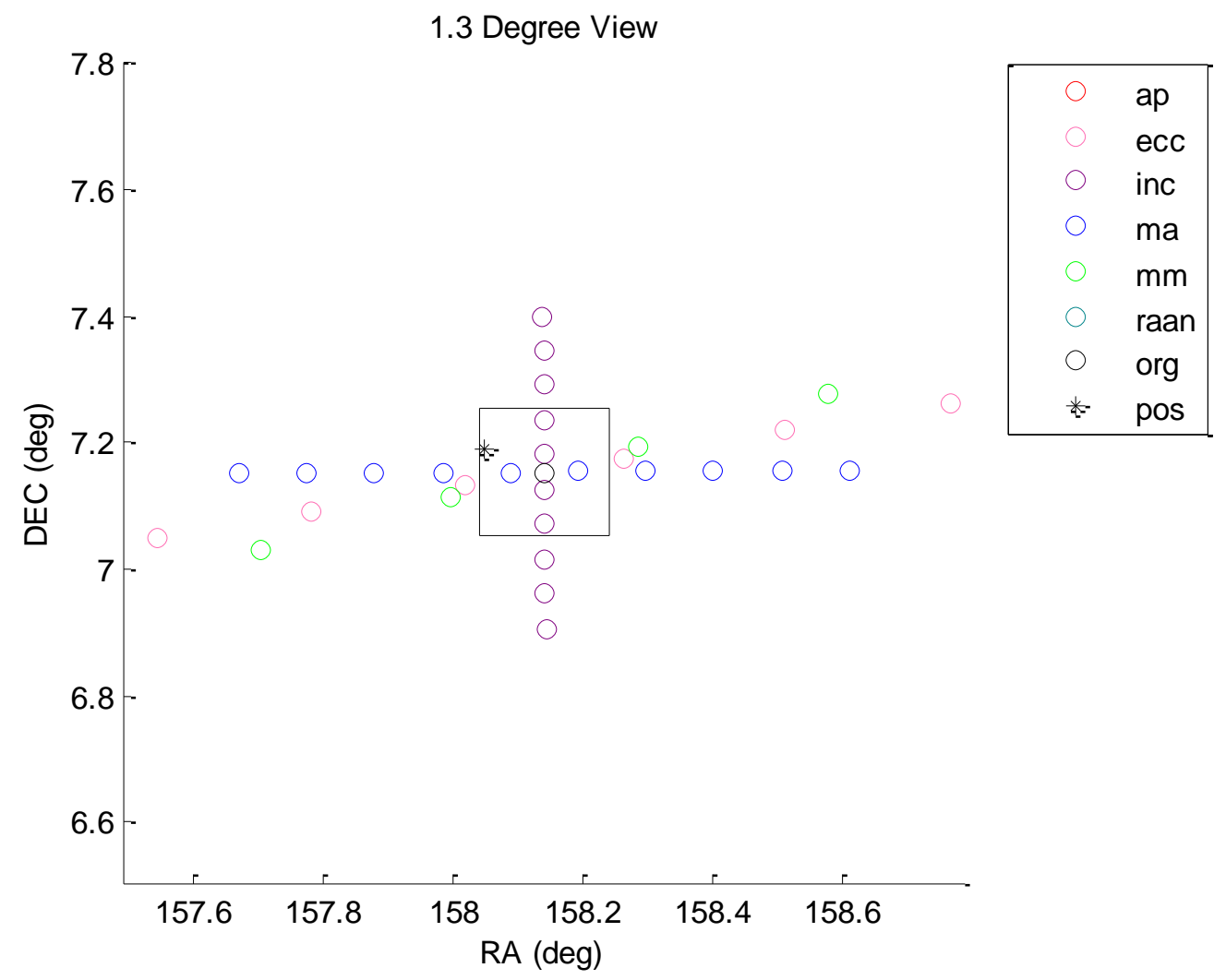




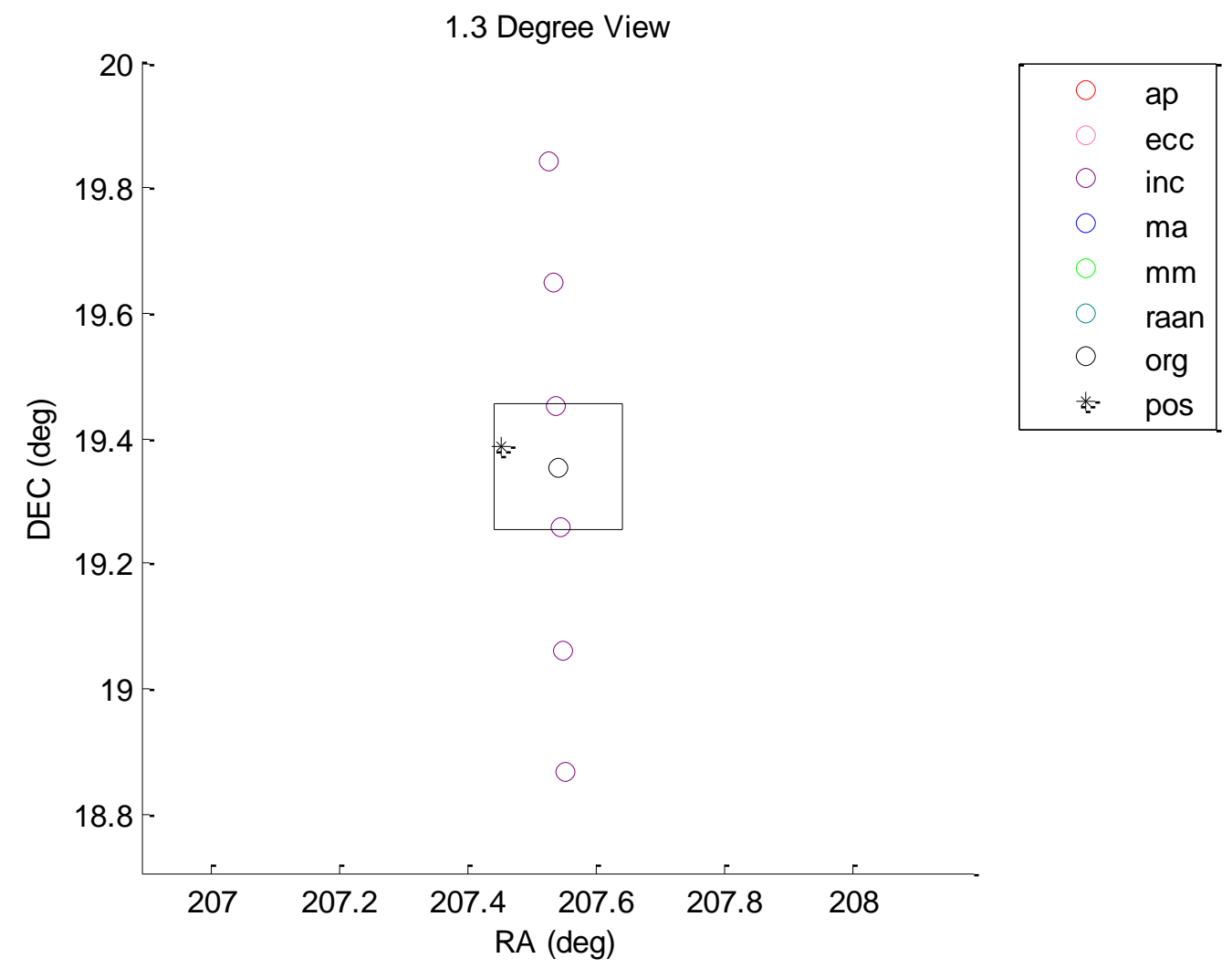




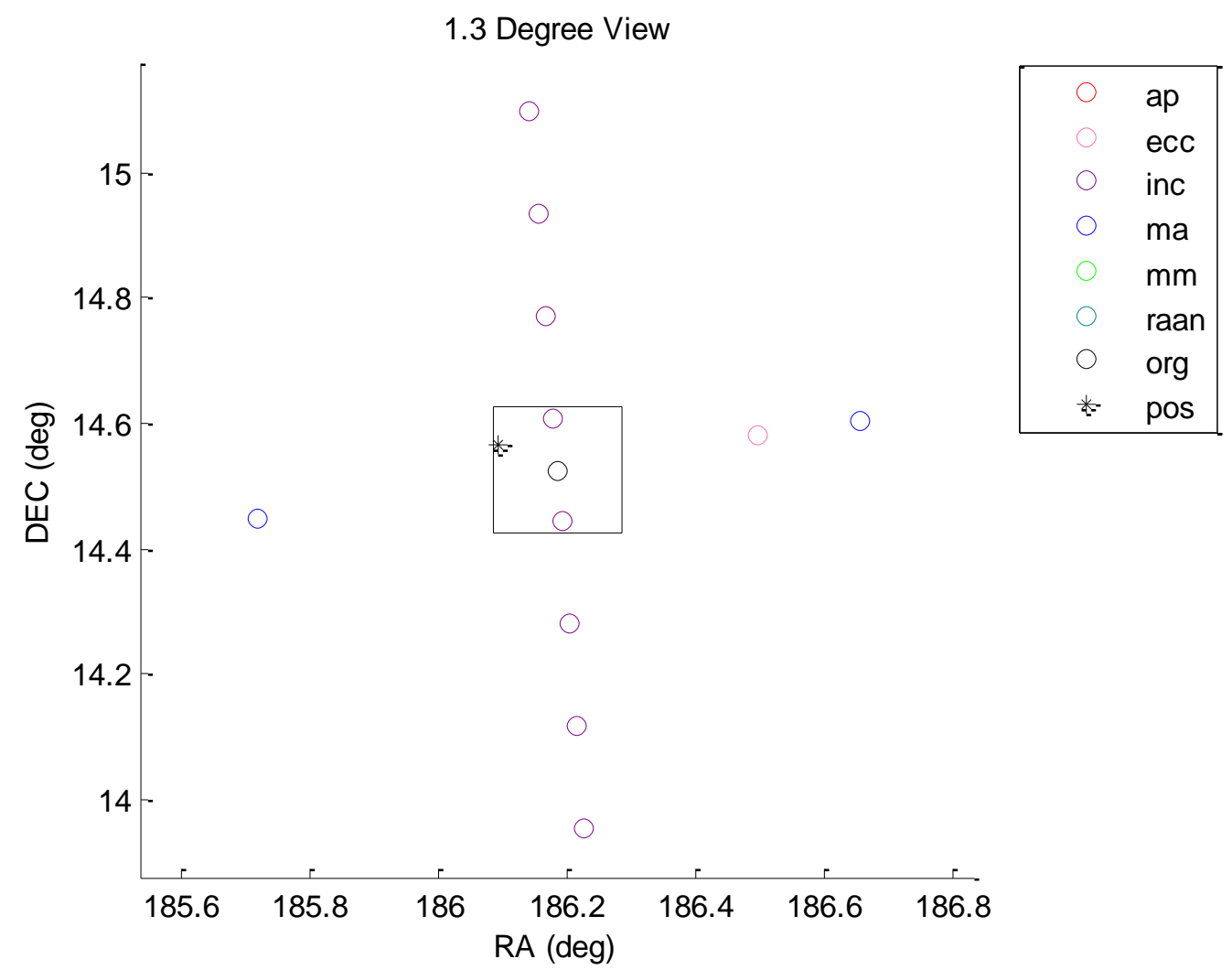




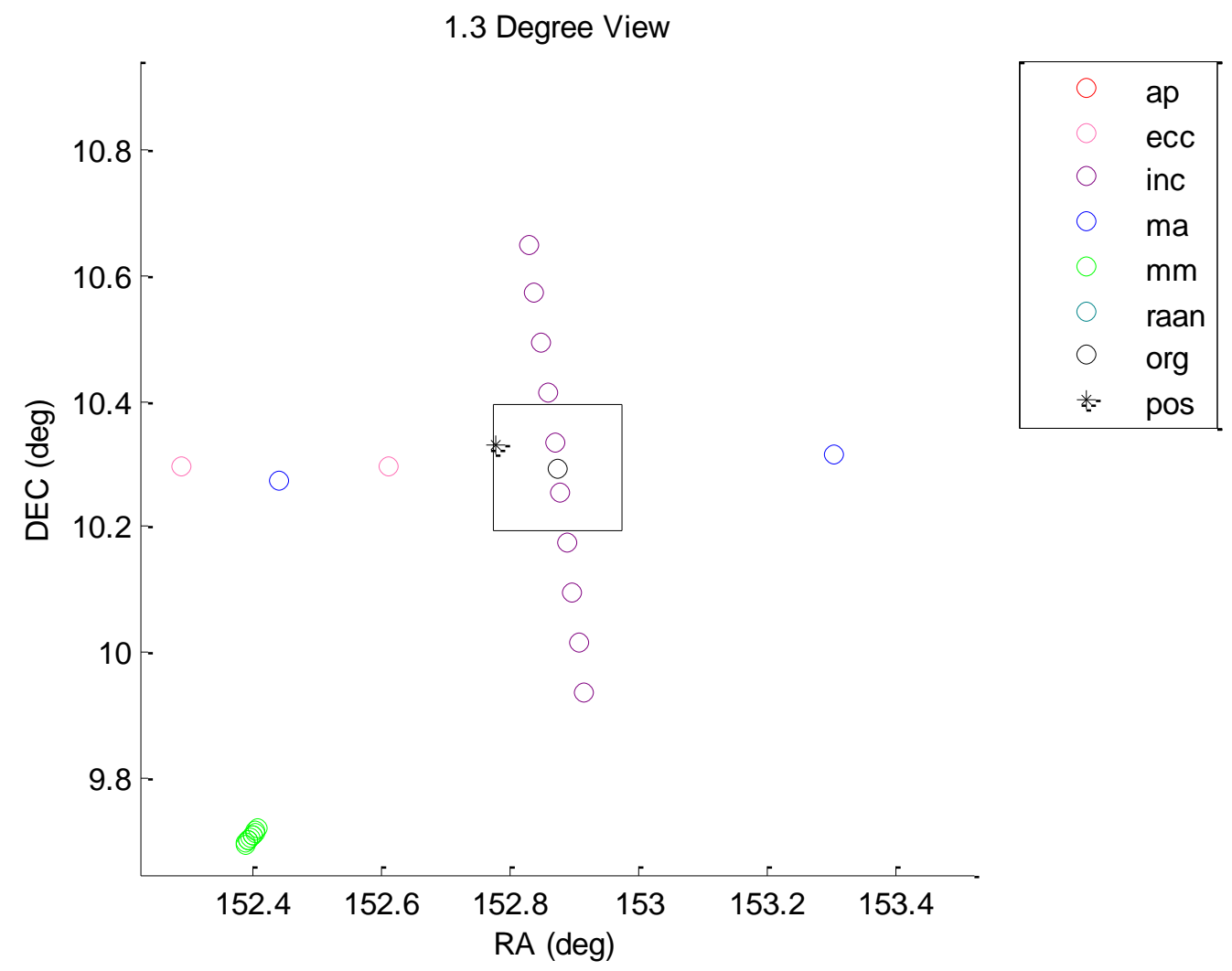




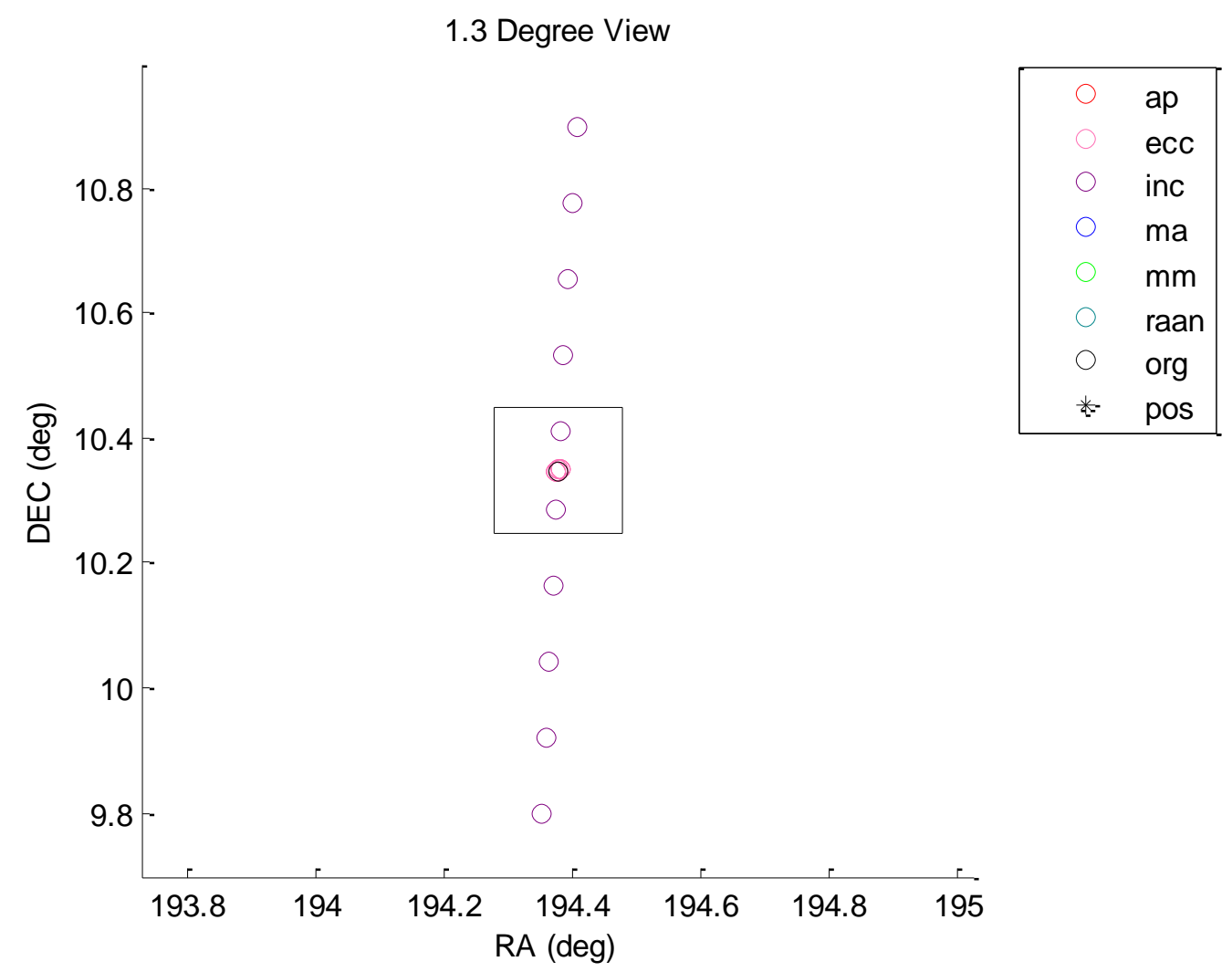




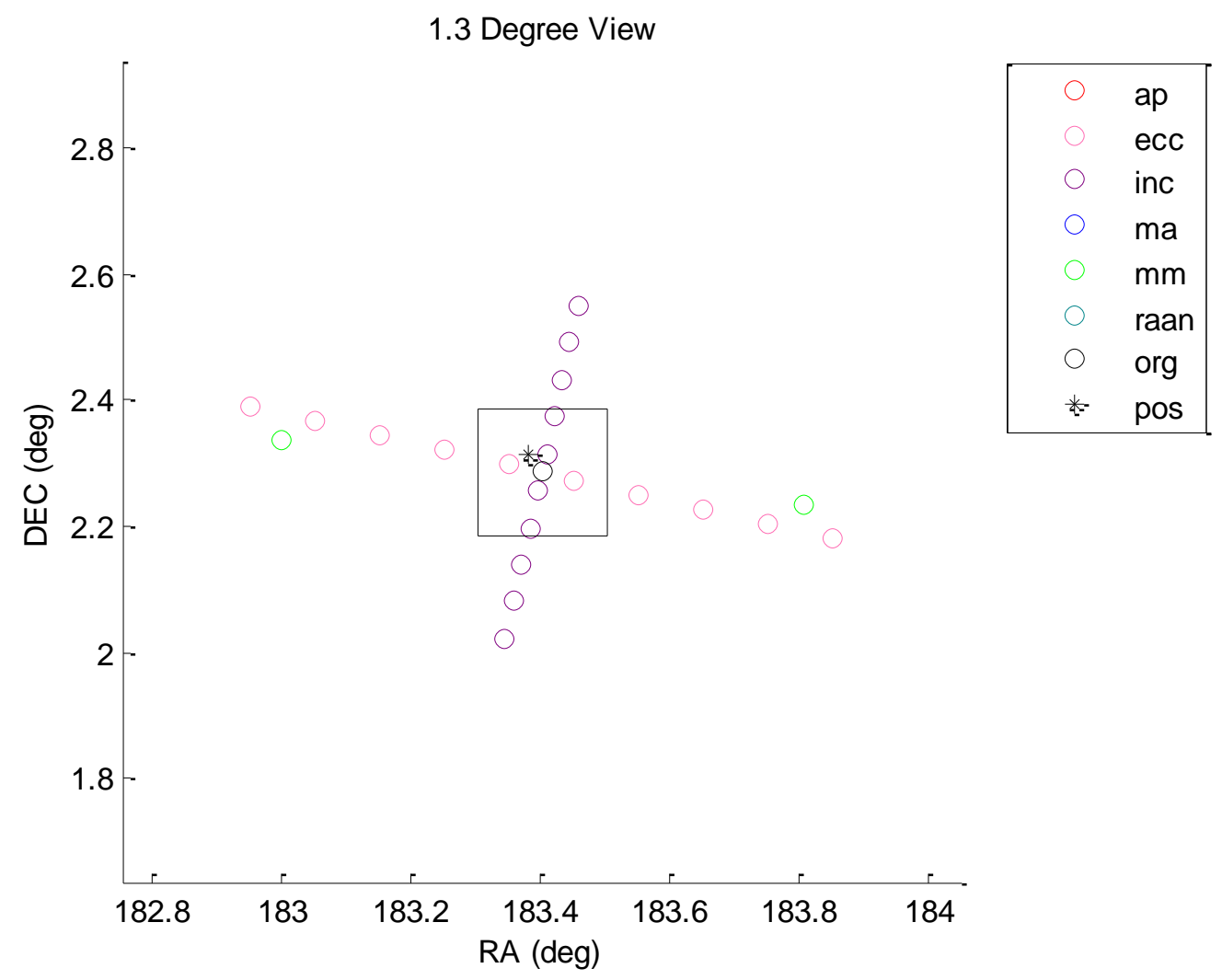




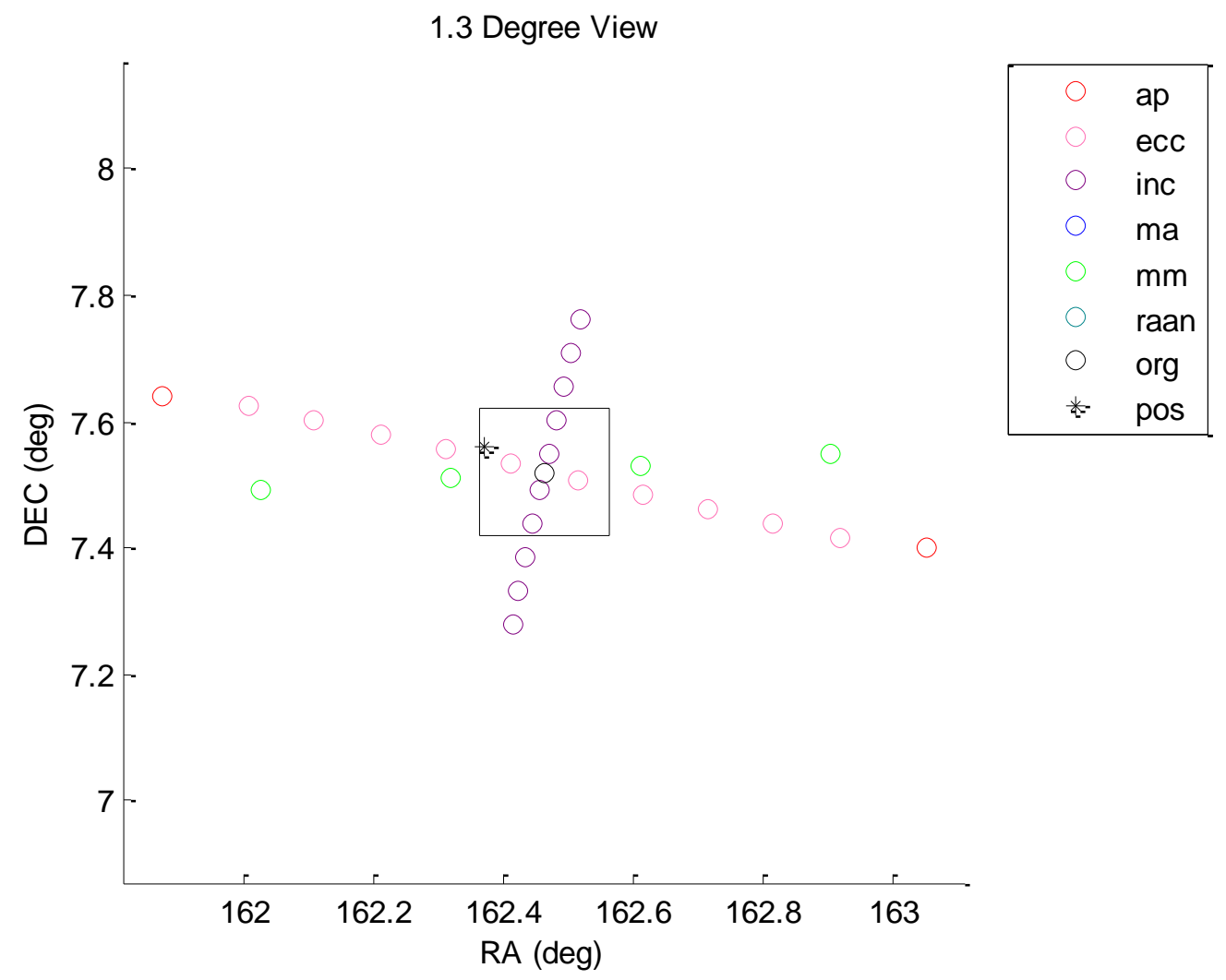




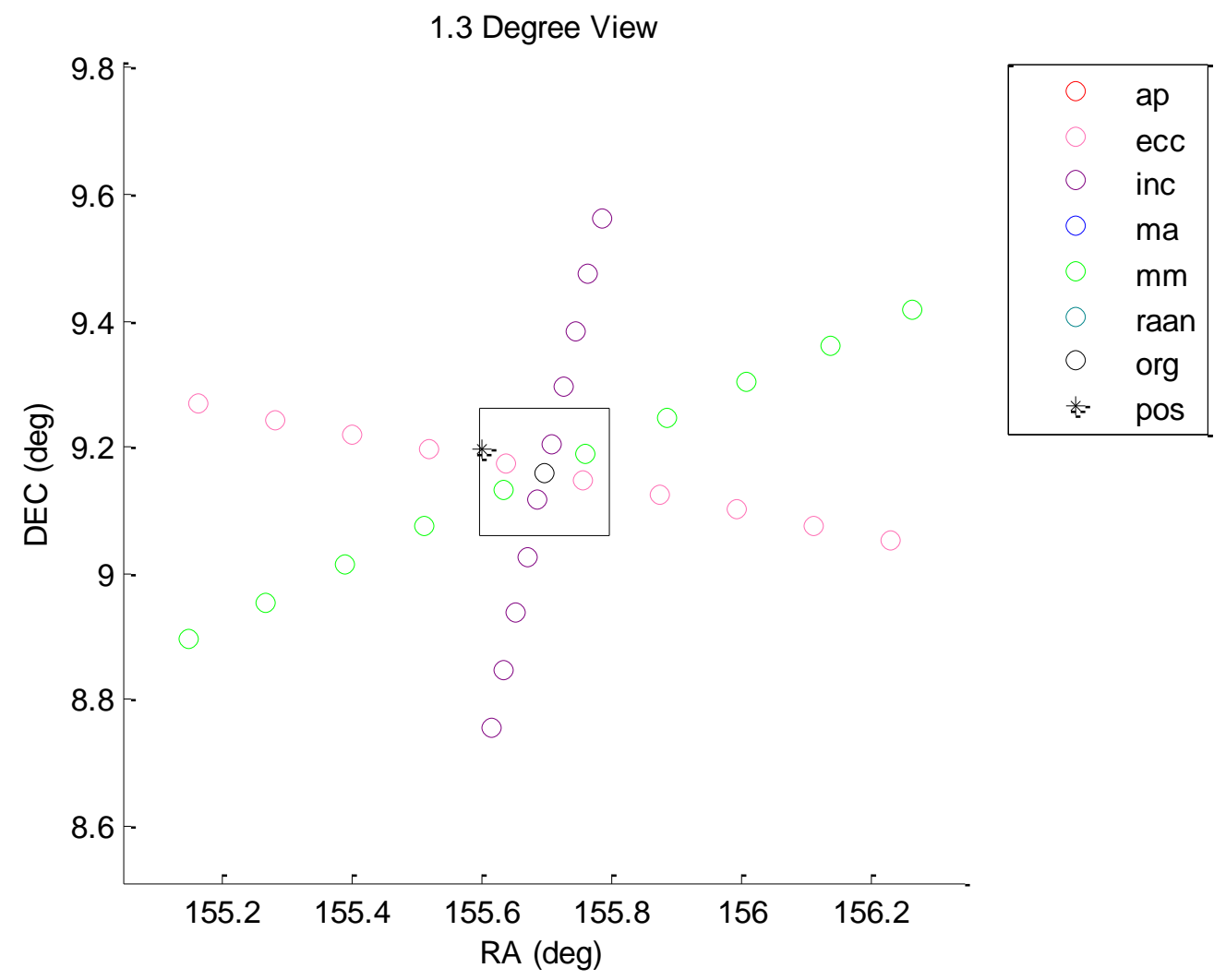




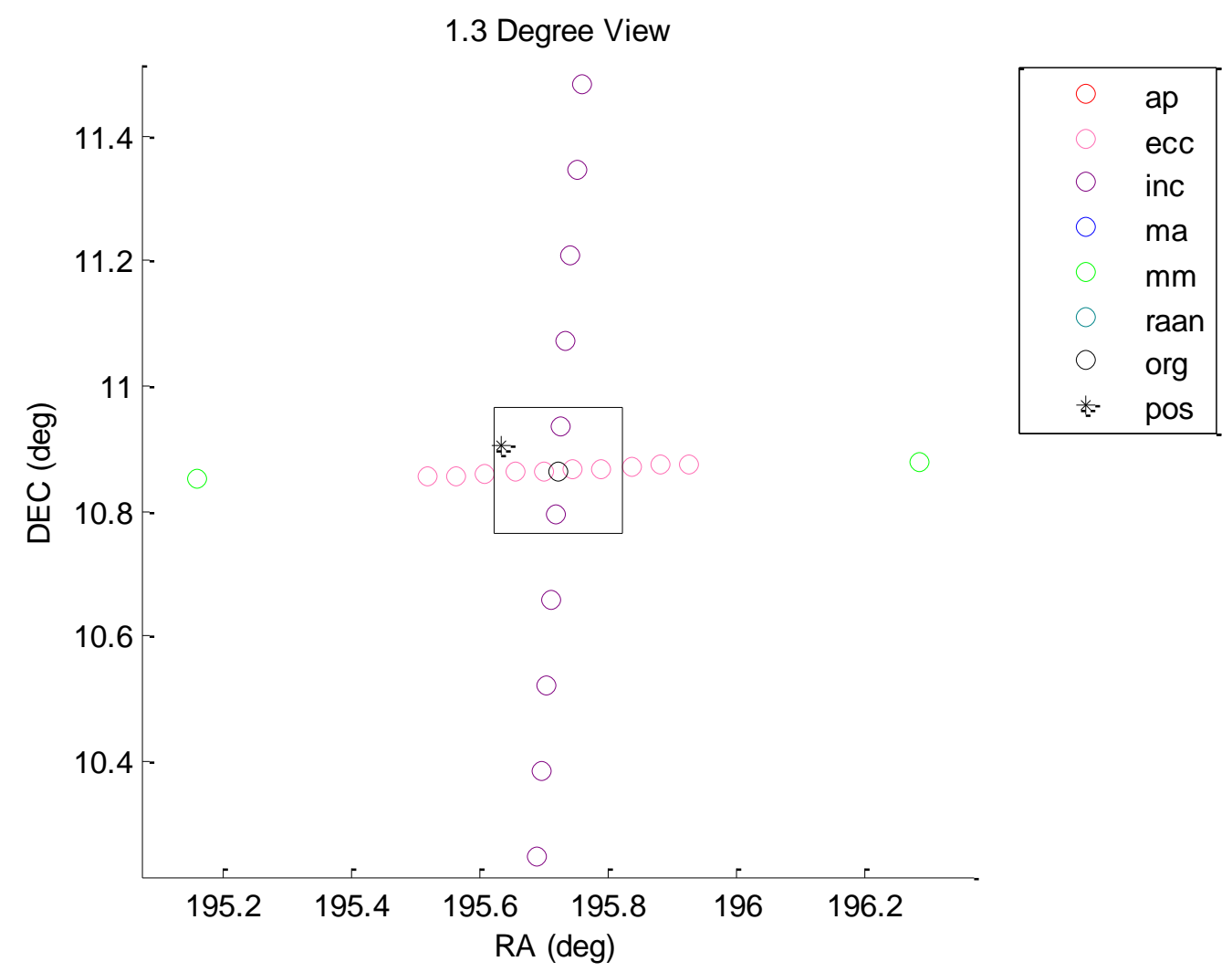




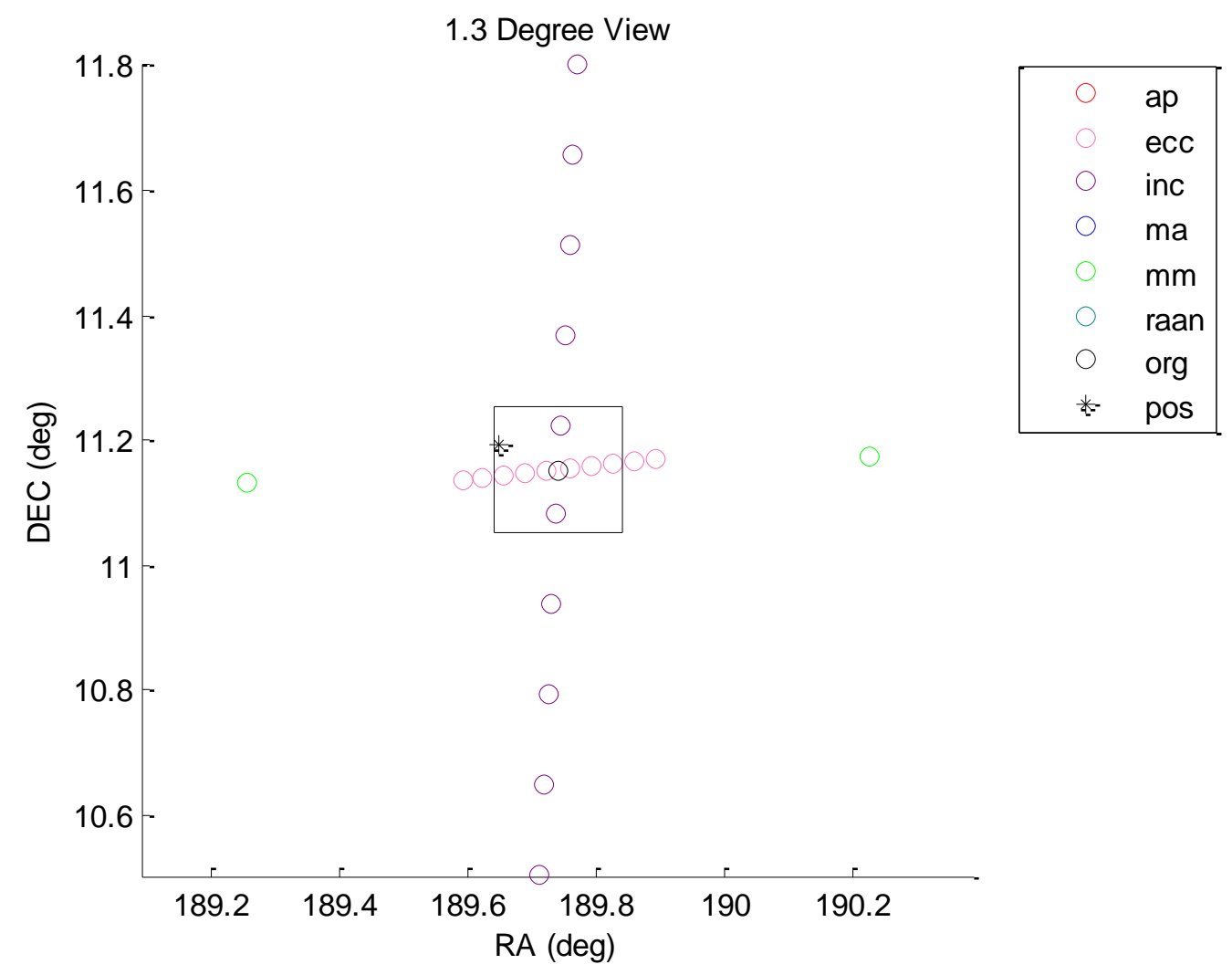




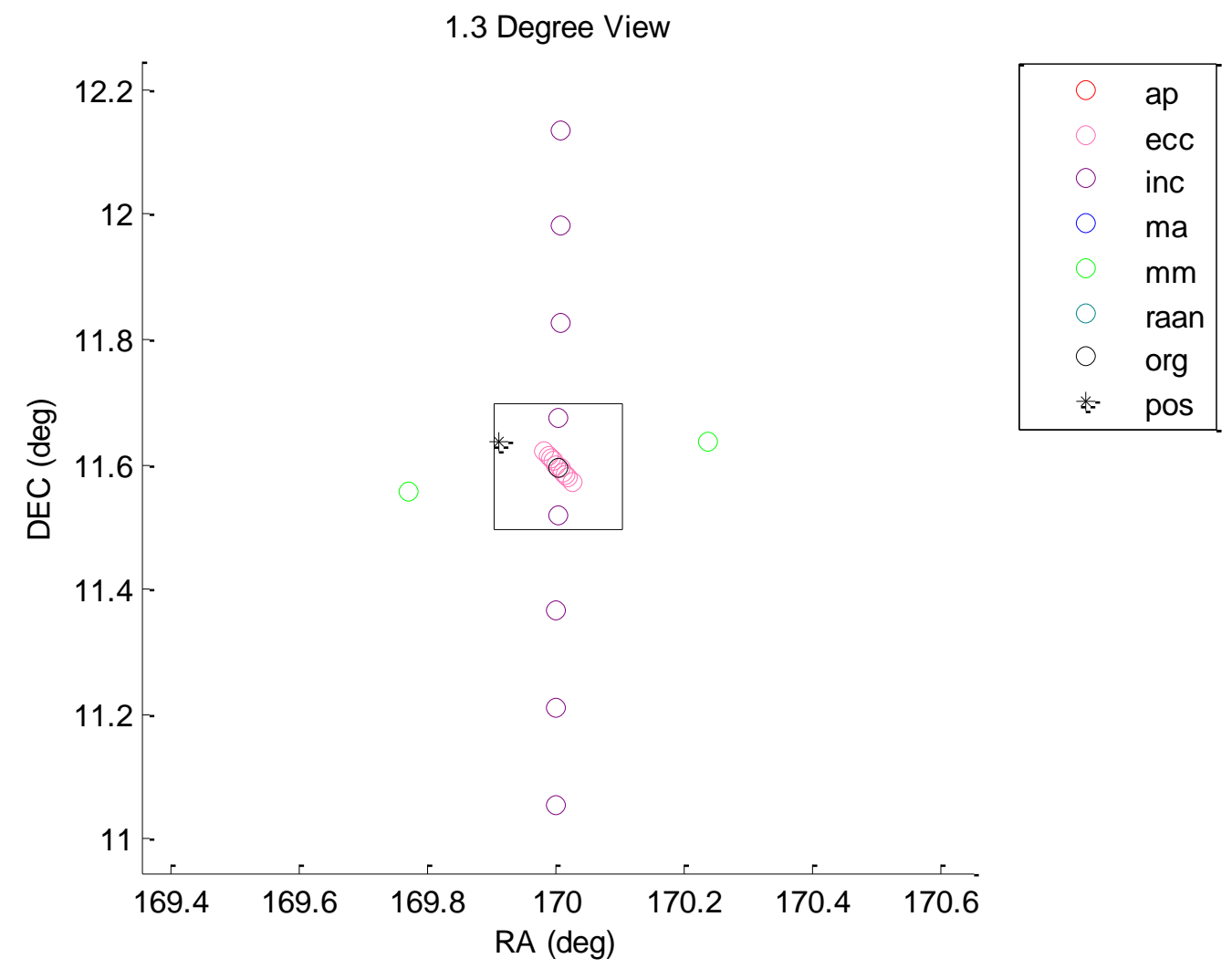




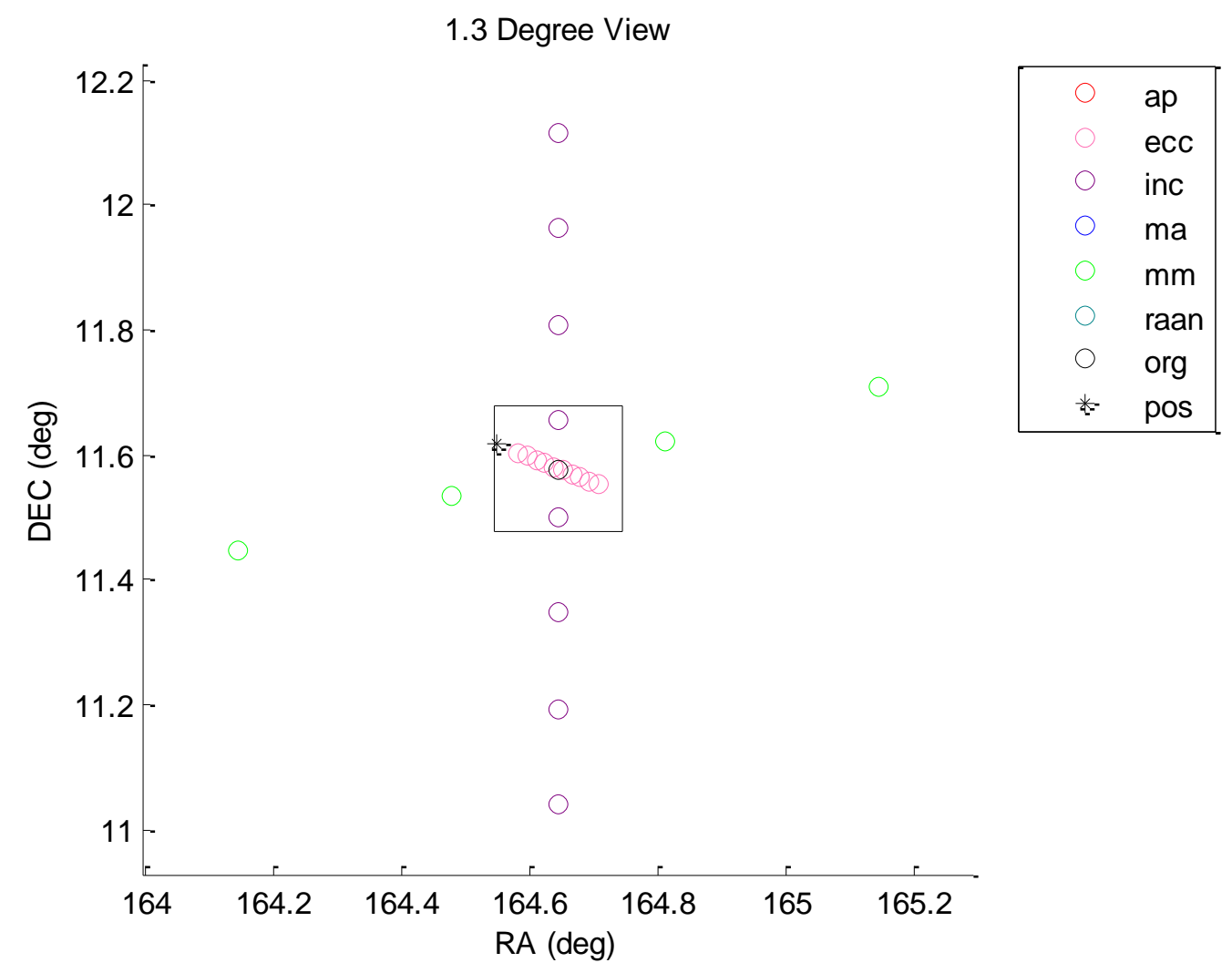




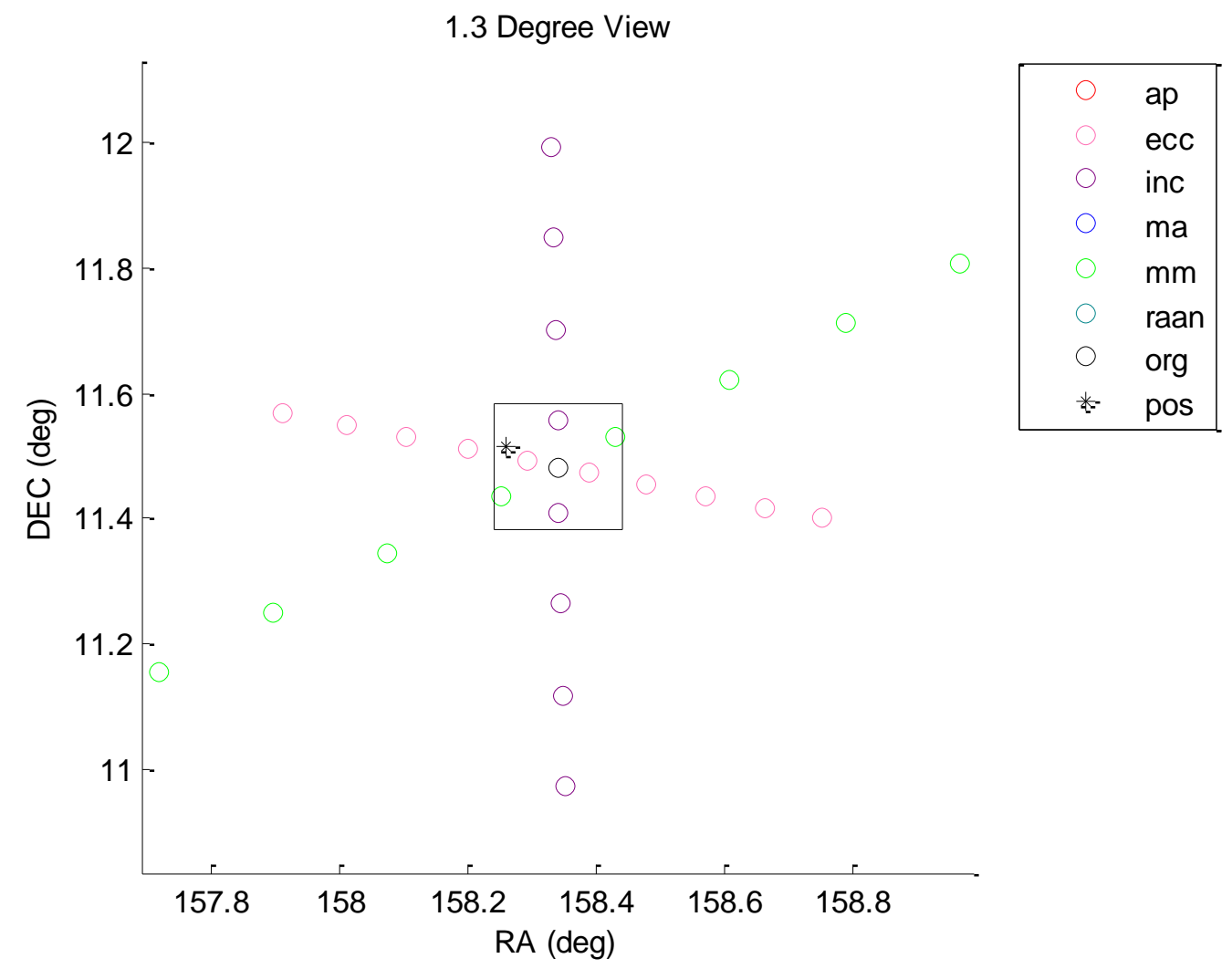




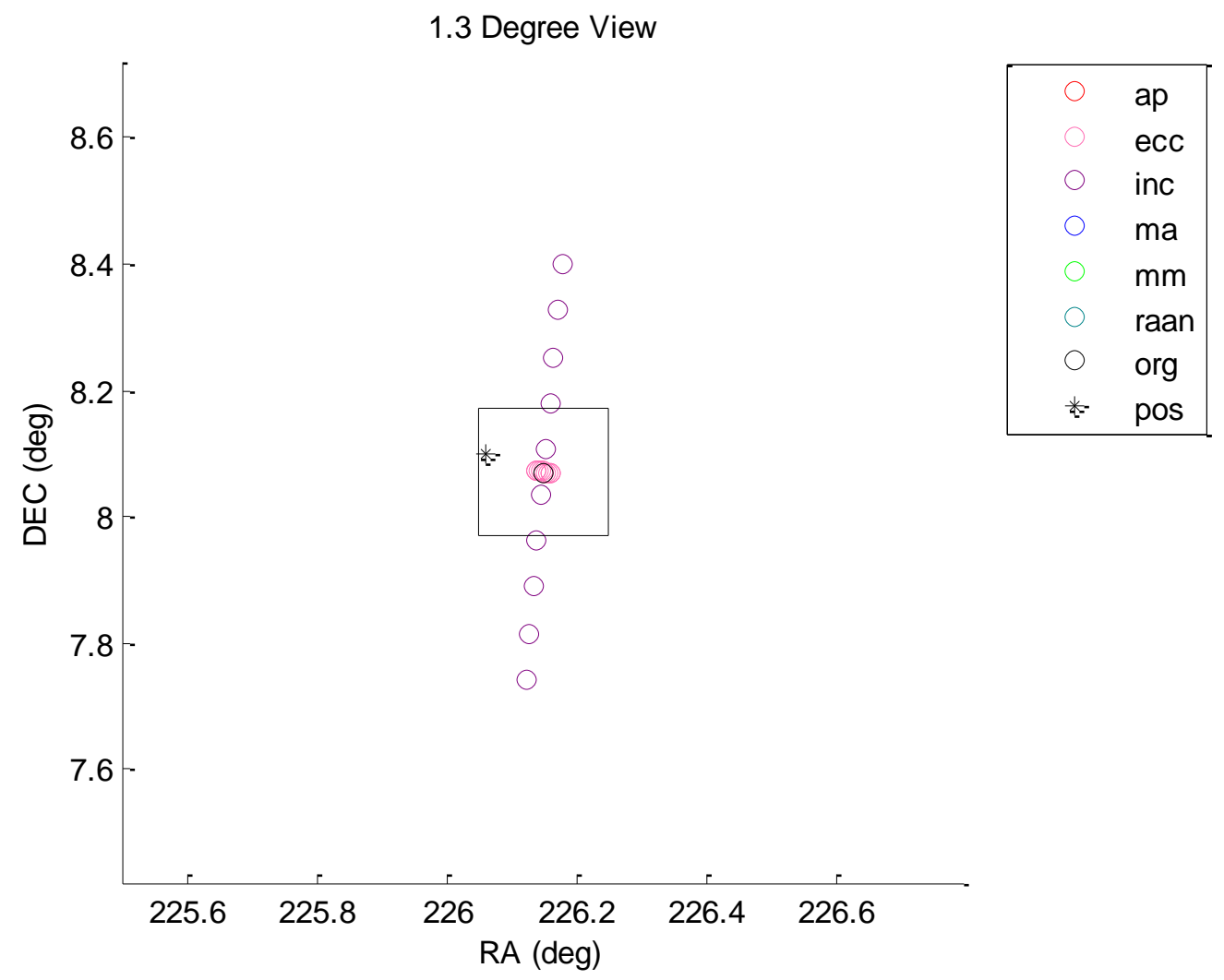




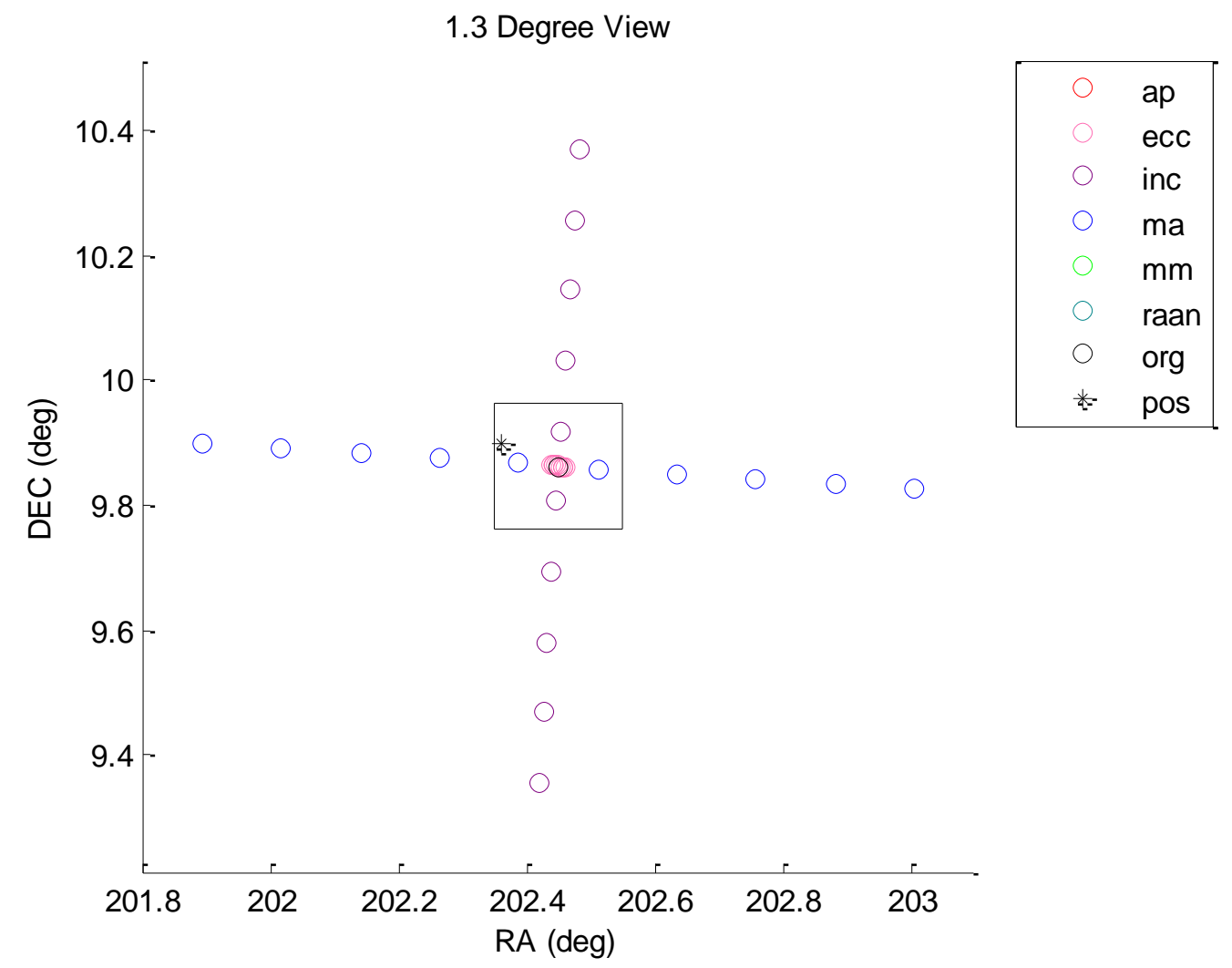




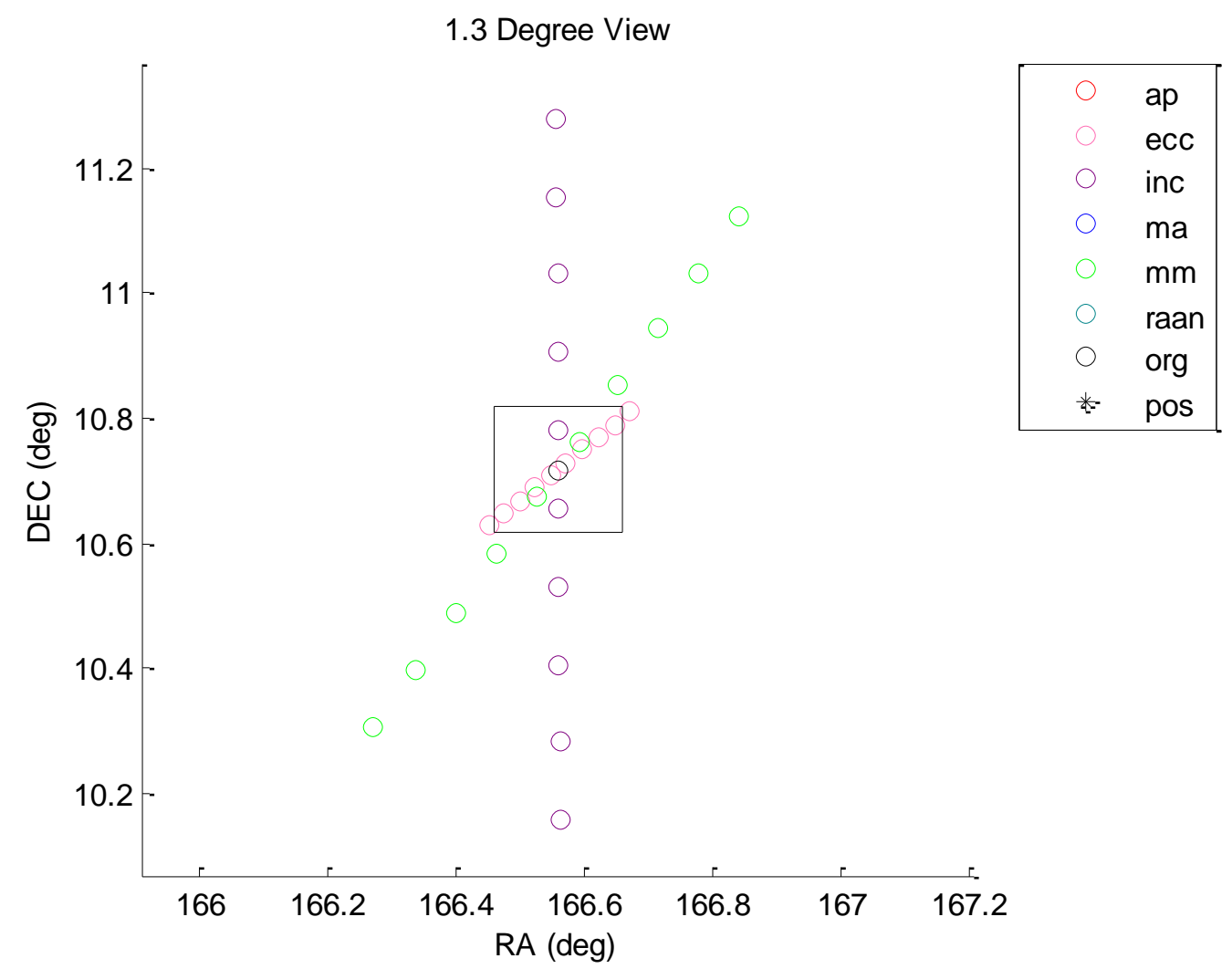




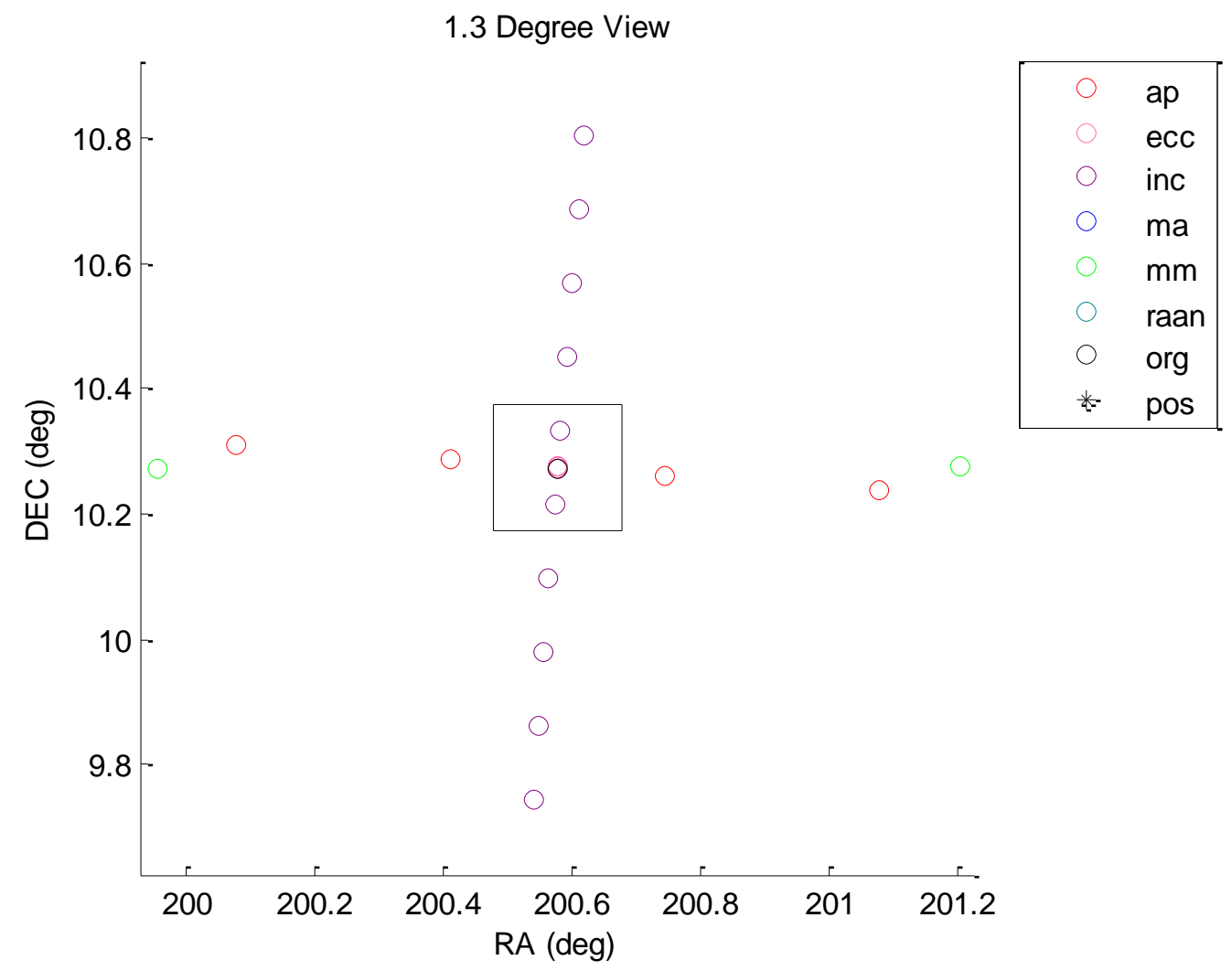




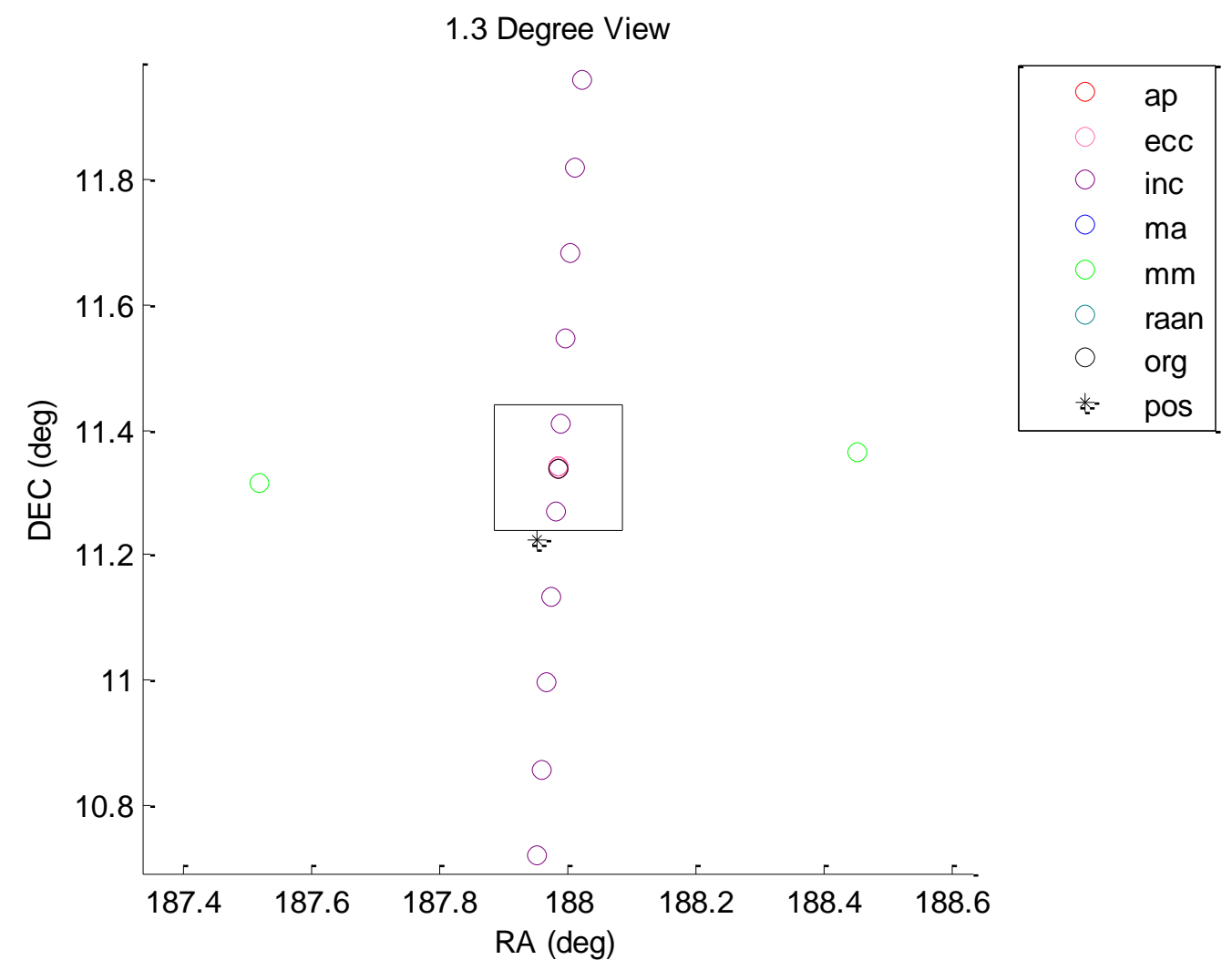




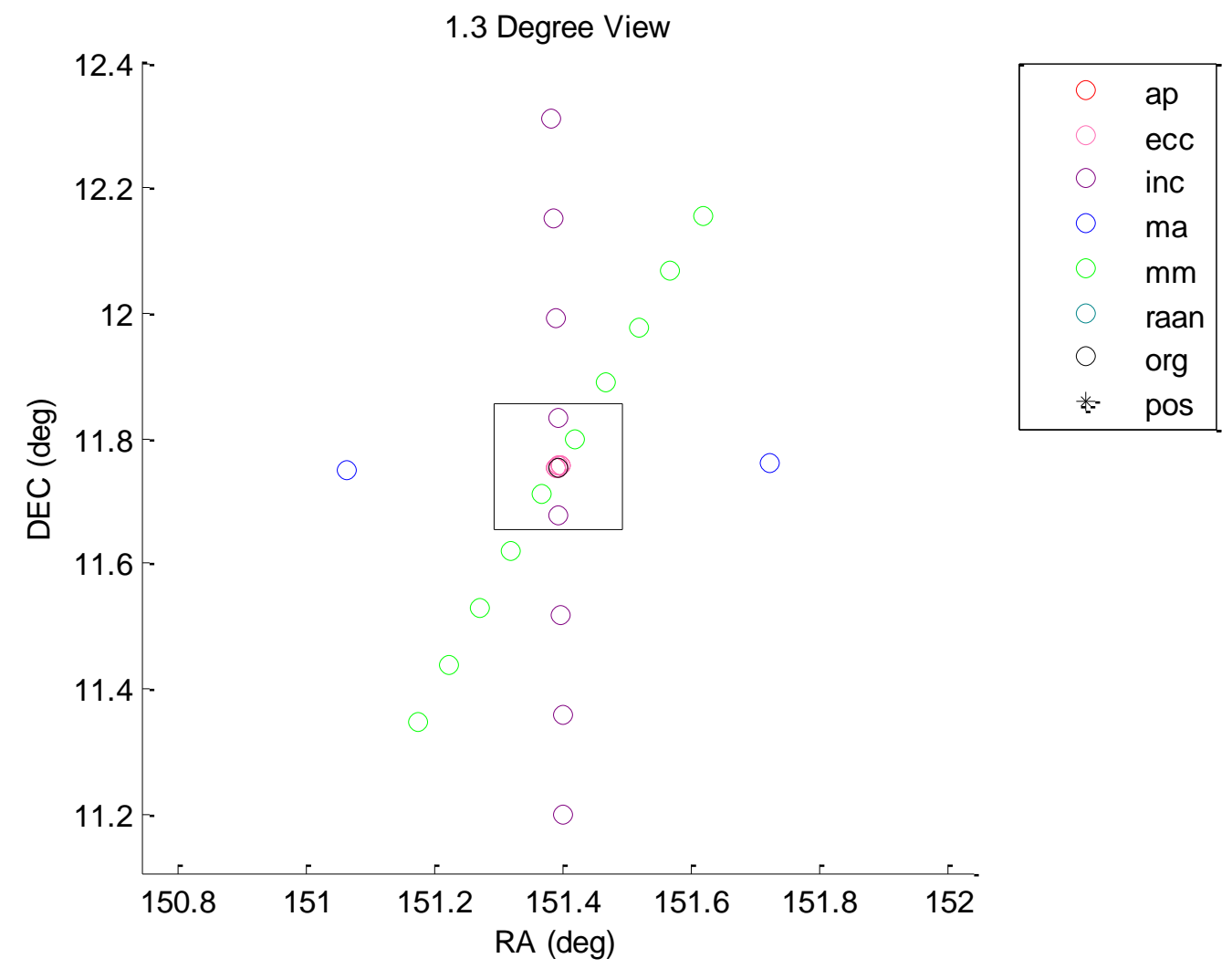




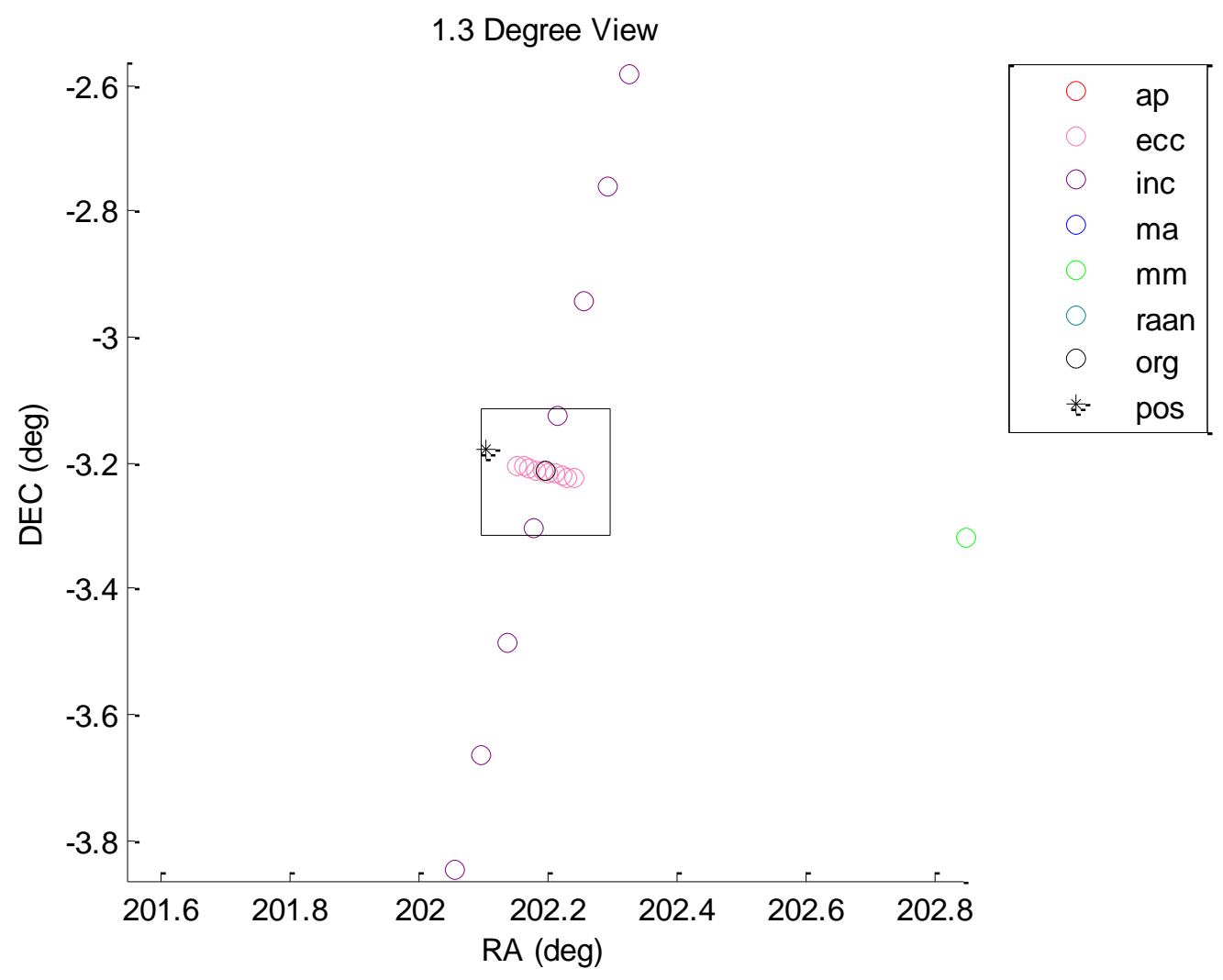




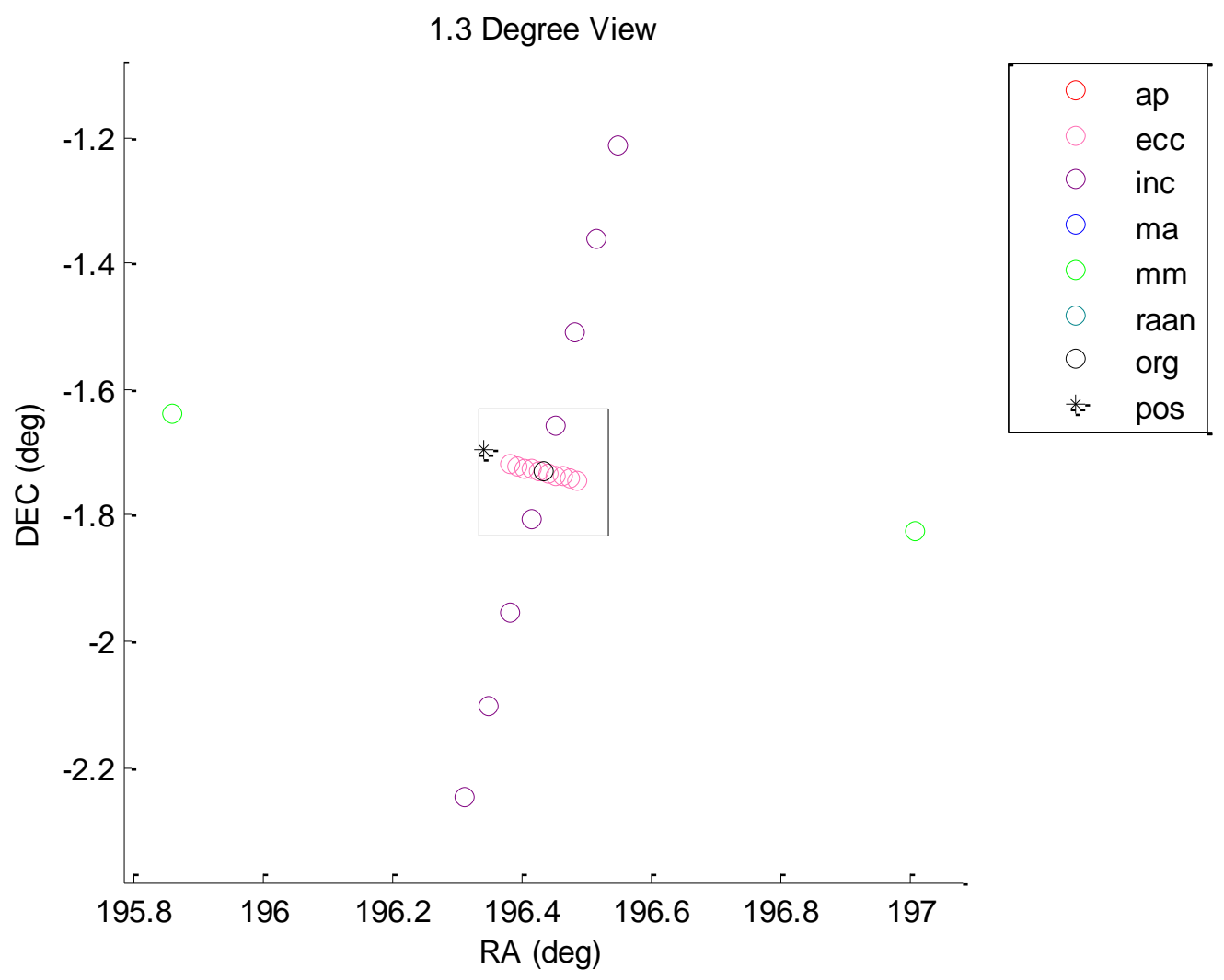




\subsection{Degree View}

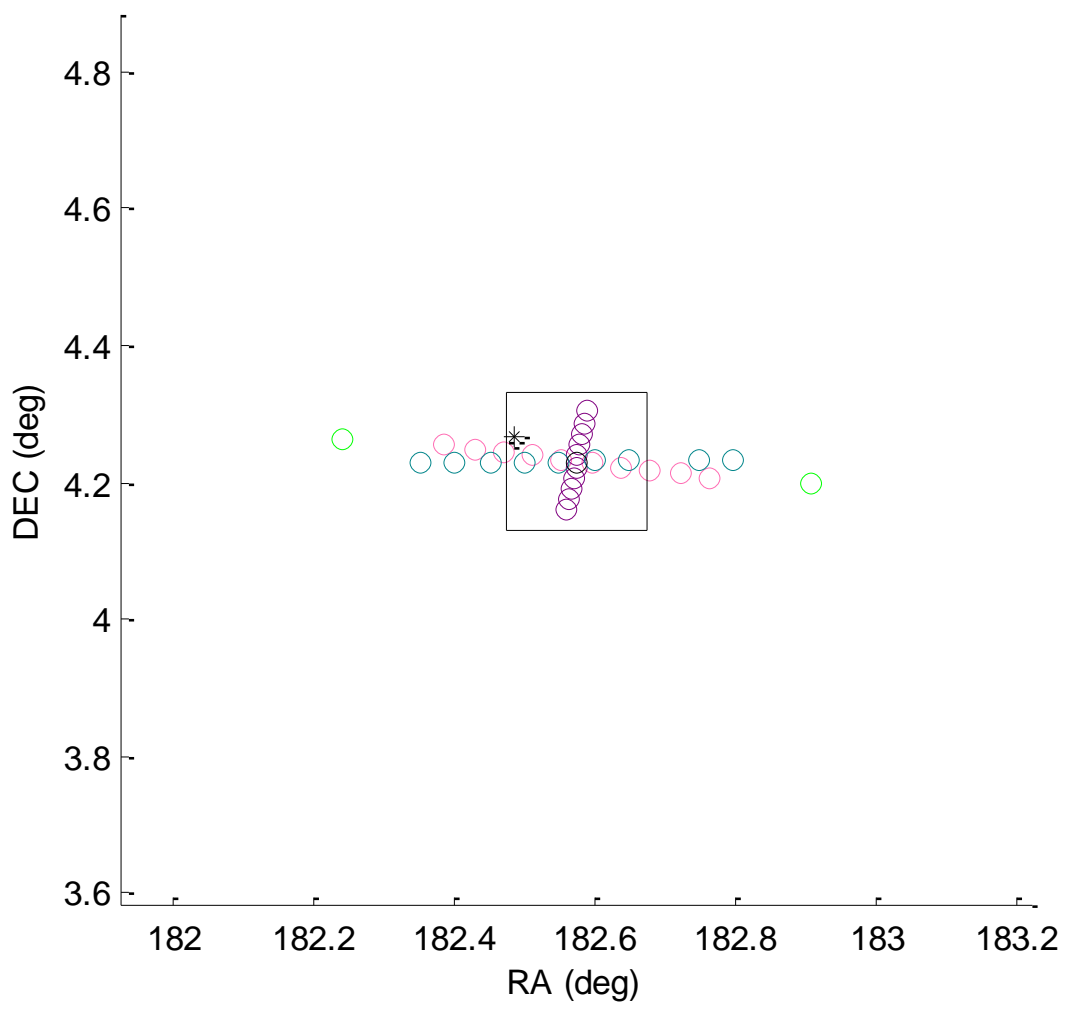

\begin{tabular}{|ll|}
\hline 0 & ap \\
ecc \\
inc \\
ma \\
mm \\
raan \\
org \\
* pos \\
\hline
\end{tabular}




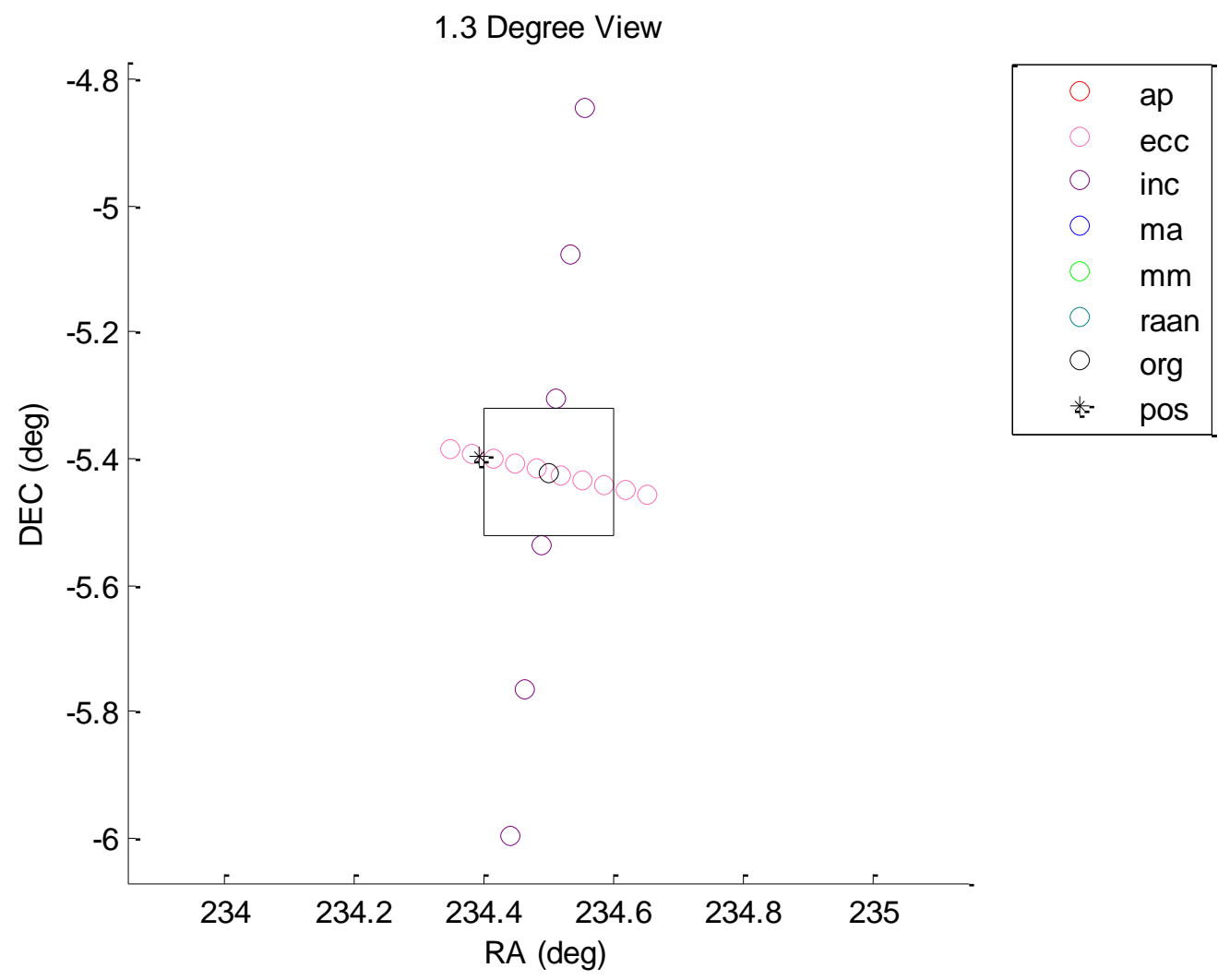




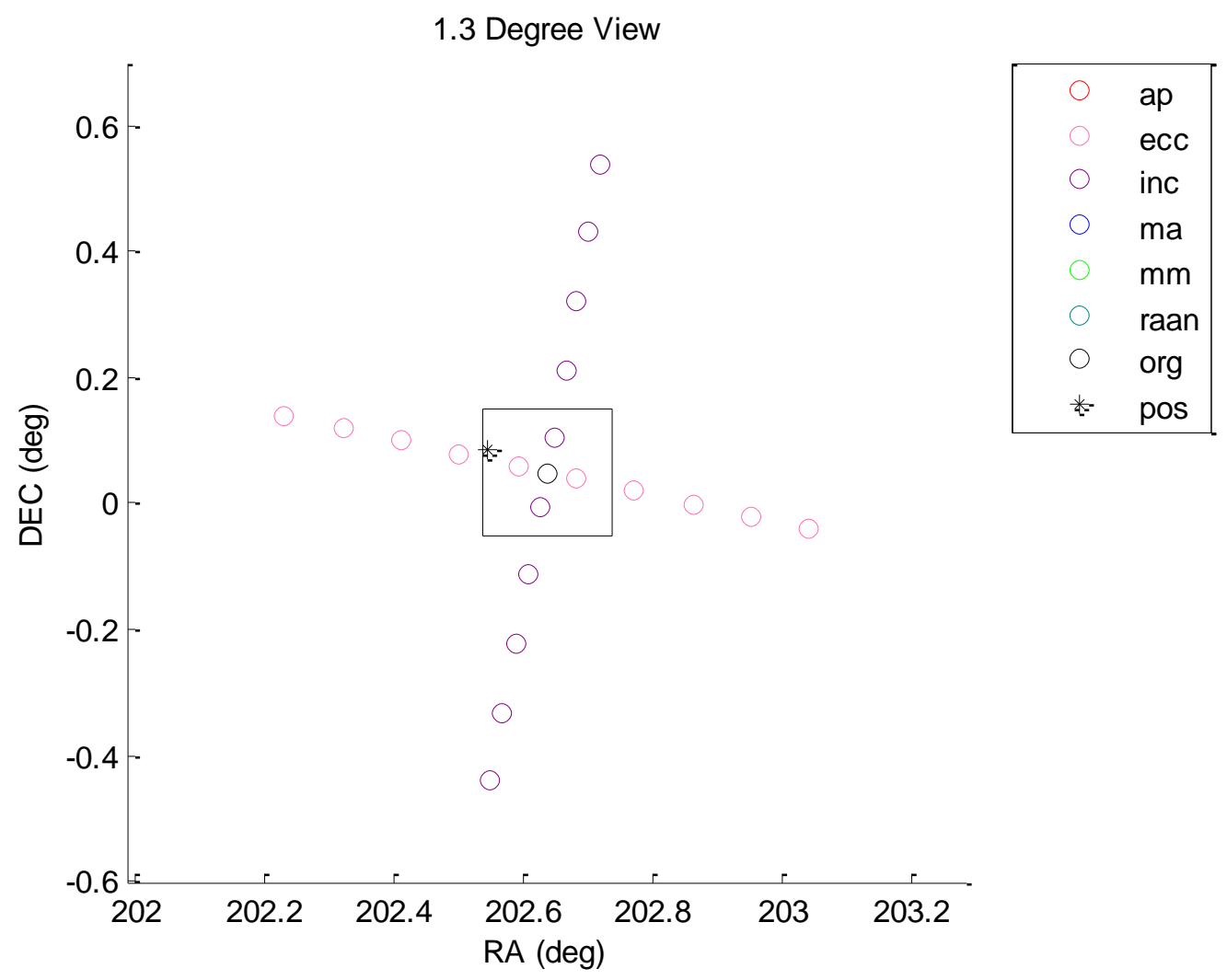




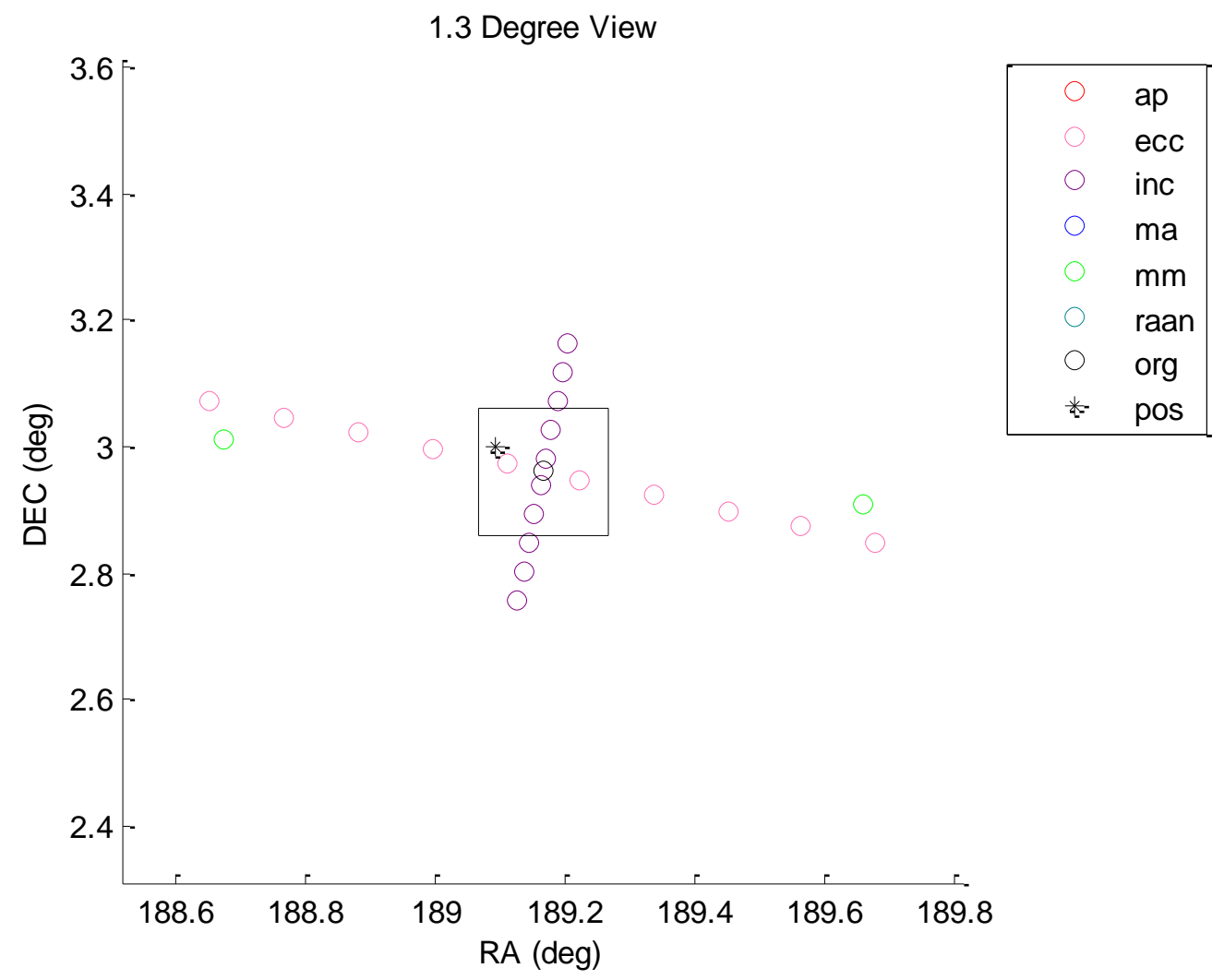




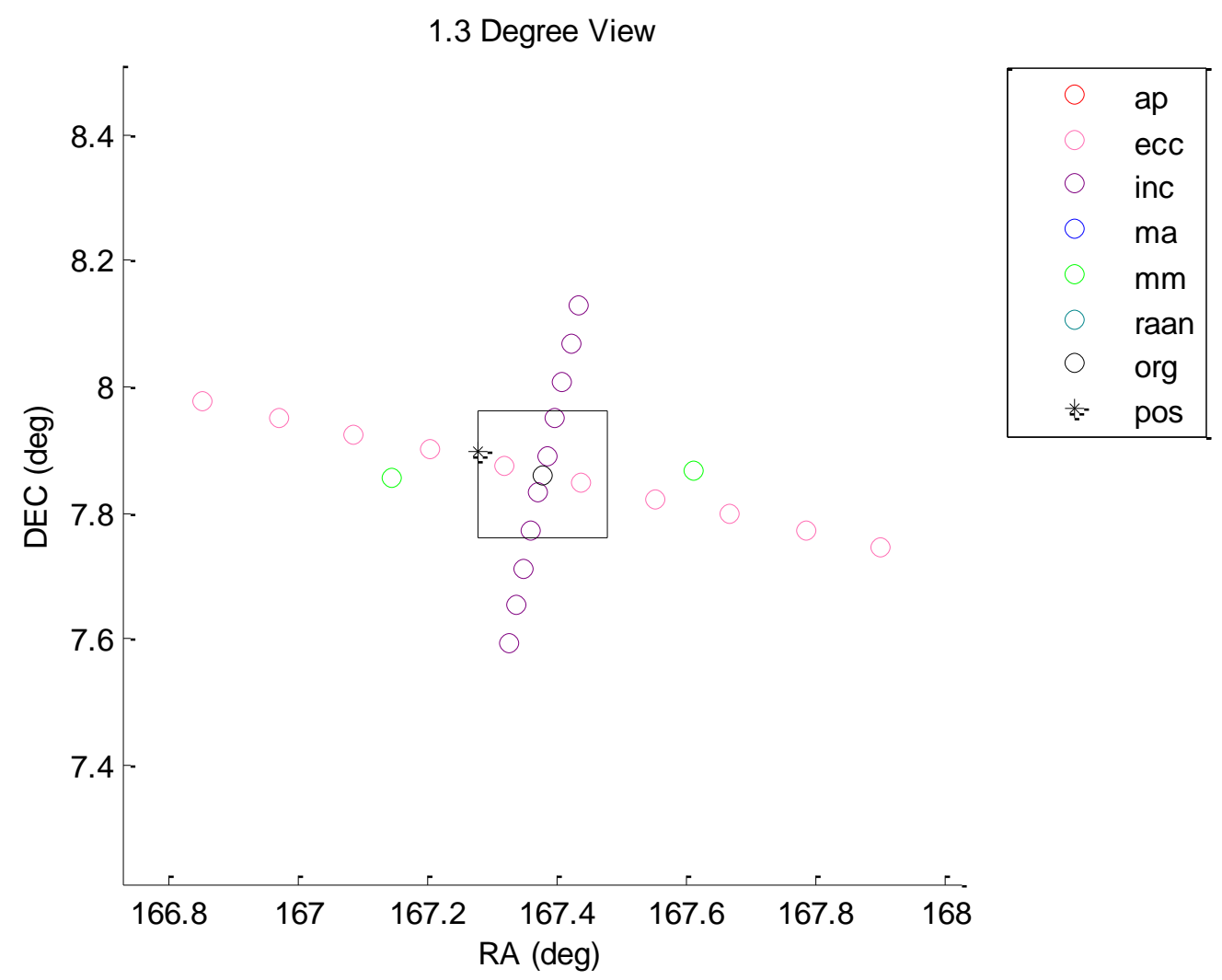




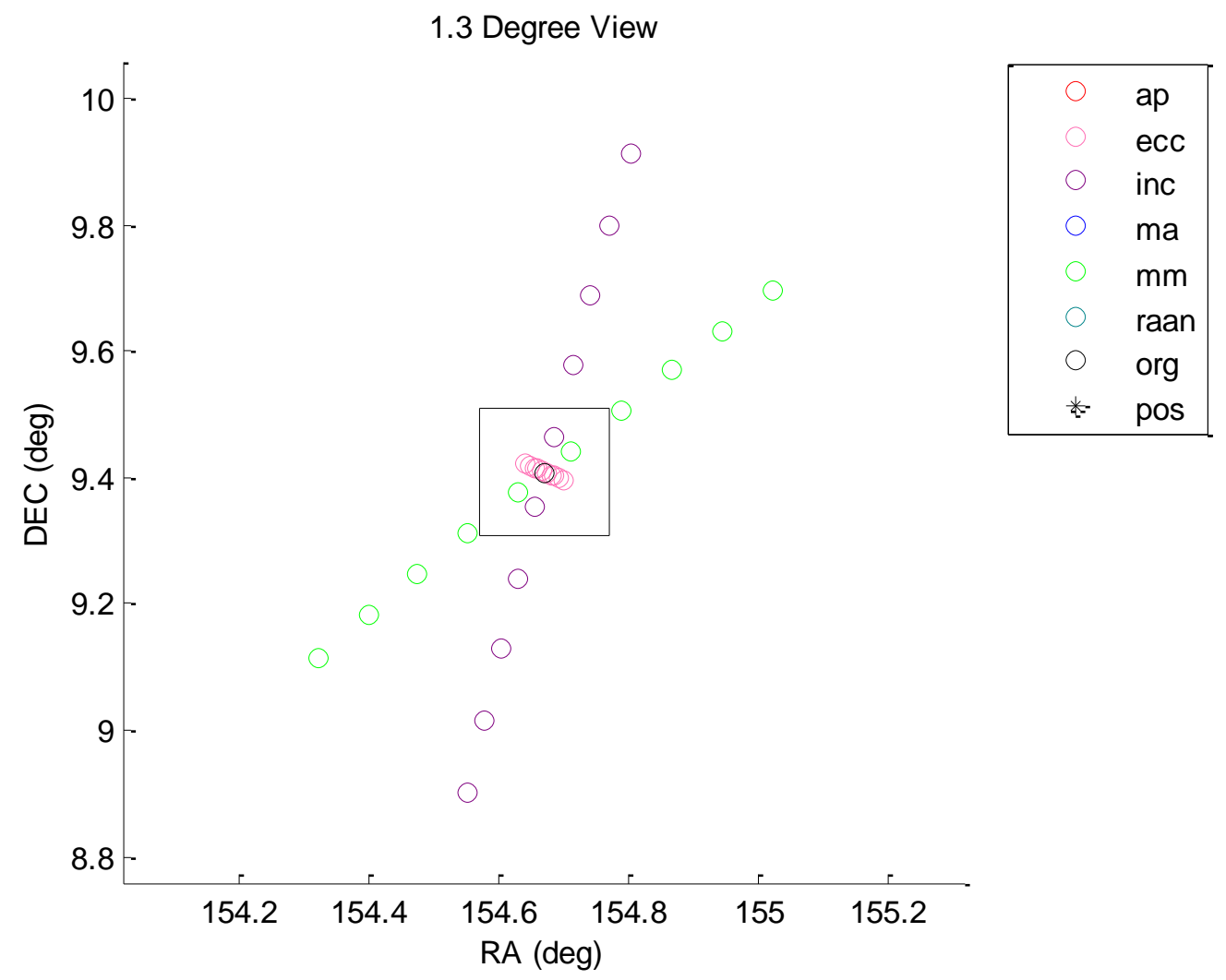




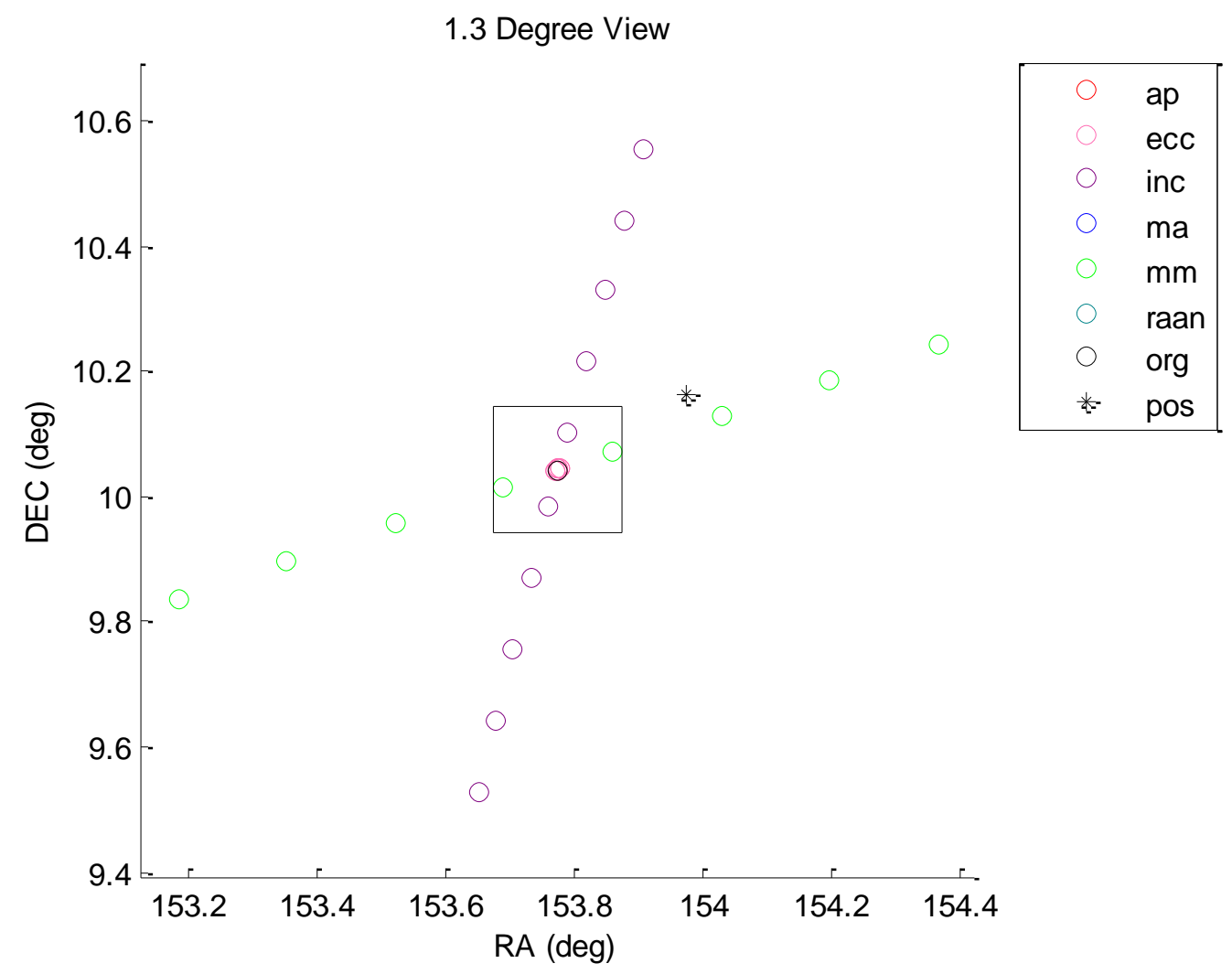




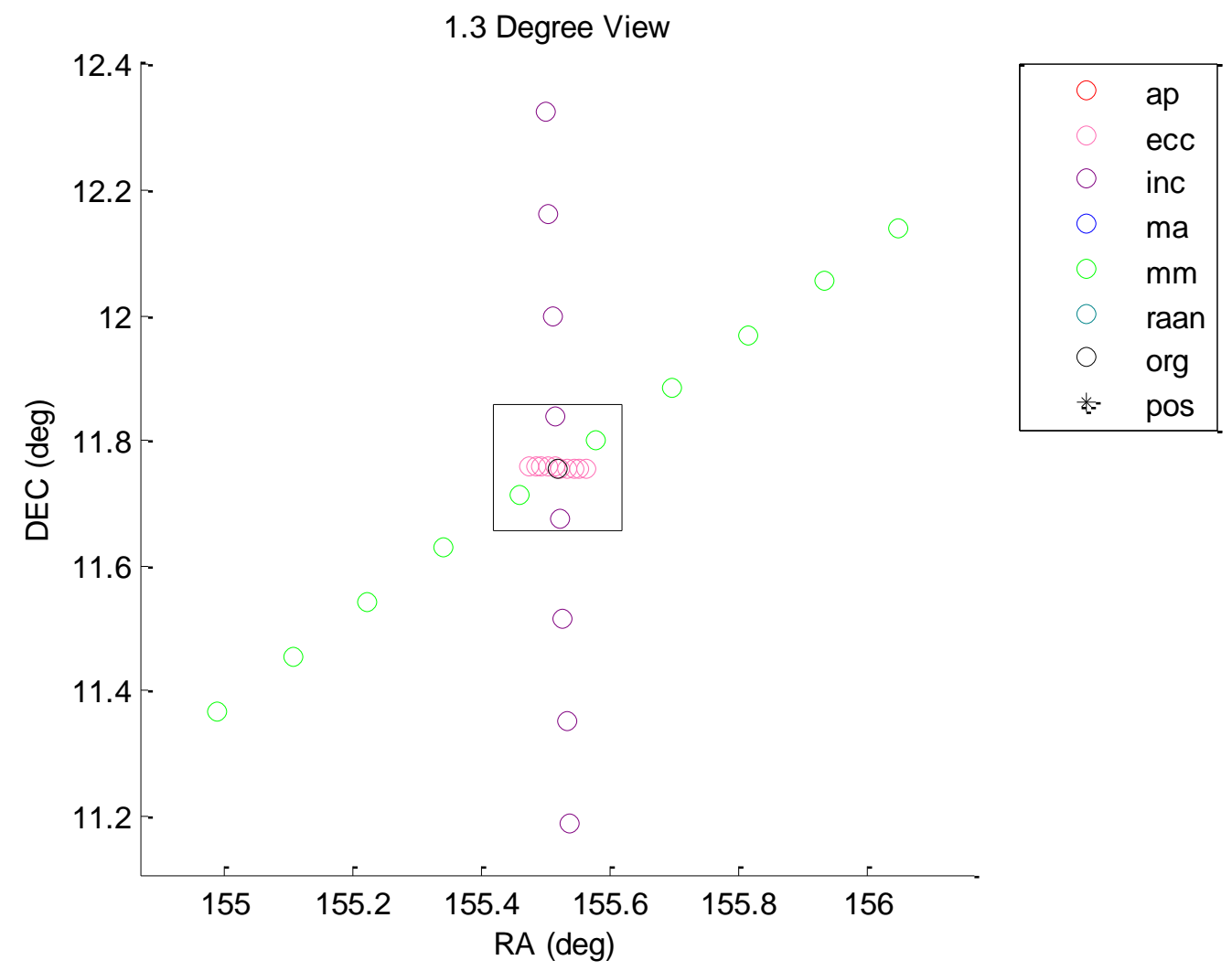




\section{APPENDIX C: FULL ORBIT SELECT PLOTS 2\% VARIATION}

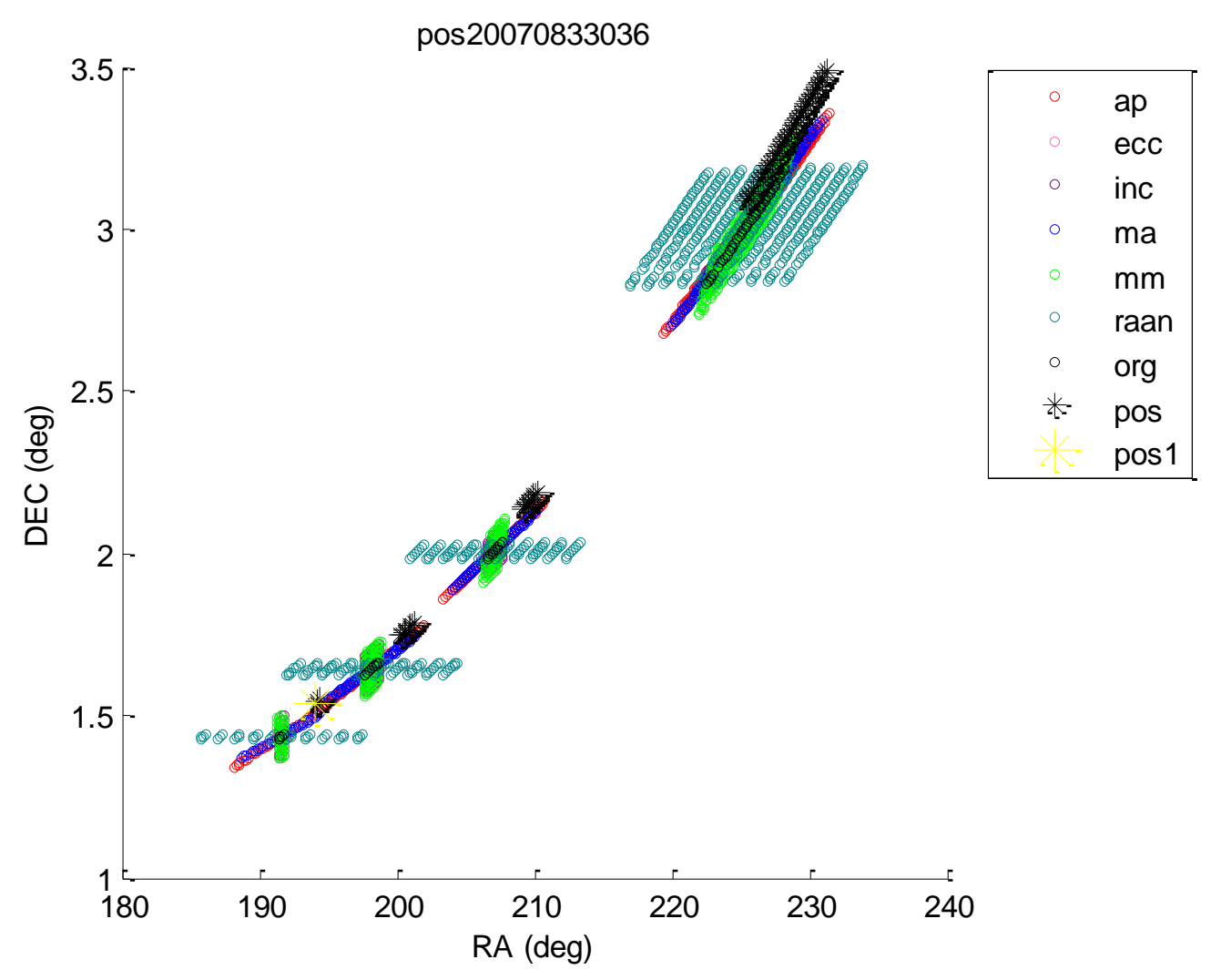



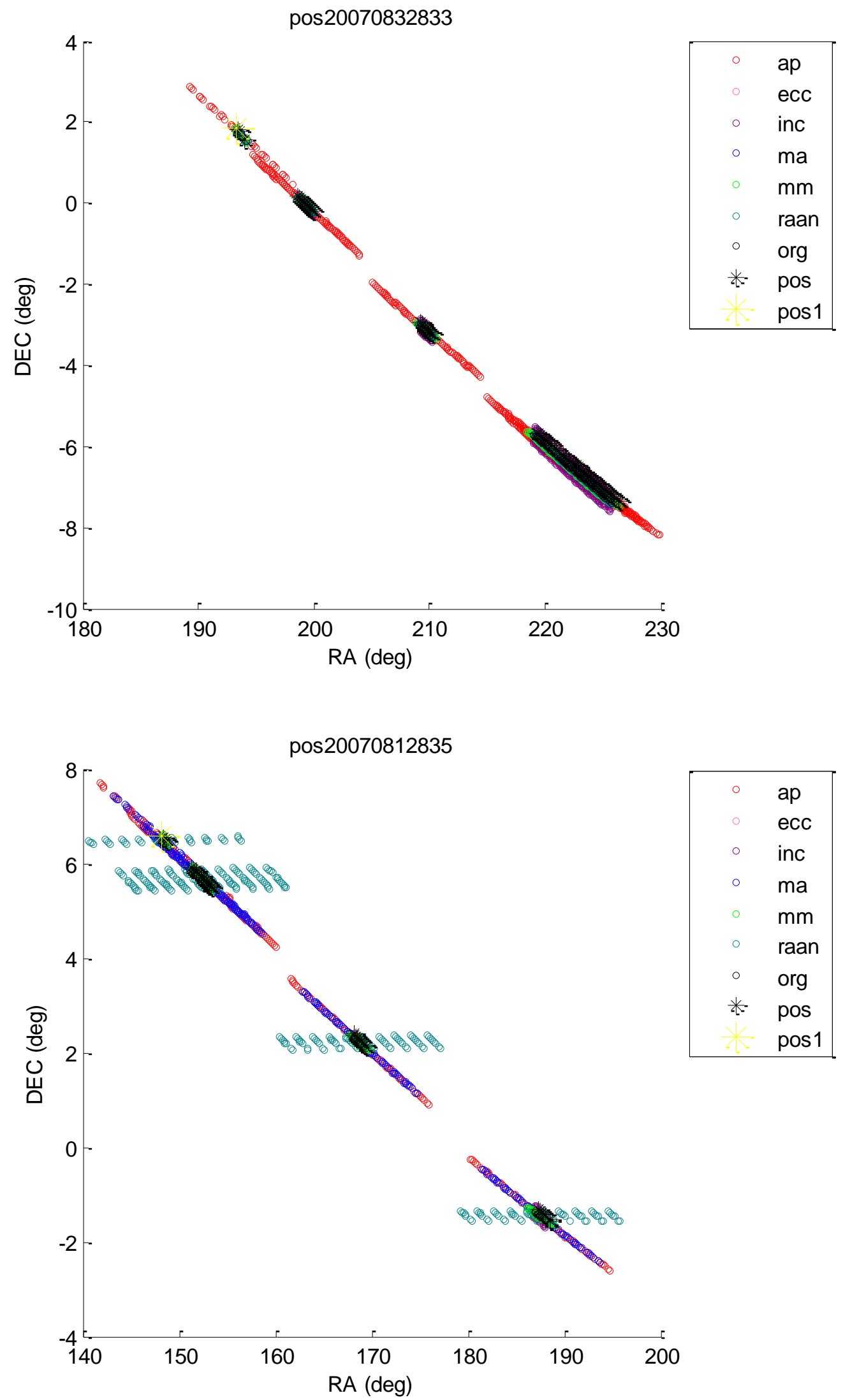

\begin{tabular}{|ll|}
\hline & ap \\
& ecc \\
& inc \\
& ma \\
& mm \\
& raan \\
$\circ$ & org \\
$*$ & pos \\
& pos1 \\
\hline
\end{tabular}



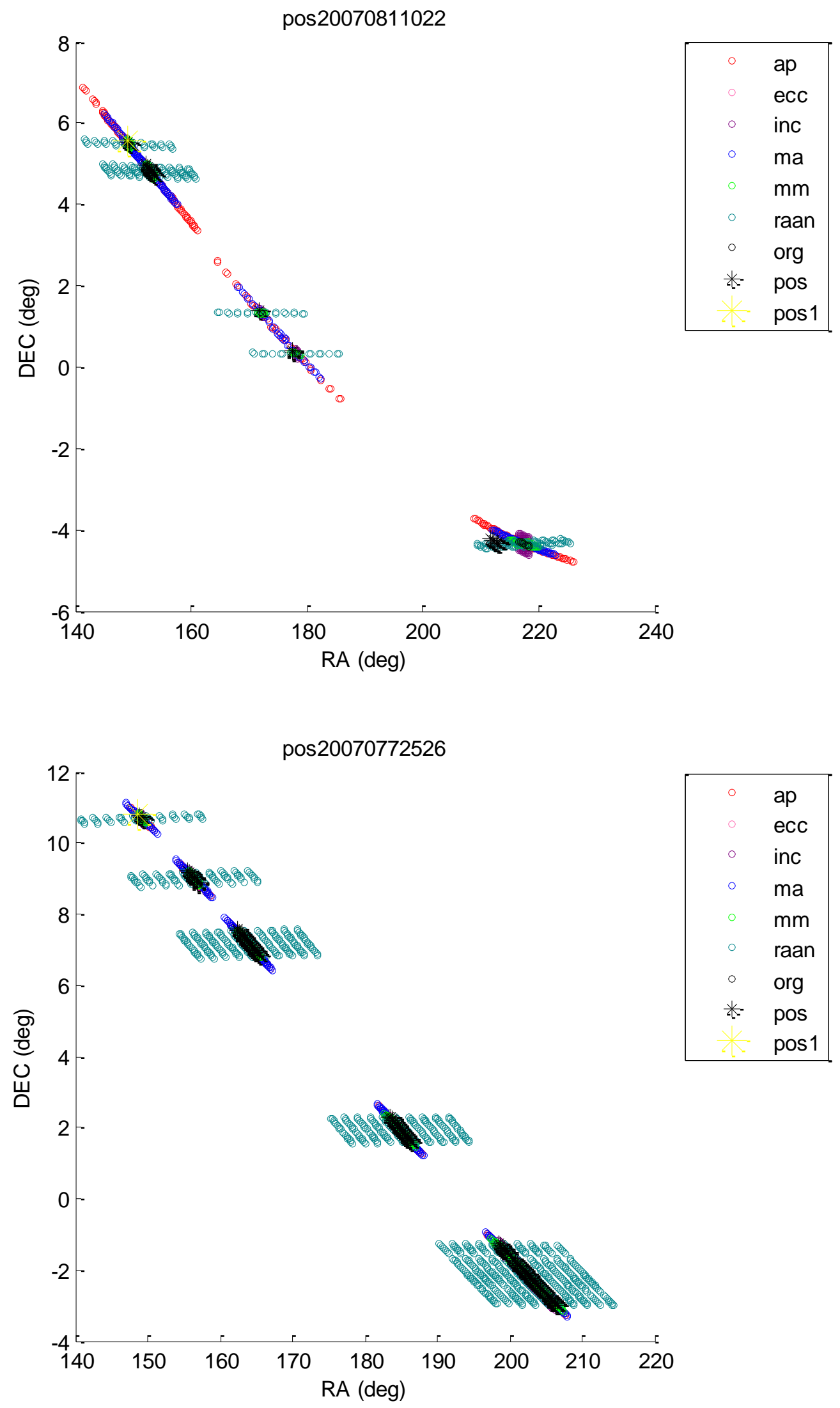

\begin{tabular}{|ll|}
\hline & ap \\
ecc \\
inc \\
ma \\
mm \\
raan \\
org \\
*- pos \\
\\
\end{tabular}




\section{APPENDIX D: FIRST PREDICTED POINT 2\% VARIATION}

The box in these images is the C9 telescope view; the images were combined to shorten the appendix. 


\subsection{Degree View}

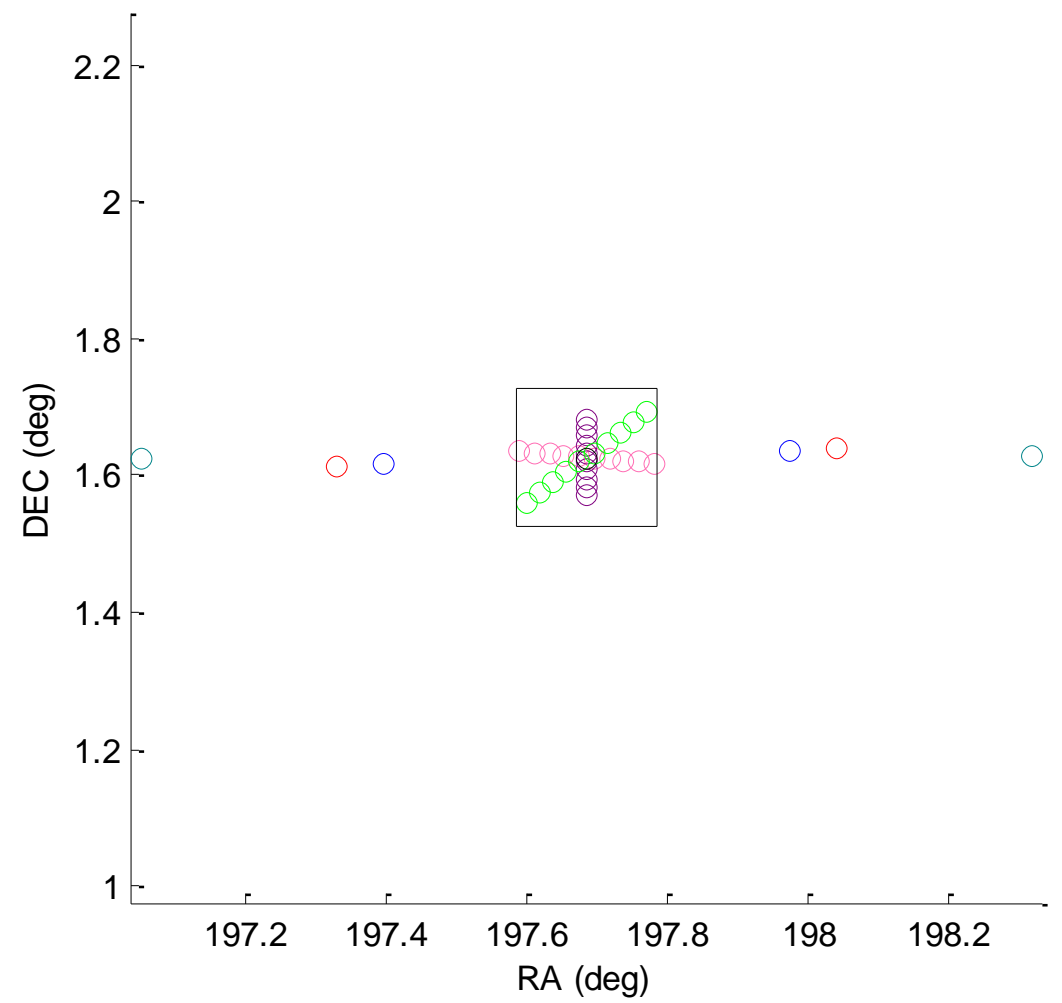

\begin{tabular}{|ll|}
\hline ap \\
ecc \\
inc \\
ma \\
mm \\
raan \\
org \\
pos \\
\hline
\end{tabular}

RA (deg) 
1.3 Degree View

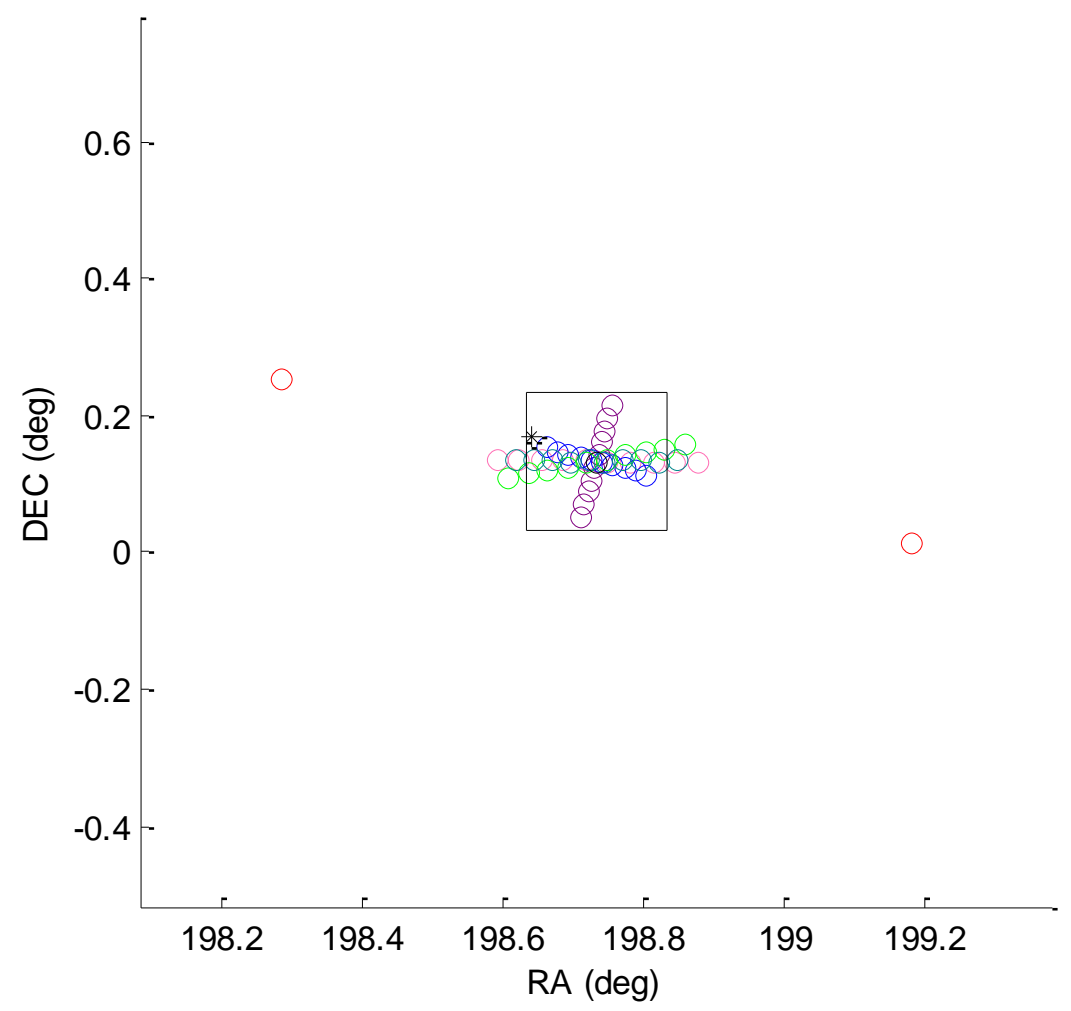

1.3 Degree View

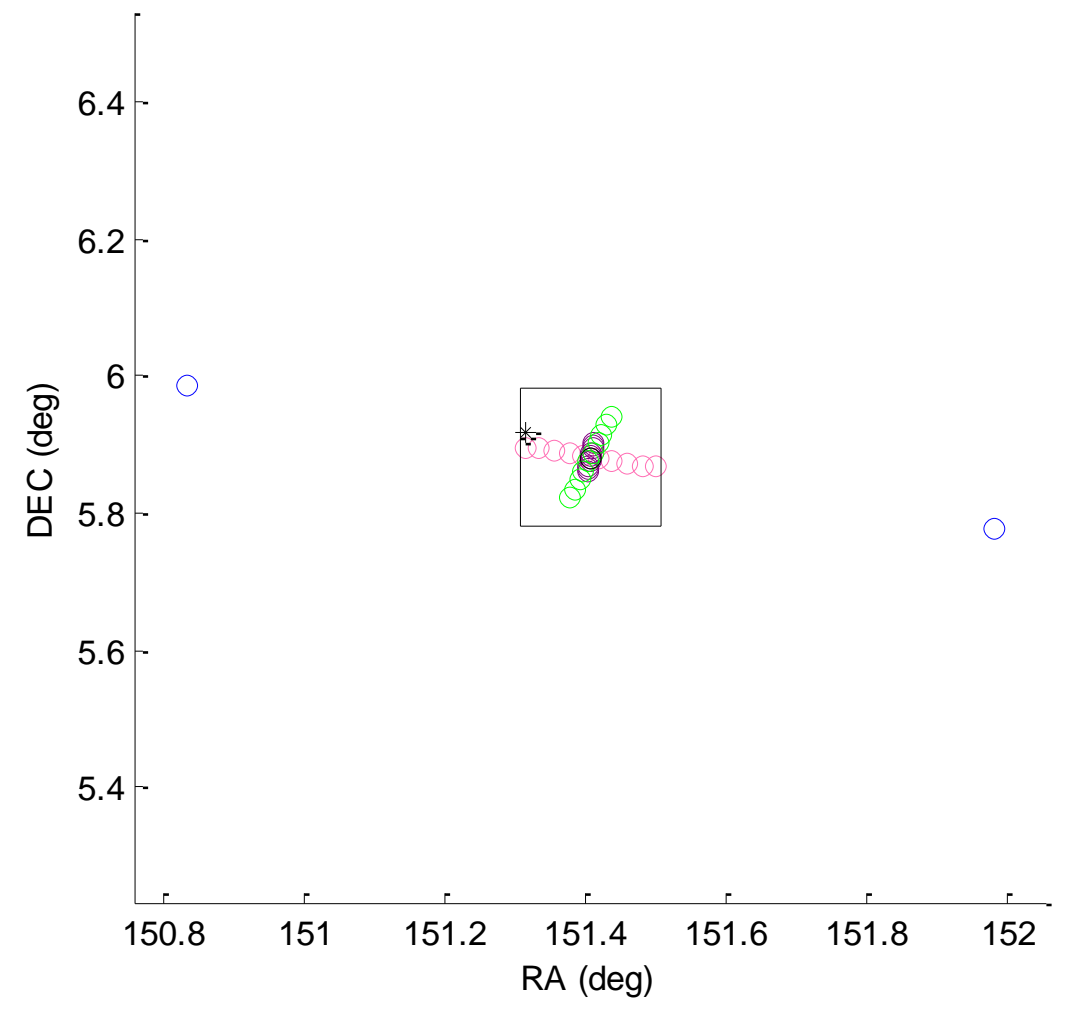

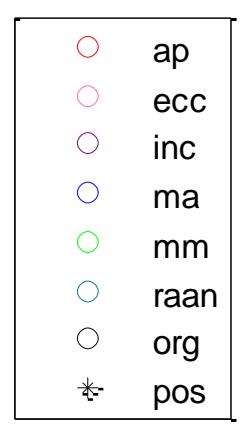

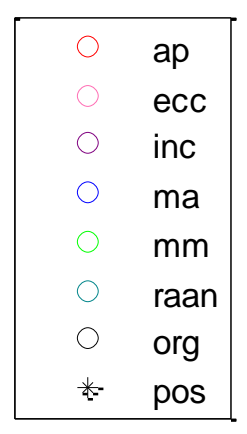


1.3 Degree View

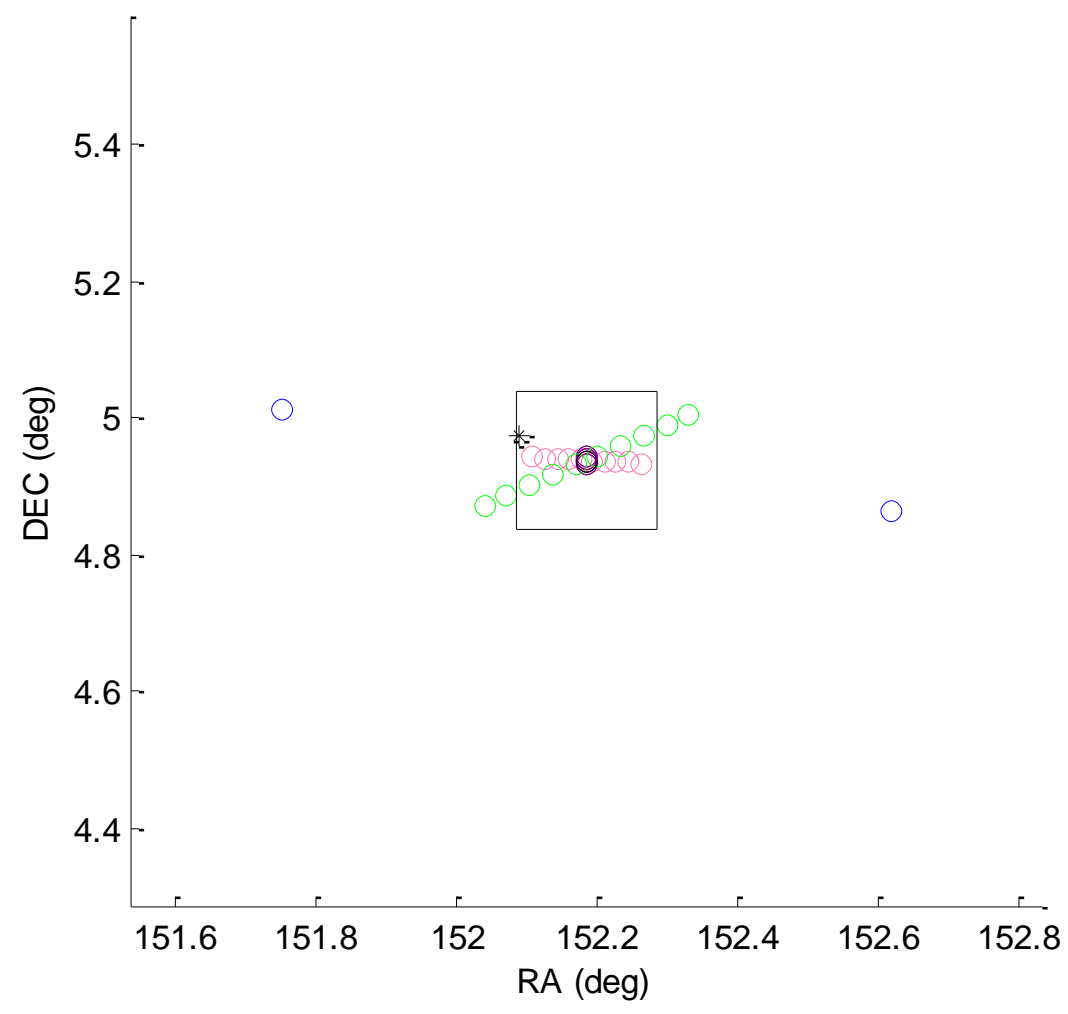

1.3 Degree View

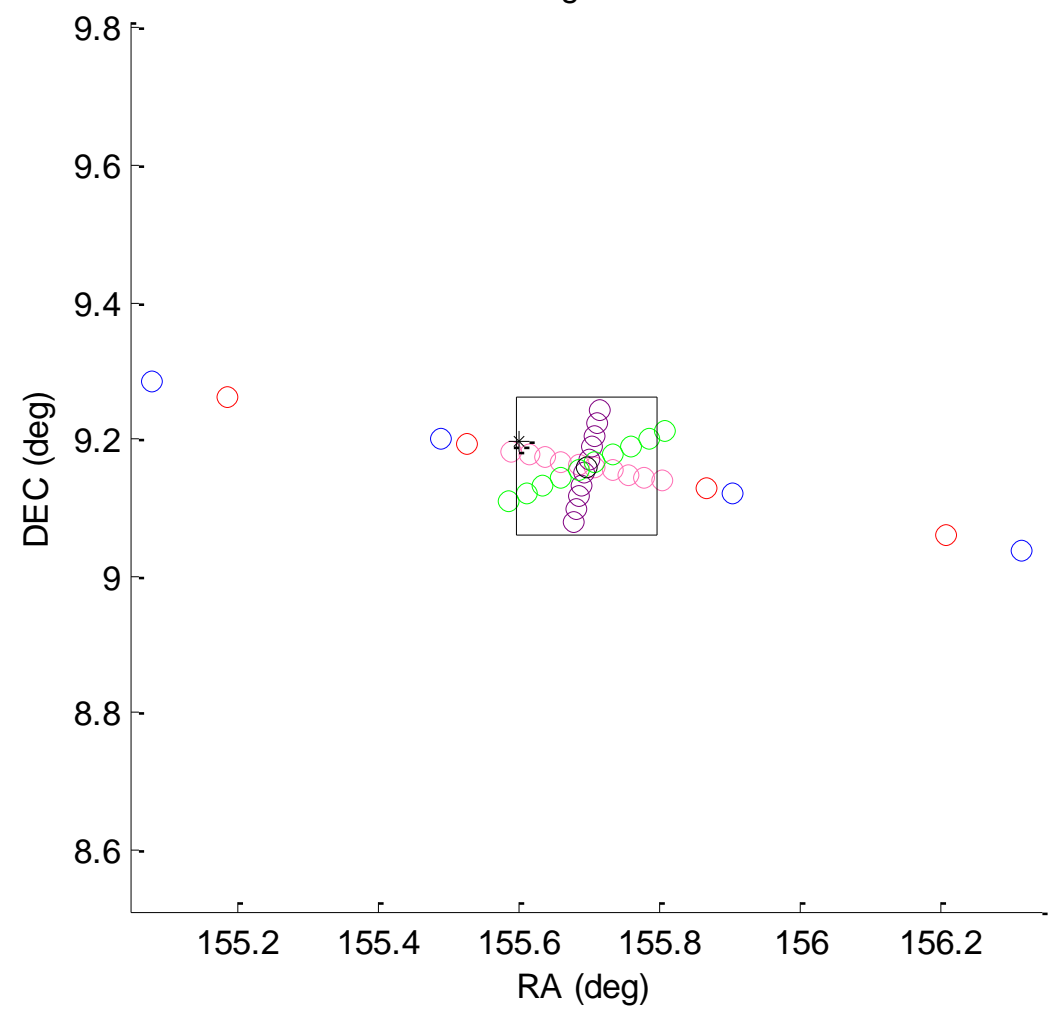

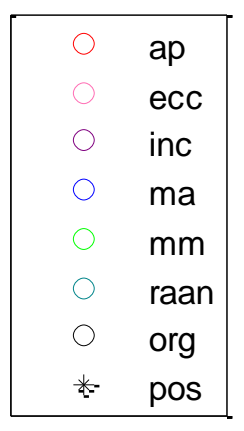

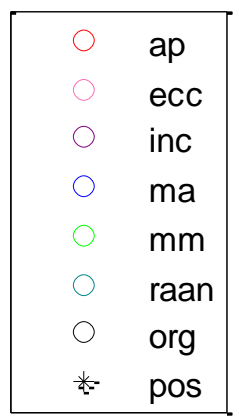




\section{APPENDIX E: CODE}

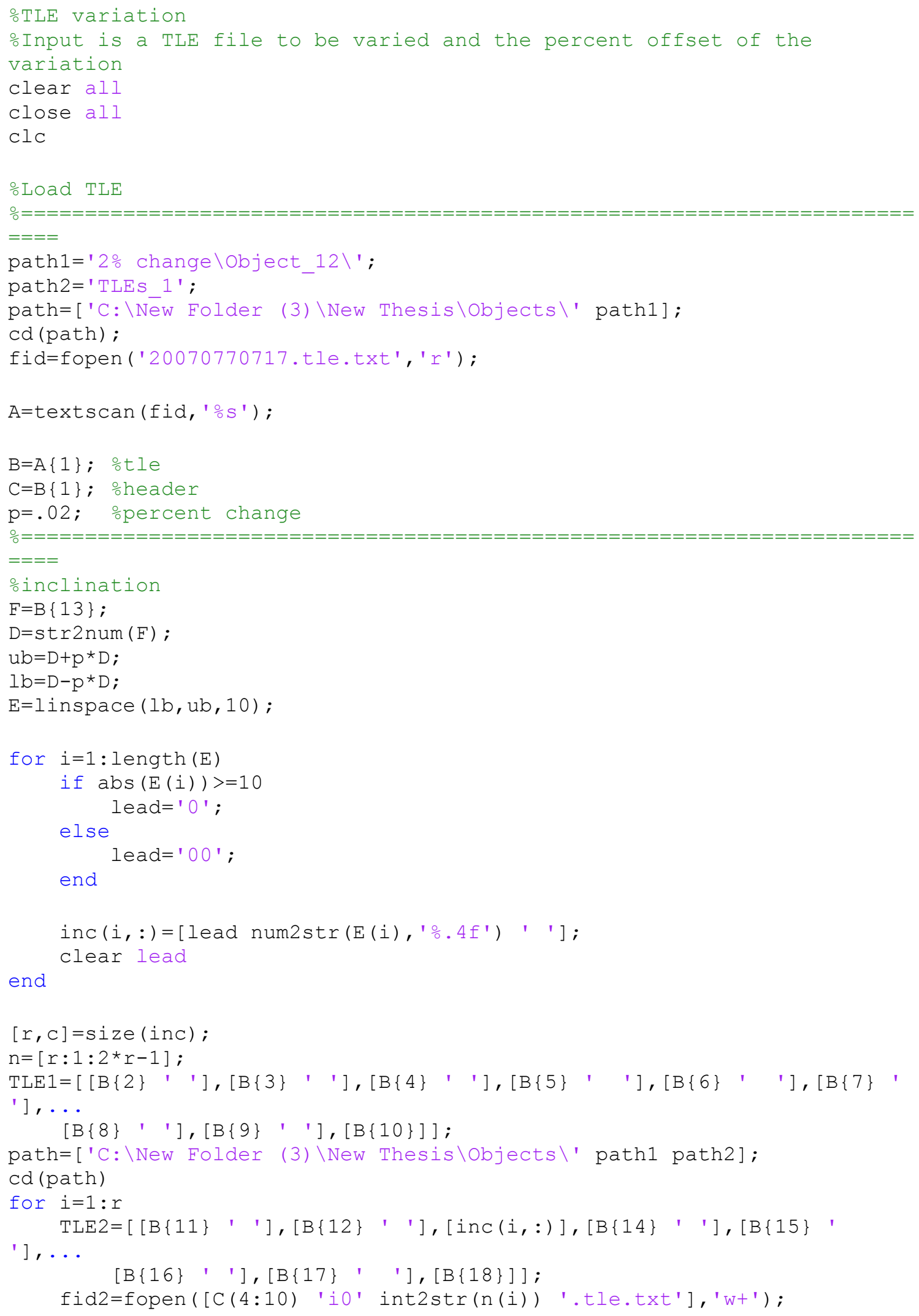




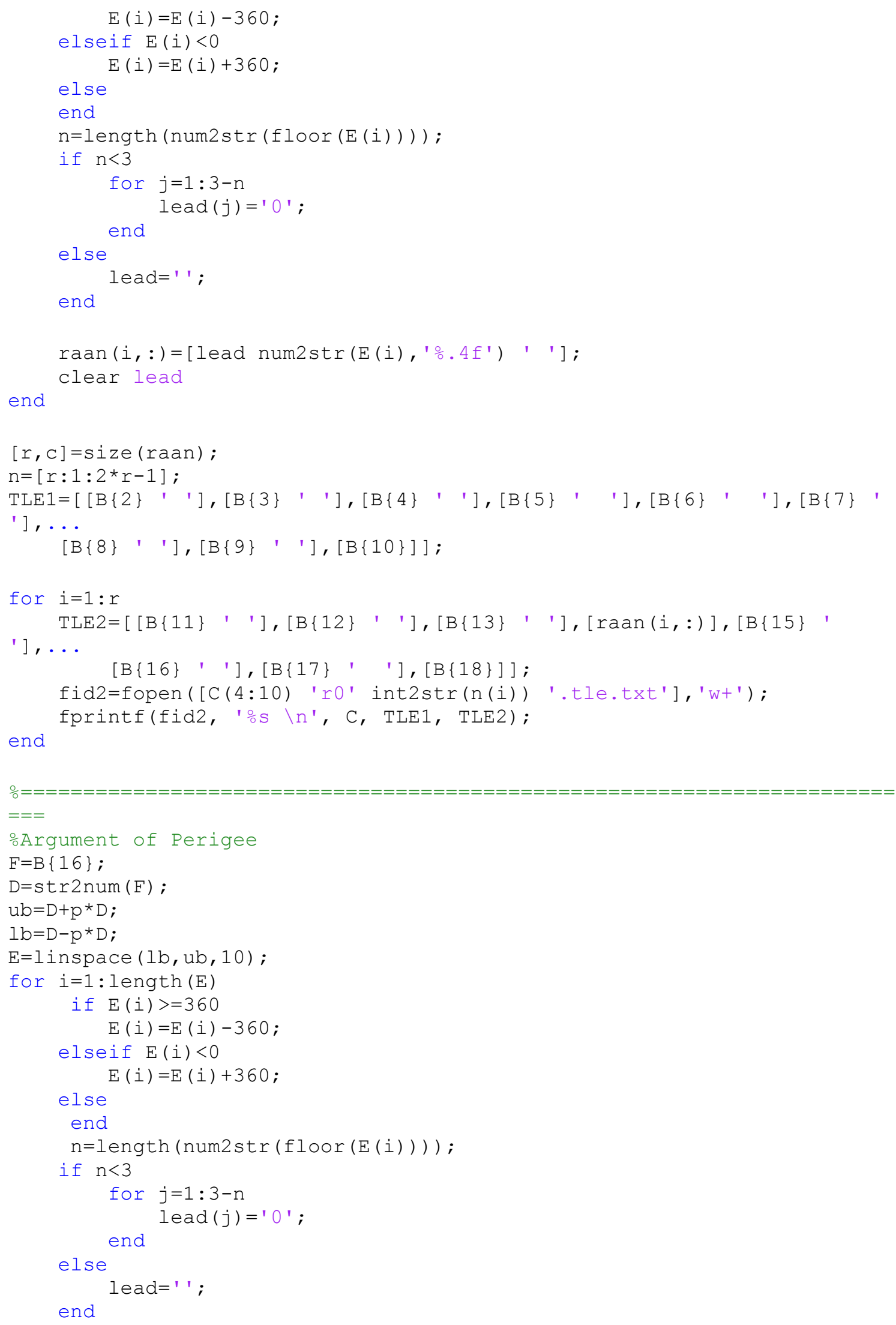




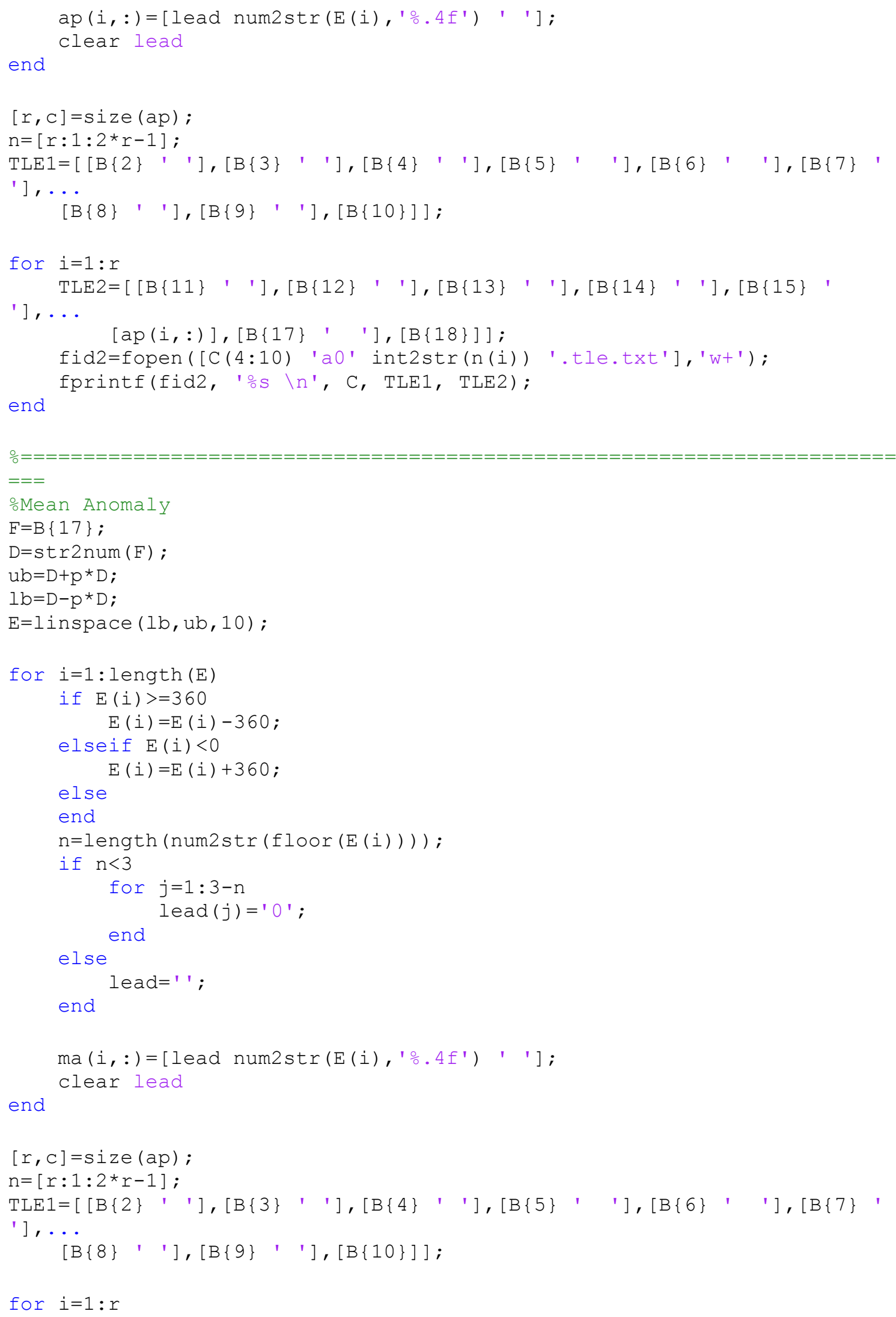




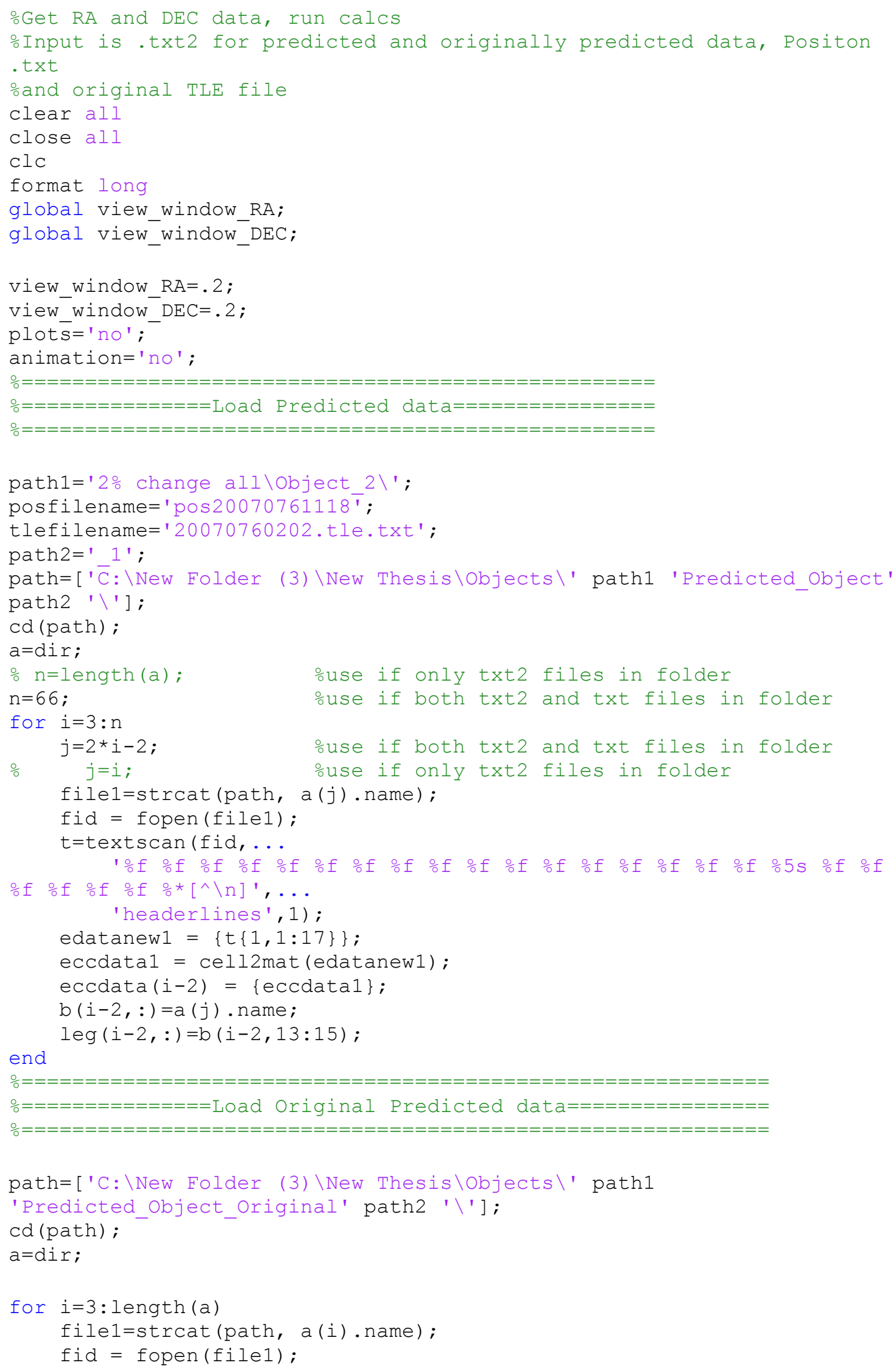




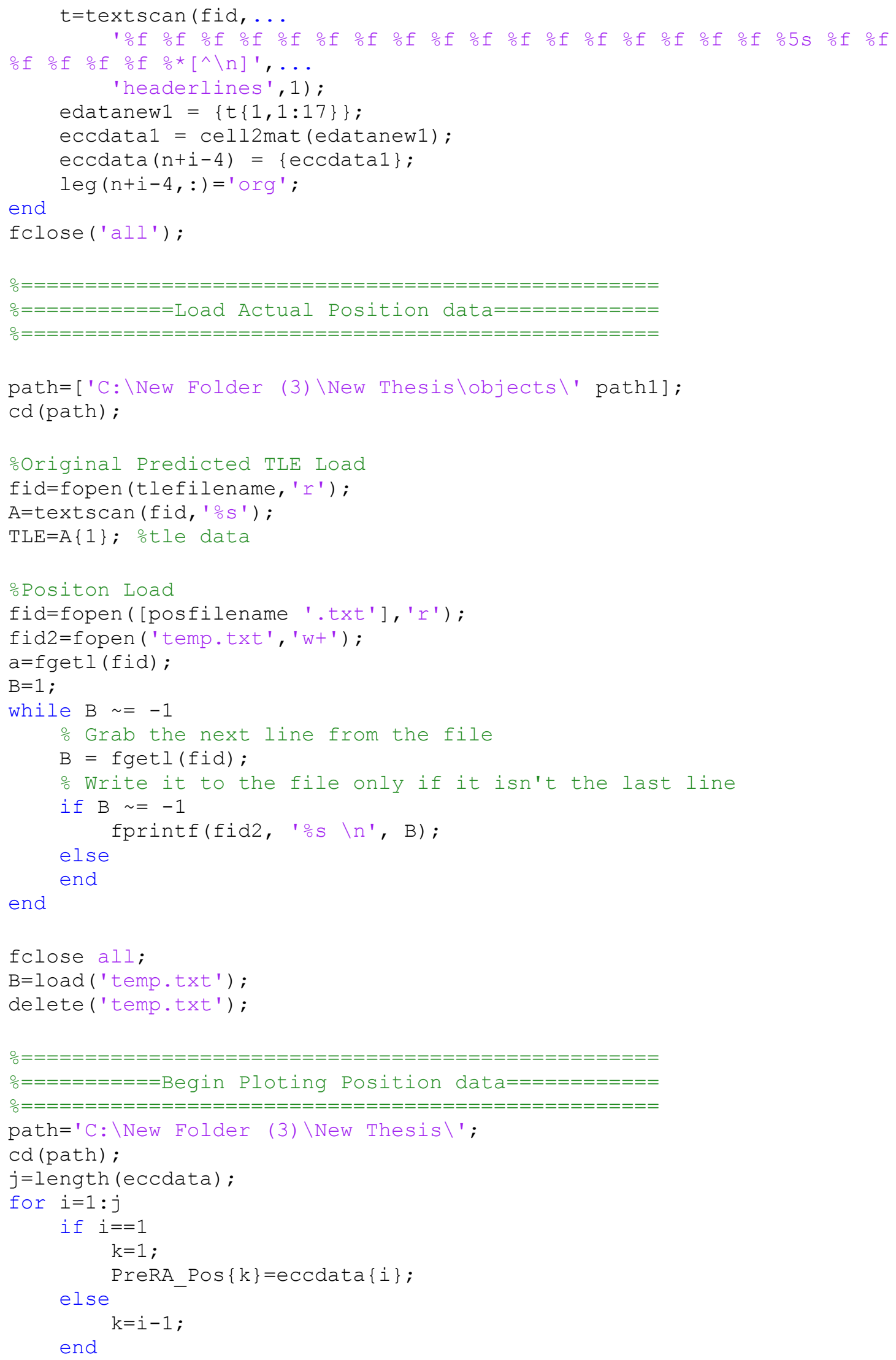




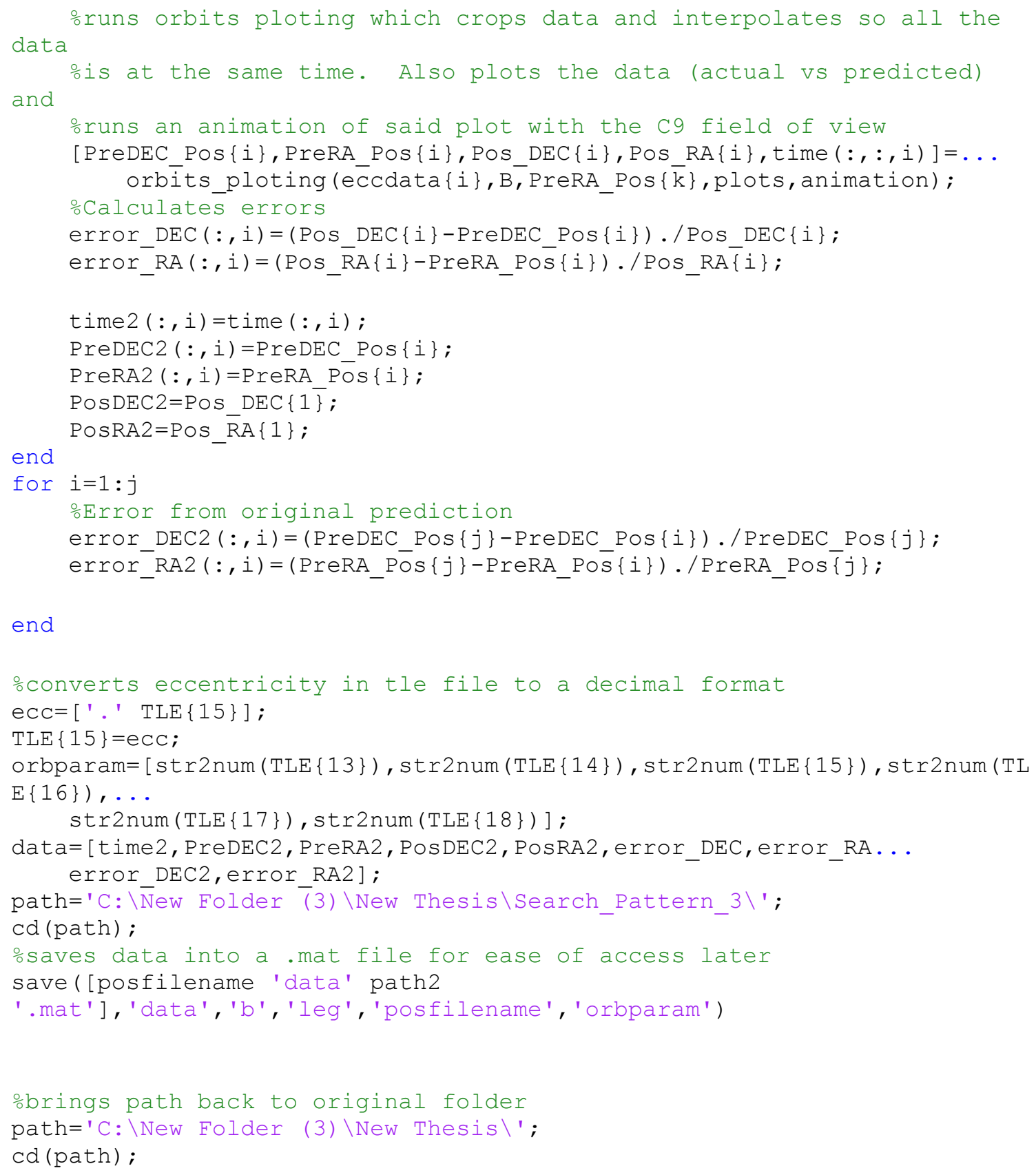




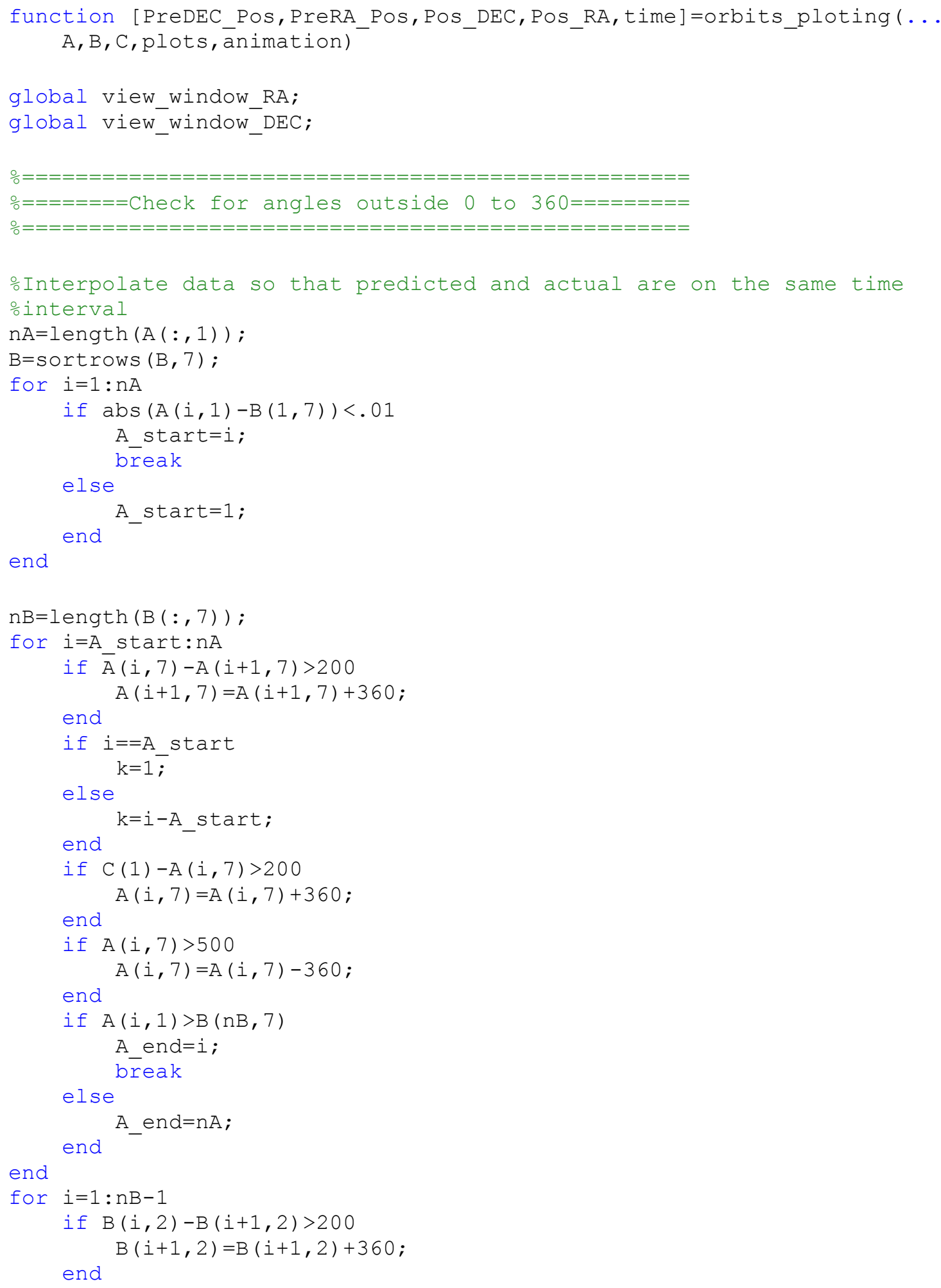




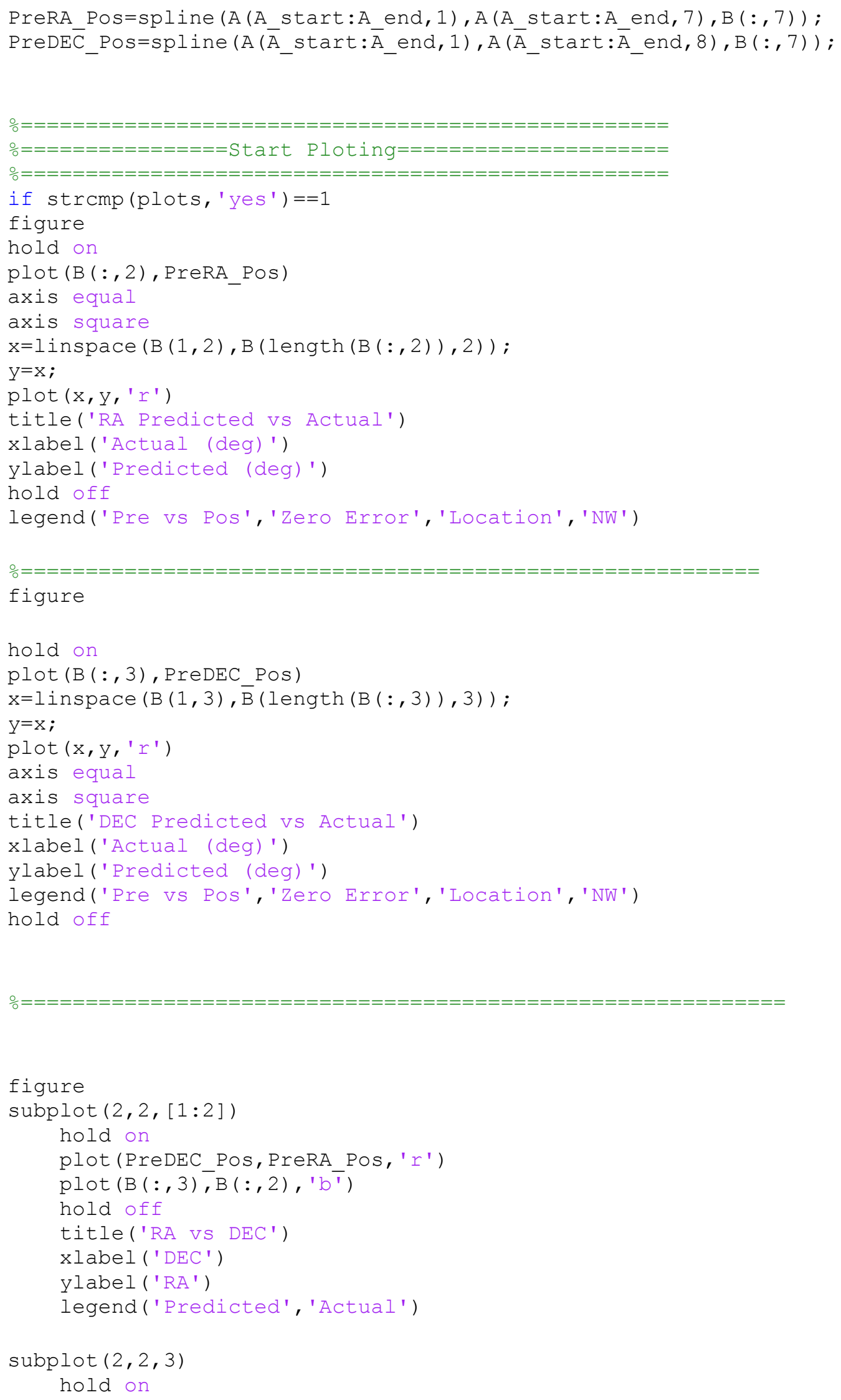




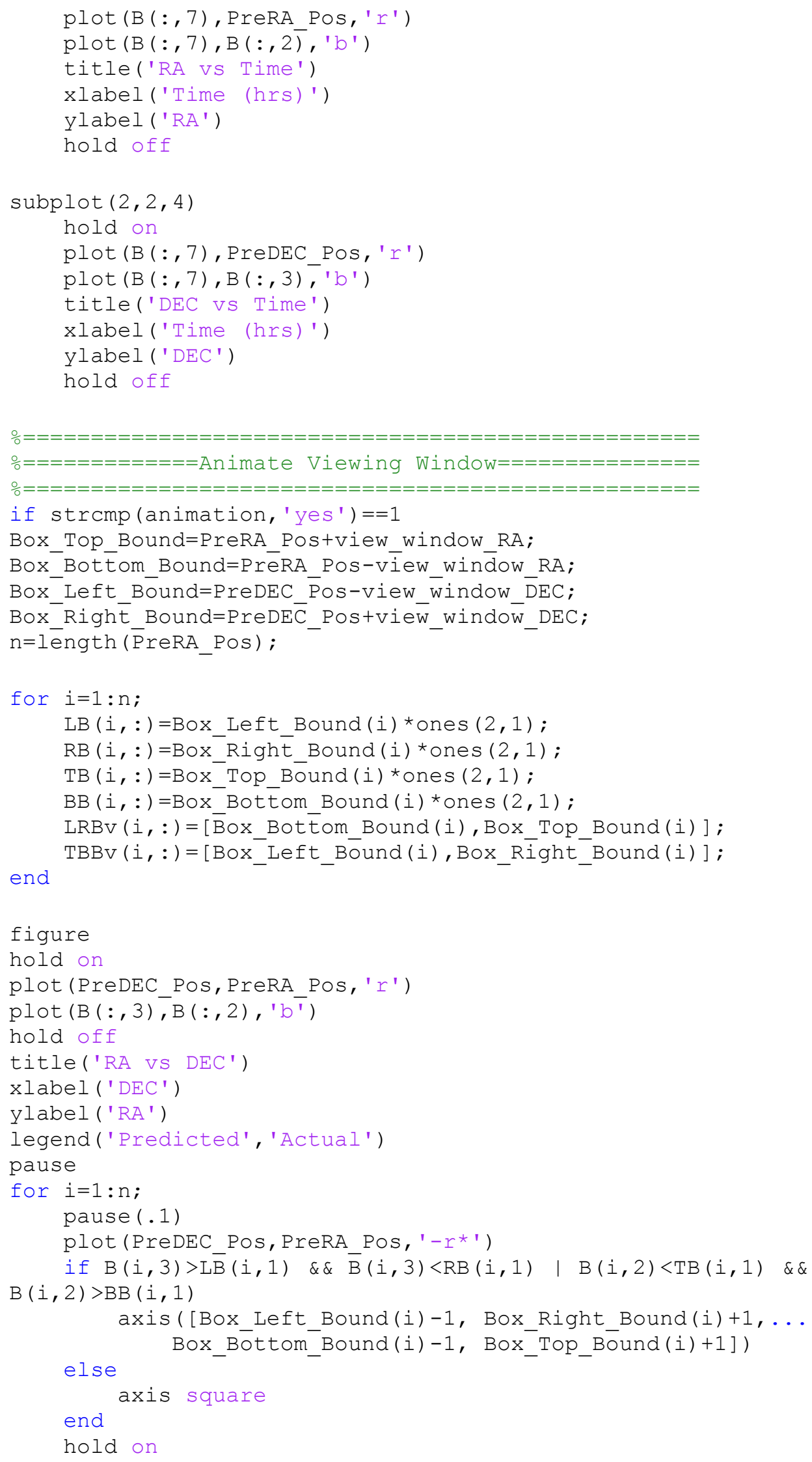




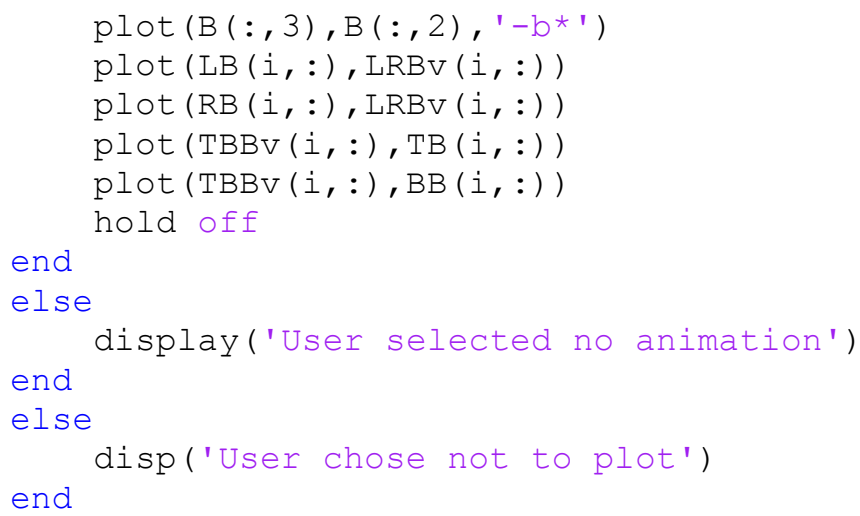




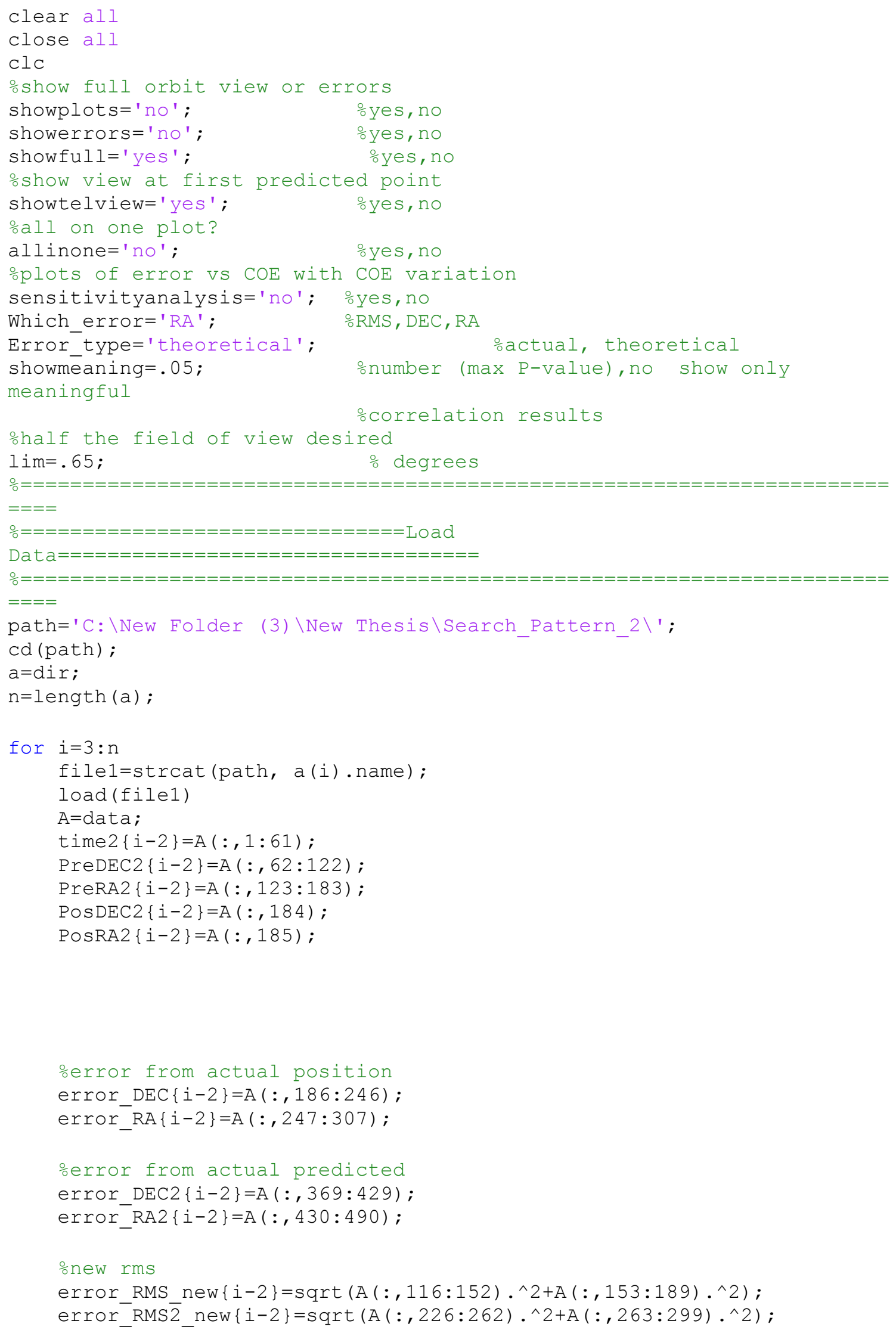


$\mathrm{b} 2\{\mathrm{i}-2\}=\mathrm{b}$;

$\operatorname{leg} 2\{i-2\}=\operatorname{leg} ;$

posfilename $2\{i-2\}=$ posfilename;

orbparam1 (:,i-2)=orbparam' ;

filename $\{i-2\}=a(i)$. name;

end

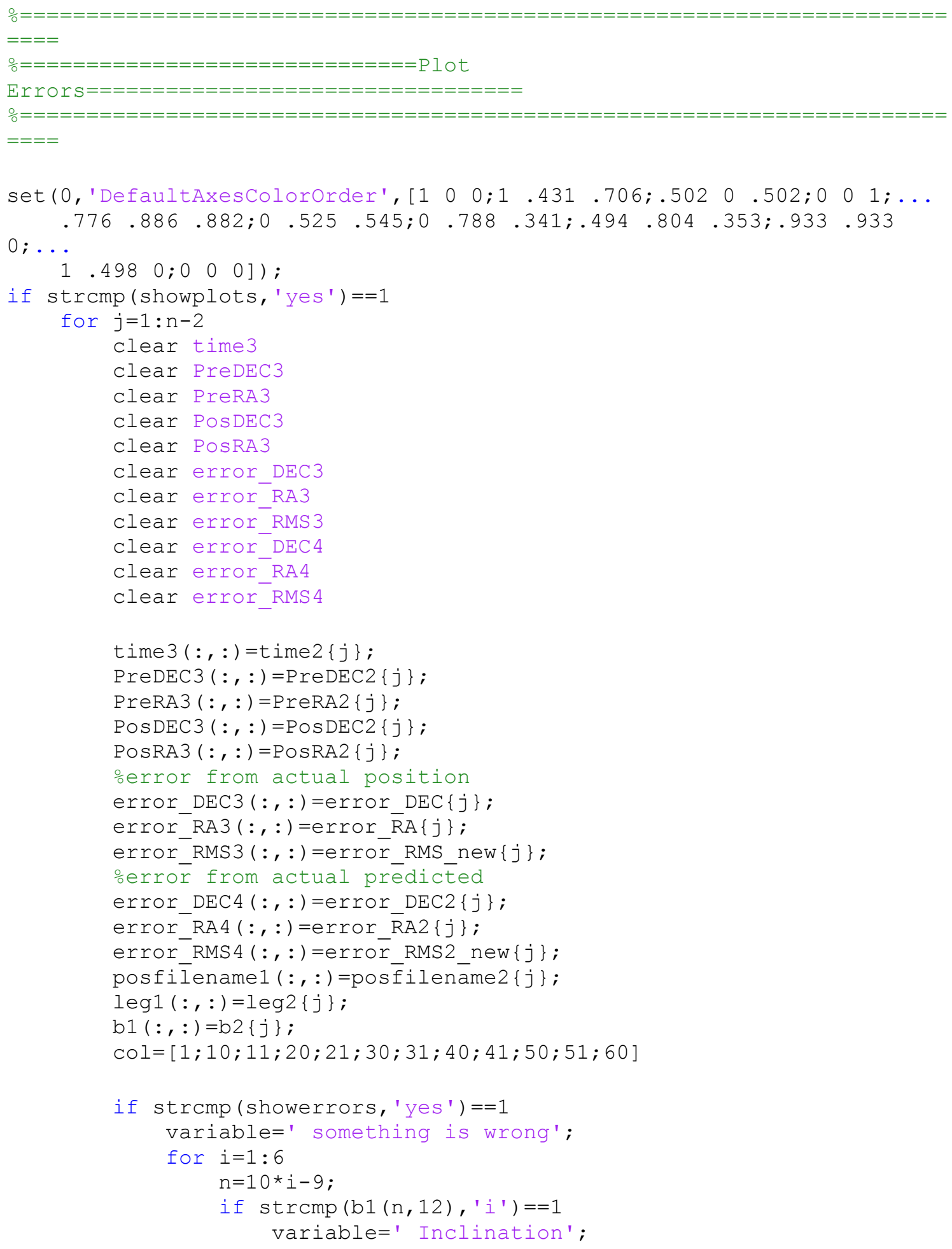




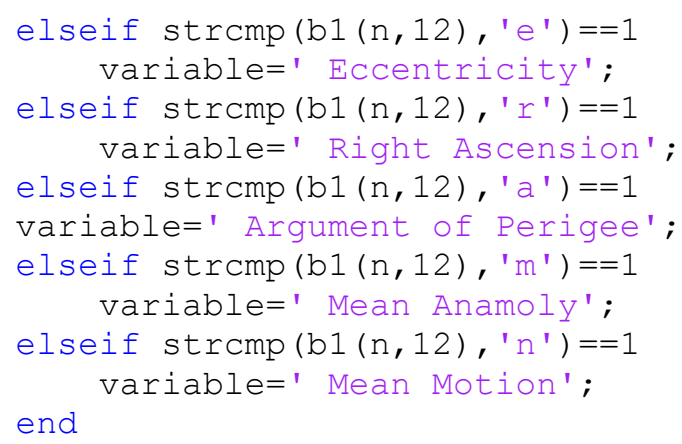




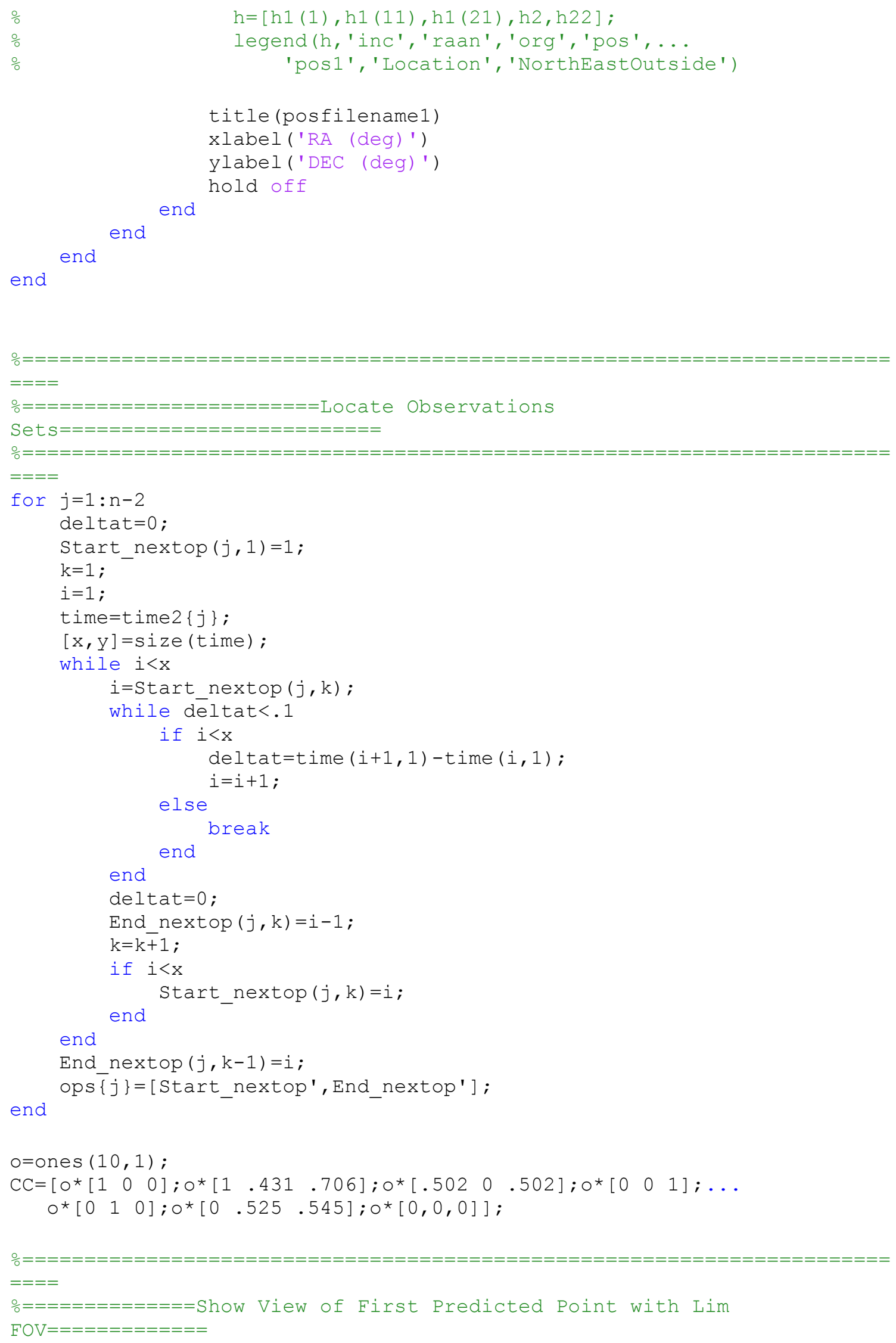




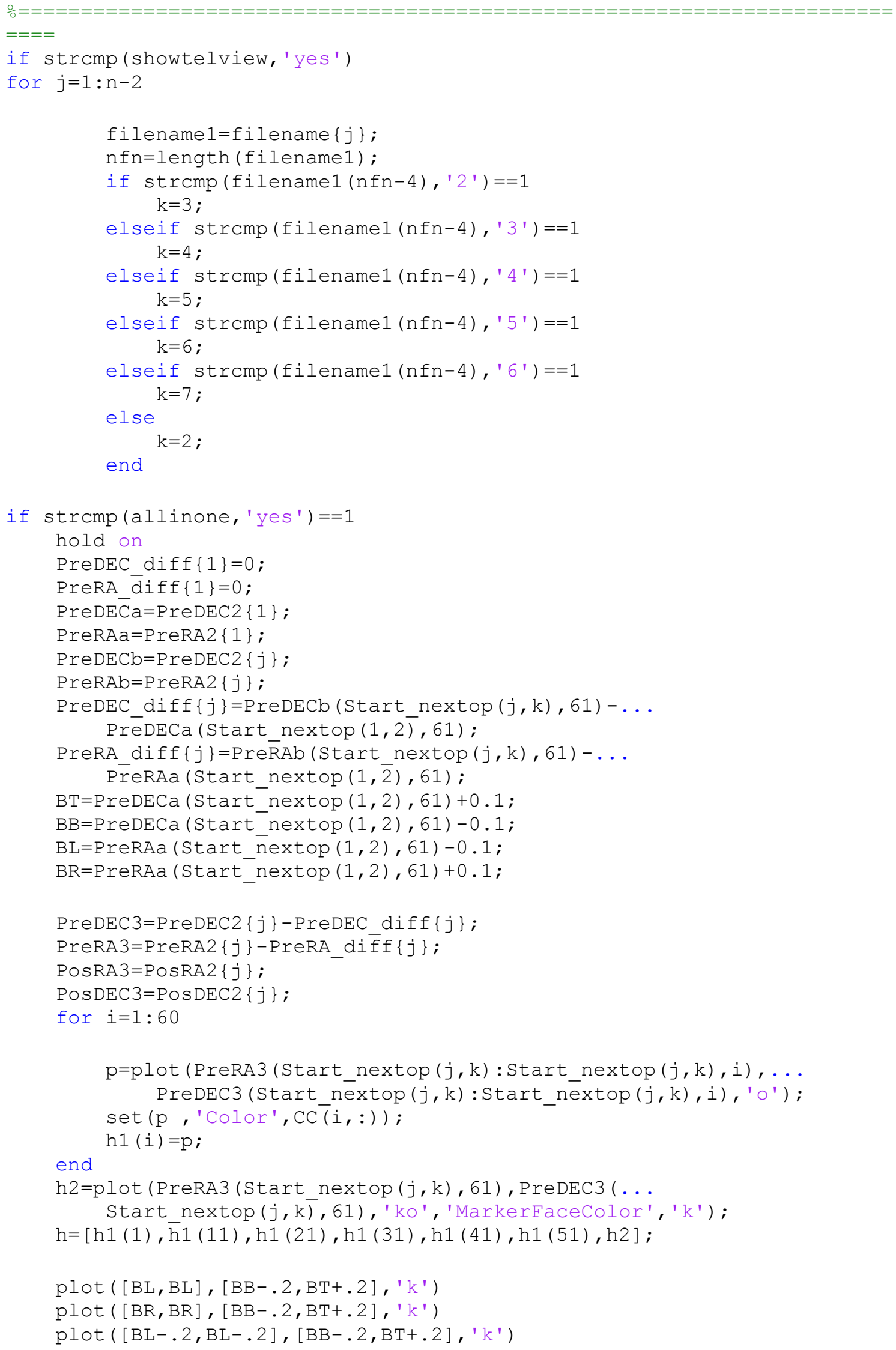




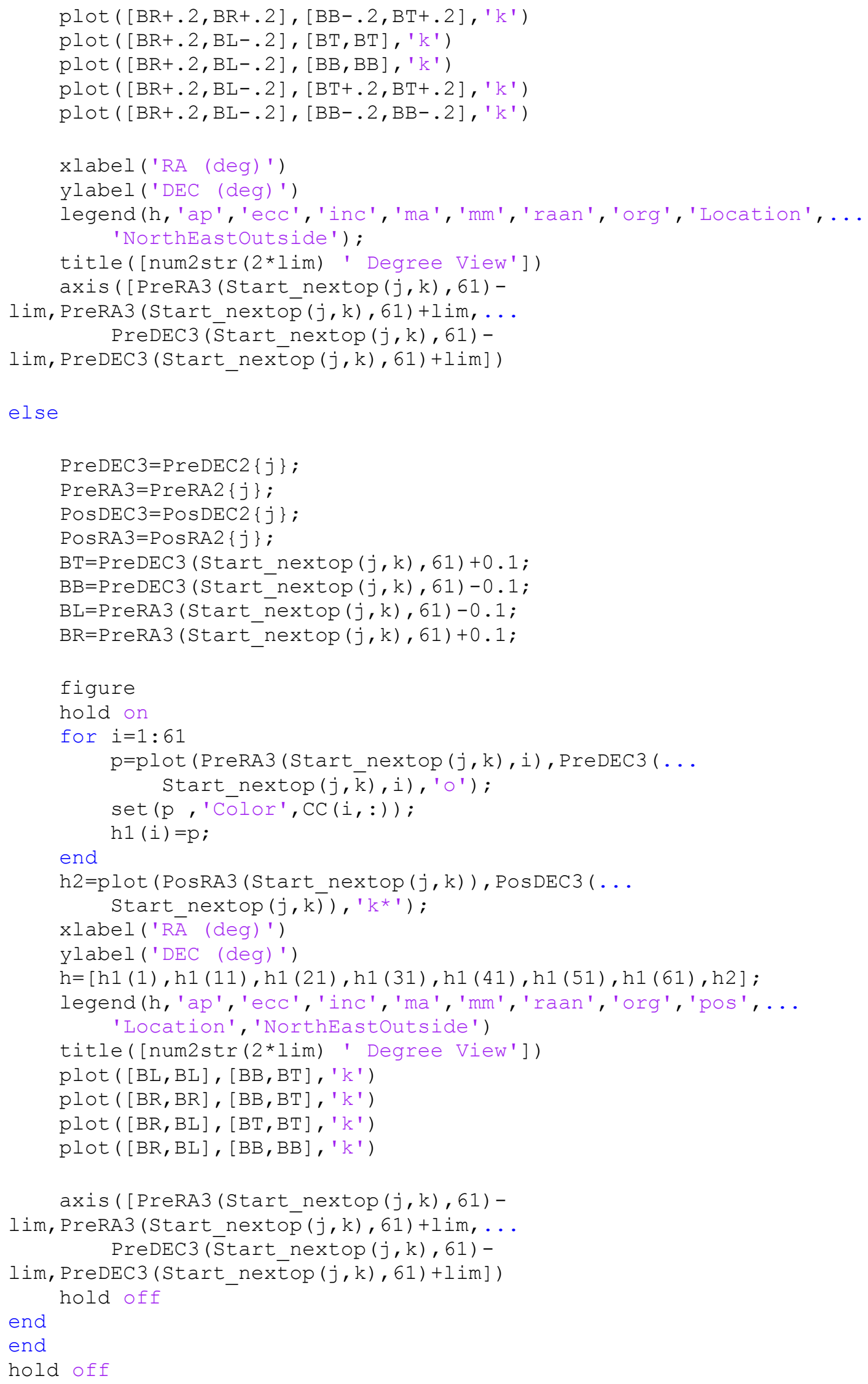


end

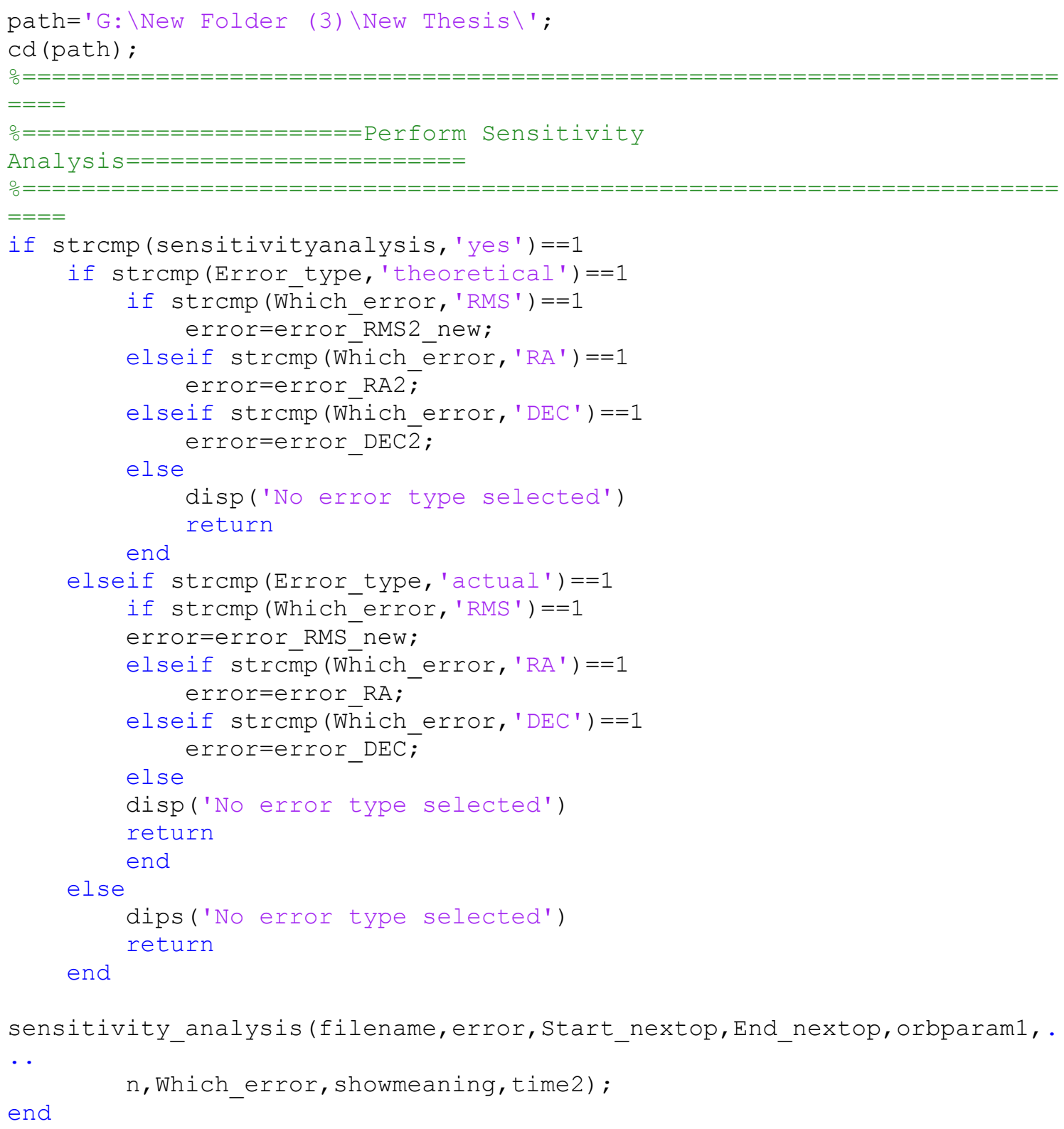




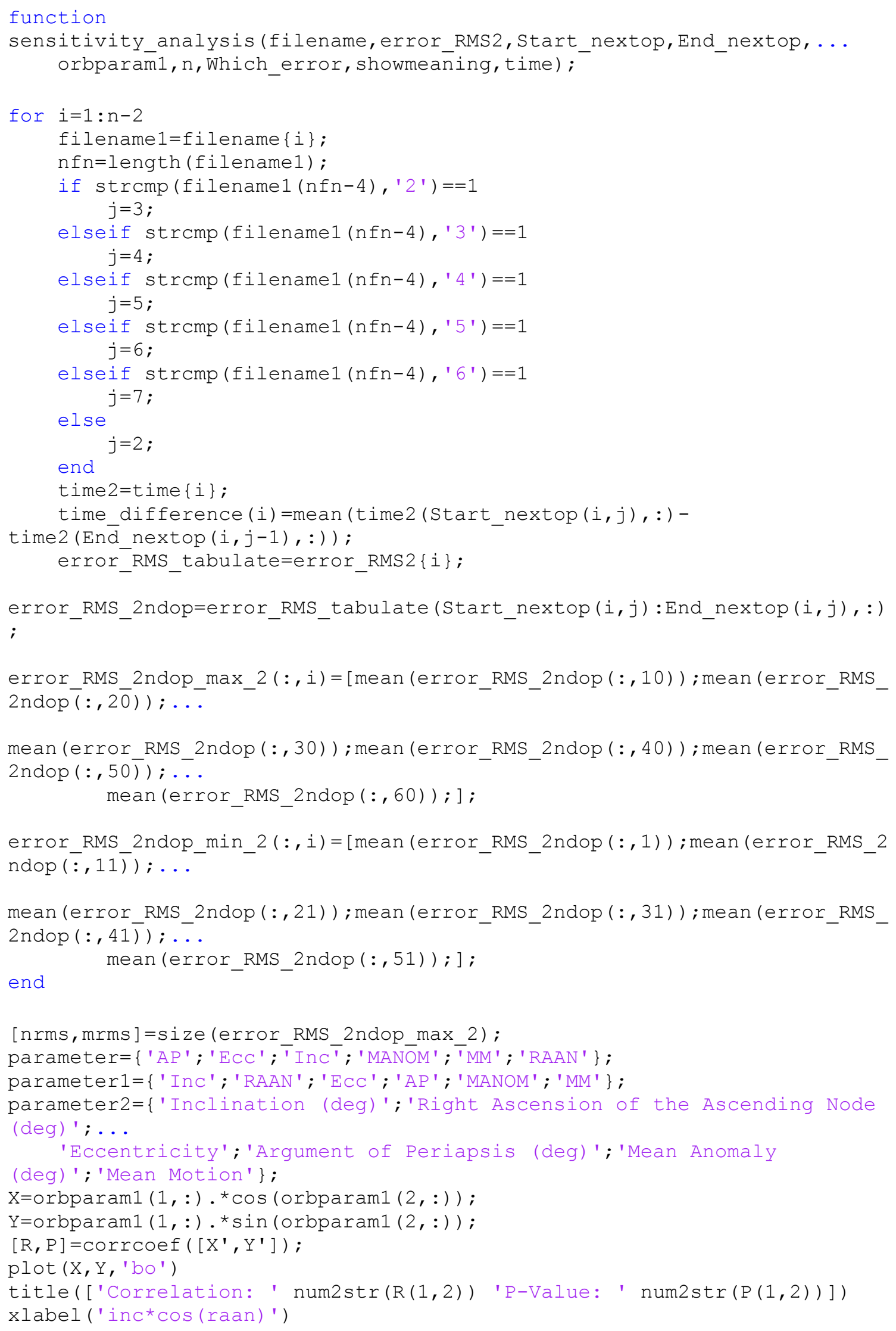




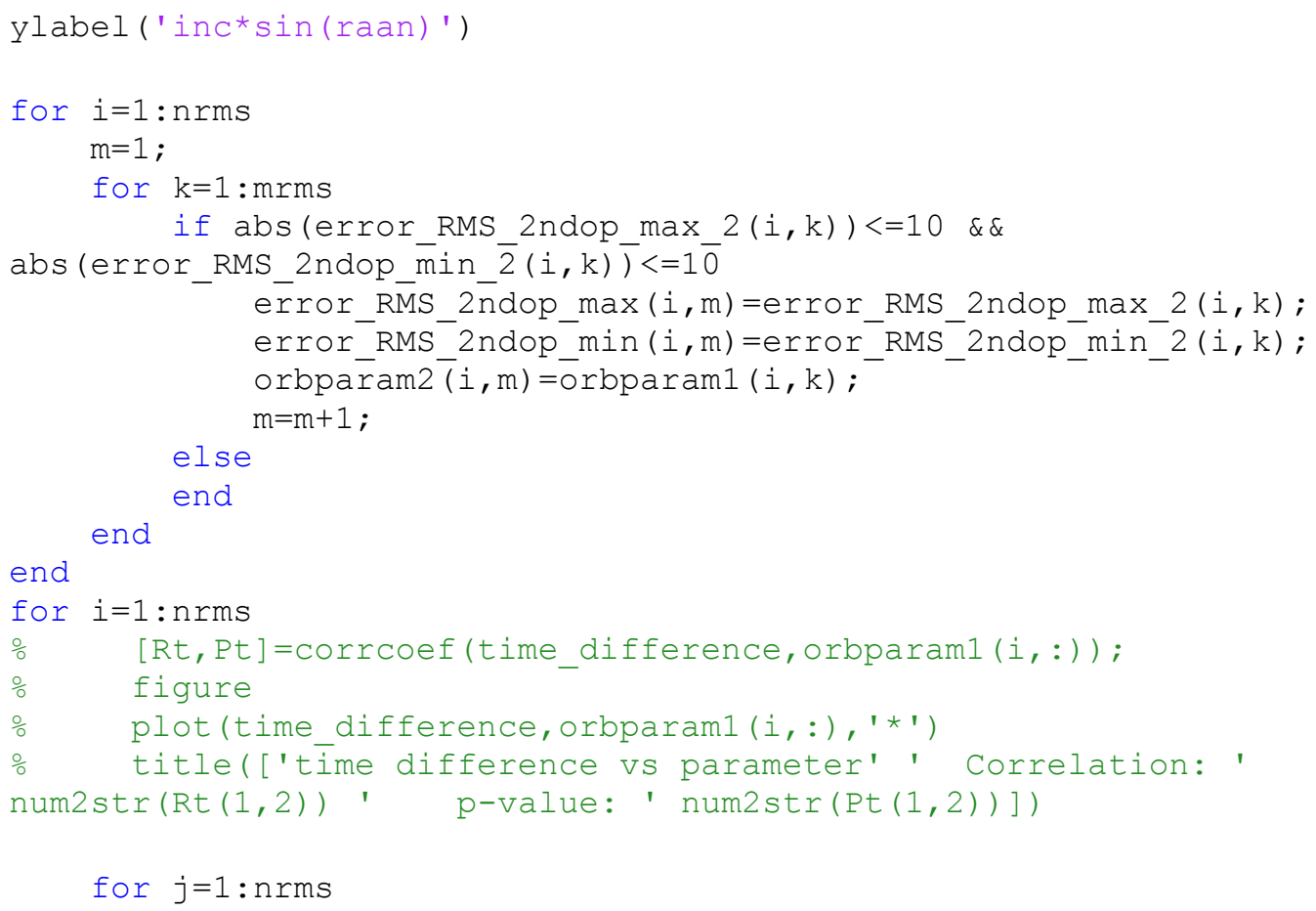


end

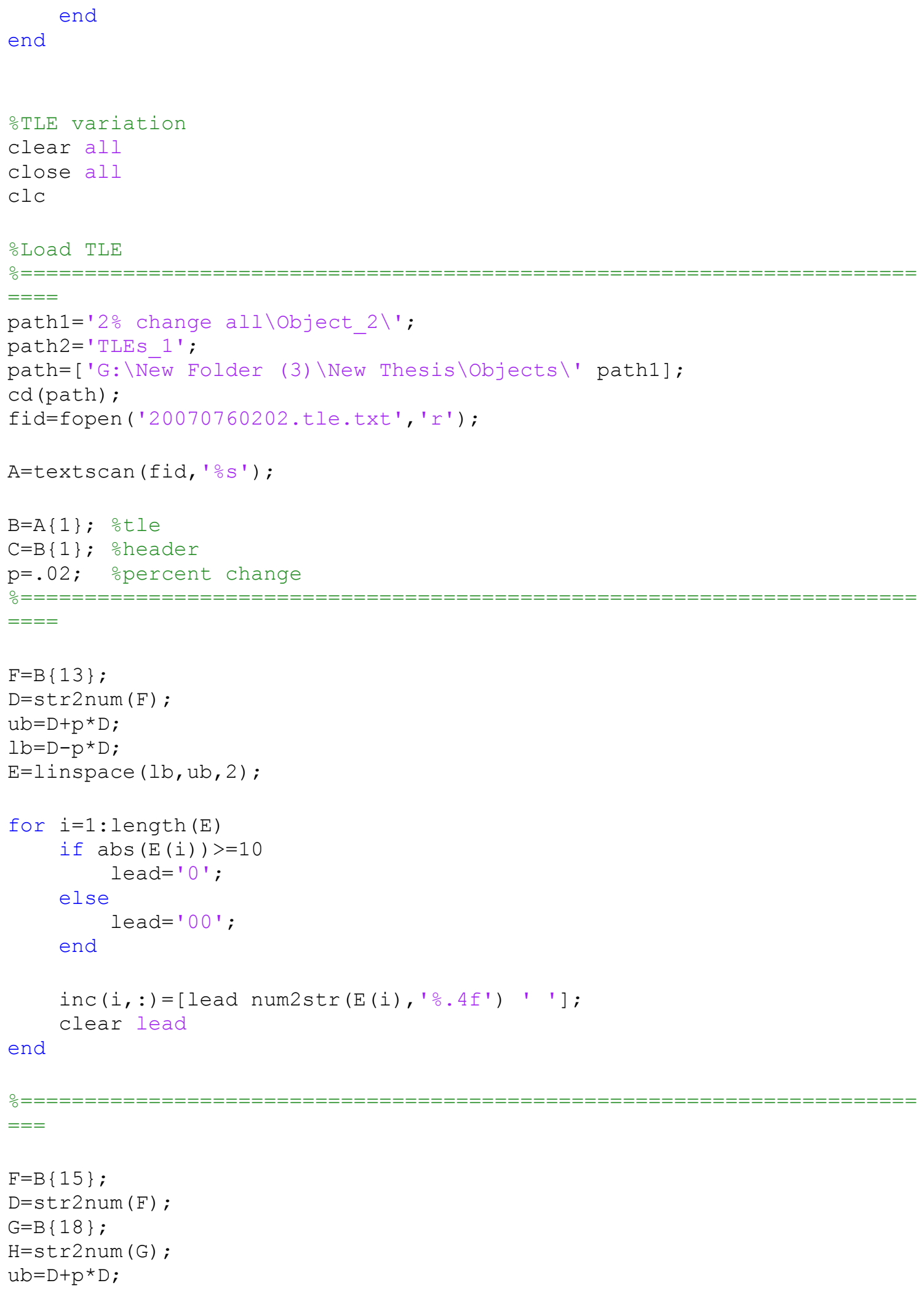




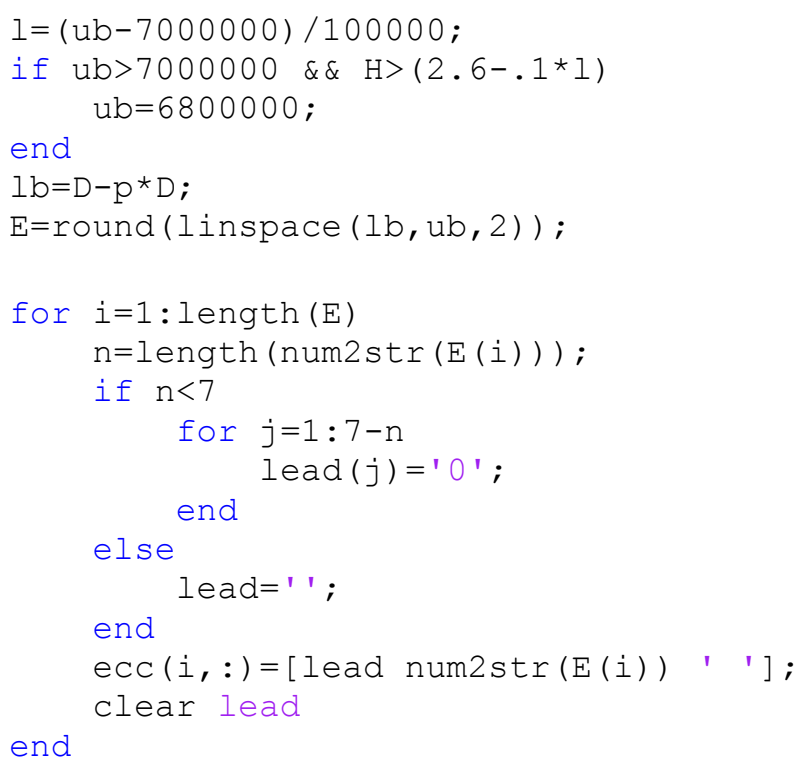




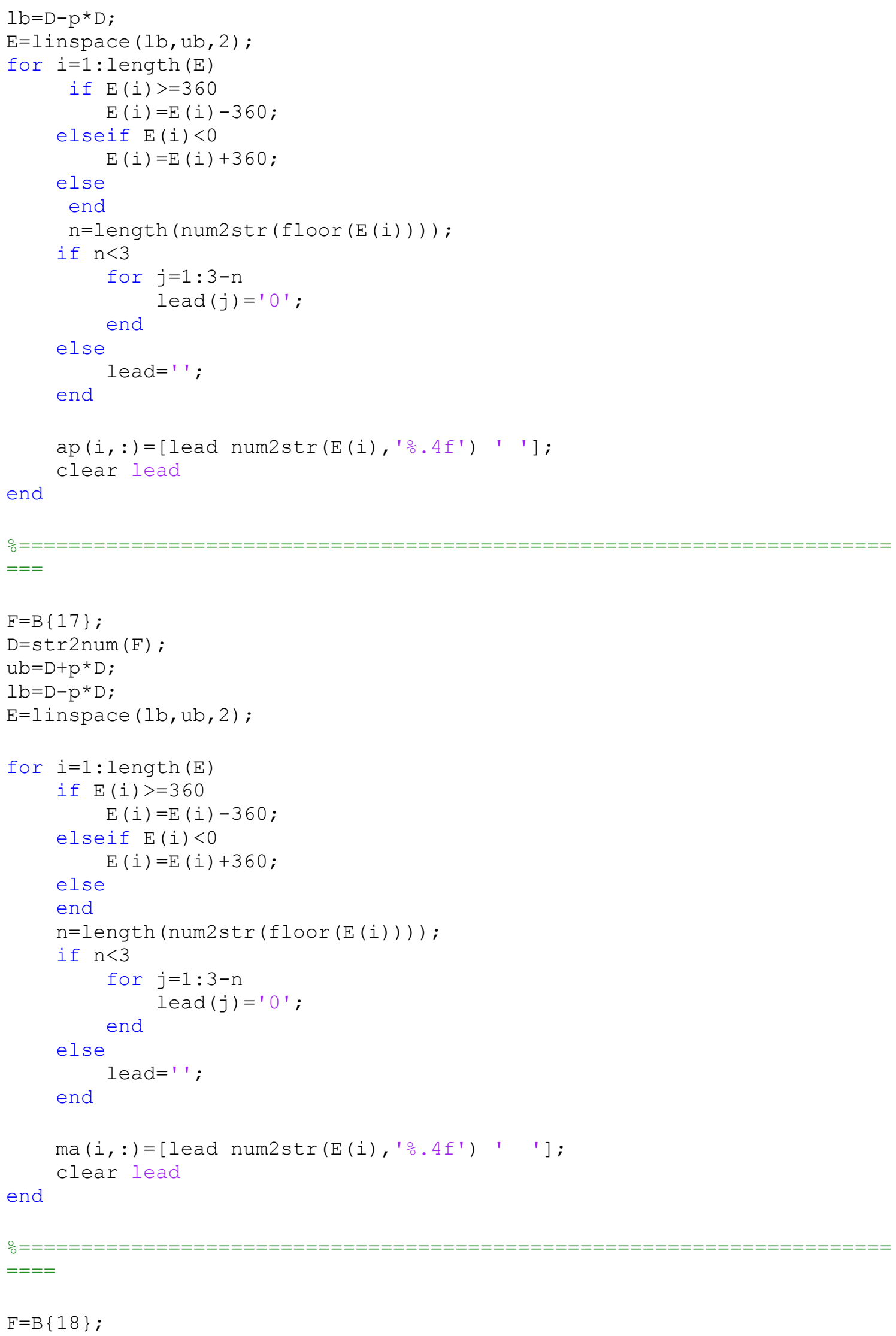




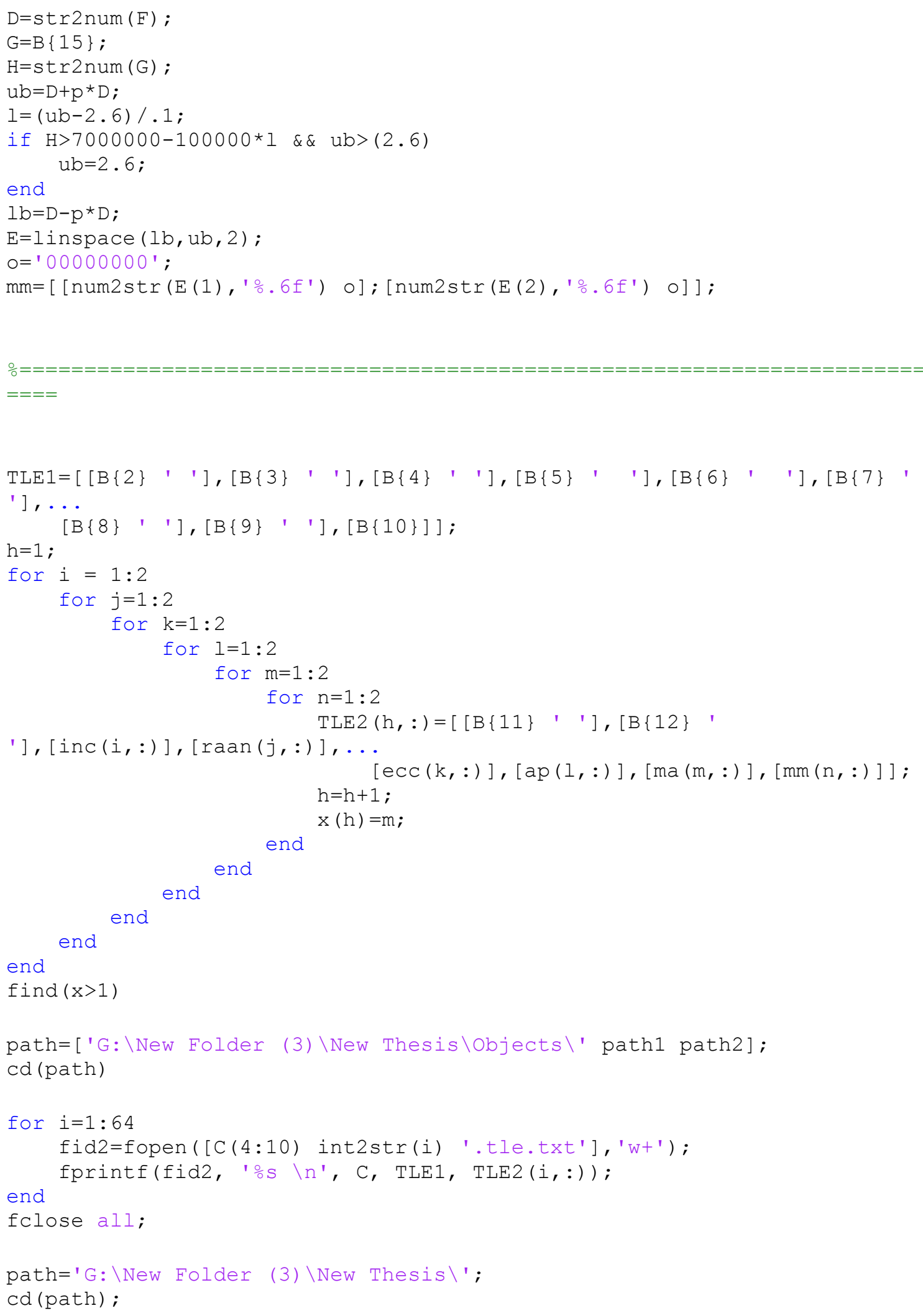




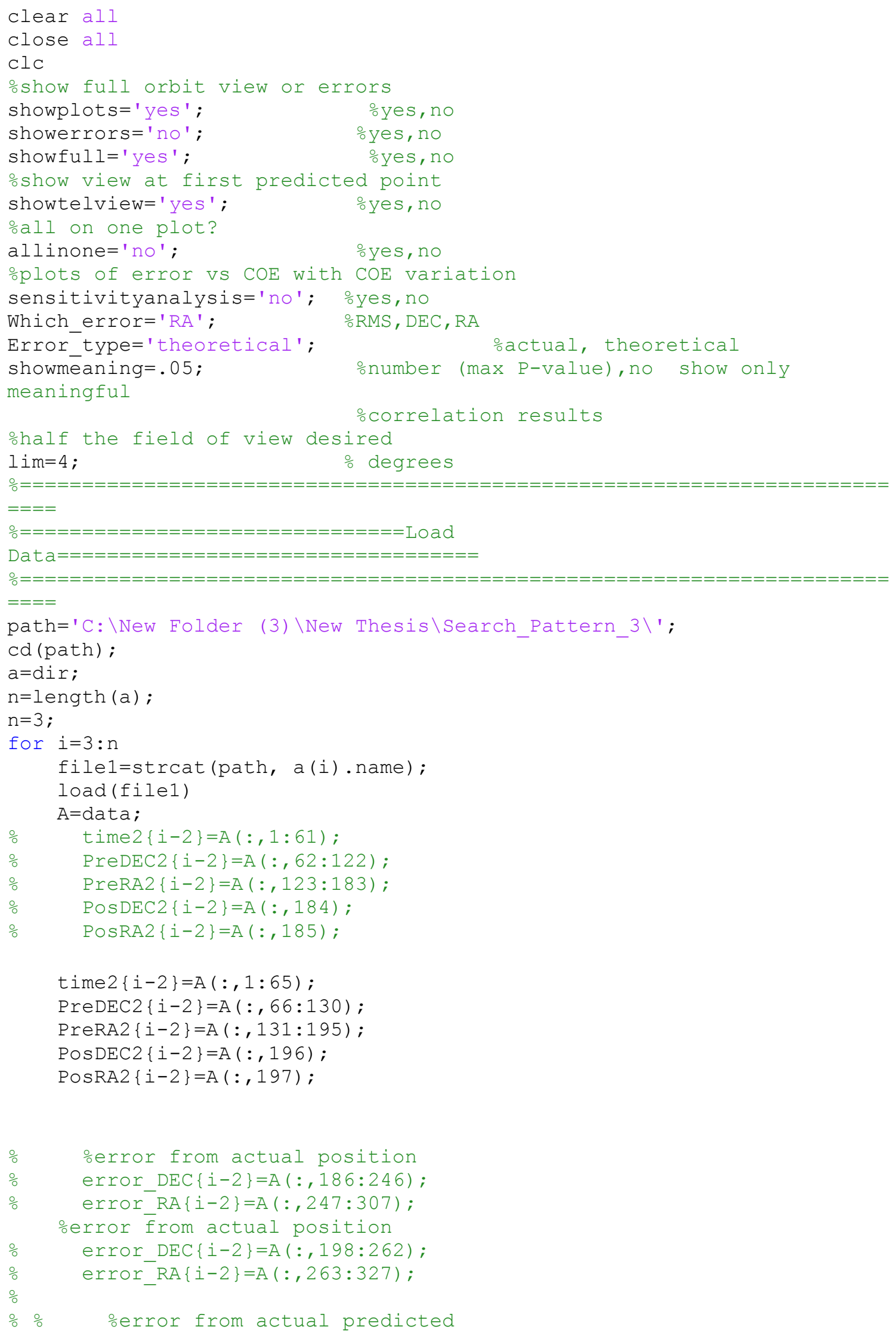




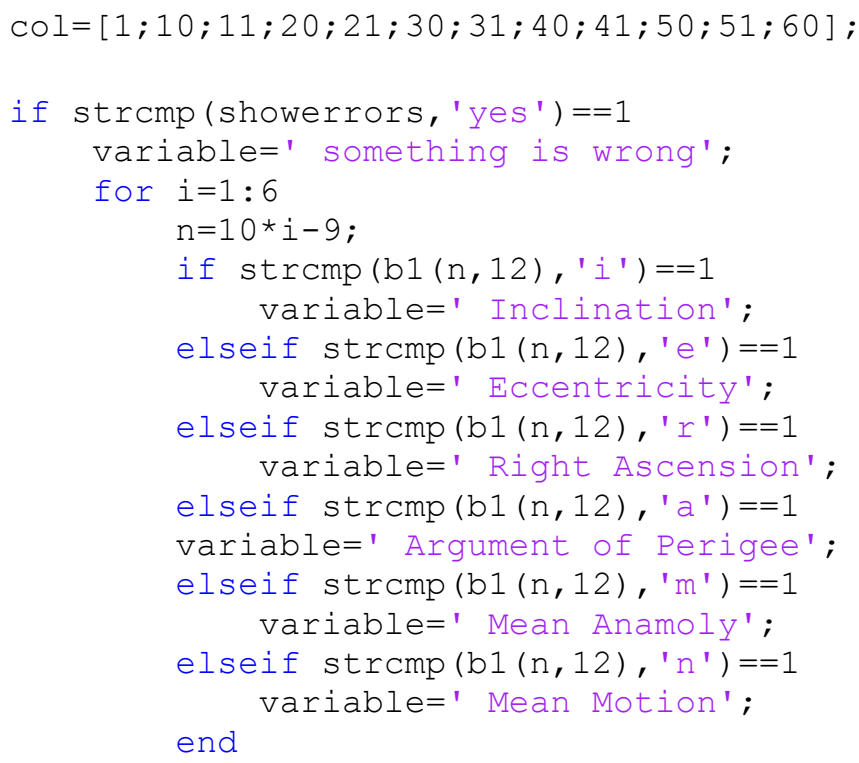


h1=plot (PreRA3, PreDEC3, 'o', 'MarkerSize', 3);

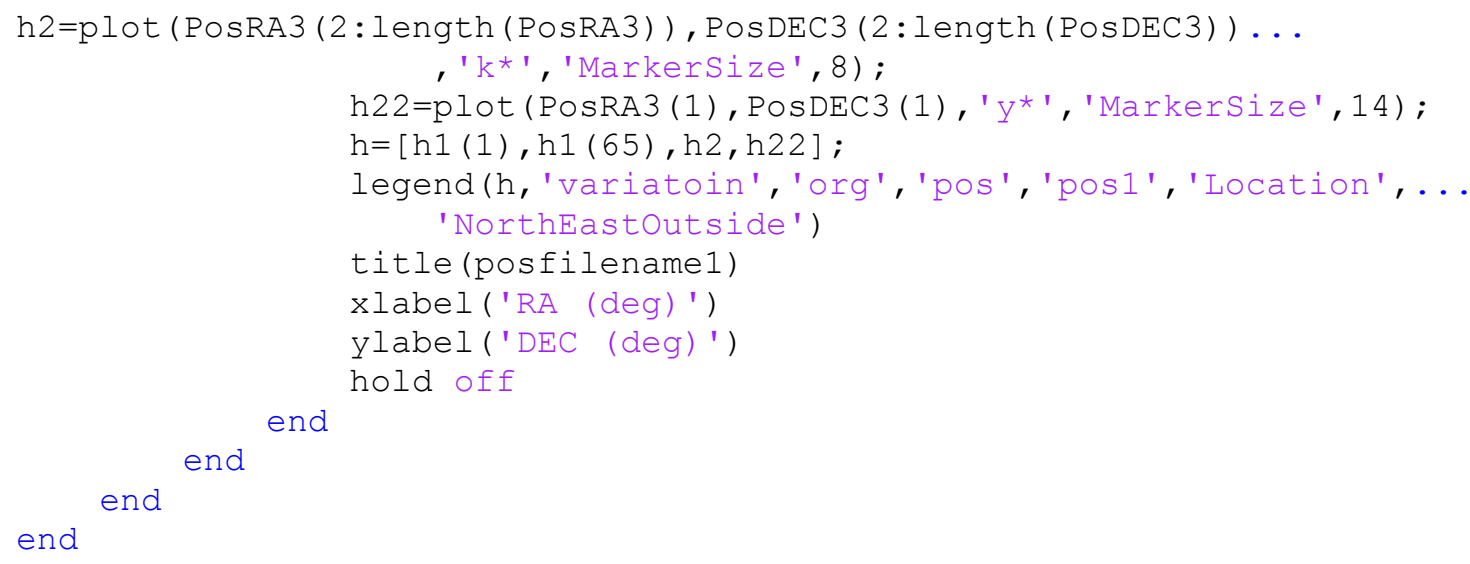

end

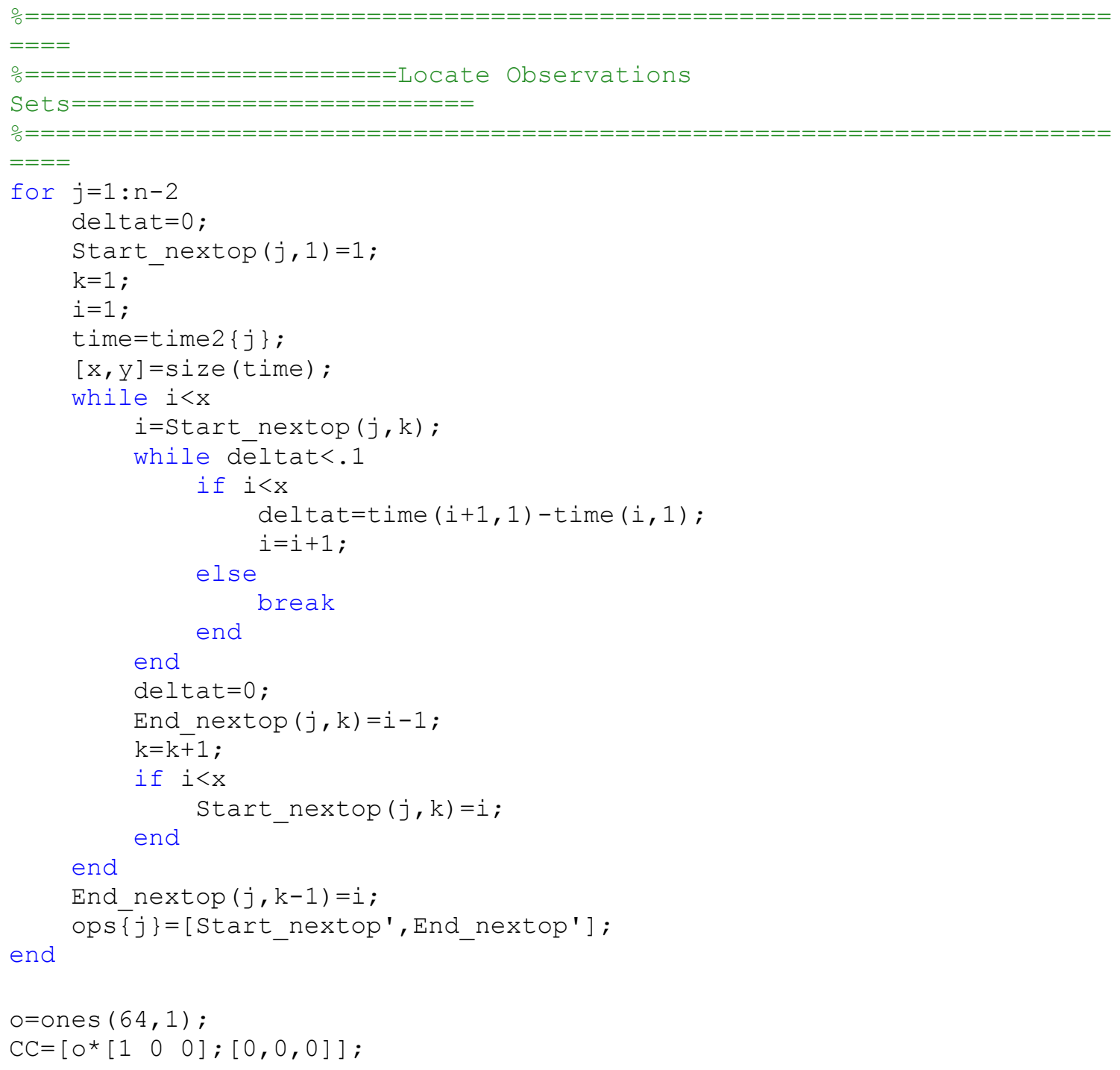




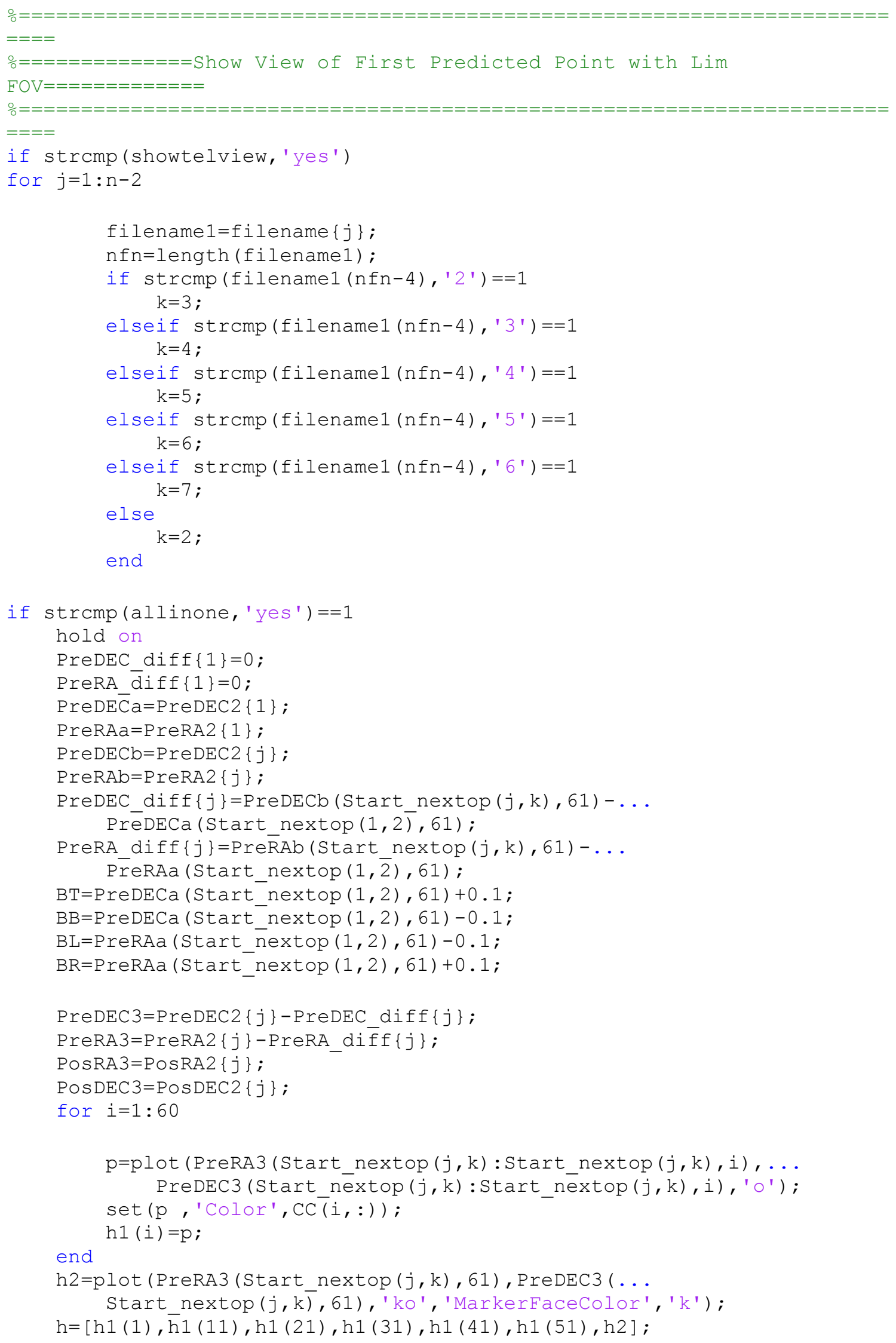




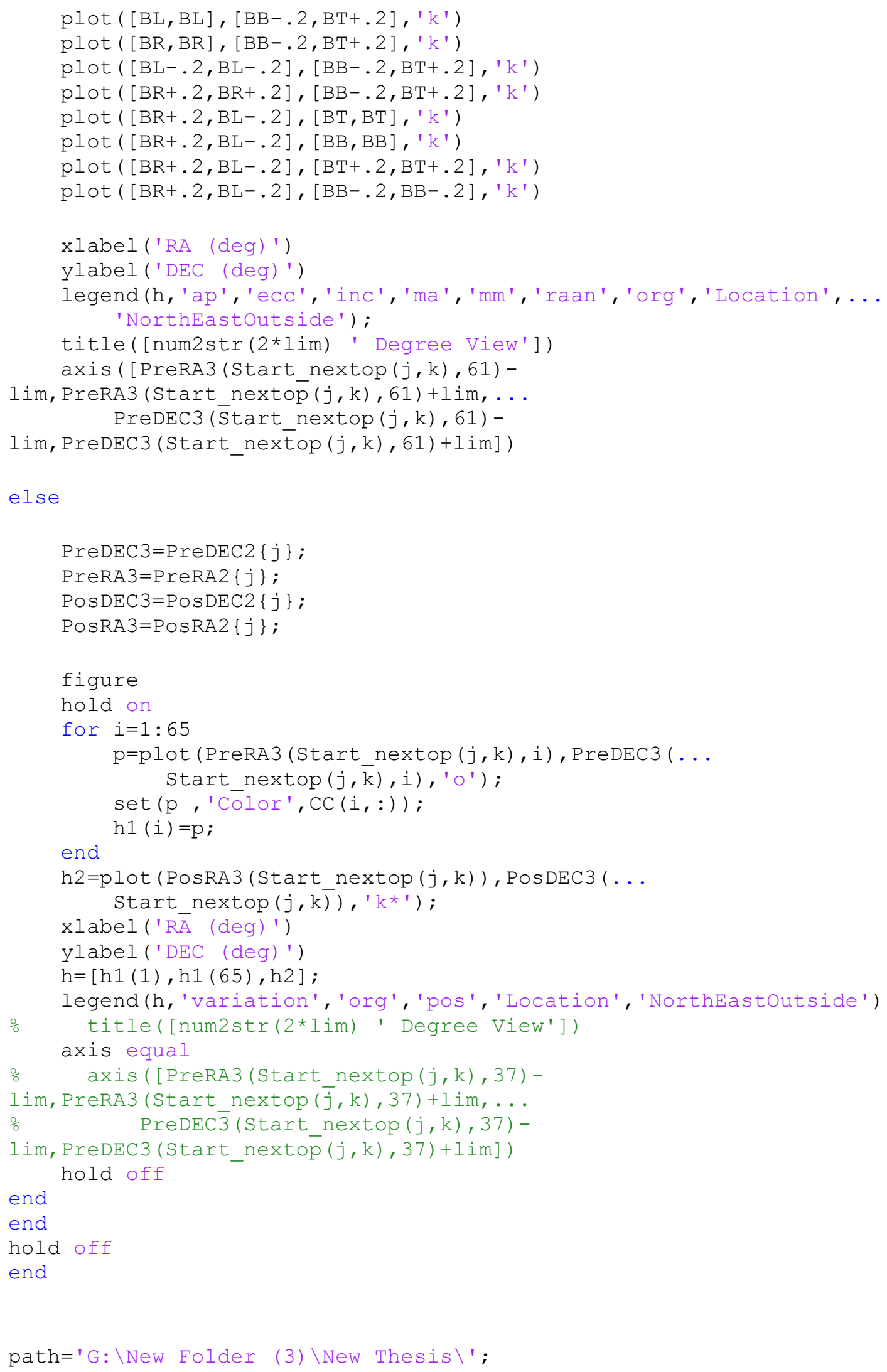




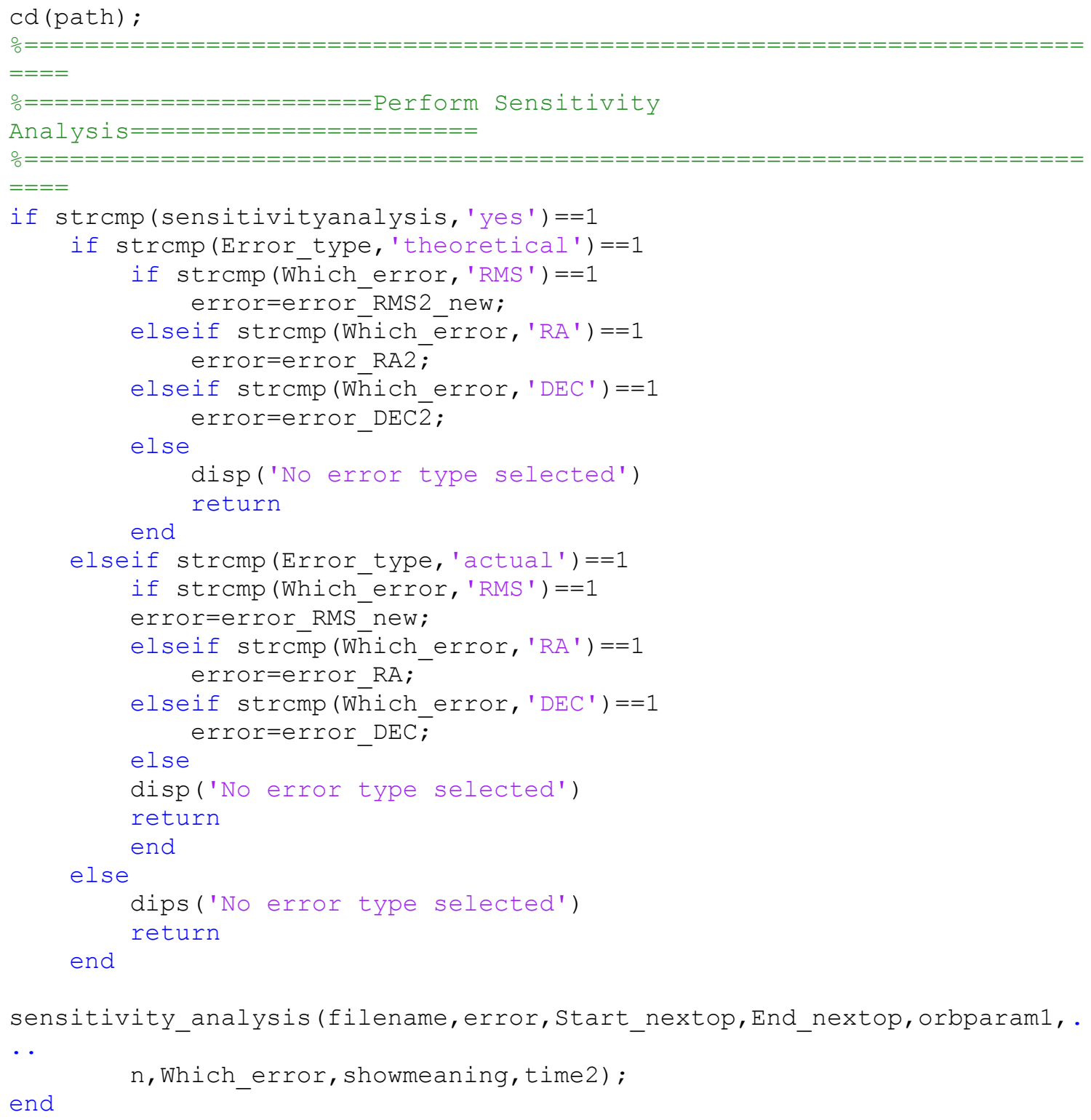

